\title{
Imine or Enamine? Insights and Predictive Guidelines from the Electronic Effect of Substituents in H-Bonded Salicylimines
}

\author{
R. Fernando Martínez,* Esther Matamoros, Pedro Cintas, and Juan C. Palacios* \\ Departamento de Química Orgánica e Inorgánica, Facultad de Ciencias, and IACYS-Unidad de Química Verde y \\ Desarrollo Sostenible, Universidad de Extremadura, E-06006 Badajoz, Spain. \\ *E-mails: rmarvaz@unex.es, palacios@unex.es.
}

\section{Supporting Information}

Content

\author{
Pages Supporting Data \\ S2 Synthesis of Salicyliden Derivatives of TRIS. Table 1S. \\ S5 Structure and Tautomeric Equilibrium in the Solid State. \\ S6 Structure and Tautomeric Equilibrium in Solution. Table 2S. \\ S7 Substituent Effects on the Tautomeric Equilibrium: Predominant Structures in Solution. \\ Schemes $1 \mathrm{~S}$ and $2 \mathrm{~S}$. \\ S8 Electronic Effects of Substituents on Chemical Shifts. Tables 3S-6S and Figures 1S-3S. \\ S12 Electronic Effect of the Substituents on the Tautomeric Equilibrium. Tables 7S-9S and Figures \\ $4 \mathrm{~S}-6 \mathrm{~S}$. \\ S15 Analysis of the Influence of Substituents on Examples Drawn from the Scientific Literature. \\ Tables 10S-14S and Figures 7S-31S. \\ S23 Imine/Enamine-Oxazolidine Equilibria. Schemes 3S, 4S and Figure 32S. \\ S25 Imine-Enamine Tautomerizations. Tables 15S-19S and Figures 33S and 34S. \\ S30 Hydrogen Bonding Analysis. Tables 20S-23S. \\ S32 Pseudo-Aromaticity in H-bonded Tautomeric Salicylimines. Tables 24S-33S and Figures 35S \\ and $36 \mathrm{~S}$. \\ S37 Synthesis of Starting Materials. Scheme 5S. \\ S38 NMR spectra of tautomeric species (only one form shown for clarity). \\ S69 Cartesian coordinates for all optimized structures. Computational calculations at the M06-2X/6- \\ $311++G(d, p)$ level in the gas phase. \\ S123 Cartesian coordinates for all optimized structures. Computational calculations at the M06-2X/6- \\ $311++\mathrm{G}(\mathrm{d}, \mathrm{p})$ level including the solvent effect (SMD model, DMSO as solvent).




\section{Synthesis of Salicyliden Derivatives of TRIS.}

A wide series of Schiff bases 4-33 could easily be obtained by reaction of TRIS whith both monosubstituted (1S-20S) and polysubstituted (21S-30S) salicylaldehydes. Most of such aldehydes were commercially available, although compounds 6S, 11S, and 13S were synthesized (see experimental section).

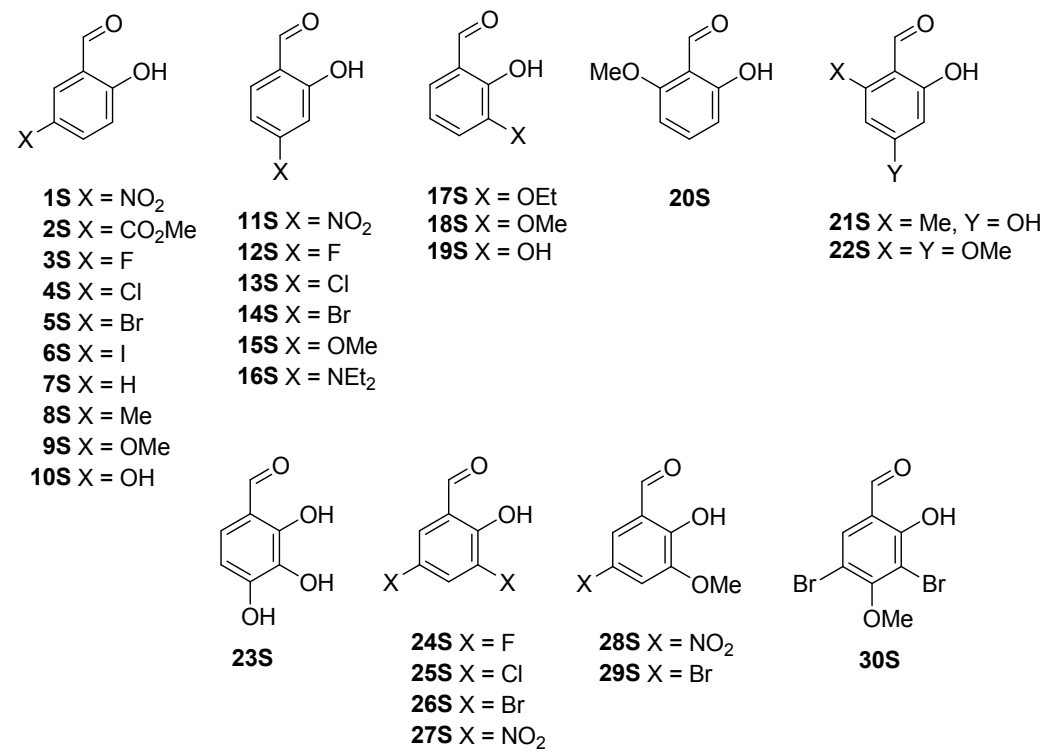

All condensation reactions were conducted by mixing equimolar amounts of the corresponding aldehyde and TRIS in $\mathrm{MeOH}-\mathrm{H}_{2} \mathrm{O}$ at room temperature. Schiff bases 4-33 were separated as insoluble solids in a few min with variable yields, although the procedure has not been optimized. Yields ranged from $98 \%$ for $\mathbf{2 8}$ to $\mathbf{1 8 \%}$ for $\mathbf{2 4}$, most remaining above $50 \%$ (Table $1 \mathrm{~S}$ ).

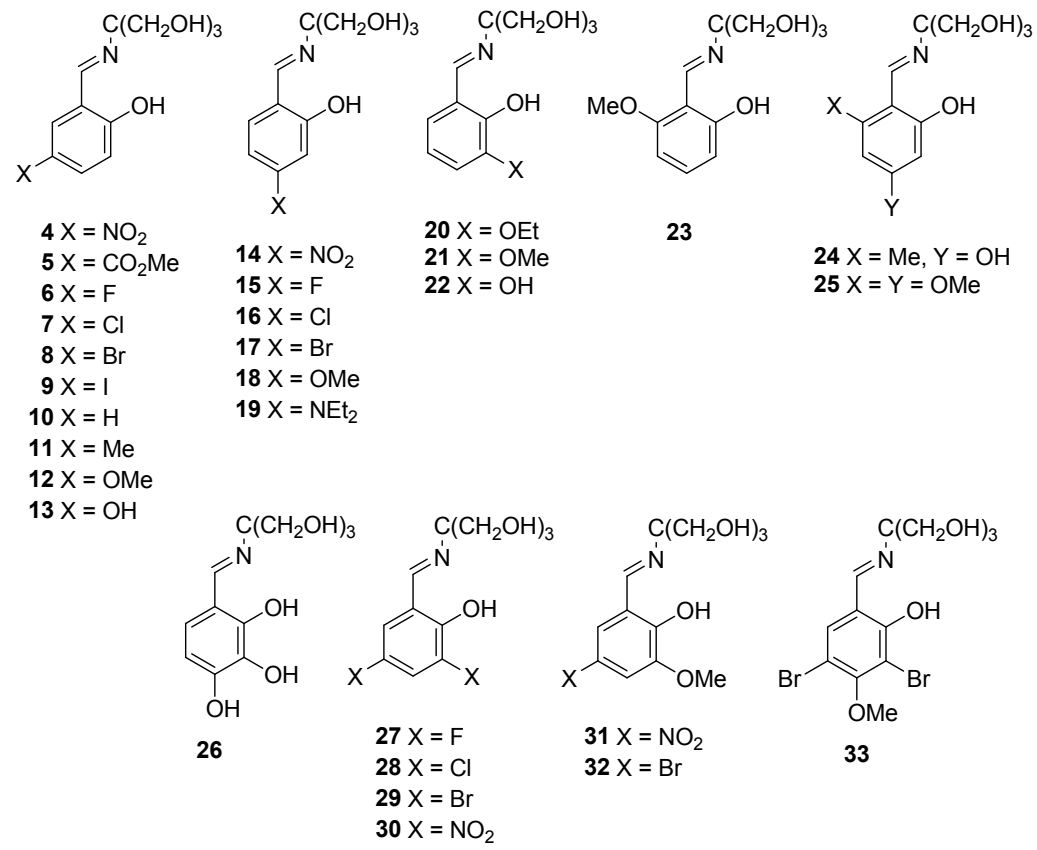




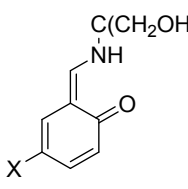

$34 \mathrm{X}=\mathrm{NO}_{2}$

$35 \mathrm{X}=\mathrm{CO}_{2} \mathrm{Me}$

$36 \mathrm{X}=\mathrm{F}$

$37 \mathrm{X}=\mathrm{Cl}$

$38 \mathrm{X}=\mathrm{Br}$

$39 X=1$

$40 \mathrm{X}=\mathrm{H}$

$41 \mathrm{X}=\mathrm{Me}$

$42 \mathrm{X}=\mathrm{OMe}$

$43 \mathrm{X}=\mathrm{OH}$

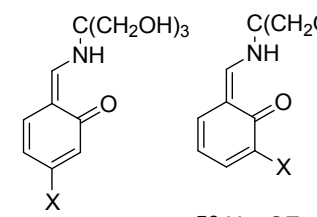

$\begin{array}{ll}44 X=\mathrm{NO}_{2} & \mathbf{5 0} X=\mathrm{OEt}\end{array}$

$45 \mathrm{X}=\mathrm{F} \quad 52 \mathrm{X}=\mathrm{OH}$

$46 \mathrm{X}=\mathrm{Cl}$

$47 \mathrm{X}=\mathrm{Br}$

$48 X=O M e$

$49 \mathrm{X}=\mathrm{NEt}_{2}$

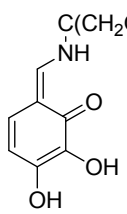

56 
Table 1S. Yields and IR absorptions of 4/34-33/63 and 64/65. ${ }^{\text {a,b }}$

\begin{tabular}{|c|c|c|c|c|c|c|}
\hline Comp. & Yield (\%) & $\mathrm{OH} / \mathrm{NH}$ & $\mathrm{C}=\mathrm{O}$ & $\mathrm{C}=\mathrm{CN}$ & $\mathrm{C}-\mathrm{O}^{\mathrm{c}}$ & $\mathrm{C}-\mathrm{O}^{\mathrm{d}}$ \\
\hline $4 / 34$ & 85 & $3500-2500$ & $1651+$ & & $1347+$ & $\begin{array}{l}1064 \\
1051\end{array}$ \\
\hline $5 / 35$ & 50 & $3400-3100$ & $1635+$ & & $1341-$ & 1054 \\
\hline $6 / 36$ & 72 & $3400-3200$ & $1644+$ & & $1347-$ & 1063 \\
\hline $7 / 37$ & 59 & $3500-2500$ & $1640+$ & & $1328-$ & 1050 \\
\hline $8 / 38$ & 56 & $3500-2600$ & $1638+$ & 1600 & $1325-$ & $\begin{array}{l}1062 \\
1024\end{array}$ \\
\hline 9/39 & 93 & $3500-3000$ & $1636+$ & 1593 & $1326-$ & $\begin{array}{l}1060 \\
1023\end{array}$ \\
\hline $10 / 40$ & 84 & $3400-2500$ & $1636+$ & 1608 & 1336- & $\begin{array}{l}1059 \\
1027\end{array}$ \\
\hline $11 / 41$ & 61 & $3500-3200$ & 1657 & & $1352-$ & 1069 \\
\hline $12 / 42$ & 43 & $3550-2600$ & $1638+$ & & $1331-$ & 1054 \\
\hline $13 / 43$ & 36 & $3500-2000$ & $1646+$ & & $1341-$ & 1035 \\
\hline $14 / 44$ & 55 & $3400-3100$ & 1648 & & $1349+$ & 1068 \\
\hline $15 / 45$ & 84 & $3300-3100$ & $1633+$ & & $1347-$ & 1054 \\
\hline $16 / 46$ & 72 & $3500-3100$ & $1647+$ & & $1340-$ & 1073 \\
\hline $17 / 47$ & 91 & $3500-3100$ & $1641+$ & 1596 & $1366-$ & 1049 \\
\hline $18 / 48$ & 50 & $3400-2400$ & $1633+$ & 1613 & $\begin{array}{l}1357- \\
1325-\end{array}$ & $\begin{array}{l}1036 \\
1020\end{array}$ \\
\hline $19 / 49$ & 70 & $3500-2500$ & $1630+$ & 1600 & $1345-$ & $\begin{array}{l}1058 \\
1043\end{array}$ \\
\hline $20 / 50$ & 87 & $3500-2400$ & 1629 & 1608 & 1373- & 1030 \\
\hline $21 / 51$ & 64 & $3400-2700$ & 1644 & 1613 & $\begin{array}{c}1372 \\
1344-\end{array}$ & $\begin{array}{l}1071 \\
1023\end{array}$ \\
\hline $22 / 52$ & 89 & $3500-2400$ & $1640+$ & & $1356-$ & 1038 \\
\hline $23 / 53$ & 91 & $3400-3100$ & $1631+$ & & $1351+$ & 1057 \\
\hline $24 / 54$ & 18 & $3500-2400$ & $1626+$ & $\begin{array}{c}1594+ \\
1567\end{array}$ & $1356+$ & $\begin{array}{l}1058 \\
1025\end{array}$ \\
\hline $25 / 55$ & 77 & $3300-3100$ & $1633+$ & & $1346+$ & 1053 \\
\hline $26 / 56$ & 62 & $3500-2400$ & $1634+$ & & 1327 & $\begin{array}{l}1051 \\
1036\end{array}$ \\
\hline $27 / 57$ & 74 & $3400-3200$ & 1645 & & $1359-$ & 1037 \\
\hline $28 / 58$ & 98 & $3500-2500$ & $1642+$ & 1597 & $1340-$ & 1063 \\
\hline 29/59 & 91 & $3400-3100$ & $1640+$ & & $1360-$ & 1062 \\
\hline $30 / 60$ & 76 & $3600-2500$ & $1644+$ & 1620 & $1374-$ & $\begin{array}{l}1072 \\
1051\end{array}$ \\
\hline $31 / 61$ & 75 & $3500-3100$ & $1641+$ & 1608 & $1347+$ & 1048 \\
\hline $32 / 62$ & 57 & $3400-3100$ & $1644+$ & 1598 & $1334-$ & 1023 \\
\hline $33 / 63$ & 87 & $3500-2700$ & $1641+$ & 1590 & $1340-$ & $\begin{array}{l}1052 \\
1016\end{array}$ \\
\hline $64 / 65$ & 70 & $3400-2500$ & $1636+$ & 1608 & 1336 & $\begin{array}{l}1059 \\
1027 \\
\end{array}$ \\
\hline
\end{tabular}

${ }^{\mathrm{a}}$ In $\mathrm{KBr} .{ }^{\mathrm{b}}+$ and - are indicative of high and low intensity, respectively. ${ }^{\mathrm{c}}$ Ar-O bond. ${ }^{\mathrm{d}}$ Bond of aliphatic alcoholic groups.

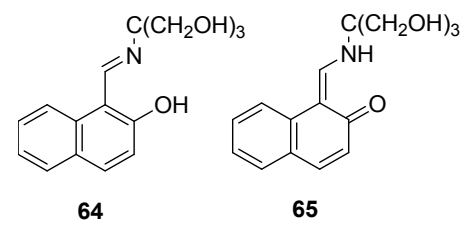




\section{Structure and Tautomeric Equilibrium in the Solid State.}

Some salicylaldehyde derivatives have been previously prepared and their solid state structures studied by X-ray diffractometry. Compound $\mathbf{1 0}$ has been a recurring example with contradictory results. Thus, Chumakov et al. ${ }^{1 a}$ found two molecules in the unit cell at $292 \mathrm{~K}$, one with imine structure and the other as enamine. However, Rao et al. ${ }^{1 \mathrm{~b}}$ and Cungen et al. ${ }^{1 \mathrm{c}}$ only found a molecule with enamine structure at $298 \mathrm{~K}(\mathbf{4 0})$, which is the same result as others reported at $208 \mathrm{~K} .{ }^{1 \mathrm{~d}}$ For compound $7^{\text {1a }}$ two molecules were found in the crystal lattice at $293 \mathrm{~K}$, both having however an enamine structure (37). Likewise, compounds $4^{\text {le }}$ and $8^{\text {le }}$ have been described as enamine tautomers in crystalline state at $293 \mathrm{~K}$ (i.e. 34 and 38, respectively); although we found a zwitterionic structure for 8 and 9 at $120 \mathrm{~K}^{2}{ }^{2}$ The structures of 21 at $213 \mathrm{~K}$ and $\mathbf{2 2}$ at $223 \mathrm{~K}$, are also consistent with enamine tautomers (51 and $\mathbf{5 2}$, respectively). ${ }^{1 \mathrm{~d}} \mathrm{In}$ addition, for the naphthylderivative 64, from 2-hydroxy-1-naphthaldehyde, its enamine structure (65) in solid state and in solution has been established previously. ${ }^{2}$ 
Structure and Tautomeric Equilibrium in Solution.

Table 2S. Selection of NMR ${ }^{\mathrm{a}, \mathrm{b}}$ data and hydrogen-bonding energy ${ }^{\mathrm{c}}$ of 4/34-33/63 and 64/65.

\begin{tabular}{|c|c|c|c|c|c|c|}
\hline Comp. & $\mathrm{OH} / \mathrm{NH}$ & $J_{\mathrm{H} 2, \mathrm{NH}}$ & $\mathrm{N}-\mathrm{CH}$ & $\mathrm{N}-\mathrm{CH}$ & $\mathrm{C}-2$ & $-E_{\mathrm{HB}}{ }^{\mathrm{d}}$ \\
\hline $4 / 34$ & $14.33 \mathrm{~d}$ & 8.8 & $8.74 d$ & 166.64 & 180.18 & 10.44 \\
\hline $5 / 35$ & $14.78 \mathrm{~d}$ & 9.2 & $8.60 \mathrm{~d}$ & 165.64 & 176.66 & 10.89 \\
\hline $6 / 36$ & $14.34 \mathrm{~s}$ & & $8.56 \mathrm{~s}$ & 164.11 & 159.51 & 10.45 \\
\hline $7 / 37$ & $14.70 \mathrm{~s}$ & & $8.54 \mathrm{~s}$ & 164.11 & 164.43 & 10.81 \\
\hline $8 / 38$ & $14.72 \mathrm{bs}$ & & $8.54 \mathrm{~s}$ & 164.06 & 165.15 & 10.82 \\
\hline 9/39 & $14.69 \mathrm{~s}$ & & $8.51 \mathrm{~s}$ & 163.99 & 165.89 & 10.79 \\
\hline $10 / 40$ & $14.53 \mathrm{bs}$ & & $8.56 \mathrm{~s}$ & 163.74 & 164.88 & 10.64 \\
\hline $11 / 41$ & $14.14 \mathrm{~s}$ & & $8.51 \mathrm{~s}$ & 164.78 & 160.61 & 10.25 \\
\hline $12 / 42$ & $13.83 \mathrm{~s}$ & & $8.56 \mathrm{~s}$ & 164.67 & 156.57 & 9.94 \\
\hline $13 / 43$ & $13.51 \mathrm{~s}$ & & $8.74 \mathrm{~s}$ & 164.60 & 154.45 & 9.62 \\
\hline $14 / 44$ & $15.32 \mathrm{~d}$ & 5.6 & $8.47 \mathrm{~d}$ & 164.91 & 168.64 & 11.43 \\
\hline $15 / 45$ & $14.77 \mathrm{~d}$ & 6.4 & $8.43 \mathrm{~d}$ & 163.99 & 174.14 & 10.88 \\
\hline $16 / 46$ & $14.85 \mathrm{bs}$ & & $8.48 \mathrm{~s}$ & 164.37 & 171.20 & 10.96 \\
\hline $17 / 47$ & $14.81 \mathrm{bs}$ & & $8.47 \mathrm{~s}$ & 164.56 & 171.29 & 10.92 \\
\hline $18 / 48$ & $14.27 \mathrm{~d}$ & 6.0 & $8.27 \mathrm{~d}$ & 162.45 & 174.24 & 10.38 \\
\hline $19 / 49$ & $13.88 \mathrm{bs}$ & & $8.08 \mathrm{~s}$ & 160.65 & 173.28 & 9.99 \\
\hline $20 / 50$ & $14.75 \mathrm{~d}$ & 4.4 & $8.48 \mathrm{~d}$ & 164.60 & 158.48 & 10.85 \\
\hline $21 / 51$ & $14.68 \mathrm{~d}$ & 3.2 & $8.47 \mathrm{~d}$ & 164.62 & 158.79 & 10.79 \\
\hline $22 / 52$ & $14.42 \mathrm{bs}$ & & $8.42 \mathrm{~s}$ & 164.32 & 162.26 & 10.53 \\
\hline $23 / 53$ & $15.22 \mathrm{~d}$ & 6.4 & $8.76 \mathrm{~d}$ & 159.26 & 170.83 & 11.33 \\
\hline $24 / 54$ & $14.90 \mathrm{~d}$ & 7.6 & $8.39 \mathrm{~d}$ & 158.42 & 174.43 & 11.01 \\
\hline $25 / 55$ & $14.03 \mathrm{~d}$ & 12.0 & $8.47 \mathrm{~d}$ & 155.84 & 178.96 & 10.14 \\
\hline $26 / 56$ & $13.90 \mathrm{~s}$ & & $8.17 \mathrm{~s}$ & 162.19 & 166.84 & 10.01 \\
\hline $27 / 57$ & $14.83 \mathrm{~s}$ & & $8.50 \mathrm{~s}$ & 164.14 & 157.79 & 10.94 \\
\hline $28 / 58$ & $14.80 \mathrm{~d}$ & 11.6 & $8.52 \mathrm{~d}$ & 164.64 & 168.33 & 10.91 \\
\hline $29 / 59$ & $14.77 \mathrm{~d}$ & 11.6 & $8.49 \mathrm{~d}$ & 164.63 & 169.11 & 10.88 \\
\hline $30 / 60$ & $13.84 \mathrm{~d}$ & 14.6 & $8.94 d$ & 167.44 & 170.47 & 9.95 \\
\hline $31 / 61$ & $13.83 \mathrm{~d}$ & 14.0 & $8.67 \mathrm{~d}$ & 166.19 & 173.66 & 9.94 \\
\hline $32 / 62$ & $14.56 \mathrm{~d}$ & 7.6 & $8.39 d$ & 163.52 & 162.64 & 10.67 \\
\hline $33 / 63$ & $14.41 \mathrm{~d}$ & 12.8 & $8.46 \mathrm{~d}$ & 163.86 & 171.99 & 10.52 \\
\hline $64 / 65$ & $14.02 \mathrm{~d}$ & 12.4 & $8.88 \mathrm{~d}$ & 156.22 & 179.76 & 10.13 \\
\hline
\end{tabular}

${ }^{\mathrm{a}}$ In DMSO- $d_{6},{ }^{\mathrm{b}} \delta$ in ppm and $J$ in $\mathrm{Hz},{ }^{\mathrm{c}}$ In $\mathrm{kcal} / \mathrm{mol},{ }^{\mathrm{d}}$ From equation [17]. 
Substituent Effects on the Tautomeric Equilibrium: Predominant Structures in Solution.

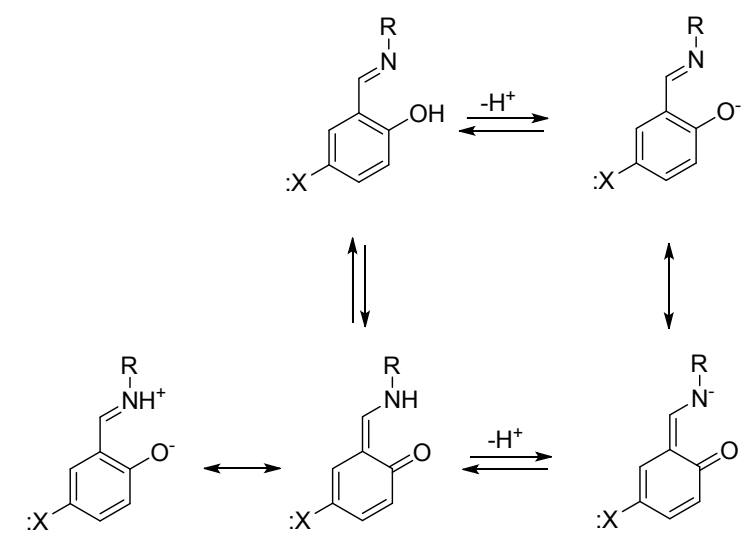

Scheme 1S

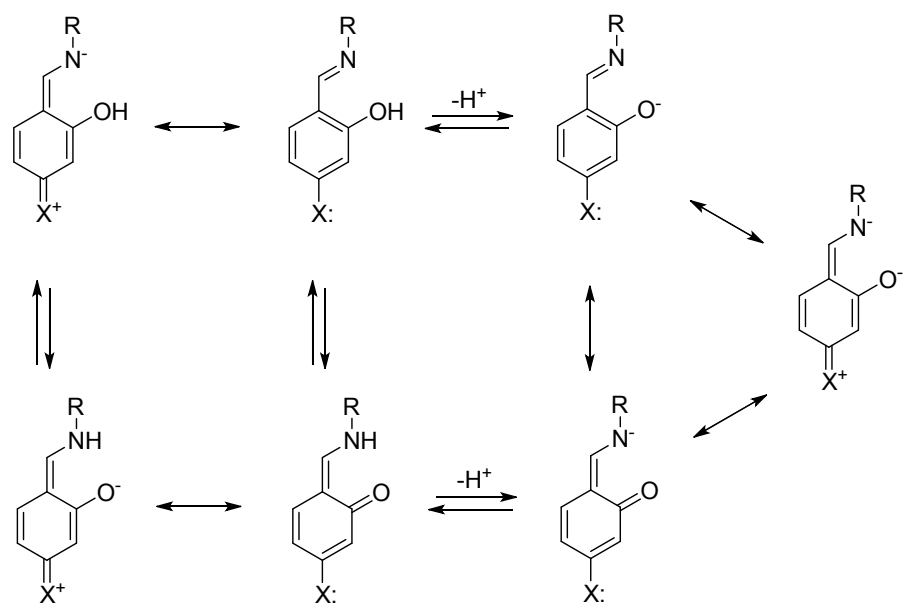

Scheme 2S 
Electronic Effects of Substituents on Chemical Shifts.

Table 3S. NMR data for the imino carbon of iminic Schiff bases. ${ }^{a}$

\begin{tabular}{ccccc}
\hline Compound & Substit. & $\delta_{\mathrm{CN}}$ & $\sigma_{\mathrm{NH}}$ & $\sigma_{\mathrm{ef}}$ \\
\hline $\mathbf{6}$ & $5-\mathrm{F}$ & 164.11 & 0.34 & -0.19 \\
$\mathbf{7}$ & $5-\mathrm{Cl}$ & 164.11 & 0.37 & -0.13 \\
$\mathbf{9}$ & $5-\mathrm{Br}$ & 164.06 & 0.37 & -0.11 \\
$\mathbf{1 1}$ & $5-\mathrm{I}$ & 163.99 & 0.34 & -0.16 \\
$\mathbf{1 2}$ & $5-\mathrm{Me}$ & 164.78 & -0.06 & -0.08 \\
$\mathbf{1 3}$ & $5-\mathrm{OMe}$ & 164.67 & 0.10 & -0.38 \\
$\mathbf{2 0}$ & $5-\mathrm{OH}$ & 164.60 & 0.13 & -0.51 \\
$\mathbf{2 1}$ & 3-OEt & 164.60 & 0.10 & -0.34 \\
$\mathbf{2 2}$ & 3-OMe & 164.62 & 0.10 & -0.38 \\
$\mathbf{2 7}$ & 3-OH & 164.32 & 0.13 & -0.51 \\
$\mathbf{3 2}$ & 3,5-diF & 164.14 & 0.68 & -0.38 \\
\hline & $5-\mathrm{Br}-3-\mathrm{OMe}$ & 163.52 & 0.47 & -0.49 \\
\hline
\end{tabular}

a $\delta$ in ppm.

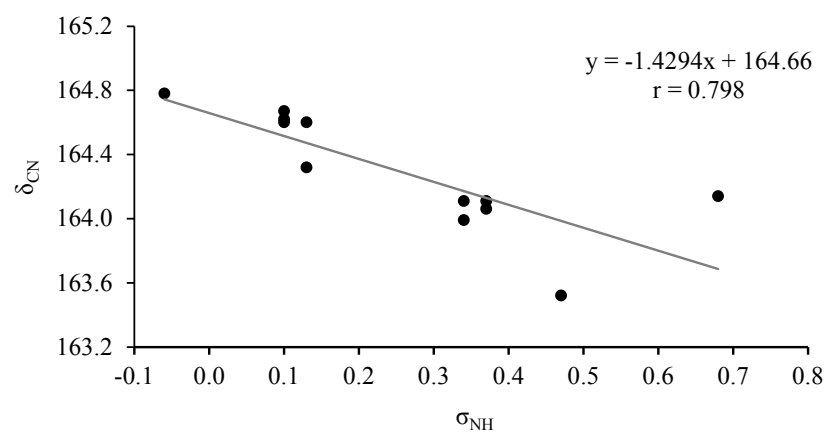

Figure 1S 
Table 4S. NMR data for the imino carbon of enaminic Schiff bases. ${ }^{\mathrm{a}}$

\begin{tabular}{|c|c|c|c|c|}
\hline Compound & Substit. & $\delta_{\mathrm{CN}}^{\mathrm{a}}$ & $\sum \sigma_{\mathrm{NH}}$ & $\sum \sigma_{\mathrm{NH}}^{+}$ \\
\hline 4 & $5-\mathrm{NO}_{2}$ & 166.64 & 0.71 & 0.71 \\
\hline 5 & $5-\mathrm{CO}_{2} \mathrm{Me}$ & 165.64 & 0.35 & 0.35 \\
\hline 14 & $4-\mathrm{NO}_{2}$ & 164.91 & 0.81 & 0.81 \\
\hline 15 & $4-\mathrm{F}$ & 163.99 & 0.15 & -0.07 \\
\hline 16 & $4-\mathrm{Cl}$ & 164.37 & 0.24 & 0.11 \\
\hline 17 & $4-\mathrm{Br}$ & 164.56 & 0.26 & 0.15 \\
\hline 18 & 4-OMe & 162.45 & -0.28 & -0.78 \\
\hline 19 & $4-\mathrm{NEt}_{2}$ & 160.65 & -0.72 & $-1.70^{\mathrm{b}}$ \\
\hline 23 & 6-OMe & 159.26 & -0.28 & -0.78 \\
\hline 24 & 4-OH-6-Me & 158.42 & -0.52 & -1.23 \\
\hline 25 & 4,6-diOMe & 155.84 & -0.56 & -1.56 \\
\hline 26 & 3,4-diOH & 162.19 & -0.25 & -0.79 \\
\hline 28 & 3,5-diCl & 164.64 & 0.74 & 0.74 \\
\hline 29 & 3,5-diBr & 164.63 & 0.74 & 0.74 \\
\hline 30 & $3,5-\mathrm{diNO}_{2}$ & 167.44 & 1.42 & 1.42 \\
\hline 31 & $5-\mathrm{NO}_{2}-3-\mathrm{OMe}$ & 166.19 & 0.81 & 0.81 \\
\hline 33 & 3,5-diBr-4-OMe & 163.86 & 0.46 & -0.04 \\
\hline $78^{c}$ & $5-\mathrm{PhN}_{2}$ & 164.70 & 0.32 & 0.32 \\
\hline
\end{tabular}

${ }^{\mathrm{a}} \delta$ in ppm. $\quad{ }^{\mathrm{b}} \sigma^{+}\left(\mathrm{NMe}_{2}\right) . \quad{ }^{\mathrm{c}}$ Reference 3.
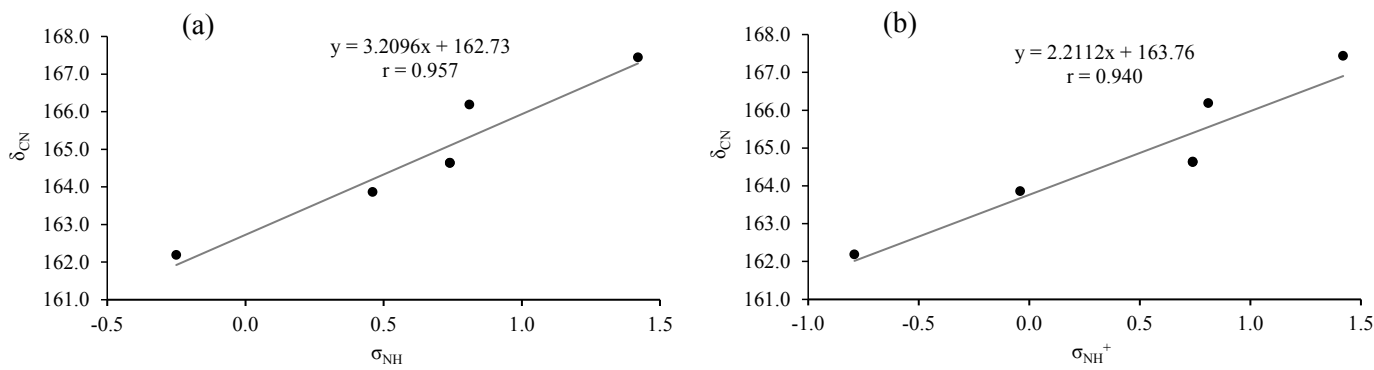

Figure 2S 
Table 5S.

\begin{tabular}{ccccc}
\hline Compound & Substit. & $\delta_{\mathrm{CO}}{ }^{\mathrm{a}}$ & $\Delta \delta_{\mathrm{CO}}{ }^{\mathrm{a}}$ & $\sigma_{\mathrm{OH}}$ \\
\hline $\mathbf{6}$ & $5-\mathrm{F}$ & 159.51 & -5.37 & 0.15 \\
$\mathbf{7}$ & $5-\mathrm{Cl}$ & 164.43 & -0.45 & 0.24 \\
$\mathbf{8}$ & $5-\mathrm{Br}$ & 165.15 & 0.27 & 0.26 \\
$\mathbf{9}$ & $5-\mathrm{I}$ & 165.89 & 1.01 & 0.18 \\
$\mathbf{1 0}$ & $\mathrm{H}$ & 164.88 & 0.00 & 0.00 \\
$\mathbf{1 1}$ & $5-\mathrm{Me}$ & 160.61 & -4.27 & -0.14 \\
$\mathbf{1 2}$ & $5-\mathrm{OMe}$ & 156.57 & -8.31 & -0.28 \\
$\mathbf{1 3}$ & $5-\mathrm{OH}$ & 154.45 & -10.43 & -0.38 \\
\hline
\end{tabular}

${ }^{\text {a }} \delta$ in ppm.

Table 6S.

\begin{tabular}{|c|c|c|c|c|c|}
\hline & Compound & Substit. & $\delta_{\mathrm{OH} / \mathrm{NH}}{ }^{\mathrm{a}}$ & $\Delta \delta_{\mathrm{OH} / \mathrm{NH}^{\mathrm{a}}}$ & $\sum \sigma_{\mathrm{OH}}$ \\
\hline \multirow{9}{*}{ 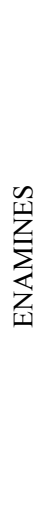 } & 4 & $5-\mathrm{NO}_{2}$ & 14.33 & -0.20 & 0.81 \\
\hline & 5 & $5-\mathrm{CO}_{2} \mathrm{Me}$ & 14.78 & 0.25 & 0.44 \\
\hline & 14 & $4-\mathrm{NO}_{2}$ & 15.32 & 0.79 & 0.71 \\
\hline & 15 & $4-\mathrm{F}$ & 14.77 & 0.24 & 0.34 \\
\hline & 16 & $4-\mathrm{Cl}$ & 14.85 & 0.32 & 0.37 \\
\hline & 17 & $4-\mathrm{Br}$ & 14.81 & 0.28 & 0.37 \\
\hline & 18 & 4-OMe & 14.27 & -0.26 & 0.10 \\
\hline & 19 & $4-\mathrm{NEt}_{2}$ & 13.88 & -0.65 & -0.23 \\
\hline & $78^{b}$ & $5-\mathrm{PhN}_{2}$ & 14.47 & -0.06 & 0.39 \\
\hline \multirow{8}{*}{ 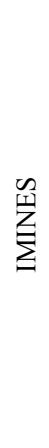 } & 6 & $5-\mathrm{F}$ & 14.34 & -0.19 & 0.15 \\
\hline & 7 & $5-\mathrm{Cl}$ & 14.70 & 0.17 & 0.24 \\
\hline & 8 & $5-\mathrm{Br}$ & 14.72 & 0.19 & 0.26 \\
\hline & 9 & $5-\mathrm{I}$ & 14.69 & 0.16 & 0.18 \\
\hline & 10 & $\mathrm{H}$ & 14.53 & 0.00 & 0.00 \\
\hline & 11 & $5-\mathrm{Me}$ & 14.14 & -0.39 & -0.14 \\
\hline & 12 & 5-OMe & 13.83 & -0.70 & -0.28 \\
\hline & 13 & $5-\mathrm{OH}$ & 13.51 & -1.02 & -0.38 \\
\hline
\end{tabular}

${ }^{\mathrm{a}} \delta$ in ppm. ${ }^{\mathrm{b}}$ Reference 3. 

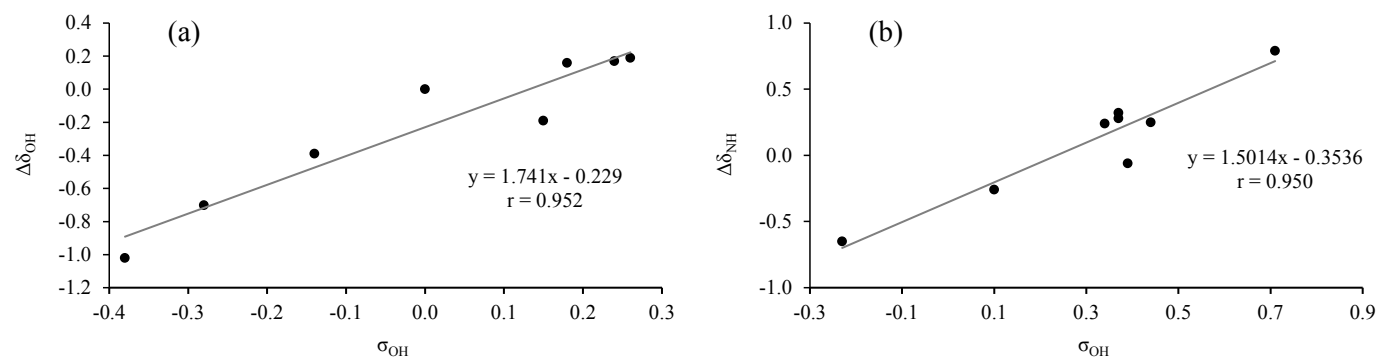

Figure 3S. Representation $\Delta \delta_{\mathrm{OH} / \mathrm{NH}}$ versus $\sigma_{\mathrm{OH}}$ : (a) imines, (b) enamines. 


\section{Electronic Effect of the Substituents on the Tautomeric Equilibrium.}

The equilibrium constant $K_{\mathrm{T}}=\mathrm{n}_{\mathrm{e}} / \mathrm{n}_{\mathrm{i}}$ is calculated by the expression:

$$
K_{\mathrm{T}}=\left(\delta_{\exp }-\delta_{\mathrm{i}}\right) /\left(\delta_{\mathrm{e}}-\delta_{\exp }\right)
$$

Table 7S. Tautomerization constants $\left(K_{\mathrm{T}}\right)$ for $\mathbf{4 / 3 4 - 3 3 / 6 3}$.

\begin{tabular}{|c|c|c|c|c|c|c|}
\hline Compound & Substit. & $\delta_{\mathrm{C}-2}{ }^{\mathrm{a}}$ & $K_{\mathrm{T}}$ & $\log K_{\mathrm{T}}$ & $\sigma_{\mathrm{ef}}$ & $\sigma_{\text {ef }}^{ \pm}$ \\
\hline $4 / 34$ & $5-\mathrm{NO}_{2}$ & 180.18 & $\infty$ & $\infty$ & 0.10 & 0.52 \\
\hline $5 / 35$ & 5-COOMe & 176.66 & 6.31 & 0.80 & 0.09 & 0.39 \\
\hline $6 / 36$ & $5-\mathrm{F}$ & 159.51 & 0.24 & -0.61 & -0.19 & -0.19 \\
\hline $7 / 37$ & $5-\mathrm{Cl}$ & 164.43 & 0.63 & -0.20 & -0.13 & -0.13 \\
\hline $8 / 38$ & $5-\mathrm{Br}$ & 165.15 & 0.71 & -0.15 & -0.11 & -0.11 \\
\hline 9/39 & $5-\mathrm{I}$ & 165.89 & 0.80 & -0.10 & -0.16 & -0.16 \\
\hline $10 / 40$ & $\mathrm{H}$ & 164.88 & 0.68 & -0.17 & 0.00 & 0.00 \\
\hline $11 / 41$ & $5-\mathrm{Me}$ & 160.61 & 0.31 & -0.50 & -0.08 & -0.08 \\
\hline $12 / 42$ & 5-OMe & 156.57 & 0.09 & -1.05 & -0.38 & -0.38 \\
\hline $13 / 43$ & $5-\mathrm{OH}$ & 154.45 & 0.00 & $-\infty$ & -0.51 & -0.51 \\
\hline $14 / 44$ & $4-\mathrm{NO}_{2}$ & 168.64 & 1.23 & 0.09 & -0.10 & -0.10 \\
\hline $15 / 45$ & $4-\mathrm{F}$ & 174.14 & 3.26 & 0.51 & 0.19 & 0.41 \\
\hline $16 / 46$ & $4-\mathrm{Cl}$ & 171.20 & 1.87 & 0.27 & 0.13 & 0.26 \\
\hline $17 / 47$ & $4-\mathrm{Br}$ & 171.29 & 1.89 & 0.28 & 0.11 & 0.22 \\
\hline $18 / 48$ & 4-OMe & 174.24 & 3.33 & 0.52 & 0.38 & 0.88 \\
\hline $19 / 49$ & $4-\mathrm{NEt}_{2}$ & 173.28 & 2.73 & 0.44 & 0.49 & 1.47 \\
\hline $20 / 50$ & 3 -OEt & 158.48 & 0.19 & -0.73 & -0.34 & -0.34 \\
\hline $21 / 51$ & 3-OMe & 158.79 & 0.20 & -0.69 & -0.38 & -0.38 \\
\hline $22 / 52$ & $3-\mathrm{OH}$ & 162.26 & 0.44 & -0.36 & -0.51 & -0.51 \\
\hline $23 / 53$ & 6-OMe & 170.83 & 1.75 & 0.24 & 0.38 & 0.88 \\
\hline $24 / 54$ & 4-OH-6-Me & 174.43 & 3.47 & 0.54 & 0.59 & 1.30 \\
\hline $25 / 55$ & 4-OMe-6-OMe & 178.96 & 20.09 & 1.30 & 0.76 & 1.76 \\
\hline $26 / 56$ & 3-OH-4-OH & 166.84 & 0.93 & -0.03 & 0.00 & 0.54 \\
\hline $27 / 57$ & $3-F-5-F$ & 157.79 & 0.15 & -0.83 & -0.38 & -0.38 \\
\hline $28 / 58$ & $3-\mathrm{Cl}-5-\mathrm{Cl}$ & 168.33 & 1.17 & 0.07 & -0.26 & -0.26 \\
\hline $29 / 59$ & $3-\mathrm{Br}-5-\mathrm{Br}$ & 169.11 & 1.32 & 0.12 & -0.22 & -0.22 \\
\hline $30 / 60$ & $3-\mathrm{NO}_{2}-5-\mathrm{NO}_{2}$ & 170.47 & 1.65 & 0.22 & 0.20 & 1.04 \\
\hline $31 / 61$ & $5-\mathrm{NO}_{2}-3-\mathrm{OMe}$ & 173.66 & 2.95 & 0.47 & -0.28 & 0.14 \\
\hline $32 / 62$ & 5-Br-3-OMe & 162.64 & 0.47 & -0.33 & -0.49 & -0.49 \\
\hline $33 / 63$ & 3-Br-5-Br-4-OMe & 171.99 & 2.14 & 0.33 & 0.16 & 0.66 \\
\hline
\end{tabular}

${ }^{\text {a }} \delta$ in ppm. 

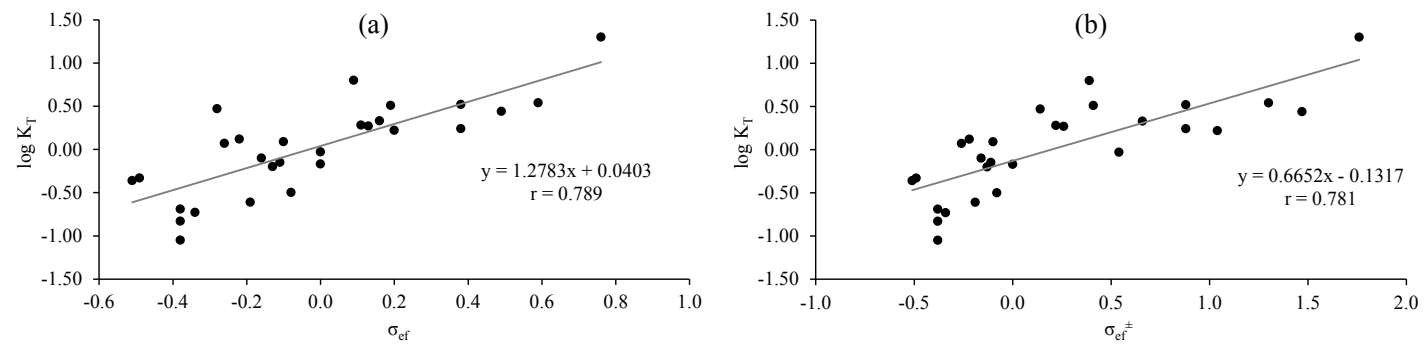

Figure 4S. Representation of $\log K_{\mathrm{T}}$ versus $\sigma_{\mathrm{ef}}(\mathrm{a})$ and $\sigma_{\mathrm{ef}}{ }^{ \pm}(\mathrm{b})$.

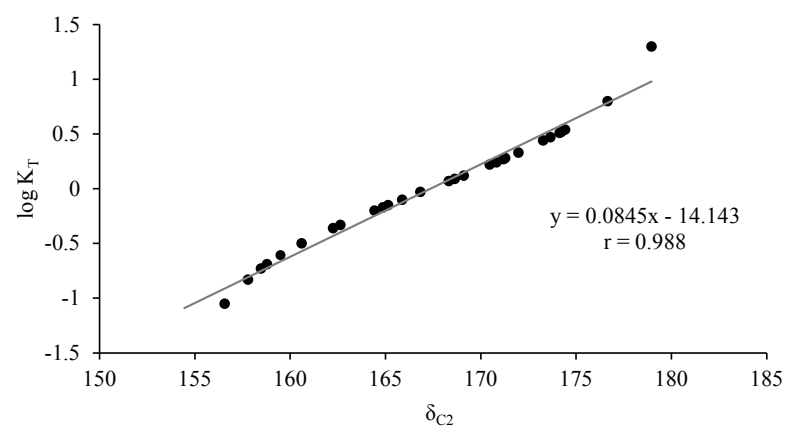

Figure 5S. Plot of $\log K_{\mathrm{T}} v s \delta_{\mathrm{C} 2}$ (by eliminating the point corresponding to the 5-nitro derivative).

Table 8S.

\begin{tabular}{|c|c|c|c|c|c|c|c|}
\hline Comp. & Subst. & $K_{\mathrm{T}}$ & $\log K_{\mathrm{T}}$ & $\delta_{\mathrm{C}-2}{ }^{\mathrm{a}}$ & $\Delta \delta_{\mathrm{C}-2}{ }^{\mathrm{a}}$ & $\sigma_{\mathrm{p}(\mathrm{H})}^{\mathrm{b}, \mathrm{d}}$ & $\sigma_{\mathrm{p}(\mathrm{N})}^{\mathrm{c}, \mathrm{d}}$ \\
\hline $4 / 34$ & $5-\mathrm{NO}_{2}$ & $\infty$ & $\infty$ & 180.18 & 15.30 & 0.44 & 0.75 \\
\hline $6 / 36$ & $5-\mathrm{H}$ & 0.68 & -0.17 & 164.88 & 0.00 & -0.05 & -0.14 \\
\hline $10 / 40$ & $5-\mathrm{F}$ & 0.24 & -0.61 & 159.51 & -5.37 & -0.45 & -0.51 \\
\hline $11 / 41$ & $5-\mathrm{CH}_{3}$ & 0.31 & -0.50 & 160.61 & -4.27 & -0.33 & -0.36 \\
\hline $12 / 42$ & $5-\mathrm{OCH}_{3}$ & 0.09 & -1.05 & 156.57 & -8.31 & -0.91 & -0.80 \\
\hline $13 / 43$ & $5-\mathrm{OH}$ & 0 & $\infty$ & 154.45 & -10.43 & -0.83 & -0.75 \\
\hline
\end{tabular}

${ }^{\mathrm{a}}$ In ppm; ${ }^{\mathrm{b}}$ From HOMA index; ${ }^{\mathrm{c}}$ From NICS index; ${ }^{\mathrm{d}}$ Reference 4. 
The representation of $\log K_{\mathrm{T}} v s \sigma_{\mathrm{ef}}$ for the Schiff bases 124-126 ${ }^{5}$ (Table 9S) gives an excellent fit at 303 $\mathrm{K}(r=0.994)$ and at $223 \mathrm{~K}(r=0.999)$ (Fig. $6 \mathrm{~S})$.<smiles>C/N=C/c1cc(OC)ccc1O</smiles>

124<smiles>[X]c1cc(OC)cc(O)c1/C=N/C</smiles>

$125 \mathrm{X}=\mathrm{H}$

$126 \mathrm{X}=\mathrm{OMe}$

Table 9S. Tautomerization constants $\left(K_{\mathrm{T}}\right)$ for 124-126.

\begin{tabular}{|c|c|c|c|c|c|c|c|}
\cline { 2 - 7 } \multicolumn{1}{c|}{} & \multicolumn{3}{c|}{$303 \mathrm{~K}$} & \multicolumn{3}{c|}{$223 \mathrm{~K}$} & \multicolumn{1}{c}{} \\
\hline Compound & Enamine & $K_{\mathrm{T}}$ & $\log K_{\mathrm{T}}$ & Enamine & $K_{\mathrm{T}}$ & $\log K_{\mathrm{T}}$ & $\sigma_{\text {ef }}$ \\
\hline $\mathbf{1 2 4}$ & $1 \%$ & 0.01 & -2.00 & $1 \%$ & 0.01 & -2.00 & -0.38 \\
$\mathbf{1 2 5}$ & $15 \%$ & 0.18 & -0.75 & $46 \% \mathrm{~b}^{\mathrm{b}}$ & $0.85^{\mathrm{b}}$ & $-0.07^{\mathrm{b}}$ & 0.38 \\
$\mathbf{1 2 6}$ & $50 \%$ & 1.00 & 0.00 & $80 \%$ & 4.00 & 0.60 & 0.76 \\
\hline
\end{tabular}

${ }^{a}$ Reference 5; b at $213 \mathrm{~K}$.

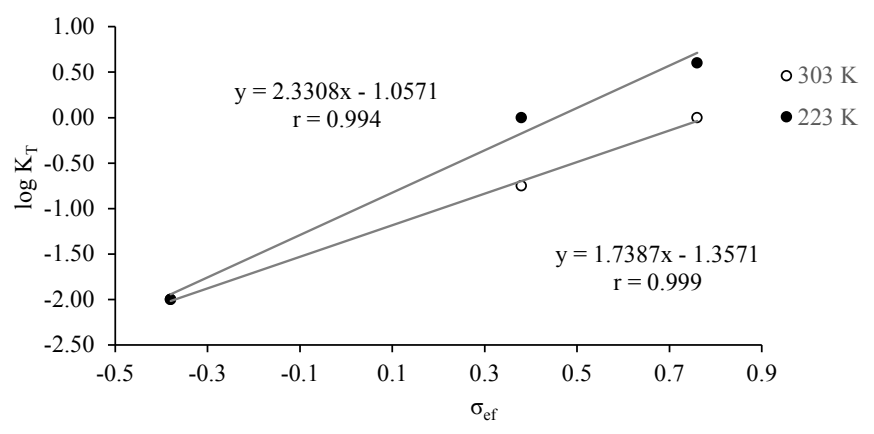

Figure 6S

$\log K_{\mathrm{T}}=1.7379 \sigma_{\mathrm{ef}}+1.3584(\mathrm{r}=0.999,303 \mathrm{~K})$

$\log K_{\mathrm{T}}=2.3192 \sigma_{\mathrm{ef}}+1.0767(\mathrm{r}=0.994,223 \mathrm{~K})$ 


\section{Analysis of the Influence of Substituents on Examples Drawn from the Scientific}

\section{Literature.}

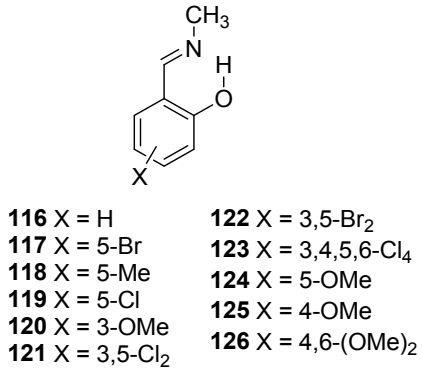

Table 10S. ${ }^{1} \mathrm{H}$ and ${ }^{13} \mathrm{C}-\mathrm{NMR}$ spectroscopic data of 116-126.

\begin{tabular}{|c|c|c|c|c|c|c|c|c|c|c|}
\hline Comp. & $\mathbf{X}$ & $\boldsymbol{\delta}_{\mathrm{C} 2}$ & $\boldsymbol{\delta}_{\mathrm{CN}}$ & $\sigma_{\mathrm{OH}}$ & $\sigma_{\mathrm{NH}}$ & $\sigma_{\mathrm{ef}}^{b}$ & $K_{\mathrm{T}}{ }^{\mathbf{c}}$ & $\log K_{\mathrm{T}} \mathrm{c}$ & $K_{\mathrm{T}}{ }^{d}$ & $\log K_{\mathrm{T}}{ }^{\mathrm{d}}$ \\
\hline 116 & $\mathrm{H}$ & 161.16 & 166.17 & 0.00 & 0.00 & 0.00 & 0.58 & -0.24 & 0.48 & -0.32 \\
\hline 117 & $5-\mathrm{Br}$ & 160.33 & 164.95 & 0.26 & 0.37 & -0.11 & 0.50 & -0.30 & 0.42 & -0.38 \\
\hline 118 & $5-\mathrm{Me}$ & 158.83 & 166.15 & -0.14 & -0.06 & -0.08 & 0.37 & -0.43 & 0.32 & -0.49 \\
\hline 119 & $5-\mathrm{Cl}$ & 159.81 & 165.03 & 0.24 & 0.37 & -0.13 & 0.45 & -0.35 & 0.38 & -0.42 \\
\hline 120 & 3-OMe & 152.19 & 166.27 & -0.28 & 0.10 & -0.38 & 0.00 & $-\infty$ & 0.01 & -2.17 \\
\hline 121 & $3,5-\mathrm{Cl}_{2}$ & 157.72 & 164.72 & 0.48 & 0.74 & -0.26 & 0.29 & -0.54 & 0.25 & -0.59 \\
\hline 122 & $3,5-\mathrm{Br}_{2}$ & 159.33 & 164.55 & 0.52 & 0.74 & -0.22 & 0.41 & -0.39 & 0.35 & -0.45 \\
\hline 123 & $3,4,5,6-\mathrm{Cl}_{4}$ & 166.50 & 163.90 & 1.22 & 1.22 & 0.00 & 1.40 & 0.15 & 1.06 & 0.03 \\
\hline 124 & 5-OMe & 155.13 & 165.27 & -0.28 & 0.10 & -0.38 & 0.14 & -0.87 & 0.12 & -0.90 \\
\hline 125 & 4-OMe & 166.76 & 164.95 & 0.10 & -0.28 & 0.38 & 1.46 & 0.17 & 1.10 & 0.04 \\
\hline 126 & $4,6-\mathrm{OMe}_{2}$ & 176.71 & 159.37 & 0.20 & -0.56 & 0.76 & $\infty$ & $\infty$ & 7.12 & 0.85 \\
\hline
\end{tabular}

${ }^{\mathrm{a}}$ In $\mathrm{CD}_{2} \mathrm{Cl}_{2}, 300 \mathrm{~K}, 400 \mathrm{MHz} ;{ }^{\mathrm{b}} \sigma_{\mathrm{ef}}=\sigma_{\mathrm{OH}}-\sigma_{\mathrm{NH}} ;{ }^{\mathrm{c}} \delta_{\mathrm{e}}=176.71 \mathrm{ppm}, \delta_{\mathrm{i}}=152.19 \mathrm{ppm} ;{ }^{\mathrm{d}} \delta_{\mathrm{e}}=180.18 \mathrm{ppm}, \delta_{\mathrm{i}}=152.00 \mathrm{ppm}$.

Also, with these Schiff bases, the representation of $\log K_{\mathrm{T}}$ versus $\delta_{\mathrm{C} 2}$ leads to a perfect fit $(\mathrm{r}=$ 0.9958) (Figure 7S, without item 120).

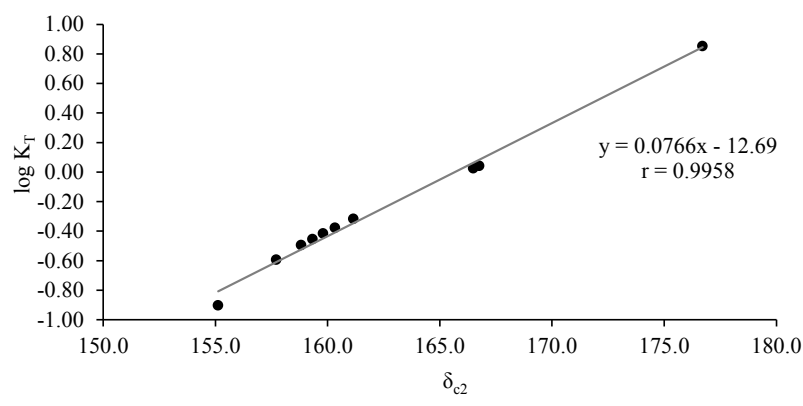

Figure 7S. Plot of $\log K_{\mathrm{T}}$ versus $\delta_{\mathrm{C} 2}\left(\sigma_{\mathrm{e}}=180.18 \mathrm{ppm}, \sigma_{\mathrm{i}}=152.00 \mathrm{ppm}\right)$ 
Table 11S. ${ }^{15} \mathrm{~N}$ chemical shifts (ppm) of 119-122 and 124-126. ${ }^{\mathrm{a}, \mathrm{b}}$

\begin{tabular}{|c|c|c|c|c|c|c|c|c|c|}
\hline Comp. & $\mathbf{X}$ & $\delta^{15}{ }_{N}^{b c}$ & $\delta^{15_{\mathrm{N}} \text { bd }}$ & $\delta^{15}{ }_{N}^{\text {be }}$ & $\delta^{5}{ }_{N}^{h}$ & $\sigma_{\text {ef }}$ & $\sigma_{\text {ef }}^{\text {ortho }}$ & $\sigma_{\text {ef }}^{ \pm}$ & $\sigma_{\text {ef }}^{ \pm}$ortho \\
\hline 119 & $5-\mathrm{Cl}$ & $-91.9^{f}$ & $-92.8^{g}$ & -93.4 & -100.3 & -0.13 & -0.13 & -0.13 & -0.13 \\
\hline 120 & 3-OMe & $-103.2^{f}$ & $-106.0^{\mathrm{g}}$ & -109.3 & -97.6 & -0.38 & -0.47 & -0.38 & -0.47 \\
\hline 121 & $3,5-\mathrm{Cl}_{2}$ & $-116.7^{f}$ & -122.8 & -134.3 & -214.4 & -0.26 & 0.18 & -0.26 & 0.00 \\
\hline 122 & $3,5-\mathrm{Br}_{2}$ & -121.3 & -130.6 & -145.5 & -213.6 & -0.22 & 0.22 & -0.22 & 0.07 \\
\hline 124 & 5-OMe & -91.0 & -91.5 & -91.7 & -86.3 & -0.38 & -0.38 & -0.38 & -0.38 \\
\hline 125 & 4-OMe & -147.0 & -176.0 & -186.5 & -235.3 & 0.38 & 0.38 & 0.88 & 0.88 \\
\hline 126 & $4,6-(\mathrm{OMe})_{2}$ & -226.9 & -242.5 & -244.2 & -239.4 & 0.76 & 0.76 & 1.76 & 1.76 \\
\hline
\end{tabular}

a At $50.7 \mathrm{MHz} ;{ }^{\mathrm{b}}$ In $\mathrm{Cl}_{3} \mathrm{CD},{ }^{\mathrm{c}}$ at $266 \mathrm{~K} ;{ }^{\mathrm{d}}$ at $217 \mathrm{~K} ;{ }^{\mathrm{e}}$ a $205.5 \mathrm{~K} ;{ }^{\mathrm{f}}$ at $254 \mathrm{~K} ;{ }^{\mathrm{g}}$ at $229 \mathrm{~K} ;{ }^{\mathrm{h}}$ In solid state at $297 \mathrm{~K}$.

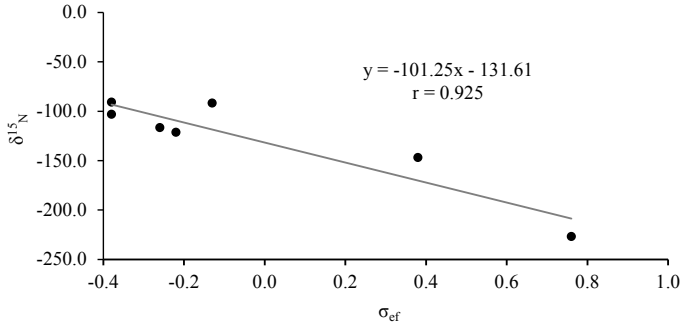

At $266 \mathrm{~K}$

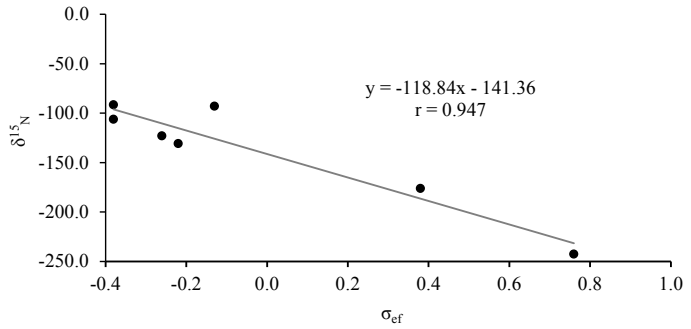

At $217 \mathrm{~K}$

Figure 8S.

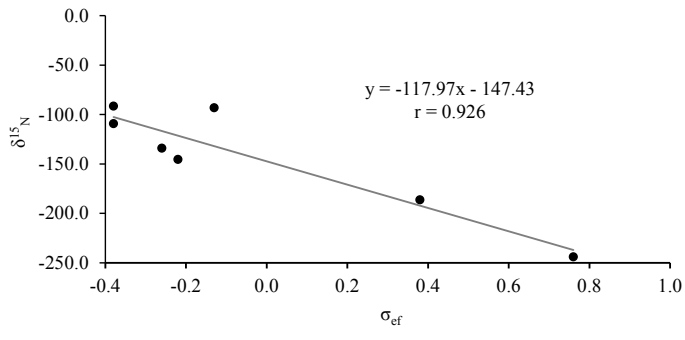

At $205.5 \mathrm{~K}$

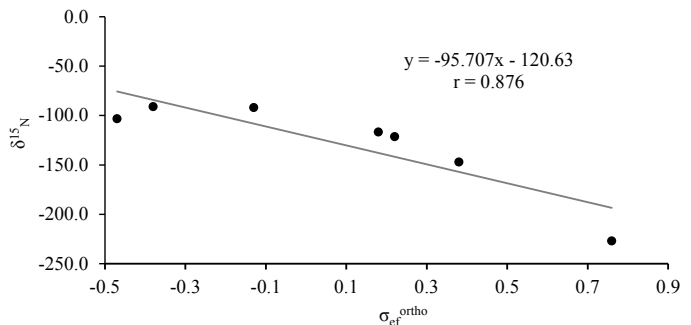

At $266 \mathrm{~K}$

Figure 9S.
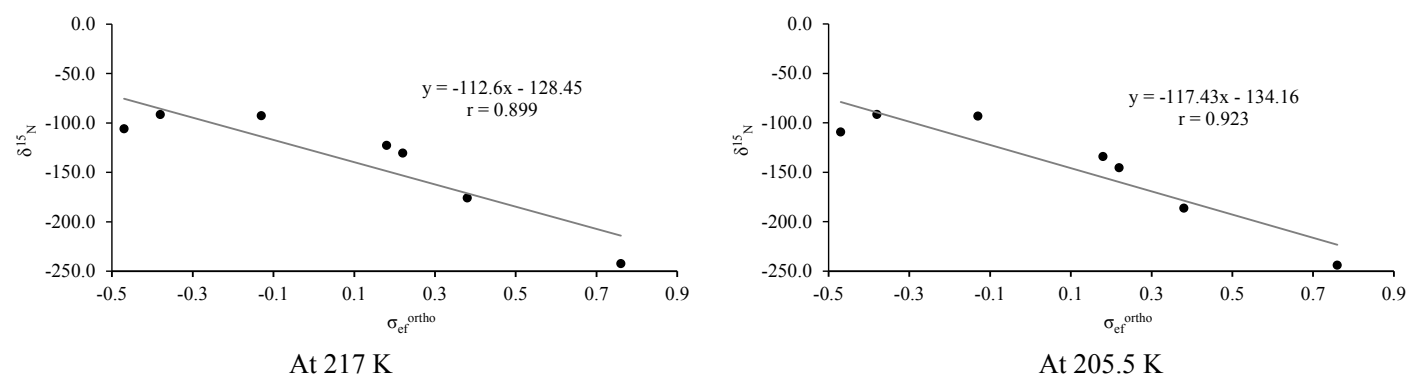

Figure 10S.

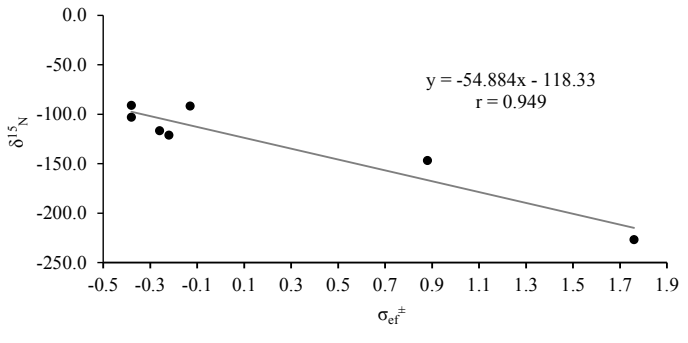

At $266 \mathrm{~K}$

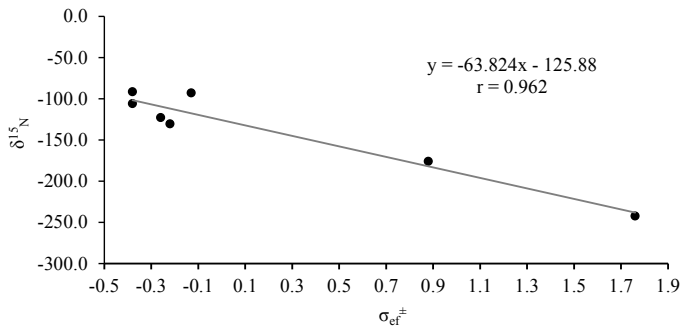

At $217 \mathrm{~K}$

Figure 11S. 


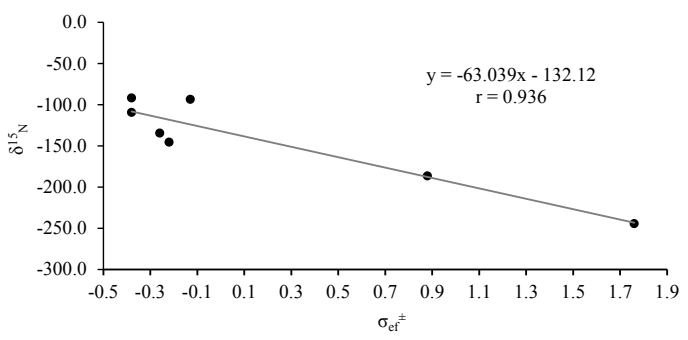

At $205.5 \mathrm{~K}$

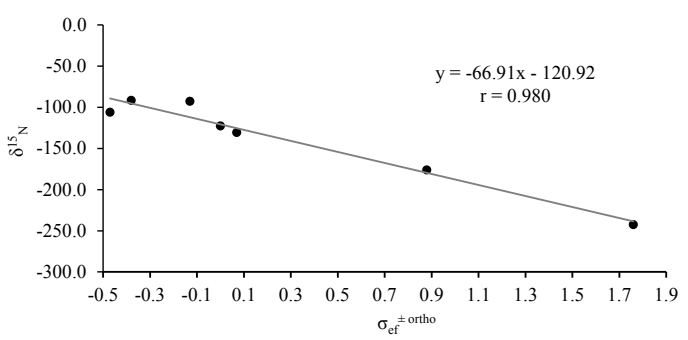

At $217 \mathrm{~K}$

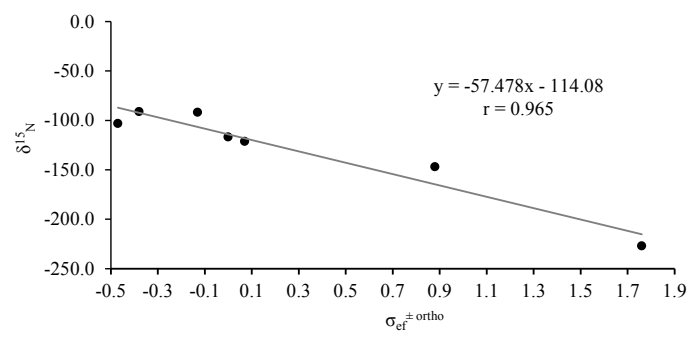

At $266 \mathrm{~K}$

Figure 12S.

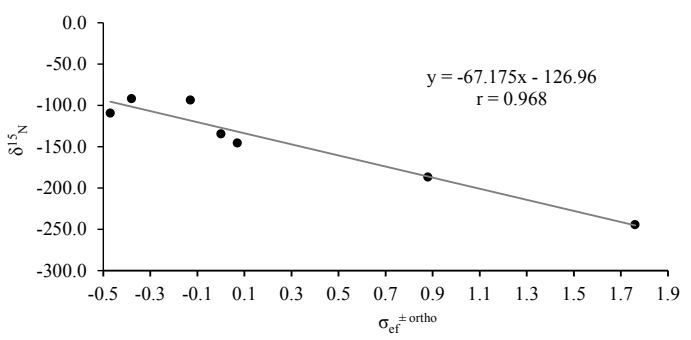

At $205.5 \mathrm{~K}$

Figure 13S.

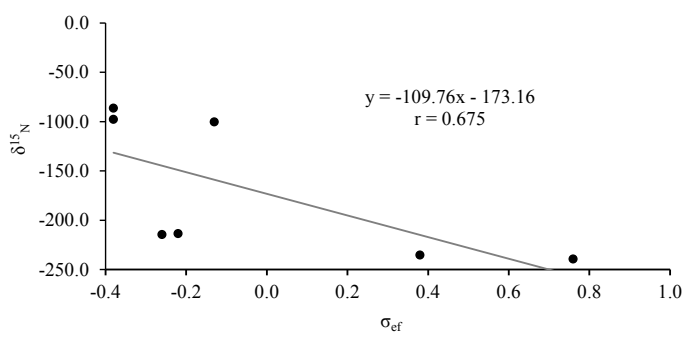

In solid state at $297 \mathrm{~K}$

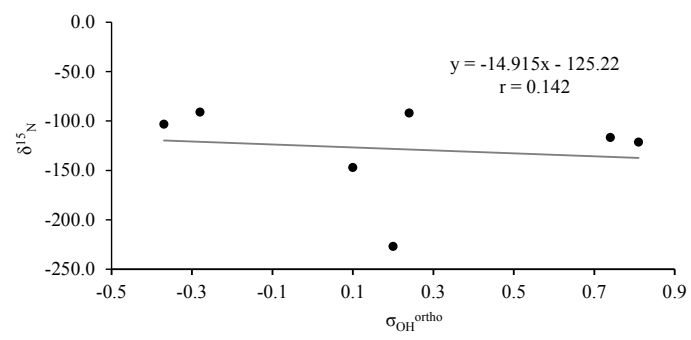

At $266 \mathrm{~K}$

Figure 14S.

Table 12S. Physicochemical parameters of compounds 124-126.

\begin{tabular}{|c|c|c|c|c|c|c|c|c|c|c|}
\hline \multirow[b]{2}{*}{ Comp. } & \multirow[b]{2}{*}{ Subst. } & \multicolumn{3}{|c|}{$\mathbf{Y}=\mathbf{H}, \mathbf{D}^{\mathrm{a}}$} & \multicolumn{2}{|c|}{$\mathbf{Y}=\mathbf{H}, \mathbf{T}^{\mathbf{b}}$} & \multirow{2}{*}{$\begin{array}{c}\mathbf{Y}=\mathbf{H}^{\mathrm{c}} \\
\delta^{15}{ }_{\mathrm{N}}\end{array}$} & \multirow[b]{2}{*}{$\sigma_{\text {ef }}$} & \multirow[b]{2}{*}{$\sigma_{\mathrm{OH}}$} & \multirow[b]{2}{*}{$\sigma_{\text {ef }}^{ \pm}$} \\
\hline & & $\delta_{\mathrm{OH}}$ & $\delta_{\mathrm{OD}}$ & $\delta^{15} \mathrm{~N}$ & $\delta_{\mathrm{OH}}$ & $\delta_{\text {OT }}$ & & & & \\
\hline 124 & 5-OMe & 12.833 & 12.597 & -87.66 & 12.940 & 12.565 & -90.56 & -0.38 & -0.28 & -0.38 \\
\hline 125 & 4-OMe & 13.856 & 13.448 & -128.58 & 13.952 & 13.378 & -167.65 & 0.38 & 0.10 & 0.88 \\
\hline 126 & $4,6-(\mathrm{OMe})_{2}$ & 14.048 & 13.540 & -208.52 & 14.051 & 13.413 & -243.30 & 0.76 & 0.20 & 1.76 \\
\hline
\end{tabular}<smiles></smiles>

$124 \mathrm{Y}=\mathrm{H}, \mathrm{D}, \mathrm{T}$<smiles>COc1ccc2c(c1)ON(C)C=C2</smiles>

$125 \mathrm{Y}=\mathrm{H}, \mathrm{D}, \mathrm{T}$

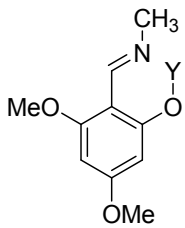

$126 \mathrm{Y}=\mathrm{H}, \mathrm{D}, \mathrm{T}$ 

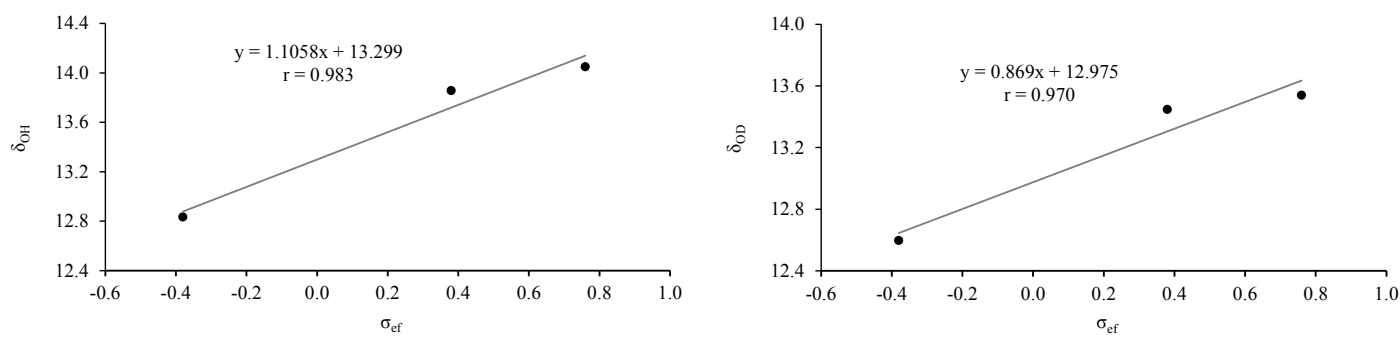

Figure 15S.
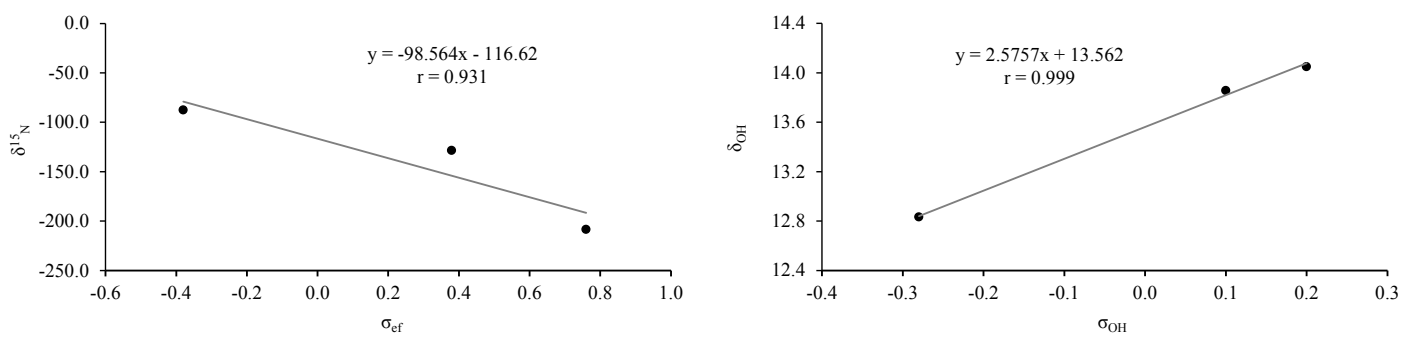

Figure 16S.
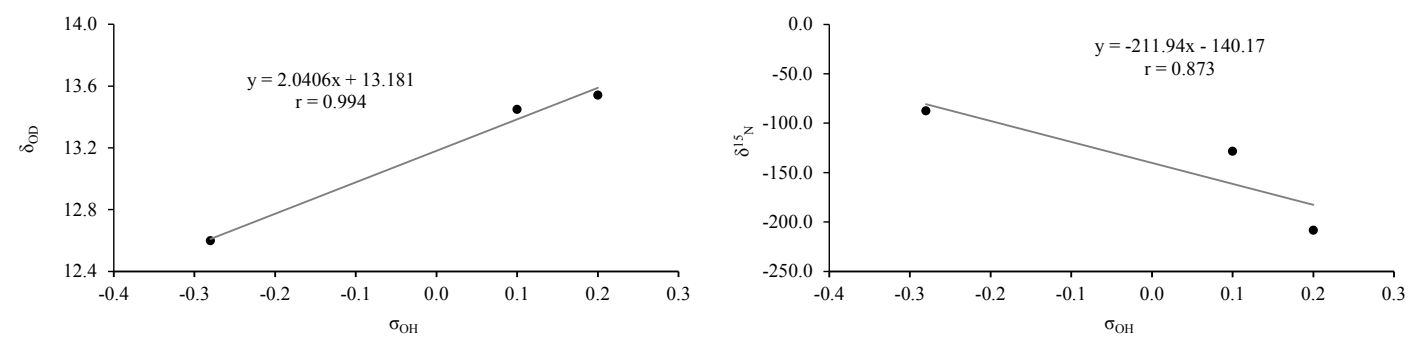

Figure 17S.
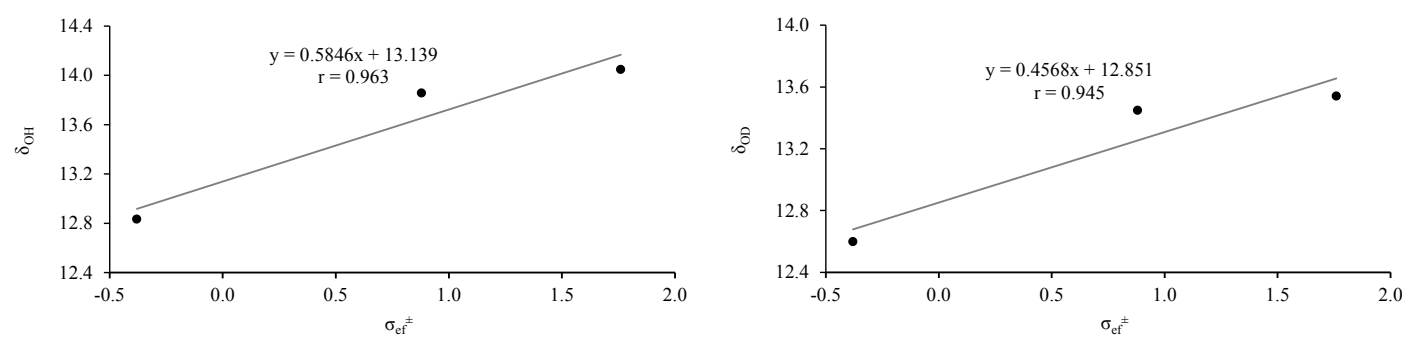

Figure 18S.
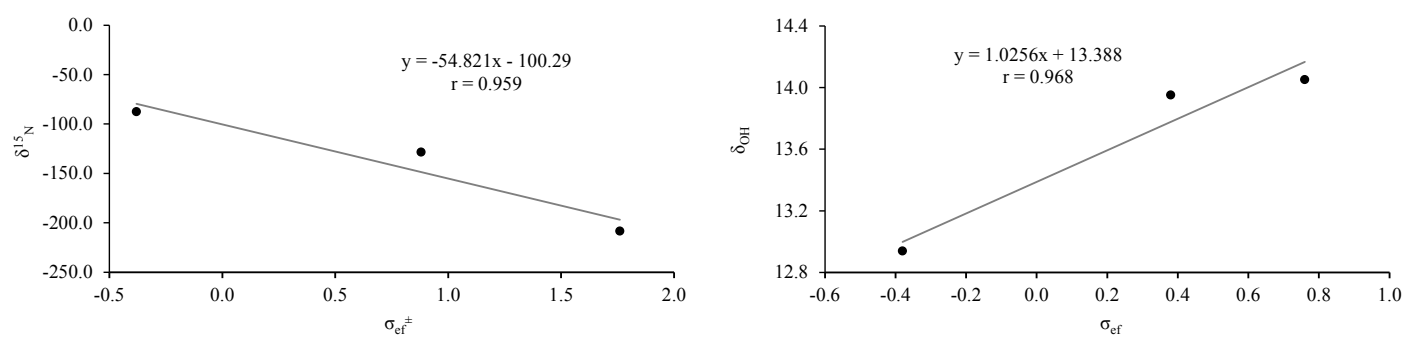

Figure 19S. 

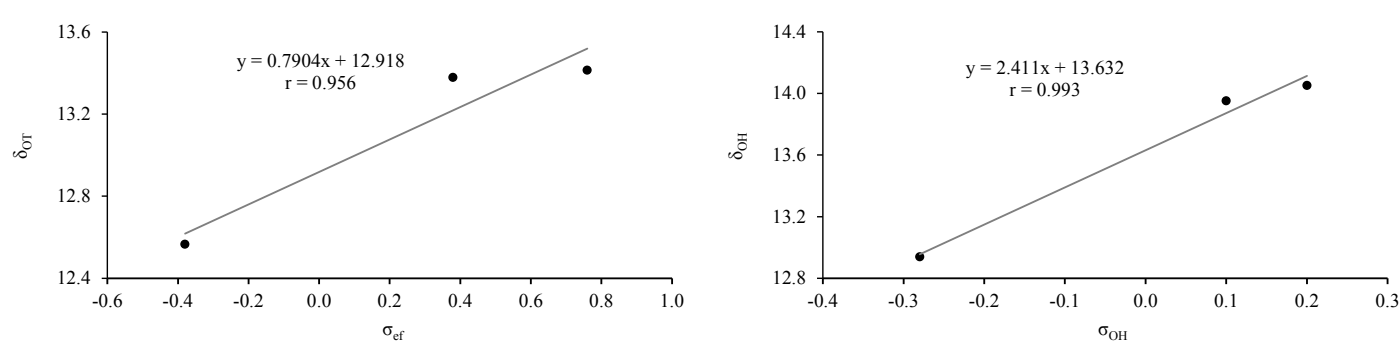

Figure 20S.
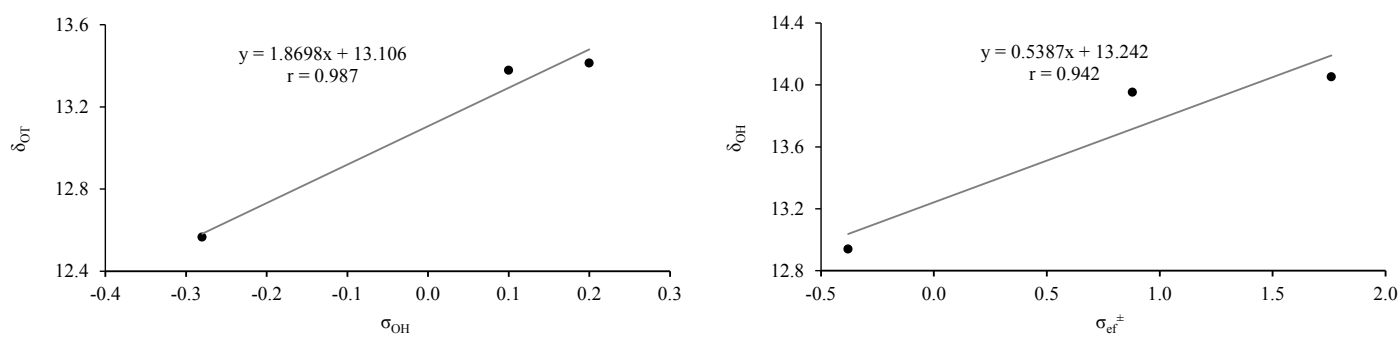

Figure 21S.
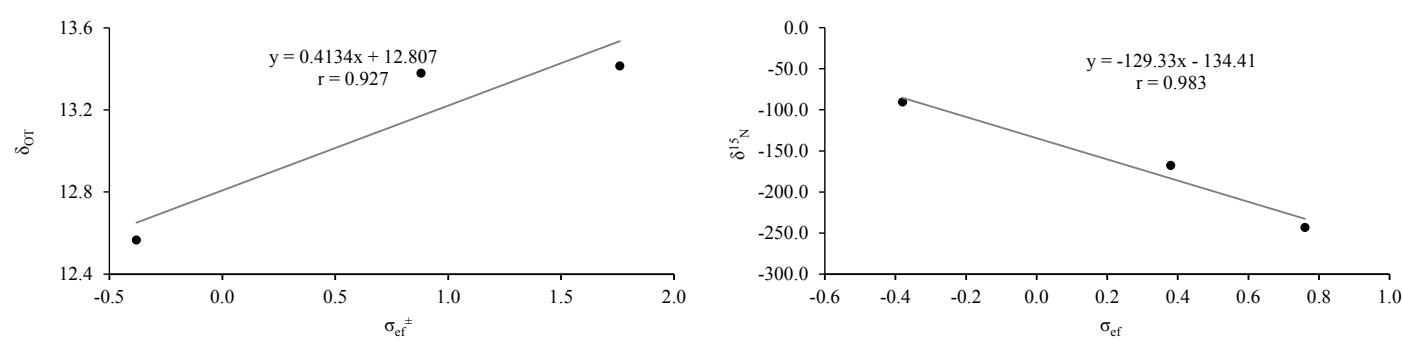

Figure 22S.
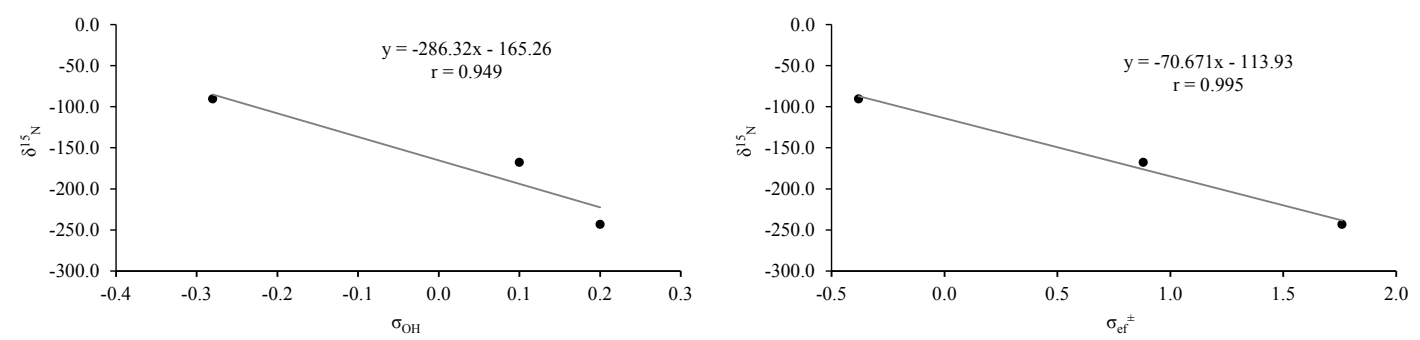

Figure 23S. 
Table 13S. Chemical shits (ppm) of 127-134. ${ }^{\mathrm{a}}$

\begin{tabular}{|c|c|c|c|c|c|c|c|c|}
\hline Comp. & $\mathbf{X}$ & $\delta_{\mathrm{OH}}^{\mathrm{b}}$ & $\delta_{\mathrm{C} 2}{ }^{\mathrm{c}}$ & $\delta^{15} \mathrm{~N}^{\mathrm{d}}$ & $\sigma_{\text {ef }}$ & $\sigma_{\mathrm{OH}}$ & $\sigma_{\mathrm{NH}}$ & $\sigma_{\text {eff }}^{ \pm}$ \\
\hline 127 & $5-\mathrm{H}$ & 13.4 & 161.0 & -84.1 & 0.00 & 0.00 & 0.00 & 0.00 \\
\hline 128 & $5-\mathrm{NO}_{2}$ & 14.5 & 175.8 & -161.3 & 0.10 & 0.81 & 0.71 & 0.52 \\
\hline 129 & 5-OMe & 12.8 & 154.8 & -80.3 & -0.38 & -0.28 & 0.10 & -0.38 \\
\hline 130 & $5-\mathrm{Br}$ & 13.5 & 160.5 & -82.8 & -0.11 & 0.26 & 0.37 & -0.11 \\
\hline 131 & $5-\mathrm{H}$ & 13.7 & 161.4 & -87.6 & 0.00 & 0.00 & 0.00 & 0.00 \\
\hline 132 & $5-\mathrm{NO}_{2}$ & 15.5 & 178.0 & -182.5 & 0.10 & 0.81 & 0.71 & 0.52 \\
\hline 133 & 5-OMe & 13.0 & 154.9 & -82.5 & -0.38 & -0.28 & 0.10 & -0.38 \\
\hline 134 & $5-\mathrm{Br}$ & 13.8 & 161.2 & -89.1 & -0.11 & 0.26 & 0.37 & -0.11 \\
\hline
\end{tabular}
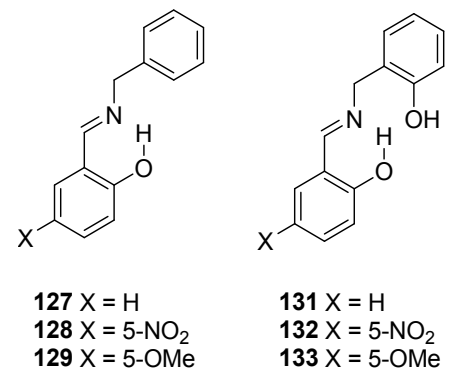

$129 X=5-\mathrm{OMe}$

$131 \mathrm{X}=\mathrm{H}$

$132 \mathrm{X}=5-\mathrm{NO}_{2}$

$133 \mathrm{X}=5-\mathrm{OMe}$

$130 \mathrm{X}=5-\mathrm{Br}$

$134 \mathrm{X}=5-\mathrm{Br}$

For compounds 127-130:
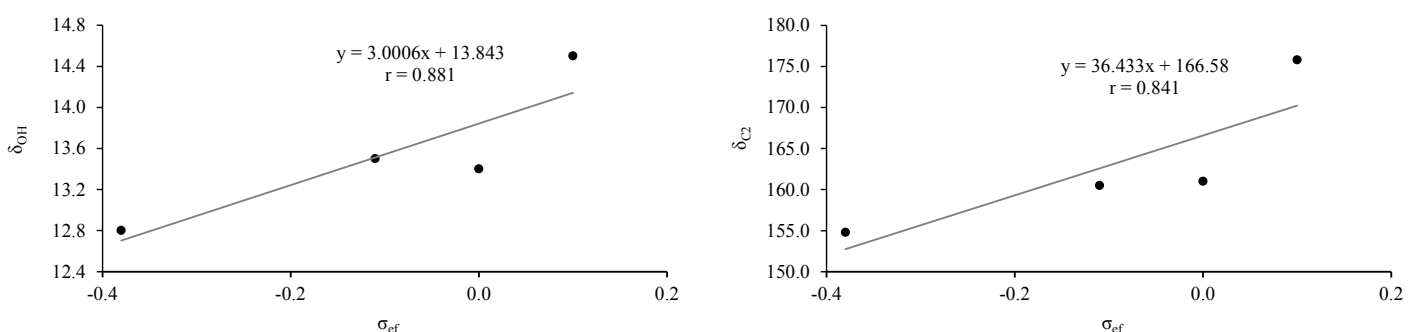

Figure 24S.
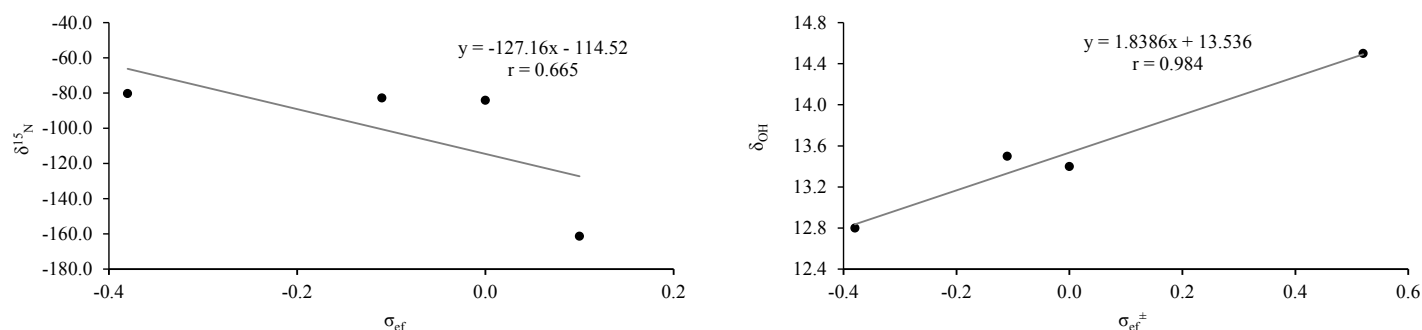

Figure 25S.
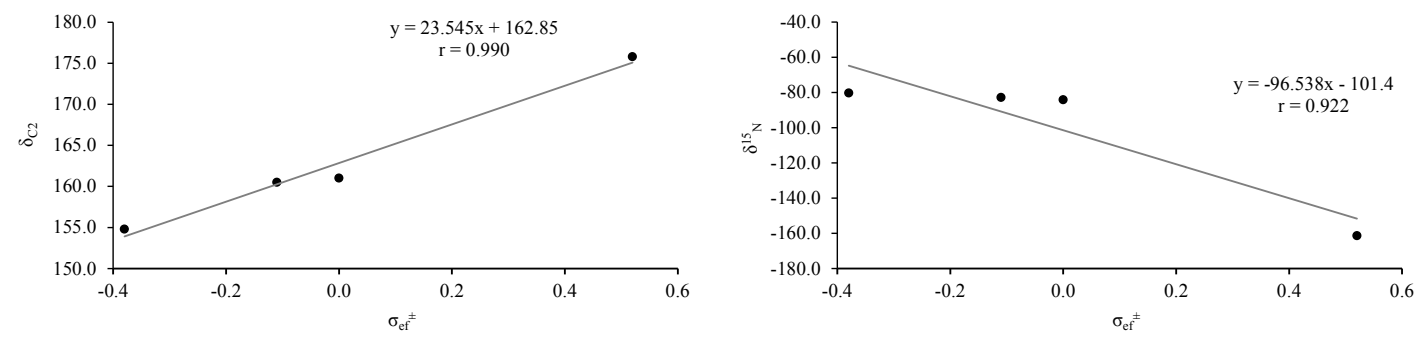

Figure 26S. 
For compounds 131-134:
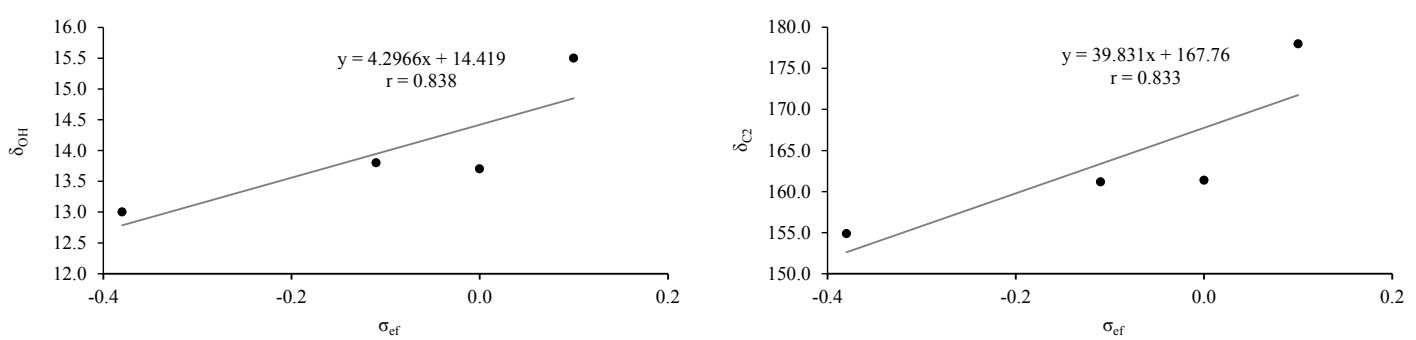

Figure 27S.
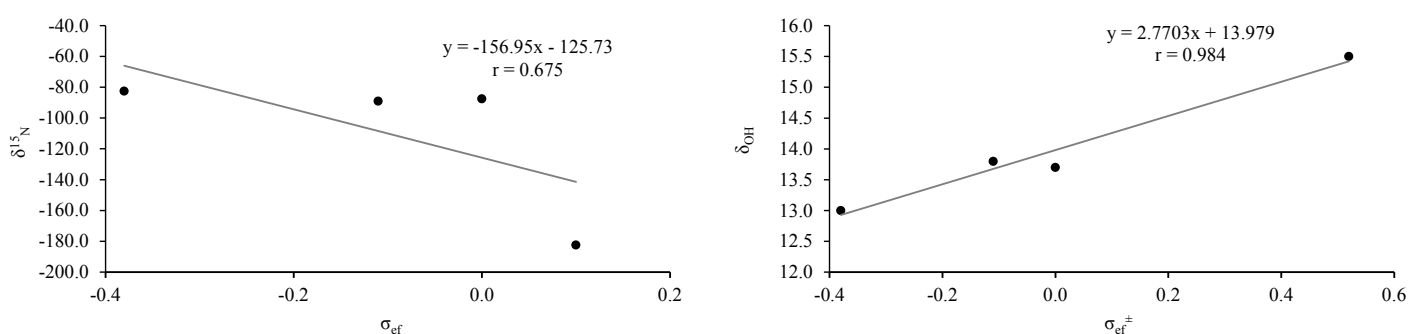

Figure 28S.
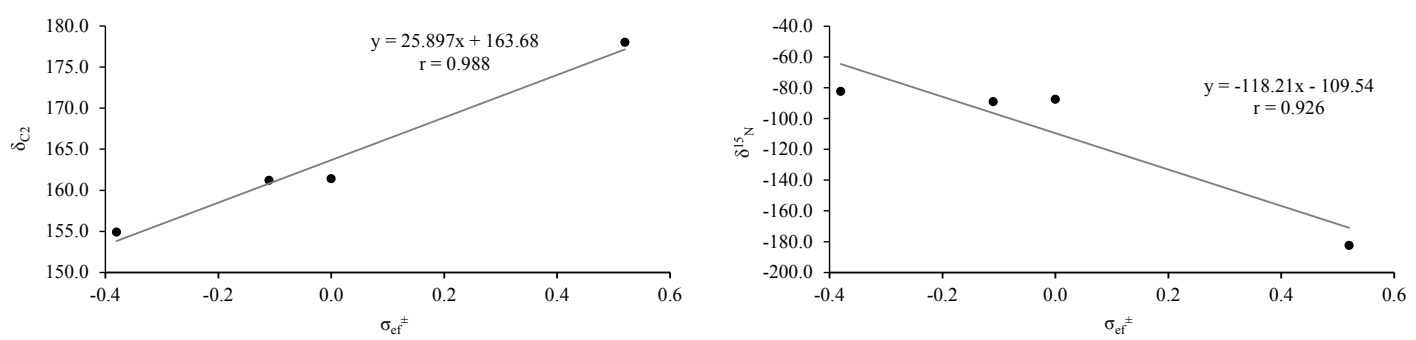

Figure 29S.

Table 14S. Physicochemical parameters of compounds 127-130, 135, and 136. ${ }^{\mathrm{a}}$

\begin{tabular}{clcccccc}
\hline Comp. & $\mathbf{X}$ & $\mathbf{K}_{\mathbf{a}} / \mathbf{1 0}^{-\mathbf{9}}$ & $\mathbf{p K}_{\mathbf{a}}$ & $\boldsymbol{\sigma}_{\text {ef }}$ & $\boldsymbol{\sigma}_{\mathbf{O H}}$ & $\boldsymbol{\sigma}_{\mathbf{O H}^{-}}$ & $\boldsymbol{\sigma}_{\text {ef }}{ }^{ \pm}$ \\
\hline $\mathbf{1 2 7}$ & $5-\mathrm{H}$ & 2.13 & 8.6 & 0.00 & 0.00 & 0.00 & 0.00 \\
$\mathbf{1 2 8}$ & $5-\mathrm{NO}_{2}$ & 15.1 & 7.8 & 0.10 & 0.81 & 1.23 & 0.52 \\
$\mathbf{1 2 9}$ & $5-\mathrm{OMe}$ & 1.23 & 8.9 & -0.38 & -0.28 & -0.28 & -0.38 \\
$\mathbf{1 3 0}$ & $5-\mathrm{Br}$ & 1.44 & 8.8 & -0.11 & 0.26 & 0.26 & -0.11 \\
$\mathbf{1 3 5}$ & $5-\mathrm{Cl}$ & 3.33 & 8.4 & -0.13 & 0.24 & 0.24 & -0.13 \\
$\mathbf{1 3 6}$ & $5-\mathrm{OH}$ & 0.17 & 9.7 & -0.51 & -0.38 & -0.38 & -0.51 \\
\hline
\end{tabular}

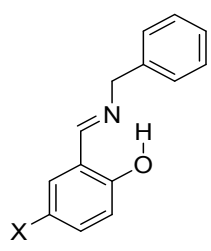

$135 \mathrm{X}=5-\mathrm{Cl}$

$136 \mathrm{X}=5-\mathrm{OH}$ 

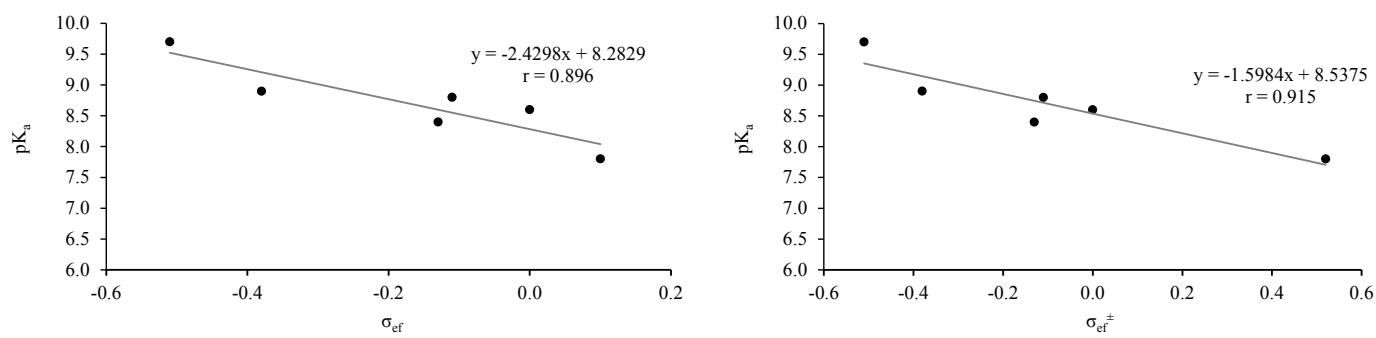

Figure 30S.
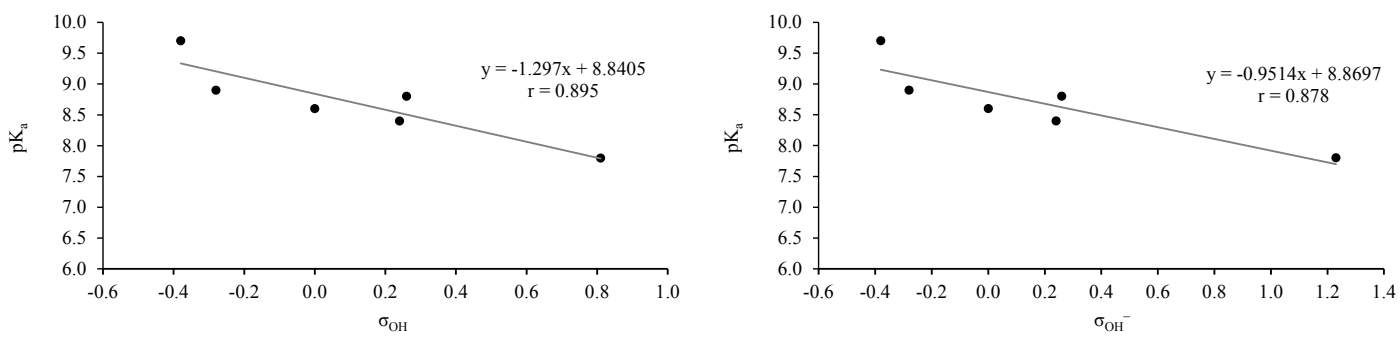

Figure 31S. 


\section{Imine/Enamine-Oxazolidine Equilibria.}

Schiff bases derived from TRIS (31S/32S) may exhibit open chain-cyclic isomerism (oxazolidine 33S). ${ }^{6}$ Thus, when DMSO- $d_{6}$ solutions of these Schiff bases are allowed to stand at room temperature, partial isomerization to a cyclic oxazolidine occurs (Scheme 3S).

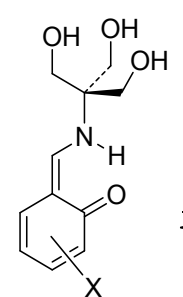

315

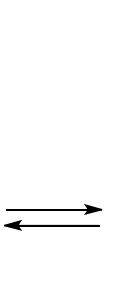

$1 \Rightarrow$

$32 S$

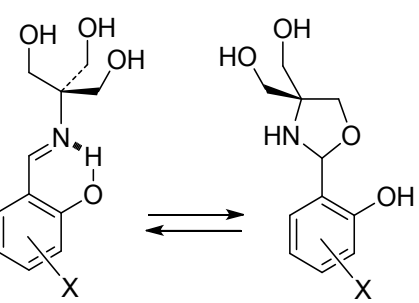

335

Scheme 3S

Ring formation manifests itself by the resonance of $\mathrm{H}-2$ that appears at $\sim 5.57 \mathrm{ppm}$. However, the proportion of oxazolidine at equilibrium is very small $(\sim 5 \%)$; a typical ${ }^{1} \mathrm{H}$ NMR spectrum is shown in Figure $32 \mathrm{~S}$ for $7 / 37$. Previous work $^{6}$ on some arylidene derivatives from TRIS evidences that oxazolidines are predominant species in the equilibrium ( $>$ 89\%). Compounds $\mathbf{3 4 S}$ and $35 \mathrm{~S}$ (Scheme $4 \mathrm{~S}$ ) coexist at equilibrium in an approximate 1:1 ratio. $^{7}$ It is apparent that the intramolecular hydrogen bridge of TRIS-salicylidene derivatives stabilizes the imine/enamine structure (36S) and extensively inhibits the formation of the oxazolidine moiety (37S), thus reversing the percentages in the equilibria.
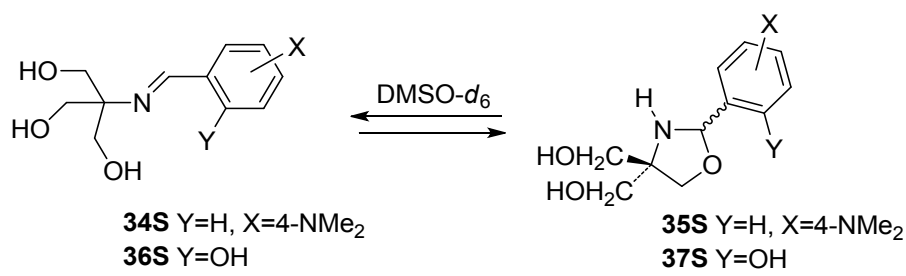

Scheme 4S 


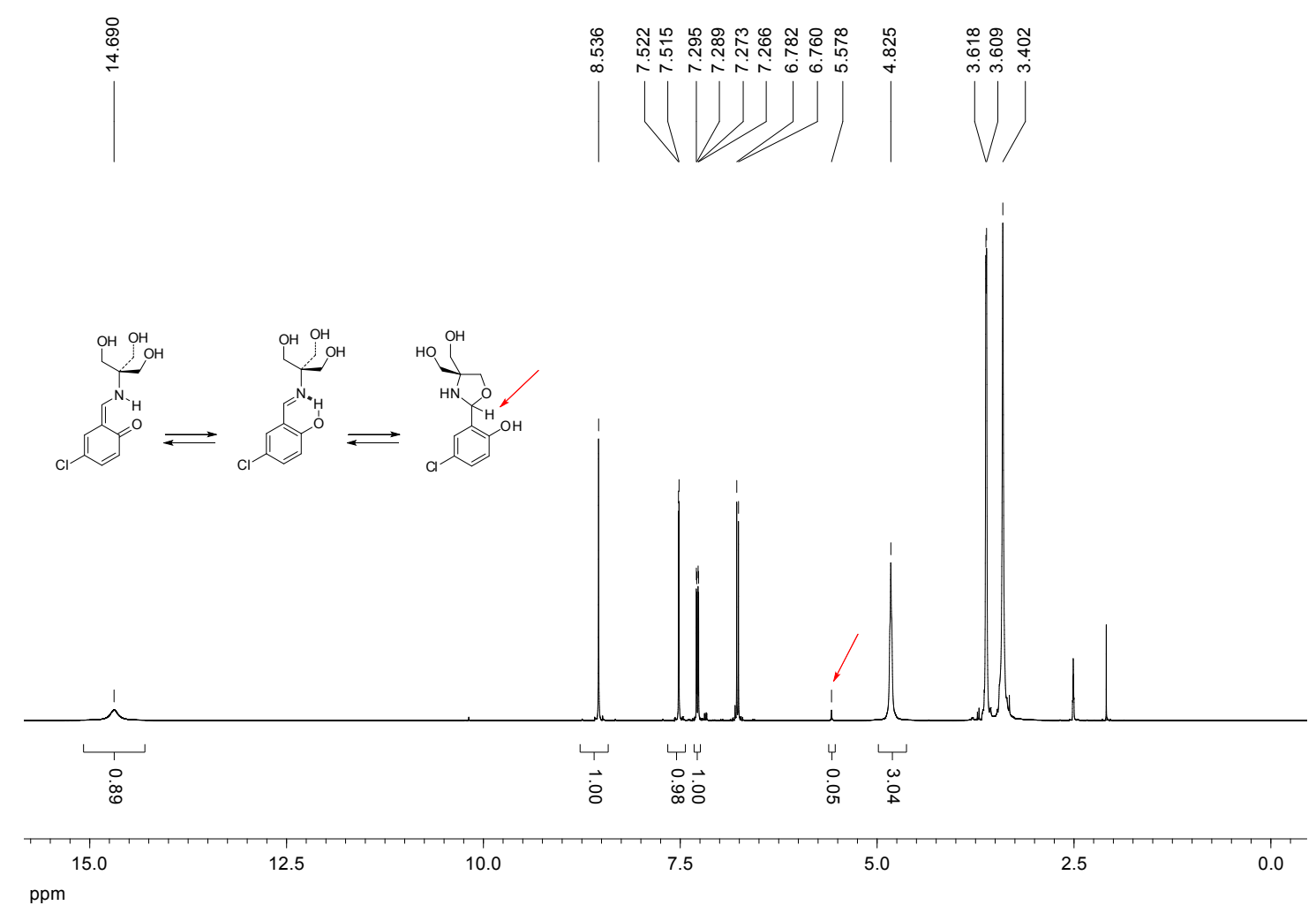

Figure 32S. ${ }^{1} \mathrm{H}$ NMR spectrum corresponding to the imine/enamine-oxazolidine equilibrium for compound $7 / 37$. 


\section{Imine-Enamine Tautomerizations.}

Table 15S. Relative energies calculated in gas phase $(\Delta \mathrm{E}$ and $\Delta \mathrm{G}, \mathrm{kcal} / \mathrm{mol}){ }^{\mathrm{a}}$

\begin{tabular}{|c|c|c|c|c|c|c|c|c|c|}
\hline \multirow{2}{*}{ Comp. } & \multicolumn{2}{|c|}{137} & \multicolumn{2}{|c|}{138} & \multicolumn{2}{|c|}{139} & \multicolumn{2}{|c|}{140} & \multirow{2}{*}{$\bar{v}_{\mathrm{i}}\left(\mathrm{cm}^{-1}\right)$} \\
\hline & $\Delta \mathbf{E}$ & $\Delta \mathbf{G}$ & $\Delta \mathbf{E}$ & $\Delta \mathbf{G}$ & $\Delta \mathbf{E}^{*}$ & $\Delta \mathbf{G}^{\star}$ & $\Delta \mathbf{E}$ & $\Delta \mathbf{G}$ & \\
\hline $4 / 34$ & 13.49 & 12.23 & 0.00 & 0.00 & 5.95 & 3.02 & 5.17 & 4.57 & 812 \\
\hline $6 / 36$ & 12.74 & 12.21 & 0.00 & 0.00 & 8.01 & 6.09 & 7.55 & 7.35 & 666 \\
\hline $7 / 37$ & 12.88 & 11.66 & 0.00 & 0.00 & 7.64 & 6.68 & 7.11 & 5.27 & 658 \\
\hline $8 / 38$ & 13.02 & 11.85 & 0.00 & 0.00 & 7.49 & 5.20 & 6.95 & 6.38 & 712 \\
\hline $10 / 40$ & 12.85 & 11.48 & 0.00 & 0.00 & 7.90 & 5.33 & 7.53 & 6.50 & 560 \\
\hline $12 / 42$ & 12.43 & 11.22 & 0.00 & 0.00 & 9.39 & 7.33 & 9.29 & 8.14 & 345 \\
\hline $13 / 43$ & 12.64 & 12.38 & 0.00 & 0.00 & 8.74 & 6.86 & 8.44 & 6.34 & 552 \\
\hline $14 / 44$ & 12.55 & 10.99 & 0.00 & 0.00 & 7.34 & 5.06 & 6.89 & 5.94 & 603 \\
\hline $15 / 45$ & 13.46 & 12.11 & 0.00 & 0.00 & 6.76 & 4.18 & 6.05 & 5.29 & 752 \\
\hline $16 / 46$ & 13.16 & 11.80 & 0.00 & 0.00 & 6.99 & 4.57 & 6.32 & 5.52 & 730 \\
\hline $17 / 47$ & 13.08 & 11.60 & 0.00 & 0.00 & 7.00 & 4.64 & 6.30 & 5.54 & 734 \\
\hline $18 / 48$ & 12.82 & 12.10 & 0.00 & 0.00 & 7.69 & 4.88 & 7.43 & 5.96 & 493 \\
\hline $21 / 51$ & 7.47 & 7.01 & 0.00 & 0.00 & 7.25 & 4.93 & 6.39 & 6.02 & 781 \\
\hline $23 / 53$ & 13.98 & 12.66 & 0.00 & 0.00 & 6.22 & 4.18 & 5.52 & 5.07 & 784 \\
\hline $24 / 54$ & 13.71 & 12.72 & 0.00 & 0.00 & 6.29 & 4.01 & 5.71 & 5.05 & 739 \\
\hline $27 / 57$ & 9.67 & 8.81 & 0.00 & 0.00 & 7.16 & 5.08 & 6.34 & 5.86 & 772 \\
\hline $30 / 60$ & 5.34 & 4.35 & 0.00 & 0.00 & 3.84 & 0.98 & 2.51 & 2.10 & 880 \\
\hline $31 / 61$ & 8.13 & 7.48 & 0.00 & 0.00 & 5.32 & 3.07 & 3.97 & 3.58 & 931 \\
\hline $84 / 38 S$ & 13.13 & 11.82 & 0.00 & 0.00 & 7.11 & 4.49 & 6.53 & 5.58 & 686 \\
\hline
\end{tabular}

${ }^{a}$ DFT calculations at M06-2X/6-311++G(d,p) level.

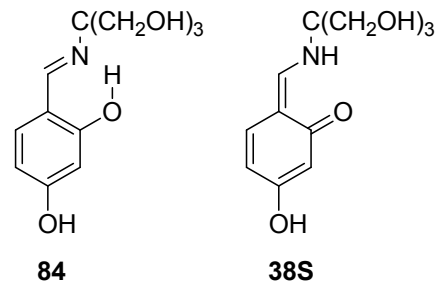

Calculations on 124-126 indicate that imine is the most stable tautomer; but the difference in stability with the enamine structure is significantly reduced in the order $124>125>126$, as predicted by the increased $\sigma_{\text {ef }}$ (Table $\left.16 \mathrm{~S}\right)$.

Table 16S. Relative energies calculated for 124-126 ( $\Delta \mathrm{E}$ and $\Delta \mathrm{G}, \mathrm{kcal} / \mathrm{mol}){ }^{\mathrm{a}, \mathrm{b}}$

\begin{tabular}{|c|c|c|c|c|c|c|c|c|}
\hline \multicolumn{3}{|c|}{ Imine } & \multicolumn{3}{|c|}{ TS } & \multicolumn{3}{|c|}{ Enamine } \\
\hline Comp. & $\Delta \mathbf{E}$ & $\Delta \mathbf{G}$ & $\Delta \mathbf{E}$ & $\Delta \mathbf{G}$ & $v_{i}^{c}$ & $\Delta \mathbf{E}$ & $\Delta \mathbf{G}$ & $\sigma_{\text {ef }}$ \\
\hline 124 & 0.00 & 0.00 & -- & -- & -- & 10.18 & 8.54 & -0.38 \\
\hline 125 & 0.00 & 0.00 & 7.19 & 4.56 & -882.5 & 6.01 & 5.40 & 0.38 \\
\hline 126 & 0.00 & 0.00 & 5.71 & 3.30 & -979.0 & 4.08 & 3.75 & 0.76 \\
\hline
\end{tabular}

Again, the activation energies in gas phase are always negative in the endothermic direction of equilibrium (transformation from enamine to imine, $\Delta \mathrm{G}_{\mathrm{e}}^{*}=\Delta \mathrm{G}_{\mathrm{TS}} \mathrm{s}^{\ddagger}-\Delta \mathrm{G}_{\text {enamine }}<0.0 \mathrm{kcal} \mathrm{mol}^{-1}$ ). 
Table 17S. Relative energies calculated in DMSO ( $\Delta \mathrm{E}$ and $\Delta \mathrm{G}$, in $\mathrm{kcal} / \mathrm{mol}){ }^{\mathrm{a}}$

\begin{tabular}{|c|c|c|c|c|c|c|c|c|c|}
\hline \multirow{2}{*}{ Compds. } & \multicolumn{2}{|c|}{137} & \multicolumn{2}{|c|}{138} & \multicolumn{2}{|c|}{139} & \multicolumn{2}{|c|}{140} & \multirow{2}{*}{$\bar{v}_{\mathrm{i}}\left(\mathbf{c m}^{-1}\right)$} \\
\hline & $\Delta \mathbf{E}$ & $\Delta \mathbf{G}$ & $\Delta \mathbf{E}$ & $\Delta \mathbf{G}$ & $\Delta \mathbf{E}^{*}$ & $\Delta \mathbf{G}^{\ddagger}$ & $\Delta \mathbf{E}$ & $\Delta \mathbf{G}$ & \\
\hline $4 / 34$ & 10.97 & 10.50 & 0.00 & 0.00 & 3.34 & 1.00 & 0.39 & 0.15 & 1024 \\
\hline $6 / 36$ & 10.38 & 10.03 & 0.00 & 0.00 & 5.53 & 3.36 & 3.93 & 3.79 & 951 \\
\hline $7 / 37$ & 10.48 & 9.74 & 0.00 & 0.00 & 5.05 & 2.63 & 3.23 & 2.67 & 937 \\
\hline $8 / 38$ & 10.68 & 9.09 & 0.00 & 0.00 & 5.05 & 2.24 & 3.09 & 2.94 & 943 \\
\hline $10 / 40$ & 10.41 & 10.11 & 0.00 & 0.00 & 5.32 & 2.96 & 3.57 & 3.78 & 965 \\
\hline $12 / 42$ & 10.06 & 9.92 & 0.00 & 0.00 & 6.62 & 5.14 & 5.67 & 5.34 & 809 \\
\hline $13 / 43$ & 10.18 & 10.28 & 0.00 & 0.00 & 6.28 & 4.34 & 4.98 & 5.23 & 890 \\
\hline $14 / 44$ & 10.67 & 10.92 & 0.00 & 0.00 & 4.71 & 2.66 & 2.77 & 2.76 & 940 \\
\hline $15 / 45$ & 10.91 & 9.88 & 0.00 & 0.00 & 4.34 & 1.99 & 1.92 & 2.02 & 987 \\
\hline $16 / 46$ & 10.79 & 9.50 & 0.00 & 0.00 & 4.47 & 2.36 & 2.17 & 1.92 & 976 \\
\hline $17 / 47$ & 10.76 & 11.57 & 0.00 & 0.00 & 4.48 & 2.39 & 2.14 & 1.64 & 974 \\
\hline $18 / 48$ & 10.55 & 9.89 & 0.00 & 0.00 & 4.94 & 2.48 & 3.00 & 3.02 & 982 \\
\hline $21 / 51$ & 7.19 & 6.79 & 0.00 & 0.00 & 4.90 & 2.88 & 2.65 & 2.71 & 990 \\
\hline $23 / 53$ & 11.06 & 9.44 & 0.00 & 0.00 & 4.23 & 1.80 & 2.12 & 1.51 & 961 \\
\hline $24 / 54$ & 11.25 & 11.11 & 0.00 & 0.00 & 3.97 & 1.27 & 1.80 & 1.48 & 969 \\
\hline $27 / 57$ & 9.76 & 9.57 & 0.00 & 0.00 & 4.40 & 2.37 & 1.94 & 2.02 & 968 \\
\hline $30 / 60$ & 11.40 & 9.70 & 3.57 & 2.49 & 4.80 & 1.49 & 0.00 & 0.00 & 1049 \\
\hline $31 / 61$ & 8.74 & 7.85 & 0.76 & 0.31 & 3.64 & 0.94 & 0.00 & 0.00 & 1073 \\
\hline 84/38S & 10.74 & 10.36 & 0.00 & 0.00 & 5.22 & 2.41 & 2.25 & 1.86 & 1008 \\
\hline
\end{tabular}

aDT calculations at the M06-2X/6-311++G(d,p) level using the SMD method (DMSO as solvent). 
Table 18S. Thermochemical and geometrical data of each structure along the reaction path for transformation of $\mathbf{1 0} / \mathbf{4 0} .^{\text {a }}$

\begin{tabular}{|c|c|c|c|c|c|c|}
\hline $\begin{array}{l}\text { Path } \\
\text { steps }\end{array}$ & $\begin{array}{c}\text { Electronic } \\
\text { energy, E } \\
(\mathrm{kcal} / \mathrm{mol})\end{array}$ & $\begin{array}{c}\text { Enthalpy, H } \\
\text { (kcal/mol) }\end{array}$ & $\begin{array}{l}\text { Gibbs Free } \\
\text { Energy, G } \\
(\mathrm{kcal} / \mathrm{mol})\end{array}$ & $\begin{array}{l}\text { Frequency } \\
\qquad\left(\mathrm{cm}^{-1}\right)\end{array}$ & $\begin{array}{l}\mathrm{d}_{\mathrm{N}-\mathrm{H}} \\
(\AA)\end{array}$ & $\begin{array}{c}\mathrm{d}_{\mathrm{O}-\mathrm{H}} \\
(\AA)\end{array}$ \\
\hline 1 & -491818.83 & -491647.54 & -491685.18 & 17.64 & 1.650 & 1.006 \\
\hline 2 & -491818.81 & -491647.53 & -491685.23 & 15.88 & 1.646 & 1.007 \\
\hline 3 & -491818.78 & -491647.49 & -491685.33 & 12.68 & 1.642 & 1.005 \\
\hline 4 & -491818.74 & -491647.48 & -491685.35 & 11.45 & 1.635 & 1.007 \\
\hline 5 & -491818.70 & -491647.45 & -491685.47 & 9.01 & 1.629 & 1.007 \\
\hline 6 & -491818.65 & -491647.42 & -491685.46 & 8.19 & 1.620 & 1.009 \\
\hline 7 & -491818.60 & -491647.38 & -491685.47 & 7.38 & 1.611 & 1.011 \\
\hline 8 & -491818.54 & -491647.32 & -491685.59 & 5.32 & 1.601 & 1.012 \\
\hline 9 & -491818.46 & -491647.83 & -491683.34 & -6.87 & 1.593 & 1.012 \\
\hline 10 & -491818.37 & -491647.76 & -491683.24 & -9.87 & 1.577 & 1.015 \\
\hline 11 & -491818.27 & -491647.67 & -491683.13 & -12.80 & 1.561 & 1.018 \\
\hline 12 & -491818.13 & -491647.57 & -491683.00 & -15.77 & 1.543 & 1.021 \\
\hline 13 & -491817.97 & -491647.45 & -491682.84 & -18.98 & 1.524 & 1.025 \\
\hline 14 & -491817.76 & -491647.30 & -491682.65 & -21.52 & 1.502 & 1.029 \\
\hline 15 & -491817.50 & -491647.14 & -491682.43 & -23.85 & 1.477 & 1.036 \\
\hline 16 & -491817.16 & -491646.97 & -491682.21 & -23.69 & 1.447 & 1.047 \\
\hline 17 & -491816.61 & -491646.98 & -491682.18 & -16.38 & 1.402 & 1.078 \\
\hline 18 & -491815.78 & -491647.11 & -491684.02 & -407.86 & 1.348 & 1.130 \\
\hline 19 & -491814.95 & -491646.41 & -491683.10 & -1037.74 & 1.294 & 1.185 \\
\hline 20 & -491814.42 & -491645.83 & -491682.42 & -1178.14 & 1.244 & 1.241 \\
\hline 21 & -491814.27 & -491645.58 & -491682.16 & -989.26 & 1.198 & 1.297 \\
\hline 22 & -491814.40 & -491645.52 & -491682.20 & -717.83 & 1.152 & 1.353 \\
\hline 23 & -491814.67 & -491645.45 & -491682.18 & -316.16 & 1.119 & 1.405 \\
\hline 24 & -491814.97 & -491644.70 & -491682.64 & 15.12 & 1.092 & 1.454 \\
\hline 25 & -491815.23 & -491644.53 & -491682.11 & 15.34 & 1.076 & 1.495 \\
\hline 26 & -491815.43 & -491644.50 & -491682.02 & 17.12 & 1.067 & 1.529 \\
\hline 27 & -491815.60 & -491644.58 & -491682.10 & 18.40 & 1.060 & 1.556 \\
\hline 28 & -491815.73 & -491644.62 & -491682.22 & 16.97 & 1.053 & 1.582 \\
\hline 29 & -491815.86 & -491644.73 & -491682.24 & 19.67 & 1.053 & 1.598 \\
\hline 30 & -491815.97 & -491644.76 & -491682.33 & 17.72 & 1.048 & 1.615 \\
\hline 31 & -491816.07 & -491644.86 & -491682.31 & 21.20 & 1.050 & 1.629 \\
\hline 32 & -491816.16 & -491644.91 & -491682.41 & 20.17 & 1.047 & 1.644 \\
\hline 33 & -491816.24 & -491644.95 & -491682.50 & 18.64 & 1.044 & 1.658 \\
\hline 34 & -491816.32 & -491645.02 & -491682.53 & 19.96 & 1.044 & 1.670 \\
\hline 35 & -491816.39 & -491645.03 & -491682.61 & 17.95 & 1.040 & 1.684 \\
\hline 36 & -491816.45 & -491645.11 & -491682.60 & 20.73 & 1.042 & 1.693 \\
\hline 37 & -491816.51 & -491645.13 & -491682.62 & 21.00 & 1.040 & 1.705 \\
\hline 38 & -491816.56 & -491645.14 & -491682.63 & 21.11 & 1.038 & 1.717 \\
\hline 39 & -491816.61 & -491645.19 & -491682.60 & 23.54 & 1.038 & 1.726 \\
\hline 40 & -491816.66 & -491645.19 & -491682.60 & 23.75 & 1.035 & 1.738 \\
\hline 41 & -491816.70 & -491645.24 & -491682.57 & 26.14 & 1.038 & 1.743 \\
\hline
\end{tabular}

${ }^{a}$ DFT calculations at the M06-2X/6-311++G(d,p) level using the SMD method (DMSO). 


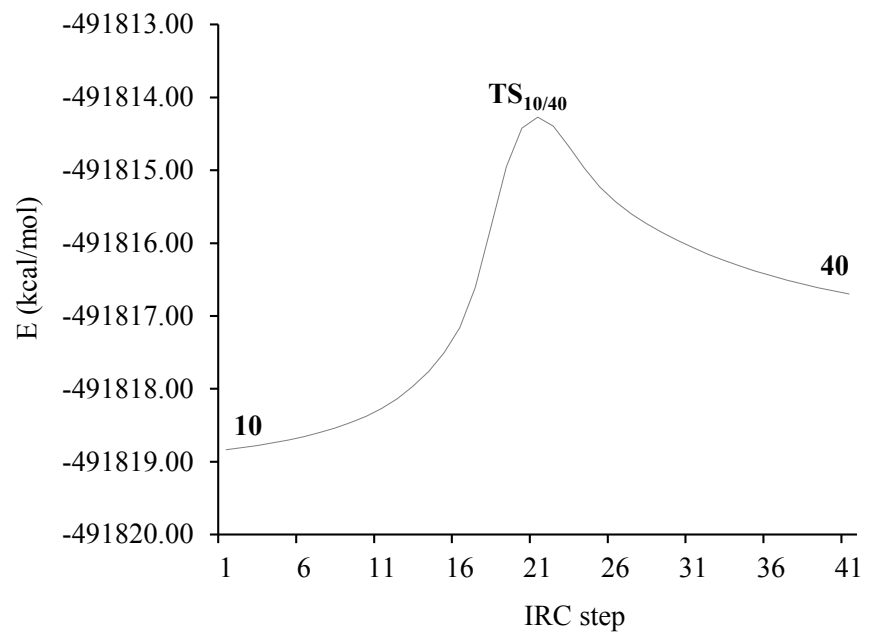

Figure 33S. IRC for tautomeric equilibrium between $\mathbf{1 0}$ and $\mathbf{4 0}$ in DMSO.

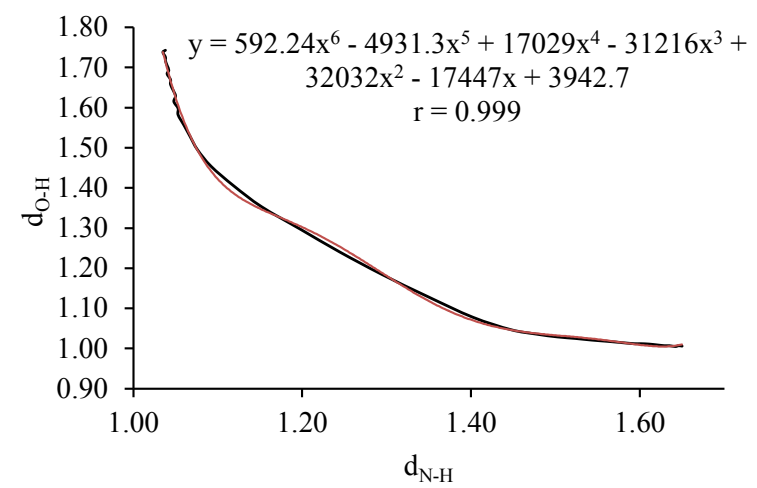

Figure 34S. Variation of the $\mathrm{NH}$ and $\mathrm{OH}$ distances along the reaction path of $\mathbf{1 0} / \mathbf{4 0}$. The Chebyshev polynomial that fits that variation is inserted (red line). 
Table 19S. Variations of relative thermodynamic parameters $(\mathrm{kcal} / \mathrm{mol})$ along the reaction coordinate for transformation of $10 / 40{ }^{a}$

\begin{tabular}{|c|c|c|c|}
\hline Path steps & $\Delta \mathrm{E}$ & $\Delta \mathrm{H}$ & $\Delta \mathrm{G}$ \\
\hline 1 & 0.00 & 0.00 & 0.00 \\
\hline 2 & 0.02 & 0.01 & -0.05 \\
\hline 3 & 0.05 & 0.05 & -0.15 \\
\hline 4 & 0.09 & 0.06 & -0.17 \\
\hline 5 & 0.13 & 0.09 & -0.29 \\
\hline 6 & 0.18 & 0.12 & -0.28 \\
\hline 7 & 0.23 & 0.16 & -0.29 \\
\hline 8 & 0.29 & 0.22 & -0.41 \\
\hline 9 & 0.37 & -0.29 & 1.84 \\
\hline 10 & 0.46 & -0.22 & 1.94 \\
\hline 11 & 0.56 & -0.13 & 2.05 \\
\hline 12 & 0.70 & -0.03 & 2.18 \\
\hline 13 & 0.86 & 0.09 & 2.34 \\
\hline 14 & 1.07 & 0.24 & 2.53 \\
\hline 15 & 1.33 & 0.40 & 2.75 \\
\hline 16 & 1.67 & 0.57 & 2.97 \\
\hline 17 & 2.22 & 0.56 & 3.00 \\
\hline 18 & 3.05 & 0.43 & 1.16 \\
\hline 19 & 3.88 & 1.13 & 2.08 \\
\hline 20 & 4.41 & 1.71 & 2.76 \\
\hline 21 & 4.56 & 1.96 & 3.02 \\
\hline 22 & 4.43 & 2.02 & 2.98 \\
\hline 23 & 4.16 & 2.09 & 3.00 \\
\hline 24 & 3.86 & 2.84 & 2.54 \\
\hline 25 & 3.60 & 3.01 & 3.07 \\
\hline 26 & 3.40 & 3.04 & 3.16 \\
\hline 27 & 3.23 & 2.96 & 3.08 \\
\hline 28 & 3.10 & 2.92 & 2.96 \\
\hline 29 & 2.97 & 2.81 & 2.94 \\
\hline 30 & 2.86 & 2.78 & 2.85 \\
\hline 31 & 2.76 & 2.68 & 2.87 \\
\hline 32 & 2.67 & 2.63 & 2.77 \\
\hline 33 & 2.59 & 2.59 & 2.68 \\
\hline 34 & 2.51 & 2.52 & 2.65 \\
\hline 35 & 2.44 & 2.51 & 2.57 \\
\hline 36 & 2.38 & 2.43 & 2.58 \\
\hline 37 & 2.32 & 2.41 & 2.56 \\
\hline 38 & 2.27 & 2.40 & 2.55 \\
\hline 39 & 2.22 & 2.35 & 2.58 \\
\hline 40 & 2.17 & 2.35 & 2.58 \\
\hline 41 & 2.13 & 2.30 & 2.61 \\
\hline
\end{tabular}

${ }^{\text {a }}$ DFT calculations at the M06-2X/6-311++G(d,p) level using the SMD method (DMSO). 


\section{Hydrogen Bonding Analysis.}

Table 20S. Hydrogen bonds $\left[\AA\right.$ and $\left.^{\circ}\right]$ and hydrogen-bonding energy ${ }^{\mathrm{a}}$ for imines 4, 6-8, 10, 12-18, 21, 23, 24, 27, 30, 31, and 84 from theoretical calculations. ${ }^{\text {b }}$

\begin{tabular}{|c|c|c|c|c|c|c|}
\hline Comp. & Substituent & $d(\mathrm{O}-\mathrm{H})$ & $d(\mathrm{H} \cdots \mathrm{N})$ & $d(\mathrm{O} \cdots \mathrm{N})$ & $\angle(\mathrm{OHN})$ & $-E_{\mathrm{HB}}{ }^{\mathrm{c}}$ \\
\hline 4 & $5-\mathrm{NO}_{2}$ & 0.994 & 1.716 & 2.611 & 147.7 & 11.82 \\
\hline 6 & $5-\mathrm{F}$ & 0.986 & 1.751 & 2.634 & 147.0 & 10.76 \\
\hline 7 & $5-\mathrm{Cl}$ & 0.987 & 1.755 & 2.638 & 147.0 & 10.58 \\
\hline 8 & $5-\mathrm{Br}$ & 0.988 & 1.741 & 2.627 & 147.2 & 11.07 \\
\hline 10 & $\mathrm{H}$ & 0.987 & 1.752 & 2.637 & 147.3 & 10.62 \\
\hline 12 & 5-OMe & 0.984 & 1.773 & 2.651 & 146.7 & 10.03 \\
\hline 13 & $5-\mathrm{OH}$ & 0.985 & 1.762 & 2.642 & 146.8 & 10.41 \\
\hline 14 & $4-\mathrm{NO}_{2}$ & 0.989 & 1.739 & 2.625 & 147.1 & 11.16 \\
\hline 15 & 4-F & 0.990 & 1.735 & 2.625 & 147.6 & 11.16 \\
\hline 16 & $4-\mathrm{Cl}$ & 0.990 & 1.739 & 2.627 & 147.4 & 11.07 \\
\hline 17 & $4-\mathrm{Br}$ & 0.989 & 1.739 & 2.628 & 147.4 & 11.02 \\
\hline 18 & 4-OMe & 0.988 & 1.748 & 2.637 & 147.7 & 10.62 \\
\hline 21 & 3-OMe & 0.993 & 1.702 & 2.599 & 148.2 & 12.42 \\
\hline 23 & 6-OMe & 0.993 & 1.696 & 2.594 & 148.4 & 12.68 \\
\hline 24 & 4-OH-6-Me & 0.989 & 1.741 & 2.631 & 147.8 & 10.89 \\
\hline 27 & 3,5-diF & 0.988 & 1.752 & 2.634 & 146.6 & 10.76 \\
\hline 30 & $3,5-\mathrm{diNO}_{2}$ & 1.005 & 1.653 & 2.569 & 149.3 & 14.06 \\
\hline 31 & $3-\mathrm{OMe}-5-\mathrm{NO}_{2}$ & 0.995 & 1.703 & 2.600 & 148.0 & 12.37 \\
\hline 84 & $4-\mathrm{OH}$ & 0.988 & 1.743 & 2.629 & 147.4 & 10.98 \\
\hline
\end{tabular}

${ }^{\mathrm{a}}$ In $\mathrm{kcal} / \mathrm{mol} ;{ }^{\mathrm{b}}$ At the M06-2X/6-311++G(d,p) level in the gas phase; ${ }^{\mathrm{c}}$ From equation [18].

Table 21S. Hydrogen bonds $\left[\AA\right.$ and $\left.{ }^{\circ}\right]$ and hydrogen-bonding energy ${ }^{a}$ for enamines 34, 36-38, 40, 42-48, 51, 53, 54, 57, 60, 61, and $\mathbf{3 8 S}$ from theoretical calculations. ${ }^{b}$

\begin{tabular}{|c|c|c|c|c|c|c|}
\hline Comp. & Substituent & $d(\mathrm{~N}-\mathrm{H})$ & $d(\mathrm{H} \cdots \mathrm{O})$ & $d(\mathrm{~N} \cdots \mathrm{O})$ & $\angle(\mathrm{NHO})$ & $-E_{\mathrm{HB}}{ }^{\mathrm{c}}$ \\
\hline 34 & $5-\mathrm{NO}_{2}$ & 1.043 & 1.685 & 2.570 & 139.7 & 14.00 \\
\hline 36 & $5-\mathrm{F}$ & 1.043 & 1.672 & 2.558 & 139.8 & 14.71 \\
\hline 37 & $5-\mathrm{Cl}$ & 1.042 & 1.683 & 2.566 & 139.5 & 14.23 \\
\hline 38 & $5-\mathrm{Br}$ & 1.043 & 1.674 & 2.561 & 140.0 & 14.53 \\
\hline 40 & $\mathrm{H}$ & 1.044 & 1.669 & 2.559 & 140.1 & 14.65 \\
\hline 42 & 5-OMe & 1.053 & 1.616 & 2.531 & 142.2 & 16.44 \\
\hline 43 & $5-\mathrm{OH}$ & 1.045 & 1.656 & 2.550 & 140.5 & 15.20 \\
\hline 44 & $4-\mathrm{NO}_{2}$ & 1.043 & 1.676 & 2.563 & 139.8 & 14.41 \\
\hline 45 & $4-\mathrm{F}$ & 1.040 & 1.695 & 2.573 & 139.0 & 13.83 \\
\hline 46 & $4-\mathrm{Cl}$ & 1.040 & 1.695 & 2.573 & 139.1 & 13.83 \\
\hline 47 & $4-\mathrm{Br}$ & 1.040 & 1.694 & 2.572 & 139.1 & 13.89 \\
\hline 48 & 4-OMe & 1.051 & 1.631 & 2.542 & 141.8 & 15.71 \\
\hline 51 & 3-OMe & 1.041 & 1.677 & 2.559 & 139.4 & 14.65 \\
\hline 53 & 6-OMe & 1.043 & 1.659 & 2.544 & 139.6 & 15.82 \\
\hline 54 & 4-OH-6-Me & 1.042 & 1.685 & 2.568 & 139.5 & 14.12 \\
\hline 57 & 3,5-diF & 1.038 & 1.705 & 2.576 & 138.6 & 13.66 \\
\hline 60 & 3,5-diNO ${ }_{2}$ & 1.039 & 1.703 & 2.576 & 138.5 & 13.66 \\
\hline 61 & 3-OMe-5- $\mathrm{NO}_{2}$ & 1.036 & 1.721 & 2.584 & 137.8 & 13.22 \\
\hline $38 S$ & $4-\mathrm{OH}$ & 1.037 & 1.709 & 2.575 & 138.0 & 13.71 \\
\hline
\end{tabular}


Table 22S. Hydrogen bonds $\left[\AA\right.$ and $\left.^{\circ}\right]$ and hydrogen-bonding energy ${ }^{a}$ for imines 4, 6-8, 10, 12-18, 21, 23, 24, 27, 30, 31, and 84 from theoretical calculations. ${ }^{\mathrm{b}}$

\begin{tabular}{|c|c|c|c|c|c|c|}
\hline Compd. & Substituent & $d(\mathrm{O}-\mathrm{H})$ & $d\left(\mathrm{H}^{\cdots} \mathrm{N}\right)$ & $d(\mathrm{O} \cdots \mathrm{N})$ & $\angle(\mathrm{OHN})$ & $-E_{\mathrm{HB}}{ }^{\mathrm{c}}$ \\
\hline 4 & $5-\mathrm{NO}_{2}$ & 1.005 & 1.660 & 2.574 & 149.0 & 13.77 \\
\hline 6 & $5-\mathrm{F}$ & 0.994 & 1.708 & 2.606 & 148.1 & 12.07 \\
\hline 7 & $5-\mathrm{Cl}$ & 0.996 & 1.703 & 2.603 & 148.2 & 12.22 \\
\hline 8 & $5-\mathrm{Br}$ & 0.997 & 1.698 & 2.599 & 148.2 & 12.38 \\
\hline 10 & $\mathrm{H}$ & 0.995 & 1.710 & 2.609 & 148.4 & 11.93 \\
\hline 12 & $5-\mathrm{OMe}$ & 0.991 & 1.731 & 2.622 & 147.8 & 11.30 \\
\hline 13 & $5-\mathrm{OH}$ & 0.991 & 1.725 & 2.618 & 147.9 & 11.49 \\
\hline 14 & $4-\mathrm{NO}_{2}$ & 0.998 & 1.691 & 2.594 & 148.3 & 12.68 \\
\hline 15 & $4-\mathrm{F}$ & 0.999 & 1.689 & 2.596 & 148.8 & 12.58 \\
\hline 16 & $4-\mathrm{Cl}$ & 0.998 & 1.693 & 2.597 & 148.6 & 12.53 \\
\hline 17 & $4-\mathrm{Br}$ & 0.998 & 1.693 & 2.597 & 148.5 & 12.53 \\
\hline 18 & 4-OMe & 0.996 & 1.703 & 2.607 & 148.9 & 12.02 \\
\hline 21 & 3-OMe & 0.996 & 1.695 & 2.598 & 148.7 & 12.47 \\
\hline 23 & 6-OMe & 1.000 & 1.663 & 2.574 & 149.2 & 13.77 \\
\hline 24 & 4-OH-6-Me & 1.002 & 1.650 & 2.565 & 149.6 & 14.29 \\
\hline 27 & 3,5-diF & 0.998 & 1.694 & 2.595 & 148.0 & 12.62 \\
\hline 30 & 3,5-diNO & 1.027 & 1.572 & 2.520 & 150.9 & 17.20 \\
\hline 31 & 3-OMe-5- $\mathrm{NO}_{2}$ & 1.007 & 1.647 & 2.564 & 149.2 & 14.35 \\
\hline 84 & $4-\mathrm{OH}$ & 0.997 & 1.697 & 2.603 & 148.9 & 12.22 \\
\hline
\end{tabular}

Table 23S. Hydrogen bonds $\left[\AA\right.$ and $\left.^{\circ}\right]$ and hydrogen-bonding energy ${ }^{\mathrm{a}}$ for enamines , 36-38, 40, 42-48, 51, 53, 54, 57, 60, 61, and $38 \mathrm{~S}$ from theoretical calculations. ${ }^{\mathrm{b}}$

\begin{tabular}{|c|c|c|c|c|c|c|}
\hline Compd. & Substituent & $d(\mathrm{~N}-\mathrm{H})$ & $d(\mathrm{H} \cdots \mathrm{O})$ & $d(\mathrm{~N} \cdots \mathrm{O})$ & $\angle(\mathrm{NHO})$ & $-E_{\mathrm{HB}}{ }^{\mathrm{c}}$ \\
\hline 34 & $5-\mathrm{NO}_{2}$ & 1.029 & 1.841 & 2.661 & 133.9 & 9.62 \\
\hline 36 & $5-\mathrm{F}$ & 1.032 & 1.785 & 2.623 & 135.6 & 11.25 \\
\hline 37 & $5-\mathrm{Cl}$ & 1.030 & 1.819 & 2.644 & 134.4 & 10.32 \\
\hline 38 & $5-\mathrm{Br}$ & 1.032 & 1.790 & 2.629 & 135.7 & 10.98 \\
\hline 40 & $\mathrm{H}$ & 1.033 & 1.783 & 2.627 & 136.1 & 11.07 \\
\hline 42 & $5-\mathrm{OMe}$ & 1.034 & 1.777 & 2.619 & 135.9 & 11.44 \\
\hline 43 & $5-\mathrm{OH}$ & 1.033 & 1.773 & 2.616 & 136.0 & 11.58 \\
\hline 44 & $4-\mathrm{NO}_{2}$ & 1.033 & 1.789 & 2.631 & 135.8 & 10.89 \\
\hline 45 & $4-\mathrm{F}$ & 1.030 & 1.809 & 2.641 & 135.1 & 10.45 \\
\hline 46 & $4-\mathrm{Cl}$ & 1.031 & 1.809 & 2.641 & 135.1 & 10.45 \\
\hline 47 & $4-\mathrm{Br}$ & 1.031 & 1.813 & 2.643 & 134.9 & 10.36 \\
\hline 48 & 4-OMe & 1.033 & 1.786 & 2.630 & 136.2 & 10.93 \\
\hline 51 & 3-OMe & 1.028 & 1.817 & 2.638 & 134.1 & 10.58 \\
\hline 53 & 6-OMe & 1.029 & 1.808 & 2.627 & 133.8 & 11.07 \\
\hline 54 & 4-OH-6-Me & 1.029 & 1.786 & 2.613 & 134.6 & 11.73 \\
\hline 57 & 3,5-diF & 1.030 & 1.818 & 2.647 & 134.8 & 10.19 \\
\hline 60 & $3,5-\mathrm{diNO}_{2}$ & 1.028 & 1.868 & 2.672 & 132.5 & 9.20 \\
\hline 61 & 3-OMe-5- $\mathrm{NO}_{2}$ & 1.028 & 1.839 & 2.653 & 133.5 & 9.95 \\
\hline $38 \mathrm{~S}$ & $4-\mathrm{OH}$ & 1.030 & 1.813 & 2.643 & 135.0 & 10.36 \\
\hline
\end{tabular}
${ }^{\mathrm{a}}$ In $\mathrm{kcal} / \mathrm{mol} ;{ }^{\mathrm{b}}$ At the M06-2X/6-311++G(d,p) level, including the solvent effect (SMD model, DMSO as solvent); ${ }^{\mathrm{C}}$ From
equation [18]. 


\section{Pseudo-Aromaticity in H-bonded Tautomeric Salicylimines.}

The HOMA values have been determined by means of equation: ${ }^{8}$

$$
\mathrm{HOMA}=1-\frac{1}{n} \sum_{j=1}^{n} \alpha_{i}\left(R_{o p t, i}-R_{j}\right)^{2}
$$

were, $R_{\mathrm{opt}, \mathrm{CC}}=1.388 \AA, \alpha_{\mathrm{C}-\mathrm{C}}=257.7 ; R_{\mathrm{opt}, \mathrm{CN}}=1.334 \AA, \alpha_{\mathrm{C}-\mathrm{N}}=93.52 ; R_{\mathrm{opt}, \mathrm{CO}}=1.265 \AA$, $\alpha_{\mathrm{C}-\mathrm{O}}=157.38$.

Table 24S. HOMA values of pseudo heterocyclic fragment in imines (M06-2X/6-311++G(d,p) in gas phase).

\begin{tabular}{|c|c|c|c|c|c|}
\hline & $d_{1}$ & $d_{2}$ & $d_{3}$ & $d_{4}$ & \\
\hline Substit. & $\mathbf{d}_{\mathrm{C}-\mathrm{O}}$ & $\mathbf{d}_{\mathrm{C}-\mathrm{C}}$ & $\mathbf{d}_{\mathrm{C}-\mathrm{C}}$ & $d_{C-N}$ & HOMA \\
\hline $5-\mathrm{NO}_{2}$ & 1.328 & 1.416 & 1.463 & 1.277 & 0.36 \\
\hline $5-\mathrm{F}$ & 1.340 & 1.410 & 1.462 & 1.278 & 0.32 \\
\hline $5-\mathrm{Cl}$ & 1.337 & 1.410 & 1.462 & 1.278 & 0.34 \\
\hline $5-\mathrm{Br}$ & 1.336 & 1.411 & 1.462 & 1.278 & 0.34 \\
\hline $\mathrm{H}$ & 1.338 & 1.412 & 1.460 & 1.279 & 0.35 \\
\hline 5-OMe & 1.345 & 1.411 & 1.462 & 1.278 & 0.29 \\
\hline $5-\mathrm{OH}$ & 1.344 & 1.409 & 1.462 & 1.278 & 0.30 \\
\hline $4-\mathrm{NO}_{2}$ & 1.333 & 1.413 & 1.464 & 1.277 & 0.33 \\
\hline $4-\mathrm{F}$ & 1.334 & 1.414 & 1.458 & 1.279 & 0.38 \\
\hline $4-\mathrm{Cl}$ & 1.335 & 1.412 & 1.459 & 1.279 & 0.37 \\
\hline $4-\mathrm{Br}$ & 1.335 & 1.412 & 1.460 & 1.279 & 0.36 \\
\hline 4-OMe & 1.337 & 1.418 & 1.455 & 1.280 & 0.38 \\
\hline $4-\mathrm{OH}$ & 1.336 & 1.416 & 1.454 & 1.281 & 0.40 \\
\hline
\end{tabular}
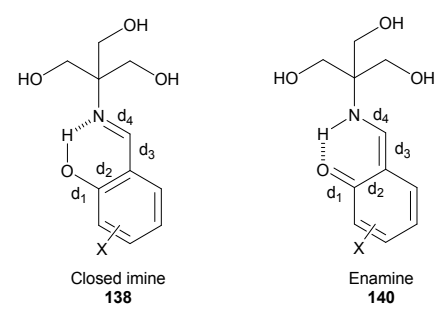
Table 25S. HOMA values of pseudo heterocyclic fragment in enamines (M06-2X/6-311++G(d,p) in gas phase).

\begin{tabular}{|c|c|c|c|c|c|}
\hline & $d_{1}$ & $d_{2}$ & $d_{3}$ & $d_{4}$ & \\
\hline Substit & $d_{C-O}$ & $\mathbf{d}_{\mathrm{C}-\mathrm{C}}$ & $\mathbf{d}_{\mathrm{C}-\mathrm{C}}$ & $\mathbf{d}_{\mathrm{C}-\mathrm{N}}$ & HOMA \\
\hline $5-\mathrm{NO}_{2}$ & 1.250 & 1.461 & 1.406 & 1.313 & 0.62 \\
\hline $5-\mathrm{F}$ & 1.256 & 1.455 & 1.398 & 1.321 & 0.69 \\
\hline $5-\mathrm{Cl}$ & 1.254 & 1.456 & 1.399 & 1.320 & 0.69 \\
\hline $5-\mathrm{Br}$ & 1.254 & 1.455 & 1.400 & 1.319 & 0.69 \\
\hline $\mathrm{H}$ & 1.256 & 1.457 & 1.398 & 1.321 & 0.68 \\
\hline 5-OMe & 1.263 & 1.452 & 1.401 & 1.319 & 0.72 \\
\hline $5-\mathrm{OH}$ & 1.259 & 1.452 & 1.398 & 1.321 & 0.72 \\
\hline $4-\mathrm{NO}_{2}$ & 1.253 & 1.453 & 1.404 & 1.315 & 0.70 \\
\hline $4-\mathrm{F}$ & 1.253 & 1.461 & 1.396 & 1.321 & 0.64 \\
\hline $4-\mathrm{Cl}$ & 1.253 & 1.458 & 1.397 & 1.320 & 0.67 \\
\hline $4-\mathrm{Br}$ & 1.253 & 1.457 & 1.398 & 1.320 & 0.68 \\
\hline 4-OMe & 1.260 & 1.462 & 1.397 & 1.320 & 0.64 \\
\hline $4-\mathrm{OH}$ & 1.255 & 1.464 & 1.393 & 1.322 & 0.62 \\
\hline
\end{tabular}

Table 26S. HOMA values of pseudo heterocyclic fragment in open imines (M06-2X/6-311++G(d,p) in gas phase).

\begin{tabular}{cccccc} 
& $\mathbf{d}_{\mathbf{1}}$ & $\mathbf{d}_{\mathbf{2}}$ & $\mathbf{d}_{\mathbf{3}}$ & $\mathbf{d}_{\mathbf{4}}$ & \\
\cline { 2 - 4 } Substit. & $\mathbf{d}_{\mathbf{C}-\mathbf{O}}$ & $\mathbf{d}_{\mathbf{C}-\mathbf{C}}$ & $\mathbf{d}_{\mathbf{C}-\mathbf{C}}$ & $\mathbf{d}_{\mathbf{C}-\mathbf{N}}$ & HOMA \\
\hline $5-\mathrm{NO}_{2}$ & 1.343 & 1.411 & 1.477 & 1.267 & 0.11 \\
$5-\mathrm{F}$ & 1.354 & 1.407 & 1.476 & 1.267 & 0.06 \\
$5-\mathrm{Cl}$ & 1.352 & 1.407 & 1.476 & 1.267 & 0.08 \\
$5-\mathrm{Br}$ & 1.351 & 1.407 & 1.476 & 1.267 & 0.08 \\
$\mathrm{H}$ & 1.352 & 1.407 & 1.474 & 1.268 & 0.10 \\
$5-\mathrm{OMe}$ & 1.358 & 1.409 & 1.476 & 1.268 & 0.03 \\
$5-\mathrm{OH}$ & 1.358 & 1.407 & 1.476 & 1.268 & 0.04 \\
$4-\mathrm{NO} 2$ & 1.348 & 1.410 & 1.477 & 1.267 & 0.08 \\
$4-\mathrm{F}$ & 1.349 & 1.409 & 1.473 & 1.268 & 0.13 \\
$4-\mathrm{Cl}$ & 1.349 & 1.408 & 1.474 & 1.268 & 0.12 \\
$4-\mathrm{Br}$ & 1.349 & 1.408 & 1.474 & 1.268 & 0.12 \\
$4-\mathrm{OMe}$ & 1.351 & 1.412 & 1.470 & 1.269 & 0.14 \\
$4-\mathrm{OH}$ & 1.350 & 1.410 & 1.470 & 1.269 & 0.15 \\
\hline
\end{tabular}
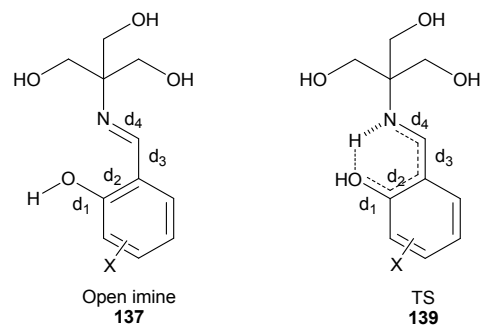

Table 27S. HOMA values of pseudo heterocyclic fragment in TS (M06-2X/6-311++G(d,p) in gas phase).

\begin{tabular}{cccccc}
\cline { 2 - 5 } & $\mathbf{d}_{\mathbf{1}}$ & $\mathbf{d}_{\mathbf{2}}$ & $\mathbf{d}_{\mathbf{3}}$ & $\mathbf{d}_{\mathbf{4}}$ & \\
\hline Substit. & $\mathbf{d}_{\mathrm{C}-\mathrm{O}}$ & $\mathbf{d}_{\mathrm{C}-\mathrm{C}}$ & $\mathbf{d}_{\mathrm{C}-\mathrm{C}}$ & $\mathbf{d}_{\mathrm{C}-\mathrm{N}}$ & HOMA \\
\hline $5-\mathrm{NO}_{2}$ & 1.277 & 1.444 & 1.428 & 1.298 & 0.66 \\
$5-\mathrm{F}$ & 1.280 & 1.440 & 1.418 & 1.305 & 0.74 \\
$5-\mathrm{Cl}$ & 1.279 & 1.440 & 1.419 & 1.304 & 0.73 \\
\hline
\end{tabular}




\begin{tabular}{cccccc}
\hline $5-\mathrm{Br}$ & 1.279 & 1.440 & 1.420 & 1.304 & 0.73 \\
$\mathrm{H}$ & 1.279 & 1.443 & 1.416 & 1.306 & 0.73 \\
$5-\mathrm{OMe}$ & 1.278 & 1.443 & 1.414 & 1.309 & 0.74 \\
$5-\mathrm{OH}$ & 1.281 & 1.439 & 1.416 & 1.308 & 0.75 \\
$4-\mathrm{NO}_{2}$ & 1.277 & 1.440 & 1.424 & 1.301 & 0.71 \\
$4-\mathrm{F}$ & 1.280 & 1.444 & 1.418 & 1.304 & 0.71 \\
$4-\mathrm{Cl}$ & 1.280 & 1.441 & 1.419 & 1.304 & 0.73 \\
$4-\mathrm{Br}$ & 1.280 & 1.440 & 1.420 & 1.303 & 0.73 \\
$4-\mathrm{OMe}$ & 1.279 & 1.450 & 1.412 & 1.308 & 0.69 \\
$4-\mathrm{OH}$ & 1.280 & 1.448 & 1.414 & 1.307 & 0.70 \\
\hline
\end{tabular}

Table 28S. HOMA values of benzene rings in imines (M06-2X/6-311++G(d,p) in gas phase).

\begin{tabular}{|c|c|c|c|c|c|c|c|}
\hline Substit. & $d_{1}$ & $d_{2}$ & $d_{3}$ & $d_{4}$ & $d_{5}$ & $d_{6}$ & HOMA \\
\hline $5-\mathrm{NO}_{2}$ & 1.416 & 1.402 & 1.379 & 1.395 & 1.381 & 1.394 & 0.95 \\
\hline $5-\mathrm{F}$ & 1.410 & 1.398 & 1.384 & 1.389 & 1.374 & 1.402 & 0.96 \\
\hline $5-\mathrm{Cl}$ & 1.410 & 1.398 & 1.383 & 1.394 & 1.379 & 1.400 & 0.96 \\
\hline $5-\mathrm{Br}$ & 1.411 & 1.398 & 1.383 & 1.395 & 1.380 & 1.401 & 0.96 \\
\hline $\mathrm{H}$ & 1.412 & 1.397 & 1.384 & 1.398 & 1.383 & 1.401 & 0.96 \\
\hline $5-\mathrm{OMe}$ & 1.411 & 1.390 & 1.390 & 1.397 & 1.387 & 1.396 & 0.97 \\
\hline $5-\mathrm{OH}$ & 1.409 & 1.394 & 1.386 & 1.396 & 1.383 & 1.400 & 0.97 \\
\hline $4-\mathrm{NO}_{2}$ & 1.413 & 1.397 & 1.379 & 1.391 & 1.382 & 1.400 & 0.96 \\
\hline $4-\mathrm{F}$ & 1.414 & 1.396 & 1.378 & 1.390 & 1.382 & 1.400 & 0.96 \\
\hline $4-\mathrm{Cl}$ & 1.412 & 1.397 & 1.382 & 1.395 & 1.382 & 1.400 & 0.96 \\
\hline $4-\mathrm{Br}$ & 1.412 & 1.398 & 1.382 & 1.395 & 1.383 & 1.399 & 0.96 \\
\hline 4-OMe & 1.418 & 1.388 & 1.392 & 1.402 & 1.384 & 1.396 & 0.95 \\
\hline $4-\mathrm{OH}$ & 1.416 & 1.392 & 1.387 & 1.401 & 1.381 & 1.399 & 0.95 \\
\hline
\end{tabular}

Table 29S. HOMA values of benzene rings in enamines (M06-2X/6-311++G(d,p) in gas phase).

\begin{tabular}{cccccccc}
\hline Substit. & $\mathbf{d}_{\mathbf{1}}$ & $\mathbf{d}_{\mathbf{2}}$ & $\mathbf{d}_{\mathbf{3}}$ & $\mathbf{d}_{\mathbf{4}}$ & $\mathbf{d}_{\mathbf{5}}$ & $\mathbf{d}_{\mathbf{6}}$ & HOMA \\
\hline $5-\mathrm{NO}_{2}$ & 1.461 & 1.447 & 1.358 & 1.423 & 1.365 & 1.411 & 0.48 \\
$5-\mathrm{F}$ & 1.455 & 1.447 & 1.361 & 1.419 & 1.351 & 1.429 & 0.46 \\
$5-\mathrm{Cl}$ & 1.456 & 1.446 & 1.361 & 1.425 & 1.357 & 1.425 & 0.47 \\
$5-\mathrm{Br}$ & 1.455 & 1.445 & 1.361 & 1.425 & 1.358 & 1.426 & 0.47 \\
$\mathrm{H}$ & 1.457 & 1.444 & 1.363 & 1.427 & 1.362 & 1.426 & 0.48 \\
$5-\mathrm{OMe}$ & 1.452 & 1.438 & 1.367 & 1.426 & 1.363 & 1.423 & 0.56 \\
$5-\mathrm{OH}$ & 1.452 & 1.444 & 1.362 & 1.426 & 1.358 & 1.428 & 0.49 \\
$4-\mathrm{NO}_{2}$ & 1.453 & 1.446 & 1.357 & 1.418 & 1.363 & 1.422 & 0.52 \\
\hline
\end{tabular}




\begin{tabular}{cccccccc}
\hline $4-\mathrm{F}$ & 1.461 & 1.441 & 1.356 & 1.42 & 1.361 & 1.424 & 0.48 \\
$4-\mathrm{Cl}$ & 1.458 & 1.444 & 1.359 & 1.425 & 1.361 & 1.423 & 0.48 \\
$4-\mathrm{Br}$ & 1.457 & 1.446 & 1.359 & 1.425 & 1.361 & 1.423 & 0.47 \\
$4-\mathrm{OMe}$ & 1.462 & 1.428 & 1.372 & 1.431 & 1.364 & 1.419 & 0.54 \\
$4-\mathrm{OH}$ & 1.464 & 1.434 & 1.365 & 1.432 & 1.359 & 1.423 & 0.47 \\
\hline
\end{tabular}

Table 30S. HOMA values of benzene rings in open imines (M06-2X/6-311++G(d,p) in gas phase).

\begin{tabular}{cccccccc}
\hline Substit. & $\mathbf{d}_{\mathbf{1}}$ & $\mathbf{d}_{\mathbf{2}}$ & $\mathbf{d}_{\mathbf{3}}$ & $\mathbf{d}_{\mathbf{4}}$ & $\mathbf{d}_{\mathbf{5}}$ & $\mathbf{d}_{\mathbf{6}}$ & HOMA \\
\hline $5-\mathrm{NO}_{2}$ & 1.411 & 1.399 & 1.382 & 1.387 & 1.384 & 1.393 & 0.97 \\
$5-\mathrm{F}$ & 1.407 & 1.395 & 1.388 & 1.382 & 1.378 & 1.399 & 0.97 \\
$5-\mathrm{Cl}$ & 1.407 & 1.395 & 1.387 & 1.387 & 1.384 & 1.398 & 0.98 \\
$5-\mathrm{Br}$ & 1.407 & 1.395 & 1.387 & 1.387 & 1.384 & 1.399 & 0.98 \\
$\mathrm{H}$ & 1.407 & 1.395 & 1.388 & 1.390 & 1.387 & 1.399 & 0.98 \\
$5-\mathrm{OMe}$ & 1.409 & 1.388 & 1.394 & 1.390 & 1.392 & 1.393 & 0.98 \\
$5-\mathrm{OH}$ & 1.407 & 1.391 & 1.391 & 1.389 & 1.387 & 1.396 & 0.98 \\
$4-\mathrm{NO}{ }_{2}$ & 1.410 & 1.393 & 1.384 & 1.383 & 1.387 & 1.398 & 0.97 \\
$4-\mathrm{F}$ & 1.409 & 1.394 & 1.382 & 1.382 & 1.387 & 1.398 & 0.97 \\
$4-\mathrm{Cl}$ & 1.408 & 1.395 & 1.386 & 1.387 & 1.387 & 1.398 & 0.98 \\
$4-\mathrm{Br}$ & 1.408 & 1.395 & 1.386 & 1.387 & 1.387 & 1.397 & 0.98 \\
$4-\mathrm{OMe}$ & 1.412 & 1.387 & 1.395 & 1.394 & 1.389 & 1.394 & 0.97 \\
$4-\mathrm{OH}$ & 1.410 & 1.391 & 1.390 & 1.393 & 1.386 & 1.397 & 0.97 \\
\hline
\end{tabular}

Table 31S. HOMA values of benzene rings in TS (M06-2X/6-311++G(d,p) in gas phase).

\begin{tabular}{cccccccc}
\hline Substit. & $\mathbf{d}_{\mathbf{1}}$ & $\mathbf{d}_{\mathbf{2}}$ & $\mathbf{d}_{\mathbf{3}}$ & $\mathbf{d}_{\mathbf{4}}$ & $\mathbf{d}_{\mathbf{5}}$ & $\mathbf{d}_{\mathbf{6}}$ & HOMA \\
\hline $5-\mathrm{NO}_{2}$ & 1.444 & 1.429 & 1.367 & 1.411 & 1.372 & 1.401 & 0.73 \\
$5-\mathrm{F}$ & 1.440 & 1.430 & 1.369 & 1.408 & 1.359 & 1.417 & 0.70 \\
$5-\mathrm{Cl}$ & 1.440 & 1.428 & 1.369 & 1.414 & 1.365 & 1.414 & 0.72 \\
$5-\mathrm{Br}$ & 1.440 & 1.428 & 1.369 & 1.414 & 1.366 & 1.414 & 0.72 \\
$\mathrm{H}$ & 1.443 & 1.428 & 1.370 & 1.417 & 1.369 & 1.415 & 0.70 \\
$5-\mathrm{OMe}$ & 1.443 & 1.427 & 1.372 & 1.419 & 1.369 & 1.416 & 0.70 \\
$5-\mathrm{OH}$ & 1.439 & 1.428 & 1.370 & 1.416 & 1.365 & 1.418 & 0.71 \\
$4-\mathrm{NO} 2$ & 1.440 & 1.429 & 1.366 & 1.408 & 1.370 & 1.411 & 0.74 \\
$4-\mathrm{F}$ & 1.444 & 1.423 & 1.365 & 1.408 & 1.370 & 1.412 & 0.73 \\
$4-\mathrm{Cl}$ & 1.441 & 1.425 & 1.369 & 1.413 & 1.370 & 1.411 & 0.74 \\
$4-\mathrm{Br}$ & 1.440 & 1.427 & 1.368 & 1.413 & 1.370 & 1.411 & 0.74 \\
$4-\mathrm{OMe}$ & 1.450 & 1.416 & 1.378 & 1.423 & 1.370 & 1.410 & 0.71 \\
$4-\mathrm{OH}$ & 1.448 & 1.418 & 1.374 & 1.420 & 1.368 & 1.411 & 0.71 \\
\hline
\end{tabular}

Table 32S. HOMA of carbocyclic (benzene) rings. ${ }^{a}$

\begin{tabular}{cccccc}
\hline $\mathbf{X}$ & $\mathbf{1 3 7}$ & $\mathbf{1 3 8}$ & $\mathbf{1 3 9}$ & $\mathbf{1 4 0}$ & $\boldsymbol{\Sigma}_{\text {номА }}$ \\
\hline $5-\mathrm{NO}_{2}$ & 0.97 & 0.95 & 0.73 & 0.48 & 1.43 \\
$5-\mathrm{F}$ & 0.97 & 0.96 & 0.70 & 0.46 & 1.42 \\
$5-\mathrm{Cl}$ & 0.98 & 0.96 & 0.72 & 0.47 & 1.43 \\
$5-\mathrm{Br}$ & 0.98 & 0.96 & 0.72 & 0.47 & 1.43 \\
$5-\mathrm{OMe}$ & 0.98 & 0.96 & 0.70 & 0.48 & 1.44 \\
$5-\mathrm{OH}$ & 0.98 & 0.97 & 0.70 & 0.56 & 1.53 \\
$\mathrm{H}$ & 0.98 & 0.97 & 0.71 & 0.49 & 1.46 \\
$4-\mathrm{NO}_{2}$ & 0.97 & 0.96 & 0.74 & 0.52 & 1.48 \\
\hline
\end{tabular}




\begin{tabular}{cccccc}
\hline $4-\mathrm{F}$ & 0.97 & 0.96 & 0.73 & 0.48 & 1.44 \\
$4-\mathrm{Cl}$ & 0.98 & 0.96 & 0.74 & 0.48 & 1.44 \\
$4-\mathrm{Br}$ & 0.98 & 0.96 & 0.74 & 0.47 & 1.43 \\
$4-\mathrm{OMe}$ & 0.97 & 0.95 & 0.71 & 0.54 & 1.49 \\
$4-\mathrm{OH}$ & 0.97 & 0.95 & 0.71 & 0.47 & 1.42 \\
\hline a & $0.96-2 \mathrm{dt} / 6-311++\mathrm{G}(\mathrm{d})$ in &
\end{tabular}

Table 33S. Hydrogen bonds parameters $\left[\AA\right.$ and $\left.{ }^{\circ}\right]$ for several compounds $\mathbf{1 3 8}$ and $\mathbf{1 4 0}$ from theoretical calculations. ${ }^{\mathrm{a}}$

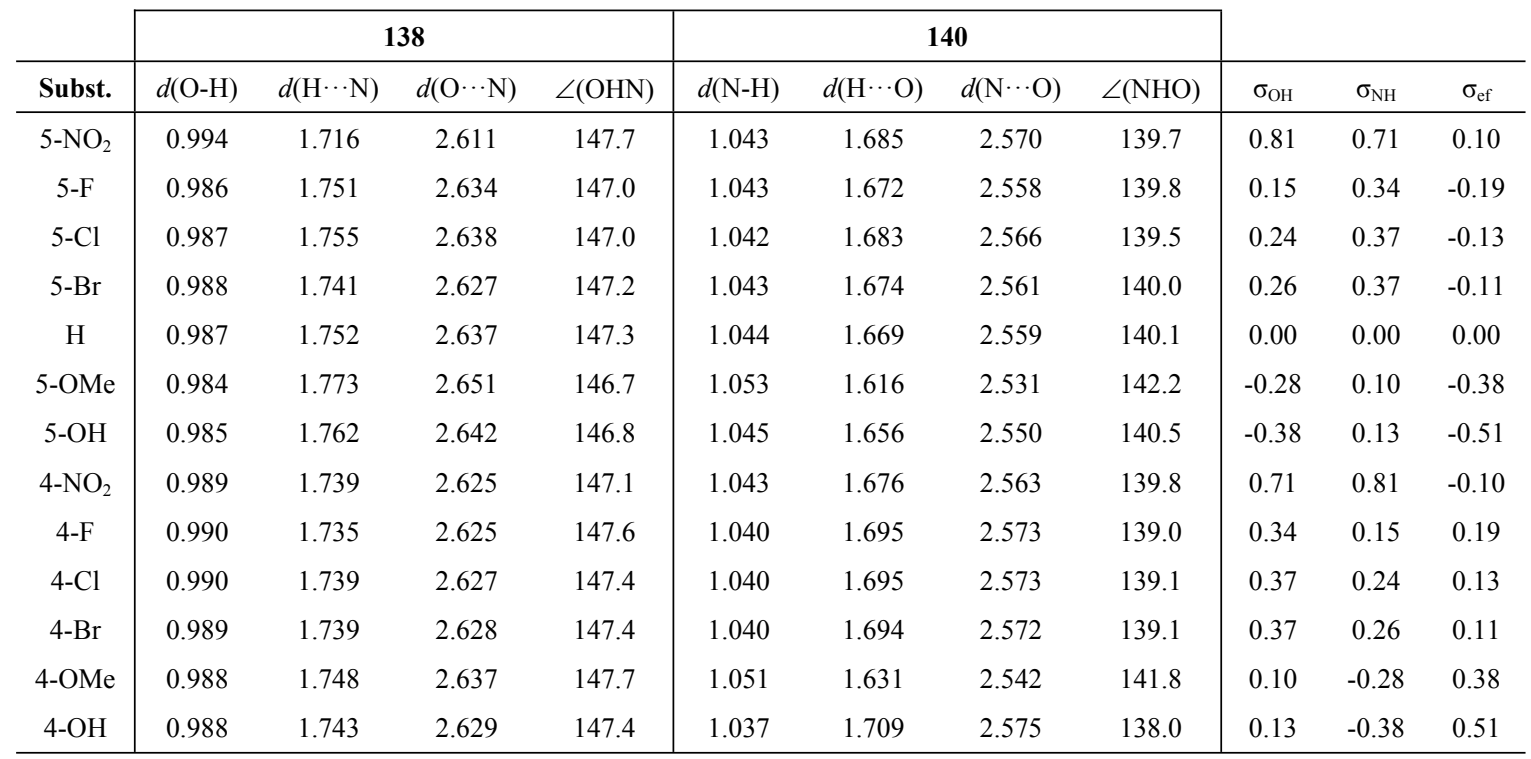

${ }^{a}$ At the M06-2X/6-311++G(d,p) level in gas phase.

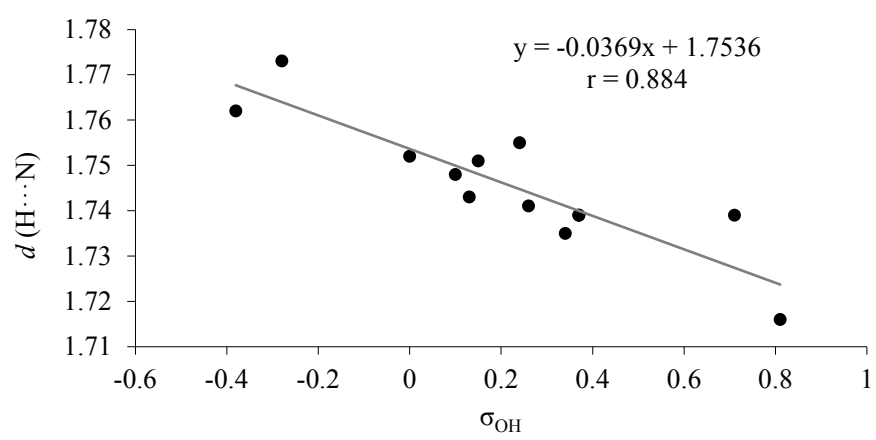

Figure 35S

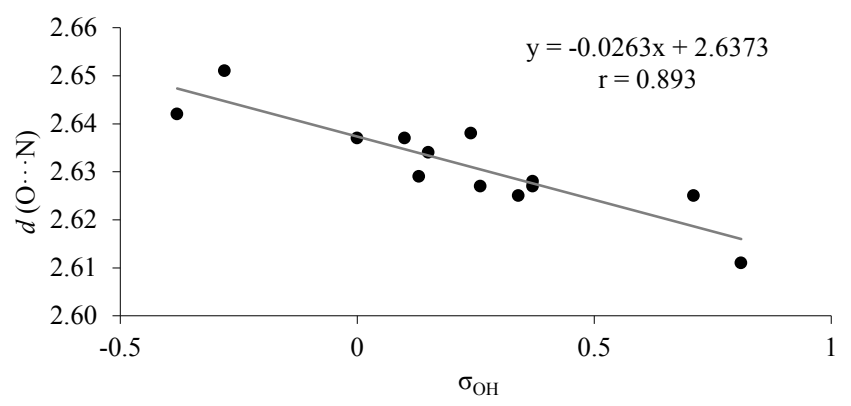

Figure 36S. 
Synthesis of Starting Materials.

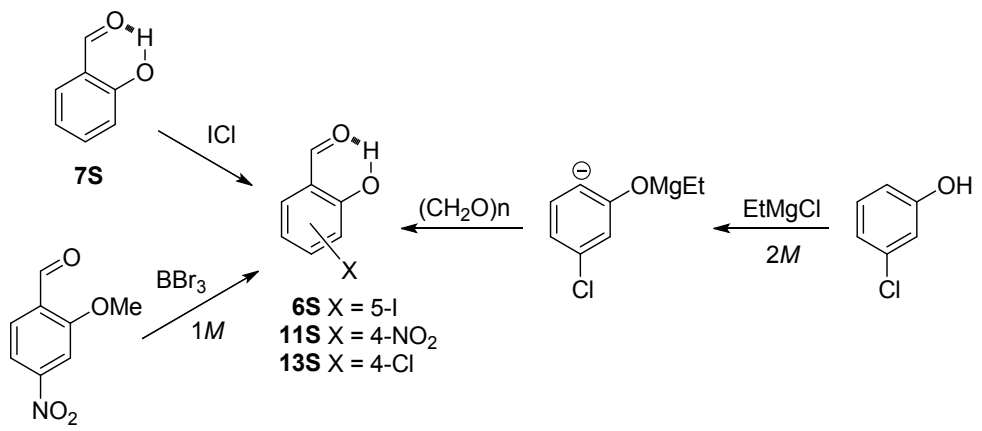

Scheme 5S 
NMR spectra of tautomeric species (only one form shown for clarity).
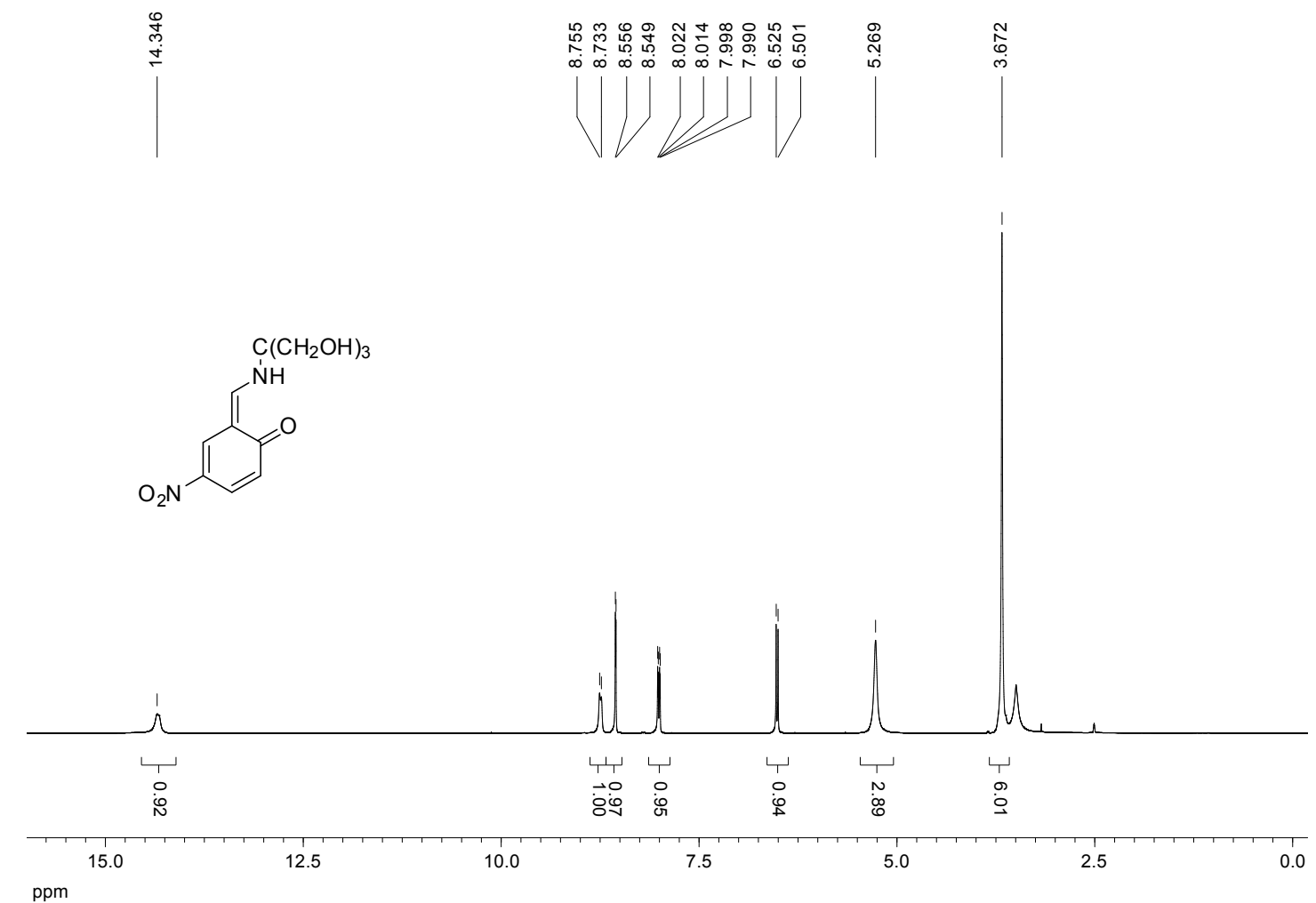

Figure 37S. ${ }^{1} \mathrm{H}$ NMR spectrum for compound 4/34 (400 MHz, DMSO- $d_{6}$ ).

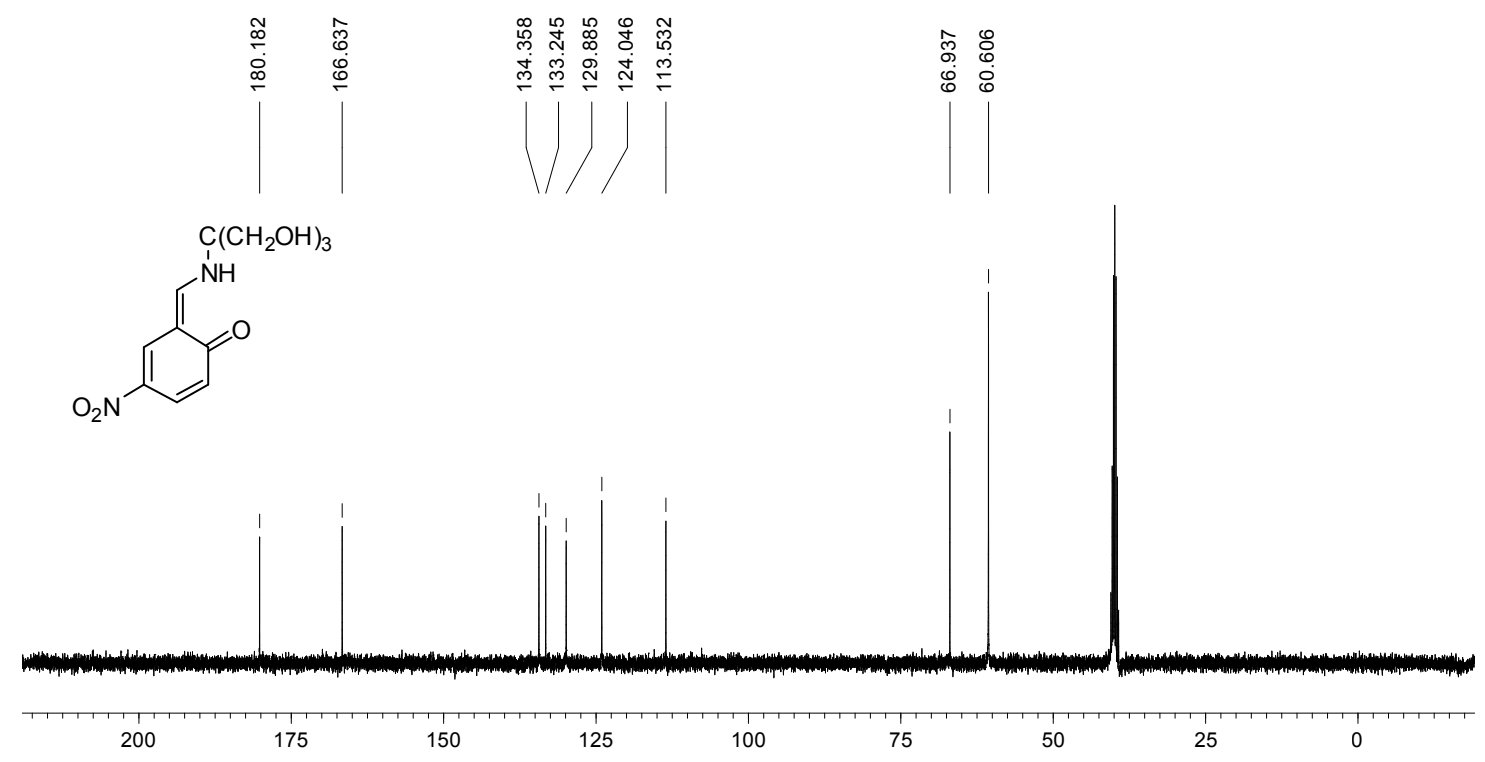

Figure 38S. ${ }^{13} \mathrm{C}\left\{{ }^{1} \mathrm{H}\right\}$ NMR spectrum for compound 4/34 (100 MHz, DMSO- $\left.d_{6}\right)$. 


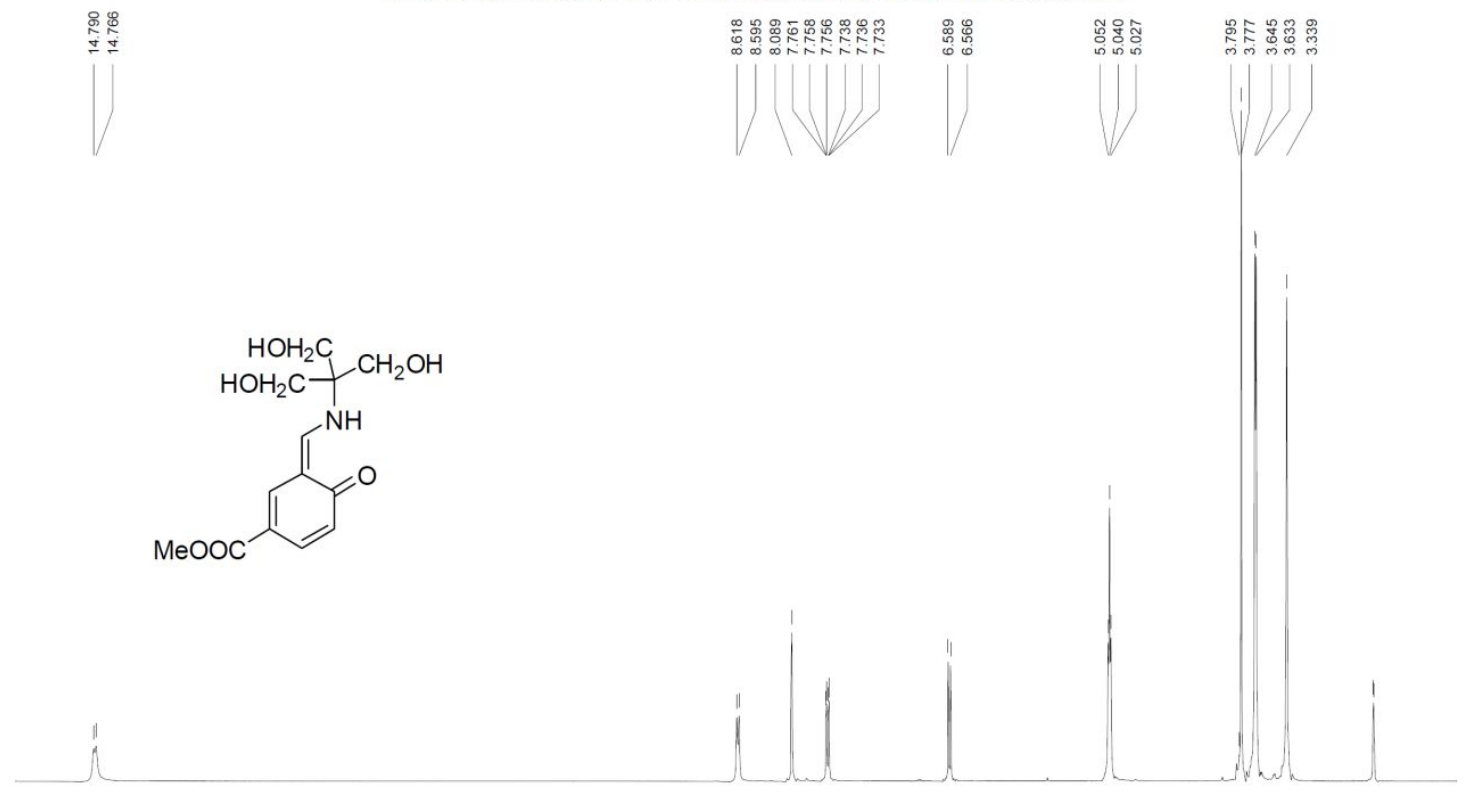

15.0
$\mathrm{pmm}$
$\mathrm{pm}$

Figure 39S. ${ }^{1} \mathrm{H}$ NMR spectrum for compound 5/35 (400 MHz, DMSO- $\left.d_{6}\right)$.
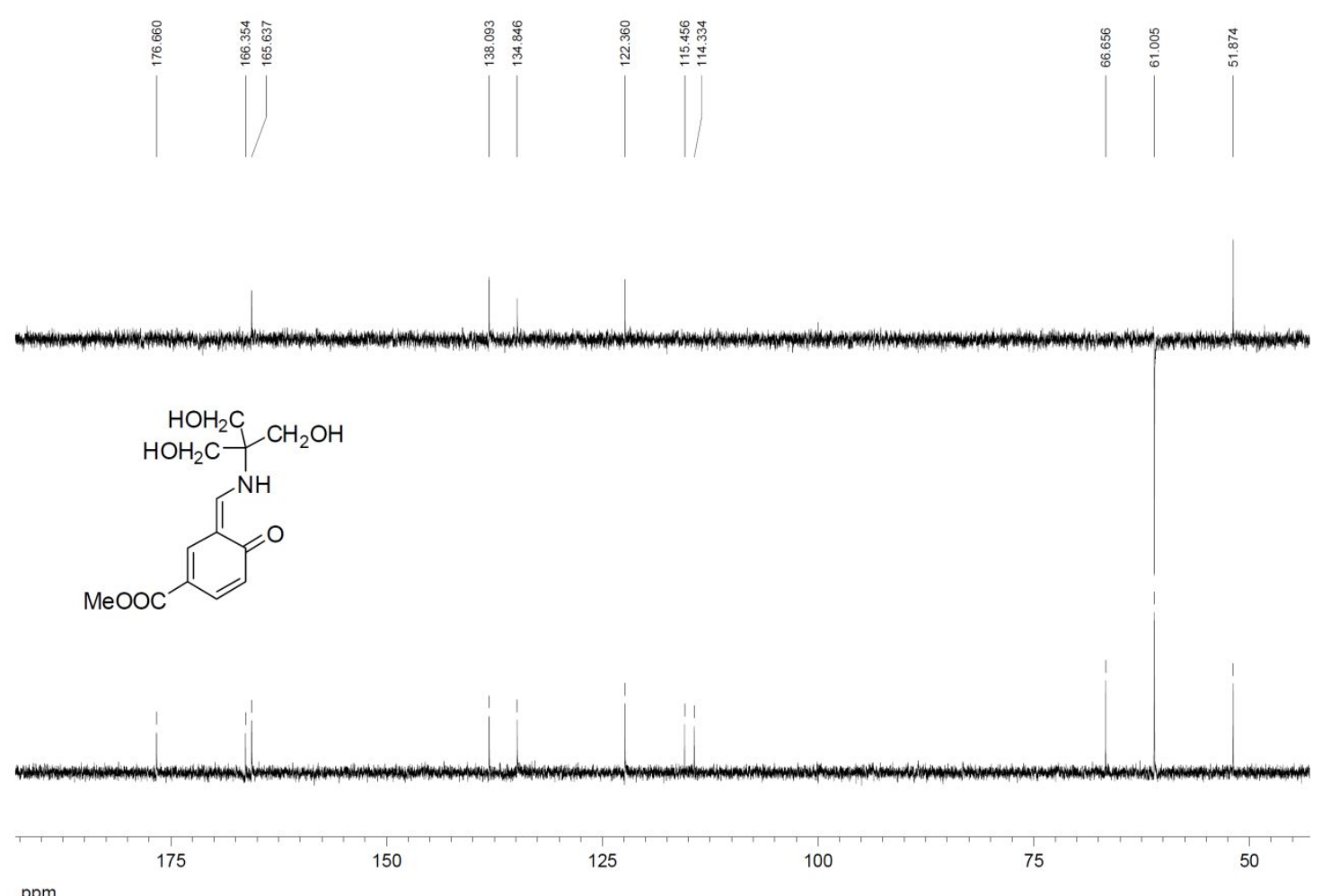

Figure 40S. ${ }^{13} \mathrm{C}\left\{{ }^{1} \mathrm{H}\right\}$ NMR spectrum for compound 5/35 (100 MHz, DMSO- $\left.d_{6}\right)$. 


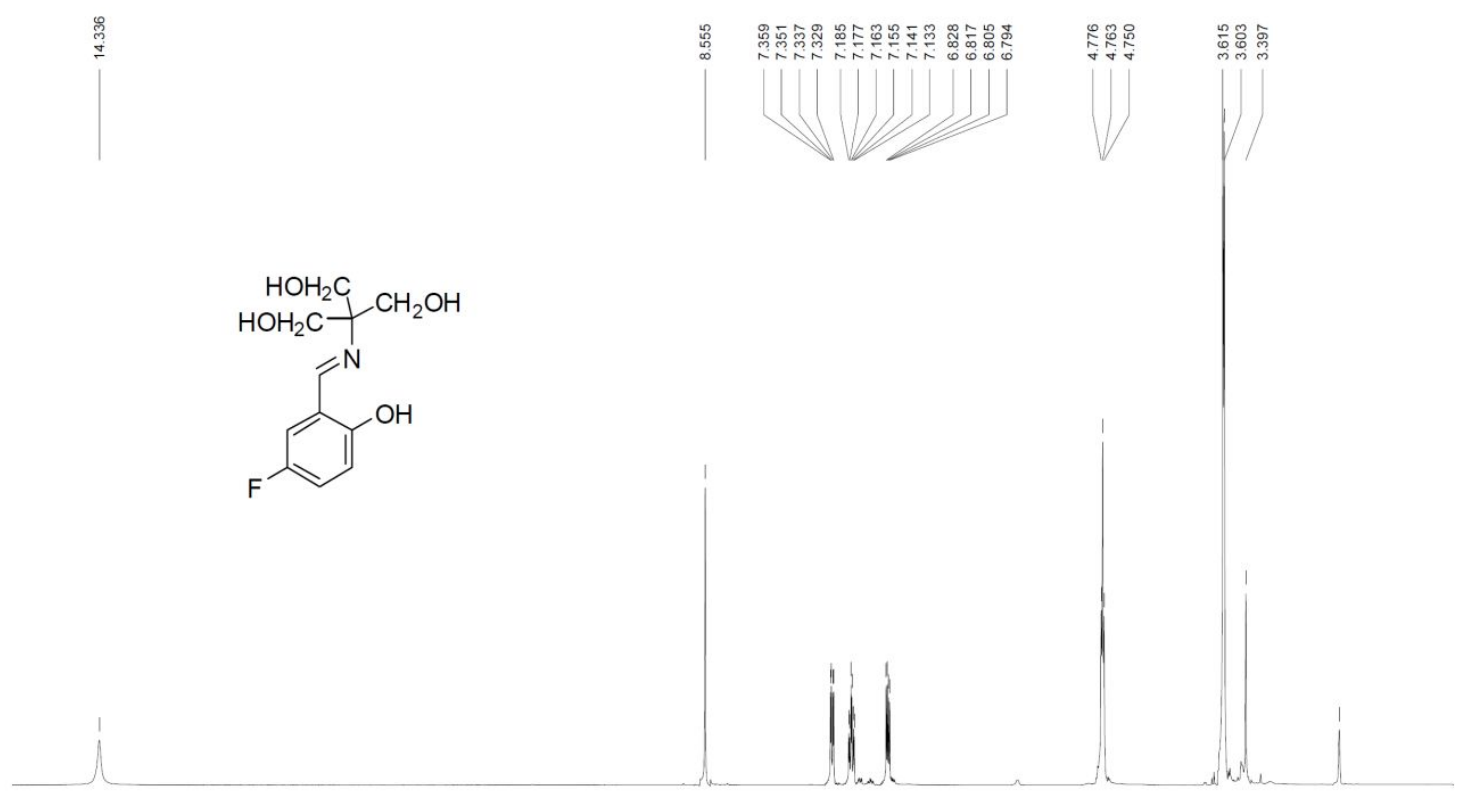

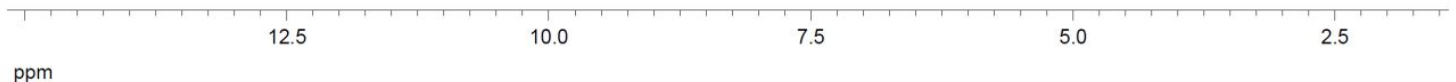

Figure 41S. ${ }^{1} \mathrm{H}$ NMR spectrum for compound 6/36 (400 MHz, DMSO- $d_{6}$ ).
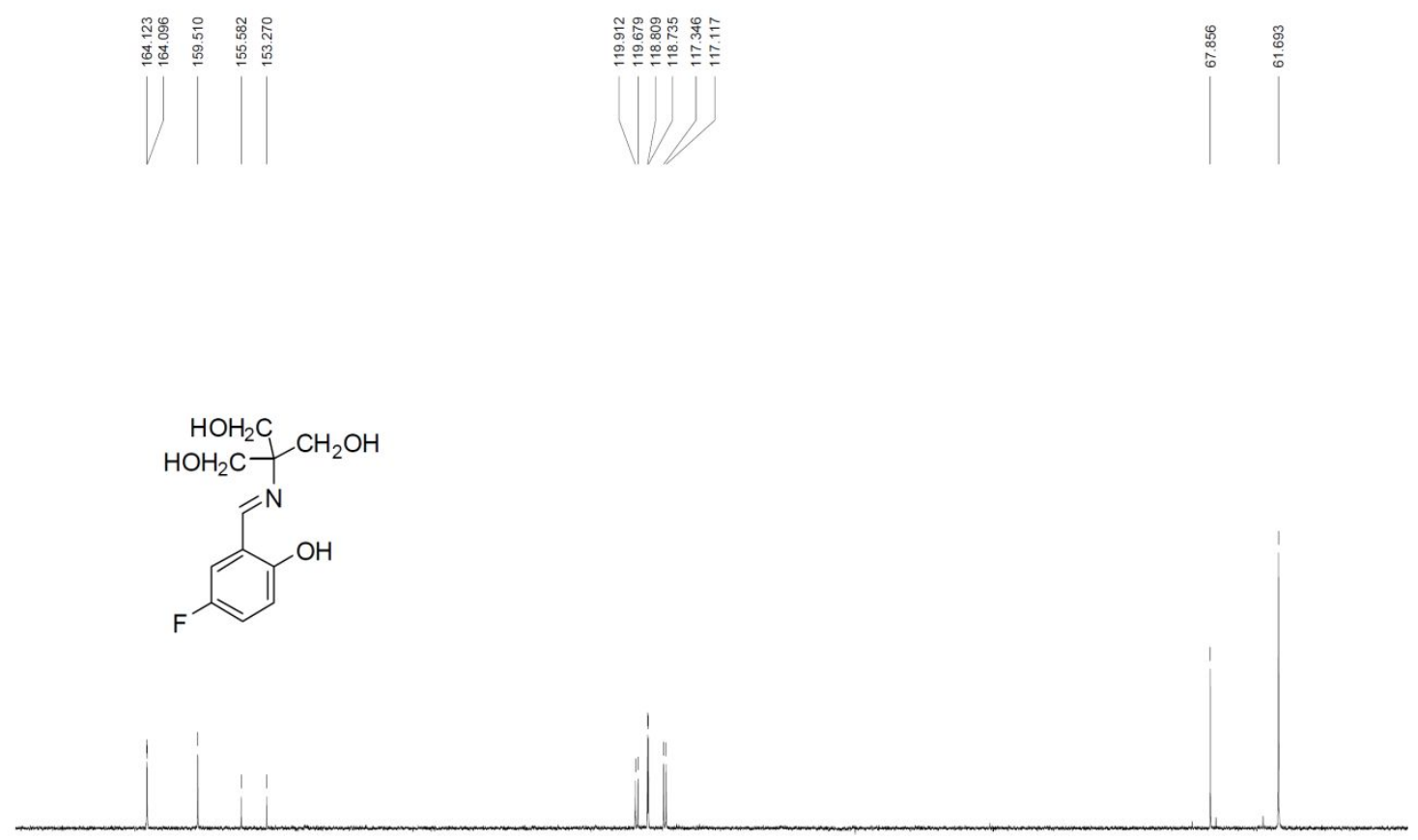

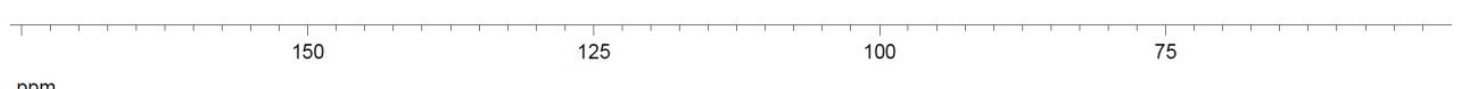

Figure 42S. ${ }^{13} \mathrm{C}\left\{{ }^{1} \mathrm{H}\right\}$ NMR spectrum for compound 6/36 (100 MHz, DMSO- $\left.d_{6}\right)$. 


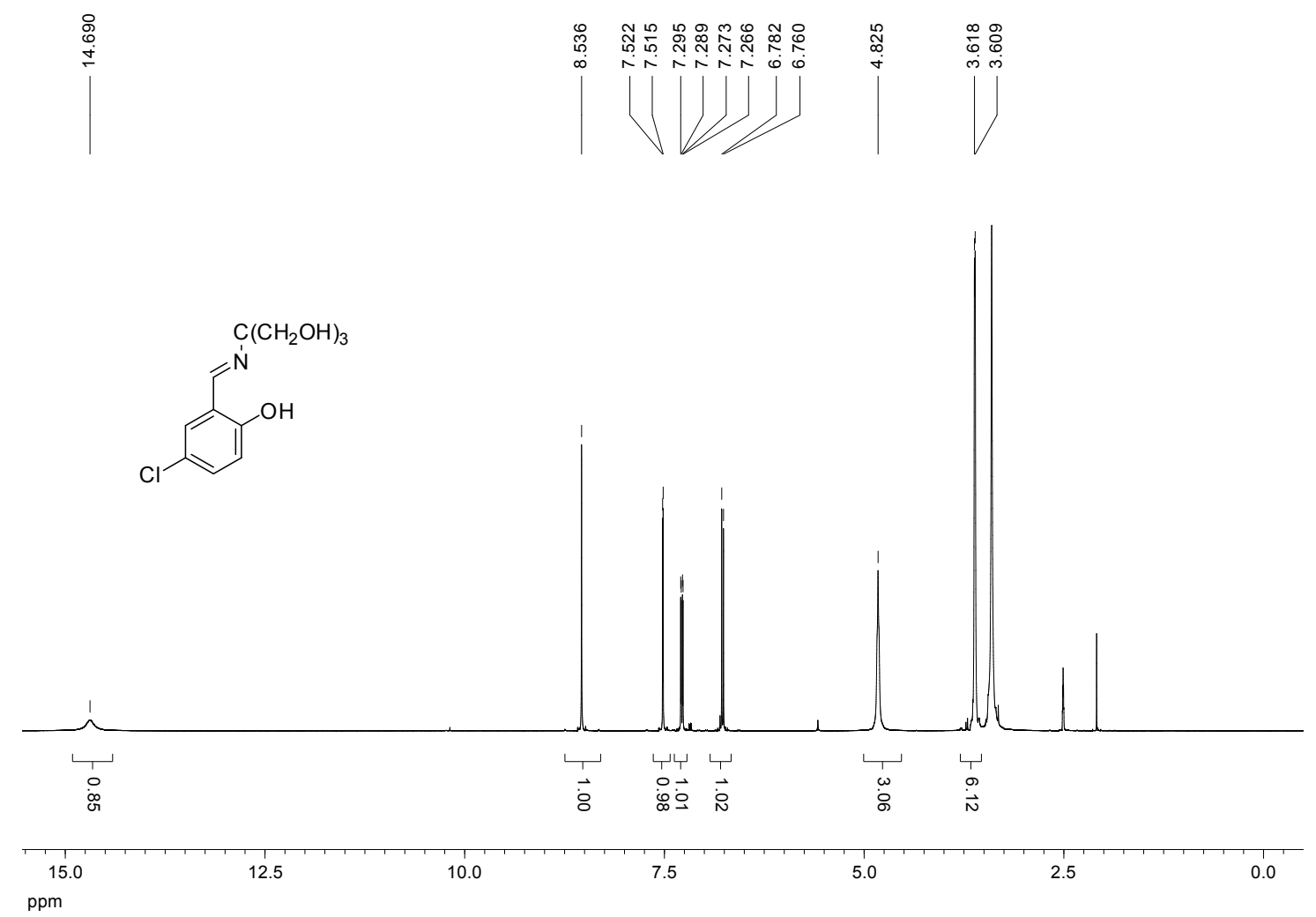

Figure 43S. ${ }^{1} \mathrm{H}$ NMR spectrum for compound 7/37 (400 MHz, DMSO- $d_{6}$ ).

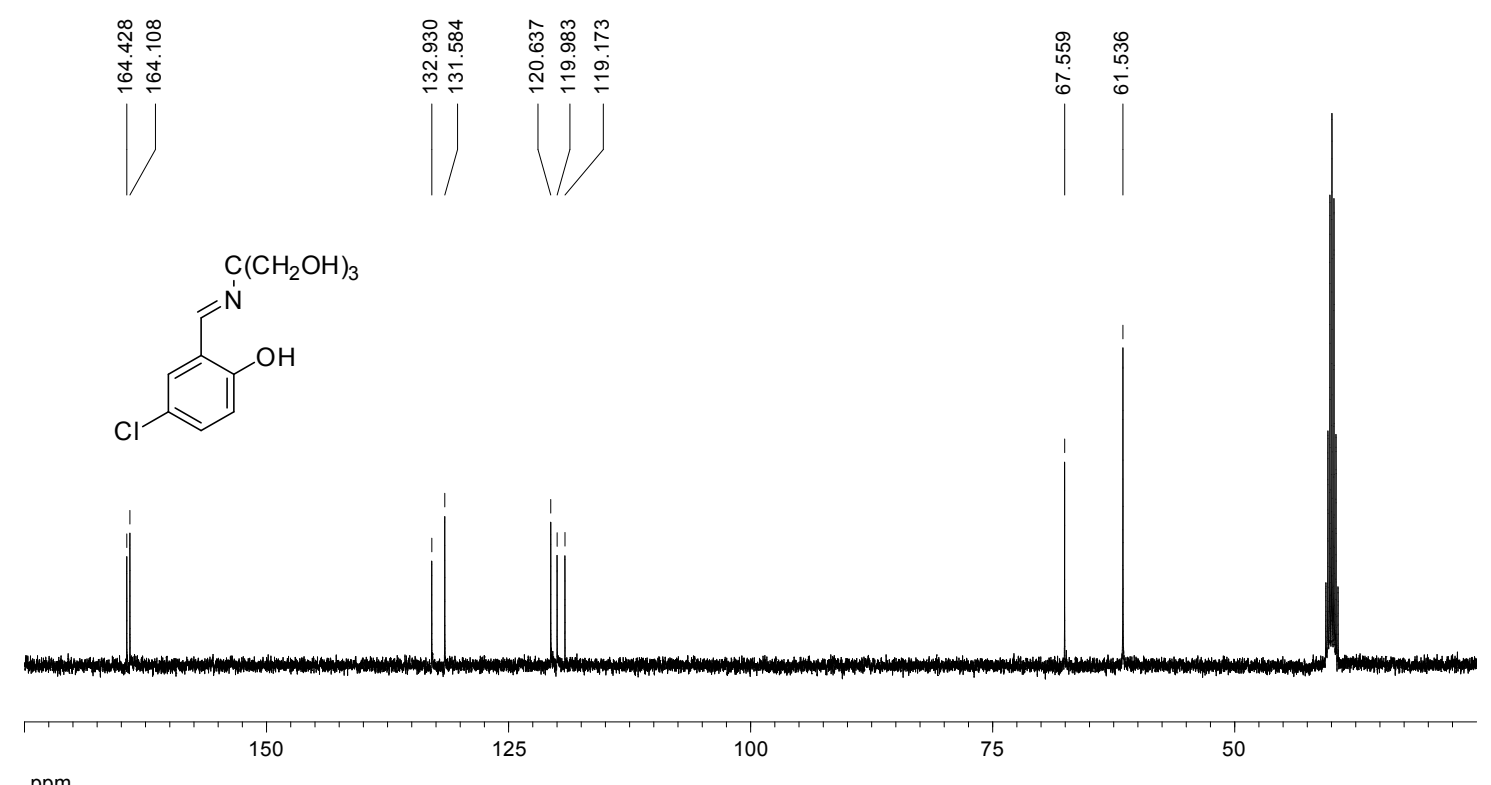

Figure 44S. ${ }^{13} \mathrm{C}\left\{{ }^{1} \mathrm{H}\right\}$ NMR spectrum for compound 7/37 (100 MHz, DMSO- $\left.d_{6}\right)$. 

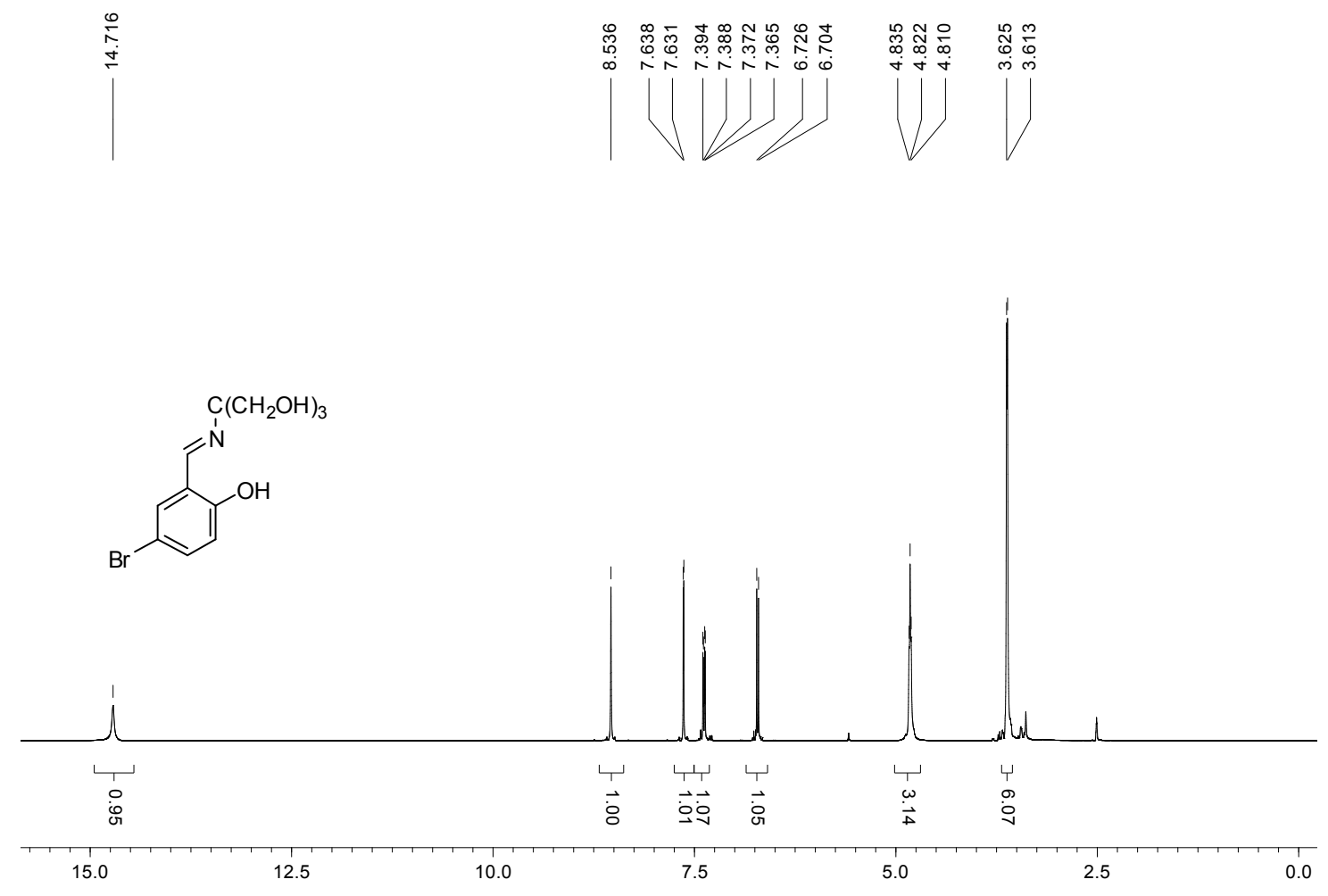

Figure 45S. ${ }^{1} \mathrm{H}$ NMR spectrum for compound 8/38 (400 MHz, DMSO- $\left.d_{6}\right)$.

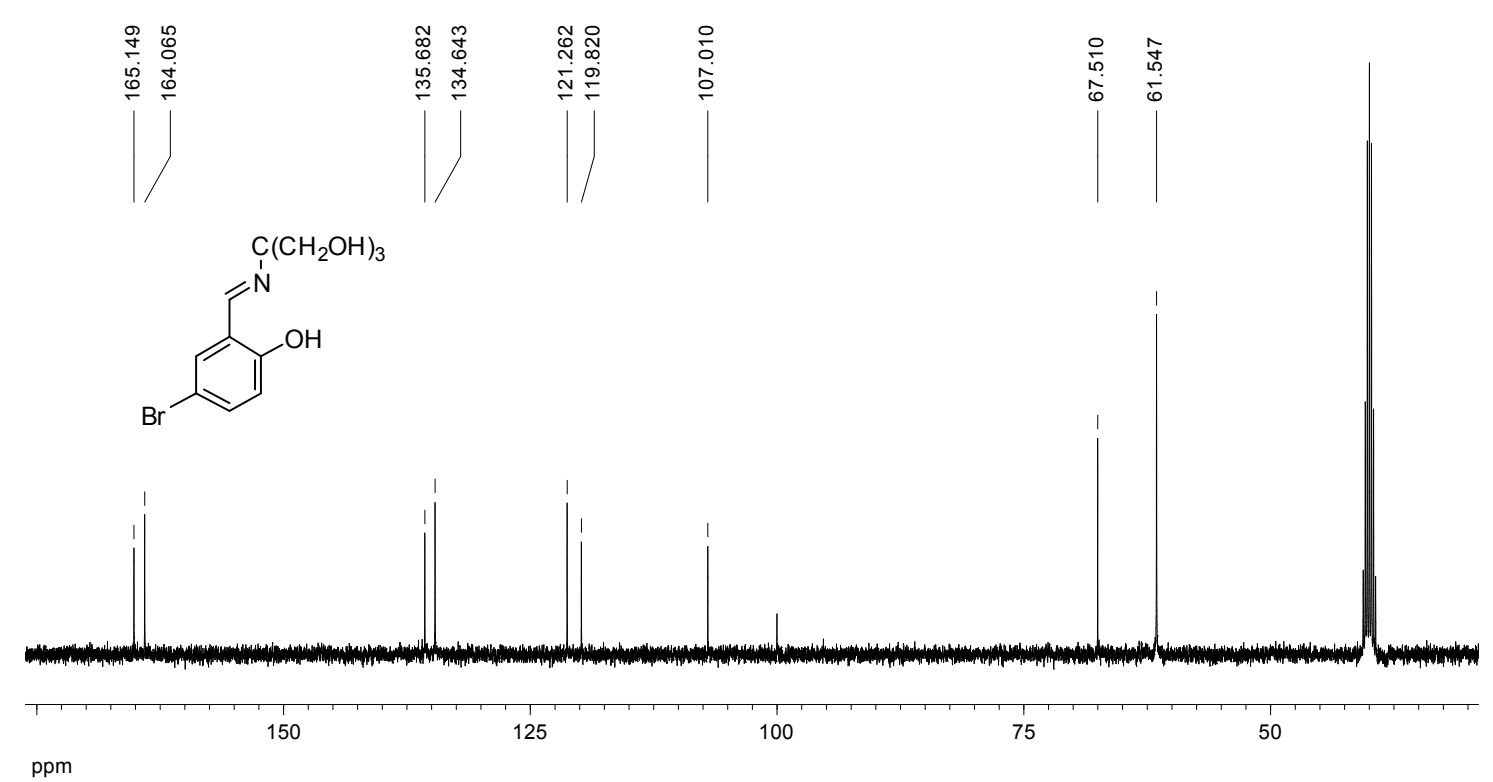

Figure 46S. ${ }^{13} \mathrm{C}\left\{{ }^{1} \mathrm{H}\right\}$ NMR spectrum for compound 8/38 (100 MHz, DMSO- $\left.d_{6}\right)$. 

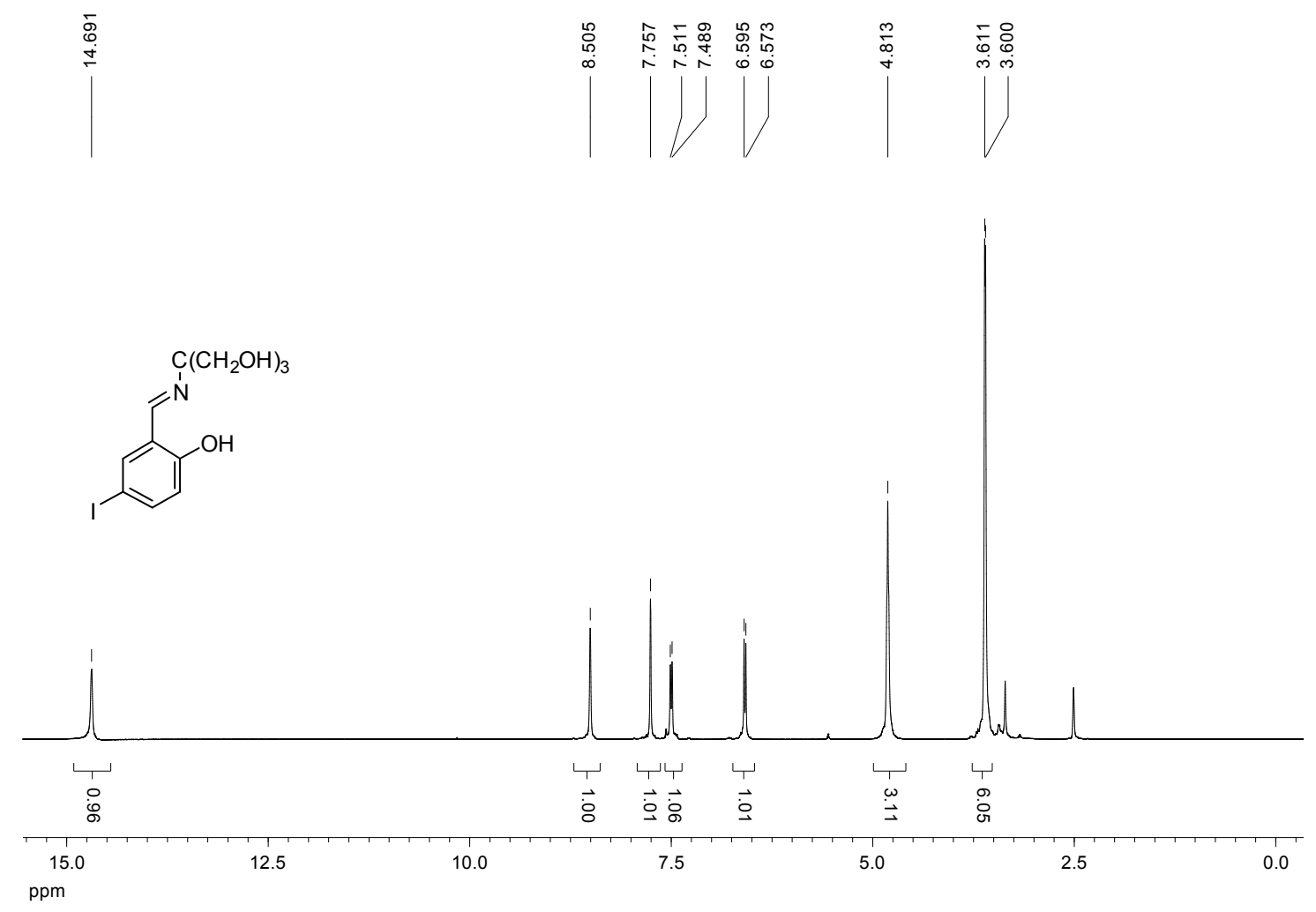

Figure 47S. ${ }^{1} \mathrm{H}$ NMR spectrum for compound 9/39 (400 MHz, DMSO- $\left.d_{6}\right)$.

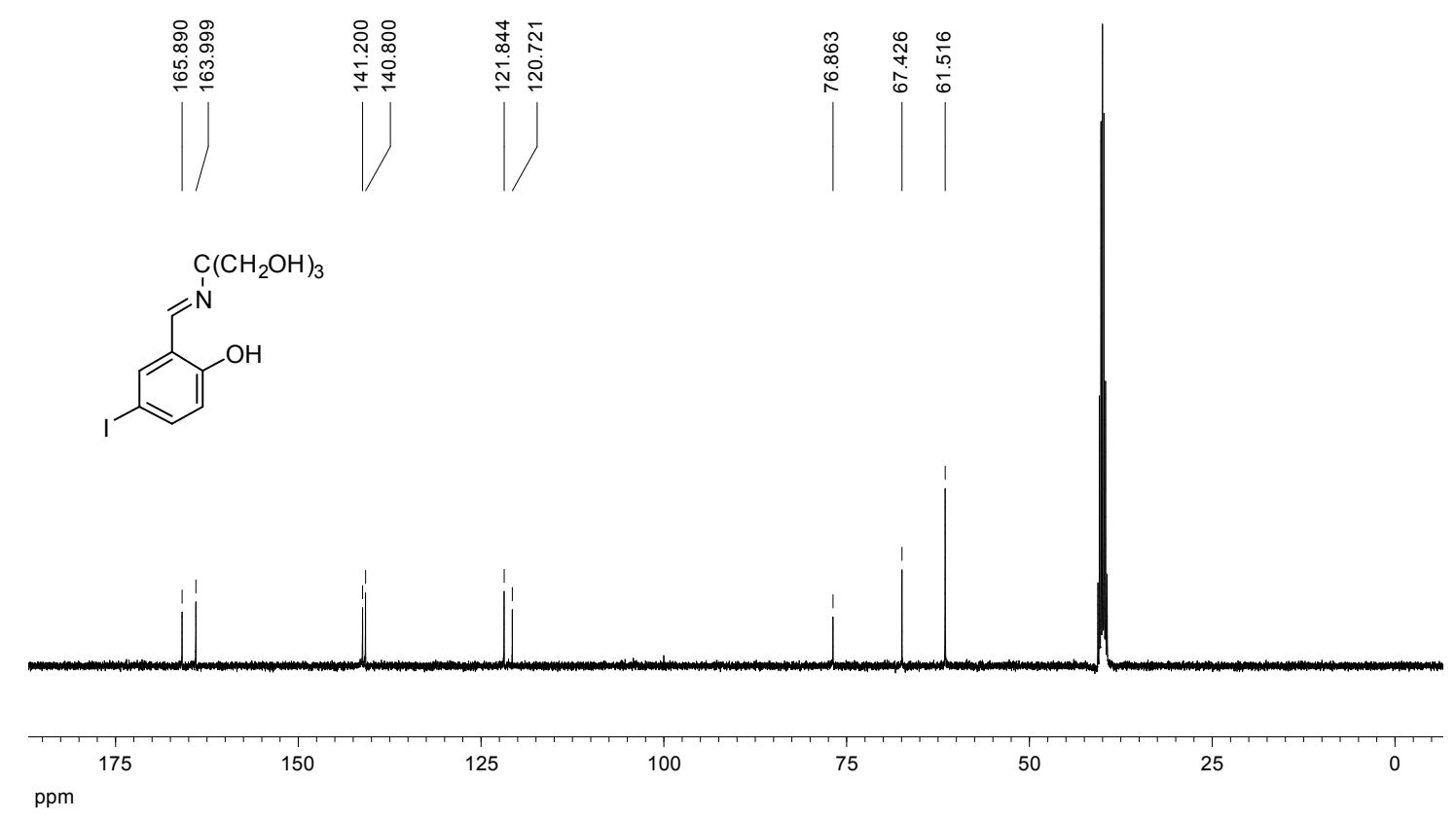

Figure 48S. ${ }^{13} \mathrm{C}\left\{{ }^{1} \mathrm{H}\right\}$ NMR spectrum for compound 9/39 (100 MHz, DMSO- $\left.d_{6}\right)$. 


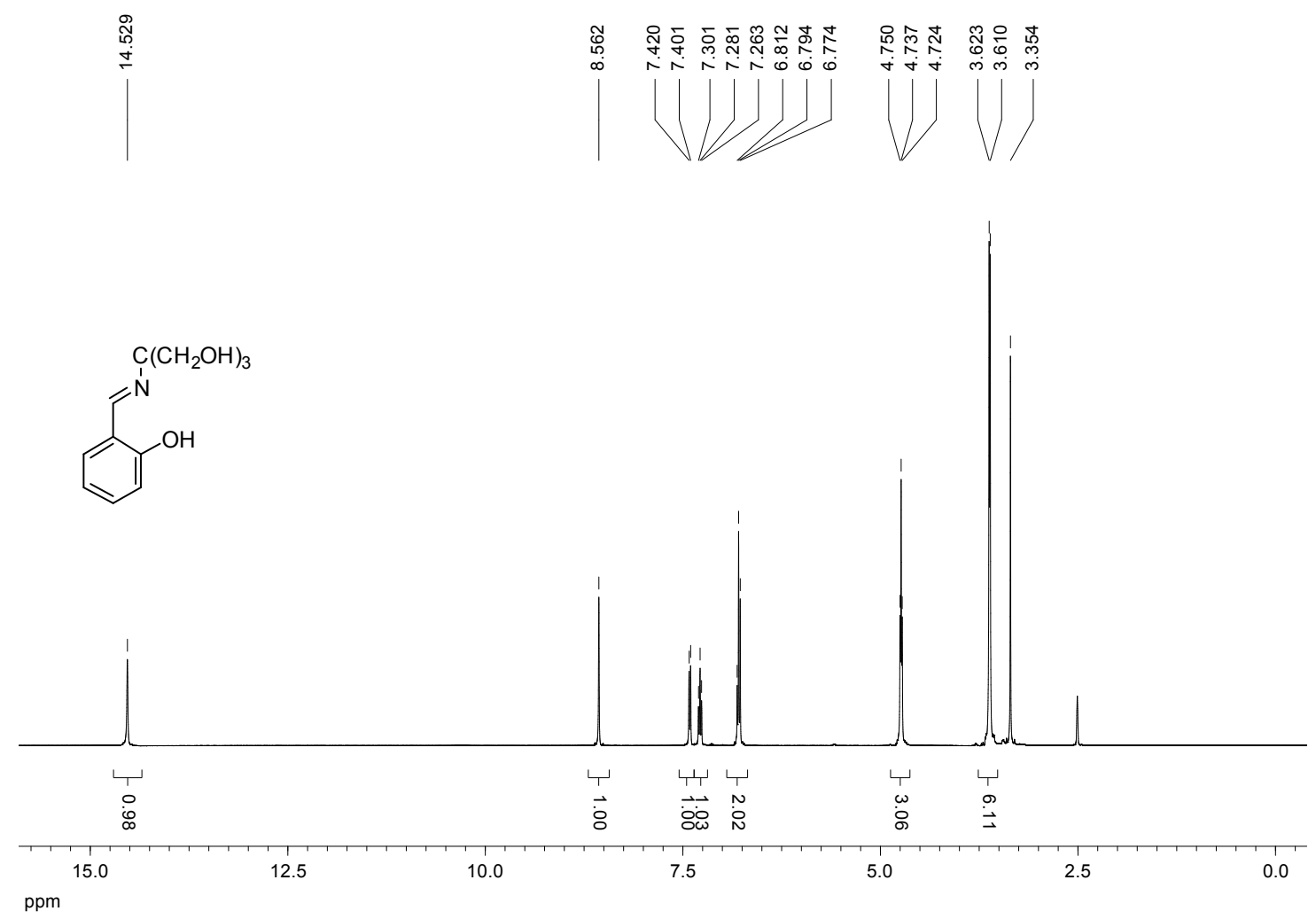

Figure 49S. ${ }^{1} \mathrm{H}$ NMR spectrum for compound 10/40 (400 MHz, DMSO- $\left.d_{6}\right)$.
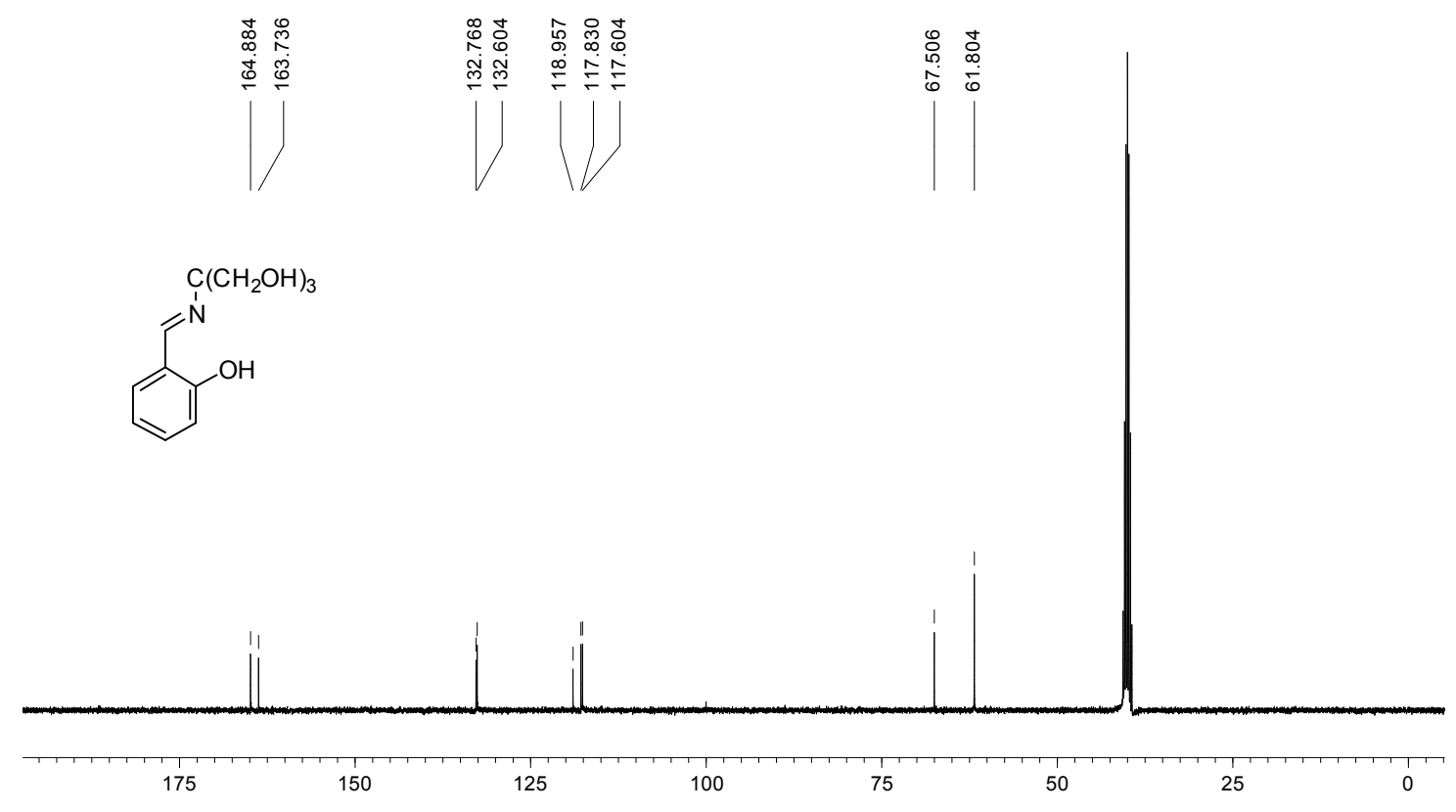

Figure 50S. ${ }^{13} \mathrm{C}\left\{{ }^{1} \mathrm{H}\right\}$ NMR spectrum for compound 10/40 (100 MHz, DMSO- $\left.d_{6}\right)$. 


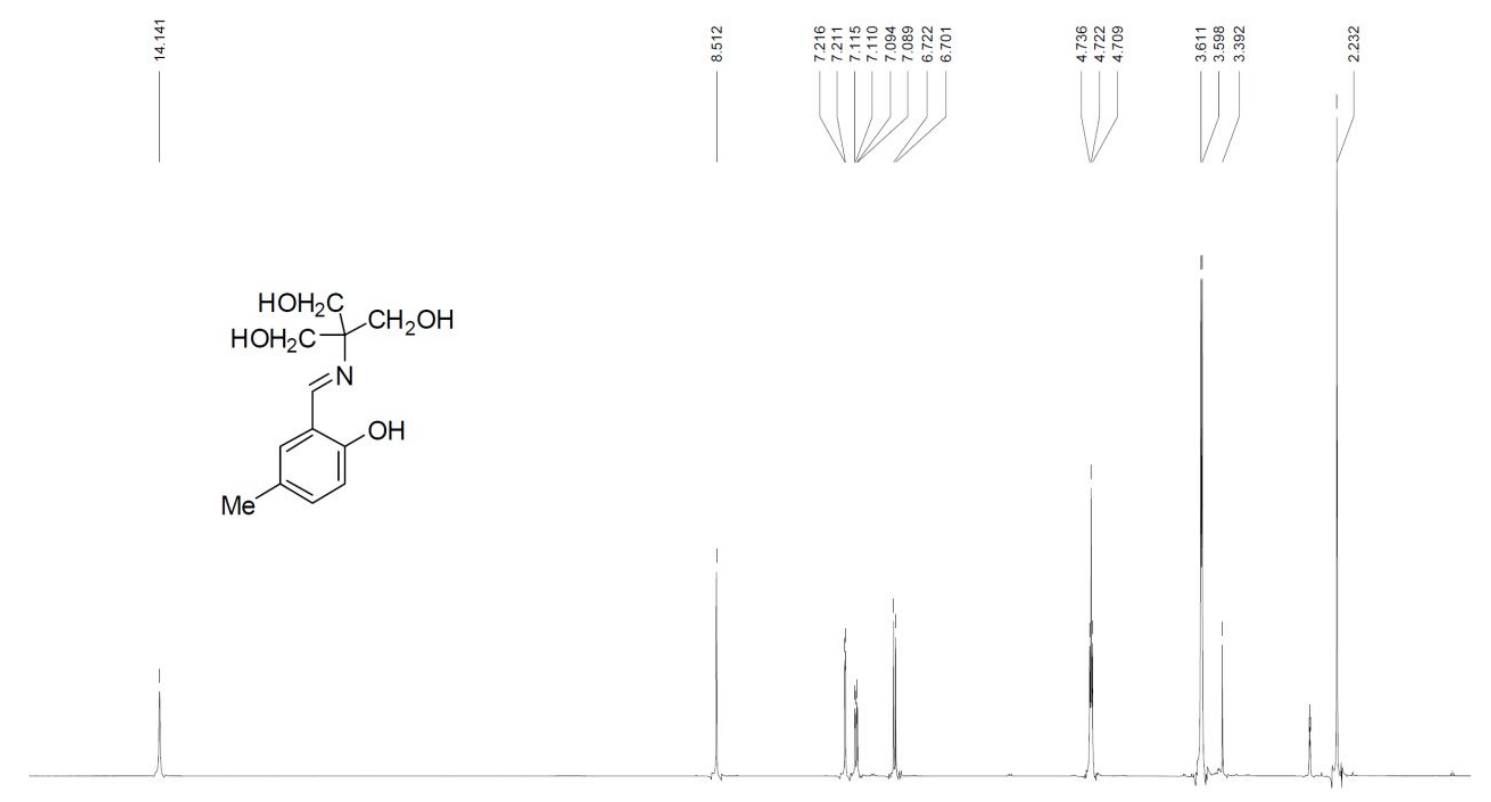

\begin{tabular}{|c|c|c|c|c|c|}
\hline 15.0 & 12.5 & 10.0 & 7.5 & 5.0 & 2.5 \\
\hline
\end{tabular}

Figure 51S. ${ }^{1} \mathrm{H}$ NMR spectrum for compound 11/41 (400 MHz, DMSO- $\left.d_{6}\right)$.

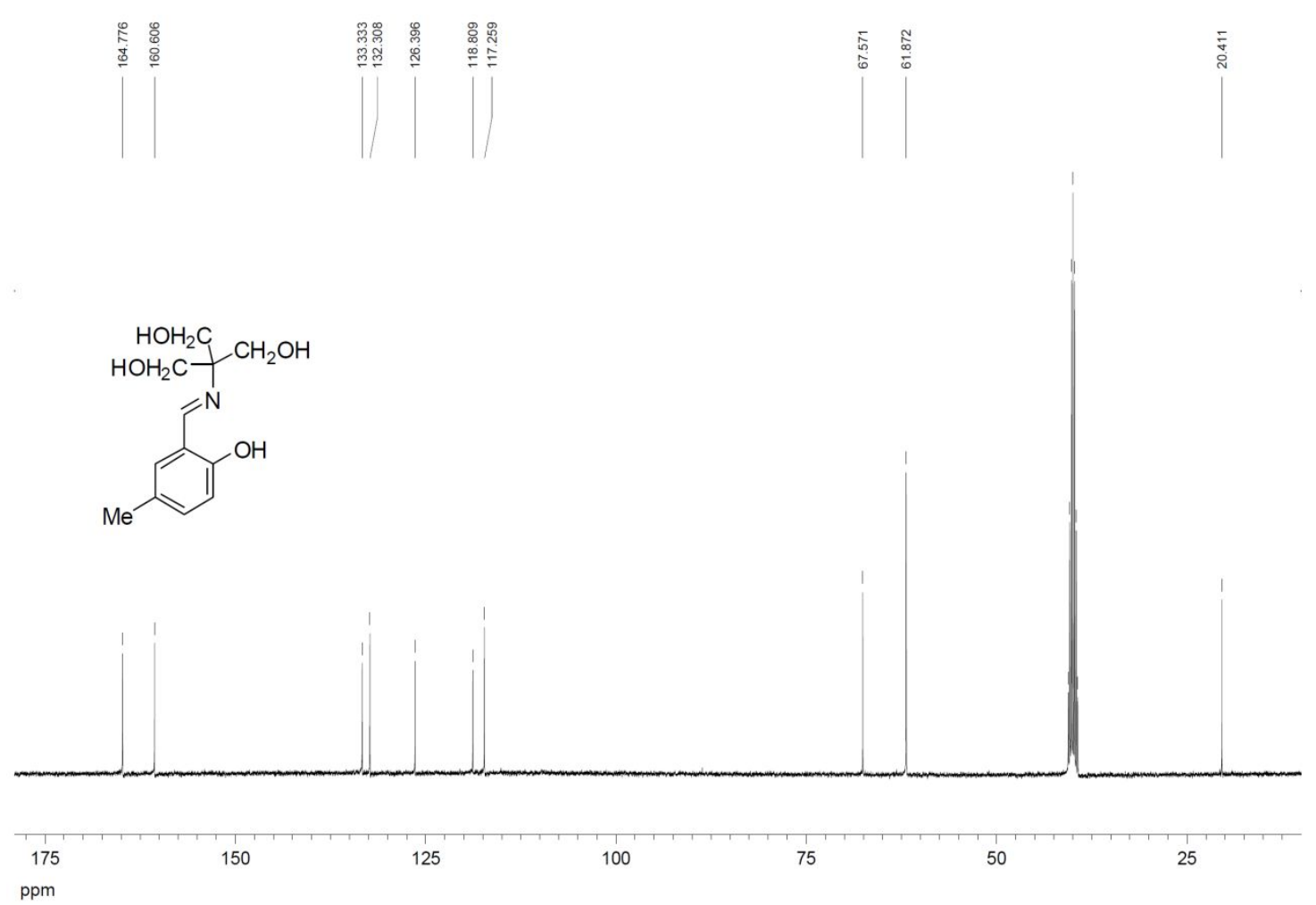

Figure 52S. ${ }^{13} \mathrm{C}\left\{{ }^{1} \mathrm{H}\right\}$ NMR spectrum for compound 11/41 (100 MHz, DMSO- $\left.d_{6}\right)$. 

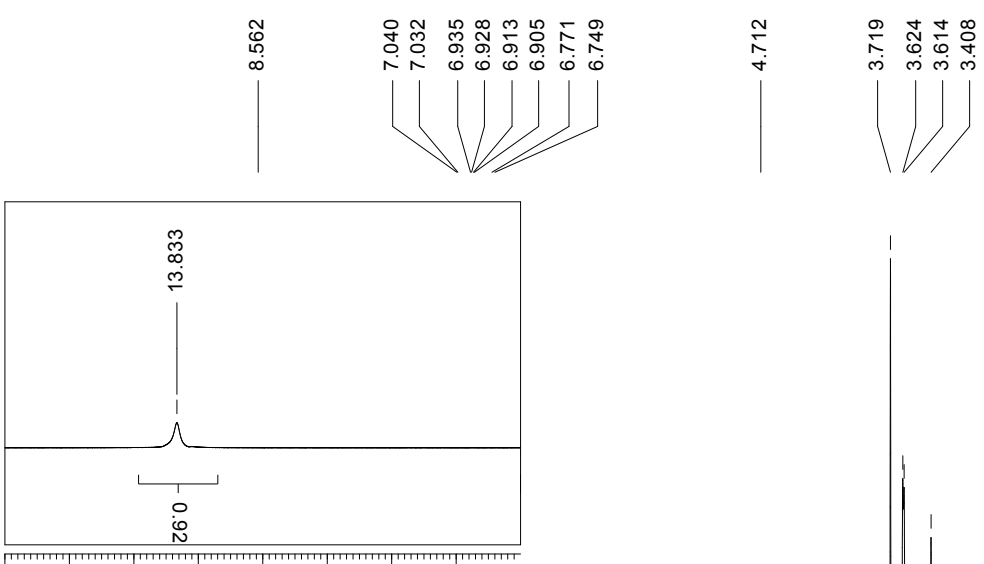

14.2514 .0013 .7513 .5013 .2513 .0012 .75

ppm

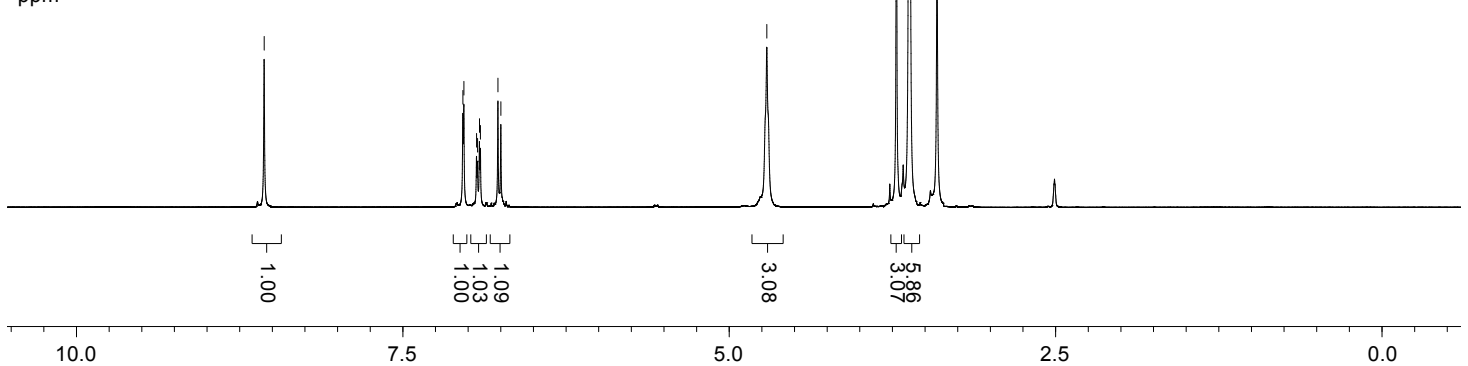

Figure 53S. ${ }^{1} \mathrm{H}$ NMR spectrum for compound 12/42 (400 MHz, DMSO- $d_{6}$ ).

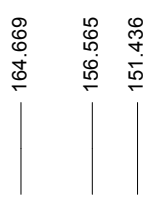

¿.

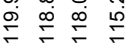

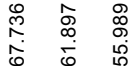

$\mathrm{C}\left(\mathrm{CH}_{2} \mathrm{OH}\right)_{3}$

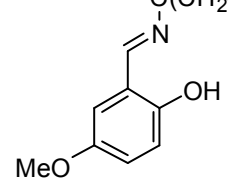
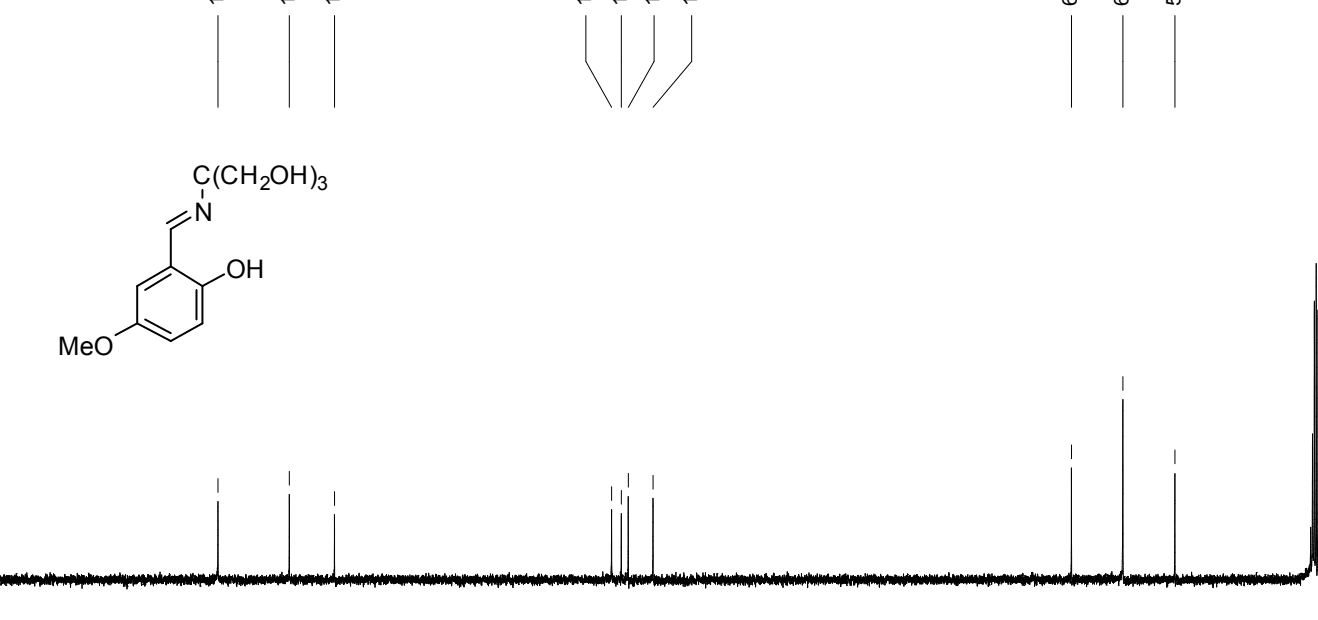

175

150

125

100

50

ppm

Figure 54S. ${ }^{13} \mathrm{C}\left\{{ }^{1} \mathrm{H}\right\}$ NMR spectrum for compound $\mathbf{1 2} / \mathbf{4 2}\left(100 \mathrm{MHz}\right.$, DMSO- $\left.d_{6}\right)$. 


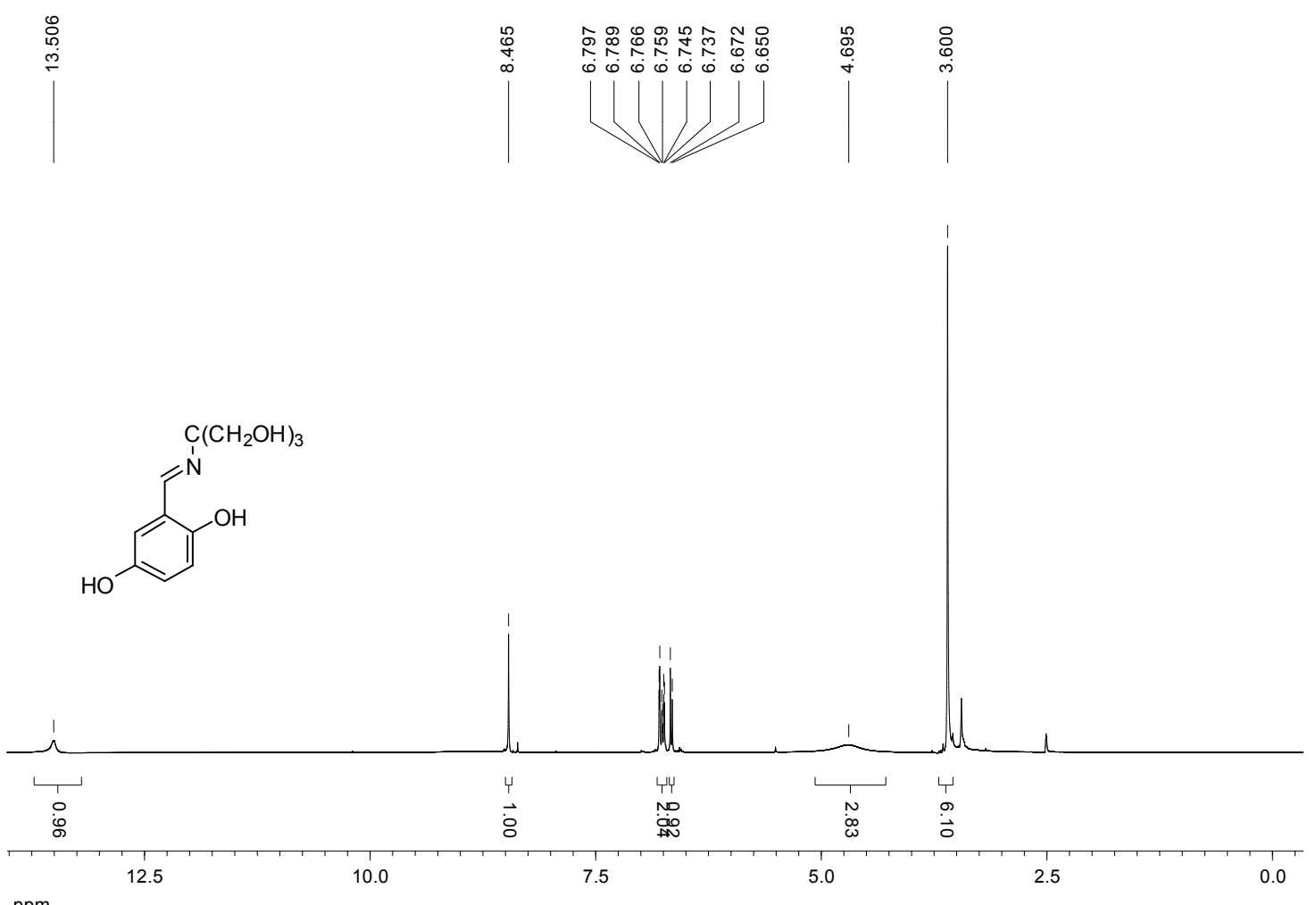

Figure 55S. ${ }^{1} \mathrm{H}$ NMR spectrum for compound 13/43 (400 MHz, DMSO- $\left.d_{6}\right)$.
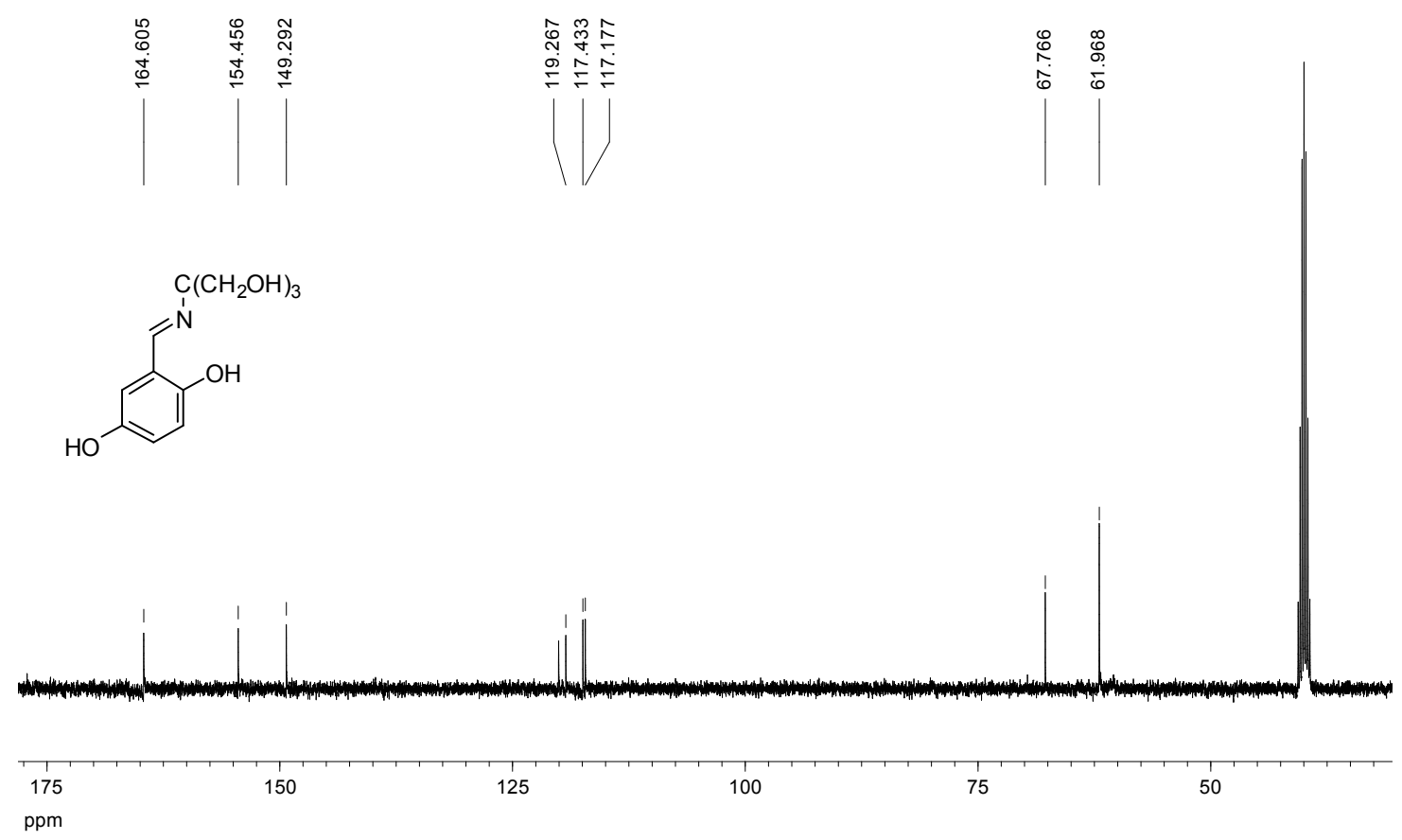

Figure 56S. ${ }^{13} \mathrm{C}\left\{{ }^{1} \mathrm{H}\right\}$ NMR spectrum for compound $\mathbf{1 3} / \mathbf{4 3}\left(100 \mathrm{MHz}\right.$, DMSO- $\left.d_{6}\right)$. 

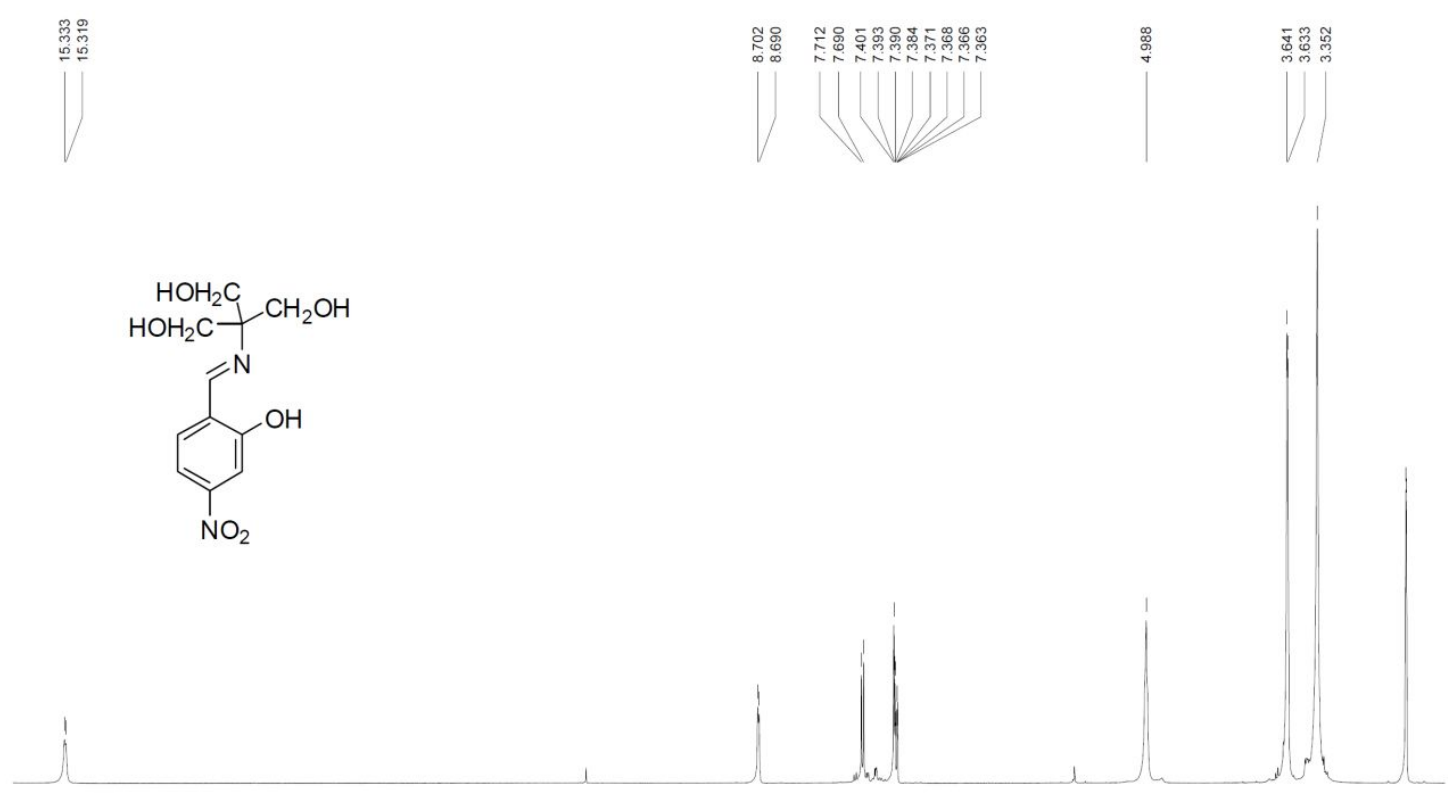

\begin{tabular}{|c|c|c|c|c|c|}
\hline 15.0 & 12.5 & 10.0 & 7.5 & 5.0 & 2.5 \\
\hline
\end{tabular}

Figure 57S. ${ }^{1} \mathrm{H}$ NMR spectrum for compound 14/44 (400 MHz, DMSO- $\left.d_{6}\right)$.
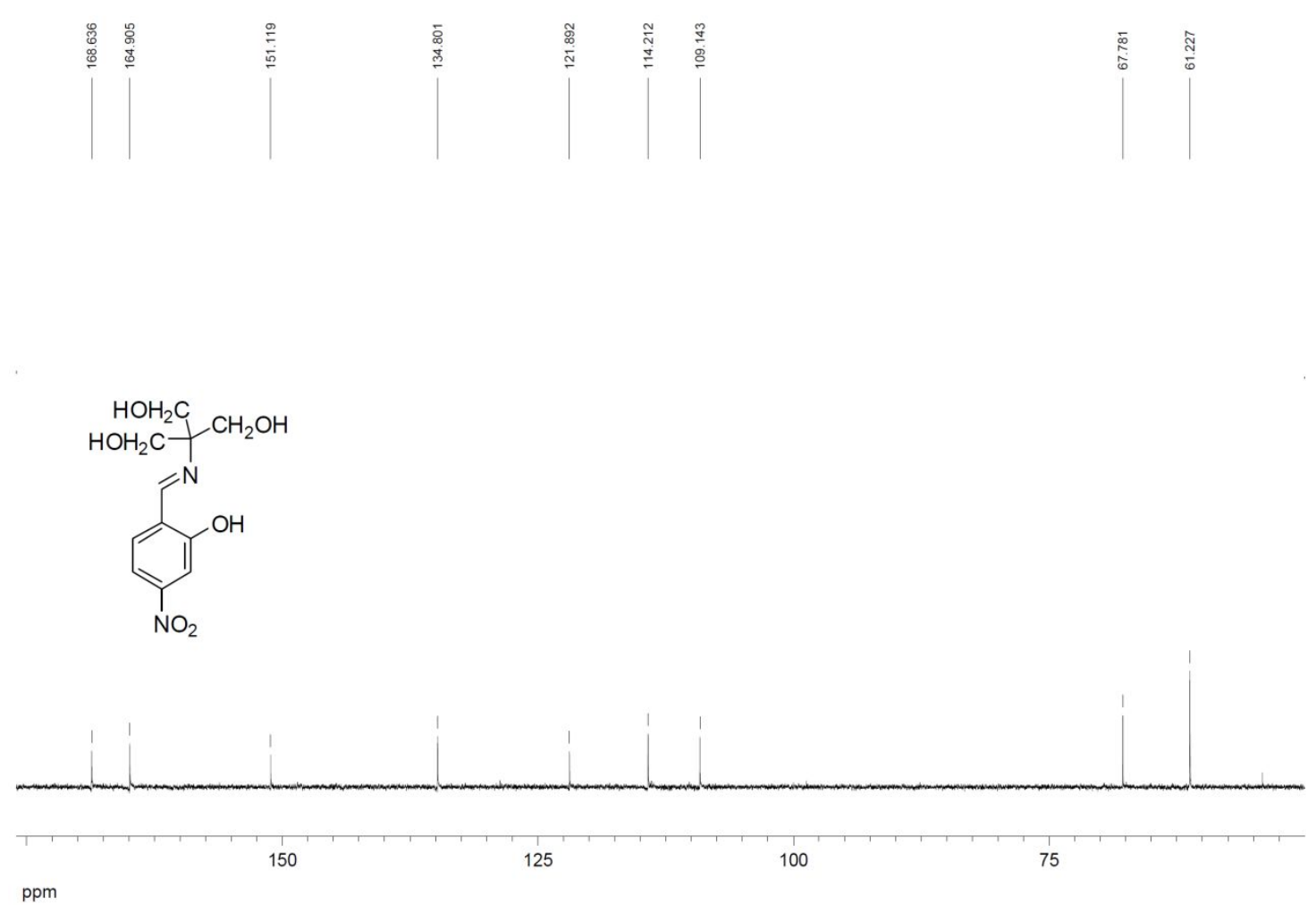

Figure 58S. ${ }^{13} \mathrm{C}\left\{{ }^{1} \mathrm{H}\right\}$ NMR spectrum for compound 14/44 (100 MHz, DMSO- $\left.d_{6}\right)$. 


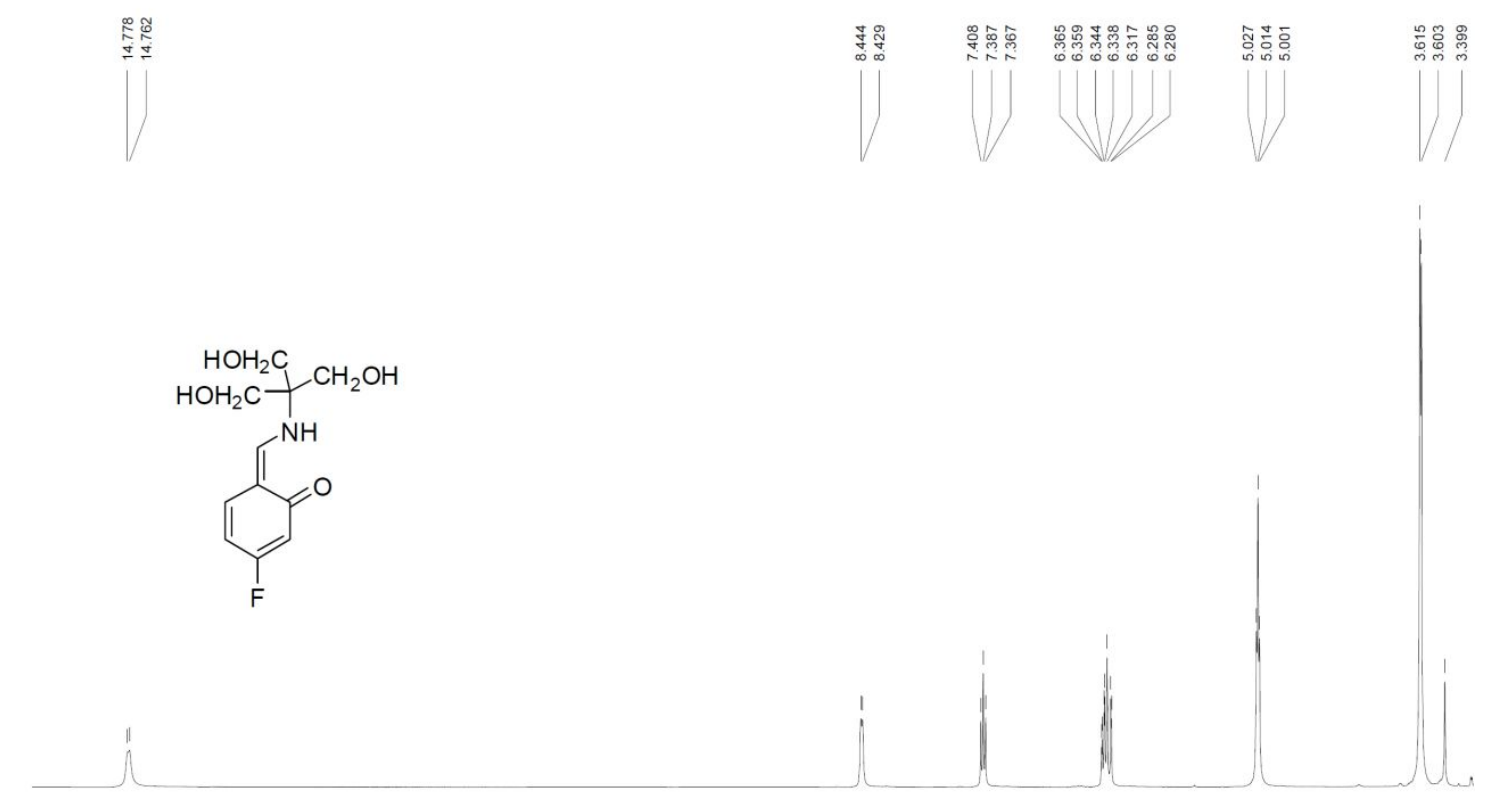

12.5
15.0
$\mathrm{ppm}$

Figure 59S. ${ }^{1} \mathrm{H}$ NMR spectrum for compound 15/45 (400 MHz, DMSO- $d_{6}$ ).
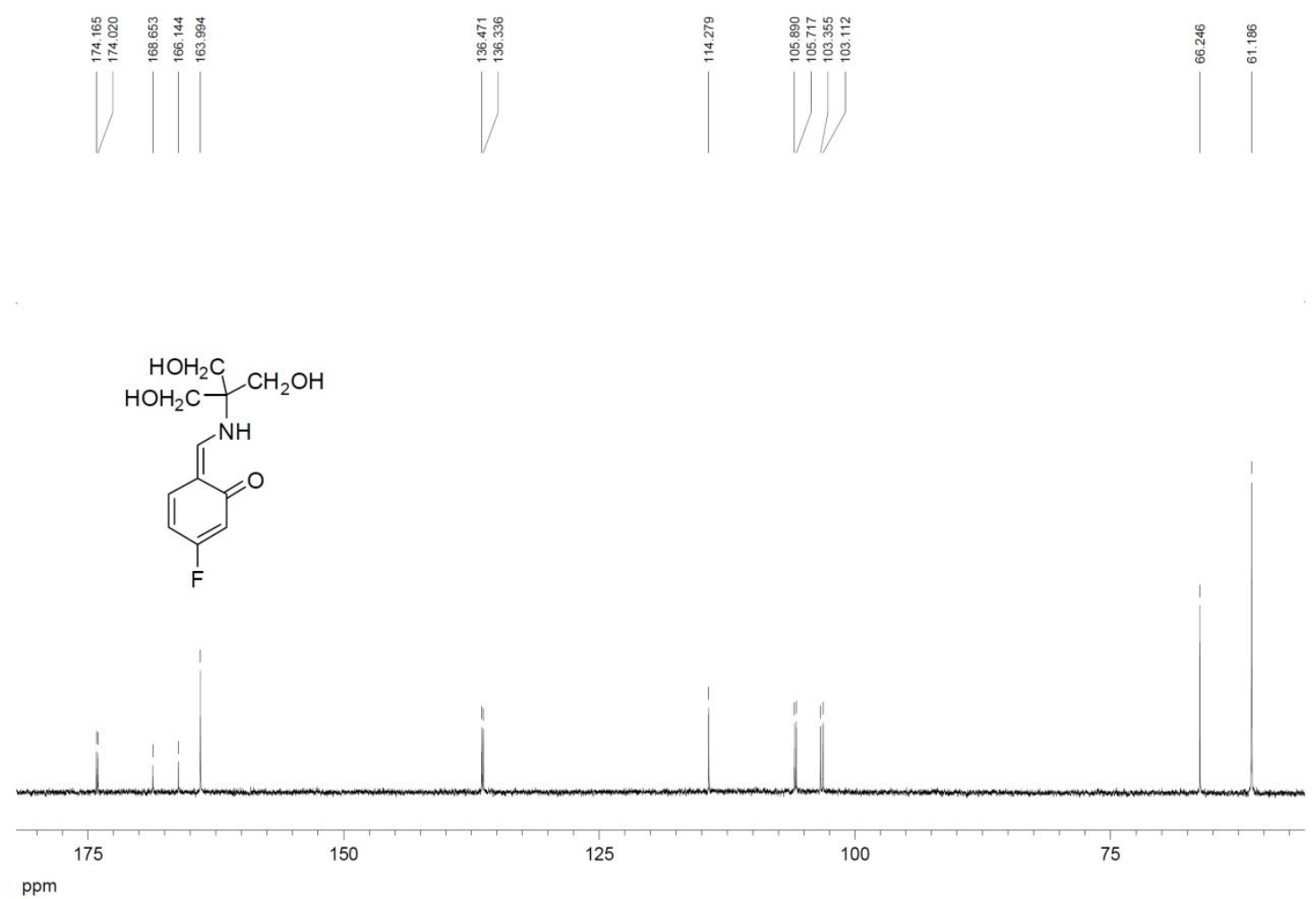

Figure 60S. ${ }^{13} \mathrm{C}\left\{{ }^{1} \mathrm{H}\right\}$ NMR spectrum for compound 15/45 (100 MHz, DMSO- $\left.d_{6}\right)$. 


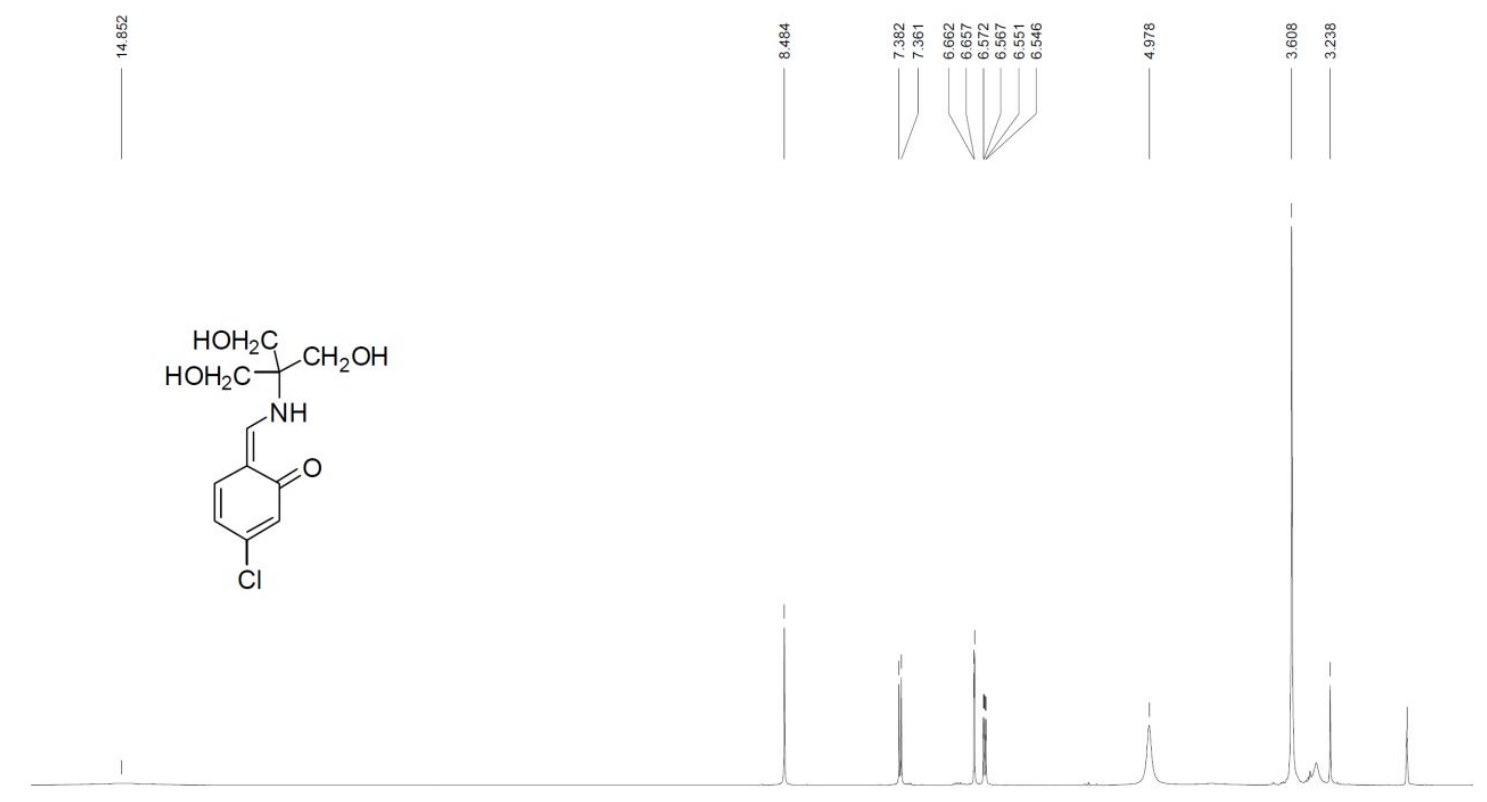

15.0
$\mathrm{ppm}$

Figure 61S. ${ }^{1} \mathrm{H}$ NMR spectrum for compound 16/46 (400 MHz, DMSO- $\left.d_{6}\right)$.

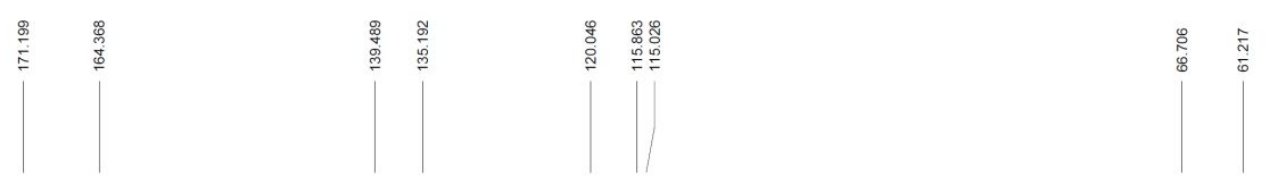

Cl

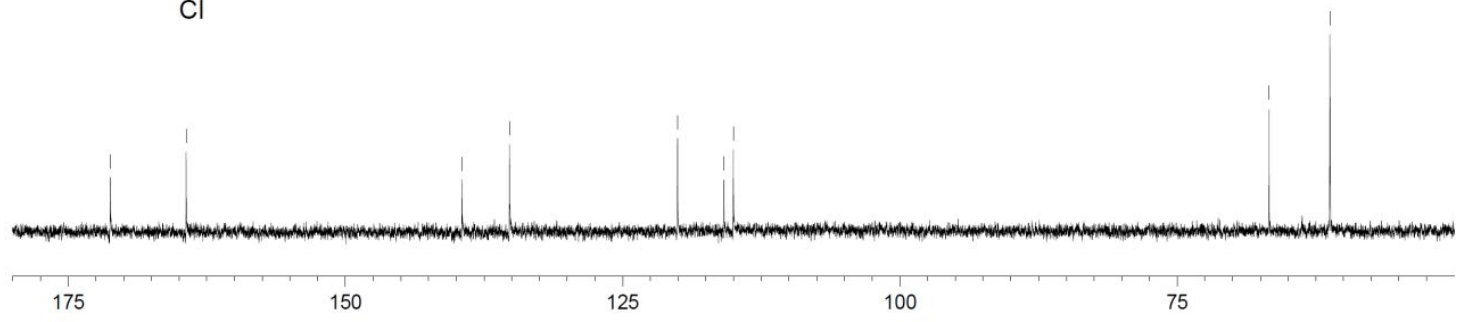

$\mathrm{ppm}$

Figure 62S. ${ }^{13} \mathrm{C}\left\{{ }^{1} \mathrm{H}\right\}$ NMR spectrum for compound 16/46 (100 MHz, DMSO- $\left.d_{6}\right)$. 


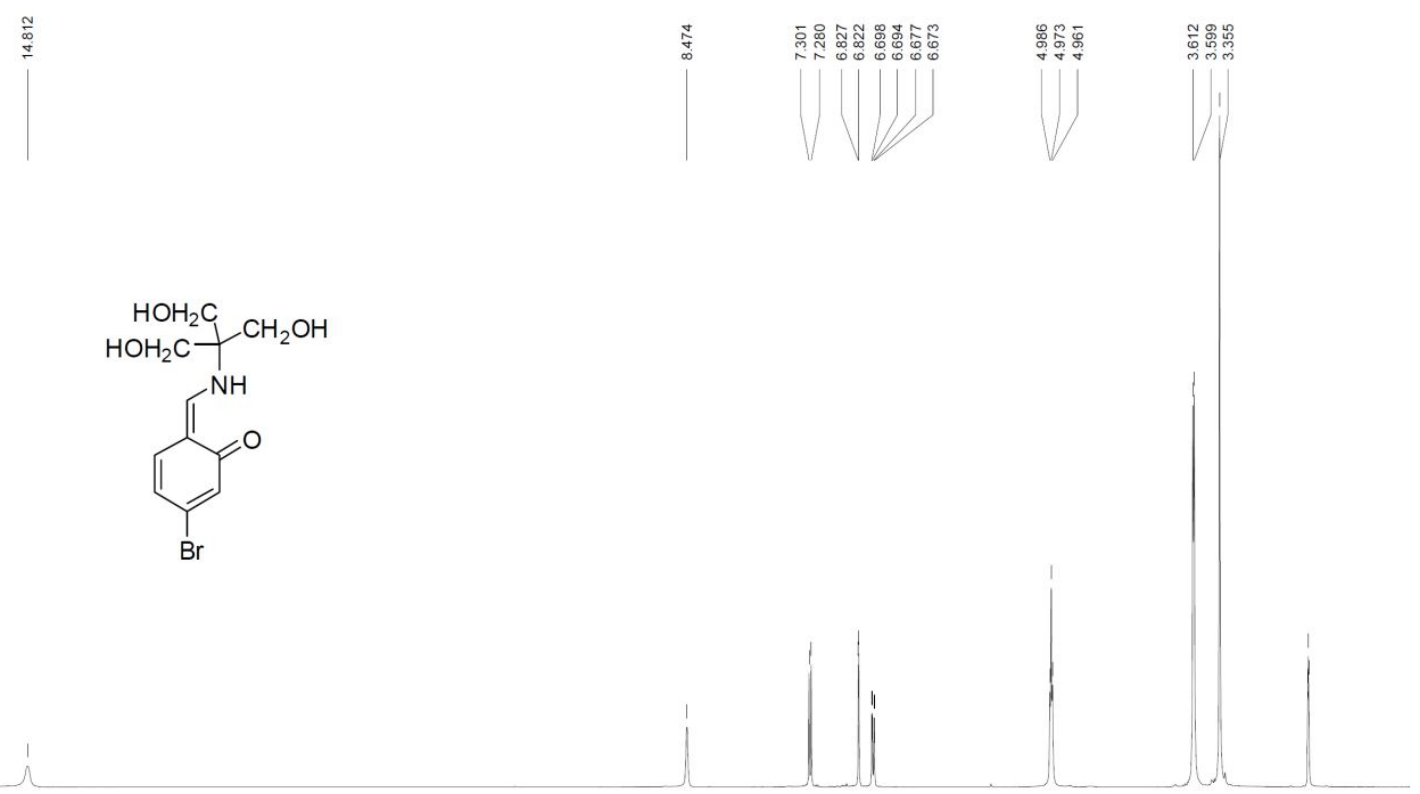

10.0
15.0
$\mathrm{ppm}$

Figure 63S. ${ }^{1} \mathrm{H}$ NMR spectrum for compound 17/47 (400 MHz, DMSO- $d_{6}$ ).
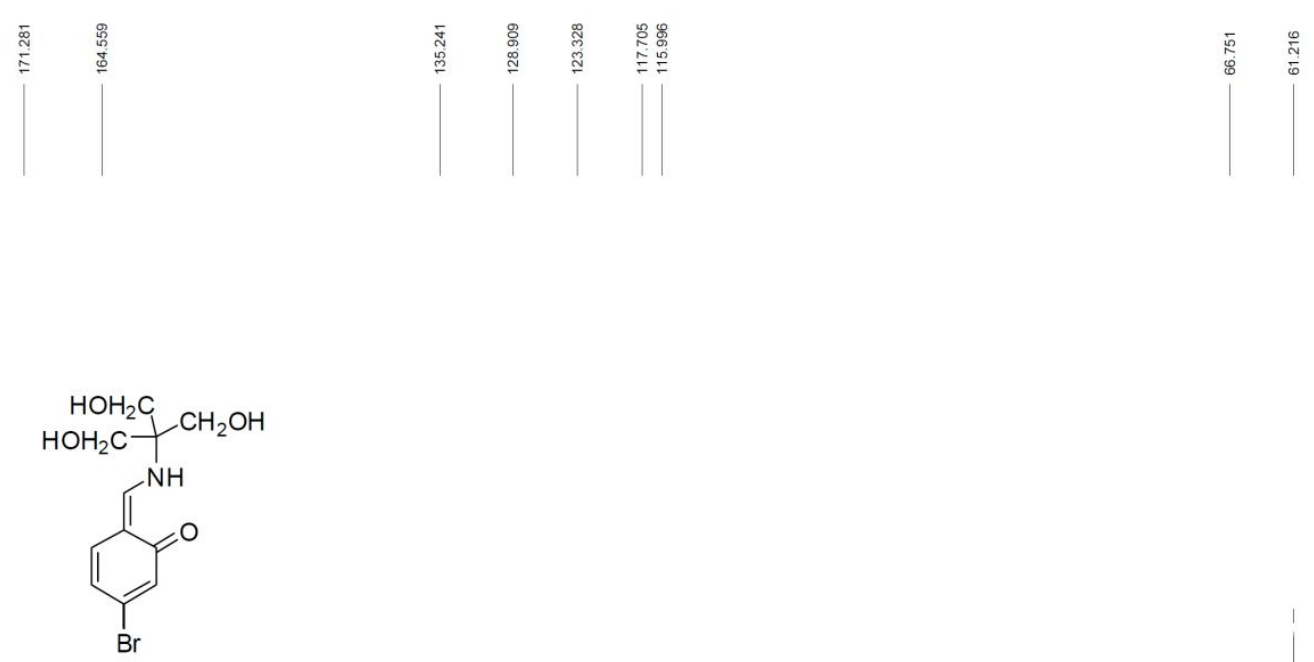

175

150

125

100

75

ppm

Figure 64S. ${ }^{13} \mathrm{C}\left\{{ }^{1} \mathrm{H}\right\}$ NMR spectrum for compound 17/47 (100 MHz, DMSO- $\left.d_{6}\right)$. 

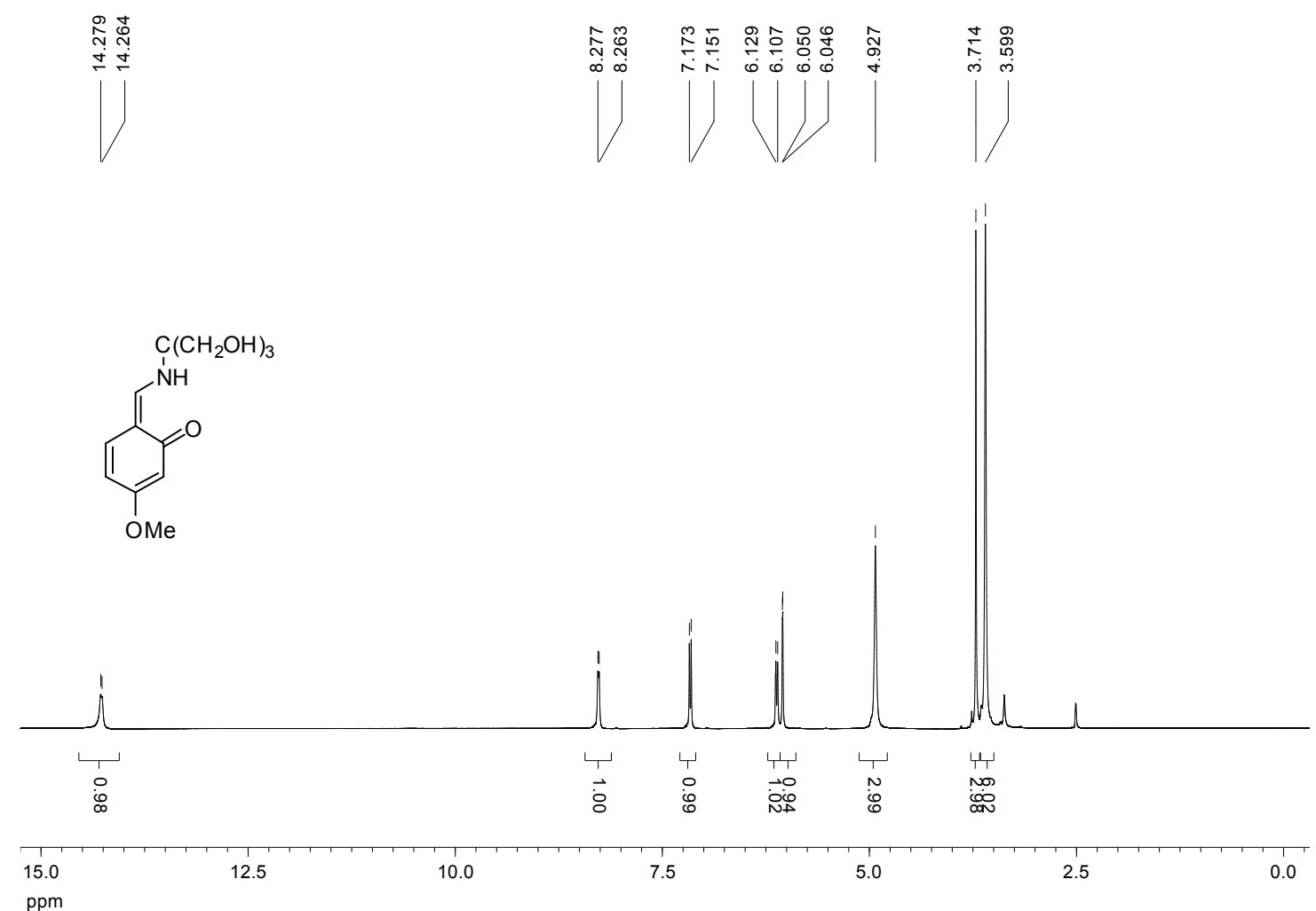

Figure 65S. ${ }^{1} \mathrm{H}$ NMR spectrum for compound 18/48 (400 MHz, DMSO- $\left.d_{6}\right)$.
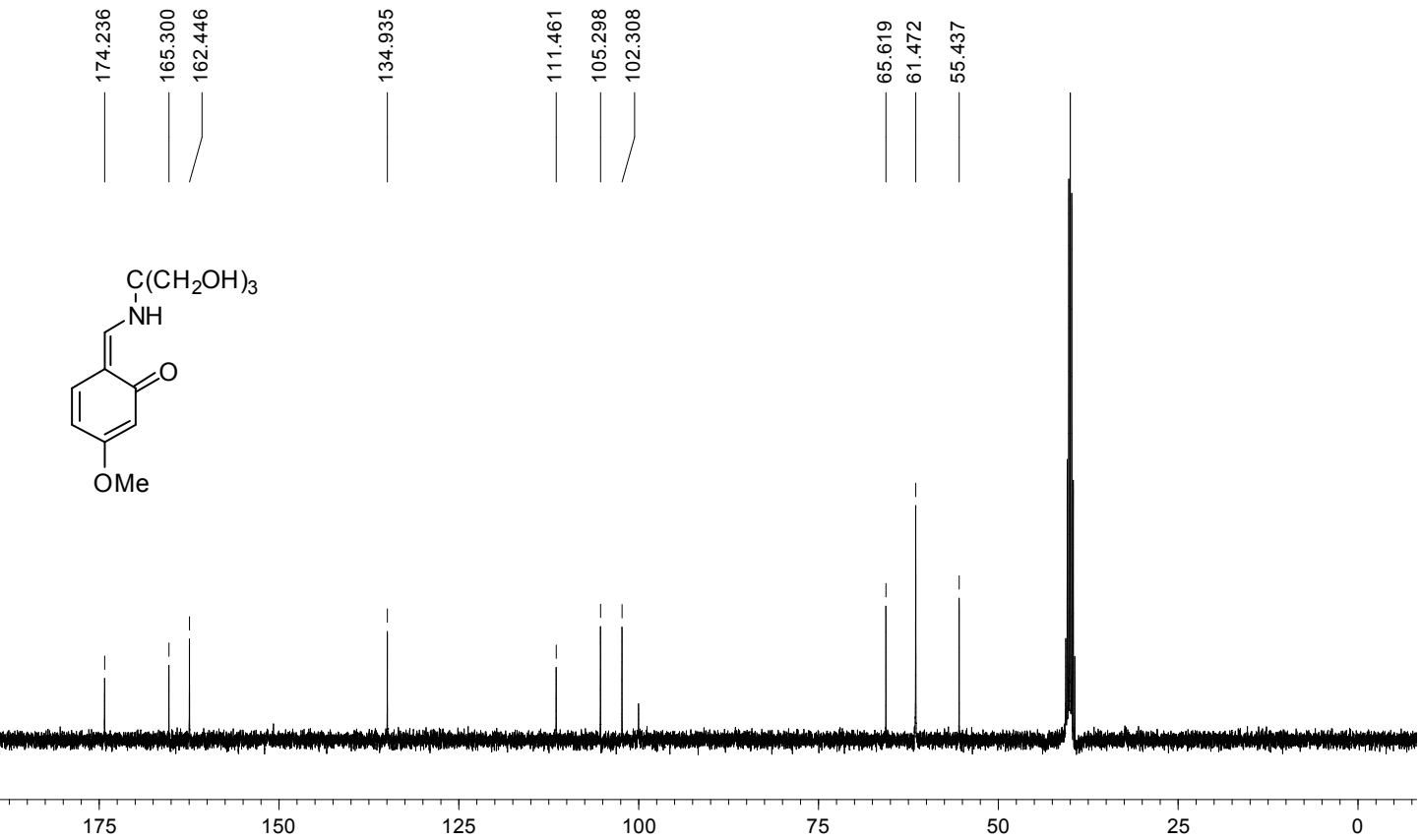

ppm

Figure 66S. ${ }^{13} \mathrm{C}\left\{{ }^{1} \mathrm{H}\right\}$ NMR spectrum for compound 18/48 (100 MHz, DMSO- $\left.d_{6}\right)$. 


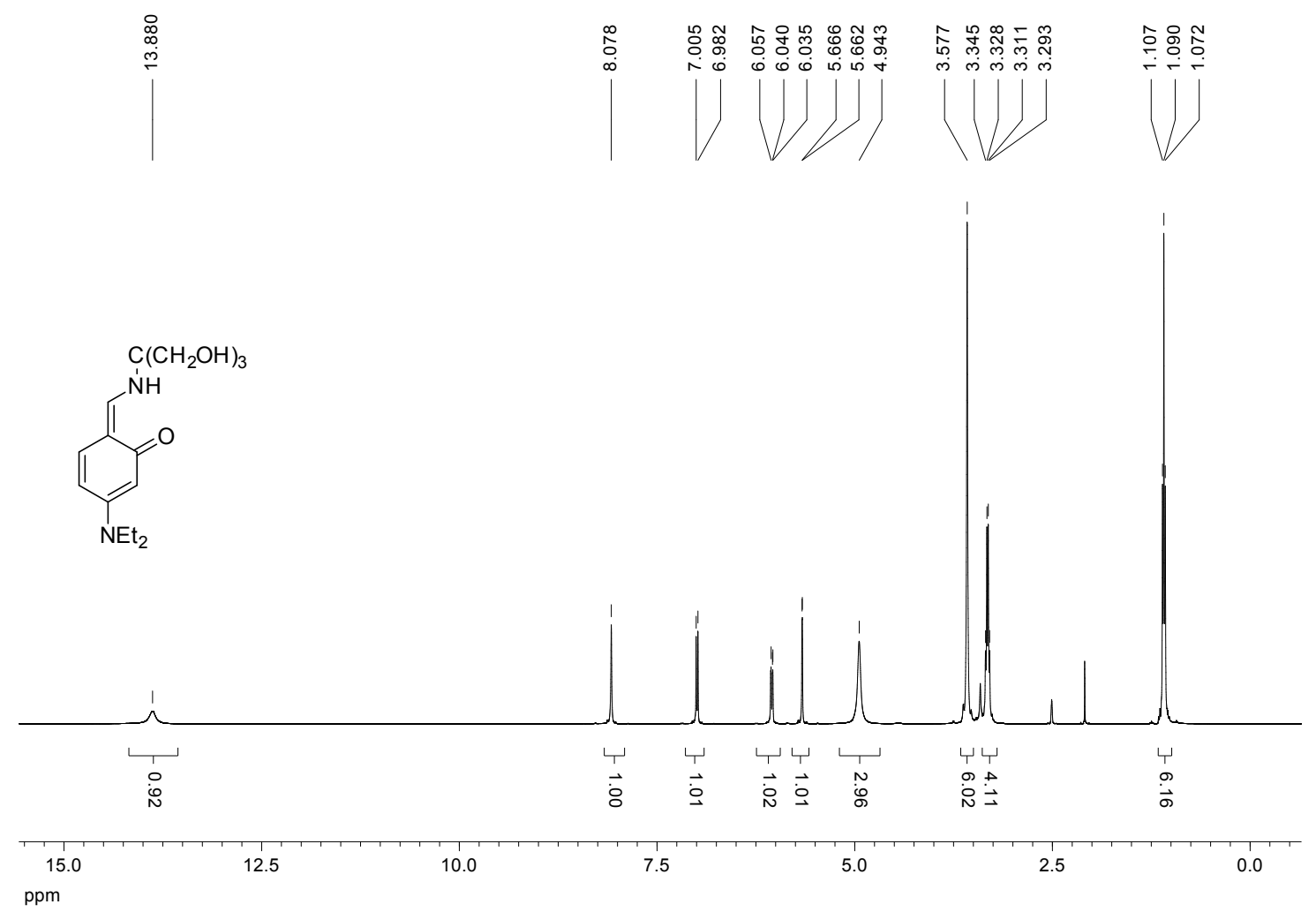

Figure 67S. ${ }^{1} \mathrm{H}$ NMR spectrum for compound 19/49 (400 MHz, DMSO- $\left.d_{6}\right)$.

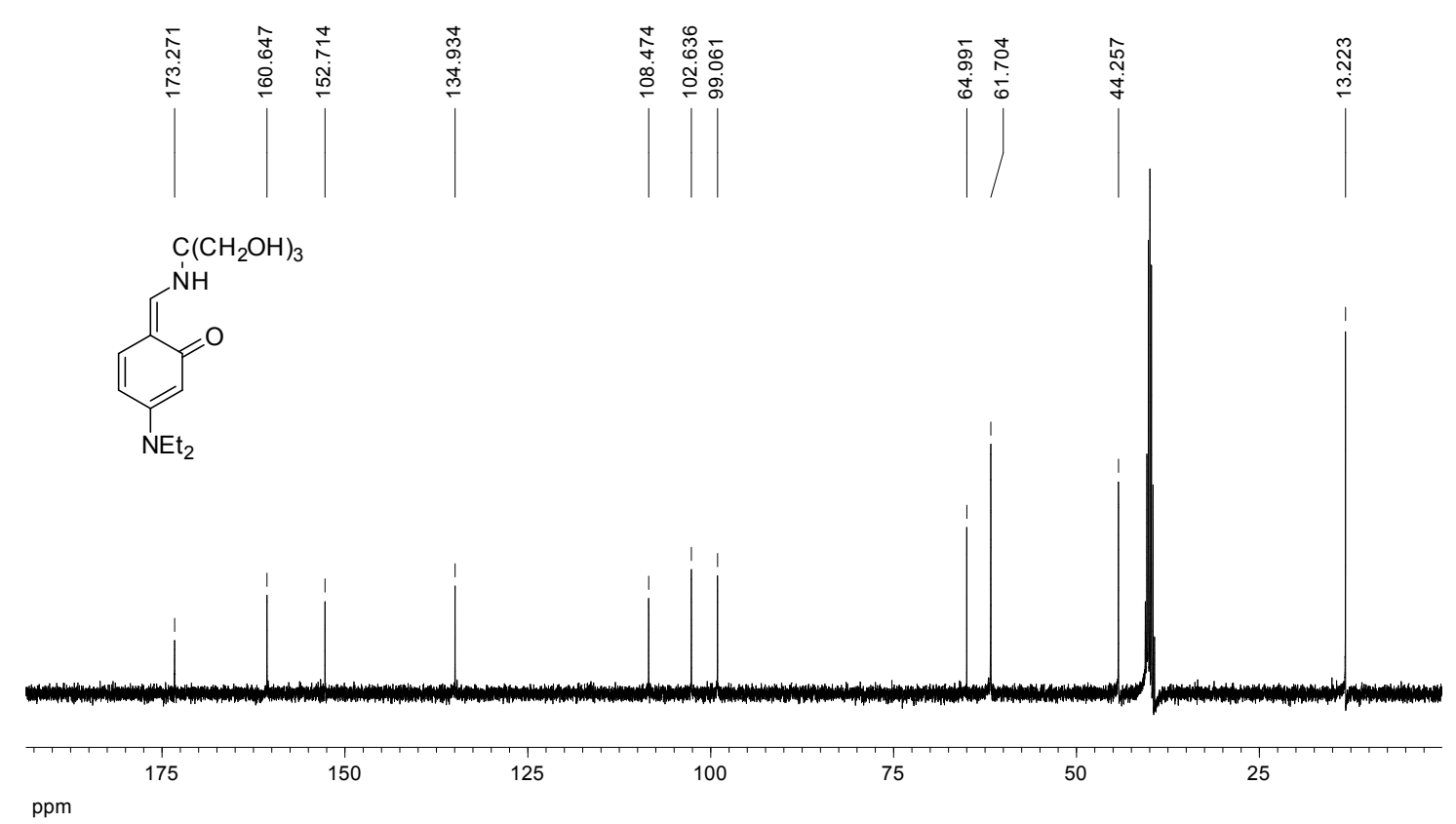

Figure 68S. ${ }^{13} \mathrm{C}\left\{{ }^{1} \mathrm{H}\right\}$ NMR spectrum for compound 19/49 (100 MHz, DMSO- $\left.d_{6}\right)$. 
<smiles>CCOc1cccc(CN=CO)c1O</smiles>

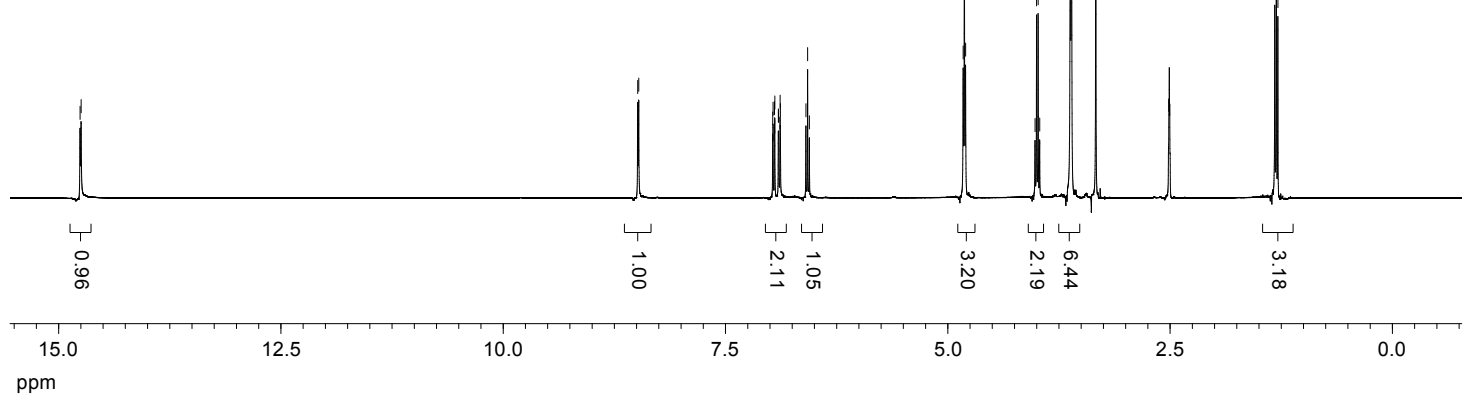

Figure 69S. ${ }^{1} \mathrm{H}$ NMR spectrum for compound 20/50 (400 MHz, DMSO- $\left.d_{6}\right)$.
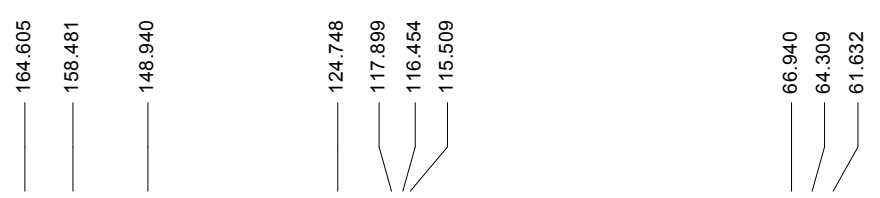<smiles>CCOc1cccc(/C=N/[CH-]CO)c1O</smiles>

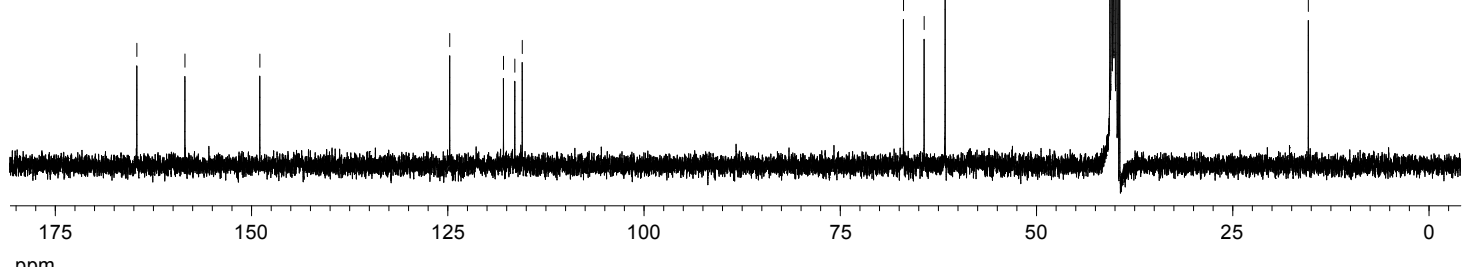

Figure 70S. ${ }^{13} \mathrm{C}\left\{{ }^{1} \mathrm{H}\right\}$ NMR spectrum for compound 20/50 (100 MHz, DMSO- $\left.d_{6}\right)$. 


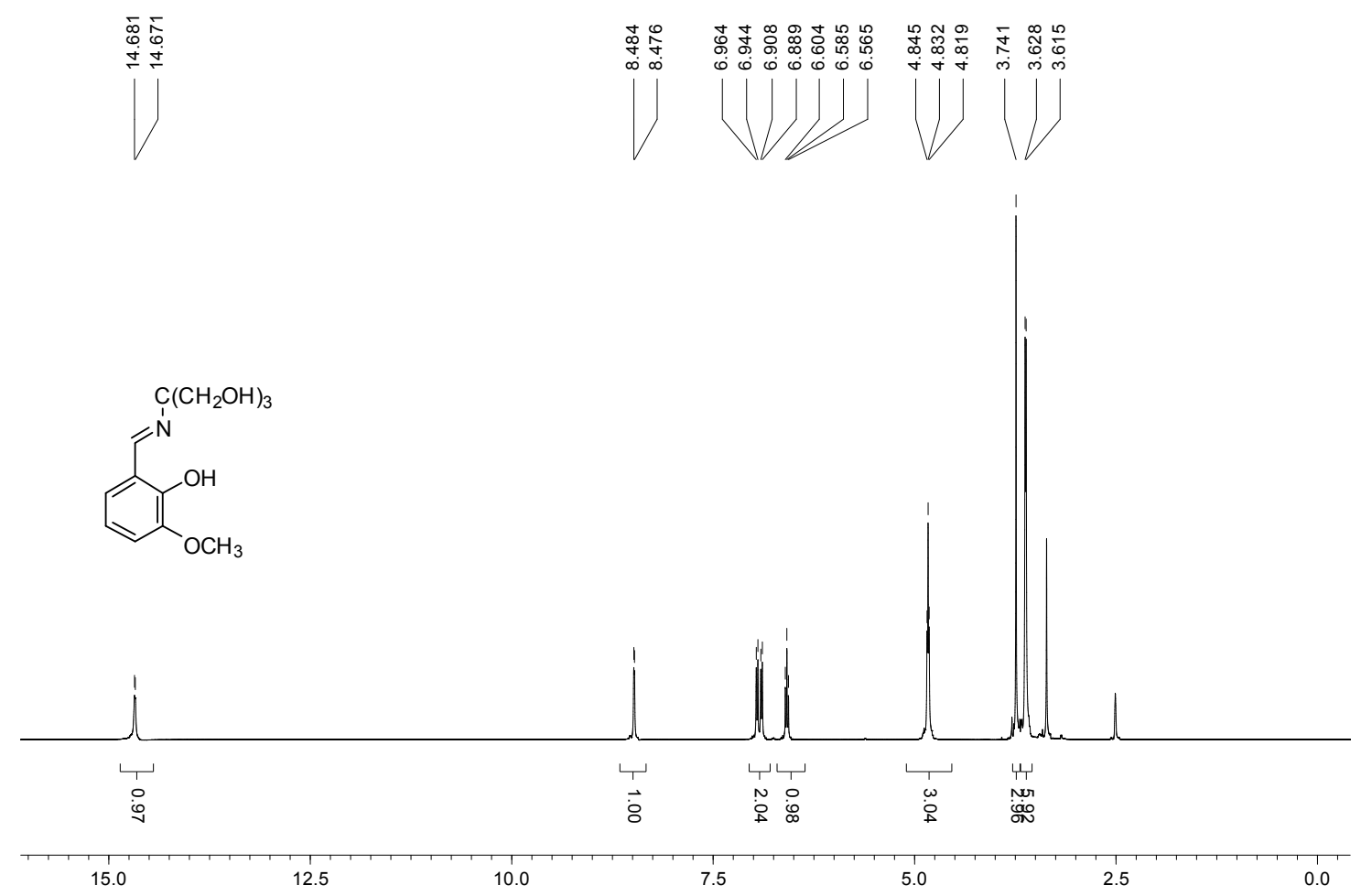

Figure 71S. ${ }^{1} \mathrm{H}$ NMR spectrum for compound 21/51 (400 MHz, DMSO- $d_{6}$ ).
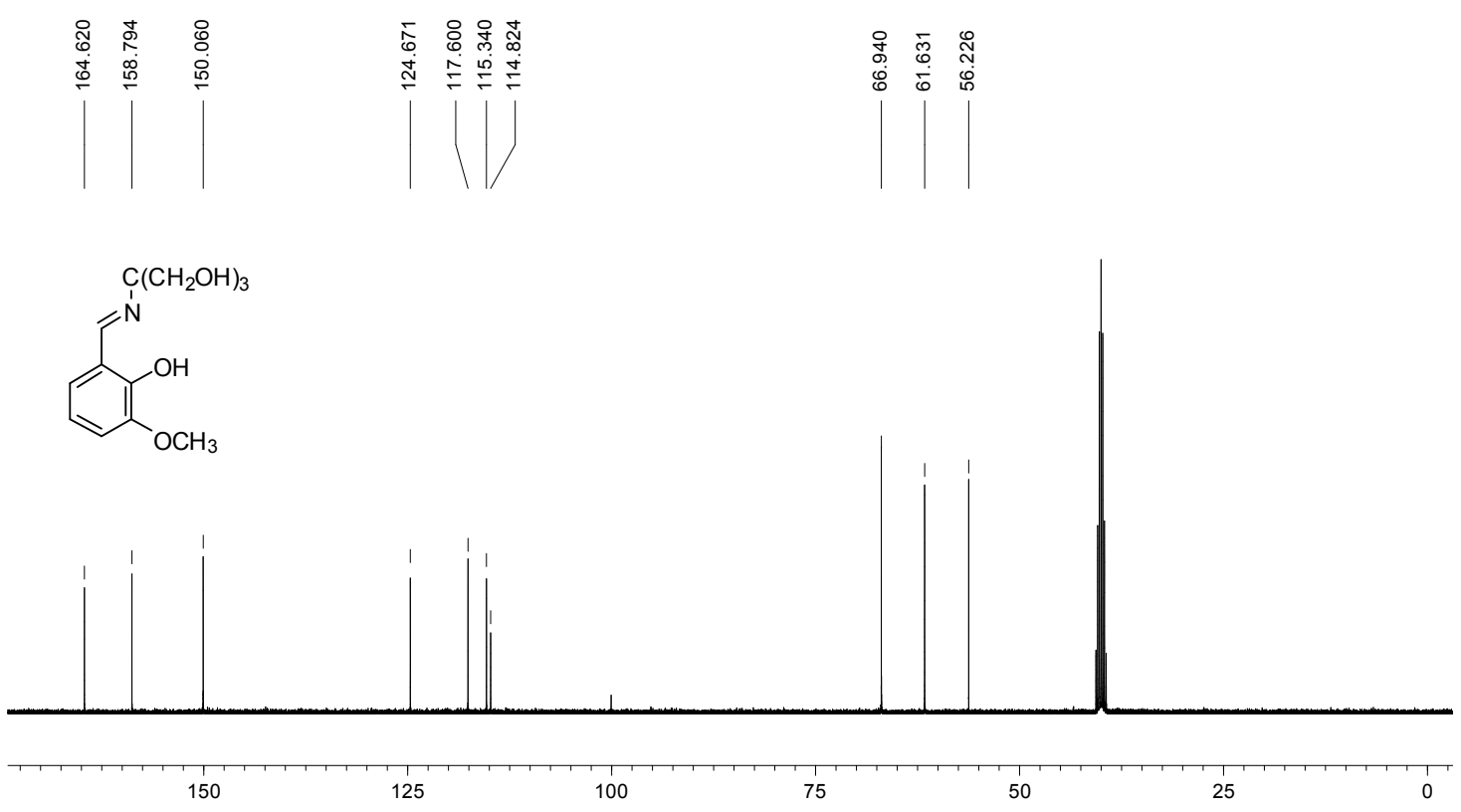

ppm

Figure 72S. ${ }^{13} \mathrm{C}\left\{{ }^{1} \mathrm{H}\right\}$ NMR spectrum for compound 21/51 (100 MHz, DMSO- $\left.d_{6}\right)$. 


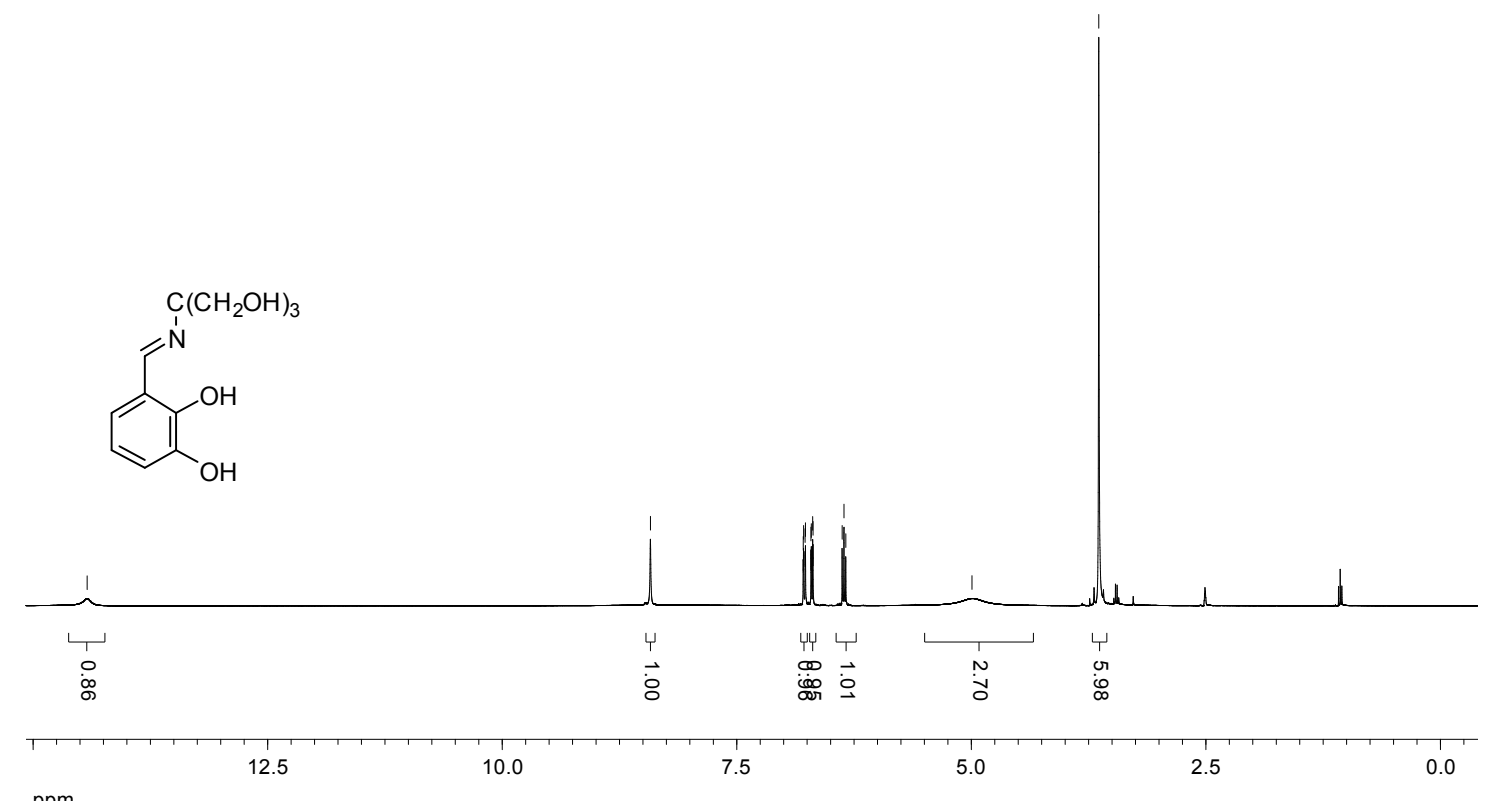

Figure 73S. ${ }^{1} \mathrm{H}$ NMR spectrum for compound 22/52 (400 MHz, DMSO- $\left.d_{6}\right)$.

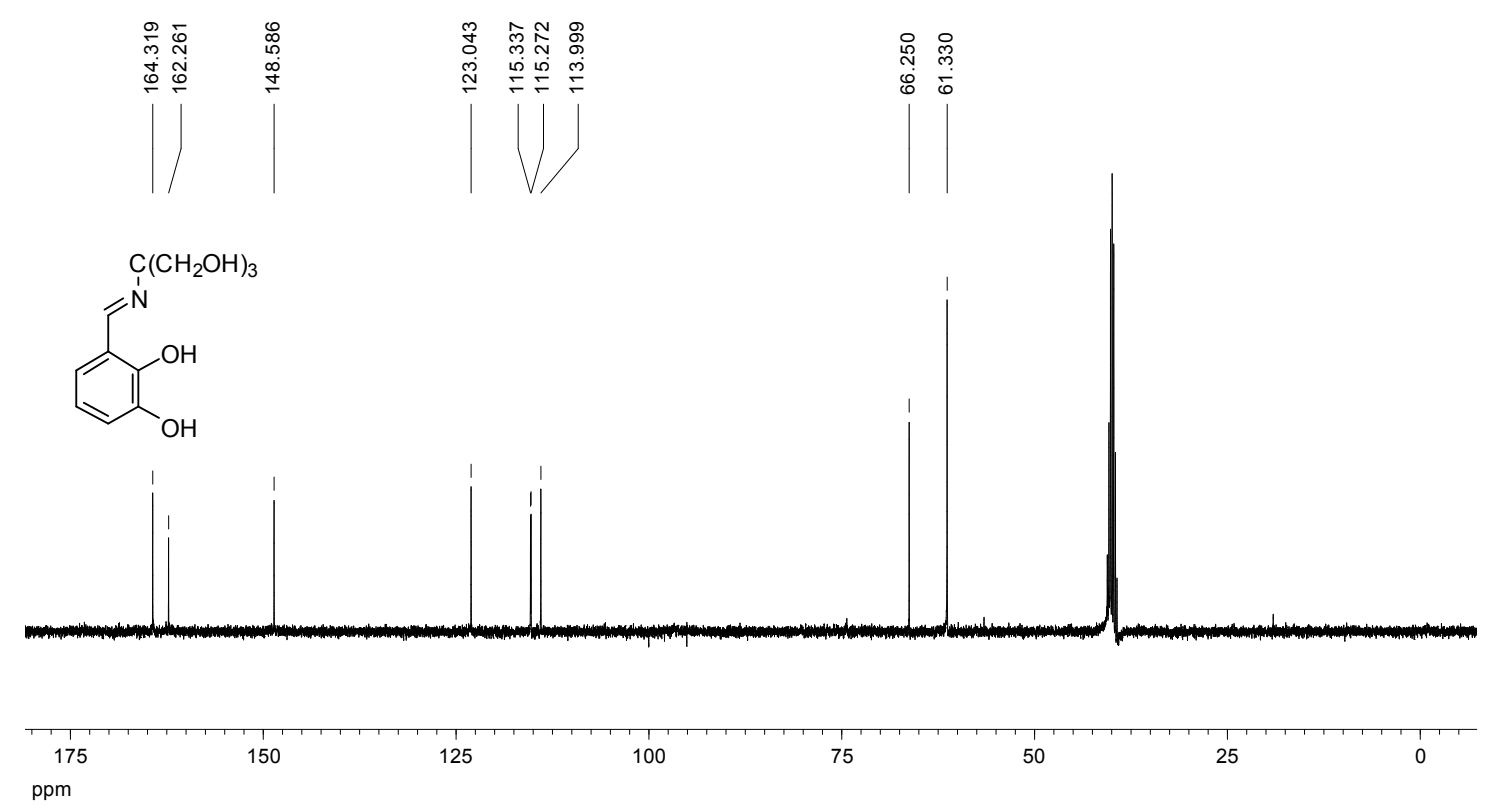

Figure 74S. ${ }^{13} \mathrm{C}\left\{{ }^{1} \mathrm{H}\right\}$ NMR spectrum for compound 22/52 (100 MHz, DMSO- $\left.d_{6}\right)$. 


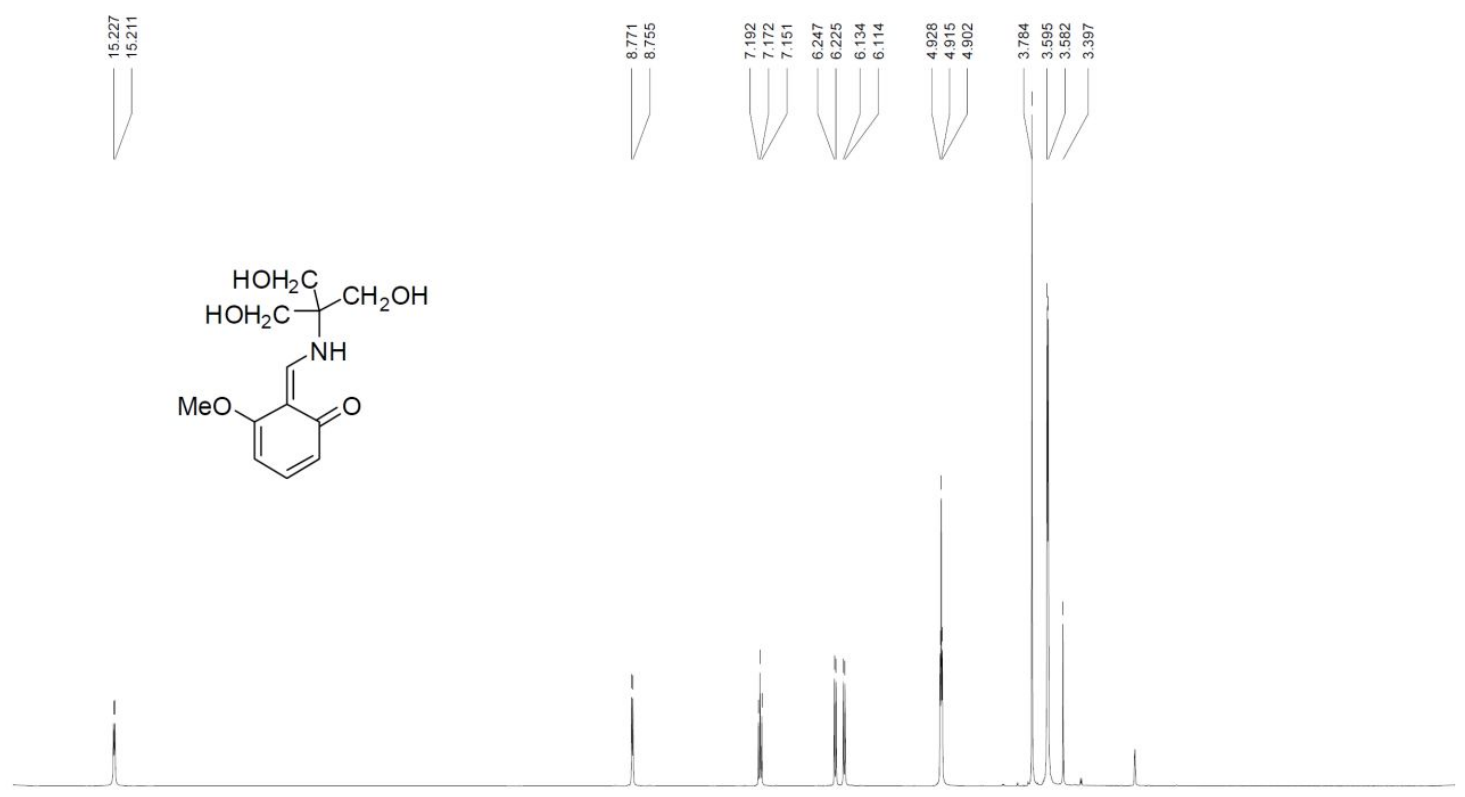

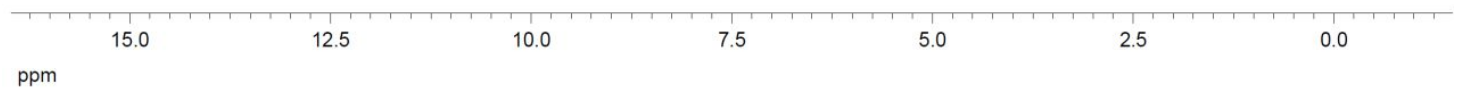

Figure 75S. ${ }^{1} \mathrm{H}$ NMR spectrum for compound 23/53 (400 MHz, DMSO- $d_{6}$ ).
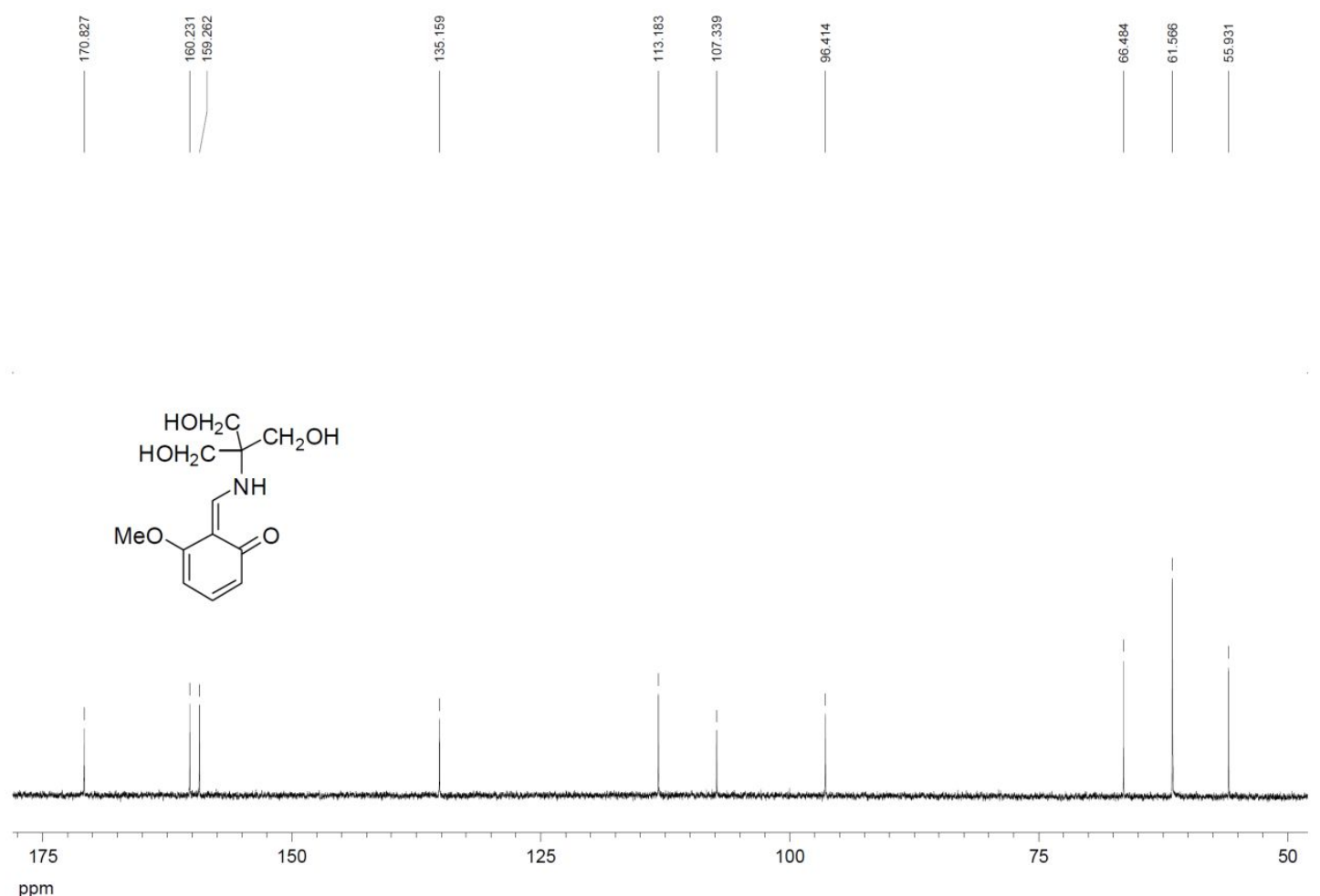

Figure 76S. ${ }^{13} \mathrm{C}\left\{{ }^{1} \mathrm{H}\right\}$ NMR spectrum for compound 23/53 (100 MHz, DMSO- $\left.d_{6}\right)$. 


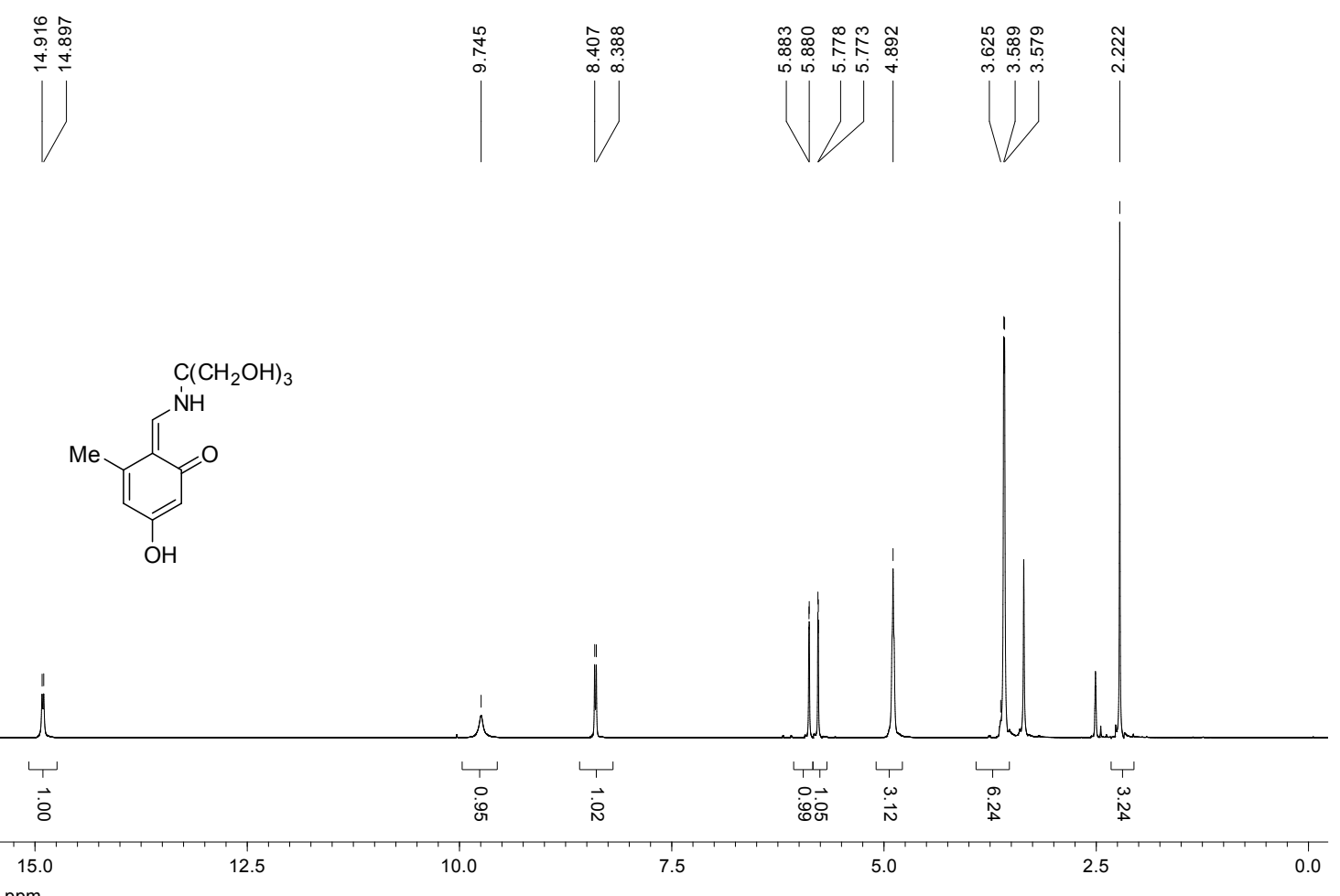

Figure 77S. ${ }^{1} \mathrm{H}$ NMR spectrum for compound 24/54 (400 MHz, DMSO- $\left.d_{6}\right)$.
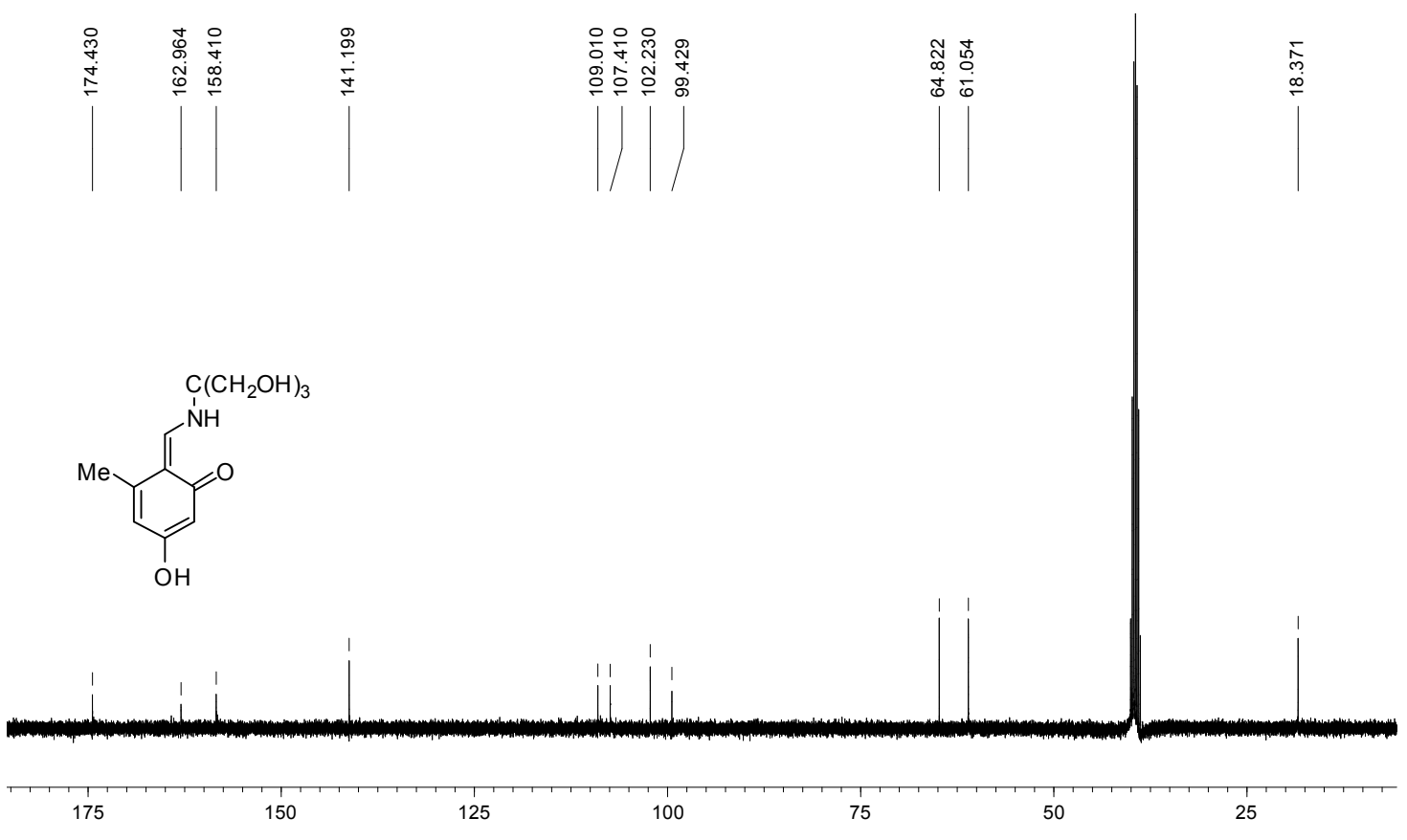

ppm

Figure 78S. ${ }^{13} \mathrm{C}\left\{{ }^{1} \mathrm{H}\right\}$ NMR spectrum for compound 24/54 (100 MHz, DMSO- $\left.d_{6}\right)$. 


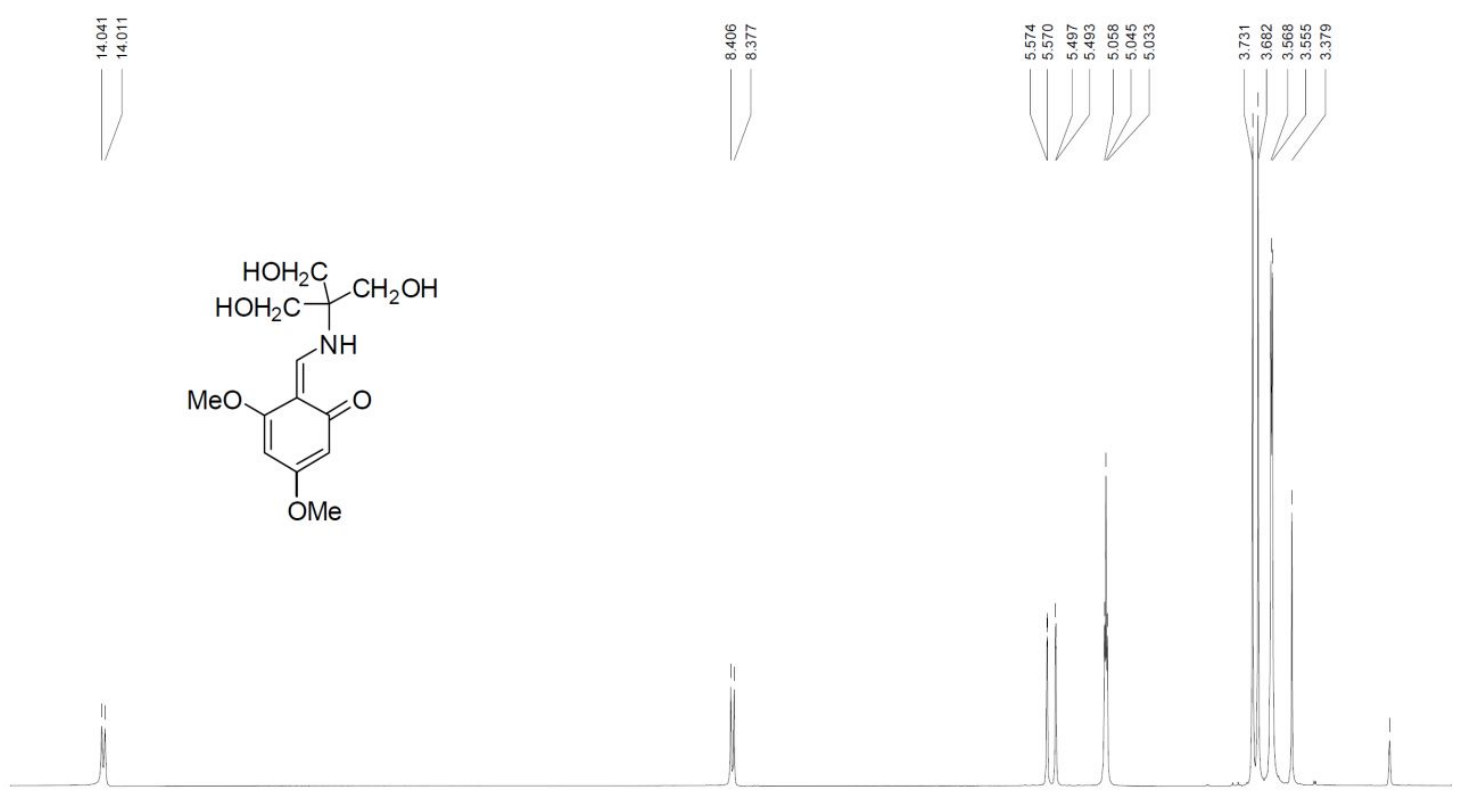

10.0
12.5
5.5

$\mathrm{ppm}$

Figure 79S. ${ }^{1} \mathrm{H}$ NMR spectrum for compound 25/55 (400 MHz, DMSO- $\left.d_{6}\right)$.
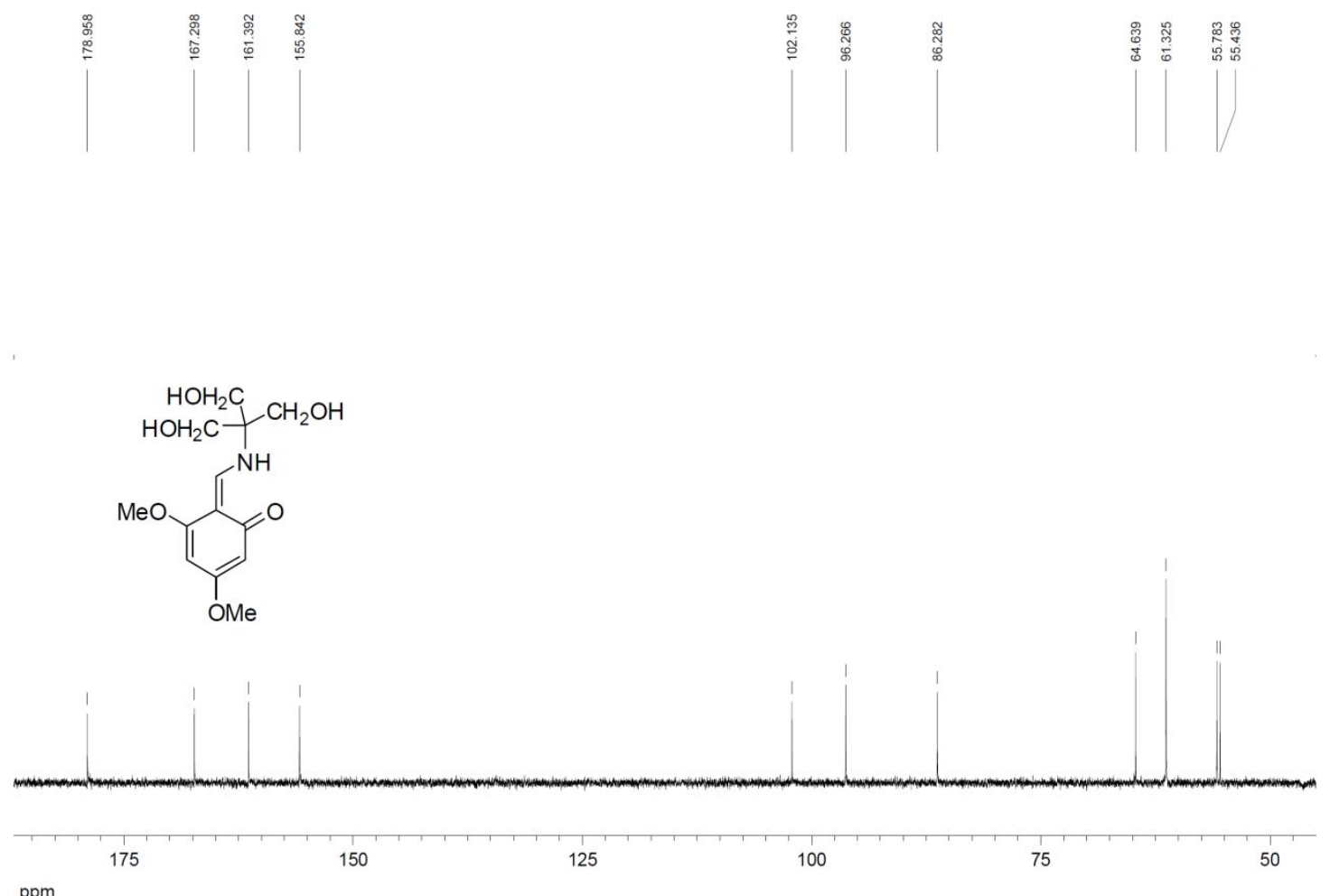

Figure 80S. ${ }^{13} \mathrm{C}\left\{{ }^{1} \mathrm{H}\right\}$ NMR spectrum for compound 25/55 (100 MHz, DMSO- $\left.d_{6}\right)$. 

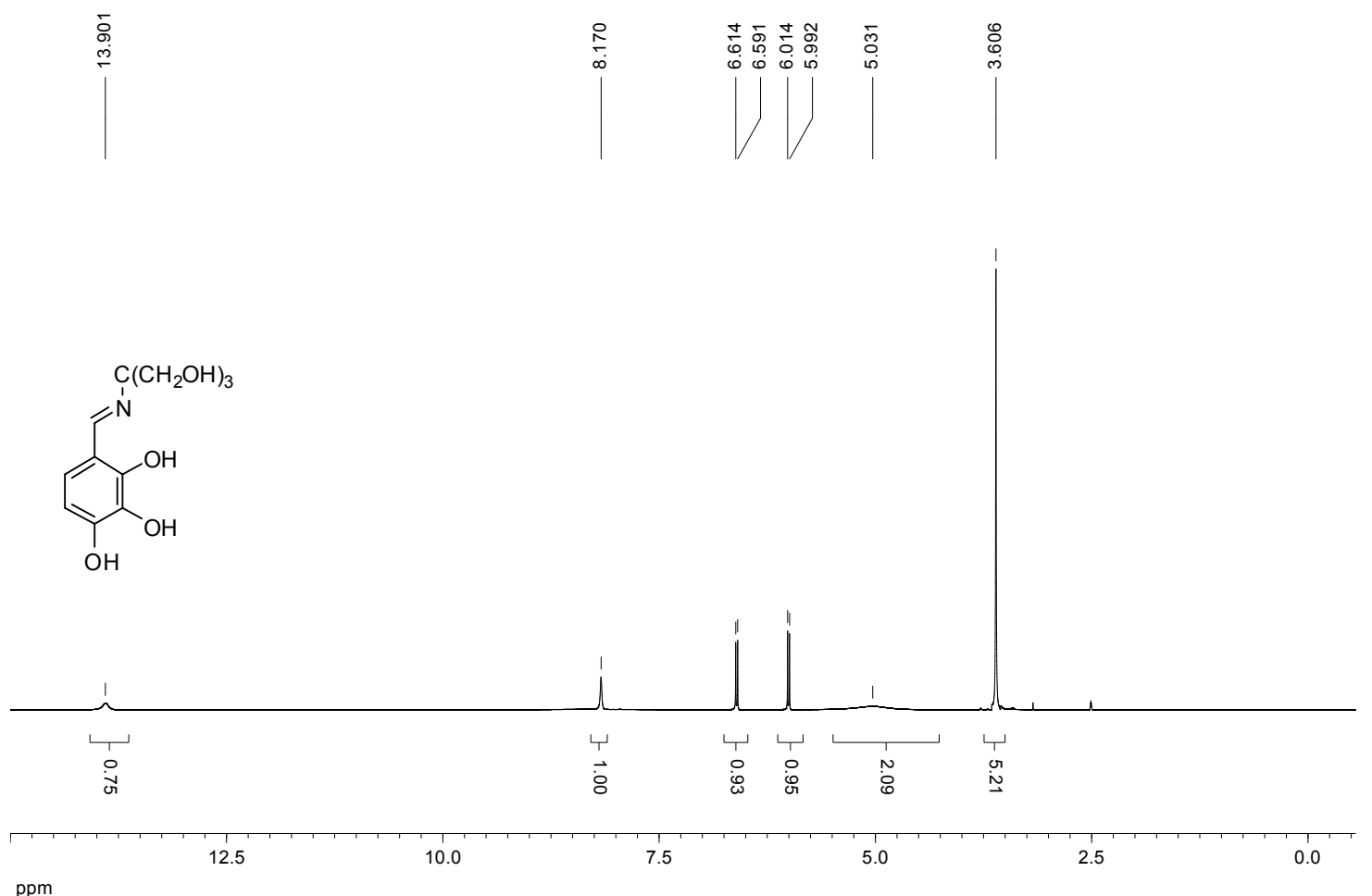

ppm

Figure 81S. ${ }^{1} \mathrm{H}$ NMR spectrum for compound 26/56 (400 MHz, DMSO- $\left.d_{6}\right)$.
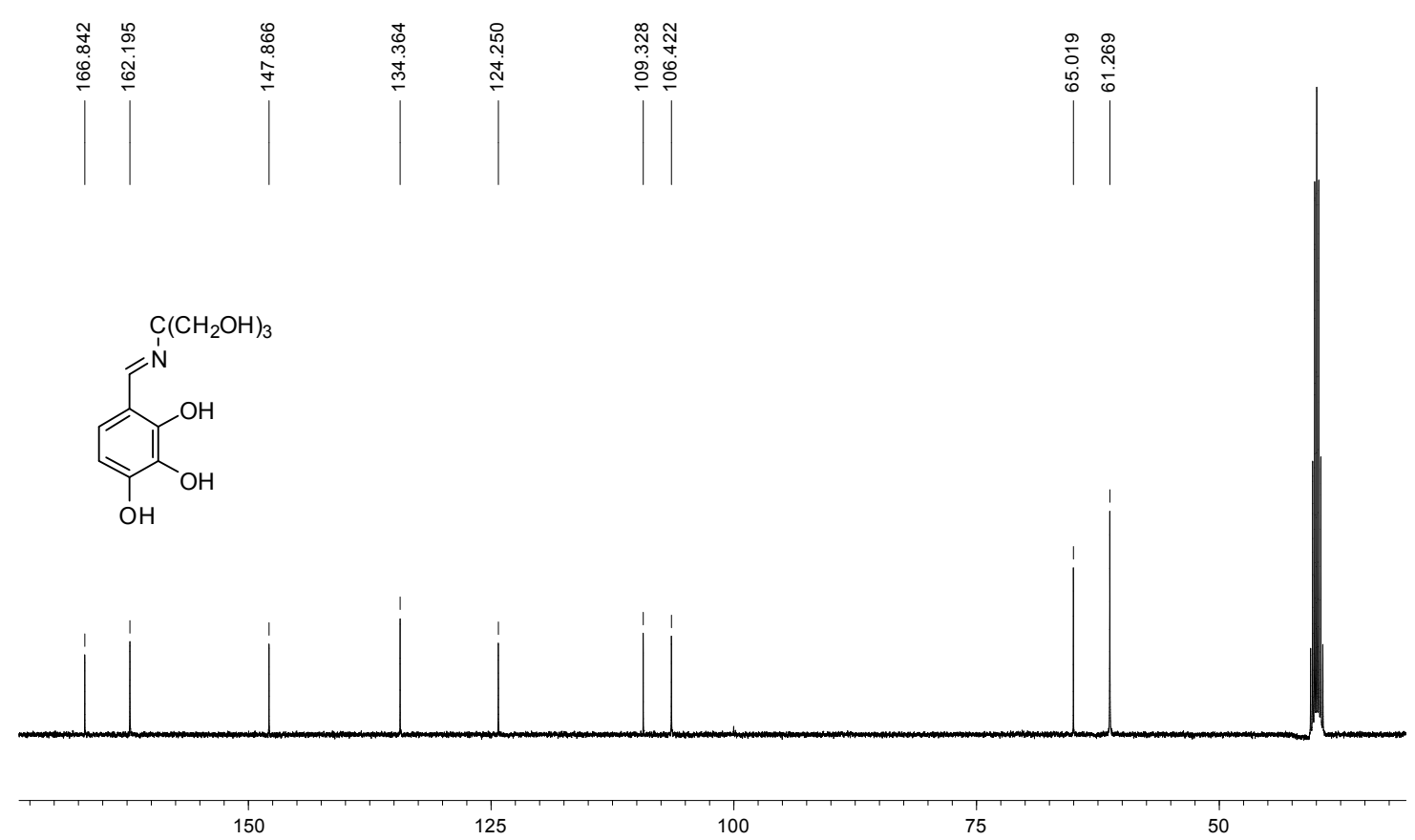

$\mathrm{ppm}$

Figure 82S. ${ }^{13} \mathrm{C}\left\{{ }^{1} \mathrm{H}\right\}$ NMR spectrum for compound 26/56 (100 MHz, DMSO- $\left.d_{6}\right)$. 


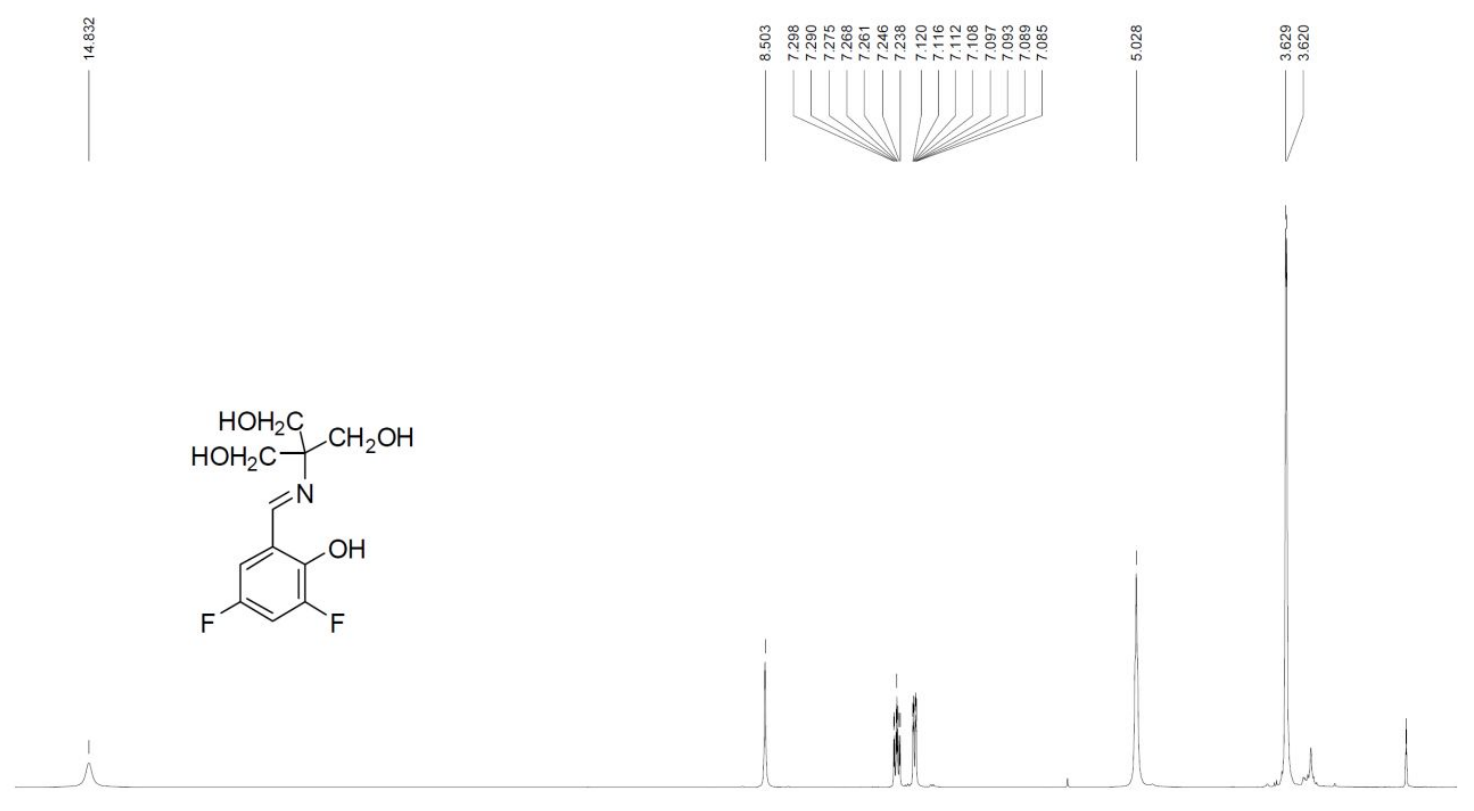

12.5
15.0
$\mathrm{ppm}$

Figure 83S. ${ }^{1} \mathrm{H}$ NMR spectrum for compound 27/57 (400 MHz, DMSO- $d_{6}$ ).
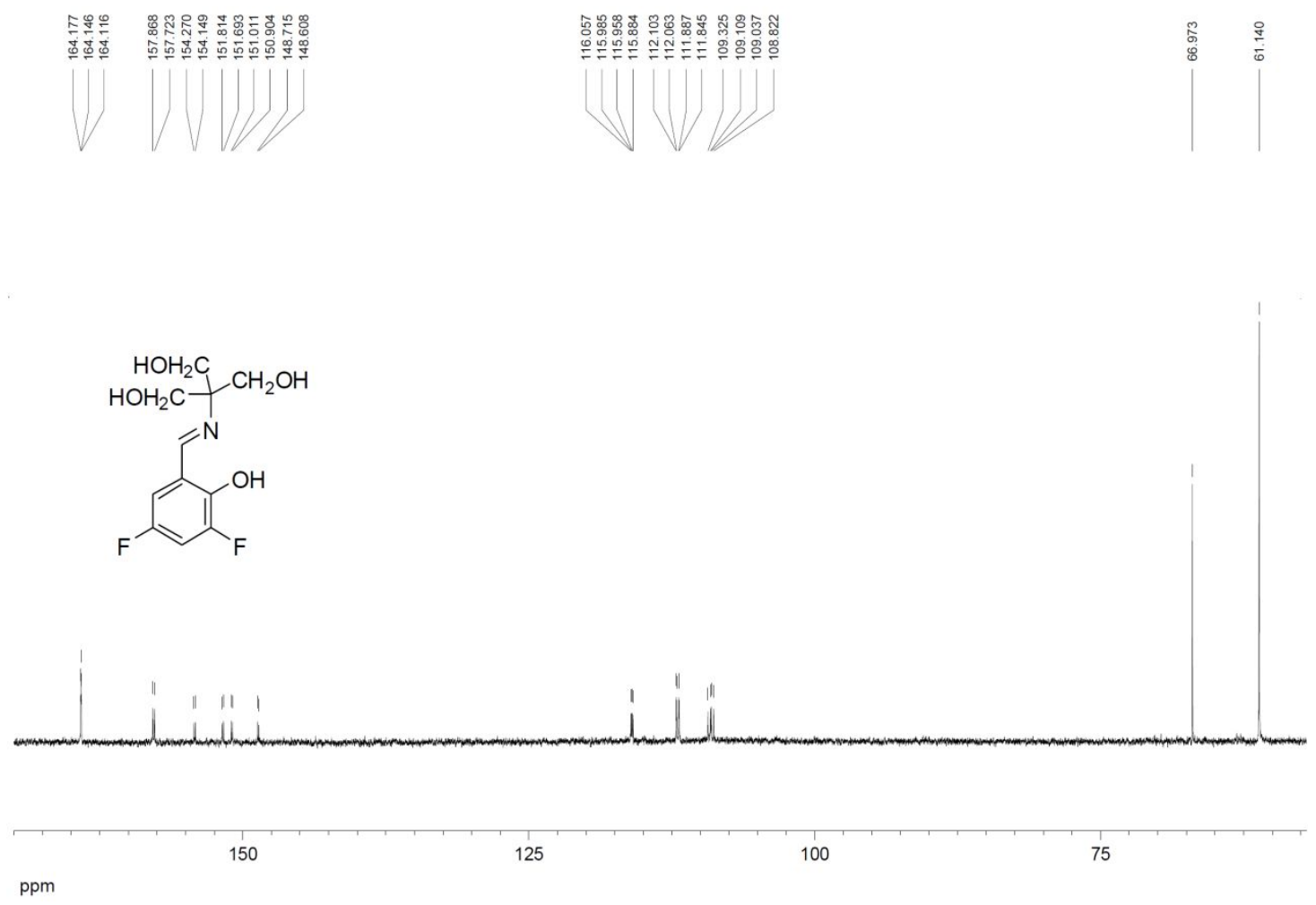

Figure 84S. ${ }^{13} \mathrm{C}\left\{{ }^{1} \mathrm{H}\right\}$ NMR spectrum for compound 27/57 (100 MHz, DMSO- $\left.d_{6}\right)$. 


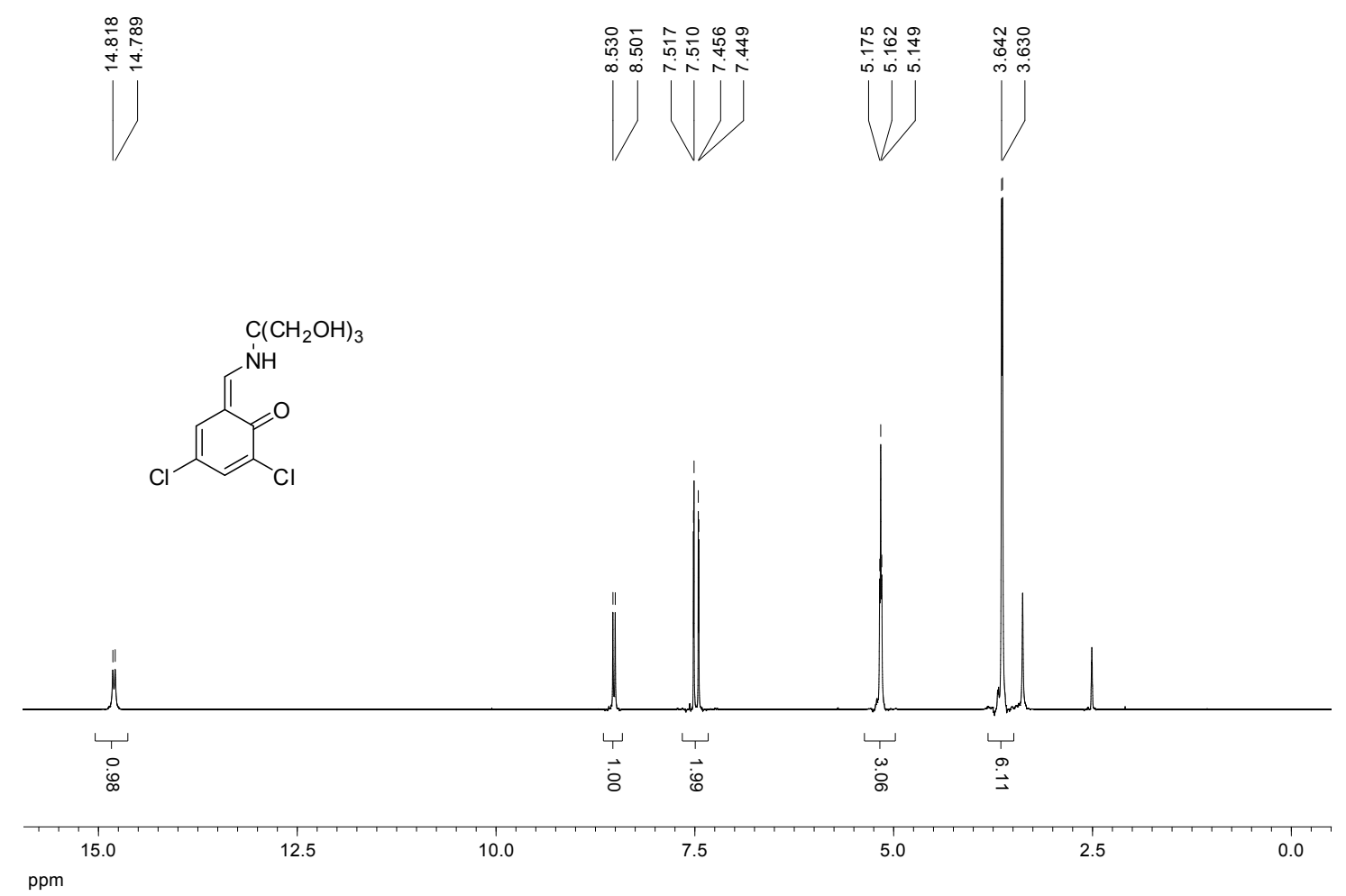

Figure 85S. ${ }^{1} \mathrm{H}$ NMR spectrum for compound 28/58 (400 MHz, DMSO- $\left.d_{6}\right)$.

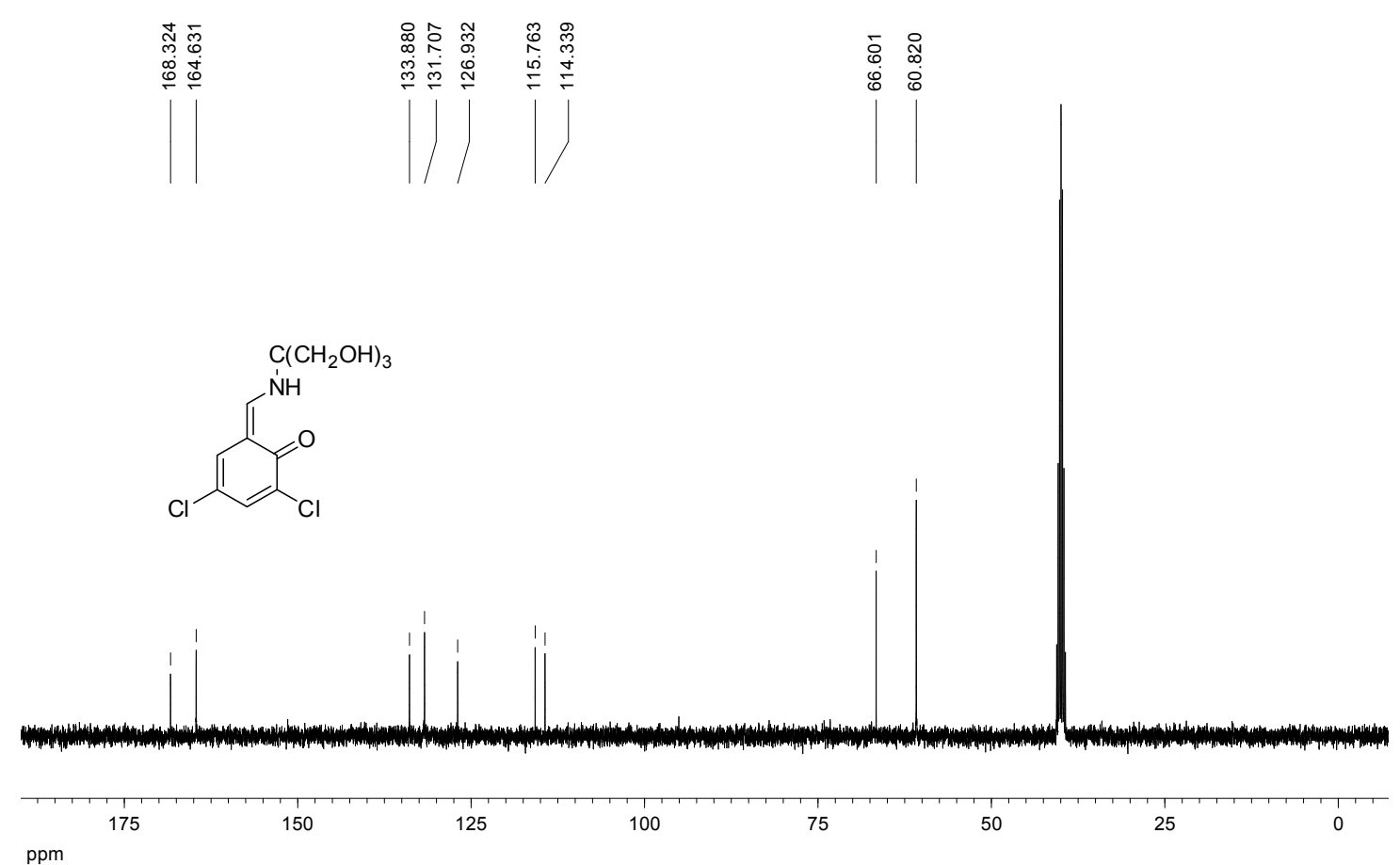

Figure 86S. ${ }^{13} \mathrm{C}\left\{{ }^{1} \mathrm{H}\right\}$ NMR spectrum for compound 28/58 (100 MHz, DMSO- $\left.d_{6}\right)$. 


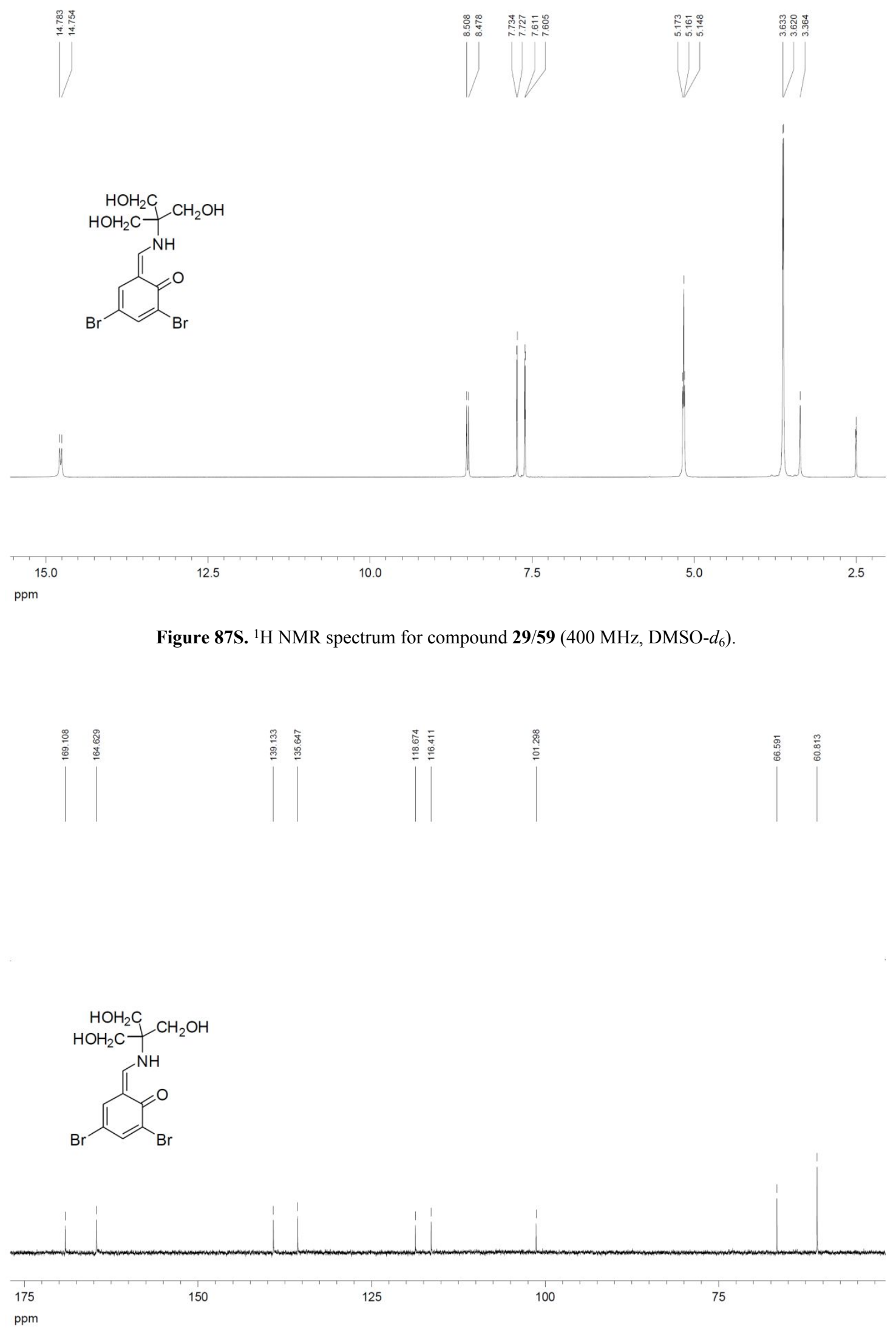

Figure 88S. ${ }^{13} \mathrm{C}\left\{{ }^{1} \mathrm{H}\right\}$ NMR spectrum for compound 29/59 (100 MHz, DMSO- $\left.d_{6}\right)$. 

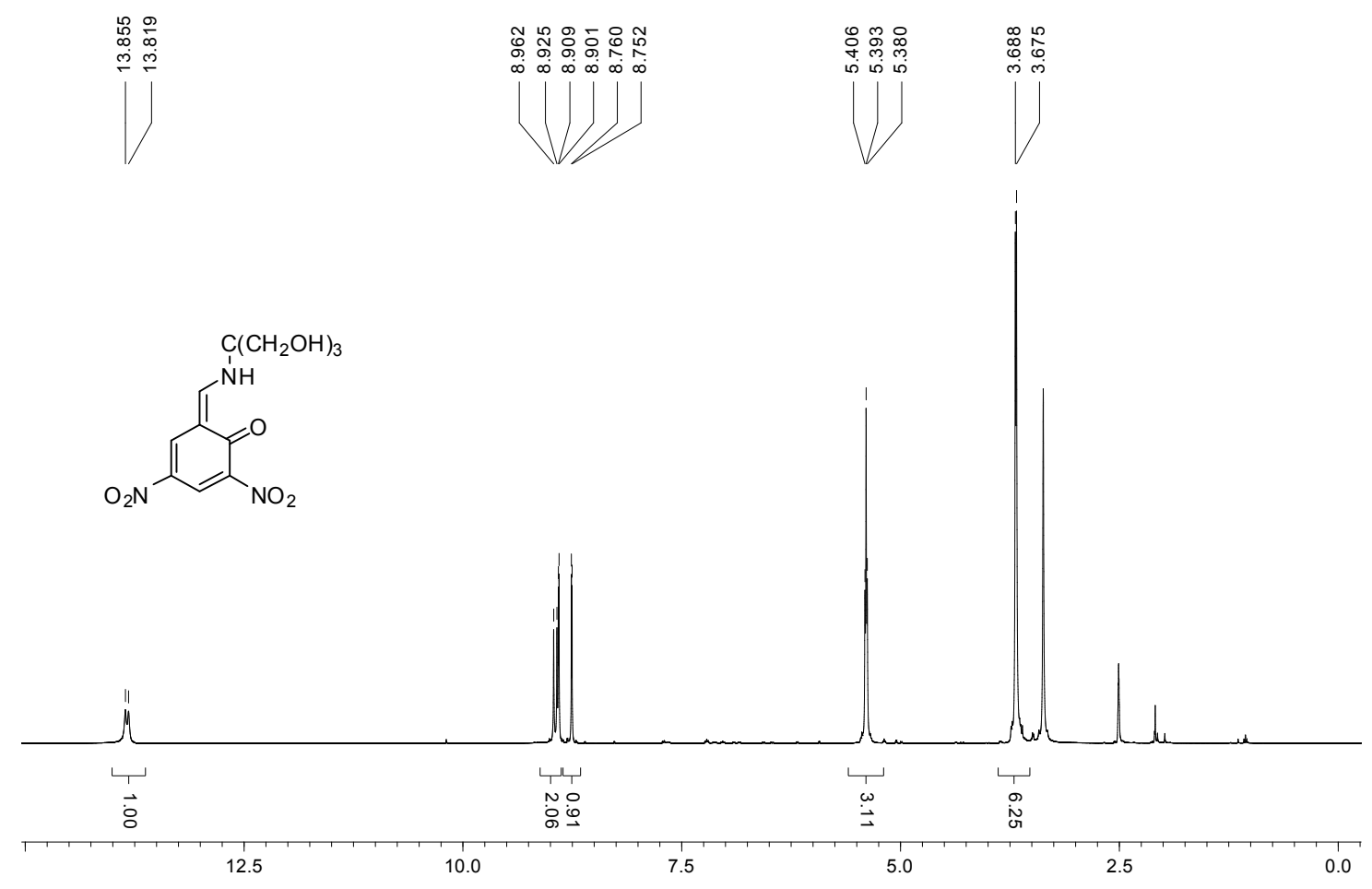

ppm

Figure 89S. ${ }^{1} \mathrm{H}$ NMR spectrum for compound 30/60 (400 MHz, DMSO- $d_{6}$ ).
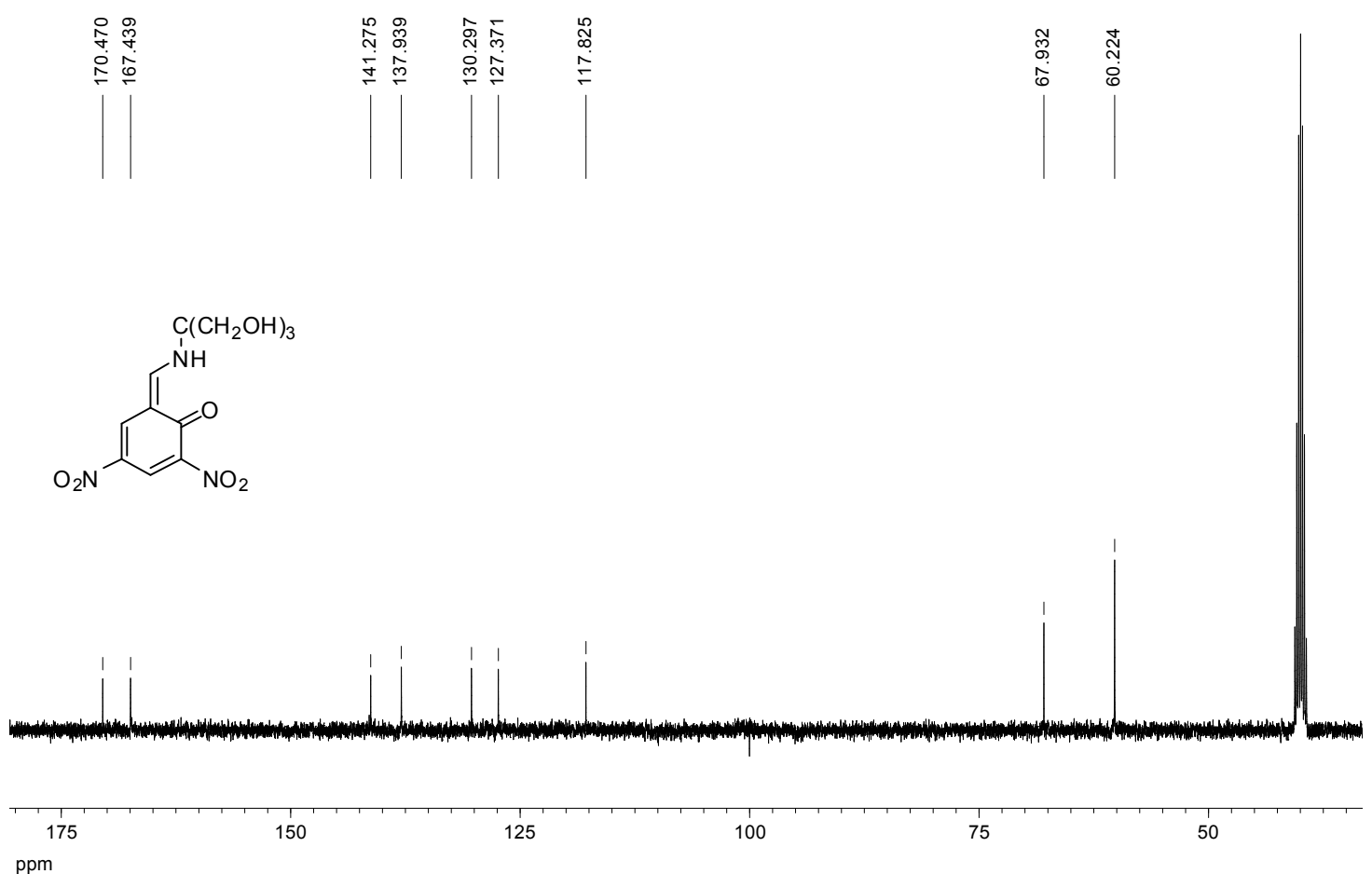

Figure 90S. ${ }^{13} \mathrm{C}\left\{{ }^{1} \mathrm{H}\right\}$ NMR spectrum for compound 30/60 (100 MHz, DMSO- $\left.d_{6}\right)$. 

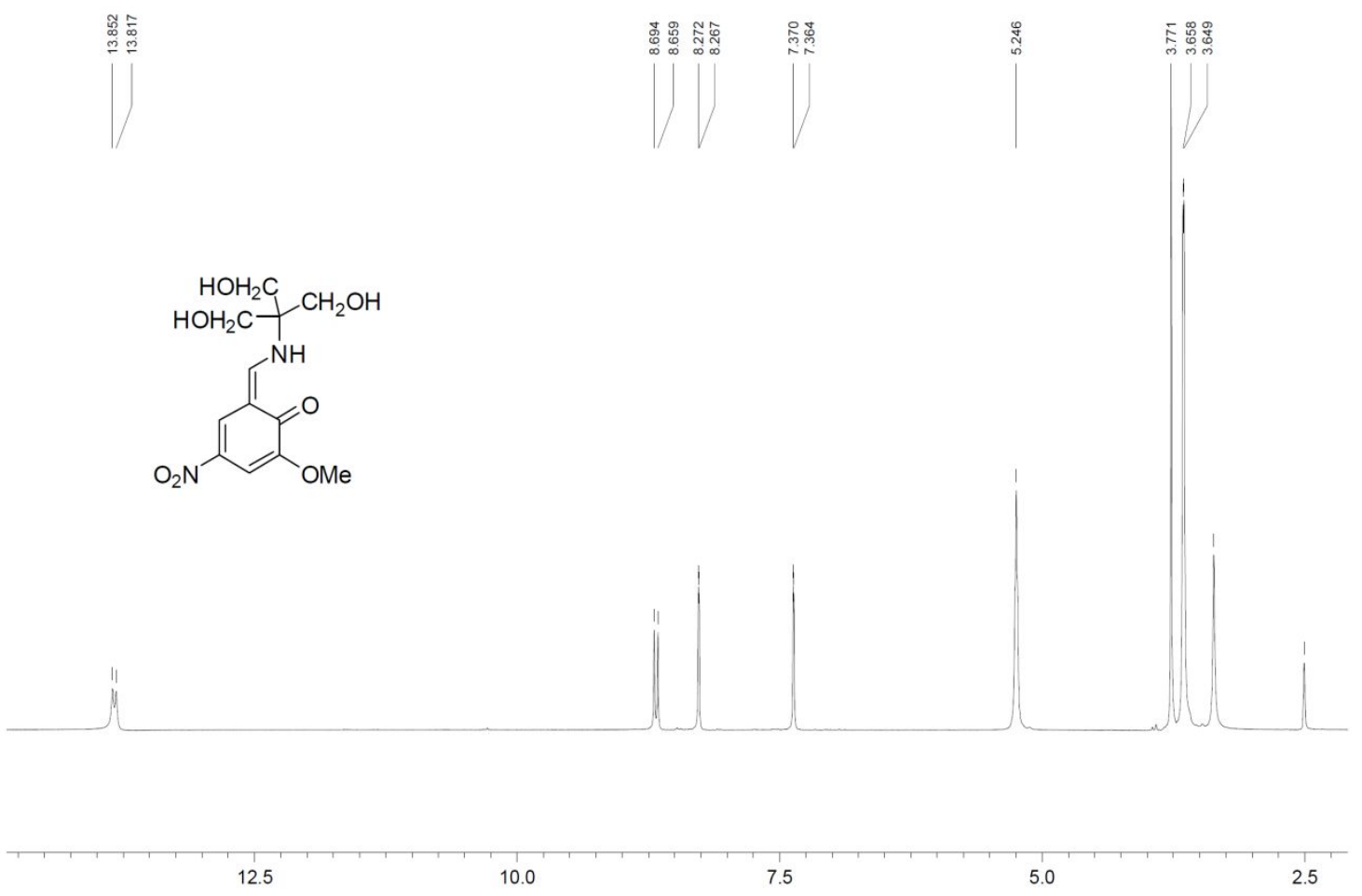

$\mathrm{ppm}$

Figure 91S. ${ }^{1} \mathrm{H}$ NMR spectrum for compound 31/61 (400 MHz, DMSO- $\left.d_{6}\right)$.
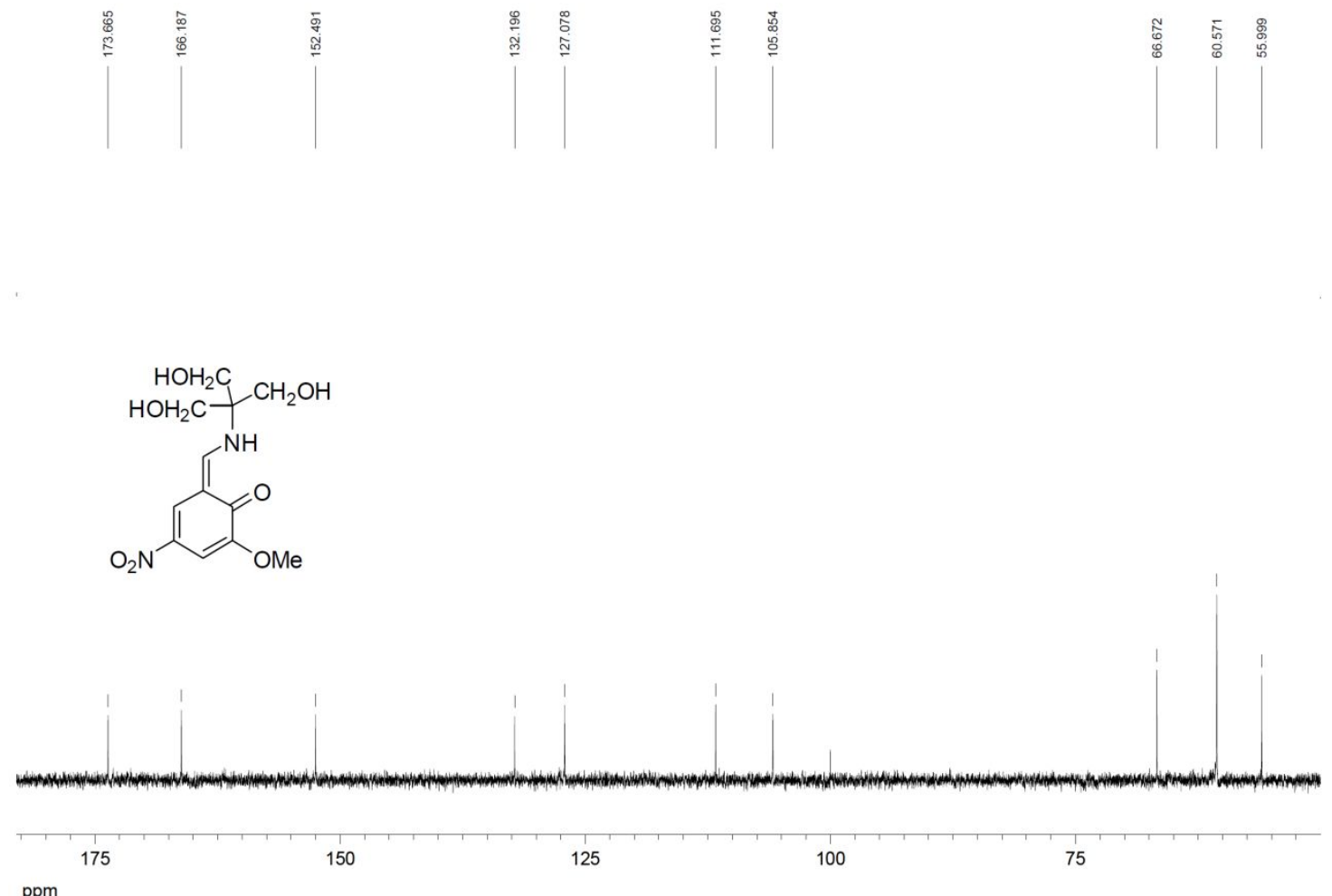

Figure 92S. ${ }^{13} \mathrm{C}\left\{{ }^{1} \mathrm{H}\right\}$ NMR spectrum for compound 31/61 (100 MHz, DMSO- $\left.d_{6}\right)$. 


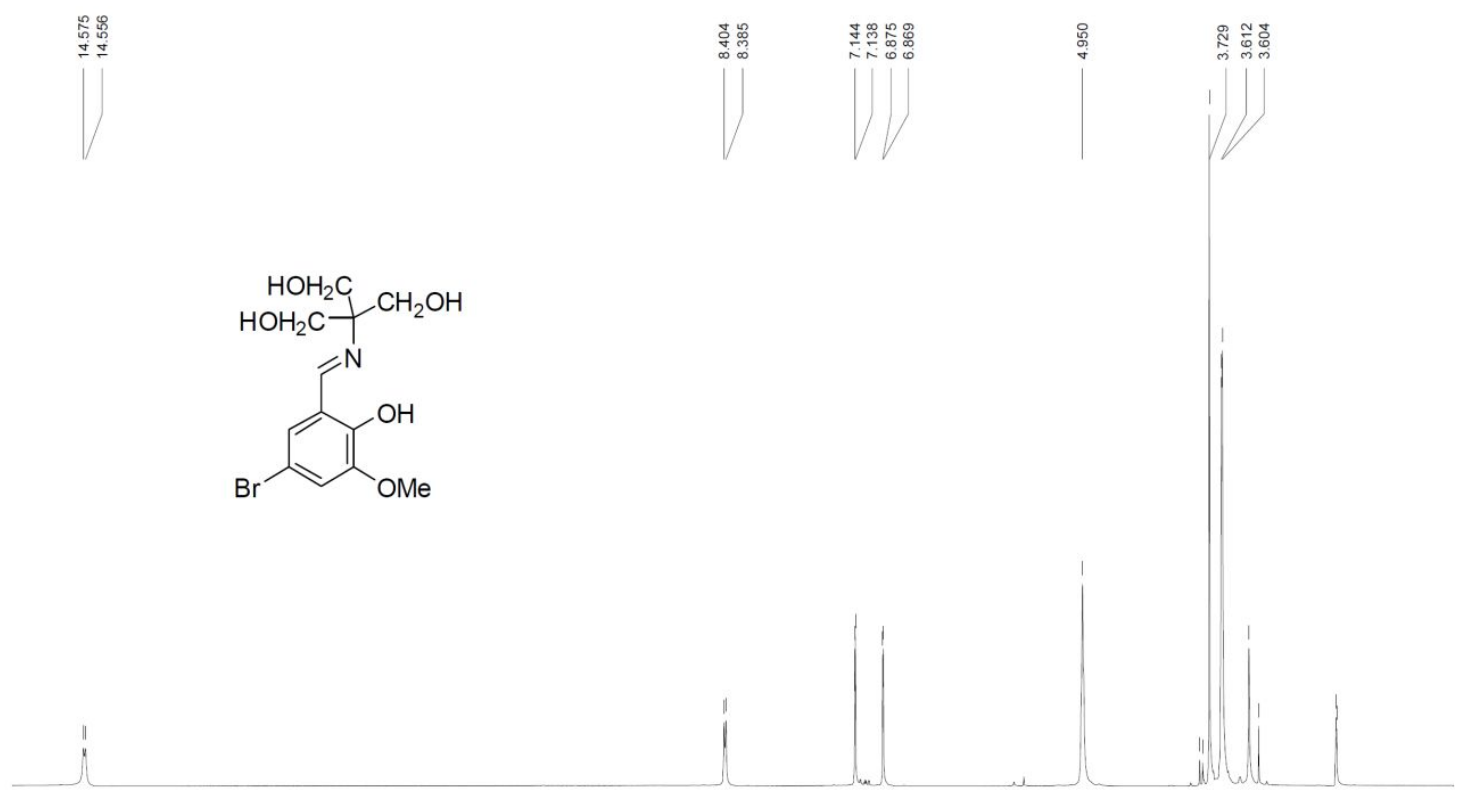

\begin{tabular}{|c|c|c|c|c|c|}
\hline 15.0 & 12.5 & 10.0 & 7.5 & 5.0 & 2.5 \\
\hline
\end{tabular}

Figure 93S. ${ }^{1} \mathrm{H}$ NMR spectrum for compound 32/62 (400 MHz, DMSO- $\left.d_{6}\right)$.
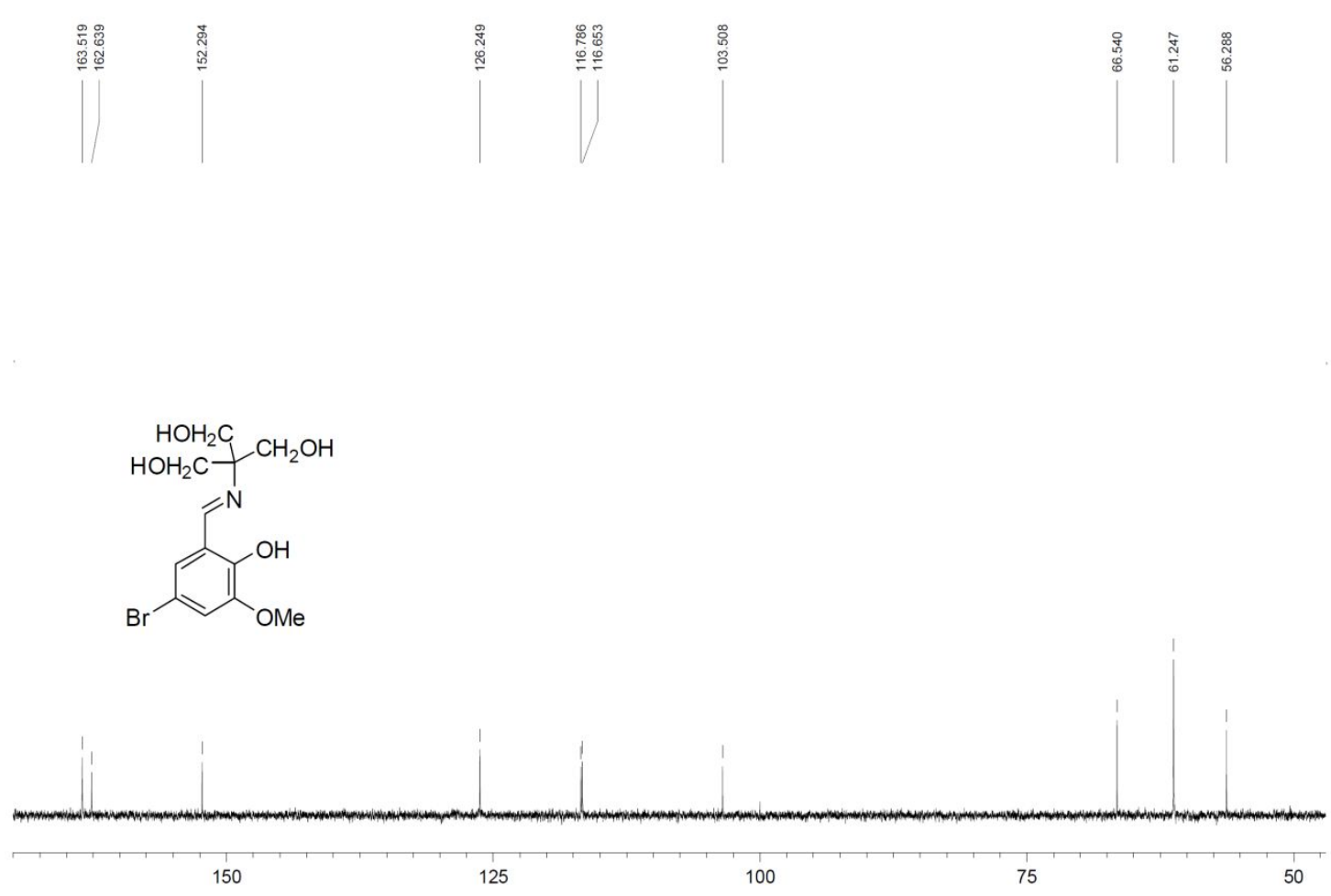

ppm

Figure 94S. ${ }^{13} \mathrm{C}\left\{{ }^{1} \mathrm{H}\right\}$ NMR spectrum for compound 32/62 (100 MHz, DMSO- $\left.d_{6}\right)$. 


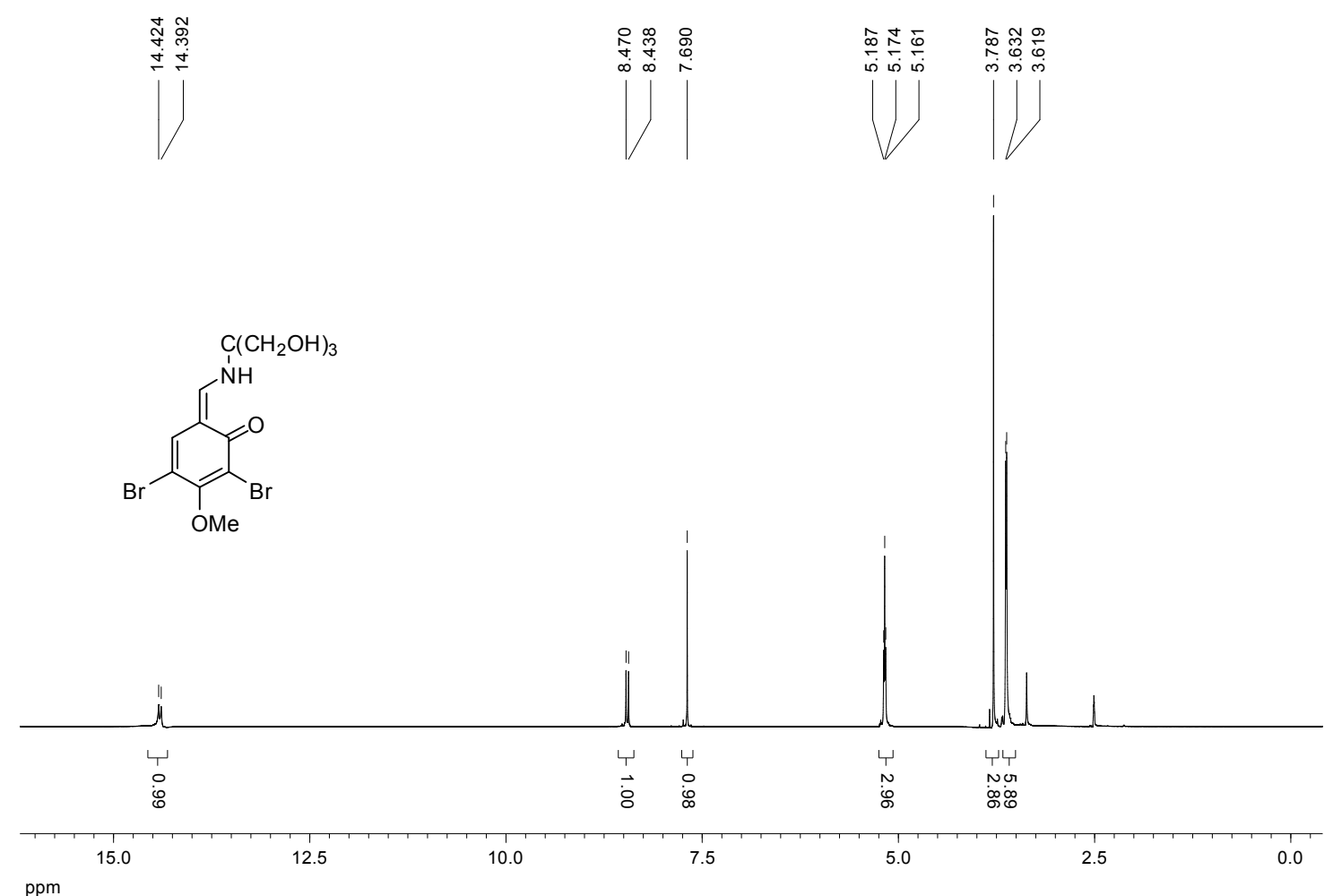

Figure 95S. ${ }^{1} \mathrm{H}$ NMR spectrum for compound 33/63 (400 MHz, DMSO- $d_{6}$ )
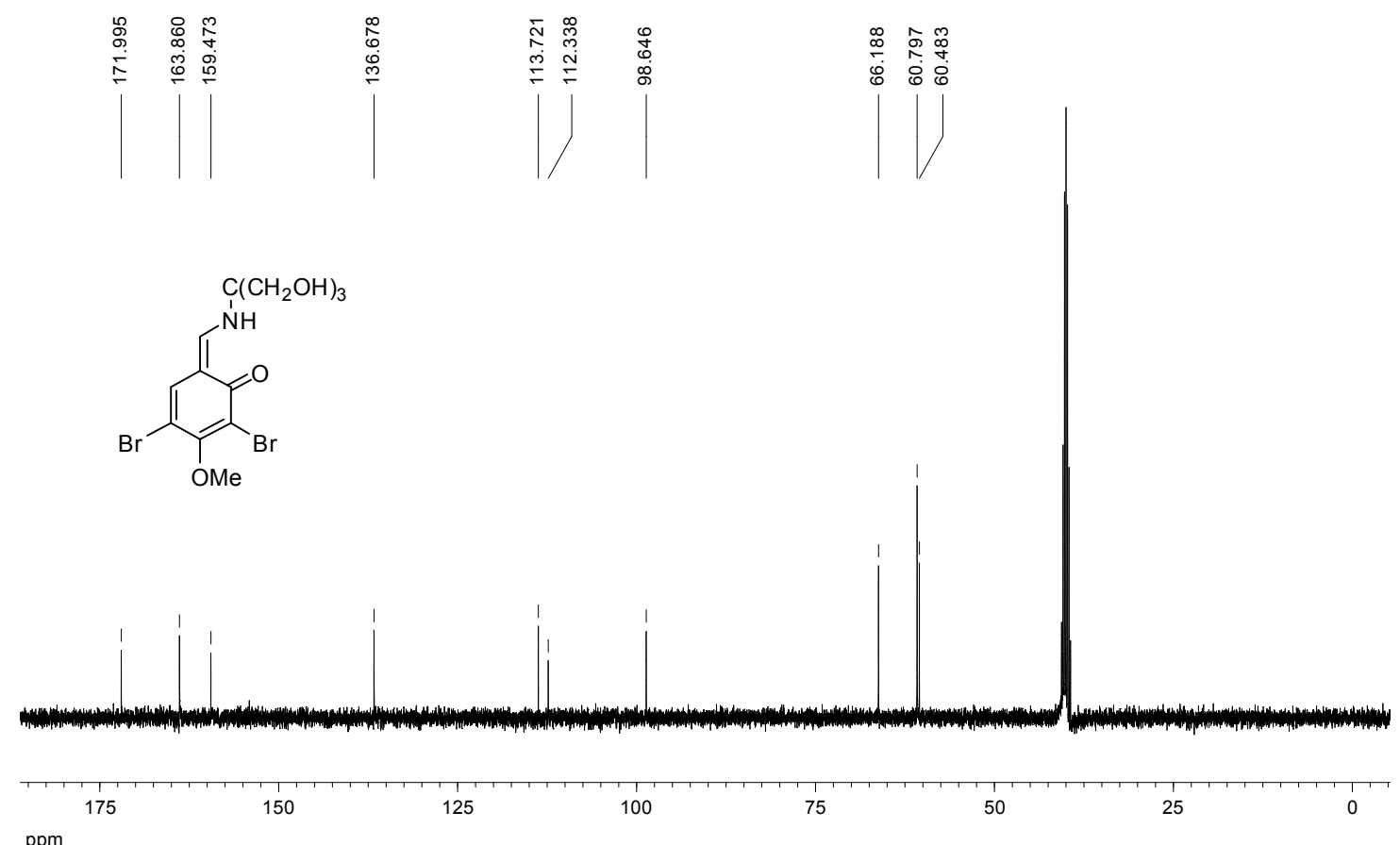

Figure 96S. ${ }^{13} \mathrm{C}\left\{{ }^{1} \mathrm{H}\right\}$ NMR spectrum for compound 33/63 (100 MHz, DMSO- $\left.d_{6}\right)$. 


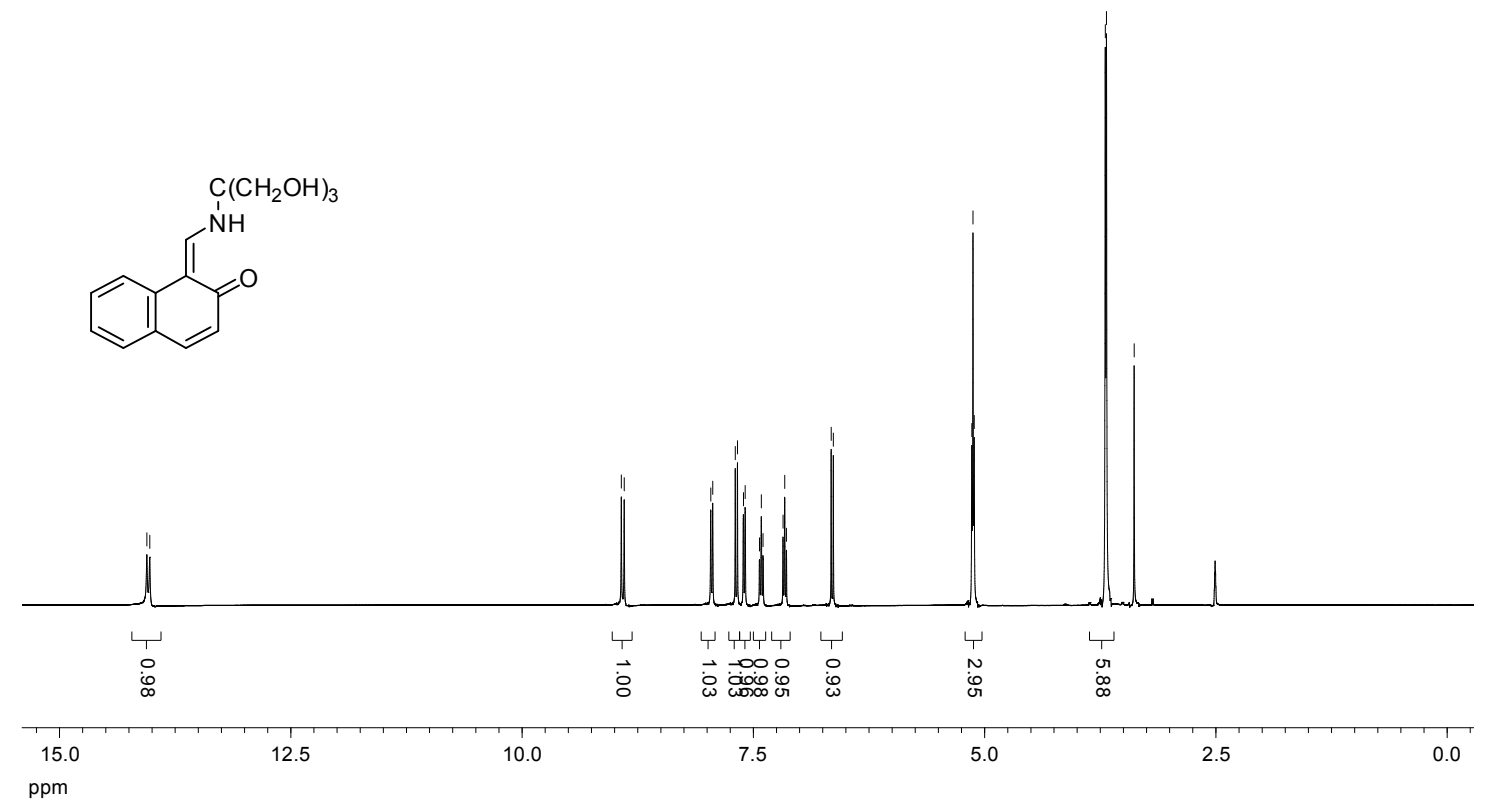

Figure 97S. ${ }^{1} \mathrm{H}$ NMR spectrum for compound 64/65 (400 MHz, DMSO- $\left.d_{6}\right)$.
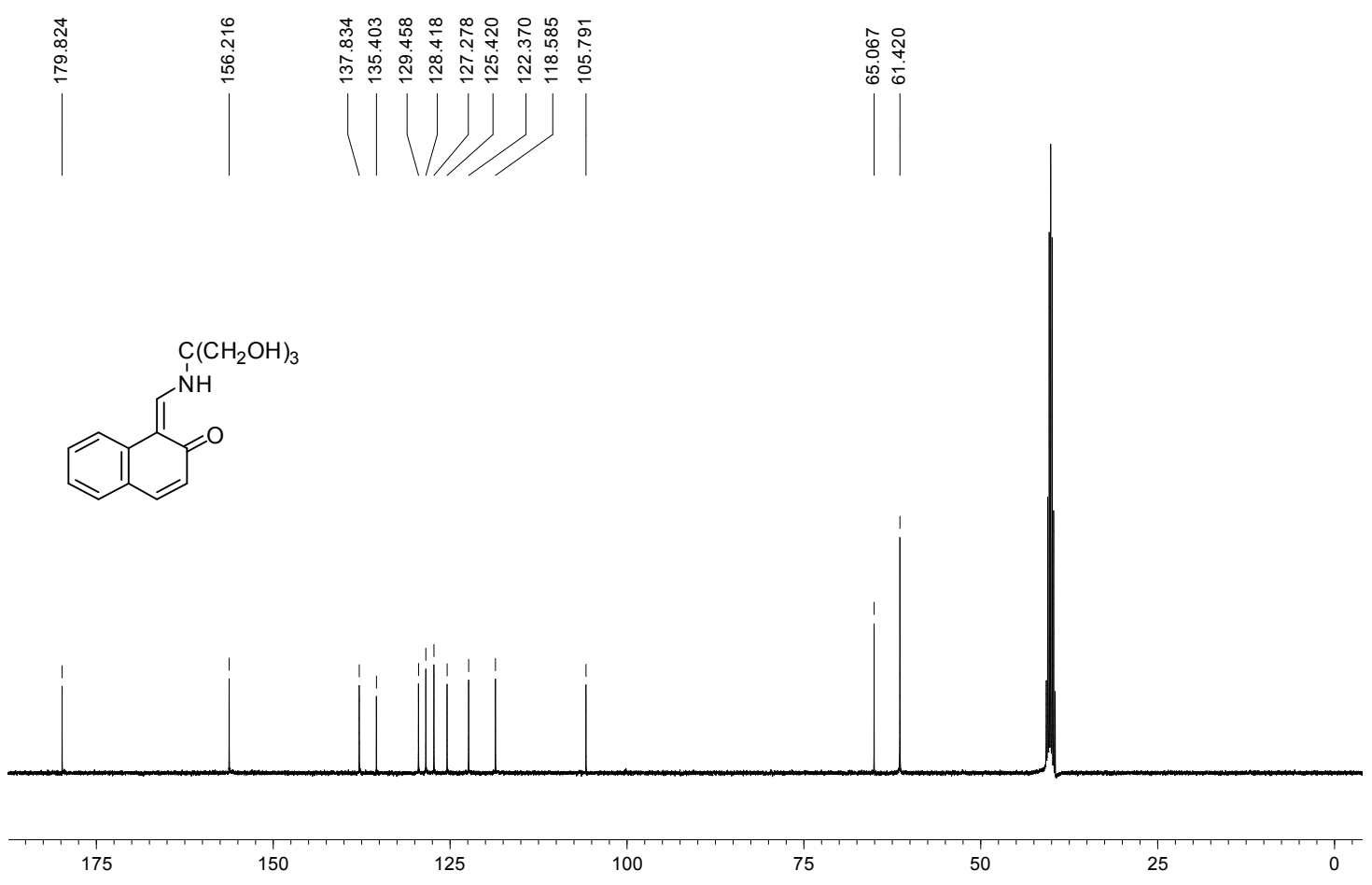

Figure 98S. ${ }^{13} \mathrm{C}\left\{{ }^{1} \mathrm{H}\right\}$ NMR spectrum for compound 64/65 (100 MHz, DMSO- $\left.d_{6}\right)$. 


\title{
Cartesian coordinates for all optimized structures.
}

\section{Computational calculations at the M06-2X/6-311++G(d,p) level in the gas phase.}

\author{
$\underline{\text { Structure of } 4 \text { (open form) }}$
}

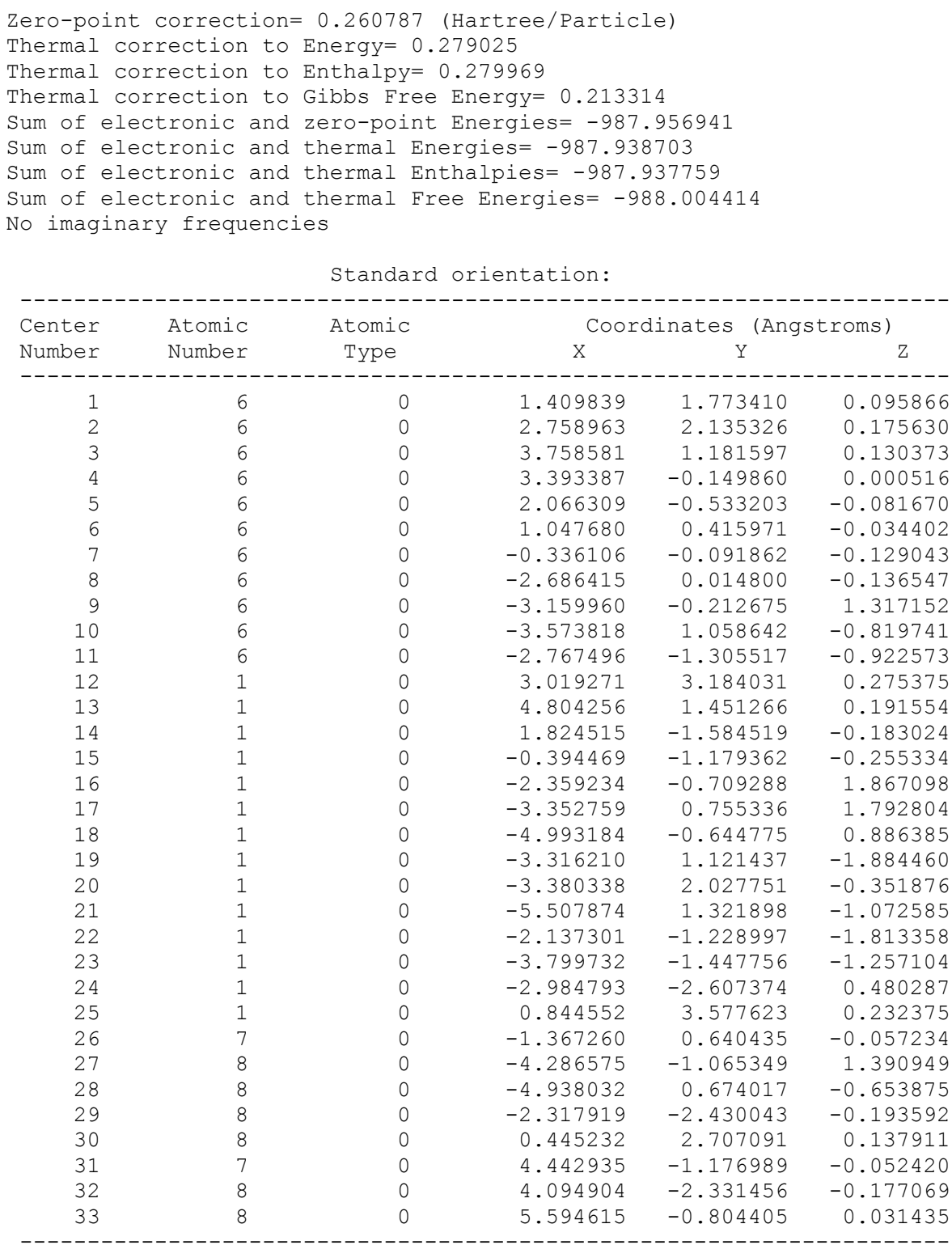

\section{$\underline{\text { Structure of } 4 \text { (closed form) }}$}

\footnotetext{
Zero-point correction $=0.261636$ (Hartree/Particle)

Thermal correction to Energy $=0.279255$

Thermal correction to Enthalpy $=0.280200$

Thermal correction to Gibbs Free Energy $=0.215322$

Sum of electronic and zero-point Energies $=-987.977589$

Sum of electronic and thermal Energies= -987.959970

Sum of electronic and thermal Enthalpies= -987.959026

Sum of electronic and thermal Free Energies= -988.023903

No imaginary frequencies
} 


\begin{tabular}{|c|c|c|c|c|c|}
\hline \multirow{2}{*}{$\begin{array}{l}\text { Center } \\
\text { Number }\end{array}$} & \multirow{2}{*}{$\begin{array}{l}\text { Atomic } \\
\text { Number }\end{array}$} & \multirow{2}{*}{$\begin{array}{c}\text { Atomic } \\
\text { Type }\end{array}$} & \multicolumn{3}{|c|}{ Coordinates (Angstroms) } \\
\hline & & & $\mathrm{X}$ & Y & Z \\
\hline & & & & & 0118360 \\
\hline 1 & 6 & 0 & 1.305728 & 1.674391 & 0.118360 \\
\hline 2 & 6 & 0 & 2.623482 & 2.142680 & 0.217802 \\
\hline 3 & 6 & 0 & 3.682376 & 1.261630 & 0.158412 \\
\hline 4 & 6 & 0 & 3.428145 & -0.100161 & -0.001372 \\
\hline 5 & 6 & 0 & 2.141410 & -0.591902 & -0.099107 \\
\hline 6 & 6 & 0 & 1.062499 & 0.288337 & -0.041353 \\
\hline 7 & 6 & 0 & -0.298330 & -0.240906 & -0.139981 \\
\hline 8 & 6 & 0 & -2.671018 & -0.006663 & -0.156212 \\
\hline 9 & 6 & 0 & -3.169941 & -0.115048 & 1.304295 \\
\hline 10 & 6 & 0 & -3.492440 & 1.035223 & -0.920046 \\
\hline 11 & 6 & 0 & -2.805324 & -1.368744 & -0.854497 \\
\hline 12 & 1 & 0 & 2.781401 & 3.206265 & 0.341485 \\
\hline 13 & 1 & 0 & 4.705775 & 1.604639 & 0.232523 \\
\hline 14 & 1 & 0 & 1.980576 & -1.656840 & -0.219330 \\
\hline 15 & 1 & 0 & -0.394412 & -1.326834 & -0.232915 \\
\hline 16 & 1 & 0 & -2.395995 & -0.604834 & 1.897068 \\
\hline 17 & 1 & 0 & -3.327901 & 0.890129 & 1.711855 \\
\hline 18 & 1 & 0 & -5.021804 & -0.491092 & 0.898141 \\
\hline 19 & 1 & 0 & -3.220909 & 1.014835 & -1.982795 \\
\hline 20 & 1 & 0 & -3.259473 & 2.025431 & -0.515510 \\
\hline 21 & 1 & 0 & -5.408272 & 1.366041 & -1.220436 \\
\hline 22 & 1 & 0 & -2.183569 & -1.372636 & -1.754440 \\
\hline 23 & 1 & 0 & -3.845560 & -1.494618 & -1.168255 \\
\hline 24 & 1 & 0 & -3.061575 & -2.582525 & 0.621534 \\
\hline 25 & 1 & 0 & -0.538208 & 2.051041 & 0.097931 \\
\hline 26 & 7 & 0 & -1.313271 & 0.532988 & -0.100238 \\
\hline 27 & 8 & 0 & -4.329061 & -0.914158 & 1.419215 \\
\hline 28 & 8 & 0 & -4.871664 & 0.730055 & -0.743025 \\
\hline 29 & 8 & 0 & -2.384620 & -2.452868 & -0.053161 \\
\hline 30 & 8 & 0 & 0.314245 & 2.555120 & 0.179028 \\
\hline 31 & 7 & 0 & 4.556059 & -1.036551 & -0.066610 \\
\hline 32 & 8 & 0 & 4.303281 & -2.213556 & -0.219493 \\
\hline 33 & 8 & 0 & 5.674180 & -0.576729 & 0.035345 \\
\hline
\end{tabular}

\section{$\underline{\text { Structure of } \mathbf{T S}} \underline{4}_{4 / 34}$}

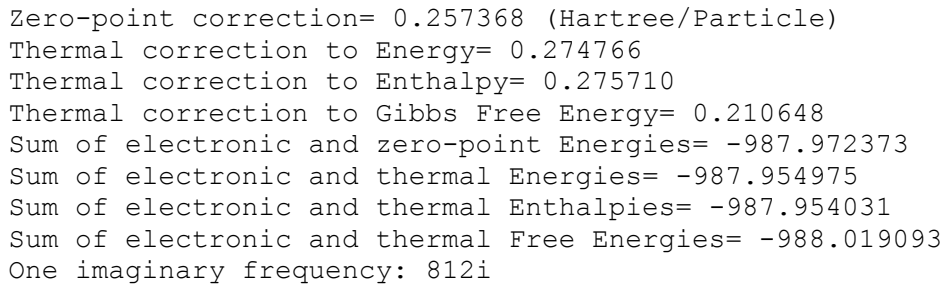

Standard orientation:

\begin{tabular}{|c|c|c|c|c|c|}
\hline \multirow{2}{*}{$\begin{array}{l}\text { Center } \\
\text { Number }\end{array}$} & \multirow{2}{*}{$\begin{array}{l}\text { Atomic } \\
\text { Number }\end{array}$} & \multirow{2}{*}{$\begin{array}{c}\text { Atomic } \\
\text { Type }\end{array}$} & \multicolumn{3}{|c|}{ Coordinates (Angstroms) } \\
\hline & & & $\mathrm{X}$ & Y & Z \\
\hline 1 & 6 & 0 & 1.197232 & 1.612631 & 0.092907 \\
\hline 2 & 6 & 0 & 2.520179 & 2.141019 & 0.200304 \\
\hline 3 & 6 & 0 & 3.610519 & 1.317839 & 0.158380 \\
\hline 4 & 6 & 0 & 3.444861 & -0.075762 & 0.008228 \\
\hline 5 & 6 & 0 & 2.200192 & -0.643921 & -0.095553 \\
\hline 6 & 6 & 0 & 1.069380 & 0.182082 & -0.056074 \\
\hline 7 & 6 & 0 & -0.242883 & -0.373040 & -0.150574 \\
\hline 8 & 6 & 0 & -2.663322 & -0.005668 & -0.145911 \\
\hline 9 & 6 & 0 & -3.134778 & -0.201884 & 1.314682 \\
\hline 10 & 6 & 0 & -3.434677 & 1.134624 & -0.814921 \\
\hline 11 & 6 & 0 & -2.868117 & -1.303238 & -0.941924 \\
\hline 12 & 1 & 0 & 2.625165 & 3.212262 & 0.315157 \\
\hline 13 & 1 & 0 & 4.615079 & 1.712854 & 0.239376 \\
\hline 14 & 1 & 0 & 2.104272 & -1.718319 & -0.205468 \\
\hline 15 & 1 & 0 & -0.386719 & -1.450172 & -0.241857 \\
\hline 16 & 1 & 0 & -2.366742 & -0.759801 & 1.852206 \\
\hline 17 & 1 & 0 & -3.247633 & 0.776072 & 1.796894 \\
\hline 18 & 1 & 0 & -5.013940 & -0.465835 & 0.952333 \\
\hline
\end{tabular}




$\begin{array}{rrrrrr}19 & 1 & 0 & -3.204637 & 1.159495 & -1.886995 \\ 20 & 1 & 0 & -3.123376 & 2.084689 & -0.366122 \\ 21 & 1 & 0 & -5.334296 & 1.604821 & -0.999177 \\ 22 & 1 & 0 & -2.269129 & -1.259047 & -1.855774 \\ 23 & 1 & 0 & -3.920869 & -1.365205 & -1.231165 \\ 24 & 1 & 0 & -3.134763 & -2.623234 & 0.439124 \\ 25 & 1 & 0 & -0.853187 & 1.499261 & 0.015136 \\ 26 & 7 & 0 & -1.267879 & 0.422698 & -0.112992 \\ 27 & 8 & 0 & -4.318144 & -0.965751 & 1.394952 \\ 28 & 8 & 0 & -4.819432 & 0.903073 & -0.594864 \\ 29 & 8 & 0 & -2.464802 & -2.454982 & -0.234223 \\ 30 & 8 & 0 & 0.163632 & 2.361167 & 0.127831 \\ 31 & 7 & 0 & 4.625193 & -0.935064 & -0.032494 \\ 32 & 8 & 0 & 5.712914 & -0.403586 & 0.067860 \\ 33 & 8 & 0 & 4.453090 & -2.131094 & -0.164372 \\ - & 1 & 0 & -10--10\end{array}$

\section{$\underline{\text { Structure of } 34}$}

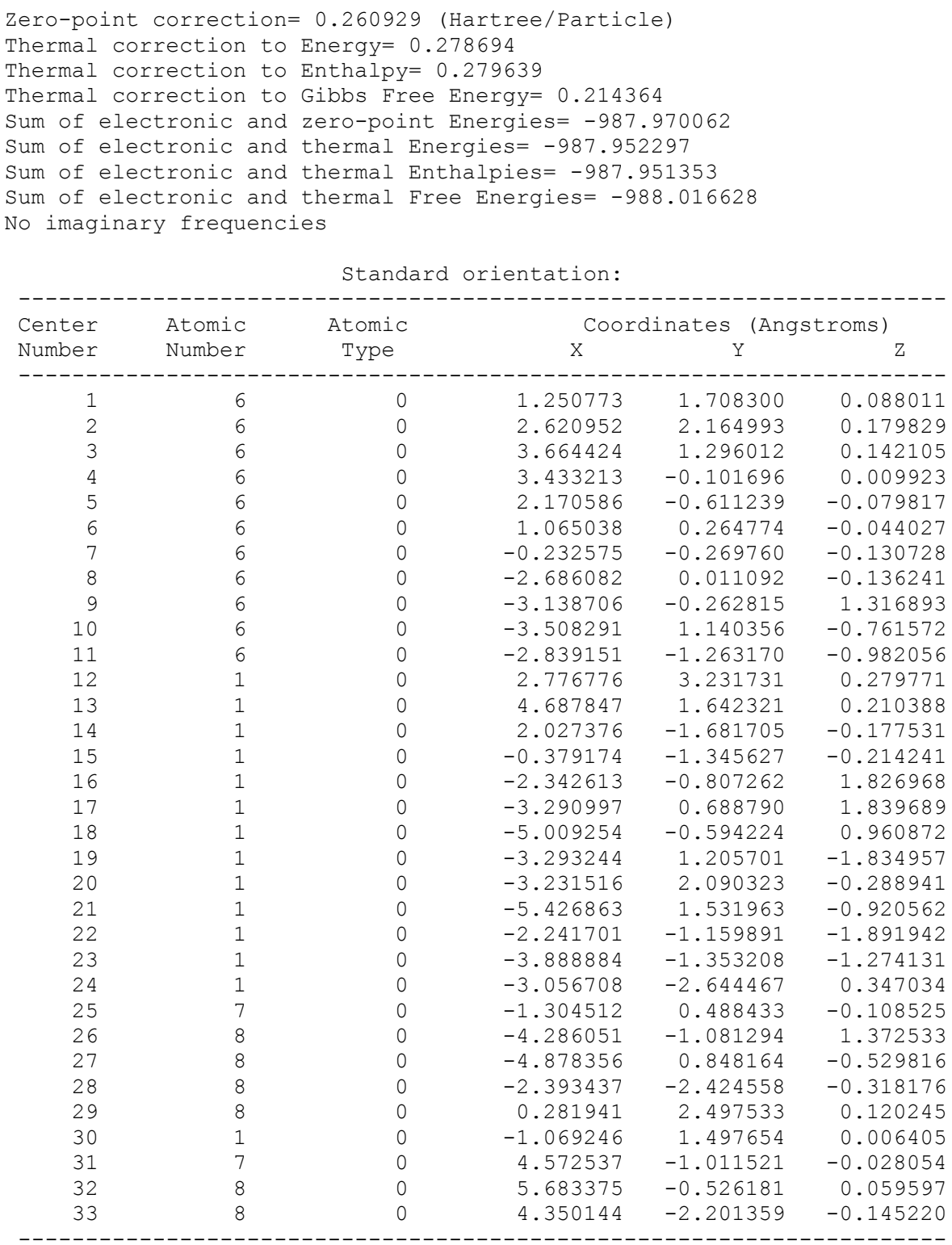

\section{$\underline{\text { Structure of } \mathbf{6} \text { (open form) }}$}

Zero-point correction= 0.249801 (Hartree/Particle) 
Thermal correction to Energy $=0.266296$

Thermal correction to Enthalpy $=0.267240$

Thermal correction to Gibbs Free Energy= 0.205406

Sum of electronic and zero-point Energies $=-882.716364$

Sum of electronic and thermal Energies $=-882.699869$

Sum of electronic and thermal Enthalpies $=-882.698925$

Sum of electronic and thermal Free Energies= -882.760758

No imaginary frequencies

\begin{tabular}{|c|c|c|c|c|c|}
\hline \multirow{2}{*}{$\begin{array}{l}\text { Center } \\
\text { Number }\end{array}$} & \multirow{2}{*}{$\begin{array}{l}\text { Atomic } \\
\text { Number }\end{array}$} & \multirow{2}{*}{$\begin{array}{c}\text { Atomic } \\
\text { Type }\end{array}$} & \multicolumn{3}{|c|}{ Coordinates (Angstroms) } \\
\hline & & & $\mathrm{X}$ & $\mathrm{Y}$ & Z \\
\hline 1 & 6 & 0 & 2.142662 & 1.336543 & 0.087161 \\
\hline 2 & 6 & 0 & 3.523286 & 1.505796 & 0.188197 \\
\hline 3 & 6 & 0 & 4.389513 & 0.421971 & 0.136625 \\
\hline 4 & 6 & 0 & 3.854139 & -0.841598 & -0.023053 \\
\hline 5 & 6 & 0 & 2.493828 & -1.038209 & -0.128405 \\
\hline 6 & 6 & 0 & 1.609726 & 0.043855 & -0.069395 \\
\hline 7 & 6 & 0 & 0.173271 & -0.277239 & -0.178360 \\
\hline 8 & 6 & 0 & -2.146390 & 0.110882 & -0.119059 \\
\hline 9 & 6 & 0 & -2.631496 & -0.143263 & 1.325643 \\
\hline 10 & 6 & 0 & -2.901866 & 1.296295 & -0.725748 \\
\hline 11 & 6 & 0 & -2.400370 & -1.139120 & -0.979406 \\
\hline 12 & 1 & 0 & 3.926551 & 2.506412 & 0.308402 \\
\hline 13 & 1 & 0 & 5.461535 & 0.549978 & 0.215604 \\
\hline 14 & 1 & 0 & 2.110723 & -2.044811 & -0.252337 \\
\hline 15 & 1 & 0 & -0.023689 & -1.335134 & -0.388791 \\
\hline 16 & 1 & 0 & -1.897430 & -0.773830 & 1.829460 \\
\hline 17 & 1 & 0 & -2.690000 & 0.809975 & 1.862643 \\
\hline 18 & 1 & 0 & -4.507588 & -0.305796 & 0.895841 \\
\hline 19 & 1 & 0 & -2.642092 & 1.394495 & -1.787206 \\
\hline 20 & 1 & 0 & -2.587739 & 2.201902 & -0.199962 \\
\hline 21 & 1 & 0 & -4.787943 & 1.820998 & -0.926333 \\
\hline 22 & 1 & 0 & -1.773090 & -1.090157 & -1.874022 \\
\hline 23 & 1 & 0 & -3.445266 & -1.129818 & -1.304824 \\
\hline 24 & 1 & 0 & -2.769456 & -2.482781 & 0.350229 \\
\hline 25 & 1 & 0 & 1.821947 & 3.202761 & 0.234498 \\
\hline 26 & 7 & 0 & -0.760560 & 0.565175 & -0.021890 \\
\hline 27 & 8 & 0 & -3.861264 & -0.843924 & 1.368393 \\
\hline 28 & 8 & 0 & -4.303768 & 1.073783 & -0.570241 \\
\hline 29 & 8 & 0 & -2.093094 & -2.354867 & -0.325295 \\
\hline 30 & 8 & 0 & 1.304793 & 2.399466 & 0.128193 \\
\hline 31 & 9 & 0 & 4.679591 & -1.903505 & -0.078175 \\
\hline
\end{tabular}

\section{$\underline{\text { Structure of } 6 \text { (closed form) }}$}

Zero-point correction $=0.250176$ (Hartree/Particle)

Thermal correction to Energy $=0.266266$

Thermal correction to Enthalpy $=0.267210$

Thermal correction to Gibbs Free Energy= 0.206255

Sum of electronic and zero-point Energies $=-882.736296$

Sum of electronic and thermal Energies= -882.720207

Sum of electronic and thermal Enthalpies= -882.719263

Sum of electronic and thermal Free Energies= -882.780218

No imaginary frequencies

Standard orientation:

\begin{tabular}{|c|c|c|c|c|c|}
\hline \multirow{2}{*}{$\begin{array}{l}\text { Center } \\
\text { Number }\end{array}$} & \multirow{2}{*}{$\begin{array}{l}\text { Atomic } \\
\text { Number }\end{array}$} & \multirow{2}{*}{$\begin{array}{c}\text { Atomic } \\
\text { Type }\end{array}$} & \multicolumn{3}{|c|}{ Coordinates (Angstroms) } \\
\hline & & & $\mathrm{X}$ & $\mathrm{Y}$ & Z \\
\hline 1 & 6 & 0 & 2.034988 & 1.288706 & 0.137497 \\
\hline 2 & 6 & 0 & 3.398416 & 1.583398 & 0.228759 \\
\hline 3 & 6 & 0 & 4.343862 & 0.577497 & 0.131575 \\
\hline 4 & 6 & 0 & 3.925525 & -0.733141 & -0.057541 \\
\hline 5 & 6 & 0 & 2.593656 & -1.057985 & -0.150934 \\
\hline 6 & 6 & 0 & 1.626731 & -0.047059 & -0.055143 \\
\hline 7 & 6 & 0 & 0.213890 & -0.410457 & -0.155971 \\
\hline 8 & 6 & 0 & -2.117623 & 0.078469 & -0.151046 \\
\hline 9 & 6 & 0 & -2.630352 & -0.010014 & 1.305340 \\
\hline 10 & 6 & 0 & -2.818633 & 1.223288 & -0.887547 \\
\hline
\end{tabular}




$\begin{array}{rrrrrr}11 & 6 & 0 & -2.401845 & -1.241355 & -0.885640 \\ 12 & 1 & 0 & 3.690736 & 2.614976 & 0.378155 \\ 13 & 1 & 0 & 5.403265 & 0.791088 & 0.200976 \\ 14 & 1 & 0 & 2.298779 & -2.090974 & -0.297134 \\ 15 & 1 & 0 & -0.006194 & -1.474595 & -0.286337 \\ 16 & 1 & 0 & -1.918255 & -0.595632 & 1.888389 \\ 17 & 1 & 0 & -2.679041 & 0.996305 & 1.736664 \\ 18 & 1 & 0 & -4.511164 & -0.168171 & 0.889077 \\ 19 & 1 & 0 & -2.550982 & 1.198048 & -1.951207 \\ 20 & 1 & 0 & -2.474941 & 2.170510 & -0.460291 \\ 21 & 1 & 0 & -4.683119 & 1.785960 & -1.164145 \\ 22 & 1 & 0 & -1.773175 & -1.294625 & -1.779045 \\ 23 & 1 & 0 & -3.445712 & -1.236087 & -1.213194 \\ 24 & 1 & 0 & -2.809943 & -2.439602 & 0.565801 \\ 25 & 1 & 0 & 0.254090 & 1.909491 & 0.160304 \\ 26 & 7 & 0 & -0.710717 & 0.467973 & -0.081686 \\ 27 & 8 & 0 & -3.872690 & -0.678739 & 1.400778 \\ 28 & 8 & 0 & -4.224853 & 1.071998 & -0.715998 \\ 29 & 8 & 0 & -2.123914 & -2.389145 & -0.110510 \\ 30 & 8 & 0 & 1.158056 & 2.296488 & 0.238919 \\ 31 & 9 & 0 & 4.852909 & -1.706158 & -0.150868 \\ ------------------------------------------------------\end{array}$

\section{$\underline{\text { Structure of } \mathbf{T S}_{\mathbf{6} / 36}}$}

Zero-point correction= 0.246466 (Hartree/Particle)
Thermal correction to Energy= 0.262067
Thermal correction to Enthalpy= 0.263011
Thermal correction to Gibbs Free Energy= 0.203198
Sum of electronic and zero-point Energies=-882.727249
Sum of electronic and thermal Energies=-882.711648
Sum of electronic and thermal Enthalpies= -882.710704
Sum of electronic and thermal Free Energies= -882.770517
One imaginary frequency: $666 i$

Standard orientation:

\begin{tabular}{|c|c|c|c|c|c|}
\hline \multirow{2}{*}{$\begin{array}{l}\text { Center } \\
\text { Number }\end{array}$} & \multirow{2}{*}{$\begin{array}{l}\text { Atomic } \\
\text { Number }\end{array}$} & \multirow{2}{*}{$\begin{array}{c}\text { Atomic } \\
\text { Type }\end{array}$} & \multicolumn{3}{|c|}{ Coordinates (Angstroms) } \\
\hline & & & $\mathrm{X}$ & Y & Z \\
\hline 1 & 6 & 0 & 1.917305 & 1.271401 & 0.088606 \\
\hline 2 & 6 & 0 & 3.296185 & 1.642516 & 0.160770 \\
\hline 3 & 6 & 0 & 4.283740 & 0.695117 & 0.116383 \\
\hline 4 & 6 & 0 & 3.960334 & -0.670379 & -0.002037 \\
\hline 5 & 6 & 0 & 2.671726 & -1.095448 & -0.076813 \\
\hline 6 & 6 & 0 & 1.630751 & -0.134569 & -0.033515 \\
\hline 7 & 6 & 0 & 0.273429 & -0.535631 & -0.113398 \\
\hline 8 & 6 & 0 & -2.098044 & 0.084582 & -0.126738 \\
\hline 9 & 6 & 0 & -2.590382 & -0.196794 & 1.311296 \\
\hline 10 & 6 & 0 & -2.750213 & 1.350897 & -0.685935 \\
\hline 11 & 6 & 0 & -2.433927 & -1.109493 & -1.036027 \\
\hline 12 & 1 & 0 & 3.527868 & 2.696163 & 0.252095 \\
\hline 13 & 1 & 0 & 5.330367 & 0.972802 & 0.170726 \\
\hline 14 & 1 & 0 & 2.448038 & -2.152441 & -0.169296 \\
\hline 15 & 1 & 0 & 0.004138 & -1.588266 & -0.203904 \\
\hline 16 & 1 & 0 & -1.891024 & -0.888025 & 1.784242 \\
\hline 17 & 1 & 0 & -2.591419 & 0.735566 & 1.887694 \\
\hline 18 & 1 & 0 & -4.483180 & -0.219651 & 0.923550 \\
\hline 19 & 1 & 0 & -2.516364 & 1.447320 & -1.753075 \\
\hline 20 & 1 & 0 & -2.345332 & 2.221181 & -0.157346 \\
\hline 21 & 1 & 0 & -4.590143 & 2.029425 & -0.817557 \\
\hline 22 & 1 & 0 & -1.816307 & -1.053174 & -1.936269 \\
\hline 23 & 1 & 0 & -3.482422 & -1.029789 & -1.336896 \\
\hline 24 & 1 & 0 & -2.867088 & -2.501904 & 0.225044 \\
\hline 25 & 1 & 0 & -0.164115 & 1.379089 & 0.034265 \\
\hline 26 & 7 & 0 & -0.668433 & 0.367647 & -0.077973 \\
\hline 27 & 8 & 0 & -3.854357 & -0.827228 & 1.330739 \\
\hline 28 & 8 & 0 & -4.153238 & 1.245285 & -0.478764 \\
\hline 29 & 8 & 0 & -2.174628 & -2.358386 & -0.431049 \\
\hline 30 & 8 & 0 & 0.979119 & 2.141419 & 0.128629 \\
\hline 31 & 9 & 0 & 4.975046 & -1.560415 & -0.041049 \\
\hline
\end{tabular}




\section{$\underline{\text { Structure of } 36}$}

Zero-point correction= 0.249743 (Hartree/Particle)

Thermal correction to Energy $=0.265772$

Thermal correction to Enthalpy $=0.266716$

Thermal correction to Gibbs Free Energy $=0.205936$

Sum of electronic and zero-point Energies $=-882.724693$

Sum of electronic and thermal Energies $=-882.708664$

Sum of electronic and thermal Enthalpies= -882.707720

Sum of electronic and thermal Free Energies $=-882.768500$

No imaginary frequencies

Standard orientation:

\begin{tabular}{|c|c|c|c|c|c|}
\hline \multirow{2}{*}{$\begin{array}{l}\text { Center } \\
\text { Number }\end{array}$} & \multirow{2}{*}{$\begin{array}{l}\text { Atomic } \\
\text { Number }\end{array}$} & \multirow{2}{*}{$\begin{array}{c}\text { Atomic } \\
\text { Type }\end{array}$} & \multicolumn{3}{|c|}{ Coordinates (Angstroms) } \\
\hline & & & $\mathrm{X}$ & Y & Z \\
\hline 1 & 6 & 0 & 1.969973 & 1.322292 & 0.093870 \\
\hline 2 & 6 & 0 & 3.383096 & 1.626195 & 0.155581 \\
\hline 3 & 6 & 0 & 4.322559 & 0.642858 & 0.107153 \\
\hline 4 & 6 & 0 & 3.939041 & -0.718127 & -0.006619 \\
\hline 5 & 6 & 0 & 2.641750 & -1.090916 & -0.072356 \\
\hline 6 & 6 & 0 & 1.626307 & -0.086760 & -0.025389 \\
\hline 7 & 6 & 0 & 0.282163 & -0.462555 & -0.103568 \\
\hline 8 & 6 & 0 & -2.124102 & 0.099020 & -0.122132 \\
\hline 9 & 6 & 0 & -2.614807 & -0.205576 & 1.311071 \\
\hline 10 & 6 & 0 & -2.810785 & 1.347029 & -0.681223 \\
\hline 11 & 6 & 0 & -2.420022 & -1.098922 & -1.040794 \\
\hline 12 & 1 & 0 & 3.658367 & 2.669784 & 0.242756 \\
\hline 13 & 1 & 0 & 5.381419 & 0.872276 & 0.153981 \\
\hline 14 & 1 & 0 & 2.377251 & -2.138797 & -0.161190 \\
\hline 15 & 1 & 0 & 0.006923 & -1.512099 & -0.194308 \\
\hline 16 & 1 & 0 & -1.895938 & -0.877395 & 1.782863 \\
\hline 17 & 1 & 0 & -2.646953 & 0.722679 & 1.893670 \\
\hline 18 & 1 & 0 & -4.505862 & -0.283917 & 0.919013 \\
\hline 19 & 1 & 0 & -2.579683 & 1.449813 & -1.748264 \\
\hline 20 & 1 & 0 & -2.432678 & 2.230109 & -0.152879 \\
\hline 21 & 1 & 0 & -4.668585 & 1.971902 & -0.815244 \\
\hline 22 & 1 & 0 & -1.797036 & -1.020697 & -1.935577 \\
\hline 23 & 1 & 0 & -3.467999 & -1.044160 & -1.348585 \\
\hline 24 & 1 & 0 & -2.826934 & -2.508337 & 0.209862 \\
\hline 25 & 7 & 0 & -0.701240 & 0.418365 & -0.074893 \\
\hline 26 & 8 & 0 & -3.858739 & -0.874963 & 1.321595 \\
\hline 27 & 8 & 0 & -4.209161 & 1.202471 & -0.472387 \\
\hline 28 & 8 & 0 & -2.133960 & -2.343701 & -0.440804 \\
\hline 29 & 8 & 0 & 1.097768 & 2.224756 & 0.139551 \\
\hline 30 & 1 & 0 & -0.349017 & 1.392619 & 0.041719 \\
\hline 31 & 9 & 0 & 4.924122 & -1.640897 & -0.048973 \\
\hline
\end{tabular}

\section{Structure of 7 (open form)}

Zero-point correction= 0.247973 (Hartree/Particle)

Thermal correction to Energy $=0.265000$

Thermal correction to Enthalpy $=0.265944$

Thermal correction to Gibbs Free Energy $=0.202401$

Sum of electronic and zero-point Energies $=-1243.078363$

Sum of electronic and thermal Energies= -1243.061336

Sum of electronic and thermal Enthalpies= -1243.060392

Sum of electronic and thermal Free Energies= -1243.123935

No imaginary frequencies

Standard orientation:

\begin{tabular}{|c|c|c|c|c|c|}
\hline \multirow{2}{*}{$\begin{array}{l}\text { Center } \\
\text { Number }\end{array}$} & \multirow{2}{*}{$\begin{array}{l}\text { Atomic } \\
\text { Number }\end{array}$} & \multirow{2}{*}{$\begin{array}{c}\text { Atomic } \\
\text { Type }\end{array}$} & \multicolumn{3}{|c|}{ Coordinates (Angstroms) } \\
\hline & & & $\mathrm{X}$ & Y & Z \\
\hline 1 & 6 & 0 & 1.687795 & 1.646592 & 0.092230 \\
\hline 2 & 6 & 0 & 3.047182 & 1.945879 & 0.180601 \\
\hline 3 & 6 & 0 & 4.009451 & 0.948124 & 0.135709 \\
\hline 4 & 6 & 0 & 3.606707 & -0.371536 & -0.004059 \\
\hline 5 & 6 & 0 & 2.262121 & -0.685254 & -0.096341 \\
\hline 6 & 6 & 0 & 1.279156 & 0.307671 & -0.046803 \\
\hline
\end{tabular}




\begin{tabular}{|c|c|c|c|c|c|}
\hline 7 & 6 & 0 & -0.121196 & -0.146694 & -0.149846 \\
\hline 8 & 6 & 0 & -2.466853 & 0.038852 & -0.126947 \\
\hline 9 & 6 & 0 & -2.933149 & -0.240118 & 1.319496 \\
\hline 10 & 6 & 0 & -3.323895 & 1.144600 & -0.748863 \\
\hline 11 & 6 & 0 & -2.604052 & -1.238447 & -0.974117 \\
\hline 12 & 1 & 0 & 3.356173 & 2.981019 & 0.287428 \\
\hline 13 & 1 & 0 & 5.061644 & 1.192033 & 0.207279 \\
\hline 14 & 1 & 0 & 1.959656 & -1.720593 & -0.206443 \\
\hline 15 & 1 & 0 & -0.221720 & -1.225339 & -0.321116 \\
\hline 16 & 1 & 0 & -2.145393 & -0.790173 & 1.836129 \\
\hline 17 & 1 & 0 & -3.085713 & 0.710737 & 1.841923 \\
\hline 18 & 1 & 0 & -4.785007 & -0.583477 & 0.891131 \\
\hline 19 & 1 & 0 & -3.075169 & 1.250177 & -1.812284 \\
\hline 20 & 1 & 0 & -3.089756 & 2.081818 & -0.236879 \\
\hline 21 & 1 & 0 & -5.248866 & 1.497547 & -0.954742 \\
\hline 22 & 1 & 0 & -1.974409 & -1.144034 & -1.863497 \\
\hline 23 & 1 & 0 & -3.642696 & -1.323552 & -1.308856 \\
\hline 24 & 1 & 0 & -2.864218 & -2.590893 & 0.371446 \\
\hline 25 & 1 & 0 & 1.198177 & 3.475251 & 0.221882 \\
\hline 26 & 7 & 0 & -1.127109 & 0.615826 & -0.035412 \\
\hline 27 & 8 & 0 & -4.090709 & -1.053873 & 1.367970 \\
\hline 28 & 8 & 0 & -4.700604 & 0.802413 & -0.586218 \\
\hline 29 & 8 & 0 & -2.197961 & -2.415425 & -0.303638 \\
\hline 30 & 8 & 0 & 0.756903 & 2.625703 & 0.131018 \\
\hline 31 & 17 & 0 & 4.802731 & -1.640580 & -0.062643 \\
\hline
\end{tabular}

\section{$\underline{\text { Structure of } 7 \text { (closed form) }}$}

Zero-point correction $=0.248966$ (Hartree/Particle)
Thermal correction to Energy $=0.265360$
Thermal correction to Enthalpy= 0.266304
Thermal correction to Gibbs Free Energy= 0.204350
Sum of electronic and zero-point Energies= -1243.097898
Sum of electronic and thermal Energies=-1243.081504
Sum of electronic and thermal Enthalpies=-1243.080560
Sum of electronic and thermal Free Energies=-1243.142514
No imaginary frequencies

Standard orientation:

\begin{tabular}{|c|c|c|c|c|c|}
\hline \multirow{2}{*}{$\begin{array}{l}\text { Center } \\
\text { Number }\end{array}$} & \multirow{2}{*}{$\begin{array}{l}\text { Atomic } \\
\text { Number }\end{array}$} & \multirow{2}{*}{$\begin{array}{c}\text { Atomic } \\
\text { Type }\end{array}$} & \multicolumn{3}{|c|}{ Coordinates (Angstroms) } \\
\hline & & & $\mathrm{X}$ & Y & Z \\
\hline 1 & 6 & 0 & 1.597735 & 1.584755 & 0.103222 \\
\hline 2 & 6 & 0 & 2.936139 & 1.980189 & 0.183179 \\
\hline 3 & 6 & 0 & 3.950972 & 1.041691 & 0.130650 \\
\hline 4 & 6 & 0 & 3.641625 & -0.311212 & -0.004688 \\
\hline 5 & 6 & 0 & 2.328076 & -0.722864 & -0.088255 \\
\hline 6 & 6 & 0 & 1.290078 & 0.215737 & -0.036004 \\
\hline 7 & 6 & 0 & -0.090088 & -0.257089 & -0.125210 \\
\hline 8 & 6 & 0 & -2.448482 & 0.031044 & -0.138556 \\
\hline 9 & 6 & 0 & -2.910526 & -0.228225 & 1.313365 \\
\hline 10 & 6 & 0 & -3.275858 & 1.155738 & -0.765472 \\
\hline 11 & 6 & 0 & -2.621686 & -1.248497 & -0.976389 \\
\hline 12 & 1 & 0 & 3.154526 & 3.035279 & 0.289703 \\
\hline 13 & 1 & 0 & 4.987216 & 1.350001 & 0.194560 \\
\hline 14 & 1 & 0 & 2.093469 & -1.776124 & -0.193919 \\
\hline 15 & 1 & 0 & -0.228315 & -1.338420 & -0.226825 \\
\hline 16 & 1 & 0 & -2.132199 & -0.794435 & 1.827016 \\
\hline 17 & 1 & 0 & -3.036736 & 0.728581 & 1.833030 \\
\hline 18 & 1 & 0 & -4.778112 & -0.524208 & 0.915344 \\
\hline 19 & 1 & 0 & -3.031473 & 1.244166 & -1.831409 \\
\hline 20 & 1 & 0 & -3.017337 & 2.094280 & -0.265154 \\
\hline 21 & 1 & 0 & -5.192757 & 1.546696 & -0.972948 \\
\hline 22 & 1 & 0 & -1.997655 & -1.173795 & -1.871434 \\
\hline 23 & 1 & 0 & -3.665206 & -1.311534 & -1.299359 \\
\hline 24 & 1 & 0 & -2.907376 & -2.595883 & 0.371249 \\
\hline 25 & 1 & 0 & -0.224687 & 2.068909 & 0.100727 \\
\hline 26 & 7 & 0 & -1.081483 & 0.548006 & -0.077358 \\
\hline 27 & 8 & 0 & -4.084619 & -1.013563 & 1.373607 \\
\hline 28 & 8 & 0 & -4.656206 & 0.852033 & -0.585952 \\
\hline 29 & 8 & 0 & -2.234509 & -2.426367 & -0.298840 \\
\hline 30 & 8 & 0 & 0.648605 & 2.524876 & 0.162167 \\
\hline
\end{tabular}




\section{$\underline{\text { Structure of } \mathbf{T S}_{7 / 37}}$}

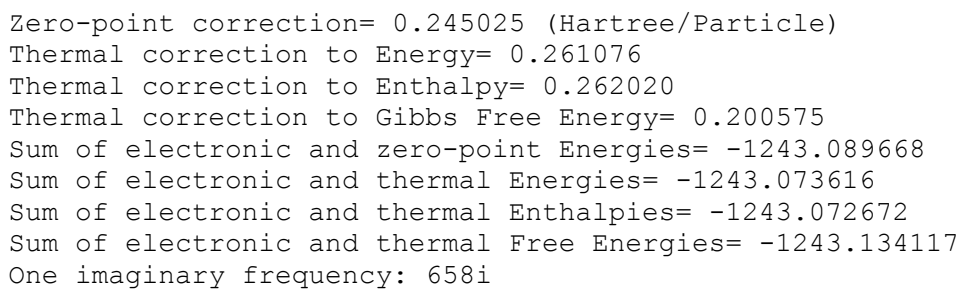

Standard orientation:

\begin{tabular}{|c|c|c|c|c|c|}
\hline \multirow{2}{*}{$\begin{array}{l}\text { Center } \\
\text { Number }\end{array}$} & \multirow{2}{*}{$\begin{array}{l}\text { Atomic } \\
\text { Number }\end{array}$} & \multirow{2}{*}{$\begin{array}{c}\text { Atomic } \\
\text { Type }\end{array}$} & \multicolumn{3}{|c|}{ Coordinates (Angstroms) } \\
\hline & & & $\mathrm{X}$ & $\mathrm{Y}$ & Z \\
\hline 1 & 6 & 0 & 1.468364 & 1.515149 & 0.099293 \\
\hline 2 & 6 & 0 & 2.811300 & 1.991797 & 0.198582 \\
\hline 3 & 6 & 0 & 3.872128 & 1.128015 & 0.152225 \\
\hline 4 & 6 & 0 & 3.673589 & -0.264222 & 0.005456 \\
\hline 5 & 6 & 0 & 2.412445 & -0.777617 & -0.091523 \\
\hline 6 & 6 & 0 & 1.298501 & 0.092664 & -0.047329 \\
\hline 7 & 6 & 0 & -0.022541 & -0.418438 & -0.139328 \\
\hline 8 & 6 & 0 & -2.436837 & 0.014162 & -0.139421 \\
\hline 9 & 6 & 0 & -2.909602 & -0.231254 & 1.311967 \\
\hline 10 & 6 & 0 & -3.180029 & 1.199805 & -0.759129 \\
\hline 11 & 6 & 0 & -2.680146 & -1.243867 & -0.988277 \\
\hline 12 & 1 & 0 & 2.959830 & 3.058507 & 0.310994 \\
\hline 13 & 1 & 0 & 4.886188 & 1.503105 & 0.227827 \\
\hline 14 & 1 & 0 & 2.257661 & -1.845596 & -0.201686 \\
\hline 15 & 1 & 0 & -0.202685 & -1.489585 & -0.234807 \\
\hline 16 & 1 & 0 & -2.159611 & -0.840658 & 1.818403 \\
\hline 17 & 1 & 0 & -2.984424 & 0.726910 & 1.839421 \\
\hline 18 & 1 & 0 & -4.795848 & -0.416408 & 0.937369 \\
\hline 19 & 1 & 0 & -2.950380 & 1.261850 & -1.829752 \\
\hline 20 & 1 & 0 & -2.842898 & 2.122226 & -0.273121 \\
\hline 21 & 1 & 0 & -5.064348 & 1.735236 & -0.915464 \\
\hline 22 & 1 & 0 & -2.071133 & -1.184206 & -1.894337 \\
\hline 23 & 1 & 0 & -3.732124 & -1.258503 & -1.287184 \\
\hline 24 & 1 & 0 & -3.001561 & -2.602008 & 0.341911 \\
\hline 25 & 1 & 0 & -0.607961 & 1.456112 & 0.025901 \\
\hline 26 & 7 & 0 & -1.032626 & 0.405763 & -0.101045 \\
\hline 27 & 8 & 0 & -4.120746 & -0.955494 & 1.365752 \\
\hline 28 & 8 & 0 & -4.571790 & 0.998984 & -0.546759 \\
\hline 29 & 8 & 0 & -2.324714 & -2.438256 & -0.325661 \\
\hline 30 & 8 & 0 & 0.462806 & 2.304628 & 0.138699 \\
\hline 31 & 17 & 0 & 5.066483 & -1.316669 & -0.048795 \\
\hline
\end{tabular}

\section{Structure of $\mathbf{3 7}$}

Zero-point correction $=0.248430$ (Hartree/Particle)

Thermal correction to Energy $=0.264893$

Thermal correction to Enthalpy $=0.265837$

Thermal correction to Gibbs Free Energy $=0.203678$

Sum of electronic and zero-point Energies= -1243.087110

Sum of electronic and thermal Energies= -1243.070647

Sum of electronic and thermal Enthalpies= -1243.069703

Sum of electronic and thermal Free Energies= -1243.131862

No imaginary frequencies

Standard orientation:

\begin{tabular}{|c|c|c|c|c|c|}
\hline \multirow{2}{*}{$\begin{array}{l}\text { Center } \\
\text { Number }\end{array}$} & \multirow{2}{*}{$\begin{array}{l}\text { Atomic } \\
\text { Number }\end{array}$} & \multirow{2}{*}{$\begin{array}{c}\text { Atomic } \\
\text { Type }\end{array}$} & \multicolumn{3}{|c|}{ Coordinates (Angstroms) } \\
\hline & & & $\mathrm{X}$ & Y & Z \\
\hline 1 & 6 & 0 & 1.522946 & 1.594627 & 0.092697 \\
\hline 2 & 6 & 0 & 2.908013 & 2.000463 & 0.180142 \\
\hline
\end{tabular}




\begin{tabular}{|c|c|c|c|c|c|}
\hline 3 & 6 & 0 & 3.919850 & 1.091840 & 0.138477 \\
\hline 4 & 6 & 0 & 3.654313 & -0.301849 & 0.005674 \\
\hline 5 & 6 & 0 & 2.379076 & -0.757136 & -0.081812 \\
\hline 6 & 6 & 0 & 1.291675 & 0.163678 & -0.041447 \\
\hline 7 & 6 & 0 & -0.017577 & -0.320560 & -0.128050 \\
\hline 8 & 6 & 0 & -2.461221 & 0.033793 & -0.132759 \\
\hline 9 & 6 & 0 & -2.912775 & -0.275689 & 1.312540 \\
\hline 10 & 6 & 0 & -3.258690 & 1.202607 & -0.715131 \\
\hline 11 & 6 & 0 & -2.657151 & -1.207906 & -1.019358 \\
\hline 12 & 1 & 0 & 3.105556 & 3.060430 & 0.281673 \\
\hline 13 & 1 & 0 & 4.952266 & 1.415967 & 0.205970 \\
\hline 14 & 1 & 0 & 2.177819 & -1.818250 & -0.182507 \\
\hline 15 & 1 & 0 & -0.203627 & -1.389634 & -0.217672 \\
\hline 16 & 1 & 0 & -2.130520 & -0.862333 & 1.796683 \\
\hline 17 & 1 & 0 & -3.029897 & 0.661585 & 1.869232 \\
\hline 18 & 1 & 0 & -4.793069 & -0.539176 & 0.951939 \\
\hline 19 & 1 & 0 & -3.046881 & 1.298440 & -1.786800 \\
\hline 20 & 1 & 0 & -2.955234 & 2.128395 & -0.212291 \\
\hline 21 & 1 & 0 & -5.165875 & 1.655742 & -0.845899 \\
\hline 22 & 1 & 0 & -2.049549 & -1.097886 & -1.921445 \\
\hline 23 & 1 & 0 & -3.707835 & -1.250539 & -1.319751 \\
\hline 24 & 1 & 0 & -2.929867 & -2.612503 & 0.272300 \\
\hline 25 & 7 & 0 & -1.070262 & 0.475098 & -0.103338 \\
\hline 26 & 8 & 0 & -4.087070 & -1.059069 & 1.352866 \\
\hline 27 & 8 & 0 & -4.636709 & 0.940245 & -0.487131 \\
\hline 28 & 8 & 0 & -2.261077 & -2.408070 & -0.392387 \\
\hline 29 & 8 & 0 & 0.582862 & 2.423966 & 0.130559 \\
\hline 30 & 1 & 0 & -0.802951 & 1.474854 & 0.017442 \\
\hline 31 & 17 & 0 & 5.004512 & -1.410033 & -0.040153 \\
\hline
\end{tabular}

\section{Structure of $\mathbf{8}$ (open form)}

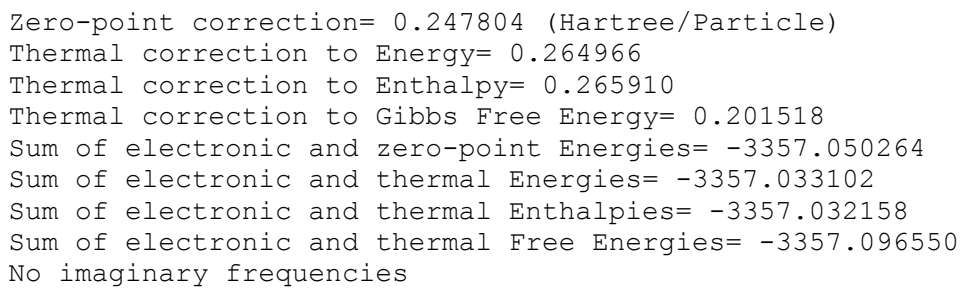

Standard orientation:

\begin{tabular}{|c|c|c|c|c|c|}
\hline \multirow{2}{*}{$\begin{array}{l}\text { Center } \\
\text { Number }\end{array}$} & \multirow{2}{*}{$\begin{array}{l}\text { Atomic } \\
\text { Number }\end{array}$} & \multirow{2}{*}{$\begin{array}{c}\text { Atomic } \\
\text { Type }\end{array}$} & \multicolumn{3}{|c|}{ Coordinates (Angstroms) } \\
\hline & & & $\mathrm{X}$ & Y & Z \\
\hline 1 & 6 & 0 & 0.909556 & 1.958075 & 0.118141 \\
\hline 2 & 6 & 0 & 2.231553 & 2.394213 & 0.206496 \\
\hline 3 & 6 & 0 & 3.290987 & 1.500181 & 0.156963 \\
\hline 4 & 6 & 0 & 3.025064 & 0.145910 & 0.014547 \\
\hline 5 & 6 & 0 & 1.718740 & -0.303252 & -0.075269 \\
\hline 6 & 6 & 0 & 0.639501 & 0.584671 & -0.023374 \\
\hline 7 & 6 & 0 & -0.706749 & -0.011627 & -0.126448 \\
\hline 8 & 6 & 0 & -3.061020 & -0.060168 & -0.141134 \\
\hline 9 & 6 & 0 & -3.556112 & -0.272552 & 1.307236 \\
\hline 10 & 6 & 0 & -3.991713 & 0.909110 & -0.875086 \\
\hline 11 & 6 & 0 & -3.047177 & -1.404985 & -0.888034 \\
\hline 12 & 1 & 0 & 2.433314 & 3.455324 & 0.315885 \\
\hline 13 & 1 & 0 & 4.310949 & 1.855347 & 0.226753 \\
\hline 14 & 1 & 0 & 1.518508 & -1.362877 & -0.186905 \\
\hline 15 & 1 & 0 & -0.693663 & -1.099158 & -0.265126 \\
\hline 16 & 1 & 0 & -2.742002 & -0.705703 & 1.890222 \\
\hline 17 & 1 & 0 & -3.815547 & 0.695988 & 1.748912 \\
\hline 18 & 1 & 0 & -5.349163 & -0.822989 & 0.844117 \\
\hline 19 & 1 & 0 & -3.713979 & 0.954657 & -1.935584 \\
\hline 20 & 1 & 0 & -3.863803 & 1.900934 & -0.433411 \\
\hline 21 & 1 & 0 & -5.931755 & 1.056502 & -1.172725 \\
\hline 22 & 1 & 0 & -2.403096 & -1.318184 & -1.767751 \\
\hline 23 & 1 & 0 & -4.061528 & -1.617980 & -1.239598 \\
\hline 24 & 1 & 0 & -3.218157 & -2.668794 & 0.553915 \\
\hline 25 & 1 & 0 & 0.234536 & 3.725628 & 0.261939 \\
\hline 26 & 7 & 0 & -1.786081 & 0.647745 & -0.048074 \\
\hline
\end{tabular}




$\begin{array}{rrrrrr}27 & 8 & 0 & -4.634824 & -1.186773 & 1.380931 \\ 28 & 8 & 0 & -5.336000 & 0.451888 & -0.726535 \\ 29 & 8 & 0 & -2.550080 & -2.479867 & -0.115551 \\ 30 & 8 & 0 & -0.116242 & 2.835775 & 0.161902 \\ 31 & 35 & 0 & 4.462267 & -1.091101 & -0.061552 \\ -\end{array}$

\section{Structure of $\mathbf{8}$ (closed form)}

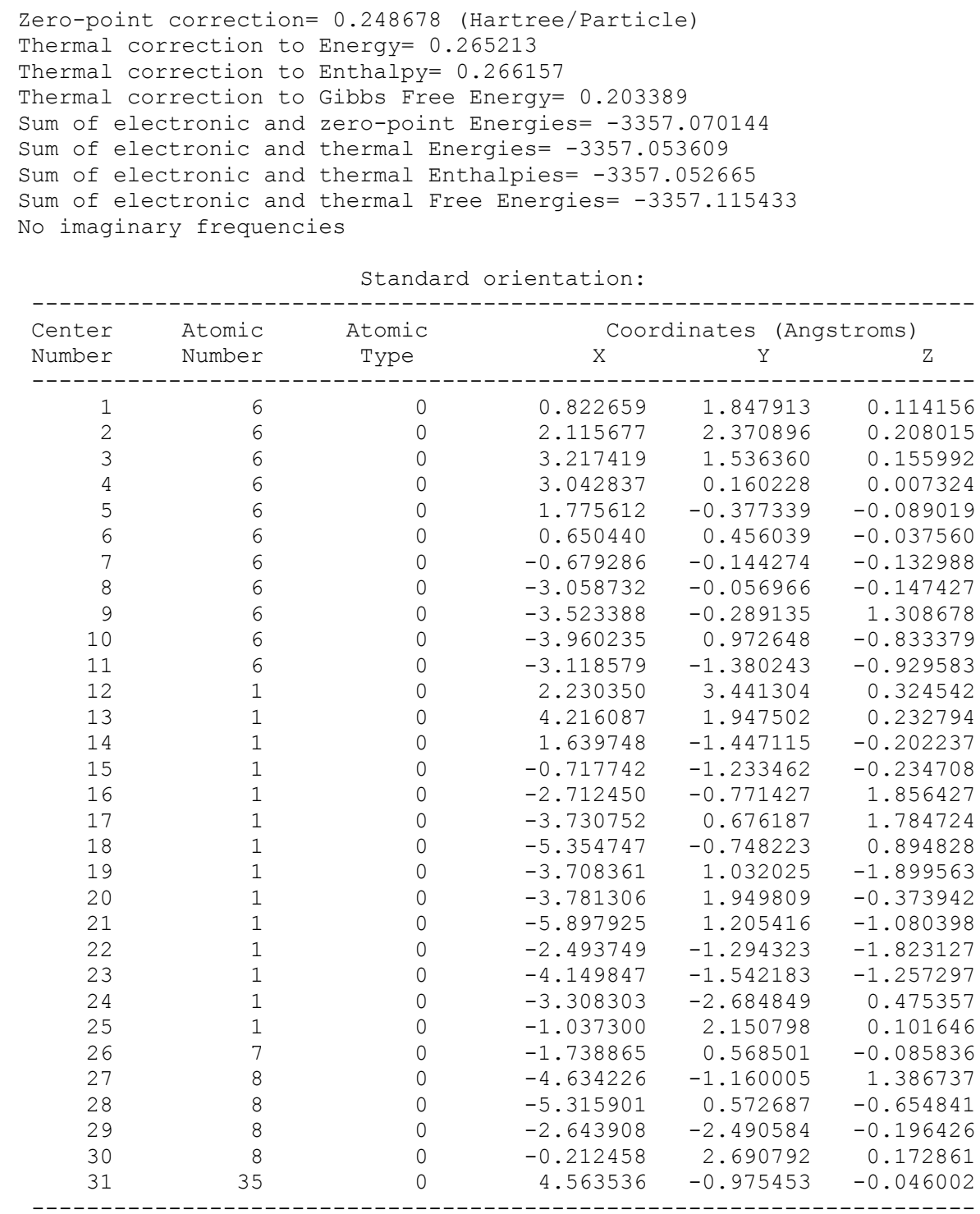

\section{$\underline{\text { Structure of } \mathbf{T S}} \underline{8}_{8 / 38}$}

Zero-point correction= 0.244601 (Hartree/Particle)
Thermal correction to Energy= 0.260820
Thermal correction to Enthalpy= 0.261765
Thermal correction to Gibbs Free Energy= 0.199743
Sum of electronic and zero-point Energies=-3357.062291
Sum of electronic and thermal Energies=-3357.046071
Sum of electronic and thermal Enthalpies= -3357.045127
Sum of electronic and thermal Free Energies=-3357.107149
One imaginary frequency: 712i

Standard orientation:

Center Atomic Atomic Coordinates (Angstroms)




\begin{tabular}{|c|c|c|c|c|c|}
\hline Number & Number & Type & $\mathrm{X}$ & Y & Z \\
\hline 1 & 6 & 0 & 0.712454 & 1.758939 & 0.091521 \\
\hline 2 & 6 & 0 & 2.013204 & 2.338909 & 0.195258 \\
\hline 3 & 6 & 0 & 3.139317 & 1.560675 & 0.157267 \\
\hline 4 & 6 & 0 & 3.050441 & 0.156395 & 0.013783 \\
\hline 5 & 6 & 0 & 1.832516 & -0.453576 & -0.087904 \\
\hline 6 & 6 & 0 & 0.654602 & 0.327691 & -0.051713 \\
\hline 7 & 6 & 0 & -0.622484 & -0.286575 & -0.142120 \\
\hline 8 & 6 & 0 & -3.060976 & -0.051737 & -0.139842 \\
\hline 9 & 6 & 0 & -3.505153 & -0.345670 & 1.311789 \\
\hline 10 & 6 & 0 & -3.905185 & 1.071106 & -0.746474 \\
\hline 11 & 6 & 0 & -3.201344 & -1.320412 & -0.996905 \\
\hline 12 & 1 & 0 & 2.077389 & 3.414226 & 0.306327 \\
\hline 13 & 1 & 0 & 4.118151 & 2.018277 & 0.240450 \\
\hline 14 & 1 & 0 & 1.757235 & -1.530458 & -0.193752 \\
\hline 15 & 1 & 0 & -0.718172 & -1.368948 & -0.234349 \\
\hline 16 & 1 & 0 & -2.702699 & -0.888060 & 1.813802 \\
\hline 17 & 1 & 0 & -3.664017 & 0.599236 & 1.844253 \\
\hline 18 & 1 & 0 & -5.369842 & -0.694753 & 0.945094 \\
\hline 19 & 1 & 0 & -3.690337 & 1.159306 & -1.818317 \\
\hline 20 & 1 & 0 & -3.643500 & 2.015468 & -0.256187 \\
\hline 21 & 1 & 0 & -5.829285 & 1.442938 & -0.892064 \\
\hline 22 & 1 & 0 & -2.600684 & -1.203643 & -1.903015 \\
\hline 23 & 1 & 0 & -4.248874 & -1.419891 & -1.295193 \\
\hline 24 & 1 & 0 & -3.405015 & -2.705201 & 0.328499 \\
\hline 25 & 1 & 0 & -1.350261 & 1.538321 & 0.018545 \\
\hline 26 & 7 & 0 & -1.693855 & 0.455162 & -0.104920 \\
\hline 27 & 8 & 0 & -4.646612 & -1.175277 & 1.364746 \\
\hline 28 & 8 & 0 & -5.273441 & 0.752424 & -0.524829 \\
\hline 29 & 8 & 0 & -2.747545 & -2.485672 & -0.342599 \\
\hline 30 & 8 & 0 & -0.353179 & 2.465954 & 0.126036 \\
\hline 31 & 35 & 0 & 4.647108 & -0.873053 & -0.022412 \\
\hline
\end{tabular}

\section{$\underline{\text { Structure of } \mathbf{3 8}}$}

Zero-point correction= 0.247936 (Hartree/Particle)

Thermal correction to Energy $=0.264597$

Thermal correction to Enthalpy $=0.265541$

Thermal correction to Gibbs Free Energy $=0.202476$

Sum of electronic and zero-point Energies= -3357.059814

Sum of electronic and thermal Energies $=-3357.043152$

Sum of electronic and thermal Enthalpies= -3357.042208

Sum of electronic and thermal Free Energies= -3357.105273

No imaginary frequencies

Standard orientation:

\begin{tabular}{|c|c|c|c|c|c|}
\hline \multirow{2}{*}{$\begin{array}{l}\text { Center } \\
\text { Number }\end{array}$} & \multirow{2}{*}{$\begin{array}{l}\text { Atomic } \\
\text { Number }\end{array}$} & \multirow{2}{*}{$\begin{array}{c}\text { Atomic } \\
\text { Type }\end{array}$} & \multicolumn{3}{|c|}{ Coordinates (Angstroms) } \\
\hline & & & $\mathrm{X}$ & $\mathrm{Y}$ & $\mathrm{Z}$ \\
\hline 1 & 6 & 0 & 0.751938 & 1.845417 & 0.090246 \\
\hline 2 & 6 & 0 & 2.094191 & 2.370607 & 0.196021 \\
\hline 3 & 6 & 0 & 3.183523 & 1.556124 & 0.158362 \\
\hline 4 & 6 & 0 & 3.043772 & 0.144986 & 0.012795 \\
\hline 5 & 6 & 0 & 1.813348 & -0.420487 & -0.089264 \\
\hline 6 & 6 & 0 & 0.648621 & 0.400855 & -0.053824 \\
\hline 7 & 6 & 0 & -0.613939 & -0.196643 & -0.143325 \\
\hline 8 & 6 & 0 & -3.080121 & -0.045004 & -0.139059 \\
\hline 9 & 6 & 0 & -3.503418 & -0.364333 & 1.312952 \\
\hline 10 & 6 & 0 & -3.969863 & 1.046618 & -0.737819 \\
\hline 11 & 6 & 0 & -3.177380 & -1.312879 & -1.004044 \\
\hline 12 & 1 & 0 & 2.196634 & 3.443139 & 0.307189 \\
\hline 13 & 1 & 0 & 4.180142 & 1.974132 & 0.241135 \\
\hline 14 & 1 & 0 & 1.701973 & -1.494263 & -0.196043 \\
\hline 15 & 1 & 0 & -0.708156 & -1.278021 & -0.230223 \\
\hline 16 & 1 & 0 & -2.676227 & -0.876451 & 1.806682 \\
\hline 17 & 1 & 0 & -3.696459 & 0.570175 & 1.852837 \\
\hline 18 & 1 & 0 & -5.357156 & -0.785620 & 0.962287 \\
\hline 19 & 1 & 0 & -3.771045 & 1.139119 & -1.812270 \\
\hline 20 & 1 & 0 & -3.738510 & 2.002746 & -0.253747 \\
\hline 21 & 1 & 0 & -5.907263 & 1.346329 & -0.864497 \\
\hline 22 & 1 & 0 & -2.583168 & -1.168512 & -1.910205 \\
\hline
\end{tabular}




\begin{tabular}{rrrrrr}
23 & 1 & 0 & -4.221903 & -1.445390 & -1.299611 \\
24 & 1 & 0 & -3.330427 & -2.710590 & 0.314606 \\
25 & 7 & 0 & -1.729329 & 0.506940 & -0.120507 \\
26 & 8 & 0 & -4.610082 & -1.239638 & 1.369211 \\
27 & 8 & 0 & -5.321798 & 0.680736 & -0.496854 \\
28 & 8 & 0 & -2.683694 & -2.466053 & -0.358471 \\
29 & 8 & 0 & -0.257562 & 2.589114 & 0.123652 \\
30 & 1 & 0 & -1.544455 & 1.526222 & -0.000466 \\
31 & 35 & 0 & 4.611496 & -0.927326 & -0.022648 \\
\hline
\end{tabular}

\section{$\underline{\text { Structure of } 10 \text { (open form) }}$}

Zero-point correction= 0.258097 (Hartree/Particle)
Thermal correction to Energy $=0.273735$
Thermal correction to Enthalpy= 0.274679
Thermal correction to Gibos Free Energy= 0.214521
Sum of electronic and zero-point Energies=-783.468880
Sum of electronic and thermal Energies= -783.453242
Sum of electronic and thermal Enthalpies= -783.452298
Sum of electronic and thermal Free Energies= -783.512456
No imaginary frequencies

\begin{tabular}{|c|c|c|c|c|c|}
\hline \multirow{2}{*}{$\begin{array}{l}\text { Center } \\
\text { Number }\end{array}$} & \multirow{2}{*}{$\begin{array}{l}\text { Atomic } \\
\text { Number }\end{array}$} & \multirow{2}{*}{$\begin{array}{c}\text { Atomic } \\
\text { Type }\end{array}$} & \multicolumn{3}{|c|}{ Coordinates (Angstroms) } \\
\hline & & & $\mathrm{X}$ & $\mathrm{Y}$ & Z \\
\hline 1 & 6 & 0 & -2.557267 & -0.960135 & 0.104848 \\
\hline 2 & 6 & 0 & -3.949704 & -1.004500 & 0.181508 \\
\hline 3 & 6 & 0 & -4.706849 & 0.154387 & 0.082848 \\
\hline 4 & 6 & 0 & -4.081551 & 1.383181 & -0.096540 \\
\hline 5 & 6 & 0 & -2.697426 & 1.424746 & -0.173169 \\
\hline 6 & 6 & 0 & -1.906935 & 0.274659 & -0.074539 \\
\hline 7 & 6 & 0 & -0.447202 & 0.457840 & -0.167120 \\
\hline 8 & 6 & 0 & 1.825916 & -0.155438 & -0.131678 \\
\hline 9 & 6 & 0 & 2.328418 & 0.007010 & 1.319829 \\
\hline 10 & 6 & 0 & 2.464191 & -1.391307 & -0.771617 \\
\hline 11 & 6 & 0 & 2.205633 & 1.087516 & -0.955925 \\
\hline 12 & 1 & 0 & -4.438901 & -1.964169 & 0.320439 \\
\hline 13 & 1 & 0 & -5.786879 & 0.092584 & 0.146260 \\
\hline 14 & 1 & 0 & -4.662720 & 2.292935 & -0.173884 \\
\hline 15 & 1 & 0 & -2.195064 & 2.376595 & -0.312649 \\
\hline 16 & 1 & 0 & -0.148220 & 1.499672 & -0.336672 \\
\hline 17 & 1 & 0 & 1.659761 & 0.692987 & 1.842030 \\
\hline 18 & 1 & 0 & 2.288119 & -0.962291 & 1.829012 \\
\hline 19 & 1 & 0 & 4.214692 & -0.007356 & 0.899549 \\
\hline 20 & 1 & 0 & 2.201885 & -1.431104 & -1.836216 \\
\hline 21 & 1 & 0 & 2.058248 & -2.276591 & -0.275017 \\
\hline 22 & 1 & 0 & 4.289620 & -2.095326 & -0.985933 \\
\hline 23 & 1 & 0 & 1.570301 & 1.131196 & -1.844952 \\
\hline 24 & 1 & 0 & 3.241678 & 0.978564 & -1.291969 \\
\hline 25 & 1 & 0 & 2.714994 & 2.333042 & 0.419747 \\
\hline 26 & 1 & 0 & -2.413637 & -2.839076 & 0.321019 \\
\hline 27 & 7 & 0 & 0.402786 & -0.475879 & -0.049701 \\
\hline 28 & 8 & 0 & 3.622968 & 0.578877 & 1.386293 \\
\hline 29 & 8 & 0 & 3.880726 & -1.315662 & -0.605779 \\
\hline 30 & 8 & 0 & 2.033641 & 2.308426 & -0.262687 \\
\hline 31 & 8 & 0 & -1.821925 & -2.091423 & 0.196731 \\
\hline
\end{tabular}

\section{$\underline{\text { Structure of } \mathbf{1 0} \text { (closed form) }}$}

Zero-point correction $=0.258989$ (Hartree/Particle)

Thermal correction to Energy $=0.274016$

Thermal correction to Enthalpy $=0.274960$

Thermal correction to Gibbs Free Energy= 0.216715

Sum of electronic and zero-point Energies= -783.488472

Sum of electronic and thermal Energies $=-783.473446$

Sum of electronic and thermal Enthalpies= -783.472501

Sum of electronic and thermal Free Energies= -783.530746

No imaginary frequencies 
Standard orientation:

\begin{tabular}{|c|c|c|c|c|c|}
\hline \multirow{2}{*}{$\begin{array}{l}\text { Center } \\
\text { Number }\end{array}$} & \multirow{2}{*}{$\begin{array}{l}\text { Atomic } \\
\text { Number }\end{array}$} & \multirow{2}{*}{$\begin{array}{l}\text { Atomic } \\
\text { Type }\end{array}$} & \multicolumn{3}{|c|}{ Coordinates (Angstroms) } \\
\hline & & & $\mathrm{X}$ & Y & Z \\
\hline 1 & ( & & & & 0106503 \\
\hline 1 & 6 & 0 & -2.469282 & -0.957408 & 0.106503 \\
\hline 2 & 6 & 0 & -3.855893 & -1.112298 & 0.185761 \\
\hline 3 & 6 & 0 & -4.685442 & -0.008006 & 0.092747 \\
\hline 4 & 6 & 0 & -4.160023 & 1.275759 & -0.080442 \\
\hline 5 & 6 & 0 & -2.788610 & 1.432512 & -0.158773 \\
\hline 6 & 6 & 0 & -1.924506 & 0.333081 & -0.068420 \\
\hline 7 & 6 & 0 & -0.483628 & 0.551097 & -0.153451 \\
\hline 8 & 6 & 0 & 1.789629 & -0.146197 & -0.137057 \\
\hline 9 & 6 & 0 & 2.282617 & 0.032159 & 1.316411 \\
\hline 10 & 6 & 0 & 2.406400 & -1.402875 & -0.756133 \\
\hline 11 & 6 & 0 & 2.194410 & 1.078433 & -0.977235 \\
\hline 12 & 1 & 0 & -4.252221 & -2.111098 & 0.320396 \\
\hline 13 & 1 & 0 & -5.758805 & -0.145944 & 0.156112 \\
\hline 14 & 1 & 0 & -4.816842 & 2.133010 & -0.150148 \\
\hline 15 & 1 & 0 & -2.356852 & 2.419638 & -0.291838 \\
\hline 16 & 1 & 0 & -0.156495 & 1.589150 & -0.274491 \\
\hline 17 & 1 & 0 & 1.620282 & 0.737269 & 1.820990 \\
\hline 18 & 1 & 0 & 2.224174 & -0.927542 & 1.842674 \\
\hline 19 & 1 & 0 & 4.174242 & -0.021721 & 0.921770 \\
\hline 20 & 1 & 0 & 2.155753 & -1.449012 & -1.823196 \\
\hline 21 & 1 & 0 & 1.980803 & -2.278326 & -0.255953 \\
\hline 22 & 1 & 0 & 4.222642 & -2.134013 & -0.951728 \\
\hline 23 & 1 & 0 & 1.565440 & 1.117636 & -1.870854 \\
\hline 24 & 1 & 0 & 3.231217 & 0.948304 & -1.302107 \\
\hline 25 & 1 & 0 & 2.719033 & 2.343673 & 0.376436 \\
\hline 26 & 1 & 0 & -0.758317 & -1.750672 & 0.131295 \\
\hline 27 & 7 & 0 & 0.353357 & -0.413240 & -0.080002 \\
\hline 28 & 8 & 0 & 3.583066 & 0.585560 & 1.382329 \\
\hline 29 & 8 & 0 & 3.818433 & -1.352298 & -0.570157 \\
\hline 30 & 8 & 0 & 2.033220 & 2.308906 & -0.301178 \\
\hline 31 & 8 & 0 & -1.697417 & -2.046571 & 0.200405 \\
\hline
\end{tabular}

\section{$\underline{\text { Structure of } \mathbf{T S}_{10 / 40}}$}

Zero-point correction= 0.254791 (Hartree/Particle)

Thermal correction to Energy $=0.269558$

Thermal correction to Enthalpy $=0.270502$

Thermal correction to Gibbs Free Energy= 0.212614

Sum of electronic and zero-point Energies $=-783.480080$

Sum of electronic and thermal Energies= -783.465313

Sum of electronic and thermal Enthalpies= -783.464369

Sum of electronic and thermal Free Energies= - 783.522257

One imaginary frequency: $560 i$

Standard orientation:

\begin{tabular}{rrrrr} 
Center & $\begin{array}{c}\text { Atomic } \\
\text { Number }\end{array}$ & \multicolumn{2}{c}{$\begin{array}{c}\text { Atomic } \\
\text { Type }\end{array}$} & \multicolumn{3}{c}{ Coordinates } \\
X Angstroms)
\end{tabular}




\begin{tabular}{rrrrrr}
19 & 1 & 0 & 4.158905 & -0.088007 & 0.929001 \\
20 & 1 & 0 & 2.064782 & -1.445574 & -1.822213 \\
21 & 1 & 0 & 1.812087 & -2.272467 & -0.264517 \\
22 & 1 & 0 & 4.070257 & -2.256993 & -0.910729 \\
23 & 1 & 0 & 1.589278 & 1.113258 & -1.890474 \\
24 & 1 & 0 & 3.244238 & 0.906518 & -1.297137 \\
25 & 1 & 0 & 2.758432 & 2.347850 & 0.343529 \\
26 & 1 & 0 & -0.272428 & -1.242517 & 0.049271 \\
27 & 7 & 0 & 0.312134 & -0.282309 & -0.096164 \\
28 & 8 & 0 & 3.586097 & 0.554013 & 1.364636 \\
29 & 8 & 0 & 3.704505 & -1.453420 & -0.535063 \\
30 & 8 & 0 & 2.066738 & 2.310015 & -0.327996 \\
31 & 8 & 0 & -1.479359 & -1.907170 & 0.168930 \\
\hline
\end{tabular}

\section{$\underline{\text { Structure of } 40}$}

\begin{tabular}{|c|c|c|c|c|c|}
\hline \multicolumn{2}{|c|}{ Thermal correction to Enthalpy $=0.274008$} & \multicolumn{4}{|c|}{ Thermal correction to Energy $=0.273063$} \\
\hline \multicolumn{6}{|c|}{ Thermal correction to Gibbs Free Energy $=0.215089$} \\
\hline \multicolumn{6}{|c|}{ Sum of electronic and zero-point Energies= -783.477637} \\
\hline \multicolumn{6}{|c|}{ Sum of electronic and thermal Energies $=-783.462406$} \\
\hline \multicolumn{6}{|c|}{ Sum of electronic and thermal Enthalpies $=-783.461462$} \\
\hline \multicolumn{6}{|c|}{ Sum of electronic and thermal Free Energies= -783.520380} \\
\hline \multirow{2}{*}{\multicolumn{6}{|c|}{ Standard orientation: }} \\
\hline & & & & & \\
\hline \multirow{2}{*}{$\begin{array}{l}\text { Center } \\
\text { Number }\end{array}$} & Atomic & \multirow{2}{*}{$\begin{array}{c}\text { Atomic } \\
\text { Type }\end{array}$} & \multicolumn{3}{|c|}{ Coordinates (Angstroms) } \\
\hline & Number & & $\mathrm{X}$ & Y & Z \\
\hline & & & ---------1 & ----------1 & -------- \\
\hline 1 & 6 & 0 & -2.396820 & -1.005848 & 0.100045 \\
\hline 2 & 6 & 0 & -3.827075 & -1.174627 & 0.198904 \\
\hline 3 & 6 & 0 & -4.671093 & -0.107514 & 0.125199 \\
\hline 4 & 6 & 0 & -4.198693 & 1.227973 & -0.049634 \\
\hline 5 & 6 & 0 & -2.858142 & 1.445912 & -0.145196 \\
\hline 6 & 6 & 0 & -1.932017 & 0.364283 & -0.075487 \\
\hline 7 & 6 & 0 & -0.561350 & 0.621242 & -0.166613 \\
\hline 8 & 6 & 0 & 1.788576 & -0.144162 & -0.136708 \\
\hline 9 & 6 & 0 & 2.277342 & 0.052861 & 1.316157 \\
\hline 10 & 6 & 0 & 2.375674 & -1.421358 & -0.741735 \\
\hline 11 & 6 & 0 & 2.204632 & 1.061495 & -0.995957 \\
\hline 12 & 1 & 0 & -4.193915 & -2.185170 & 0.331623 \\
\hline 13 & 1 & 0 & -5.740757 & -0.274477 & 0.201874 \\
\hline 14 & 1 & 0 & -4.902054 & 2.048310 & -0.103238 \\
\hline 15 & 1 & 0 & -2.467902 & 2.451132 & -0.276014 \\
\hline 16 & 1 & 0 & -0.199272 & 1.642322 & -0.275742 \\
\hline 17 & 1 & 0 & 1.610081 & 0.762445 & 1.807766 \\
\hline 18 & 1 & 0 & 2.218257 & -0.900076 & 1.855143 \\
\hline 19 & 1 & 0 & 4.175140 & -0.016946 & 0.957435 \\
\hline 20 & 1 & 0 & 2.159428 & -1.454103 & -1.816198 \\
\hline 21 & 1 & 0 & 1.910359 & -2.290698 & -0.262882 \\
\hline 22 & 1 & 0 & 4.175621 & -2.199231 & -0.875296 \\
\hline 23 & 1 & 0 & 1.591535 & 1.078988 & -1.900682 \\
\hline 24 & 1 & 0 & 3.247931 & 0.924430 & -1.294279 \\
\hline 25 & 1 & 0 & 2.710488 & 2.364562 & 0.330758 \\
\hline 26 & 7 & 0 & 0.343431 & -0.339468 & -0.119705 \\
\hline 27 & 8 & 0 & 3.574731 & 0.609569 & 1.377916 \\
\hline 28 & 8 & 0 & 3.776936 & -1.410322 & -0.502102 \\
\hline 29 & 8 & 0 & 2.024541 & 2.300270 & -0.344680 \\
\hline 30 & 8 & 0 & -1.602574 & -1.977116 & 0.163595 \\
\hline 31 & 1 & 0 & -0.094229 & -1.275962 & 0.025997 \\
\hline
\end{tabular}

\section{$\underline{\text { Structure of } 12 \text { (open form) }}$}

Zero-point correction $=0.290362$ (Hartree/Particle)

Thermal correction to Energy $=0.308772$

Thermal correction to Enthalpy= 0.309716

Thermal correction to Gibbs Free Energy= 0.243386

Sum of electronic and zero-point Energies= -897.946105 
Sum of electronic and thermal Energies= -897.927695

Sum of electronic and thermal Enthalpies= -897.926751

Sum of electronic and thermal Free Energies= -897.993081

No imaginary frequencies

Standard orientation:

\begin{tabular}{|c|c|c|c|c|c|}
\hline \multirow{2}{*}{$\begin{array}{l}\text { Center } \\
\text { Number }\end{array}$} & \multirow{2}{*}{$\begin{array}{l}\text { Atomic } \\
\text { Number }\end{array}$} & \multirow{2}{*}{$\begin{array}{c}\text { Atomic } \\
\text { Type }\end{array}$} & \multicolumn{3}{|c|}{ Coordinates (Angstroms) } \\
\hline & & & $\mathrm{X}$ & $\mathrm{Y}$ & Z \\
\hline 1 & 6 & 0 & 1.657525 & 1.585981 & 0.092196 \\
\hline 2 & 6 & 0 & 3.017223 & 1.849744 & 0.182589 \\
\hline 3 & 6 & 0 & 3.968777 & 0.832579 & 0.125583 \\
\hline 4 & 6 & 0 & 3.551729 & -0.484263 & -0.029827 \\
\hline 5 & 6 & 0 & 2.188821 & -0.752463 & -0.123235 \\
\hline 6 & 6 & 0 & 1.227060 & 0.252844 & -0.061246 \\
\hline 7 & 6 & 0 & -0.180556 & -0.178320 & -0.165216 \\
\hline 8 & 6 & 0 & -2.523699 & 0.034612 & -0.123307 \\
\hline 9 & 6 & 0 & -2.990879 & -0.243372 & 1.322543 \\
\hline 10 & 6 & 0 & -3.366168 & 1.154806 & -0.739208 \\
\hline 11 & 6 & 0 & -2.682031 & -1.236802 & -0.975812 \\
\hline 12 & 1 & 0 & 3.349687 & 2.876688 & 0.300510 \\
\hline 13 & 1 & 0 & 5.016644 & 1.089289 & 0.202200 \\
\hline 14 & 1 & 0 & 1.878537 & -1.784632 & -0.244389 \\
\hline 15 & 1 & 0 & -0.296475 & -1.252335 & -0.354953 \\
\hline 16 & 1 & 0 & -2.210791 & -0.808621 & 1.834353 \\
\hline 17 & 1 & 0 & -3.125873 & 0.707570 & 1.849630 \\
\hline 18 & 1 & 0 & -4.847817 & -0.555711 & 0.893417 \\
\hline 19 & 1 & 0 & -3.116569 & 1.261901 & -1.802256 \\
\hline 20 & 1 & 0 & -3.118449 & 2.085993 & -0.222832 \\
\hline 21 & 1 & 0 & -5.285836 & 1.538761 & -0.939673 \\
\hline 22 & 1 & 0 & -2.051486 & -1.148897 & -1.865143 \\
\hline 23 & 1 & 0 & -3.722253 & -1.303558 & -1.310286 \\
\hline 24 & 1 & 0 & -2.961200 & -2.587656 & 0.367014 \\
\hline 25 & 1 & 0 & 1.199891 & 3.423073 & 0.239273 \\
\hline 26 & 7 & 0 & -1.176782 & 0.594089 & -0.030443 \\
\hline 27 & 8 & 0 & -4.162574 & -1.037894 & 1.371472 \\
\hline 28 & 8 & 0 & -4.748345 & 0.831831 & -0.578006 \\
\hline 29 & 8 & 0 & -2.295591 & -2.424037 & -0.311574 \\
\hline 30 & 8 & 0 & 0.739408 & 2.585218 & 0.140293 \\
\hline 31 & 8 & 0 & 4.382442 & -1.561257 & -0.102275 \\
\hline 32 & 6 & 0 & 5.768071 & -1.319342 & 0.000300 \\
\hline 33 & 1 & 0 & 6.020231 & -0.861944 & 0.962925 \\
\hline 34 & 1 & 0 & 6.251802 & -2.290669 & -0.075702 \\
\hline 35 & 1 & 0 & 6.119473 & -0.674784 & -0.812423 \\
\hline
\end{tabular}

\section{Structure of 12 (closed form)}

Zero-point correction= 0.291335 (Hartree/Particle)

Thermal correction to Energy $=0.309111$

Thermal correction to Enthalpy $=0.310055$

Thermal correction to Gibbs Free Energy $=0.245323$

Sum of electronic and zero-point Energies $=\mathbf{- 8 9 7 . 9 6 4 9 4 6}$

Sum of electronic and thermal Energies= -897.947171

Sum of electronic and thermal Enthalpies= -897.946226

Sum of electronic and thermal Free Energies= -898.010958

No imaginary frequencies

Standard orientation:

\begin{tabular}{|c|c|c|c|c|c|}
\hline \multirow{2}{*}{$\begin{array}{l}\text { Center } \\
\text { Number }\end{array}$} & \multirow{2}{*}{$\begin{array}{l}\text { Atomic } \\
\text { Number }\end{array}$} & \multirow{2}{*}{$\begin{array}{c}\text { Atomic } \\
\text { Type }\end{array}$} & \multicolumn{3}{|c|}{ Coordinates (Angstroms) } \\
\hline & & & $\mathrm{X}$ & Y & Z \\
\hline 1 & 6 & 0 & 1.577347 & 1.513021 & 0.101435 \\
\hline 2 & 6 & 0 & 2.920643 & 1.866947 & 0.171763 \\
\hline 3 & 6 & 0 & 3.920119 & 0.903166 & 0.110264 \\
\hline 4 & 6 & 0 & 3.590360 & -0.447393 & -0.024738 \\
\hline 5 & 6 & 0 & 2.253240 & -0.809224 & -0.099652 \\
\hline 6 & 6 & 0 & 1.240200 & 0.149728 & -0.039045 \\
\hline 7 & 6 & 0 & -0.150312 & -0.294896 & -0.125587 \\
\hline 8 & 6 & 0 & -2.503042 & 0.034606 & -0.132630 \\
\hline 9 & 6 & 0 & -2.965997 & -0.241150 & 1. 315162 \\
\hline 10 & 6 & 0 & -3.309468 & 1.186444 & -0.737475 \\
\hline
\end{tabular}




$\begin{array}{rrrrrr}11 & 6 & 0 & -2.704030 & -1.226227 & -0.992794 \\ 12 & 1 & 0 & 3.169530 & 2.915467 & 0.278293 \\ 13 & 1 & 0 & 4.952874 & 1.220463 & 0.169630 \\ 14 & 1 & 0 & 2.003815 & -1.859428 & -0.206567 \\ 15 & 1 & 0 & -0.308551 & -1.372711 & -0.237420 \\ 16 & 1 & 0 & -2.200934 & -0.838505 & 1.813345 \\ 17 & 1 & 0 & -3.063825 & 0.707930 & 1.854845 \\ 18 & 1 & 0 & -4.840252 & -0.482452 & 0.911846 \\ 19 & 1 & 0 & -3.062631 & 1.290763 & -1.801391 \\ 20 & 1 & 0 & -3.032357 & 2.109523 & -0.218925 \\ 21 & 1 & 0 & -5.217403 & 1.623206 & -0.935109 \\ 22 & 1 & 0 & -2.075040 & -1.150850 & -1.884192 \\ 23 & 1 & 0 & -3.747857 & -1.258789 & -1.319847 \\ 24 & 1 & 0 & -3.024528 & -2.586261 & 0.333420 \\ 25 & 1 & 0 & -0.233790 & 2.046681 & 0.114279 \\ 26 & 7 & 0 & -1.127553 & 0.526729 & -0.067409 \\ 27 & 8 & 0 & -4.161334 & -0.995883 & 1.365489 \\ 28 & 8 & 0 & -4.696631 & 0.907847 & -0.564886 \\ 29 & 8 & 0 & -2.348404 & -2.425432 & -0.335467 \\ 30 & 8 & 0 & 0.646974 & 2.481321 & 0.169115 \\ 31 & 8 & 0 & 4.497986 & -1.463895 & -0.091619 \\ 32 & 6 & 0 & 5.863634 & -1.122331 & -0.009023 \\ 33 & 1 & 0 & 6.094083 & -0.632299 & 0.942884 \\ 34 & 1 & 0 & 6.413939 & -2.058443 & -0.074095 \\ 35 & 1 & 0 & 6.160906 & -0.469207 & -0.836265 \\ ----------------------------------------19\end{array}$

\section{$\underline{\text { Structure of } \mathbf{T S}_{12 / 42}}$}

Zero-point correction= 0.287889 (Hartree/Particle)
Thermal correction to Energy= 0.305262
Thermal correction to Enthalpy= 0.306206
Thermal correction to Gibbs Free Energy= 0.242053
Sum of electronic and zero-point Energies=-897.953435
Sum of electronic and thermal Energies= -897.936062
Sum of electronic and thermal Enthalpies= -897.935118
Sum of electronic and thermal Free Energies= -897.999271
One imaginary frequency: 345i

\begin{tabular}{|c|c|c|c|c|c|}
\hline \multirow{2}{*}{$\begin{array}{l}\text { Center } \\
\text { Number }\end{array}$} & \multirow{2}{*}{$\begin{array}{l}\text { Atomic } \\
\text { Number }\end{array}$} & \multirow{2}{*}{$\begin{array}{c}\text { Atomic } \\
\text { Type }\end{array}$} & \multicolumn{3}{|c|}{ Coordinates (Angstroms) } \\
\hline & & & $\mathrm{X}$ & Y & Z \\
\hline 1 & 6 & 0 & 1.458194 & 1.443845 & 0.088912 \\
\hline 2 & 6 & 0 & 2.819037 & 1.866059 & 0.171201 \\
\hline 3 & 6 & 0 & 3.854396 & 0.966951 & 0.122566 \\
\hline 4 & 6 & 0 & 3.624859 & -0.426832 & -0.011545 \\
\hline 5 & 6 & 0 & 2.336728 & -0.881544 & -0.095847 \\
\hline 6 & 6 & 0 & 1.248574 & 0.022597 & -0.048780 \\
\hline 7 & 6 & 0 & -0.080035 & -0.452801 & -0.132158 \\
\hline 8 & 6 & 0 & -2.491318 & 0.029982 & -0.129785 \\
\hline 9 & 6 & 0 & -2.962000 & -0.258399 & 1.313698 \\
\hline 10 & 6 & 0 & -3.213661 & 1.250851 & -0.703620 \\
\hline 11 & 6 & 0 & -2.764163 & -1.192545 & -1.021822 \\
\hline 12 & 1 & 0 & 3.007844 & 2.927506 & 0.274499 \\
\hline 13 & 1 & 0 & 4.867842 & 1.343348 & 0.189468 \\
\hline 14 & 1 & 0 & 2.156996 & -1.946700 & -0.198540 \\
\hline 15 & 1 & 0 & -0.288916 & -1.518237 & -0.228049 \\
\hline 16 & 1 & 0 & -2.224634 & -0.905635 & 1.791068 \\
\hline 17 & 1 & 0 & -3.009152 & 0.680092 & 1.878349 \\
\hline 18 & 1 & 0 & -4.852432 & -0.386200 & 0.934949 \\
\hline 19 & 1 & 0 & -2.989140 & 1.344681 & -1.772921 \\
\hline 20 & 1 & 0 & -2.854972 & 2.149269 & -0.188881 \\
\hline 21 & 1 & 0 & -5.086526 & 1.830968 & -0.832326 \\
\hline 22 & 1 & 0 & -2.151258 & -1.116256 & -1.923805 \\
\hline 23 & 1 & 0 & -3.815819 & -1.171718 & -1.321910 \\
\hline 24 & 1 & 0 & -3.119796 & -2.584449 & 0.263114 \\
\hline 25 & 1 & 0 & -0.667961 & 1.412238 & 0.027842 \\
\hline 26 & 7 & 0 & -1.080057 & 0.391510 & -0.091672 \\
\hline 27 & 8 & 0 & -4.191462 & -0.954023 & 1.348241 \\
\hline 28 & 8 & 0 & -4.608728 & 1.073089 & -0.489157 \\
\hline 29 & 8 & 0 & -2.439993 & -2.417852 & -0.400734 \\
\hline 30 & 8 & 0 & 0.482409 & 2.267353 & 0.133555 \\
\hline
\end{tabular}




$\begin{array}{rrrrrr}31 & 8 & 0 & 4.624948 & -1.362341 & -0.060344 \\ 32 & 6 & 0 & 5.955735 & -0.905980 & 0.013873 \\ 33 & 1 & 0 & 6.150698 & -0.388218 & 0.959303 \\ 34 & 1 & 0 & 6.582658 & -1.793679 & -0.041624 \\ 35 & 1 & 0 & 6.199495 & -0.240509 & -0.821468 \\ -\end{array}$

\section{Structure of $\mathbf{4 2}$}

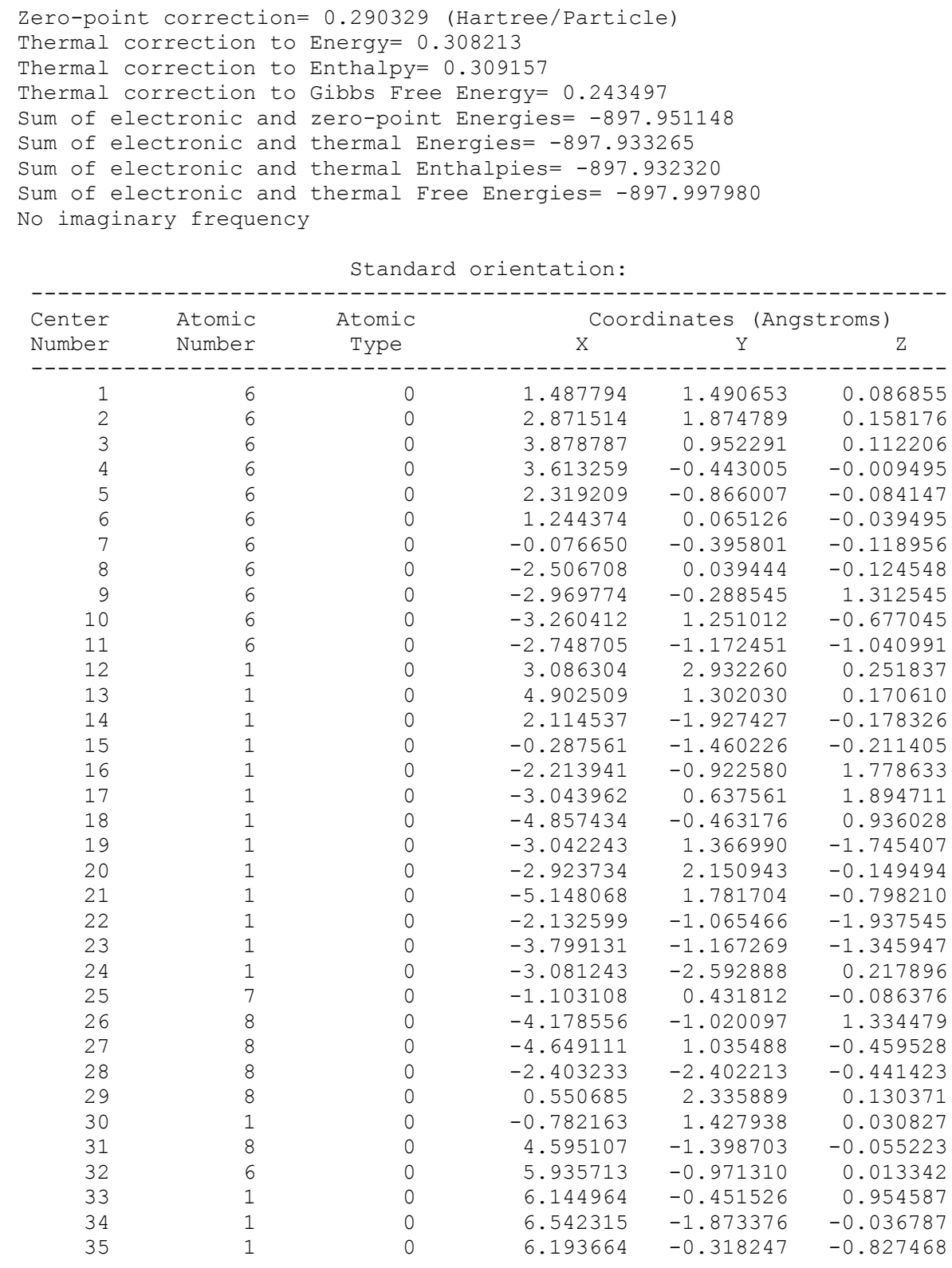

\section{$\underline{\text { Structure of } 13 \text { (open form) }}$}

Zero-point correction $=0.262222$ (Hartree/Particle)

Thermal correction to Energy $=0.279162$

Thermal correction to Enthalpy= 0.280107

Thermal correction to Gibbs Free Energy= 0.217635

Sum of electronic and zero-point Energies $=-858.685482$

Sum of electronic and thermal Energies $=-858.668541$

Sum of electronic and thermal Enthalpies= -858.667597

Sum of electronic and thermal Free Energies= -858.730068

No imaginary fregqencies 
Standard orientation:

\begin{tabular}{|c|c|c|c|c|c|}
\hline \multirow{2}{*}{$\begin{array}{l}\text { Center } \\
\text { Number }\end{array}$} & \multirow{2}{*}{$\begin{array}{l}\text { Atomic } \\
\text { Number }\end{array}$} & \multirow{2}{*}{$\begin{array}{c}\text { Atomic } \\
\text { Type }\end{array}$} & \multicolumn{3}{|c|}{ Coordinates (Angstroms) } \\
\hline & & & $\mathrm{X}$ & Y & Z \\
\hline & & & & & \\
\hline 1 & 6 & 0 & 2.130484 & 1.354898 & 0.084520 \\
\hline 2 & 6 & 0 & 3.507591 & 1.521483 & 0.187628 \\
\hline 3 & 6 & 0 & 4.374287 & 0.435223 & 0.138190 \\
\hline 4 & 6 & 0 & 3.864741 & -0.846666 & -0.021646 \\
\hline 5 & 6 & 0 & 2.492235 & -1.019485 & -0.128523 \\
\hline 6 & 6 & 0 & 1.606694 & 0.058270 & -0.072105 \\
\hline 7 & 6 & 0 & 0.171773 & -0.269237 & -0.183368 \\
\hline 8 & 6 & 0 & -2.150393 & 0.105479 & -0.117045 \\
\hline 9 & 6 & 0 & -2.633371 & -0.156877 & 1.326536 \\
\hline 10 & 6 & 0 & -2.910951 & 1.290557 & -0.717998 \\
\hline 11 & 6 & 0 & -2.400546 & -1.141416 & -0.983230 \\
\hline 12 & 1 & 0 & 3.913801 & 2.521191 & 0.307779 \\
\hline 13 & 1 & 0 & 5.444421 & 0.595733 & 0.221946 \\
\hline 14 & 1 & 0 & 2.104635 & -2.024662 & -0.252800 \\
\hline 15 & 1 & 0 & -0.020038 & -1.326368 & -0.402265 \\
\hline 16 & 1 & 0 & -1.897435 & -0.788620 & 1.826099 \\
\hline 17 & 1 & 0 & -2.692636 & 0.793522 & 1.868483 \\
\hline 18 & 1 & 0 & -4.508660 & -0.322362 & 0.894474 \\
\hline 19 & 1 & 0 & -2.651829 & 1.394486 & -1.779054 \\
\hline 20 & 1 & 0 & -2.599847 & 2.194810 & -0.188205 \\
\hline 21 & 1 & 0 & -4.798557 & 1.811054 & -0.915543 \\
\hline 22 & 1 & 0 & -1.771760 & -1.087227 & -1.876441 \\
\hline 23 & 1 & 0 & -3.445013 & -1.132741 & -1.310473 \\
\hline 24 & 1 & 0 & -2.766687 & -2.488890 & 0.342756 \\
\hline 25 & 1 & 0 & 1.805079 & 3.221576 & 0.224681 \\
\hline 26 & 7 & 0 & -0.767081 & 0.566425 & -0.018179 \\
\hline 27 & 8 & 0 & -3.862453 & -0.859816 & 1.367946 \\
\hline 28 & 8 & 0 & -4.312654 & 1.063114 & -0.563494 \\
\hline 29 & 8 & 0 & -2.092447 & -2.359852 & -0.334621 \\
\hline 30 & 8 & 0 & 1.287549 & 2.418361 & 0.121804 \\
\hline 31 & 8 & 0 & 4.650685 & -1.962245 & -0.082820 \\
\hline 32 & 1 & 0 & 5.573397 & -1.716344 & 0.017540 \\
\hline
\end{tabular}

\section{$\underline{\text { Structure of } 13 \text { (closed form) }}$}

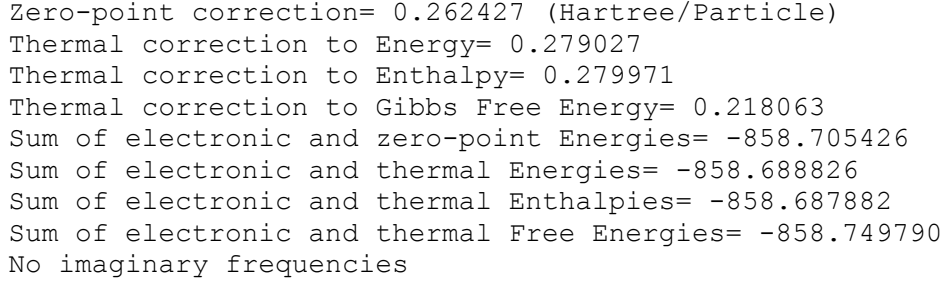

Standard orientation:

\begin{tabular}{|c|c|c|c|c|c|}
\hline \multirow{2}{*}{$\begin{array}{l}\text { Center } \\
\text { Number }\end{array}$} & \multirow{2}{*}{$\begin{array}{l}\text { Atomic } \\
\text { Number }\end{array}$} & \multirow{2}{*}{$\begin{array}{c}\text { Atomic } \\
\text { Type }\end{array}$} & \multicolumn{3}{|c|}{ Coordinates (Angstroms) } \\
\hline & & & $\mathrm{X}$ & $\mathrm{Y}$ & Z \\
\hline 1 & 6 & 0 & -2.031393 & -1.312132 & 0.113014 \\
\hline 2 & 6 & 0 & -3.394594 & -1.595931 & 0.186632 \\
\hline 3 & 6 & 0 & -4.332214 & -0.578146 & 0.112739 \\
\hline 4 & 6 & 0 & -3.930407 & 0.750084 & -0.035912 \\
\hline 5 & 6 & 0 & -2.581578 & 1.044151 & -0.111801 \\
\hline 6 & 6 & 0 & -1.621455 & 0.027610 & -0.040438 \\
\hline 7 & 6 & 0 & -0.207750 & 0.390994 & -0.130265 \\
\hline 8 & 6 & 0 & 2.122579 & -0.086778 & -0.135769 \\
\hline 9 & 6 & 0 & 2.615793 & 0.116383 & 1.314327 \\
\hline 10 & 6 & 0 & 2.843237 & -1.273845 & -0.780081 \\
\hline 11 & 6 & 0 & 2.402249 & 1.180484 & -0.962816 \\
\hline 12 & 1 & 0 & -3.699575 & -2.628252 & 0.303229 \\
\hline 13 & 1 & 0 & -5.389678 & -0.818141 & 0.171779 \\
\hline 14 & 1 & 0 & -2.272412 & 2.077147 & -0.227765 \\
\hline 15 & 1 & 0 & 0.014049 & 1.456468 & -0.249723 \\
\hline 16 & 1 & 0 & 1.898489 & 0.751144 & 1.836757 \\
\hline 17 & 1 & 0 & 2.652301 & -0.851865 & 1.826954 \\
\hline
\end{tabular}




$\begin{array}{rrrrrr}18 & 1 & 0 & 4.498849 & 0.239367 & 0.897003 \\ 19 & 1 & 0 & 2.579005 & -1.332440 & -1.843300 \\ 20 & 1 & 0 & 2.509710 & -2.189998 & -0.282806 \\ 21 & 1 & 0 & 4.714173 & -1.839537 & -0.999857 \\ 22 & 1 & 0 & 1.767729 & 1.171039 & -1.853360 \\ 23 & 1 & 0 & 3.444897 & 1.153508 & -1.293735 \\ 24 & 1 & 0 & 2.817110 & 2.480444 & 0.397248 \\ 25 & 1 & 0 & -0.253556 & -1.943743 & 0.131652 \\ 26 & 7 & 0 & 0.718454 & -0.487385 & -0.065728 \\ 27 & 8 & 0 & 3.861112 & 0.784944 & 1.372412 \\ 28 & 8 & 0 & 4.247580 & -1.093780 & -0.617393 \\ 29 & 8 & 0 & 2.128071 & 2.381433 & -0.270478 \\ 30 & 8 & 0 & -1.157548 & -2.329727 & 0.191085 \\ 31 & 8 & 0 & -4.817044 & 1.788731 & -0.112874 \\ 32 & 1 & 0 & -5.714446 & 1.456482 & -0.033797 \\ -------1.0374\end{array}$

\section{$\underline{\text { Structure of } \mathbf{T S}_{13 / 43}}$}

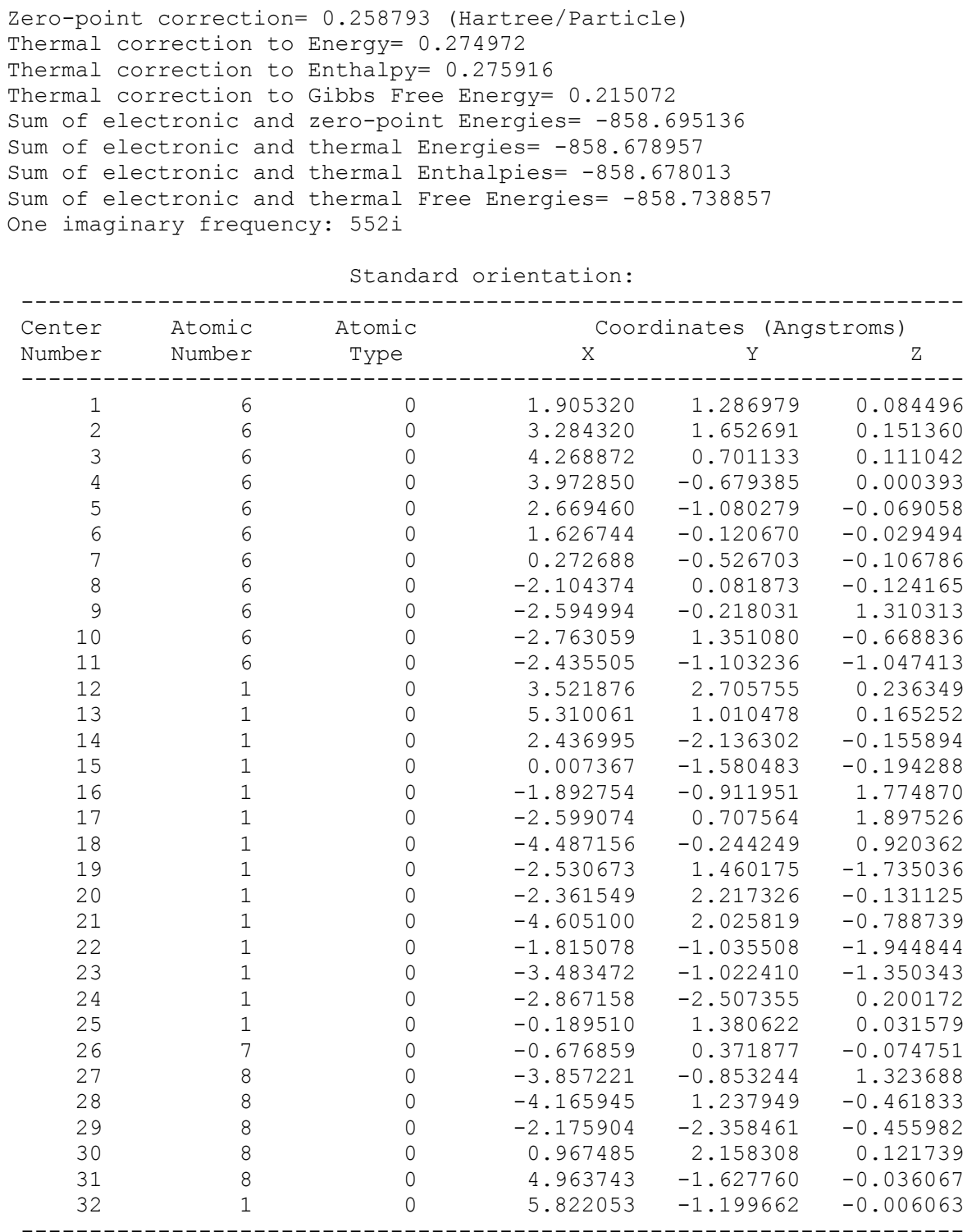

\section{Structure of $\mathbf{4 3}$}

Zero-point correction $=0.261356$ (Hartree/Particle)

Thermal correction to Energy $=0.278335$ 
Thermal correction to Enthalpy $=0.279279$

Thermal correction to Gibbs Free Energy= 0.214711

Sum of electronic and zero-point Energies= -858.693043

Sum of electronic and thermal Energies= -858.676064

Sum of electronic and thermal Enthalpies= -858.675119

Sum of electronic and thermal Free Energies= -858.739688

No imaginary frequencies

Standard orientation:

\begin{tabular}{|c|c|c|c|c|c|}
\hline \multirow{2}{*}{$\begin{array}{l}\text { Center } \\
\text { Number }\end{array}$} & \multirow{2}{*}{$\begin{array}{l}\text { Atomic } \\
\text { Number }\end{array}$} & \multirow{2}{*}{$\begin{array}{c}\text { Atomic } \\
\text { Type }\end{array}$} & \multicolumn{3}{|c|}{ Coordinates (Angstroms) } \\
\hline & & & $\mathrm{X}$ & Y & Z \\
\hline 1 & 6 & 0 & 1.951517 & 1.331485 & 0.089239 \\
\hline 2 & 6 & 0 & 3.360874 & 1.639054 & 0.145105 \\
\hline 3 & 6 & 0 & 4.302788 & 0.656327 & 0.099770 \\
\hline 4 & 6 & 0 & 3.953849 & -0.722056 & -0.003234 \\
\hline 5 & 6 & 0 & 2.643854 & -1.076534 & -0.062320 \\
\hline 6 & 6 & 0 & 1.622620 & -0.078852 & -0.021087 \\
\hline 7 & 6 & 0 & 0.279934 & -0.461927 & -0.096759 \\
\hline 8 & 6 & 0 & -2.127432 & 0.094781 & -0.119529 \\
\hline 9 & 6 & 0 & -2.616765 & -0.227978 & 1.309814 \\
\hline 10 & 6 & 0 & -2.816972 & 1.348305 & -0.662346 \\
\hline 11 & 6 & 0 & -2.422888 & -1.092119 & -1.053260 \\
\hline 12 & 1 & 0 & 3.636352 & 2.683381 & 0.224581 \\
\hline 13 & 1 & 0 & 5.356335 & 0.924096 & 0.143257 \\
\hline 14 & 1 & 0 & 2.375164 & -2.124407 & -0.143199 \\
\hline 15 & 1 & 0 & 0.009178 & -1.512922 & -0.184808 \\
\hline 16 & 1 & 0 & -1.897428 & -0.905572 & 1.772440 \\
\hline 17 & 1 & 0 & -2.648151 & 0.692910 & 1.904016 \\
\hline 18 & 1 & 0 & -4.507181 & -0.302586 & 0.914588 \\
\hline 19 & 1 & 0 & -2.586807 & 1.464772 & -1.728154 \\
\hline 20 & 1 & 0 & -2.439683 & 2.225097 & -0.123194 \\
\hline 21 & 1 & 0 & -4.675246 & 1.973601 & -0.787784 \\
\hline 22 & 1 & 0 & -1.796731 & -1.003657 & -1.944787 \\
\hline 23 & 1 & 0 & -3.470054 & -1.032380 & -1.363508 \\
\hline 24 & 1 & 0 & -2.832694 & -2.512723 & 0.182716 \\
\hline 25 & 7 & 0 & -0.706289 & 0.417267 & -0.070248 \\
\hline 26 & 8 & 0 & -3.861346 & -0.897354 & 1.313780 \\
\hline 27 & 8 & 0 & -4.215672 & 1.200012 & -0.454795 \\
\hline 28 & 8 & 0 & -2.140710 & -2.344818 & -0.468143 \\
\hline 29 & 8 & 0 & 1.073222 & 2.232444 & 0.131733 \\
\hline 30 & 1 & 0 & -0.351283 & 1.393627 & 0.041518 \\
\hline 31 & 8 & 0 & 4.919057 & -1.696749 & -0.046906 \\
\hline 32 & 1 & 0 & 5.787583 & -1.295299 & 0.026352 \\
\hline
\end{tabular}

\section{Structure of $\mathbf{1 4}$ (open form)}

Zero-point correction= 0.260752 (Hartree/Particle)

Thermal correction to Energy $=0.279009$

Thermal correction to Enthalpy $=0.279953$

Thermal correction to Gibbs Free Energy $=0.212666$

Sum of electronic and zero-point Energies= $\mathbf{- 9 8 7 . 9 5 5 0 7 6}$

Sum of electronic and thermal Energies= -987.936819

Sum of electronic and thermal Enthalpies= -987.935875

Sum of electronic and thermal Free Energies= -988.003162

No imaginary frequencies

Standard orientation:

\begin{tabular}{|c|c|c|c|c|c|}
\hline \multirow{2}{*}{$\begin{array}{l}\text { Center } \\
\text { Number }\end{array}$} & \multirow{2}{*}{$\begin{array}{l}\text { Atomic } \\
\text { Number }\end{array}$} & \multirow{2}{*}{$\begin{array}{c}\text { Atomic } \\
\text { Type }\end{array}$} & \multicolumn{3}{|c|}{ Coordinates (Angstroms) } \\
\hline & & & $\mathrm{X}$ & $\mathrm{Y}$ & Z \\
\hline 1 & 6 & 0 & -1.529699 & -0.977977 & 0.017834 \\
\hline 2 & 6 & 0 & -2.919247 & -1.038275 & 0.086961 \\
\hline 3 & 6 & 0 & -3.652316 & 0.132535 & 0.007129 \\
\hline 4 & 6 & 0 & -3.067535 & 1.376906 & -0.143764 \\
\hline 5 & 6 & 0 & -1.683279 & 1.417840 & -0.213360 \\
\hline 6 & 6 & 0 & -0.890027 & 0.269793 & -0.131637 \\
\hline 7 & 6 & 0 & 0.572018 & 0.464295 & -0.210618 \\
\hline 8 & 6 & 0 & 2.843146 & -0.151556 & -0.117178 \\
\hline 9 & 6 & 0 & 3.317960 & 0.027316 & 1. 343452 \\
\hline 10 & 6 & 0 & 3.491849 & -1.396015 & -0.729631 \\
\hline
\end{tabular}




\begin{tabular}{|c|c|c|c|c|c|}
\hline 11 & 6 & 0 & 3.235915 & 1.082560 & -0.946898 \\
\hline 12 & 1 & 0 & -3.435192 & -1.984010 & 0.202207 \\
\hline 13 & 1 & 0 & -3.678348 & 2.266249 & -0.201531 \\
\hline 14 & 1 & 0 & -1.187153 & 2.374829 & -0.332484 \\
\hline 15 & 1 & 0 & 0.867928 & 1.503419 & -0.397189 \\
\hline 16 & 1 & 0 & 2.635224 & 0.713184 & 1.847286 \\
\hline 17 & 1 & 0 & 3.276244 & -0.937813 & 1.860138 \\
\hline 18 & 1 & 0 & 5.212198 & 0.019475 & 0.963033 \\
\hline 19 & 1 & 0 & 3.252201 & -1.448632 & -1.799020 \\
\hline 20 & 1 & 0 & 3.075558 & -2.275545 & -0.231083 \\
\hline 21 & 1 & 0 & 5.323628 & -2.094825 & -0.901367 \\
\hline 22 & 1 & 0 & 2.627467 & 1.108803 & -1.855370 \\
\hline 23 & 1 & 0 & 4.282129 & 0.978610 & -1.250401 \\
\hline 24 & 1 & 0 & 3.698654 & 2.357586 & 0.419857 \\
\hline 25 & 1 & 0 & -1.365927 & -2.862219 & 0.186213 \\
\hline 26 & 7 & 0 & 1.417742 & -0.465807 & -0.055210 \\
\hline 27 & 8 & 0 & 4.604906 & 0.609804 & 1.424957 \\
\hline 28 & 8 & 0 & 4.902774 & -1.315428 & -0.53361 \\
\hline 29 & 8 & 0 & 3.029853 & 2.308945 & -0.273696 \\
\hline 30 & 8 & 0 & -0.787104 & -2.100528 & 0.085012 \\
\hline 31 & 7 & 0 & -5.126868 & 0.037193 & 0.086971 \\
\hline 32 & 8 & 0 & -5.756522 & 1.068726 & 0.018992 \\
\hline 33 & 8 & 0 & -5.610588 & -1.066936 & 0.215052 \\
\hline
\end{tabular}

\section{$\underline{\text { Structure of } 14 \text { (closed form) }}$}

Zero-point correction= 0.261735 (Hartree/Particle) Thermal correction to Energy $=0.279335$

Thermal correction to Enthalpy $=0.280279$

Thermal correction to Gibbs Free Energy $=0.215151$

Sum of electronic and zero-point Energies= -987.974097

Sum of electronic and thermal Energies= -987.956497

Sum of electronic and thermal Enthalpies= -987.955553

Sum of electronic and thermal Free Energies= -988.020681

No imaginary frequencies

Standard orientation:

\begin{tabular}{|c|c|c|c|c|c|}
\hline \multirow{2}{*}{$\begin{array}{l}\text { Center } \\
\text { Number }\end{array}$} & \multirow{2}{*}{$\begin{array}{l}\text { Atomic } \\
\text { Number }\end{array}$} & \multirow{2}{*}{$\begin{array}{c}\text { Atomic } \\
\text { Type }\end{array}$} & \multicolumn{3}{|c|}{ Coordinates (Angstroms) } \\
\hline & & & $\mathrm{X}$ & $\mathrm{Y}$ & Z \\
\hline 1 & 6 & 0 & -1.462964 & -0.889867 & 0.045313 \\
\hline 2 & 6 & 0 & -2.851084 & -1.034822 & 0.105049 \\
\hline 3 & 6 & 0 & -3.636090 & 0.095101 & 0.007628 \\
\hline 4 & 6 & 0 & -3.126330 & 1.379556 & -0.147535 \\
\hline 5 & 6 & 0 & -1.751286 & 1.509492 & -0.205449 \\
\hline 6 & 6 & 0 & -0.905355 & 0.398471 & -0.111811 \\
\hline 7 & 6 & 0 & 0.543673 & 0.599204 & -0.176985 \\
\hline 8 & 6 & 0 & 2.800226 & -0.153155 & -0.134157 \\
\hline 9 & 6 & 0 & 3.278251 & -0.033758 & 1.332077 \\
\hline 10 & 6 & 0 & 3.393795 & -1.405757 & -0.783998 \\
\hline 11 & 6 & 0 & 3.242076 & 1.087366 & -0.929132 \\
\hline 12 & 1 & 0 & -3.291439 & -2.015019 & 0.225391 \\
\hline 13 & 1 & 0 & -3.793637 & 2.225964 & -0.216184 \\
\hline 14 & 1 & 0 & -1.308046 & 2.492300 & -0.325549 \\
\hline 15 & 1 & 0 & 0.889275 & 1.632175 & -0.283878 \\
\hline 16 & 1 & 0 & 2.620627 & 0.662497 & 1.854736 \\
\hline 17 & 1 & 0 & 3.200691 & -1.010676 & 1.823020 \\
\hline 18 & 1 & 0 & 5.174665 & -0.107699 & 0.966525 \\
\hline 19 & 1 & 0 & 3.160396 & -1.412671 & -1.855930 \\
\hline 20 & 1 & 0 & 2.938940 & -2.285917 & -0.318636 \\
\hline 21 & 1 & 0 & 5.196354 & -2.170667 & -0.972571 \\
\hline 22 & 1 & 0 & 2.637787 & 1.159703 & -1.837868 \\
\hline 23 & 1 & 0 & 4.284734 & 0.951244 & -1.230836 \\
\hline 24 & 1 & 0 & 3.754786 & 2.314659 & 0.464820 \\
\hline 25 & 1 & 0 & 0.236498 & -1.700116 & 0.085538 \\
\hline 26 & 7 & 0 & 1.356405 & -0.382601 & -0.099459 \\
\hline 27 & 8 & 0 & 4.583588 & 0.498502 & 1.428691 \\
\hline 28 & 8 & 0 & 4.801580 & -1.393366 & -0.572114 \\
\hline 29 & 8 & 0 & 3.076749 & 2.298515 & -0.221236 \\
\hline 30 & 8 & 0 & -0.709049 & -1.985274 & 0.140341 \\
\hline 31 & 7 & 0 & -5.108720 & -0.074735 & 0.074224 \\
\hline 32 & 8 & 0 & -5.787136 & 0.926695 & 0.012284 \\
\hline
\end{tabular}




\section{$\underline{\text { Structure of } \mathbf{T S}} \underline{\mathbf{1}}_{\mathbf{1 / 4 4}}$}

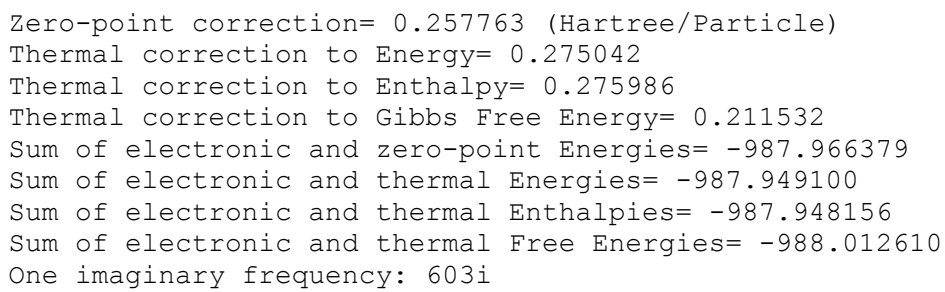

Standard orientation:

\begin{tabular}{|c|c|c|c|c|c|}
\hline \multirow{2}{*}{$\begin{array}{l}\text { Center } \\
\text { Number }\end{array}$} & \multirow{2}{*}{$\begin{array}{l}\text { Atomic } \\
\text { Number }\end{array}$} & \multirow{2}{*}{$\begin{array}{l}\text { Atomic } \\
\text { Type }\end{array}$} & \multicolumn{3}{|c|}{ Coordinates (Angstroms) } \\
\hline & & & $\mathrm{X}$ & Y & Z \\
\hline 1 & 6 & 0 & -1.368662 & -0.821522 & 0.034028 \\
\hline 2 & 6 & 0 & -2.781824 & -1.020448 & 0.098274 \\
\hline 3 & 6 & 0 & -3.608980 & 0.063193 & 0.016831 \\
\hline 4 & 6 & 0 & -3.178052 & 1.395766 & -0.127126 \\
\hline 5 & 6 & 0 & -1.825918 & 1.609366 & -0.190002 \\
\hline 6 & 6 & 0 & -0.913005 & 0.536195 & -0.113084 \\
\hline 7 & 6 & 0 & 0.491120 & 0.766796 & -0.177220 \\
\hline 8 & 6 & 0 & 2.763623 & -0.153745 & -0.129016 \\
\hline 9 & 6 & 0 & 3.256920 & -0.043710 & 1.333277 \\
\hline 10 & 6 & 0 & 3.254021 & -1.455560 & -0.767816 \\
\hline 11 & 6 & 0 & 3.271838 & 1.044544 & -0.945129 \\
\hline 12 & 1 & 0 & -3.173811 & -2.021939 & 0.207970 \\
\hline 13 & 1 & 0 & -3.899920 & 2.196028 & -0.183422 \\
\hline 14 & 1 & 0 & -1.439638 & 2.617477 & -0.300842 \\
\hline 15 & 1 & 0 & 0.887983 & 1.777497 & -0.274471 \\
\hline 16 & 1 & 0 & 2.638727 & 0.690419 & 1.851858 \\
\hline 17 & 1 & 0 & 3.133430 & -1.010327 & 1.835280 \\
\hline 18 & 1 & 0 & 5.146983 & -0.232507 & 0.978852 \\
\hline 19 & 1 & 0 & 3.036106 & -1.447178 & -1.842552 \\
\hline 20 & 1 & 0 & 2.725043 & -2.297801 & -0.307891 \\
\hline 21 & 1 & 0 & 4.994650 & -2.354658 & -0.925636 \\
\hline 22 & 1 & 0 & 2.681268 & 1.125641 & -1.861792 \\
\hline 23 & 1 & 0 & 4.310201 & 0.850626 & -1.227889 \\
\hline 24 & 1 & 0 & 3.834745 & 2.281433 & 0.421896 \\
\hline 25 & 1 & 0 & 0.675964 & -1.184681 & 0.013007 \\
\hline 26 & 7 & 0 & 1.307147 & -0.244268 & -0.110769 \\
\hline 27 & 8 & 0 & 4.586841 & 0.422360 & 1.411895 \\
\hline 28 & 8 & 0 & 4.652174 & -1.550072 & -0.530346 \\
\hline 29 & 8 & 0 & 3.151650 & 2.271719 & -0.259339 \\
\hline 30 & 8 & 0 & -0.548797 & -1.797866 & 0.103424 \\
\hline 31 & 7 & 0 & -5.077170 & -0.181055 & 0.085945 \\
\hline 32 & 8 & 0 & -5.802091 & 0.789107 & 0.057615 \\
\hline 33 & 8 & 0 & -5.455784 & -1.327501 & 0.165663 \\
\hline
\end{tabular}

\section{$\underline{\text { Structure of } 44}$}

Zero-point correction= 0.260782 (Hartree/Particle)

Thermal correction to Energy $=0.278575$

Thermal correction to Enthalpy $=0.279520$

Thermal correction to Gibbs Free Energy $=0.213648$

Sum of electronic and zero-point Energies= -987.964078

Sum of electronic and thermal Energies= -987.946284

Sum of electronic and thermal Enthalpies= -987.945340

Sum of electronic and thermal Free Energies= -988.011212

No imaginary frequencies

Standard orientation:

$\begin{array}{llll}\text { Center } & \text { Atomic } & \text { Atomic } & \text { Coordinates (Angstroms) } \\ \text { Number } & \text { Number } & \text { Type } & \text { X }\end{array}$




\begin{tabular}{|c|c|c|c|c|c|}
\hline 1 & 6 & 0 & -1.409299 & -0.906465 & 0.025894 \\
\hline 2 & 6 & 0 & -2.847692 & -1.041597 & 0.094096 \\
\hline 3 & 6 & 0 & -3.629210 & 0.065649 & 0.015727 \\
\hline 4 & 6 & 0 & -3.148320 & 1.391840 & -0.129487 \\
\hline 5 & 6 & 0 & -1.797271 & 1.557159 & -0.195041 \\
\hline 6 & 6 & 0 & -0.908280 & 0.449955 & -0.121074 \\
\hline 7 & 6 & 0 & 0.477137 & 0.669416 & -0.183084 \\
\hline 8 & 6 & 0 & 2.800890 & -0.164450 & -0.126350 \\
\hline 9 & 6 & 0 & 3.274360 & 0.001808 & 1.336516 \\
\hline 10 & 6 & 0 & 3.357784 & -1.452645 & -0.737229 \\
\hline 11 & 6 & 0 & 3.259745 & 1.039162 & -0.965238 \\
\hline 12 & 1 & 0 & -3.275379 & -2.028631 & 0.204573 \\
\hline 13 & 1 & 0 & -3.843349 & 2.215499 & -0.182320 \\
\hline 14 & 1 & 0 & -1.378043 & 2.552252 & -0.306032 \\
\hline 15 & 1 & 0 & 0.870137 & 1.680756 & -0.273647 \\
\hline 16 & 1 & 0 & 2.616961 & 0.719062 & 1.830365 \\
\hline 17 & 1 & 0 & 3.188736 & -0.956998 & 1.861329 \\
\hline 18 & 1 & 0 & 5.175972 & -0.109389 & 1.009389 \\
\hline 19 & 1 & 0 & 3.155949 & -1.469549 & -1.814899 \\
\hline 20 & 1 & 0 & 2.862017 & -2.313225 & -0.272878 \\
\hline 21 & 1 & 0 & 5.140137 & -2.271718 & -0.857286 \\
\hline 22 & 1 & 0 & 2.667874 & 1.077576 & -1.883544 \\
\hline 23 & 1 & 0 & 4.305874 & 0.881769 & -1.241620 \\
\hline 24 & 1 & 0 & 3.769129 & 2.324825 & 0.378403 \\
\hline 25 & 7 & 0 & 1.347645 & -0.315008 & -0.128480 \\
\hline 26 & 8 & 0 & 4.580690 & 0.529702 & 1.418324 \\
\hline 27 & 8 & 0 & 4.753582 & -1.480112 & -0.476526 \\
\hline 28 & 8 & 0 & 3.089145 & 2.273491 & -0.304295 \\
\hline 29 & 8 & 0 & -0.652089 & -1.902296 & 0.092354 \\
\hline 30 & 1 & 0 & 0.884895 & -1.242132 & -0.006564 \\
\hline 31 & 7 & 0 & -5.106593 & -0.122718 & 0.090445 \\
\hline 32 & 8 & 0 & -5.529962 & -1.253527 & 0.164884 \\
\hline 33 & 8 & 0 & -5.792285 & 0.875866 & 0.071834 \\
\hline
\end{tabular}

\section{$\underline{\text { Structure of } 15 \text { (open form) }}$}

Zero-point correction= 0.250042 (Hartree/Particle)
Thermal correction to Energy= 0.266472
Thermal correction to Enthalpy= 0.267416
Thermal correction to Gibbs Free Energy= 0.205373
Sum of electronic and zero-point Energies= -882.718907
Sum of electronic and thermal Energies= -882.702477
Sum of electronic and thermal Enthalpies=-882.701533
Sum of electronic and thermal Free Energies= -882.763576
No imaginary frequencies

Standard orientation:

\begin{tabular}{|c|c|c|c|c|c|}
\hline \multirow{2}{*}{$\begin{array}{l}\text { Center } \\
\text { Number }\end{array}$} & \multirow{2}{*}{$\begin{array}{l}\text { Atomic } \\
\text { Number }\end{array}$} & \multirow{2}{*}{$\begin{array}{c}\text { Atomic } \\
\text { Type }\end{array}$} & \multicolumn{3}{|c|}{ Coordinates (Angstroms) } \\
\hline & & & $\mathrm{X}$ & Y & $\mathrm{Z}$ \\
\hline 1 & 6 & 0 & -2.176807 & -0.974714 & 0.077572 \\
\hline 2 & 6 & 0 & -3.568095 & -1.029578 & 0.150880 \\
\hline 3 & 6 & 0 & -4.299171 & 0.138449 & 0.048008 \\
\hline 4 & 6 & 0 & -3.708687 & 1.375237 & -0.129917 \\
\hline 5 & 6 & 0 & -2.324046 & 1.409537 & -0.201312 \\
\hline 6 & 6 & 0 & -1.529102 & 0.263914 & -0.099397 \\
\hline 7 & 6 & 0 & -0.070186 & 0.451444 & -0.183650 \\
\hline 8 & 6 & 0 & 2.204260 & -0.154354 & -0.128265 \\
\hline 9 & 6 & 0 & 2.693211 & 0.015095 & 1.327268 \\
\hline 10 & 6 & 0 & 2.853662 & -1.389337 & -0.758468 \\
\hline 11 & 6 & 0 & 2.585351 & 1.087848 & -0.952803 \\
\hline 12 & 1 & 0 & -4.087368 & -1.971361 & 0.288322 \\
\hline 13 & 1 & 0 & -4.317391 & 2.265781 & -0.204670 \\
\hline 14 & 1 & 0 & -1.826663 & 2.363708 & -0.339184 \\
\hline 15 & 1 & 0 & 0.226393 & 1.494021 & -0.352462 \\
\hline 16 & 1 & 0 & 2.015880 & 0.698504 & 1.841658 \\
\hline 17 & 1 & 0 & 2.654372 & -0.953019 & 1.838837 \\
\hline 18 & 1 & 0 & 4.583317 & 0.010648 & 0.924746 \\
\hline 19 & 1 & 0 & 2.600894 & -1.434082 & -1.825188 \\
\hline 20 & 1 & 0 & 2.447733 & -2.274967 & -0.262352 \\
\hline 21 & 1 & 0 & 4.684768 & -2.083805 & -0.954957 \\
\hline 22 & 1 & 0 & 1.959606 & 1.124589 & -1.848941 \\
\hline
\end{tabular}




\begin{tabular}{rrrrrr}
23 & 1 & 0 & 3.625633 & 0.984447 & -1.277065 \\
24 & 1 & 0 & 3.072735 & 2.342481 & 0.422988 \\
25 & 1 & 0 & -2.023987 & -2.854641 & 0.292968 \\
26 & 7 & 0 & 0.781435 & -0.479481 & -0.058010 \\
27 & 8 & 0 & 3.983232 & 0.595101 & 1.403241 \\
28 & 8 & 0 & 4.267897 & -1.305753 & -0.580161 \\
29 & 8 & 0 & 2.397603 & 2.309775 & -0.265318 \\
30 & 8 & 0 & -1.439838 & -2.100696 & 0.170686 \\
31 & 9 & 0 & -5.635496 & 0.055045 & 0.126033 \\
\hline
\end{tabular}

\title{
$\underline{\text { Structure of } 15 \text { (closed form) }}$
}

\begin{abstract}
Zero-point correction $=0.250870$ (Hartree/Particle)
Thermal correction to Energy $=0.266685$

Thermal correction to Enthalpy $=0.267629$

Thermal correction to Gibbs Free Energy $=0.207532$

Sum of electronic and zero-point Energies= -882.739529

Sum of electronic and thermal Energies= -882.723714

Sum of electronic and thermal Enthalpies $=-882.722770$

Sum of electronic and thermal Free Energies $=-882.782867$

No imaginary frequencies
\end{abstract}

\begin{tabular}{|c|c|c|c|c|c|}
\hline \multirow{2}{*}{$\begin{array}{l}\text { Center } \\
\text { Number }\end{array}$} & \multirow{2}{*}{$\begin{array}{l}\text { Atomic } \\
\text { Number }\end{array}$} & \multirow{2}{*}{$\begin{array}{c}\text { Atomic } \\
\text { Type }\end{array}$} & \multicolumn{3}{|c|}{ Coordinates (Angstroms) } \\
\hline & & & $\mathrm{X}$ & $\mathrm{Y}$ & Z \\
\hline & & & ----------- & ----------- & ---------- \\
\hline 1 & 6 & 0 & -2.095962 & -0.929443 & 0.082573 \\
\hline 2 & 6 & 0 & -3.481080 & -1.086815 & 0.157858 \\
\hline 3 & 6 & 0 & -4.278846 & 0.031952 & 0.053992 \\
\hline 4 & 6 & 0 & -3.779827 & 1.317467 & -0.122913 \\
\hline 5 & 6 & 0 & -2.406950 & 1.460560 & -0.194427 \\
\hline 6 & 6 & 0 & -1.545654 & 0.361328 & -0.095352 \\
\hline 7 & 6 & 0 & -0.104656 & 0.570209 & -0.171705 \\
\hline 8 & 6 & 0 & 2.162921 & -0.149347 & -0.135818 \\
\hline 9 & 6 & 0 & 2.648160 & 0.005195 & 1.323276 \\
\hline 10 & 6 & 0 & 2.771719 & -1.404021 & -0.766812 \\
\hline 11 & 6 & 0 & 2.584613 & 1.081708 & -0.957378 \\
\hline 12 & 1 & 0 & -3.912563 & -2.069231 & 0.295819 \\
\hline 13 & 1 & 0 & -4.458534 & 2.155754 & -0.196312 \\
\hline 14 & 1 & 0 & -1.975416 & 2.446928 & -0.329470 \\
\hline 15 & 1 & 0 & 0.231140 & 1.605443 & -0.291214 \\
\hline 16 & 1 & 0 & 1.987094 & 0.706849 & 1.834284 \\
\hline 17 & 1 & 0 & 2.580623 & -0.961670 & 1.835303 \\
\hline 18 & 1 & 0 & 4.542298 & -0.056643 & 0.942740 \\
\hline 19 & 1 & 0 & 2.529442 & -1.433633 & -1.836398 \\
\hline 20 & 1 & 0 & 2.333521 & -2.281925 & -0.281872 \\
\hline 21 & 1 & 0 & 4.582600 & -2.149595 & -0.956627 \\
\hline 22 & 1 & 0 & 1.966729 & 1.134446 & -1.858102 \\
\hline 23 & 1 & 0 & 3.624289 & 0.949135 & -1.271627 \\
\hline 24 & 1 & 0 & 3.102269 & 2.330436 & 0.414516 \\
\hline 25 & 1 & 0 & -0.386009 & -1.718441 & 0.116516 \\
\hline 26 & 7 & 0 & 0.723547 & -0.401428 & -0.090775 \\
\hline 27 & 8 & 0 & 3.951072 & 0.549628 & 1.404572 \\
\hline 28 & 8 & 0 & 4.182136 & -1.369201 & -0.568292 \\
\hline 29 & 8 & 0 & 2.421650 & 2.304871 & -0.268743 \\
\hline 30 & 8 & 0 & -1.328216 & -2.015659 & 0.183723 \\
\hline 31 & 9 & 0 & -5.608009 & -0.128636 & 0.129782 \\
\hline
\end{tabular}

\section{$\underline{\text { Structure of } \mathbf{T S}_{15 / 45}}$}

Zero-point correction $=0.246538$ (Hartree/Particle)

Thermal correction to Energy $=0.262093$

Thermal correction to Enthalpy $=0.263038$

Thermal correction to Gibbs Free Energy= 0.203425

Sum of electronic and zero-point Energies $=-882.733088$

Sum of electronic and thermal Energies $=-882.717532$

Sum of electronic and thermal Enthalpies $=-882.716588$

Sum of electronic and thermal Free Energies= -882.776201

One imaginary frequency: $752 i$ 
Standard orientation:

\begin{tabular}{|c|c|c|c|c|c|}
\hline \multirow{2}{*}{$\begin{array}{l}\text { Center } \\
\text { Number }\end{array}$} & \multirow{2}{*}{$\begin{array}{l}\text { Atomic } \\
\text { Number }\end{array}$} & \multirow{2}{*}{$\begin{array}{l}\text { Atomic } \\
\text { Type }\end{array}$} & \multicolumn{3}{|c|}{ Coordinates (Angstroms) } \\
\hline & & & $\mathrm{X}$ & Y & Z \\
\hline 1 & ( & & & & 0072686 \\
\hline 1 & 6 & 0 & -1.987991 & -0.900469 & $\begin{array}{l}0.072686 \\
0.156539\end{array}$ \\
\hline 2 & 6 & 0 & -3.389213 & -1.131626 & 0.156539 \\
\hline 3 & 6 & 0 & -4.243700 & -0.070127 & 0.071482 \\
\hline 4 & 6 & 0 & -3.842290 & 1.269115 & -0.095513 \\
\hline 5 & 6 & 0 & -2.497548 & 1.516909 & -0.175947 \\
\hline 6 & 6 & 0 & -1.556877 & 0.467352 & -0.096439 \\
\hline 7 & 6 & 0 & -0.164254 & 0.724916 & -0.175137 \\
\hline 8 & 6 & 0 & 2.129209 & -0.138296 & -0.133648 \\
\hline 9 & 6 & 0 & 2.628672 & 0.003632 & 1.322646 \\
\hline 10 & 6 & 0 & 2.649994 & -1.434787 & -0.758539 \\
\hline 11 & 6 & 0 & 2.605706 & 1.060333 & -0.969244 \\
\hline 12 & 1 & 0 & -3.760764 & -2.139228 & 0.285155 \\
\hline 13 & 1 & 0 & -4.589366 & 2.048066 & -0.154508 \\
\hline 14 & 1 & 0 & -2.137048 & 2.532766 & -0.302651 \\
\hline 15 & 1 & 0 & 0.208330 & 1.743884 & -0.285446 \\
\hline 16 & 1 & 0 & 1.999565 & 0.734311 & 1.832901 \\
\hline 17 & 1 & 0 & 2.524369 & -0.957100 & 1.840179 \\
\hline 18 & 1 & 0 & 4.518969 & -0.152171 & 0.953356 \\
\hline 19 & 1 & 0 & 2.424108 & -1.445483 & -1.831594 \\
\hline 20 & 1 & 0 & 2.145120 & -2.283514 & -0.284166 \\
\hline 21 & 1 & 0 & 4.409175 & -2.297930 & -0.915555 \\
\hline 22 & 1 & 0 & 2.000157 & 1.121324 & -1.877500 \\
\hline 23 & 1 & 0 & 3.643540 & 0.882505 & -1.265424 \\
\hline 24 & 1 & 0 & 3.160475 & 2.315405 & 0.383120 \\
\hline 25 & 1 & 0 & 0.051806 & -1.225472 & 0.034519 \\
\hline 26 & 7 & 0 & 0.677570 & -0.268565 & -0.106701 \\
\hline 27 & 8 & 0 & 3.951972 & 0.494169 & 1.390221 \\
\hline 28 & 8 & 0 & 4.052999 & -1.493910 & -0.531684 \\
\hline 29 & 8 & 0 & 2.475500 & 2.294066 & -0.295966 \\
\hline 30 & 8 & 0 & -1.138049 & -1.854785 & 0.143870 \\
\hline 31 & 9 & 0 & -5.563450 & -0.301716 & 0.152186 \\
\hline
\end{tabular}

\section{Structure of $\mathbf{4 5}$}

Zero-point correction $=0.250063$ (Hartree/Particle)

Thermal correction to Energy $=0.266073$

Thermal correction to Enthalpy $=0.267017$

Thermal correction to Gibbs Free Energy= 0.206319

Sum of electronic and zero-point Energies $=-882.730697$

Sum of electronic and thermal Energies $=-882.714687$

Sum of electronic and thermal Enthalpies= -882.713743

Sum of electronic and thermal Free Energies= -882.774441

No imaginary frequencies

Standard orientation:

\begin{tabular}{|c|c|c|c|c|c|}
\hline \multirow{2}{*}{$\begin{array}{l}\text { Center } \\
\text { Number }\end{array}$} & \multirow{2}{*}{$\begin{array}{l}\text { Atomic } \\
\text { Number }\end{array}$} & \multirow{2}{*}{$\begin{array}{c}\text { Atomic } \\
\text { Type }\end{array}$} & \multicolumn{3}{|c|}{ Coordinates (Angstroms) } \\
\hline & & & $\mathrm{X}$ & $\mathrm{Y}$ & Z \\
\hline 1 & 6 & 0 & -2.043709 & -0.974818 & 0.066091 \\
\hline 2 & 6 & 0 & -3.473785 & -1.123098 & 0.156381 \\
\hline 3 & 6 & 0 & -4.268672 & -0.026927 & 0.075326 \\
\hline 4 & 6 & 0 & -3.801476 & 1.302828 & -0.094775 \\
\hline 5 & 6 & 0 & -2.455881 & 1.487600 & -0.180216 \\
\hline 6 & 6 & 0 & -1.551278 & 0.390523 & -0.105059 \\
\hline 7 & 6 & 0 & -0.177459 & 0.627152 & -0.184158 \\
\hline 8 & 6 & 0 & 2.165562 & -0.155057 & -0.133463 \\
\hline 9 & 6 & 0 & 2.639341 & 0.035154 & 1.325523 \\
\hline 10 & 6 & 0 & 2.752753 & -1.433349 & -0.736034 \\
\hline 11 & 6 & 0 & 2.596420 & 1.051272 & -0.984431 \\
\hline 12 & 1 & 0 & -3.893718 & -2.111539 & 0.286666 \\
\hline 13 & 1 & 0 & -4.514324 & 2.113535 & -0.149149 \\
\hline 14 & 1 & 0 & -2.049705 & 2.486187 & -0.307626 \\
\hline 15 & 1 & 0 & 0.197915 & 1.643913 & -0.286872 \\
\hline 16 & 1 & 0 & 1.968863 & 0.744704 & 1.812900 \\
\hline 17 & 1 & 0 & 2.571978 & -0.919601 & 1.860387 \\
\hline 18 & 1 & 0 & 4.541396 & -0.040109 & 0.990472 \\
\hline
\end{tabular}




\begin{tabular}{rrrrrr}
19 & 1 & 0 & 2.547954 & -1.462214 & -1.812878 \\
20 & 1 & 0 & 2.278598 & -2.302126 & -0.264637 \\
21 & 1 & 0 & 4.551468 & -2.216456 & -0.854198 \\
22 & 1 & 0 & 1.994582 & 1.073444 & -1.896619 \\
23 & 1 & 0 & 3.642636 & 0.910789 & -1.270360 \\
24 & 1 & 0 & 3.091850 & 2.349661 & 0.351174 \\
25 & 7 & 0 & 0.718413 & -0.341676 & -0.133092 \\
26 & 8 & 0 & 3.937010 & 0.588192 & 1.402674 \\
27 & 8 & 0 & 4.150798 & -1.428574 & -0.480704 \\
28 & 8 & 0 & 2.412726 & 2.288642 & -0.331600 \\
29 & 8 & 0 & -1.267329 & -1.955873 & 0.133052 \\
30 & 1 & 0 & 0.279827 & -1.274496 & 0.006568 \\
31 & 9 & 0 & -5.599416 & -0.181193 & 0.160816 \\
\hline
\end{tabular}

\section{$\underline{\text { Structure of } 16 \text { (open form) }}$}

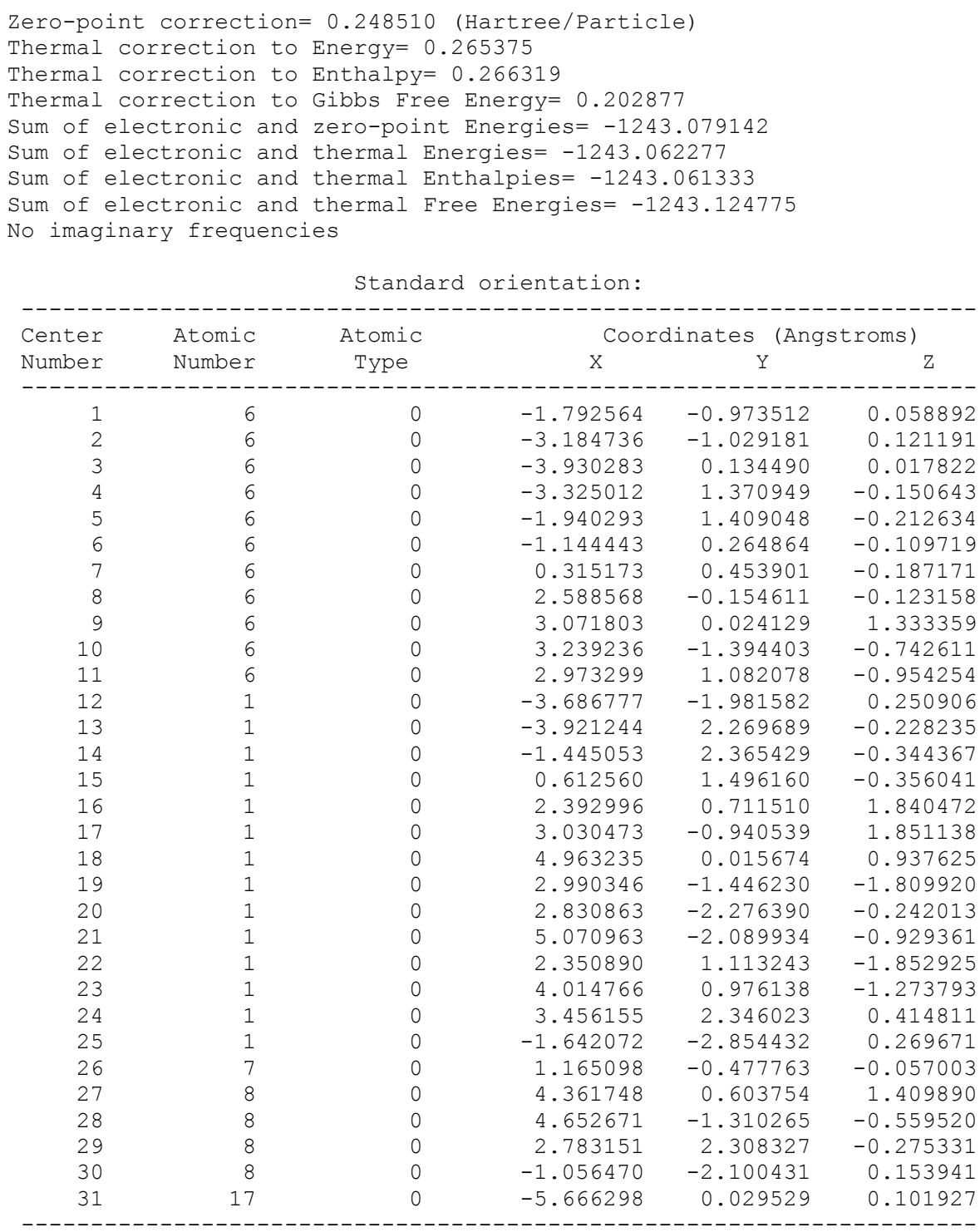

\section{$\underline{\text { Structure of } 16 \text { (closed form) }}$}

Zero-point correction $=0.249367$ (Hartree/Particle)

Thermal correction to Energy $=0.265608$

Thermal correction to Enthalpy $=0.266552$

Thermal correction to Gibbs Free Energy= 0.205053

Sum of electronic and zero-point Energies= -1243.099260 
Sum of electronic and thermal Energies= -1243.083019

Sum of electronic and thermal Enthalpies= -1243.082075

Sum of electronic and thermal Free Energies= -1243.143574

No imaginary frequencies

Standard orientation:

\begin{tabular}{|c|c|c|c|c|c|}
\hline \multirow{2}{*}{$\begin{array}{l}\text { Center } \\
\text { Number }\end{array}$} & \multirow{2}{*}{$\begin{array}{l}\text { Atomic } \\
\text { Number }\end{array}$} & \multirow{2}{*}{$\begin{array}{c}\text { Atomic } \\
\text { Type }\end{array}$} & \multicolumn{3}{|c|}{ Coordinates (Angstroms) } \\
\hline & & & $\mathrm{X}$ & Y & Z \\
\hline 1 & 6 & 0 & -1.721557 & -0.898686 & 0.062080 \\
\hline 2 & 6 & 0 & -3.109510 & -1.044759 & 0.125798 \\
\hline 3 & 6 & 0 & -3.911155 & 0.076044 & 0.022693 \\
\hline 4 & 6 & 0 & -3.386557 & 1.358083 & -0.142926 \\
\hline 5 & 6 & 0 & -2.012395 & 1.491854 & -0.204170 \\
\hline 6 & 6 & 0 & -1.159732 & 0.386384 & -0.105025 \\
\hline 7 & 6 & 0 & 0.284350 & 0.584429 & -0.173488 \\
\hline 8 & 6 & 0 & 2.544438 & -0.156819 & -0.131364 \\
\hline 9 & 6 & 0 & 3.024881 & 0.000504 & 1.329246 \\
\hline 10 & 6 & 0 & 3.143990 & -1.420535 & -0.753214 \\
\hline 11 & 6 & 0 & 2.980402 & 1.066412 & -0.957196 \\
\hline 12 & 1 & 0 & -3.531943 & -2.032374 & 0.254757 \\
\hline 13 & 1 & 0 & -1.574243 & 2.476556 & -0.331476 \\
\hline 14 & 1 & 0 & 0.629813 & 1.616943 & -0.288555 \\
\hline 15 & 1 & 0 & 2.368296 & 0.710787 & 1.834055 \\
\hline 16 & 1 & 0 & 2.946606 & -0.963213 & 1.845595 \\
\hline 17 & 1 & 0 & 4.919835 & -0.080975 & 0.956734 \\
\hline 18 & 1 & 0 & 2.906062 & -1.453611 & -1.823672 \\
\hline 19 & 1 & 0 & 2.695375 & -2.291623 & -0.265481 \\
\hline 20 & 1 & 0 & 4.949340 & -2.181385 & -0.935094 \\
\hline 21 & 1 & 0 & 2.365966 & 1.120595 & -1.860198 \\
\hline 22 & 1 & 0 & 4.019892 & 0.922494 & -1.267081 \\
\hline 23 & 1 & 0 & 3.505130 & 2.317130 & 0.410328 \\
\hline 24 & 1 & 0 & -0.020883 & -1.707410 & 0.103909 \\
\hline 25 & 7 & 0 & 1.102545 & -0.395133 & -0.091839 \\
\hline 26 & 8 & 0 & 4.332284 & 0.533329 & 1.412652 \\
\hline 27 & 8 & 0 & 4.553565 & -1.397766 & -0.548415 \\
\hline 28 & 8 & 0 & 2.826261 & 2.294077 & -0.274770 \\
\hline 29 & 8 & 0 & -0.966071 & -1.994294 & 0.163288 \\
\hline 30 & 1 & 0 & -4.045386 & 2.211960 & -0.217688 \\
\hline 31 & 17 & 0 & -5.640172 & -0.120010 & 0.104124 \\
\hline
\end{tabular}

\section{$\underline{\text { Structure of }} \mathbf{T S}_{16 / 46}$}

Zero-point correction= 0.245184 (Hartree/Particle)
Thermal correction to Energy= 0.261134
Thermal correction to Enthalpy= 0.262078
Thermal correction to Gibbs Free Energy= 0.201191
Sum of electronic and zero-point Energies= -1243.092303
Sum of electronic and thermal Energies=-1243.076353
Sum of electronic and thermal Enthalpies=-1243.075409
Sum of electronic and thermal Free Energies=-1243.136296
One imaginary frequency: 730i

Standard orientation:

\begin{tabular}{|c|c|c|c|c|c|}
\hline \multirow{2}{*}{$\begin{array}{l}\text { Center } \\
\text { Number }\end{array}$} & \multirow{2}{*}{$\begin{array}{l}\text { Atomic } \\
\text { Number }\end{array}$} & \multirow{2}{*}{$\begin{array}{c}\text { Atomic } \\
\text { Type }\end{array}$} & \multicolumn{3}{|c|}{ Coordinates (Angstroms) } \\
\hline & & & $\mathrm{X}$ & $\mathrm{Y}$ & Z \\
\hline 1 & 6 & 0 & -1.622216 & -0.842497 & 0.051621 \\
\hline 2 & 6 & 0 & -3.030499 & -1.051572 & 0.122235 \\
\hline 3 & 6 & 0 & -3.879415 & 0.018329 & 0.035519 \\
\hline 4 & 6 & 0 & -3.441685 & 1.352847 & -0.120448 \\
\hline 5 & 6 & 0 & -2.092778 & 1.579491 & -0.188392 \\
\hline 6 & 6 & 0 & -1.168555 & 0.516081 & -0.107030 \\
\hline 7 & 6 & 0 & 0.229421 & 0.751280 & -0.176751 \\
\hline 8 & 6 & 0 & 2.507211 & -0.152612 & -0.129062 \\
\hline 9 & 6 & 0 & 3.004171 & -0.020900 & 1.329259 \\
\hline 10 & 6 & 0 & 3.007168 & -1.457522 & -0.753521 \\
\hline 11 & 6 & 0 & 3.007015 & 1.038405 & -0.961828 \\
\hline 12 & 1 & 0 & -3.399306 & -2.061475 & 0.241705 \\
\hline 13 & 1 & 0 & -4.163286 & 2.154824 & -0.182866 \\
\hline 14 & 1 & 0 & -1.717156 & 2.591107 & -0.306978 \\
\hline
\end{tabular}




\begin{tabular}{|c|c|c|c|c|c|}
\hline 15 & 1 & 0 & 0.620312 & 1.763795 & -0.281728 \\
\hline 16 & 1 & 0 & 2.385025 & 0.718620 & 1.838951 \\
\hline 17 & 1 & 0 & 2.883683 & -0.980809 & 1.844747 \\
\hline 18 & 1 & 0 & 4.893200 & -0.206451 & 0.967325 \\
\hline 19 & 1 & 0 & 2.784982 & -1.463420 & -1.827380 \\
\hline 20 & 1 & 0 & 2.486135 & -2.297859 & -0.281623 \\
\hline 21 & 1 & 0 & 4.752068 & -2.349685 & -0.906196 \\
\hline 22 & 1 & 0 & 2.407371 & 1.109203 & -1.873310 \\
\hline 23 & 1 & 0 & 4.043373 & 0.843859 & -1.252416 \\
\hline 24 & 1 & 0 & 3.575691 & 2.285272 & 0.392596 \\
\hline 25 & 1 & 0 & 0.413431 & -1.202644 & 0.026070 \\
\hline 26 & 7 & 0 & 1.053115 & -0.256756 & -0.107221 \\
\hline 27 & 8 & 0 & 4.334473 & 0.449037 & 1.401188 \\
\hline 28 & 8 & 0 & 4.407983 & -1.540757 & -0.521529 \\
\hline 29 & 8 & 0 & 2.892801 & 2.273744 & -0.28878 \\
\hline 30 & 8 & 0 & -0.791826 & -1.813487 & 0.125006 \\
\hline 31 & 17 & 0 & -5.600224 & -0.261372 & 0.118528 \\
\hline
\end{tabular}

\section{$\underline{\text { Structure of } 46}$}

Zero-point correction $=0.248581$ (Hartree/Particle)
Thermal correction to Energy $=0.265018$
Thermal correction to Enthalpy= 0.265963
Thermal correction to Gibbs Free Energy= 0.203784
Sum of electronic and zero-point Energies= -1243.089975
Sum of electronic and thermal Energies=-1243.073537
Sum of electronic and thermal Enthalpies=-1243.072593
Sum of electronic and thermal Free Energies= -1243.134771
No imaginary frequencies

Standard orientation:

\begin{tabular}{|c|c|c|c|c|c|}
\hline \multirow{2}{*}{$\begin{array}{l}\text { Center } \\
\text { Number }\end{array}$} & \multirow{2}{*}{$\begin{array}{l}\text { Atomic } \\
\text { Number }\end{array}$} & \multirow{2}{*}{$\begin{array}{c}\text { Atomic } \\
\text { Type }\end{array}$} & \multicolumn{3}{|c|}{ Coordinates (Angstroms) } \\
\hline & & & $\mathrm{X}$ & $\mathrm{Y}$ & Z \\
\hline & & & & ----------1 & ---------- \\
\hline 1 & 6 & 0 & -1.670126 & -0.932836 & 0.040174 \\
\hline 2 & 6 & 0 & -3.105494 & -1.068670 & 0.117811 \\
\hline 3 & 6 & 0 & -3.902431 & 0.029659 & 0.036536 \\
\hline 4 & 6 & 0 & -3.407643 & 1.356485 & -0.122640 \\
\hline 5 & 6 & 0 & -2.059659 & 1.528602 & -0.196134 \\
\hline 6 & 6 & 0 & -1.164103 & 0.424930 & -0.119598 \\
\hline 7 & 6 & 0 & 0.213678 & 0.647545 & -0.188472 \\
\hline 8 & 6 & 0 & 2.546478 & -0.164339 & -0.128239 \\
\hline 9 & 6 & 0 & 3.015544 & 0.021893 & 1.332893 \\
\hline 10 & 6 & 0 & 3.119929 & -1.451220 & -0.725920 \\
\hline 11 & 6 & 0 & 2.996512 & 1.035129 & -0.978869 \\
\hline 12 & 1 & 0 & -3.514941 & -2.062773 & 0.239000 \\
\hline 13 & 1 & 0 & -4.099467 & 2.184623 & -0.179497 \\
\hline 14 & 1 & 0 & -1.645746 & 2.525333 & -0.315327 \\
\hline 15 & 1 & 0 & 0.601348 & 1.660244 & -0.285644 \\
\hline 16 & 1 & 0 & 2.351371 & 0.739891 & 1.816505 \\
\hline 17 & 1 & 0 & 2.934555 & -0.931437 & 1.868405 \\
\hline 18 & 1 & 0 & 4.918012 & -0.076663 & 1.006800 \\
\hline 19 & 1 & 0 & 2.920335 & -1.479405 & -1.803751 \\
\hline 20 & 1 & 0 & 2.632368 & -2.312953 & -0.255260 \\
\hline 21 & 1 & 0 & 4.909302 & -2.257121 & -0.832995 \\
\hline 22 & 1 & 0 & 2.399600 & 1.063230 & -1.894131 \\
\hline 23 & 1 & 0 & 4.042329 & 0.880994 & -1.259141 \\
\hline 24 & 1 & 0 & 3.500775 & 2.328841 & 0.357864 \\
\hline 25 & 7 & 0 & 1.096778 & -0.332104 & -0.134402 \\
\hline 26 & 8 & 0 & 4.319187 & 0.559487 & 1.415084 \\
\hline 27 & 8 & 0 & 4.516429 & -1.463550 & -0.463222 \\
\hline 28 & 8 & 0 & 2.824737 & 2.275387 & -0.328581 \\
\hline 29 & 8 & 0 & -0.908598 & -1.925352 & 0.109099 \\
\hline 30 & 1 & 0 & 0.646333 & -1.260488 & -0.002346 \\
\hline 31 & 17 & 0 & -5.634339 & -0.165202 & 0.130355 \\
\hline
\end{tabular}

\section{$\underline{\text { Structure of } 17 \text { (open form) }}$}

Zero-point correction= 0.247959 (Hartree/Particle) 
Thermal correction to Energy $=0.265047$

Thermal correction to Enthalpy $=0.265992$

Thermal correction to Gibbs Free Energy= 0.201178

Sum of electronic and zero-point Energies $=-3357.051240$

Sum of electronic and thermal Energies= -3357.034151

Sum of electronic and thermal Enthalpies= -3357.033207

Sum of electronic and thermal Free Energies= $\mathbf{- 3 3 5 7 . 0 9 8 0 2 1}$

No imaginary frequencies

\begin{tabular}{|c|c|c|c|c|c|}
\hline \multirow{2}{*}{$\begin{array}{l}\text { Center } \\
\text { Number }\end{array}$} & \multirow{2}{*}{$\begin{array}{l}\text { Atomic } \\
\text { Number }\end{array}$} & \multirow{2}{*}{$\begin{array}{c}\text { Atomic } \\
\text { Type }\end{array}$} & \multicolumn{3}{|c|}{ Coordinates (Angstroms) } \\
\hline & & & $\mathrm{X}$ & $Y$ & Z \\
\hline & & & 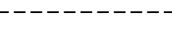 & 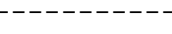 & ---------- \\
\hline 1 & 6 & 0 & -1.094028 & -0.972140 & 0.072288 \\
\hline 2 & 6 & 0 & -2.487413 & -1.030710 & 0.118230 \\
\hline 3 & 6 & 0 & -3.233943 & 0.130372 & -0.007346 \\
\hline 4 & 6 & 0 & -2.629065 & 1.367113 & -0.177366 \\
\hline 5 & 6 & 0 & -1.243334 & 1.407226 & -0.221940 \\
\hline 6 & 6 & 0 & -0.446584 & 0.265601 & -0.102383 \\
\hline 7 & 6 & 0 & 1.013245 & 0.457020 & -0.174149 \\
\hline 8 & 6 & 0 & 3.286919 & -0.151351 & -0.128308 \\
\hline 9 & 6 & 0 & 3.776251 & -0.010746 & 1.330349 \\
\hline 10 & 6 & 0 & 3.935443 & -1.374252 & -0.782659 \\
\hline 11 & 6 & 0 & 3.667742 & 1.106318 & -0.929181 \\
\hline 12 & 1 & 0 & -2.984027 & -1.985480 & 0.251455 \\
\hline 13 & 1 & 0 & -3.221571 & 2.266406 & -0.271301 \\
\hline 14 & 1 & 0 & -0.748812 & 2.363795 & -0.355659 \\
\hline 15 & 1 & 0 & 1.309869 & 1.502356 & -0.323796 \\
\hline 16 & 1 & 0 & 3.098515 & 0.661154 & 1.859144 \\
\hline 17 & 1 & 0 & 3.739131 & -0.989043 & 1.822316 \\
\hline 18 & 1 & 0 & 5.666523 & -0.005527 & 0.928393 \\
\hline 19 & 1 & 0 & 3.682772 & -1.397893 & -1.850071 \\
\hline 20 & 1 & 0 & 3.529099 & -2.269357 & -0.304224 \\
\hline 21 & 1 & 0 & 5.765587 & -2.068559 & -0.986338 \\
\hline 22 & 1 & 0 & 3.042742 & 1.158668 & -1.825108 \\
\hline 23 & 1 & 0 & 4.708291 & 1.009671 & -1.254493 \\
\hline 24 & 1 & 0 & 4.153079 & 2.336759 & 0.469046 \\
\hline 25 & 1 & 0 & -0.941640 & -2.849756 & 0.310425 \\
\hline 26 & 7 & 0 & 1.863714 & -0.476658 & -0.064182 \\
\hline 27 & 8 & 0 & 5.065279 & 0.569419 & 1.416868 \\
\hline 28 & 8 & 0 & 5.349531 & -1.294340 & -0.602711 \\
\hline 29 & 8 & 0 & 3.477867 & 2.314961 & -0.219566 \\
\hline 30 & 8 & 0 & -0.356652 & -2.095992 & 0.189801 \\
\hline 31 & 35 & 0 & -5.125862 & 0.013838 & 0.049118 \\
\hline
\end{tabular}

\section{Structure of 17 (closed form)}

Zero-point correction= 0.248805 (Hartree/Particle)

Thermal correction to Energy $=0.265283$

Thermal correction to Enthalpy $=0.266228$

Thermal correction to Gibbs Free Energy= 0.203533

Sum of electronic and zero-point Energies $=-3357.071237$

Sum of electronic and thermal Energies= -3357.054758

Sum of electronic and thermal Enthalpies= -3357.053814

Sum of electronic and thermal Free Energies= -3357.116509

No imaginary frequencies

Standard orientation:

\begin{tabular}{|c|c|c|c|c|c|}
\hline \multirow{2}{*}{$\begin{array}{l}\text { Center } \\
\text { Number }\end{array}$} & \multirow{2}{*}{$\begin{array}{l}\text { Atomic } \\
\text { Number }\end{array}$} & \multirow{2}{*}{$\begin{array}{c}\text { Atomic } \\
\text { Type }\end{array}$} & \multicolumn{3}{|c|}{ Coordinates (Angstroms) } \\
\hline & & & $\mathrm{X}$ & $\mathrm{Y}$ & Z \\
\hline 1 & 6 & 0 & -1.034138 & -0.871398 & 0.057898 \\
\hline 2 & 6 & 0 & -2.424337 & -1.008200 & 0.109189 \\
\hline 3 & 6 & 0 & -3.216793 & 0.118729 & -0.000496 \\
\hline 4 & 6 & 0 & -2.681730 & 1.397888 & -0.156801 \\
\hline 5 & 6 & 0 & -1.305578 & 1.521290 & -0.205548 \\
\hline 6 & 6 & 0 & -0.461912 & 0.409526 & -0.102911 \\
\hline 7 & 6 & 0 & 0.984182 & 0.596697 & -0.167291 \\
\hline 8 & 6 & 0 & 3.238075 & -0.163260 & -0.128235 \\
\hline 9 & 6 & 0 & 3.723003 & -0.010153 & 1.331295 \\
\hline 10 & 6 & 0 & 3.825641 & -1.431993 & -0.751433 \\
\hline
\end{tabular}




$\begin{array}{rrrrrr}11 & 6 & 0 & 3.682577 & 1.056218 & -0.955052 \\ 12 & 1 & 0 & -2.849725 & -1.995372 & 0.231832 \\ 13 & 1 & 0 & -3.329303 & 2.259717 & -0.236150 \\ 14 & 1 & 0 & -0.859652 & 2.503261 & -0.327605 \\ 15 & 1 & 0 & 1.337739 & 1.626614 & -0.280875 \\ 16 & 1 & 0 & 3.073548 & 0.705376 & 1.837889 \\ 17 & 1 & 0 & 3.638183 & -0.973347 & 1.847591 \\ 18 & 1 & 0 & 5.616306 & -0.107355 & 0.954200 \\ 19 & 1 & 0 & 3.585275 & -1.462912 & -1.821410 \\ 20 & 1 & 0 & 3.370632 & -2.299289 & -0.262880 \\ 21 & 1 & 0 & 5.624373 & -2.207145 & -0.938349 \\ 22 & 1 & 0 & 3.067171 & 1.115093 & -1.857114 \\ 23 & 1 & 0 & 4.720351 & 0.903712 & -1.266589 \\ 24 & 1 & 0 & 4.220199 & 2.303326 & 0.410707 \\ 25 & 1 & 0 & 0.659875 & -1.693691 & 0.107067 \\ 26 & 7 & 0 & 1.794227 & -0.389512 & -0.086448 \\ 27 & 8 & 0 & 5.034925 & 0.511883 & 1.411390 \\ 28 & 8 & 0 & 5.235705 & -1.421096 & -0.549362 \\ 29 & 8 & 0 & 3.539444 & 2.285150 & -0.272651 \\ 30 & 8 & 0 & -0.287716 & -1.972943 & 0.162538 \\ 31 & 35 & 0 & -5.102496 & -0.081926 & 0.057366 \\ ---------------------------------------------------------\end{array}$

\section{$\underline{\text { Structure of } \mathbf{T S}_{17 / 47}}$}

Zero-point correction= 0.244663 (Hartree/Particle) Thermal correction to Energy $=0.260860$

Thermal correction to Enthalpy $=0.261804$

Thermal correction to Gibbs Free Energy= 0.199772

Sum of electronic and zero-point Energies= -3357.064223

Sum of electronic and thermal Energies= -3357.048026

Sum of electronic and thermal Enthalpies= -3357.047082

Sum of electronic and thermal Free Energies= -3357.109114

one imaginary frequency: $734 i$

Standard orientation:

\begin{tabular}{|c|c|c|c|c|c|}
\hline \multirow{2}{*}{$\begin{array}{l}\text { Center } \\
\text { Number }\end{array}$} & \multirow{2}{*}{$\begin{array}{l}\text { Atomic } \\
\text { Number }\end{array}$} & \multirow{2}{*}{$\begin{array}{c}\text { Atomic } \\
\text { Type }\end{array}$} & \multicolumn{3}{|c|}{ Coordinates (Angstroms) } \\
\hline & & & $\mathrm{X}$ & Y & Z \\
\hline 1 & 6 & 0 & -0.945121 & -0.789028 & 0.037952 \\
\hline 2 & 6 & 0 & -2.358018 & -0.977317 & 0.096100 \\
\hline 3 & 6 & 0 & -3.189283 & 0.106024 & 0.005899 \\
\hline 4 & 6 & 0 & -2.730489 & 1.434989 & -0.139218 \\
\hline 5 & 6 & 0 & -1.376985 & 1.640049 & -0.195387 \\
\hline 6 & 6 & 0 & -0.469843 & 0.562415 & -0.112192 \\
\hline 7 & 6 & 0 & 0.932473 & 0.775752 & -0.175073 \\
\hline 8 & 6 & 0 & 3.194973 & -0.165876 & -0.125077 \\
\hline 9 & 6 & 0 & 3.692742 & -0.042830 & 1.333743 \\
\hline 10 & 6 & 0 & 3.673416 & -1.478950 & -0.749364 \\
\hline 11 & 6 & 0 & 3.715408 & 1.016593 & -0.957292 \\
\hline 12 & 1 & 0 & -2.737538 & -1.984107 & 0.207433 \\
\hline 13 & 1 & 0 & -3.435092 & 2.251643 & -0.203576 \\
\hline 14 & 1 & 0 & -0.985080 & 2.646440 & -0.306766 \\
\hline 15 & 1 & 0 & 1.340063 & 1.781966 & -0.276505 \\
\hline 16 & 1 & 0 & 3.084913 & 0.705963 & 1.843501 \\
\hline 17 & 1 & 0 & 3.557118 & -1.001073 & 1.848569 \\
\hline 18 & 1 & 0 & 5.579074 & -0.258115 & 0.974353 \\
\hline 19 & 1 & 0 & 3.451927 & -1.481104 & -1.823384 \\
\hline 20 & 1 & 0 & 3.138173 & -2.310563 & -0.277877 \\
\hline 21 & 1 & 0 & 5.403179 & -2.399990 & -0.901088 \\
\hline 22 & 1 & 0 & 3.119313 & 1.096130 & -1.870400 \\
\hline 23 & 1 & 0 & 4.749313 & 0.805639 & -1.245032 \\
\hline 24 & 1 & 0 & 4.300997 & 2.256225 & 0.396706 \\
\hline 25 & 1 & 0 & 1.083833 & -1.181635 & 0.022721 \\
\hline 26 & 7 & 0 & 1.739192 & -0.245542 & -0.104953 \\
\hline 27 & 8 & 0 & 5.030023 & 0.406414 & 1.406773 \\
\hline 28 & 8 & 0 & 5.072454 & -1.585545 & -0.516340 \\
\hline 29 & 8 & 0 & 3.618685 & 2.253986 & -0.285296 \\
\hline 30 & 8 & 0 & -0.131059 & -1.773503 & 0.113484 \\
\hline 31 & 35 & 0 & -5.068028 & -0.174582 & 0.070757 \\
\hline
\end{tabular}




\section{$\underline{\text { Structure of } \mathbf{4 7}}$}

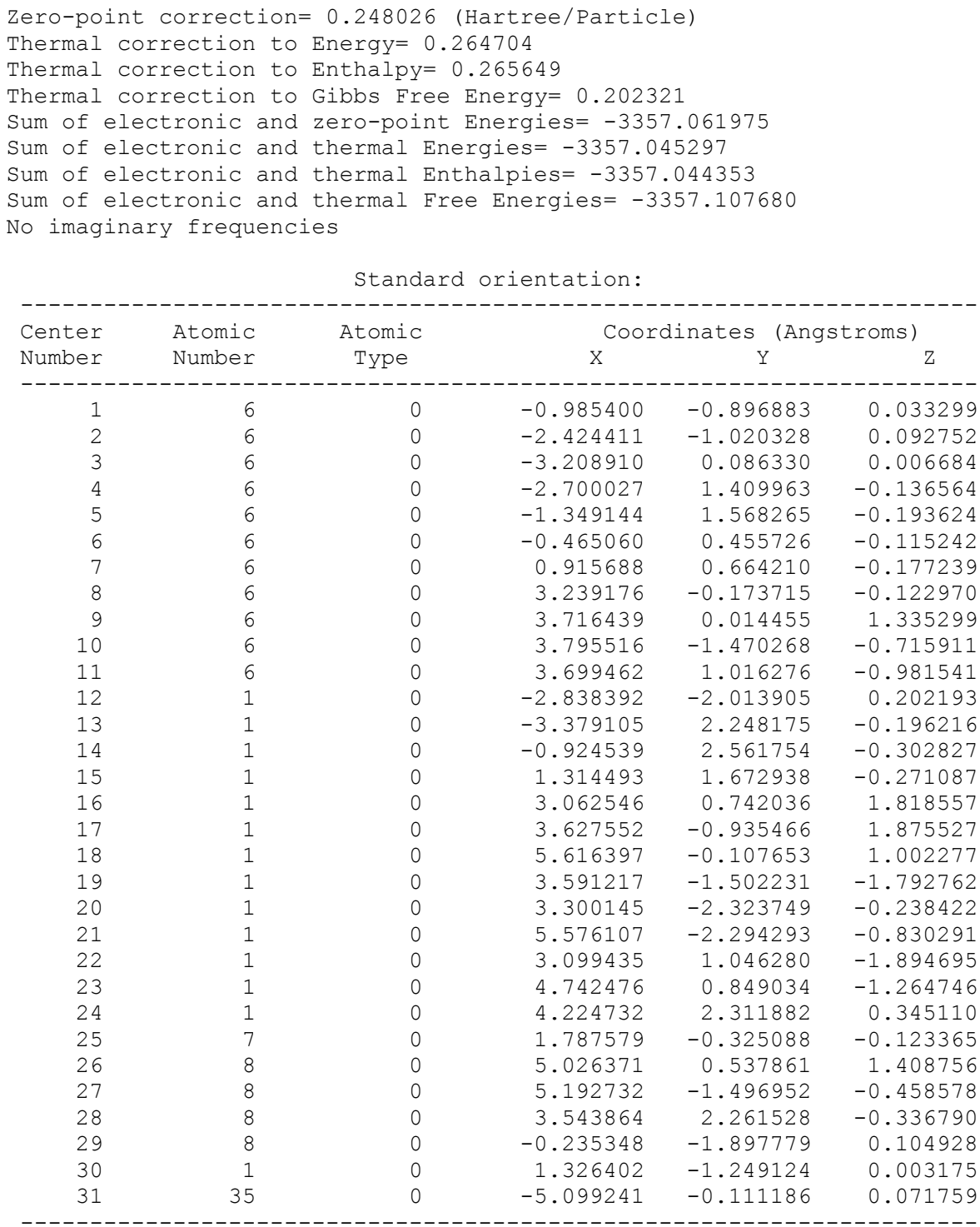

\section{$\underline{\text { Structure of } 18 \text { (open form) }}$}

Zero-point correction= 0.291522 (Hartree/Particle)

Thermal correction to Energy $=0.309584$

Thermal correction to Enthalpy $=0.310528$

Thermal correction to Gibbs Free Energy $=0.245238$

Sum of electronic and zero-point Energies= -897.950076

Sum of electronic and thermal Energies= -897.932014

Sum of electronic and thermal Enthalpies= -897.931070

Sum of electronic and thermal Free Energies= -897.996360

No imaginary frequencies

Standard orientation:

\begin{tabular}{|c|c|c|c|c|c|}
\hline \multirow{2}{*}{$\begin{array}{l}\text { Center } \\
\text { Number }\end{array}$} & \multirow{2}{*}{$\begin{array}{l}\text { Atomic } \\
\text { Number }\end{array}$} & \multirow{2}{*}{$\begin{array}{c}\text { Atomic } \\
\text { Type }\end{array}$} & \multicolumn{3}{|c|}{ Coordinates (Angstroms) } \\
\hline & & & $\mathrm{X}$ & $\mathrm{Y}$ & Z \\
\hline 1 & 6 & 0 & 1.743664 & 1.129098 & 0.079809 \\
\hline 2 & 6 & 0 & 3.125156 & 1.234537 & 0.150515 \\
\hline 3 & 6 & 0 & 3.937607 & 0.105448 & 0.045752 \\
\hline 4 & 6 & 0 & 3.364346 & -1.152426 & -0.136664 \\
\hline 5 & 6 & 0 & 1.979721 & -1.238139 & -0.205990 \\
\hline 6 & 6 & 0 & 1.136565 & -0.132720 & -0.100512 \\
\hline
\end{tabular}




$\begin{array}{rrrrrr}7 & 6 & 0 & -0.310341 & -0.379719 & -0.183553 \\ 8 & 6 & 0 & -2.610719 & 0.113847 & -0.125577 \\ 9 & 6 & 0 & -3.094607 & -0.076271 & 1.328376 \\ 10 & 6 & 0 & -3.317860 & 1.314074 & -0.760497 \\ 11 & 6 & 0 & -2.931255 & -1.146792 & -0.948714 \\ 12 & 1 & 0 & 3.601868 & 2.199311 & 0.288919 \\ 13 & 1 & 0 & 3.965024 & -2.046387 & -0.220347 \\ 14 & 1 & 0 & 1.523633 & -2.213034 & -0.345800 \\ 15 & 1 & 0 & -0.560943 & -1.433523 & -0.357675 \\ 16 & 1 & 0 & -2.387537 & -0.727466 & 1.844489 \\ 17 & 1 & 0 & -3.100831 & 0.893160 & 1.838902 \\ 18 & 1 & 0 & -4.980843 & -0.160745 & 0.917345 \\ 19 & 1 & 0 & -3.061550 & 1.370452 & -1.825820 \\ 20 & 1 & 0 & -2.958981 & 2.218848 & -0.262761 \\ 21 & 1 & 0 & -5.178160 & 1.925201 & -0.957365 \\ 22 & 1 & 0 & -2.300516 & -1.158314 & -1.841937 \\ 23 & 1 & 0 & -3.973837 & -1.091141 & -1.277698 \\ 24 & 1 & 0 & -3.365410 & -2.415281 & 0.431744 \\ 25 & 1 & 0 & 1.521529 & 2.999707 & 0.297573 \\ 26 & 7 & 0 & -1.206004 & 0.509744 & -0.052954 \\ 27 & 8 & 0 & -4.358066 & -0.714079 & 1.403789 \\ 28 & 8 & 0 & -4.728279 & 1.161789 & -0.590951 \\ 29 & 8 & 0 & -2.693407 & -2.358605 & -0.258053 \\ 30 & 8 & 0 & 0.962189 & 2.227061 & 0.176421 \\ 31 & 8 & 0 & 5.268493 & 0.336078 & 0.133551 \\ 32 & 6 & 0 & 6.138048 & -0.772990 & 0.016549 \\ 33 & 1 & 0 & 7.145633 & -0.374762 & 0.110106 \\ 34 & 1 & 0 & 5.958028 & -1.500912 & 0.813092 \\ 35 & 1 & 0 & 6.025535 & -1.258942 & -0.957102 \\ ---------------------------------------1907\end{array}$

\section{$\underline{\text { Structure of } 18 \text { (closed form) }}$}

Zero-point correction= 0.291953 (Hartree/Particle) Thermal correction to Energy $=0.309528$

Thermal correction to Enthalpy $=0.310472$

Thermal correction to Gibbs Free Energy= 0.246385

Sum of electronic and zero-point Energies= -897.970075

Sum of electronic and thermal Energies= -897.952500

Sum of electronic and thermal Enthalpies= -897.951556

Sum of electronic and thermal Free Energies= $\mathbf{- 8 9 8 . 0 1 5 6 4 2}$

No imaginary frequencies

Standard orientation:

\begin{tabular}{|c|c|c|c|c|c|}
\hline \multirow{2}{*}{$\begin{array}{l}\text { Center } \\
\text { Number }\end{array}$} & \multirow{2}{*}{$\begin{array}{l}\text { Atomic } \\
\text { Number }\end{array}$} & \multirow{2}{*}{$\begin{array}{l}\text { Atomic } \\
\text { Type }\end{array}$} & \multicolumn{3}{|c|}{ Coordinates (Angstroms) } \\
\hline & & & $\mathrm{X}$ & Y & Z \\
\hline 1 & 6 & 0 & -1.670658 & -1.047924 & 0.078696 \\
\hline 2 & 6 & 0 & -3.042944 & -1.243831 & 0.149470 \\
\hline 3 & 6 & 0 & -3.912680 & -0.162049 & 0.045165 \\
\hline 4 & 6 & 0 & -3.422928 & 1.139737 & -0.134224 \\
\hline 5 & 6 & 0 & -2.052348 & 1.322781 & -0.202865 \\
\hline 6 & 6 & 0 & -1.152949 & 0.259780 & -0.100644 \\
\hline 7 & 6 & 0 & 0.277028 & 0.516718 & -0.174718 \\
\hline 8 & 6 & 0 & 2.570046 & -0.116980 & -0.132801 \\
\hline 9 & 6 & 0 & 3.047337 & 0.059282 & 1.325792 \\
\hline 10 & 6 & 0 & 3.225078 & -1.349969 & -0.760025 \\
\hline 11 & 6 & 0 & 2.949643 & 1.125933 & -0.957685 \\
\hline 12 & 1 & 0 & -3.439147 & -2.241280 & 0.287549 \\
\hline 13 & 1 & 0 & -4.085891 & 1.988289 & -0.216917 \\
\hline 14 & 1 & 0 & -1.655695 & 2.324018 & -0.338710 \\
\hline 15 & 1 & 0 & 0.576121 & 1.563057 & -0.297684 \\
\hline 16 & 1 & 0 & 2.362566 & 0.741416 & 1.832065 \\
\hline 17 & 1 & 0 & 3.009404 & -0.906996 & 1.841906 \\
\hline 18 & 1 & 0 & 4.942435 & 0.059981 & 0.944864 \\
\hline 19 & 1 & 0 & 2.981802 & -1.392324 & -1.828965 \\
\hline 20 & 1 & 0 & 2.820474 & -2.241483 & -0.271017 \\
\hline 21 & 1 & 0 & 5.059923 & -2.035719 & -0.943542 \\
\hline 22 & 1 & 0 & 2.328029 & 1.157211 & -1.856729 \\
\hline 23 & 1 & 0 & 3.992435 & 1.026951 & -1.274369 \\
\hline 24 & 1 & 0 & 3.426176 & 2.389391 & 0.415060 \\
\hline 25 & 1 & 0 & 0.064267 & -1.784284 & 0.120580 \\
\hline 26 & 7 & 0 & 1.141835 & -0.423641 & -0.089196 \\
\hline
\end{tabular}




\begin{tabular}{rrrrrr}
27 & 8 & 0 & 4.332720 & 0.645672 & 1.409147 \\
28 & 8 & 0 & 4.634638 & -1.263526 & -0.565585 \\
29 & 8 & 0 & 2.750030 & 2.345696 & -0.271739 \\
30 & 8 & 0 & -0.866019 & -2.110672 & 0.184588 \\
31 & 8 & 0 & -5.228241 & -0.465346 & 0.129880 \\
32 & 6 & 0 & -6.164586 & 0.589984 & 0.040563 \\
33 & 1 & 0 & -7.144501 & 0.129769 & 0.144204 \\
34 & 1 & 0 & -6.017383 & 1.318092 & 0.843705 \\
35 & 1 & 0 & -6.099131 & 1.094033 & -0.928306 \\
\hline
\end{tabular}

\section{$\underline{\text { Structure of } \mathbf{T S}_{18 / 48}}$} Zero-point correction $=0.287578$ (Hartree/Particle)
Thermal correction to Energy $=0.305032$
Thermal correction to Enthalpy= 0.305976
Thermal correction to Gibbs Free Energy= 0.241908
Sum of electronic and zero-point Energies=-897.962193
Sum of electronic and thermal Energies=-897.944740
Sum of electronic and thermal Enthalpies= -897.943795
Sum of electronic and thermal Free Energies= -898.007863
One imaginary frequency: 493i

\begin{tabular}{|c|c|c|c|c|c|}
\hline \multirow{2}{*}{$\begin{array}{l}\text { Center } \\
\text { Number }\end{array}$} & \multirow{2}{*}{$\begin{array}{l}\text { Atomic } \\
\text { Number }\end{array}$} & \multirow{2}{*}{$\begin{array}{c}\text { Atomic } \\
\text { Type }\end{array}$} & \multicolumn{3}{|c|}{ Coordinates (Angstroms) } \\
\hline & & & $\mathrm{X}$ & $\mathrm{Y}$ & Z \\
\hline & & & 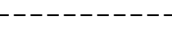 & ----------- & ---------- \\
\hline 1 & 6 & 0 & -1.570595 & -0.992492 & 0.055945 \\
\hline 2 & 6 & 0 & -2.961674 & -1.246476 & 0.125752 \\
\hline 3 & 6 & 0 & -3.876320 & -0.218645 & 0.049834 \\
\hline 4 & 6 & 0 & -3.471409 & 1.136991 & -0.102152 \\
\hline 5 & 6 & 0 & -2.131321 & 1.412317 & -0.172077 \\
\hline 6 & 6 & 0 & -1.162871 & 0.390304 & -0.097823 \\
\hline 7 & 6 & 0 & 0.216150 & 0.685008 & -0.168520 \\
\hline 8 & 6 & 0 & 2.536736 & -0.117147 & -0.126426 \\
\hline 9 & 6 & 0 & 3.027037 & 0.077853 & 1.326286 \\
\hline 10 & 6 & 0 & 3.094521 & -1.414754 & -0.715693 \\
\hline 11 & 6 & 0 & 2.984343 & 1.071473 & -0.992953 \\
\hline 12 & 1 & 0 & -3.306443 & -2.265473 & 0.241784 \\
\hline 13 & 1 & 0 & -4.194755 & 1.936222 & -0.164087 \\
\hline 14 & 1 & 0 & -1.797250 & 2.439038 & -0.287749 \\
\hline 15 & 1 & 0 & 0.564446 & 1.713197 & -0.272042 \\
\hline 16 & 1 & 0 & 2.378771 & 0.808261 & 1.812578 \\
\hline 17 & 1 & 0 & 2.941896 & -0.869764 & 1.870727 \\
\hline 18 & 1 & 0 & 4.921065 & -0.043256 & 0.964409 \\
\hline 19 & 1 & 0 & 2.873238 & -1.458571 & -1.788855 \\
\hline 20 & 1 & 0 & 2.609648 & -2.264014 & -0.221853 \\
\hline 21 & 1 & 0 & 4.873026 & -2.243941 & -0.831999 \\
\hline 22 & 1 & 0 & 2.374142 & 1.096624 & -1.899544 \\
\hline 23 & 1 & 0 & 4.025376 & 0.910307 & -1.288071 \\
\hline 24 & 1 & 0 & 3.512570 & 2.369178 & 0.328935 \\
\hline 25 & 1 & 0 & 0.512330 & -1.248965 & 0.024976 \\
\hline 26 & 7 & 0 & 1.090311 & -0.286011 & -0.104865 \\
\hline 27 & 8 & 0 & 4.339357 & 0.600086 & 1.386221 \\
\hline 28 & 8 & 0 & 4.498178 & -1.432415 & -0.483320 \\
\hline 29 & 8 & 0 & 2.829207 & 2.319049 & -0.350304 \\
\hline 30 & 8 & 0 & -0.698767 & -1.926038 & 0.124366 \\
\hline 31 & 8 & 0 & -5.178799 & -0.580274 & 0.127746 \\
\hline 32 & 6 & 0 & -6.169736 & 0.426926 & 0.082513 \\
\hline 33 & 1 & 0 & -7.122552 & -0.088967 & 0.177636 \\
\hline 34 & 1 & 0 & -6.056137 & 1.133562 & 0.909748 \\
\hline 35 & 1 & 0 & -6.144103 & 0.967216 & -0.868322 \\
\hline
\end{tabular}

\section{$\underline{\text { Structure of } 48}$}

Zero-point correction $=0.290358$ (Hartree/Particle)

Thermal correction to Energy $=0.308223$

Thermal correction to Enthalpy= 0.309167

Thermal correction to Gibbs Free Energy= 0.244035

Sum of electronic and zero-point Energies $=-897.959823$ 
Sum of electronic and thermal Energies= -897.941959

Sum of electronic and thermal Enthalpies= -897.941015

Sum of electronic and thermal Free Energies= -898.006146

No imaginary frequencies

Standard orientation:

\begin{tabular}{|c|c|c|c|c|c|}
\hline \multirow{2}{*}{$\begin{array}{l}\text { Center } \\
\text { Number }\end{array}$} & \multirow{2}{*}{$\begin{array}{l}\text { Atomic } \\
\text { Number }\end{array}$} & \multirow{2}{*}{$\begin{array}{c}\text { Atomic } \\
\text { Type }\end{array}$} & \multicolumn{3}{|c|}{ Coordinates (Angstroms) } \\
\hline & & & $\mathrm{X}$ & $\mathrm{Y}$ & Z \\
\hline 1 & 6 & 0 & -1.602307 & -1.057337 & 0.057389 \\
\hline 2 & 6 & 0 & -3.013452 & -1.260787 & 0.136075 \\
\hline 3 & 6 & 0 & -3.894565 & -0.212981 & 0.052970 \\
\hline 4 & 6 & 0 & -3.451289 & 1.137350 & -0.116956 \\
\hline 5 & 6 & 0 & -2.110979 & 1.375646 & -0.194097 \\
\hline 6 & 6 & 0 & -1.160158 & 0.325777 & -0.112578 \\
\hline 7 & 6 & 0 & 0.204507 & 0.614026 & -0.186902 \\
\hline 8 & 6 & 0 & 2.563125 & -0.121568 & -0.128215 \\
\hline 9 & 6 & 0 & 3.034613 & 0.081245 & 1.329475 \\
\hline 10 & 6 & 0 & 3.169961 & -1.393016 & -0.725801 \\
\hline 11 & 6 & 0 & 2.975455 & 1.087810 & -0.983837 \\
\hline 12 & 1 & 0 & -3.386605 & -2.268405 & 0.264253 \\
\hline 13 & 1 & 0 & -4.154768 & 1.953680 & -0.184277 \\
\hline 14 & 1 & 0 & -1.750410 & 2.392148 & -0.320905 \\
\hline 15 & 1 & 0 & 0.548419 & 1.641824 & -0.293266 \\
\hline 16 & 1 & 0 & 2.355234 & 0.784529 & 1.813479 \\
\hline 17 & 1 & 0 & 2.978743 & -0.871889 & 1.868402 \\
\hline 18 & 1 & 0 & 4.936201 & 0.030219 & 0.989142 \\
\hline 19 & 1 & 0 & 2.963502 & -1.429849 & -1.802078 \\
\hline 20 & 1 & 0 & 2.709245 & -2.266180 & -0.249711 \\
\hline 21 & 1 & 0 & 4.977611 & -2.156363 & -0.835623 \\
\hline 22 & 1 & 0 & 2.372031 & 1.097816 & -1.895141 \\
\hline 23 & 1 & 0 & 4.023522 & 0.963004 & -1.270772 \\
\hline 24 & 1 & 0 & 3.450357 & 2.396410 & 0.348658 \\
\hline 25 & 7 & 0 & 1.121381 & -0.333357 & -0.126577 \\
\hline 26 & 8 & 0 & 4.326135 & 0.650594 & 1.405003 \\
\hline 27 & 8 & 0 & 4.569339 & -1.366809 & -0.474344 \\
\hline 28 & 8 & 0 & 2.773829 & 2.325355 & -0.335604 \\
\hline 29 & 8 & 0 & -0.775581 & -2.005460 & 0.131583 \\
\hline 30 & 1 & 0 & 0.679063 & -1.276577 & 0.014274 \\
\hline 31 & 8 & 0 & -5.208317 & -0.530048 & 0.140257 \\
\hline 32 & 6 & 0 & -6.169982 & 0.504892 & 0.085977 \\
\hline 33 & 1 & 0 & -7.136400 & 0.017507 & 0.194110 \\
\hline 34 & 1 & 0 & -6.032448 & 1.219923 & 0.902189 \\
\hline 35 & 1 & 0 & -6.135773 & 1.030306 & -0.872865 \\
\hline
\end{tabular}

\section{Structure of $\mathbf{2 1}$ (open form)}

Zero-point correction= 0.291612 (Hartree/Particle)

Thermal correction to Energy $=0.309716$

Thermal correction to Enthalpy $=0.310660$

Thermal correction to Gibbs Free Energy $=0.245007$

Sum of electronic and zero-point Energies $=-897.951035$

Sum of electronic and thermal Energies= -897.932931

Sum of electronic and thermal Enthalpies= -897.931986

Sum of electronic and thermal Free Energies= -897.997640

No imaginary frequencies

Standard orientation:

\begin{tabular}{|c|c|c|c|c|c|}
\hline \multirow{2}{*}{$\begin{array}{l}\text { Center } \\
\text { Number }\end{array}$} & \multirow{2}{*}{$\begin{array}{l}\text { Atomic } \\
\text { Number }\end{array}$} & \multirow{2}{*}{$\begin{array}{c}\text { Atomic } \\
\text { Type }\end{array}$} & \multicolumn{3}{|c|}{ Coordinates (Angstroms) } \\
\hline & & & $\mathrm{X}$ & $\mathrm{Y}$ & Z \\
\hline 1 & 6 & 0 & -1.998248 & -0.367486 & -0.011191 \\
\hline 2 & 6 & 0 & -3.396421 & -0.201320 & 0.034970 \\
\hline 3 & 6 & 0 & -3.972211 & 1.055722 & -0.012597 \\
\hline 4 & 6 & 0 & -3.149877 & 2.181108 & -0.112043 \\
\hline 5 & 6 & 0 & -1.779306 & 2.026163 & -0.160914 \\
\hline 6 & 6 & 0 & -1.176482 & 0.758268 & -0.109681 \\
\hline 7 & 6 & 0 & 0.295365 & 0.713671 & -0.169734 \\
\hline 8 & 6 & 0 & 2.441409 & -0.251309 & -0.124385 \\
\hline 9 & 6 & 0 & 2.941314 & -0.219581 & 1.336642 \\
\hline 10 & 6 & 0 & 2.888195 & -1.549495 & -0.802180 \\
\hline
\end{tabular}




\begin{tabular}{|c|c|c|c|c|c|}
\hline 11 & 6 & 0 & 3.023965 & 0.943299 & -0.900742 \\
\hline 12 & 1 & 0 & -5.047260 & 1.172669 & 0.023913 \\
\hline 13 & 1 & 0 & -3.593087 & 3.167690 & -0.151509 \\
\hline 14 & 1 & 0 & -1.136887 & 2.896465 & -0.237400 \\
\hline 15 & 1 & 0 & 0.758544 & 1.699399 & -0.297770 \\
\hline 16 & 1 & 0 & 2.377032 & 0.540697 & 1.878458 \\
\hline 17 & 1 & 0 & 2.749097 & -1.189365 & 1.808816 \\
\hline 18 & 1 & 0 & 4.807417 & -0.504468 & 0.928625 \\
\hline 19 & 1 & 0 & 2.636521 & -1.513230 & -1.869413 \\
\hline 20 & 1 & 0 & 2.343435 & -2.376294 & -0.338918 \\
\hline 21 & 1 & 0 & 4.582918 & -2.525414 & -1.020832 \\
\hline 22 & 1 & 0 & 2.419372 & 1.114298 & -1.795913 \\
\hline 23 & 1 & 0 & 4.036425 & 0.684703 & -1.226815 \\
\hline 24 & 1 & 0 & 3.696410 & 2.051538 & 0.521640 \\
\hline 25 & 1 & 0 & -2.241108 & -2.224811 & 0.099318 \\
\hline 26 & 7 & 0 & 0.984104 & -0.346716 & -0.070999 \\
\hline 27 & 8 & 0 & 4.306692 & 0.146462 & 1.434679 \\
\hline 28 & 8 & 0 & 4.297605 & -1.700768 & -0.623254 \\
\hline 29 & 8 & 0 & 3.031649 & 2.154274 & -0.169731 \\
\hline 30 & 8 & 0 & -1.495070 & -1.614931 & 0.035208 \\
\hline 31 & 8 & 0 & -4.078725 & -1.382875 & 0.127759 \\
\hline 32 & 6 & 0 & -5.488736 & -1.323432 & 0.207168 \\
\hline 33 & 1 & 0 & -5.833110 & -2.351685 & 0.289375 \\
\hline 34 & 1 & 0 & -5.804601 & -0.758942 & 1.089326 \\
\hline 35 & 1 & 0 & -5.910850 & -0.867174 & -0.693089 \\
\hline
\end{tabular}

\title{
$\underline{\text { Structure of } \mathbf{2 1} \text { (closed form) }}$
}

\begin{abstract}
Zero-point correction= 0.291698 (Hartree/Particle) Thermal correction to Energy $=0.309410$ Thermal correction to Enthalpy $=0.310354$ Thermal correction to Gibbs Free Energy $=0.245753$ Sum of electronic and zero-point Energies $=-897.962860$ Sum of electronic and thermal Energies $=-897.945148$ Sum of electronic and thermal Enthalpies= -897.944204 Sum of electronic and thermal Free Energies= -898.008805 No imaginary frequencies
\end{abstract}

Standard orientation:

\begin{tabular}{|c|c|c|c|c|c|}
\hline \multirow{2}{*}{$\begin{array}{l}\text { Center } \\
\text { Number }\end{array}$} & \multirow{2}{*}{$\begin{array}{l}\text { Atomic } \\
\text { Number }\end{array}$} & \multirow{2}{*}{$\begin{array}{c}\text { Atomic } \\
\text { Type }\end{array}$} & \multicolumn{3}{|c|}{ Coordinates (Angstroms) } \\
\hline & & & $\mathrm{X}$ & $\mathrm{Y}$ & Z \\
\hline 1 & 6 & 0 & -1.937421 & -0.334642 & 0.000599 \\
\hline 2 & 6 & 0 & -3.349171 & -0.267171 & 0.040778 \\
\hline 3 & 6 & 0 & -3.979947 & 0.964936 & -0.015354 \\
\hline 4 & 6 & 0 & -3.234938 & 2.149160 & -0.113452 \\
\hline 5 & 6 & 0 & -1.860755 & 2.092600 & -0.155340 \\
\hline 6 & 6 & 0 & -1.198161 & 0.853498 & -0.099512 \\
\hline 7 & 6 & 0 & 0.261233 & 0.828636 & -0.150991 \\
\hline 8 & 6 & 0 & 2.387178 & -0.238927 & -0.134306 \\
\hline 9 & 6 & 0 & 2.880840 & -0.237428 & 1.330028 \\
\hline 10 & 6 & 0 & 2.792513 & -1.541828 & -0.828286 \\
\hline 11 & 6 & 0 & 3.006325 & 0.947767 & -0.893849 \\
\hline 12 & 1 & 0 & -5.059860 & 1.022413 & 0.015730 \\
\hline 13 & 1 & 0 & -3.749733 & 3.100267 & -0.155712 \\
\hline 14 & 1 & 0 & -1.270138 & 2.999102 & -0.229979 \\
\hline 15 & 1 & 0 & 0.757668 & 1.801308 & -0.228078 \\
\hline 16 & 1 & 0 & 2.335013 & 0.532502 & 1.877134 \\
\hline 17 & 1 & 0 & 2.660229 & -1.206622 & 1.791986 \\
\hline 18 & 1 & 0 & 4.742947 & -0.575869 & 0.940181 \\
\hline 19 & 1 & 0 & 2.549434 & -1.482099 & -1.896448 \\
\hline 20 & 1 & 0 & 2.221358 & -2.361733 & -0.381917 \\
\hline 21 & 1 & 0 & 4.460420 & -2.559465 & -1.056832 \\
\hline 22 & 1 & 0 & 2.413375 & 1.143664 & -1.791611 \\
\hline 23 & 1 & 0 & 4.014193 & 0.665653 & -1.213010 \\
\hline 24 & 1 & 0 & 3.704576 & 2.029747 & 0.538230 \\
\hline 25 & 1 & 0 & -0.390117 & -1.397866 & 0.027678 \\
\hline 26 & 7 & 0 & 0.925924 & -0.262895 & -0.101818 \\
\hline 27 & 8 & 0 & 4.253106 & 0.090373 & 1.436678 \\
\hline 28 & 8 & 0 & 4.191336 & -1.739495 & -0.638228 \\
\hline 29 & 8 & 0 & 3.035814 & 2.147193 & -0.147029 \\
\hline 30 & 8 & 0 & -1.367083 & -1.539408 & 0.060643 \\
\hline
\end{tabular}




$\begin{array}{rrrrrr}31 & 8 & 0 & -3.976932 & -1.462674 & 0.135328 \\ 32 & 6 & 0 & -5.386096 & -1.453970 & 0.195932 \\ 33 & 1 & 0 & -5.689644 & -2.495238 & 0.276729 \\ 34 & 1 & 0 & -5.741095 & -0.901439 & 1.072154 \\ 35 & 1 & 0 & -5.819631 & -1.017751 & -0.710134 \\ -\end{array}$

\section{Structure of $\mathbf{T S}_{\underline{21 / 51}}$}

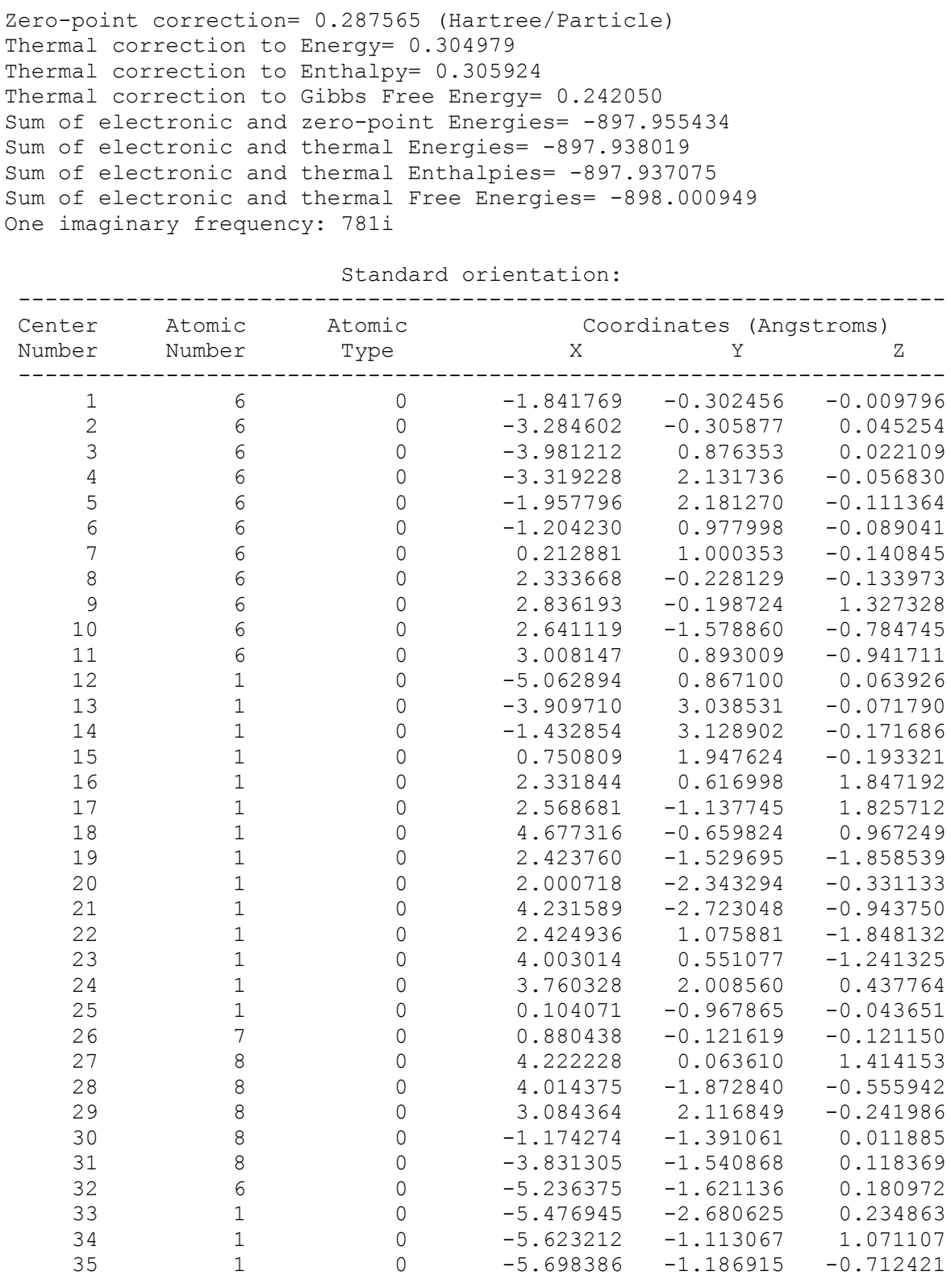

\section{$\underline{\text { Structure of } 51}$}

Zero-point correction $=0.291163$ (Hartree/Particle)

Thermal correction to Energy $=0.308982$

Thermal correction to Enthalpy= 0.309926

Thermal correction to Gibbs Free Energy $=0.245172$

Sum of electronic and zero-point Energies= -897.953215

Sum of electronic and thermal Energies= -897.935396

Sum of electronic and thermal Enthalpies= -897.934452

Sum of electronic and thermal Free Energies= -897.999206

No imaginary frequencies 
Standard orientation:

\begin{tabular}{|c|c|c|c|c|c|}
\hline \multirow{2}{*}{$\begin{array}{l}\text { Center } \\
\text { Number }\end{array}$} & \multirow{2}{*}{$\begin{array}{l}\text { Atomic } \\
\text { Number }\end{array}$} & \multirow{2}{*}{$\begin{array}{c}\text { Atomic } \\
\text { Type }\end{array}$} & \multicolumn{3}{|c|}{ Coordinates (Angstroms) } \\
\hline & & & $\mathrm{X}$ & Y & Z \\
\hline-- & & & & & \\
\hline 1 & 6 & 0 & -1.895857 & -0.374213 & -0.014232 \\
\hline 2 & 6 & 0 & -3.357110 & -0.288690 & 0.042228 \\
\hline 3 & 6 & 0 & -3.989197 & 0.920245 & 0.026871 \\
\hline 4 & 6 & 0 & -3.259525 & 2.151237 & -0.045631 \\
\hline 5 & 6 & 0 & -1.905375 & 2.138003 & -0.101258 \\
\hline 6 & 6 & 0 & -1.194183 & 0.895007 & -0.087743 \\
\hline 7 & 6 & 0 & 0.198980 & 0.896832 & -0.140068 \\
\hline 8 & 6 & 0 & 2.385195 & -0.255212 & -0.130821 \\
\hline 9 & 6 & 0 & 2.881030 & -0.149794 & 1.328636 \\
\hline 10 & 6 & 0 & 2.762294 & -1.608340 & -0.737954 \\
\hline 11 & 6 & 0 & 3.007387 & 0.869376 & -0.976982 \\
\hline 12 & 1 & 0 & -5.070100 & 0.968468 & 0.069490 \\
\hline 13 & 1 & 0 & -3.807793 & 3.084200 & -0.054356 \\
\hline 14 & 1 & 0 & -1.338142 & 3.061530 & -0.155267 \\
\hline 15 & 1 & 0 & 0.740505 & 1.840129 & -0.184897 \\
\hline 16 & 1 & 0 & 2.330862 & 0.653962 & 1.820363 \\
\hline 17 & 1 & 0 & 2.663893 & -1.084770 & 1.858655 \\
\hline 18 & 1 & 0 & 4.747311 & -0.523773 & 0.995975 \\
\hline 19 & 1 & 0 & 2.559114 & -1.599680 & -1.815404 \\
\hline 20 & 1 & 0 & 2.153451 & -2.391625 & -0.271479 \\
\hline 21 & 1 & 0 & 4.407379 & -2.676223 & -0.849981 \\
\hline 22 & 1 & 0 & 2.414936 & 0.994242 & -1.886956 \\
\hline 23 & 1 & 0 & 4.016366 & 0.561399 & -1.265819 \\
\hline 24 & 1 & 0 & 3.710052 & 2.060897 & 0.364562 \\
\hline 25 & 7 & 0 & 0.927632 & -0.209117 & -0.138035 \\
\hline 26 & 8 & 0 & 4.250257 & 0.191210 & 1.409936 \\
\hline 27 & 8 & 0 & 4.142539 & -1.831730 & -0.479479 \\
\hline 28 & 8 & 0 & 3.030996 & 2.117444 & -0.318622 \\
\hline 29 & 8 & 0 & -1.309523 & -1.475512 & 0.001962 \\
\hline 30 & 1 & 0 & 0.348834 & -1.065267 & -0.058035 \\
\hline 31 & 8 & 0 & -3.960659 & -1.495260 & 0.108702 \\
\hline 32 & 6 & 0 & -5.367532 & -1.508137 & 0.176299 \\
\hline 33 & 1 & 0 & -5.659491 & -2.554818 & 0.226732 \\
\hline 34 & 1 & 0 & -5.725086 & -0.984680 & 1.069863 \\
\hline 35 & 1 & 0 & -5.811031 & -1.047286 & -0.713261 \\
\hline
\end{tabular}

\section{$\underline{\text { Structure of } \mathbf{2 3} \text { (open form) }}$}

Zero-point correction $=0.290805$ (Hartree/Particle) Thermal correction to Energy $=0.309083$ Thermal correction to Enthalpy $=0.310027$

Thermal correction to Gibbs Free Energy= 0.244249

Sum of electronic and zero-point Energies= -897.947652

Sum of electronic and thermal Energies= -897.929374

Sum of electronic and thermal Enthalpies= -897.928430

Sum of electronic and thermal Free Energies= -897.994208

No imaginary frequencies

Standard orientation:

\begin{tabular}{|c|c|c|c|c|c|}
\hline \multirow{2}{*}{$\begin{array}{l}\text { Center } \\
\text { Number }\end{array}$} & \multirow{2}{*}{$\begin{array}{l}\text { Atomic } \\
\text { Number }\end{array}$} & \multirow{2}{*}{$\begin{array}{c}\text { Atomic } \\
\text { Type }\end{array}$} & \multicolumn{3}{|c|}{ Coordinates (Angstroms) } \\
\hline & & & $\mathrm{x}$ & Y & $\mathrm{Z}$ \\
\hline 1 & 6 & 0 & -2.022501 & -1.683747 & -0.032260 \\
\hline 2 & 6 & 0 & -3.382822 & -1.981130 & 0.089677 \\
\hline 3 & 6 & 0 & -4.306386 & -0.955957 & 0.184777 \\
\hline 4 & 6 & 0 & -3.906513 & 0.376265 & 0.159295 \\
\hline 5 & 6 & 0 & -2.549233 & 0.665054 & 0.038134 \\
\hline 6 & 6 & 0 & -1.581185 & -0.355249 & -0.055570 \\
\hline 7 & 6 & 0 & -0.167776 & 0.036976 & -0.227508 \\
\hline 8 & 6 & 0 & 2.162699 & -0.225382 & -0.023558 \\
\hline 9 & 6 & 0 & 2.605558 & 0.471123 & 1.281244 \\
\hline 10 & 6 & 0 & 2.968098 & -1.510682 & -0.231374 \\
\hline 11 & 6 & 0 & 2.398754 & 0.712201 & -1.221010 \\
\hline 12 & 1 & 0 & -3.705362 & -3.017011 & 0.104806 \\
\hline 13 & 1 & 0 & -4.643348 & 1.161907 & 0.239413 \\
\hline 14 & 1 & 0 & -0.013752 & 1.002362 & -0.716949 \\
\hline
\end{tabular}




$\begin{array}{rrrrrr}15 & 1 & 0 & 1.841715 & 1.198913 & 1.558958 \\ 16 & 1 & 0 & 2.679644 & -0.271077 & 2.083809 \\ 17 & 1 & 0 & 4.486377 & 0.560924 & 0.847864 \\ 18 & 1 & 0 & 2.740730 & -1.931774 & -1.218635 \\ 19 & 1 & 0 & 2.663732 & -2.227435 & 0.536004 \\ 20 & 1 & 0 & 4.871951 & -2.009490 & -0.230810 \\ 21 & 1 & 0 & 1.778595 & 0.380477 & -2.058292 \\ 22 & 1 & 0 & 3.446727 & 0.627232 & -1.526988 \\ 23 & 1 & 0 & 2.718618 & 2.392213 & -0.341635 \\ 24 & 1 & 0 & -1.573109 & -3.517851 & -0.201591 \\ 25 & 7 & 0 & 0.791012 & -0.683060 & 0.187612 \\ 26 & 8 & 0 & 3.814960 & 1.194324 & 1.128479 \\ 27 & 8 & 0 & 4.360016 & -1.205879 & -0.123450 \\ 28 & 8 & 0 & 2.063489 & 2.062274 & -0.968085 \\ 29 & 8 & 0 & -1.110756 & -2.676185 & -0.163986 \\ 30 & 8 & 0 & -2.055258 & 1.926853 & 0.016185 \\ 31 & 6 & 0 & -2.967636 & 3.005465 & 0.047012 \\ 32 & 1 & 0 & -3.647970 & 2.970074 & -0.809099 \\ 33 & 1 & 0 & -3.545208 & 3.005787 & 0.976091 \\ 34 & 1 & 0 & -2.363106 & 3.907955 & -0.004625 \\ 35 & 1 & 0 & -5.359270 & -1.192516 & 0.282096 \\ ------------------------------------------------------\end{array}$

\section{$\underline{\text { Structure of } \mathbf{2 3} \text { (closed form) }}$}

Zero-point correction= 0.291707 (Hartree/Particle)
Thermal correction to Energy= 0.309294
Thermal correction to Enthalpy= 0.310238
Thermal correction to Gibbs Free Energy= 0.246343
Sum of electronic and zero-point Energies=-897.969024
Sum of electronic and thermal Energies=-897.951437
Sum of electronic and thermal Enthalpies= -897.950493
Sum of electronic and thermal Free Energies= -898.014388
No imaginary frequencies

Standard orientation:

\begin{tabular}{|c|c|c|c|c|c|}
\hline \multirow{2}{*}{$\begin{array}{l}\text { Center } \\
\text { Number }\end{array}$} & \multirow{2}{*}{$\begin{array}{l}\text { Atomic } \\
\text { Number }\end{array}$} & \multirow{2}{*}{$\begin{array}{c}\text { Atomic } \\
\text { Type }\end{array}$} & \multicolumn{3}{|c|}{ Coordinates (Angstroms) } \\
\hline & & & $\mathrm{X}$ & Y & Z \\
\hline & & & 11056363 & 1701500 & $-1-----1$ \\
\hline $\begin{array}{l}1 \\
2\end{array}$ & $\begin{array}{l}6 \\
6\end{array}$ & 0 & $\begin{array}{l}-1.856363 \\
-3.181646\end{array}$ & $\begin{array}{l}-1.101540 \\
-2.141003\end{array}$ & 0.091838 \\
\hline 3 & 6 & 0 & -4.204136 & -1.215565 & 0.172691 \\
\hline 4 & 6 & 0 & -3.958675 & 0.155669 & 0.047511 \\
\hline 5 & 6 & 0 & -2.645239 & 0.592222 & -0.052248 \\
\hline 6 & 6 & 0 & -1.570552 & -0.325990 & -0.025492 \\
\hline 7 & 6 & 0 & -0.199717 & 0.162971 & -0.111881 \\
\hline 8 & 6 & 0 & 2.163747 & -0.134588 & -0.151748 \\
\hline 9 & 6 & 0 & 2.670910 & -0.093349 & 1.307496 \\
\hline 10 & 6 & 0 & 2.954815 & -1.169180 & -0.956548 \\
\hline 11 & 6 & 0 & 2.339119 & 1.247624 & -0.803189 \\
\hline 12 & 1 & 0 & -3.368271 & -3.202336 & 0.294650 \\
\hline 13 & 1 & 0 & -5.230112 & -1.556675 & 0.250855 \\
\hline 14 & 1 & 0 & -4.785554 & 0.849942 & 0.029619 \\
\hline 15 & 1 & 0 & -0.067701 & 1.244835 & -0.159433 \\
\hline 16 & 1 & 0 & 1.917975 & 0.406120 & 1.918833 \\
\hline 17 & 1 & 0 & 2.790507 & -1.116677 & 1.681560 \\
\hline 18 & 1 & 0 & 4.531791 & 0.230698 & 0.896598 \\
\hline 19 & 1 & 0 & 2.679427 & -1.101383 & -2.016385 \\
\hline 20 & 1 & 0 & 2.694121 & -2.166088 & -0.587867 \\
\hline 21 & 1 & 0 & 4.857441 & -1.556828 & -1.273771 \\
\hline 22 & 1 & 0 & 1.691043 & 1.311164 & -1.681663 \\
\hline 23 & 1 & 0 & 3.374911 & 1.338404 & -1.144686 \\
\hline 24 & 1 & 0 & 2.681348 & 2.368719 & 0.723293 \\
\hline 25 & 1 & 0 & -0.020123 & -2.130020 & 0.047748 \\
\hline 26 & 7 & 0 & 0.795364 & -0.644901 & -0.112657 \\
\hline 27 & 8 & 0 & 3.863817 & 0.655290 & 1.447778 \\
\hline 28 & 8 & 0 & 4.346118 & -0.914104 & -0.778354 \\
\hline 29 & 8 & 0 & 1.996285 & 2.323257 & 0.045695 \\
\hline 30 & 8 & 0 & -0.883346 & -2.614526 & 0.125725 \\
\hline 31 & 8 & 0 & -2.292872 & 1.894491 & -0.181840 \\
\hline 32 & 6 & 0 & -3.320084 & 2.865440 & -0.197726 \\
\hline 33 & 1 & 0 & -3.996947 & 2.706640 & -1.042342 \\
\hline 34 & 1 & 0 & -3.888824 & 2.850367 & 0.736500 \\
\hline
\end{tabular}




\section{$\underline{\text { Structure of } \mathbf{T S}} \underline{23 / 53}_{2}$}

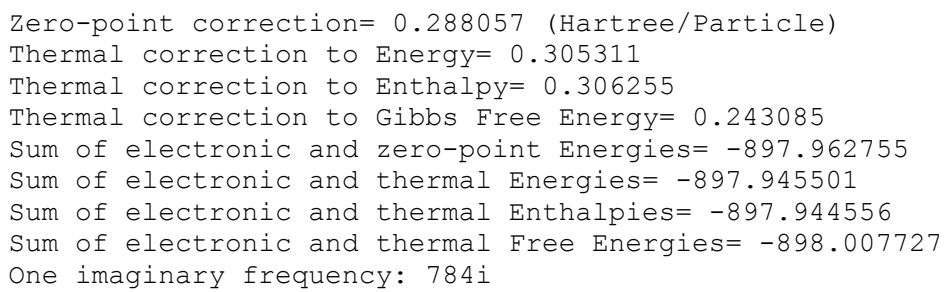

Standard orientation:

\begin{tabular}{|c|c|c|c|c|c|}
\hline \multirow{2}{*}{$\begin{array}{l}\text { Center } \\
\text { Number }\end{array}$} & \multirow{2}{*}{$\begin{array}{l}\text { Atomic } \\
\text { Number }\end{array}$} & \multirow{2}{*}{$\begin{array}{c}\text { Atomic } \\
\text { Type }\end{array}$} & \multicolumn{3}{|c|}{ Coordinates (Angstroms) } \\
\hline & & & $\mathrm{X}$ & Y & Z \\
\hline 1 & 6 & 0 & -1.693457 & -1.712675 & 0.087936 \\
\hline 2 & 6 & 0 & -3.009692 & -2.243122 & 0.217074 \\
\hline 3 & 6 & 0 & -4.089293 & -1.401717 & 0.209635 \\
\hline 4 & 6 & 0 & -3.975164 & 0.002535 & 0.077968 \\
\hline 5 & 6 & 0 & -2.717773 & 0.549214 & -0.043988 \\
\hline 6 & 6 & 0 & -1.563947 & -0.283826 & -0.037757 \\
\hline 7 & 6 & 0 & -0.263797 & 0.272767 & -0.133091 \\
\hline 8 & 6 & 0 & 2.161024 & -0.091601 & -0.154274 \\
\hline 9 & 6 & 0 & 2.658330 & -0.040249 & 1.308758 \\
\hline 10 & 6 & 0 & 2.918263 & -1.162612 & -0.943544 \\
\hline 11 & 6 & 0 & 2.364423 & 1.275259 & -0.825058 \\
\hline 12 & 1 & 0 & -3.117256 & -3.315299 & 0.317339 \\
\hline 13 & 1 & 0 & -5.084423 & -1.822788 & 0.307936 \\
\hline 14 & 1 & 0 & -4.865591 & 0.613418 & 0.076497 \\
\hline 15 & 1 & 0 & -0.127117 & 1.350910 & -0.186700 \\
\hline 16 & 1 & 0 & 1.907154 & 0.476705 & 1.907370 \\
\hline 17 & 1 & 0 & 2.760395 & -1.060623 & 1.696210 \\
\hline 18 & 1 & 0 & 4.531906 & 0.238179 & 0.926880 \\
\hline 19 & 1 & 0 & 2.670730 & -1.080469 & -2.008859 \\
\hline 20 & 1 & 0 & 2.608055 & -2.150486 & -0.585776 \\
\hline 21 & 1 & 0 & 4.809953 & -1.628244 & -1.207887 \\
\hline 22 & 1 & 0 & 1.735450 & 1.329465 & -1.717697 \\
\hline 23 & 1 & 0 & 3.408730 & 1.347895 & -1.143118 \\
\hline 24 & 1 & 0 & 2.689718 & 2.425812 & 0.684481 \\
\hline 25 & 1 & 0 & 0.363709 & -1.598829 & -0.028800 \\
\hline 26 & 7 & 0 & 0.770031 & -0.524096 & -0.137906 \\
\hline 27 & 8 & 0 & 3.858864 & 0.693532 & 1.446021 \\
\hline 28 & 8 & 0 & 4.311021 & -0.964197 & -0.727694 \\
\hline 29 & 8 & 0 & 2.010918 & 2.362155 & 0.001960 \\
\hline 30 & 8 & 0 & -0.655509 & -2.464524 & 0.086081 \\
\hline 31 & 8 & 0 & -2.464428 & 1.873290 & -0.178772 \\
\hline 32 & 6 & 0 & -3.566068 & 2.759628 & -0.184905 \\
\hline 33 & 1 & 0 & -4.120215 & 2.700786 & 0.756279 \\
\hline 34 & 1 & 0 & -3.147957 & 3.756814 & -0.301612 \\
\hline 35 & 1 & 0 & -4.237468 & 2.541777 & -1.020486 \\
\hline
\end{tabular}

\section{$\underline{\text { Structure of } 53}$}

Zero-point correction= 0.291341 (Hartree/Particle)

Thermal correction to Energy $=0.309064$

Thermal correction to Enthalpy $=0.310008$

Thermal correction to Gibbs Free Energy $=0.245635$

Sum of electronic and zero-point Energies= -897.960599

Sum of electronic and thermal Energies= -897.942877

Sum of electronic and thermal Enthalpies= -897.941933

Sum of electronic and thermal Free Energies= -898.006306

No imaginary frequencies

Standard orientation:

Center Atomic Atomic Coordinates (Angstroms)




\begin{tabular}{|c|c|c|c|c|c|}
\hline Number & Number & Type & $\mathrm{X}$ & Y & Z \\
\hline 1 & 6 & 0 & -1.788952 & -1.760960 & 0.065756 \\
\hline 2 & 6 & 0 & -3.161982 & -2.191210 & 0.157842 \\
\hline 3 & 6 & 0 & -4.173141 & -1.280744 & 0.159311 \\
\hline 4 & 6 & 0 & -3.961636 & 0.126093 & 0.072185 \\
\hline 5 & 6 & 0 & -2.677067 & 0.589156 & -0.018315 \\
\hline 6 & 6 & 0 & -1.566532 & -0.321373 & -0.023071 \\
\hline 7 & 6 & 0 & -0.266976 & 0.179821 & -0.103404 \\
\hline 8 & 6 & 0 & 2.179155 & -0.152073 & -0.132300 \\
\hline 9 & 6 & 0 & 2.624782 & 0.125751 & 1.320273 \\
\hline 10 & 6 & 0 & 2.987307 & -1.299835 & -0.741224 \\
\hline 11 & 6 & 0 & 2.370002 & 1.111553 & -0.989903 \\
\hline 12 & 1 & 0 & -3.345609 & -3.255743 & 0.224802 \\
\hline 13 & 1 & 0 & -5.197610 & -1.631808 & 0.229763 \\
\hline 14 & 1 & 0 & -4.810714 & 0.792733 & 0.079461 \\
\hline 15 & 1 & 0 & -0.097669 & 1.252311 & -0.154868 \\
\hline 16 & 1 & 0 & 1.840190 & 0.702231 & 1.812681 \\
\hline 17 & 1 & 0 & 2.737873 & -0.823924 & 1.856635 \\
\hline 18 & 1 & 0 & 4.504691 & 0.397951 & 0.966310 \\
\hline 19 & 1 & 0 & 2.777595 & -1.369958 & -1.815313 \\
\hline 20 & 1 & 0 & 2.689214 & -2.239927 & -0.262497 \\
\hline 21 & 1 & 0 & 4.896242 & -1.744100 & -0.876103 \\
\hline 22 & 1 & 0 & 1.753517 & 1.023730 & -1.888156 \\
\hline 23 & 1 & 0 & 3.418675 & 1.158933 & -1.298309 \\
\hline 24 & 1 & 0 & 2.651203 & 2.476908 & 0.339320 \\
\hline 25 & 7 & 0 & 0.794259 & -0.608467 & -0.117687 \\
\hline 26 & 8 & 0 & 3.801091 & 0.906470 & 1.385747 \\
\hline 27 & 8 & 0 & 4.364797 & -1.036393 & -0.505715 \\
\hline 28 & 8 & 0 & 1.983666 & 2.298854 & -0.333929 \\
\hline 29 & 8 & 0 & -0.833416 & -2.574239 & 0.062761 \\
\hline 30 & 1 & 0 & 0.537905 & -1.613730 & -0.030748 \\
\hline 31 & 8 & 0 & -2.335452 & 1.896759 & -0.108292 \\
\hline 32 & 6 & 0 & -3.376690 & 2.852711 & -0.106956 \\
\hline 33 & 1 & 0 & -4.050615 & 2.696681 & -0.954331 \\
\hline 34 & 1 & 0 & -3.945657 & 2.808774 & 0.826272 \\
\hline 35 & 1 & 0 & -2.894165 & 3.823266 & -0.196849 \\
\hline
\end{tabular}

\section{$\underline{\text { Structure of } \mathbf{2 4} \text { (open form) }}$}

Zero-point correction $=0.290137$ (Hartree/Particle) Thermal correction to Energy $=0.308680$

Thermal correction to Enthalpy $=0.309624$

Thermal correction to Gibbs Free Energy= 0.243303

Sum of electronic and zero-point Energies $=-897.967738$

Sum of electronic and thermal Energies= -897.949195

Sum of electronic and thermal Enthalpies= -897.948251

Sum of electronic and thermal Free Energies= -898.014572

No imaginary frequencies

Standard orientation:

\begin{tabular}{|c|c|c|c|c|c|}
\hline \multirow{2}{*}{$\begin{array}{l}\text { Center } \\
\text { Number }\end{array}$} & \multirow{2}{*}{$\begin{array}{l}\text { Atomic } \\
\text { Number }\end{array}$} & \multirow{2}{*}{$\begin{array}{c}\text { Atomic } \\
\text { Type }\end{array}$} & \multicolumn{3}{|c|}{ Coordinates (Angstroms) } \\
\hline & & & $\mathrm{X}$ & Y & Z \\
\hline 1 & 6 & 0 & -2.021999 & -1.211221 & 0.157630 \\
\hline 2 & 6 & 0 & -3.405497 & -1.349537 & 0.115911 \\
\hline 3 & 6 & 0 & -4.203852 & -0.224094 & -0.020168 \\
\hline 4 & 6 & 0 & -3.629736 & 1.041246 & -0.104992 \\
\hline 5 & 6 & 0 & -2.247634 & 1.186041 & -0.051828 \\
\hline 6 & 6 & 0 & -1.417859 & 0.056506 & 0.068612 \\
\hline 7 & 6 & 0 & 0.041199 & 0.253297 & 0.122002 \\
\hline 8 & 6 & 0 & 2.305194 & -0.245480 & -0.202011 \\
\hline 9 & 6 & 0 & 2.741966 & -0.158874 & 1.274335 \\
\hline 10 & 6 & 0 & 3.030863 & -1.397486 & -0.898036 \\
\hline 11 & 6 & 0 & 2.646255 & 1.071799 & -0.928458 \\
\hline 12 & 1 & 0 & -3.873721 & -2.324875 & 0.190116 \\
\hline 13 & 1 & 0 & -4.262351 & 1.916493 & -0.213811 \\
\hline 14 & 1 & 0 & 0.365338 & 1.221629 & 0.515275 \\
\hline 15 & 1 & 0 & 2.022573 & 0.448529 & 1.825294 \\
\hline 16 & 1 & 0 & 2.741606 & -1.163401 & 1.713711 \\
\hline 17 & 1 & 0 & 4.639415 & -0.040938 & 0.931260 \\
\hline 18 & 1 & 0 & 2.817216 & -1.367241 & -1.973262 \\
\hline
\end{tabular}




\begin{tabular}{|c|c|c|c|c|c|}
\hline 19 & 1 & 0 & 2.644543 & -2.336137 & -0.490659 \\
\hline 20 & 1 & 0 & 4.893216 & -2.018159 & -1.047513 \\
\hline 21 & 1 & 0 & 2.032314 & 1.136630 & -1.829942 \\
\hline 22 & 1 & 0 & 3.698483 & 1.045783 & -1.230273 \\
\hline 23 & 1 & 0 & 3.042870 & 2.247866 & 0.539729 \\
\hline 24 & 1 & 0 & -1.788267 & -3.073940 & 0.437678 \\
\hline 25 & 7 & 0 & 0.886514 & -0.600414 & -0.291249 \\
\hline 26 & 8 & 0 & 3.998879 & 0.476319 & 1.434322 \\
\hline 27 & 8 & 0 & 4.433213 & -1.273478 & -0.655912 \\
\hline 28 & 8 & 0 & 2.378969 & 2.229464 & -0.160219 \\
\hline 29 & 8 & 0 & -1.232679 & -2.298848 & 0.318451 \\
\hline 30 & 6 & 0 & -1.657985 & 2.573359 & -0.144647 \\
\hline 31 & 1 & 0 & -1.166464 & 2.859053 & 0.789072 \\
\hline 32 & 1 & 0 & -0.907741 & 2.641999 & -0.93491 \\
\hline 33 & 1 & 0 & -2.439588 & 3.304968 & -0.348595 \\
\hline 34 & 8 & 0 & -5.547755 & -0.420232 & -0.06334 \\
\hline 35 & 1 & 0 & -5.995545 & 0.425505 & -0.150910 \\
\hline
\end{tabular}

\title{
$\underline{\text { Structure of } 24 \text { (closed form) }}$
}

\begin{abstract}
Zero-point correction= 0.290838 (Hartree/Particle) Thermal correction to Energy $=0.308761$ Thermal correction to Enthalpy $=0.309705$ Thermal correction to Gibbs Free Energy $=0.244882$ Sum of electronic and zero-point Energies $=\mathbf{- 8 9 7 . 9 8 8 8 8 3}$ Sum of electronic and thermal Energies $=-897.970960$ Sum of electronic and thermal Enthalpies= -897.970016 Sum of electronic and thermal Free Energies $=\mathbf{- 8 9 8 . 0 3 4 8 4 0}$ No imaginary frequencies
\end{abstract}

Standard orientation:

\begin{tabular}{|c|c|c|c|c|c|}
\hline \multirow{2}{*}{$\begin{array}{l}\text { Center } \\
\text { Number }\end{array}$} & \multirow{2}{*}{$\begin{array}{l}\text { Atomic } \\
\text { Number }\end{array}$} & \multirow{2}{*}{$\begin{array}{c}\text { Atomic } \\
\text { Type }\end{array}$} & \multicolumn{3}{|c|}{ Coordinates (Angstroms) } \\
\hline & & & $\mathrm{X}$ & $\mathrm{Y}$ & Z \\
\hline 1 & 6 & 0 & -1.930348 & -1.180594 & 0.059540 \\
\hline 2 & 6 & 0 & -3.299290 & -1.427564 & 0.131007 \\
\hline 3 & 6 & 0 & -4.179280 & -0.362531 & 0.085702 \\
\hline 4 & 6 & 0 & -3.716465 & 0.955131 & -0.029935 \\
\hline 5 & 6 & 0 & -2.358860 & 1.215382 & -0.100379 \\
\hline 6 & 6 & 0 & -1.438828 & 0.145061 & -0.056850 \\
\hline 7 & 6 & 0 & -0.008535 & 0.398565 & -0.125891 \\
\hline 8 & 6 & 0 & 2.284679 & -0.232399 & -0.132246 \\
\hline 9 & 6 & 0 & 2.766900 & -0.104769 & 1.329729 \\
\hline 10 & 6 & 0 & 2.945026 & -1.438233 & -0.804805 \\
\hline 11 & 6 & 0 & 2.654751 & 1.041010 & -0.914471 \\
\hline 12 & 1 & 0 & -3.661910 & -2.442609 & 0.220415 \\
\hline 13 & 1 & 0 & -4.427760 & 1.774234 & -0.063782 \\
\hline 14 & 1 & 0 & 0.311110 & 1.440538 & -0.193349 \\
\hline 15 & 1 & 0 & 2.079391 & 0.553525 & 1.863200 \\
\hline 16 & 1 & 0 & 2.738104 & -1.089008 & 1.811474 \\
\hline 17 & 1 & 0 & 4.661273 & -0.077810 & 0.945839 \\
\hline 18 & 1 & 0 & 2.698976 & -1.444169 & -1.873898 \\
\hline 19 & 1 & 0 & 2.546697 & -2.348935 & -0.347011 \\
\hline 20 & 1 & 0 & 4.781601 & -2.113336 & -1.006385 \\
\hline 21 & 1 & 0 & 2.029155 & 1.099497 & -1.809369 \\
\hline 22 & 1 & 0 & 3.696802 & 0.959198 & -1.238104 \\
\hline 23 & 1 & 0 & 3.131602 & 2.259555 & 0.498235 \\
\hline 24 & 1 & 0 & -0.178316 & -1.881561 & 0.048205 \\
\hline 25 & 7 & 0 & 0.858109 & -0.547554 & -0.097339 \\
\hline 26 & 8 & 0 & 4.048195 & 0.488182 & 1.429703 \\
\hline 27 & 8 & 0 & 4.354716 & -1.350722 & -0.611040 \\
\hline 28 & 8 & 0 & 2.451766 & 2.236082 & -0.185963 \\
\hline 29 & 8 & 0 & -1.106608 & -2.229090 & 0.105390 \\
\hline 30 & 6 & 0 & -1.891764 & 2.645961 & -0.222486 \\
\hline 31 & 1 & 0 & -1.249319 & 2.931223 & 0.613637 \\
\hline 32 & 1 & 0 & -1.324388 & 2.805512 & -1.142315 \\
\hline 33 & 1 & 0 & -2.746816 & 3.321214 & -0.234439 \\
\hline 34 & 8 & 0 & -5.504046 & -0.645964 & 0.157476 \\
\hline 35 & 1 & 0 & -6.013064 & 0.167778 & 0.111008 \\
\hline
\end{tabular}




\section{$\underline{\text { Structure of } \mathbf{T S}_{24 / 54}}$}

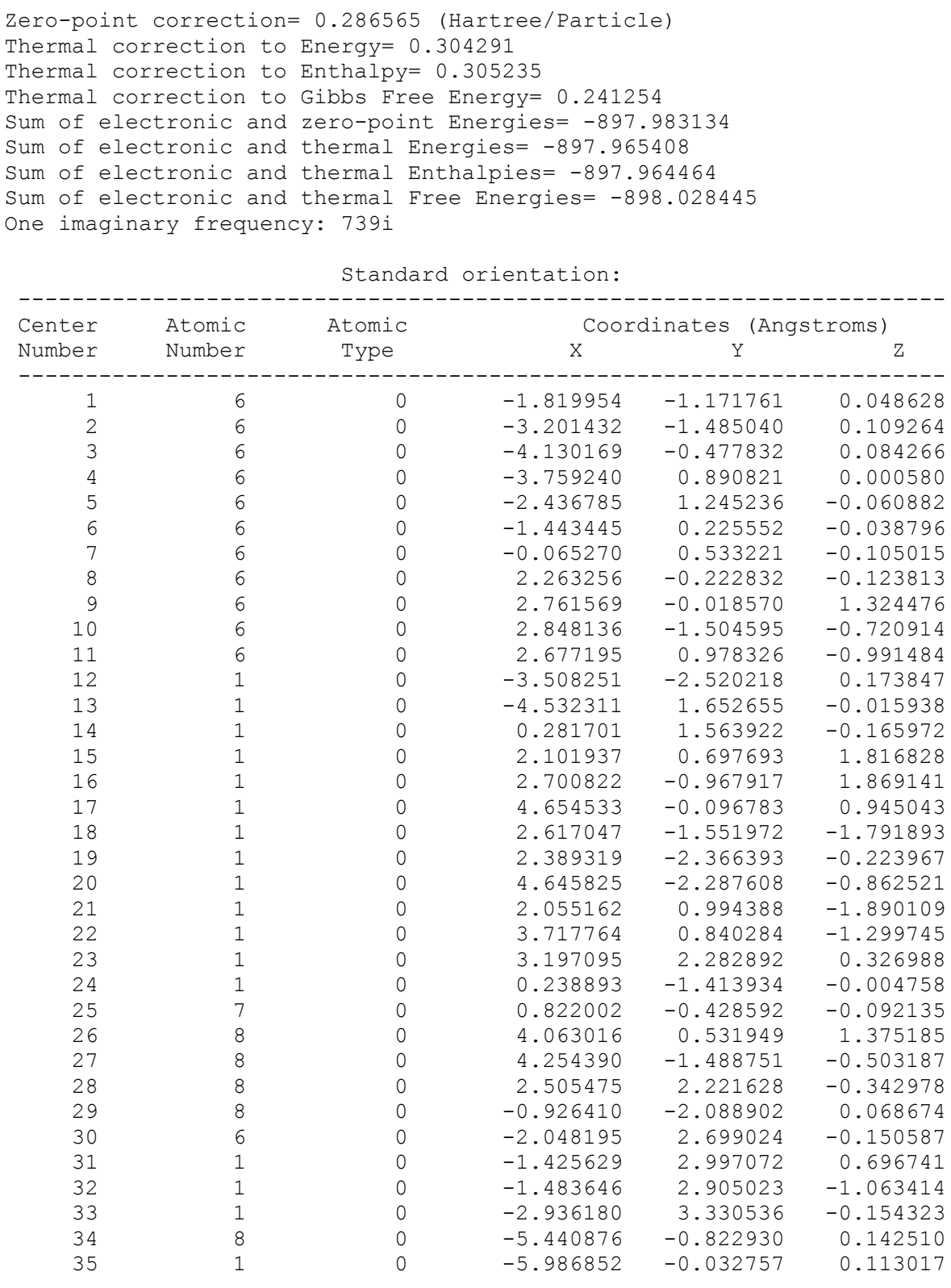

\section{$\underline{\text { Structure of } \mathbf{5 4}}$}

Zero-point correction $=0.289865$ (Hartree/Particle)

Thermal correction to Energy $=0.308001$

Thermal correction to Enthalpy $=0.308945$

Thermal correction to Gibbs Free Energy $=0.243839$

Sum of electronic and zero-point Energies= -897.980765

Sum of electronic and thermal Energies= -897.962629

Sum of electronic and thermal Enthalpies= -897.961685

Sum of electronic and thermal Free Energies= -898.026791

No imaginary frequencies

Standard orientation:

\begin{tabular}{|c|c|c|c|c|c|}
\hline \multirow{2}{*}{$\begin{array}{l}\text { Center } \\
\text { Number }\end{array}$} & \multirow{2}{*}{$\begin{array}{l}\text { Atomic } \\
\text { Number }\end{array}$} & \multirow{2}{*}{$\begin{array}{c}\text { Atomic } \\
\text { Type }\end{array}$} & \multicolumn{3}{|c|}{ Coordinates (Angstroms) } \\
\hline & & & $\mathrm{X}$ & Y & Z \\
\hline 1 & 6 & 0 & -1.872502 & -1.230320 & 0.043752 \\
\hline 2 & 6 & 0 & -3.283338 & -1.471900 & 0.114397 \\
\hline
\end{tabular}




\begin{tabular}{|c|c|c|c|c|c|}
\hline 3 & 6 & 0 & -4.161724 & -0.430972 & 0.090736 \\
\hline 4 & 6 & 0 & -3.731909 & 0.930648 & -0.001329 \\
\hline 5 & 6 & 0 & -2.404831 & 1.233160 & -0.070545 \\
\hline 6 & 6 & 0 & -1.440871 & 0.168882 & -0.050936 \\
\hline 7 & 6 & 0 & -0.078455 & 0.453935 & -0.119412 \\
\hline 8 & 6 & 0 & 2.290801 & -0.238166 & -0.124922 \\
\hline 9 & 6 & 0 & 2.766290 & -0.017412 & 1.328323 \\
\hline 10 & 6 & 0 & 2.926095 & -1.495570 & -0.721916 \\
\hline 11 & 6 & 0 & 2.670361 & 0.978019 & -0.988152 \\
\hline 12 & 1 & 0 & -3.634135 & -2.492710 & 0.184732 \\
\hline 13 & 1 & 0 & -4.476111 & 1.720974 & -0.015740 \\
\hline 14 & 1 & 0 & 0.274464 & 1.480638 & -0.176091 \\
\hline 15 & 1 & 0 & 2.073700 & 0.672719 & 1.812963 \\
\hline 16 & 1 & 0 & 2.734911 & -0.968560 & 1.872728 \\
\hline 17 & 1 & 0 & 4.666548 & -0.025082 & 0.976554 \\
\hline 18 & 1 & 0 & 2.714263 & -1.541233 & -1.796833 \\
\hline 19 & 1 & 0 & 2.490462 & -2.378583 & -0.240199 \\
\hline 20 & 1 & 0 & 4.750852 & -2.215162 & -0.840250 \\
\hline 21 & 1 & 0 & 2.057405 & 0.972498 & -1.892961 \\
\hline 22 & 1 & 0 & 3.718254 & 0.875640 & -1.284516 \\
\hline 23 & 1 & 0 & 3.132843 & 2.302345 & 0.333436 \\
\hline 24 & 7 & 0 & 0.854845 & -0.485394 & -0.118119 \\
\hline 25 & 8 & 0 & 4.044902 & 0.582140 & 1.394740 \\
\hline 26 & 8 & 0 & 4.325861 & -1.434496 & -0.479025 \\
\hline 27 & 8 & 0 & 2.449487 & 2.214313 & -0.342044 \\
\hline 28 & 8 & 0 & -1.041767 & -2.171823 & 0.062594 \\
\hline 29 & 1 & 0 & 0.444455 & -1.439534 & -0.026328 \\
\hline 30 & 8 & 0 & -5.488628 & -0.705742 & 0.157935 \\
\hline 31 & 1 & 0 & -5.991764 & 0.112094 & 0.126514 \\
\hline 32 & 6 & 0 & -1.964080 & 2.671196 & -0.165334 \\
\hline 33 & 1 & 0 & -1.329235 & 2.949526 & 0.679697 \\
\hline 34 & 1 & 0 & -1.395066 & 2.855065 & -1.080182 \\
\hline 35 & 1 & 0 & -2.829620 & 3.332976 & -0.168256 \\
\hline
\end{tabular}

\section{Structure of $\mathbf{2 7}$ (open form)}

Zero-point correction= 0.241788 (Hartree/Particle)
Thermal correction to Energy= 0.259144
Thermal correction to Enthalpy= 0.260088
Thermal correction to Gibos Free Energy= 0.196192
Sum of electronic and zero-point Energies= -981.964624
Sum of electronic and thermal Energies=-981.947267
Sum of electronic and thermal Enthalpies=-981.946323
Sum of electronic and thermal Free Energies= -982.010219
No imaginary frequencies

Standard orientation:

\begin{tabular}{|c|c|c|c|c|c|}
\hline \multirow{2}{*}{$\begin{array}{l}\text { Center } \\
\text { Number }\end{array}$} & \multirow{2}{*}{$\begin{array}{l}\text { Atomic } \\
\text { Number }\end{array}$} & \multirow{2}{*}{$\begin{array}{c}\text { Atomic } \\
\text { Type }\end{array}$} & \multicolumn{3}{|c|}{ Coordinates (Angstroms) } \\
\hline & & & $\mathrm{X}$ & Y & $\mathrm{Z}$ \\
\hline 1 & 6 & 0 & -1.961119 & -1.003542 & 0.053012 \\
\hline 2 & 6 & 0 & -3.352625 & -1.055227 & 0.117178 \\
\hline 3 & 6 & 0 & -4.170044 & 0.051908 & 0.058349 \\
\hline 4 & 6 & 0 & -3.545933 & 1.280482 & -0.074206 \\
\hline 5 & 6 & 0 & -2.174927 & 1.394604 & -0.143806 \\
\hline 6 & 6 & 0 & -1.359825 & 0.256863 & -0.080985 \\
\hline 7 & 6 & 0 & 0.097410 & 0.479483 & -0.161453 \\
\hline 8 & 6 & 0 & 2.375846 & -0.118714 & -0.147322 \\
\hline 9 & 6 & 0 & 2.882464 & -0.094178 & 1.313189 \\
\hline 10 & 6 & 0 & 3.018067 & -1.285865 & -0.902211 \\
\hline 11 & 6 & 0 & 2.743299 & 1.198395 & -0.849784 \\
\hline 12 & 1 & 0 & -1.728506 & 2.376560 & -0.247248 \\
\hline 13 & 1 & 0 & 0.377414 & 1.532817 & -0.278731 \\
\hline 14 & 1 & 0 & 2.204947 & 0.522383 & 1.905494 \\
\hline 15 & 1 & 0 & 2.863975 & -1.110689 & 1.721069 \\
\hline 16 & 1 & 0 & 4.768106 & -0.036934 & 0.896292 \\
\hline 17 & 1 & 0 & 2.756913 & -1.226698 & -1.966264 \\
\hline 18 & 1 & 0 & 2.616833 & -2.216296 & -0.491706 \\
\hline 19 & 1 & 0 & 4.845766 & -1.965185 & -1.162973 \\
\hline 20 & 1 & 0 & 2.122190 & 1.310411 & -1.743203 \\
\hline 21 & 1 & 0 & 3.785986 & 1.139633 & -1.176598 \\
\hline 22 & 1 & 0 & 3.212204 & 2.333306 & 0.633248 \\
\hline
\end{tabular}




\begin{tabular}{rrrrrr}
23 & 1 & 0 & -1.841486 & -2.885007 & 0.212948 \\
24 & 7 & 0 & 0.954302 & -0.449960 & -0.085757 \\
25 & 8 & 0 & 4.164859 & 0.493316 & 1.430589 \\
26 & 8 & 0 & 4.433172 & -1.216612 & -0.727870 \\
27 & 8 & 0 & 2.532793 & 2.346047 & -0.051126 \\
28 & 8 & 0 & -1.238128 & -2.139693 & 0.115011 \\
29 & 9 & 0 & -4.306258 & 2.385692 & -0.136671 \\
30 & 9 & 0 & -3.902125 & -2.282536 & 0.243468 \\
31 & 1 & 0 & -5.246260 & -0.042709 & 0.112199 \\
\hline
\end{tabular}

\section{$\underline{\text { Structure of } \mathbf{2 7} \text { (closed form) }}$}

Zero-point correction= 0.242382 (Hartree/Particle)
Thermal correction to Energy= 0.259228
Thermal correction to Enthalpy= 0.260173
Thermal correction to Gibos Free Energy= 0.197557
Sum of electronic and zero-point Energies=-981.979434
Sum of electronic and thermal Energies= -981.962588
Sum of electronic and thermal Enthalpies= -981.961644
Sum of electronic and thermal Free Energies= -982.024259
No imaginary frequencies

\begin{tabular}{|c|c|c|c|c|c|}
\hline \multirow{2}{*}{$\begin{array}{l}\text { Center } \\
\text { Number }\end{array}$} & \multirow{2}{*}{$\begin{array}{l}\text { Atomic } \\
\text { Number }\end{array}$} & \multirow{2}{*}{$\begin{array}{c}\text { Atomic } \\
\text { Type }\end{array}$} & \multicolumn{3}{|c|}{ Coordinates (Angstroms) } \\
\hline & & & $\mathrm{X}$ & $\mathrm{Y}$ & Z \\
\hline 1 & 6 & 0 & -1.881695 & -0.977400 & 0.049730 \\
\hline 2 & 6 & 0 & -3.270335 & -1.137641 & 0.120180 \\
\hline 3 & 6 & 0 & -4.142716 & -0.073425 & 0.066705 \\
\hline 4 & 6 & 0 & -3.612624 & 1.204572 & -0.062511 \\
\hline 5 & 6 & 0 & -2.259011 & 1.424195 & -0.135259 \\
\hline 6 & 6 & 0 & -1.381701 & 0.330590 & -0.079615 \\
\hline 7 & 6 & 0 & 0.059388 & 0.577771 & -0.152473 \\
\hline 8 & 6 & 0 & 2.339424 & -0.102450 & -0.151003 \\
\hline 9 & 6 & 0 & 2.832134 & -0.051283 & 1.313971 \\
\hline 10 & 6 & 0 & 2.962070 & -1.298268 & -0.876250 \\
\hline 11 & 6 & 0 & 2.735264 & 1.192027 & -0.880292 \\
\hline 12 & 1 & 0 & -5.211040 & -0.235800 & 0.124020 \\
\hline 13 & 1 & 0 & -1.877721 & 2.433561 & -0.233341 \\
\hline 14 & 1 & 0 & 0.369932 & 1.624197 & -0.231268 \\
\hline 15 & 1 & 0 & 2.159832 & 0.591984 & 1.883581 \\
\hline 16 & 1 & 0 & 2.791998 & -1.056695 & 1.748627 \\
\hline 17 & 1 & 0 & 4.725667 & -0.043926 & 0.926727 \\
\hline 18 & 1 & 0 & 2.714678 & -1.253522 & -1.944184 \\
\hline 19 & 1 & 0 & 2.540453 & -2.216362 & -0.455284 \\
\hline 20 & 1 & 0 & 4.781120 & -2.006529 & -1.115288 \\
\hline 21 & 1 & 0 & 2.122330 & 1.293672 & -1.780449 \\
\hline 22 & 1 & 0 & 3.779193 & 1.106395 & -1.195763 \\
\hline 23 & 1 & 0 & 3.219218 & 2.358220 & 0.574918 \\
\hline 24 & 1 & 0 & -0.173122 & -1.751568 & 0.051461 \\
\hline 25 & 7 & 0 & 0.904063 & -0.379046 & -0.111085 \\
\hline 26 & 8 & 0 & 4.121452 & 0.516576 & 1.427811 \\
\hline 27 & 8 & 0 & 4.372436 & -1.254743 & -0.681433 \\
\hline 28 & 8 & 0 & 2.537230 & 2.357737 & -0.107221 \\
\hline 29 & 8 & 0 & -1.108327 & -2.063689 & 0.109161 \\
\hline 30 & 9 & 0 & -4.460630 & 2.246257 & -0.115828 \\
\hline 31 & 9 & 0 & -3.754313 & -2.376200 & 0.244519 \\
\hline
\end{tabular}

\section{$\underline{\text { Structure of } \mathbf{T S}_{2}} \underline{2767}$}

Zero-point correction $=0.238497$ (Hartree/Particle)

Thermal correction to Energy $=0.254888$

Thermal correction to Enthalpy $=0.255832$

Thermal correction to Gibbs Free Energy= 0.194250

Sum of electronic and zero-point Energies= -981.971913

Sum of electronic and thermal Energies= -981.955522

Sum of electronic and thermal Enthalpies= -981.954577

Sum of electronic and thermal Free Energies= -982.016160

One imaginary frequency: $772 i$ 
Standard orientation:

\begin{tabular}{|c|c|c|c|c|c|}
\hline \multirow{2}{*}{$\begin{array}{l}\text { Center } \\
\text { Number }\end{array}$} & \multirow{2}{*}{$\begin{array}{l}\text { Atomic } \\
\text { Number }\end{array}$} & \multirow{2}{*}{$\begin{array}{l}\text { Atomic } \\
\text { Type }\end{array}$} & \multicolumn{3}{|c|}{ Coordinates (Angstroms) } \\
\hline & & & $\mathrm{X}$ & Y & Z \\
\hline 1 & 6 & & & 0918733 & 0040766 \\
\hline 1 & 6 & 0 & 1.765265 & 0.948733 & 0.040766 \\
\hline 2 & 6 & 0 & 3.170754 & 1.200568 & 0.112540 \\
\hline 3 & 6 & 0 & 4.103646 & 0.206988 & 0.076789 \\
\hline 4 & 6 & 0 & 3.679874 & -1.130835 & -0.034432 \\
\hline 5 & 6 & 0 & 2.364786 & -1.466389 & -0.105687 \\
\hline 6 & 6 & 0 & 1.395985 & -0.431236 & -0.069007 \\
\hline 7 & 6 & 0 & 0.006230 & -0.728859 & -0.139267 \\
\hline 8 & 6 & 0 & -2.307348 & 0.080736 & -0.142190 \\
\hline 9 & 6 & 0 & -2.811061 & -0.026369 & 1.315936 \\
\hline 10 & 6 & 0 & -2.852359 & 1.345679 & -0.810049 \\
\hline 11 & 6 & 0 & -2.749600 & -1.154796 & -0.941742 \\
\hline 12 & 1 & 0 & 5.158884 & 0.443750 & 0.134049 \\
\hline 13 & 1 & 0 & 2.066288 & -2.504762 & -0.188762 \\
\hline 14 & 1 & 0 & -0.340721 & -1.760086 & -0.210600 \\
\hline 15 & 1 & 0 & -2.168355 & -0.725828 & 1.852484 \\
\hline 16 & 1 & 0 & -2.731756 & 0.952258 & 1.803580 \\
\hline 17 & 1 & 0 & -4.702287 & 0.074711 & 0.933485 \\
\hline 18 & 1 & 0 & -2.620489 & 1.328511 & -1.881766 \\
\hline 19 & 1 & 0 & -2.369403 & 2.220073 & -0.359795 \\
\hline 20 & 1 & 0 & -4.628385 & 2.166757 & -0.995337 \\
\hline 21 & 1 & 0 & -2.139732 & -1.227857 & -1.846272 \\
\hline 22 & 1 & 0 & -3.790503 & -1.012972 & -1.245881 \\
\hline 23 & 1 & 0 & -3.278660 & -2.384905 & 0.444102 \\
\hline 24 & 1 & 0 & -0.266061 & 1.224496 & -0.010094 \\
\hline 25 & 7 & 0 & -0.858428 & 0.244794 & -0.111483 \\
\hline 26 & 8 & 0 & -4.122139 & -0.545516 & 1.390575 \\
\hline 27 & 8 & 0 & -4.257040 & 1.379267 & -0.591965 \\
\hline 28 & 8 & 0 & -2.588474 & -2.362455 & -0.229656 \\
\hline 29 & 8 & 0 & 0.904816 & 1.894003 & 0.073653 \\
\hline 30 & 9 & 0 & 4.631702 & -2.083859 & -0.067214 \\
\hline 31 & 9 & 0 & 3.560058 & 2.473259 & 0.221479 \\
\hline
\end{tabular}

\section{Structure of 67}

Zero-point correction $=0.241880$ (Hartree/Particle)

Thermal correction to Energy $=0.258776$

Thermal correction to Enthalpy $=0.259720$

Thermal correction to Gibbs Free Energy= 0.196797

Sum of electronic and zero-point Energies= -981.969834

Sum of electronic and thermal Energies= -981.952939

Sum of electronic and thermal Enthalpies= -981.951995

Sum of electronic and thermal Free Energies= -982.014917

No imaginary frequencies

Standard orientation:

\begin{tabular}{|c|c|c|c|c|c|}
\hline \multirow{2}{*}{$\begin{array}{l}\text { Center } \\
\text { Number }\end{array}$} & \multirow{2}{*}{$\begin{array}{l}\text { Atomic } \\
\text { Number }\end{array}$} & \multirow{2}{*}{$\begin{array}{c}\text { Atomic } \\
\text { Type }\end{array}$} & \multicolumn{3}{|c|}{ Coordinates (Angstroms) } \\
\hline & & & $\mathrm{X}$ & Y & Z \\
\hline 1 & 6 & 0 & -1.824744 & -1.010451 & 0.029117 \\
\hline 2 & 6 & 0 & -3.264834 & -1.171373 & 0.113197 \\
\hline 3 & 6 & 0 & -4.133653 & -0.133242 & 0.084456 \\
\hline 4 & 6 & 0 & -3.632164 & 1.190290 & -0.031594 \\
\hline 5 & 6 & 0 & -2.310999 & 1.455279 & -0.111911 \\
\hline 6 & 6 & 0 & -1.381992 & 0.366300 & -0.083031 \\
\hline 7 & 6 & 0 & -0.010070 & 0.628579 & -0.152695 \\
\hline 8 & 6 & 0 & 2.346951 & -0.112020 & -0.142095 \\
\hline 9 & 6 & 0 & 2.821986 & 0.045436 & 1.320441 \\
\hline 10 & 6 & 0 & 2.954391 & -1.362214 & -0.782584 \\
\hline 11 & 6 & 0 & 2.751167 & 1.125964 & -0.960072 \\
\hline 12 & 1 & 0 & -5.200920 & -0.306391 & 0.149283 \\
\hline 13 & 1 & 0 & -1.960120 & 2.477007 & -0.196789 \\
\hline 14 & 1 & 0 & 0.348706 & 1.654385 & -0.214135 \\
\hline 15 & 1 & 0 & 2.139899 & 0.727949 & 1.830062 \\
\hline 16 & 1 & 0 & 2.774707 & -0.925181 & 1.828371 \\
\hline 17 & 1 & 0 & 4.724251 & 0.016026 & 0.979309 \\
\hline 18 & 1 & 0 & 2.745872 & -1.364878 & -1.859110 \\
\hline
\end{tabular}




\begin{tabular}{rrrrrr}
19 & 1 & 0 & 2.498387 & -2.252715 & -0.334051 \\
20 & 1 & 0 & 4.764953 & -2.112961 & -0.919393 \\
21 & 1 & 0 & 2.147063 & 1.161103 & -1.870399 \\
22 & 1 & 0 & 3.799316 & 1.014539 & -1.251351 \\
23 & 1 & 0 & 3.224812 & 2.398450 & 0.408438 \\
24 & 7 & 0 & 0.902537 & -0.325341 & -0.141949 \\
25 & 8 & 0 & 4.108259 & 0.621265 & 1.408385 \\
26 & 8 & 0 & 4.352530 & -1.338009 & -0.531734 \\
27 & 8 & 0 & 2.543241 & 2.340027 & -0.272176 \\
28 & 8 & 0 & -1.051827 & -1.991540 & 0.056119 \\
29 & 1 & 0 & 0.490799 & -1.272669 & -0.043033 \\
30 & 9 & 0 & -4.541049 & 2.185376 & -0.056603 \\
31 & 9 & 0 & -3.717766 & -2.421655 & 0.223527 \\
\hline
\end{tabular}

\section{Structure of $\mathbf{3 0}$ (open form)}

Zero-point correction= 0.263608 (Hartree/Particle)
Thermal correction to Energy= 0.284230
Thermal correction to Enthalpy= 0.285174
Thermal correction to Gibbs Free Energy= 0.212582
Sum of electronic and zero-point Energies= -1192.442396
Sum of electronic and thermal Energies=-1192.421774
Sum of electronic and thermal Enthalpies= -1192.420830
Sum of electronic and thermal Free Energies= -1192.493422
No imaginary frequencies

Standard orientation:

\begin{tabular}{|c|c|c|c|c|c|}
\hline \multirow{2}{*}{$\begin{array}{l}\text { Center } \\
\text { Number }\end{array}$} & \multirow{2}{*}{$\begin{array}{l}\text { Atomic } \\
\text { Number }\end{array}$} & \multirow{2}{*}{$\begin{array}{c}\text { Atomic } \\
\text { Type }\end{array}$} & \multicolumn{3}{|c|}{ Coordinates (Angstroms) } \\
\hline & & & $\mathrm{X}$ & $\mathrm{Y}$ & Z \\
\hline & & & & - & ---------- \\
\hline 1 & 6 & 0 & 1.171439 & 1.060396 & -0.031113 \\
\hline 2 & 6 & 0 & 2.584299 & 1.122182 & 0.044857 \\
\hline 3 & 6 & 0 & 3.386337 & -0.011654 & 0.041243 \\
\hline 4 & 6 & 0 & 2.768225 & -1.235819 & -0.041746 \\
\hline 5 & 6 & 0 & 1.384542 & -1.350723 & -0.117410 \\
\hline 6 & 6 & 0 & 0.573825 & -0.227806 & -0.108814 \\
\hline 7 & 6 & 0 & -0.885896 & -0.465758 & -0.181848 \\
\hline 8 & 6 & 0 & -3.173997 & 0.089868 & -0.141712 \\
\hline 9 & 6 & 0 & -3.665567 & 0.086689 & 1.325759 \\
\hline 10 & 6 & 0 & -3.846439 & 1.229028 & -0.913155 \\
\hline 11 & 6 & 0 & -3.519953 & -1.249335 & -0.810788 \\
\hline 12 & 1 & 0 & 4.463077 & 0.074292 & 0.099933 \\
\hline 13 & 1 & 0 & 0.942552 & -2.338390 & -0.181744 \\
\hline 14 & 1 & 0 & -1.145181 & -1.524766 & -0.292881 \\
\hline 15 & 1 & 0 & -2.964873 & -0.495602 & 1.925798 \\
\hline 16 & 1 & 0 & -3.673650 & 1.113028 & 1.708222 \\
\hline 17 & 1 & 0 & -5.554772 & -0.028613 & 0.939585 \\
\hline 18 & 1 & 0 & -3.598490 & 1.152292 & -1.979275 \\
\hline 19 & 1 & 0 & -3.458932 & 2.175927 & -0.527508 \\
\hline 20 & 1 & 0 & -5.694255 & 1.850683 & -1.177740 \\
\hline 21 & 1 & 0 & -2.916664 & -1.362980 & -1.716370 \\
\hline 22 & 1 & 0 & -4.570421 & -1.224957 & -1.115159 \\
\hline 23 & 1 & 0 & -3.930492 & -2.374267 & 0.698485 \\
\hline 24 & 1 & 0 & 0.959995 & 2.918461 & 0.023350 \\
\hline 25 & 7 & 0 & -1.757562 & 0.446882 & -0.097095 \\
\hline 26 & 8 & 0 & -4.927086 & -0.535330 & 1.468381 \\
\hline 27 & 8 & 0 & -5.255558 & 1.133094 & -0.716224 \\
\hline 28 & 8 & 0 & -3.258234 & -2.372133 & 0.006886 \\
\hline 29 & 8 & 0 & 0.395553 & 2.122854 & -0.040407 \\
\hline 30 & 7 & 0 & 3.595865 & -2.453073 & -0.052807 \\
\hline 31 & 8 & 0 & 4.796156 & -2.310797 & 0.016494 \\
\hline 32 & 8 & 0 & 3.017778 & -3.514466 & -0.131837 \\
\hline 33 & 7 & 0 & 3.275838 & 2.409843 & 0.127215 \\
\hline 34 & 8 & 0 & 4.476903 & 2.416682 & 0.189208 \\
\hline 35 & 8 & 0 & 2.589767 & 3.429712 & 0.127808 \\
\hline
\end{tabular}

\section{$\underline{\text { Structure of } \mathbf{3 0} \text { (closed form) }}$}

Zero-point correction $=0.264168$ (Hartree/Particle) 
Thermal correction to Energy $=0.284432$

Thermal correction to Enthalpy $=0.285376$

Thermal correction to Gibbs Free Energy= 0.214159

Sum of electronic and zero-point Energies $=-1192.450349$

Sum of electronic and thermal Energies= -1192.430084

Sum of electronic and thermal Enthalpies= -1192.429140

Sum of electronic and thermal Free Energies= -1192.500357

No imaginary frequencies

\begin{tabular}{|c|c|c|c|c|c|}
\hline \multirow{2}{*}{$\begin{array}{l}\text { Center } \\
\text { Number }\end{array}$} & \multirow{2}{*}{$\begin{array}{l}\text { Atomic } \\
\text { Number }\end{array}$} & \multirow{2}{*}{$\begin{array}{c}\text { Atomic } \\
\text { Type }\end{array}$} & \multicolumn{3}{|c|}{ Coordinates (Angstroms) } \\
\hline & & & $\mathrm{X}$ & $\mathrm{Y}$ & Z \\
\hline & & & & & \\
\hline 1 & 6 & 0 & 1.072942 & 0.976090 & 0.001097 \\
\hline 2 & 6 & 0 & 2.467077 & 1.168461 & 0.045141 \\
\hline 3 & 6 & 0 & 3.360081 & 0.117490 & 0.018756 \\
\hline 4 & 6 & 0 & 2.859665 & -1.173874 & -0.015862 \\
\hline 5 & 6 & 0 & 1.498754 & -1.424856 & -0.047193 \\
\hline 6 & 6 & 0 & 0.601407 & -0.364970 & -0.049461 \\
\hline 7 & 6 & 0 & -0.836247 & -0.644399 & -0.110735 \\
\hline 8 & 6 & 0 & -3.122795 & 0.031222 & -0.171507 \\
\hline 9 & 6 & 0 & -3.622450 & 0.053681 & 1.293390 \\
\hline 10 & 6 & 0 & -3.744500 & 1.185796 & -0.961577 \\
\hline 11 & 6 & 0 & -3.502893 & -1.303378 & -0.832291 \\
\hline 12 & 1 & 0 & 4.426289 & 0.303800 & 0.025190 \\
\hline 13 & 1 & 0 & 1.144395 & -2.448762 & -0.077099 \\
\hline 14 & 1 & 0 & -1.134659 & -1.696617 & -0.119914 \\
\hline 15 & 1 & 0 & -2.946016 & -0.550511 & 1.899765 \\
\hline 16 & 1 & 0 & -3.597916 & 1.081447 & 1.673095 \\
\hline 17 & 1 & 0 & -5.516198 & 0.009788 & 0.909131 \\
\hline 18 & 1 & 0 & -3.493756 & 1.085712 & -2.025042 \\
\hline 19 & 1 & 0 & -3.328321 & 2.127110 & -0.588161 \\
\hline 20 & 1 & 0 & -5.569899 & 1.852703 & -1.261137 \\
\hline 21 & 1 & 0 & -2.892884 & -1.443008 & -1.729614 \\
\hline 22 & 1 & 0 & -4.549112 & -1.248334 & -1.145613 \\
\hline 23 & 1 & 0 & -3.972233 & -2.413906 & 0.672969 \\
\hline 24 & 1 & 0 & -0.684042 & 1.619613 & -0.077305 \\
\hline 25 & 7 & 0 & -1.686353 & 0.306990 & -0.140434 \\
\hline 26 & 8 & 0 & -4.902888 & -0.524296 & 1.427663 \\
\hline 27 & 8 & 0 & -5.152865 & 1.144160 & -0.766223 \\
\hline 28 & 8 & 0 & -3.280972 & -2.422029 & 0.000314 \\
\hline 29 & 8 & 0 & 0.246906 & 1.994866 & -0.032143 \\
\hline 30 & 7 & 0 & 3.800329 & -2.302934 & -0.023177 \\
\hline 31 & 8 & 0 & 4.982570 & -2.043833 & 0.010970 \\
\hline 32 & 8 & 0 & 3.328870 & -3.419034 & -0.063798 \\
\hline 33 & 7 & 0 & 3.032443 & 2.525555 & 0.120548 \\
\hline 34 & 8 & 0 & 4.079417 & 2.711379 & -0.463420 \\
\hline 35 & 8 & 0 & 2.436859 & 3.344551 & 0.777465 \\
\hline
\end{tabular}

\section{$\underline{\text { Structure of } \mathbf{T S}_{30 / 60}}$}

Zero-point correction $=0.260043$ (Hartree/Particle)

Thermal correction to Energy $=0.280010$

Thermal correction to Enthalpy $=0.280954$

Thermal correction to Gibbs Free Energy= 0.209614

Sum of electronic and zero-point Energies= -1192.448360

Sum of electronic and thermal Energies= -1192.428394

Sum of electronic and thermal Enthalpies $=-1192.427450$

Sum of electronic and thermal Free Energies= -1192.498789

one imaginary frequency: 880i

Standard orientation:

\begin{tabular}{|c|c|c|c|c|c|}
\hline \multirow{2}{*}{$\begin{array}{l}\text { Center } \\
\text { Number }\end{array}$} & \multirow{2}{*}{$\begin{array}{l}\text { Atomic } \\
\text { Number }\end{array}$} & \multirow{2}{*}{$\begin{array}{c}\text { Atomic } \\
\text { Type }\end{array}$} & \multicolumn{3}{|c|}{ Coordinates (Angstroms) } \\
\hline & & & $\mathrm{X}$ & $\mathrm{Y}$ & Z \\
\hline 1 & 6 & 0 & 0.984014 & 0.935464 & 0.006340 \\
\hline 2 & 6 & 0 & 2.390408 & 1.194819 & 0.047065 \\
\hline 3 & 6 & 0 & 3.330000 & 0.197100 & 0.051016 \\
\hline 4 & 6 & 0 & 2.916082 & -1.134273 & -0.034375 \\
\hline 5 & 6 & 0 & 1.580392 & -1.469733 & -0.102339 \\
\hline 6 & 6 & 0 & 0.620272 & -0.461516 & -0.075966 \\
\hline
\end{tabular}




$\begin{array}{rrrrrr}7 & 6 & 0 & -0.780147 & -0.785530 & -0.131170 \\ 8 & 6 & 0 & -3.094622 & -0.002896 & -0.124214 \\ 9 & 6 & 0 & -3.594646 & -0.104018 & 1.337332 \\ 10 & 6 & 0 & -3.651355 & 1.253413 & -0.799567 \\ 11 & 6 & 0 & -3.520076 & -1.250191 & -0.912490 \\ 12 & 1 & 0 & 4.383075 & 0.441478 & 0.116492 \\ 13 & 1 & 0 & 1.292610 & -2.512997 & -0.169373 \\ 14 & 1 & 0 & -1.104936 & -1.824544 & -0.201958 \\ 15 & 1 & 0 & -2.940556 & -0.787425 & 1.880984 \\ 16 & 1 & 0 & -3.533537 & 0.880695 & 1.815127 \\ 17 & 1 & 0 & -5.489132 & -0.034782 & 0.963818 \\ 18 & 1 & 0 & -3.418792 & 1.234646 & -1.871209 \\ 19 & 1 & 0 & -3.179331 & 2.135862 & -0.353099 \\ 20 & 1 & 0 & -5.439143 & 2.046659 & -0.989151 \\ 21 & 1 & 0 & -2.923256 & -1.313796 & -1.826723 \\ 22 & 1 & 0 & -4.567832 & -1.132849 & -1.201345 \\ 23 & 1 & 0 & -4.010812 & -2.499745 & 0.473368 \\ 24 & 1 & 0 & -1.020927 & 1.179932 & 0.007527 \\ 25 & 7 & 0 & -1.644336 & 0.174208 & -0.090538 \\ 26 & 8 & 0 & -4.893681 & -0.646177 & 1.413240 \\ 27 & 8 & 0 & -5.054510 & 1.267807 & -0.580839 \\ 28 & 8 & 0 & -3.317200 & -2.447946 & -0.195288 \\ 29 & 8 & 0 & 0.091638 & 1.836421 & 0.069070 \\ 30 & 7 & 0 & 2.878150 & 2.579125 & 0.089514 \\ 31 & 8 & 0 & 2.253566 & 3.409316 & -0.526370 \\ 32 & 8 & 0 & 3.895377 & 2.786116 & 0.720110 \\ 33 & 7 & 0 & 3.925009 & -2.194095 & -0.055623 \\ 34 & 8 & 0 & 3.531011 & -3.339825 & -0.134089 \\ 35 & 8 & 0 & 5.088659 & -1.861166 & 0.006951 \\ ---------------------------------------1\end{array}$

\section{$\underline{\text { Structure of } 60}$}

Zero-point correction $=0.263955$ (Hartree/Particle) Thermal correction to Energy $=0.284399$

Thermal correction to Enthalpy $=0.285343$

Thermal correction to Gibbs Free Energy= 0.213498

Sum of electronic and zero-point Energies $=-1192.446558$

Sum of electronic and thermal Energies= -1192.426113

Sum of electronic and thermal Enthalpies= -1192.425169

Sum of electronic and thermal Free Energies= -1192.497014

No imaginary frequencies

Standard orientation:

\begin{tabular}{|c|c|c|c|c|c|}
\hline \multirow{2}{*}{$\begin{array}{l}\text { Center } \\
\text { Number }\end{array}$} & \multirow{2}{*}{$\begin{array}{l}\text { Atomic } \\
\text { Number }\end{array}$} & \multirow{2}{*}{$\begin{array}{c}\text { Atomic } \\
\text { Type }\end{array}$} & \multicolumn{3}{|c|}{ Coordinates (Angstroms) } \\
\hline & & & $\mathrm{X}$ & $\mathrm{Y}$ & Z \\
\hline 1 & 6 & 0 & 1.039123 & 1.021444 & -0.037552 \\
\hline 2 & 6 & 0 & 2.482432 & 1.180481 & 0.038823 \\
\hline 3 & 6 & 0 & 3.355332 & 0.137970 & 0.019462 \\
\hline 4 & 6 & 0 & 2.861408 & -1.182082 & -0.017389 \\
\hline 5 & 6 & 0 & 1.516590 & -1.440623 & -0.051198 \\
\hline 6 & 6 & 0 & 0.602254 & -0.375416 & -0.067978 \\
\hline 7 & 6 & 0 & -0.778659 & -0.668736 & -0.120373 \\
\hline 8 & 6 & 0 & -3.143367 & 0.040318 & -0.164948 \\
\hline 9 & 6 & 0 & -3.629119 & 0.017271 & 1.304516 \\
\hline 10 & 6 & 0 & -3.752958 & 1.222239 & -0.923504 \\
\hline 11 & 6 & 0 & -3.521303 & -1.273458 & -0.864659 \\
\hline 12 & 1 & 0 & 4.423374 & 0.318044 & 0.037496 \\
\hline 13 & 1 & 0 & 1.171219 & -2.468557 & -0.070694 \\
\hline 14 & 1 & 0 & -1.115165 & -1.704217 & -0.116180 \\
\hline 15 & 1 & 0 & -2.938928 & -0.595258 & 1.886788 \\
\hline 16 & 1 & 0 & -3.612243 & 1.034017 & 1.714104 \\
\hline 17 & 1 & 0 & -5.531257 & -0.020968 & 0.963997 \\
\hline 18 & 1 & 0 & -3.534115 & 1.130830 & -1.994269 \\
\hline 19 & 1 & 0 & -3.311134 & 2.154887 & -0.552563 \\
\hline 20 & 1 & 0 & -5.571281 & 1.932443 & -1.139646 \\
\hline 21 & 1 & 0 & -2.928764 & -1.374170 & -1.778228 \\
\hline 22 & 1 & 0 & -4.574609 & -1.214820 & -1.150129 \\
\hline 23 & 1 & 0 & -3.955953 & -2.448072 & 0.603988 \\
\hline 24 & 7 & 0 & -1.695758 & 0.263057 & -0.166692 \\
\hline 25 & 8 & 0 & -4.899352 & -0.579488 & 1.431174 \\
\hline 26 & 8 & 0 & -5.150719 & 1.201061 & -0.681421 \\
\hline
\end{tabular}




\begin{tabular}{rrrrrr}
27 & 8 & 0 & -3.267857 & -2.411065 & -0.071413 \\
28 & 8 & 0 & 0.237748 & 1.963815 & -0.106640 \\
29 & 1 & 0 & -1.295261 & 1.221837 & -0.139612 \\
30 & 7 & 0 & 3.047285 & 2.531765 & 0.134483 \\
31 & 8 & 0 & 2.456020 & 3.332517 & 0.818388 \\
32 & 8 & 0 & 4.087203 & 2.741637 & -0.458025 \\
33 & 7 & 0 & 3.808373 & -2.293666 & -0.009719 \\
34 & 8 & 0 & 4.989899 & -2.022066 & 0.028428 \\
35 & 8 & 0 & 3.355110 & -3.420229 & -0.043338 \\
\hline
\end{tabular}

\title{
$\underline{\text { Structure of } \mathbf{3 1} \text { (open form) }}$
}

\begin{abstract}
Zero-point correction $=0.294265$ (Hartree/Particle) Thermal correction to Energy $=0.314966$ Thermal correction to Enthalpy $=0.315910$

Thermal correction to Gibbs Free Energy= 0.243869

Sum of electronic and zero-point Energies= -1102.440350

Sum of electronic and thermal Energies= -1102.419650

Sum of electronic and thermal Enthalpies= -1102.418706

Sum of electronic and thermal Free Energies= -1102.490746

No imaginary frequencies
\end{abstract}

\begin{tabular}{|c|c|c|c|c|c|}
\hline \multirow{2}{*}{$\begin{array}{l}\text { Center } \\
\text { Number }\end{array}$} & \multirow{2}{*}{$\begin{array}{l}\text { Atomic } \\
\text { Number }\end{array}$} & \multirow{2}{*}{$\begin{array}{c}\text { Atomic } \\
\text { Type }\end{array}$} & \multicolumn{3}{|c|}{ Coordinates (Angstroms) } \\
\hline & & & $\mathrm{x}$ & $\mathrm{Y}$ & Z \\
\hline 1 & 6 & 0 & 1.208087 & 1.185558 & 0.001143 \\
\hline 2 & 6 & 0 & 2.611956 & 1.350694 & 0.063102 \\
\hline 3 & 6 & 0 & 3.462720 & 0.267136 & 0.041358 \\
\hline 4 & 6 & 0 & 2.891798 & -1.002131 & -0.045309 \\
\hline 5 & 6 & 0 & 1.530547 & -1.197841 & -0.106562 \\
\hline 6 & 6 & 0 & 0.661408 & -0.100656 & -0.083005 \\
\hline 7 & 6 & 0 & -0.784568 & -0.392520 & -0.146729 \\
\hline 8 & 6 & 0 & -3.088498 & 0.096977 & -0.150117 \\
\hline 9 & 6 & 0 & -3.590292 & 0.128667 & 1.311979 \\
\hline 10 & 6 & 0 & -3.787930 & 1.187953 & -0.965644 \\
\hline 11 & 6 & 0 & -3.393669 & -1.273453 & -0.776810 \\
\hline 12 & 1 & 0 & 4.537807 & 0.363991 & 0.086927 \\
\hline 13 & 1 & 0 & 1.137321 & -2.204655 & -0.171115 \\
\hline 14 & 1 & 0 & -1.014675 & -1.462277 & -0.210673 \\
\hline 15 & 1 & 0 & -2.881835 & -0.421178 & 1.933087 \\
\hline 16 & 1 & 0 & -3.620032 & 1.165874 & 1.663241 \\
\hline 17 & 1 & 0 & -5.471902 & -0.042651 & 0.909060 \\
\hline 18 & 1 & 0 & -3.528175 & 1.080212 & -2.026259 \\
\hline 19 & 1 & 0 & -3.430143 & 2.158222 & -0.610829 \\
\hline 20 & 1 & 0 & -5.650726 & 1.736273 & -1.286520 \\
\hline 21 & 1 & 0 & -2.771214 & -1.405053 & -1.666681 \\
\hline 22 & 1 & 0 & -4.439215 & -1.282789 & -1.099662 \\
\hline 23 & 1 & 0 & -3.802115 & -2.348314 & 0.768203 \\
\hline 24 & 1 & 0 & 1.019075 & 3.047797 & 0.089442 \\
\hline 25 & 7 & 0 & -1.683958 & 0.498740 & -0.112817 \\
\hline 26 & 8 & 0 & -4.842217 & -0.512440 & 1.468480 \\
\hline 27 & 8 & 0 & -5.197039 & 1.061519 & -0.777957 \\
\hline 28 & 8 & 0 & -3.124391 & -2.363087 & 0.082289 \\
\hline 29 & 8 & 0 & 0.438274 & 2.277576 & 0.022571 \\
\hline 30 & 7 & 0 & 3.788592 & -2.165422 & -0.072189 \\
\hline 31 & 8 & 0 & 4.983046 & -1.953293 & -0.009527 \\
\hline 32 & 8 & 0 & 3.285822 & -3.264755 & -0.156195 \\
\hline 33 & 8 & 0 & 2.997189 & 2.653070 & 0.142533 \\
\hline 34 & 6 & 0 & 4.387432 & 2.925925 & 0.203408 \\
\hline 35 & 1 & 0 & 4.831450 & 2.468632 & 1.091768 \\
\hline 36 & 1 & 0 & 4.891310 & 2.558833 & -0.694831 \\
\hline 37 & 1 & 0 & 4.481215 & 4.007286 & 0.261446 \\
\hline
\end{tabular}

\section{$\underline{\text { Structure of } \mathbf{3 1} \text { (closed form) }}$}

Zero-point correction $=0.294347$ (Hartree/Particle)

Thermal correction to Energy $=0.314652$

Thermal correction to Enthalpy $=0.315596$ 
Thermal correction to Gibbs Free Energy $=0.244902$

Sum of electronic and zero-point Energies= -1102.453220

Sum of electronic and thermal Energies= -1102.432915

Sum of electronic and thermal Enthalpies= -1102.431971

Sum of electronic and thermal Free Energies= -1102.502665

No imaginary frequencies

Standard orientation:

\begin{tabular}{|c|c|c|c|c|c|}
\hline \multirow{2}{*}{$\begin{array}{l}\text { Center } \\
\text { Number }\end{array}$} & \multirow{2}{*}{$\begin{array}{l}\text { Atomic } \\
\text { Number }\end{array}$} & \multirow{2}{*}{$\begin{array}{l}\text { Atomic } \\
\text { Type }\end{array}$} & \multicolumn{3}{|c|}{ Coordinates (Angstroms) } \\
\hline & & & $\mathrm{X}$ & Y & $\mathrm{Z}$ \\
\hline--7 & $\sigma_{0}$ & & & 1720127 & 0 \\
\hline 1 & 6 & 0 & 1.119094 & 1.122437 & 0.006672 \\
\hline 2 & 6 & 0 & 2.510556 & 1.402062 & 0.061728 \\
\hline 3 & 6 & 0 & 3.419792 & 0.364248 & 0.038263 \\
\hline 4 & 6 & 0 & 2.950991 & -0.951961 & -0.039800 \\
\hline 5 & 6 & 0 & 1.613221 & -1.257965 & -0.093292 \\
\hline 6 & 6 & 0 & 0.682324 & -0.212540 & -0.070961 \\
\hline 7 & 6 & 0 & -0.745362 & -0.529252 & -0.130137 \\
\hline 8 & 6 & 0 & -3.054831 & 0.062984 & -0.151838 \\
\hline 9 & 6 & 0 & -3.552922 & 0.077586 & 1.312341 \\
\hline 10 & 6 & 0 & -3.715359 & 1.192648 & -0.946625 \\
\hline 11 & 6 & 0 & -3.397837 & -1.284661 & -0.806537 \\
\hline 12 & 1 & 0 & 4.485954 & 0.532615 & 0.077905 \\
\hline 13 & 1 & 0 & 1.291236 & -2.290151 & -0.150739 \\
\hline 14 & 1 & 0 & -1.009375 & -1.590181 & -0.170747 \\
\hline 15 & 1 & 0 & -2.859521 & -0.506051 & 1.919530 \\
\hline 16 & 1 & 0 & -3.553573 & 1.107324 & 1.687753 \\
\hline 17 & 1 & 0 & -5.443457 & -0.023217 & 0.923206 \\
\hline 18 & 1 & 0 & -3.461774 & 1.094899 & -2.009603 \\
\hline 19 & 1 & 0 & -3.328212 & 2.147931 & -0.578215 \\
\hline 20 & 1 & 0 & -5.560128 & 1.802126 & -1.253337 \\
\hline 21 & 1 & 0 & -2.782838 & -1.412014 & -1.702164 \\
\hline 22 & 1 & 0 & -4.444812 & -1.259120 & -1.122376 \\
\hline 23 & 1 & 0 & -3.834396 & -2.391751 & 0.709539 \\
\hline 24 & 1 & 0 & -0.638349 & 1.771324 & -0.010869 \\
\hline 25 & 7 & 0 & -1.630443 & 0.391889 & -0.121771 \\
\hline 26 & 8 & 0 & -4.819793 & -0.531508 & 1.454971 \\
\hline 27 & 8 & 0 & -5.123559 & 1.110248 & -0.752132 \\
\hline 28 & 8 & 0 & -3.149727 & -2.394700 & 0.030294 \\
\hline 29 & 8 & 0 & 0.281983 & 2.147112 & 0.031480 \\
\hline 30 & 7 & 0 & 3.935061 & -2.040772 & -0.063977 \\
\hline 31 & 8 & 0 & 5.109906 & -1.738700 & -0.007953 \\
\hline 32 & 8 & 0 & 3.518122 & -3.177566 & -0.138767 \\
\hline 33 & 8 & 0 & 2.828727 & 2.708730 & 0.135600 \\
\hline 34 & 6 & 0 & 4.202795 & 3.038671 & 0.202172 \\
\hline 35 & 1 & 0 & 4.668394 & 2.601431 & 1.090860 \\
\hline 36 & 1 & 0 & 4.731883 & 2.699514 & -0.693774 \\
\hline 37 & 1 & 0 & 4.246614 & 4.123042 & 0.263423 \\
\hline
\end{tabular}

\section{$\underline{\text { Structure of } \mathbf{T S}_{31 / 61}}$}

Zero-point correction= 0.290462 (Hartree/Particle)

Thermal correction to Energy $=0.310391$

Thermal correction to Enthalpy $=0.311335$

Thermal correction to Gibbs Free Energy= 0.241307

Sum of electronic and zero-point Energies= -1102.448627

Sum of electronic and thermal Energies $=-1102.428698$

Sum of electronic and thermal Enthalpies $=-1102.427754$

Sum of electronic and thermal Free Energies= -1102.497782

one imaginary frequency: 931i

Standard orientation:

\begin{tabular}{|c|c|c|c|c|c|}
\hline \multirow{2}{*}{$\begin{array}{l}\text { Center } \\
\text { Number }\end{array}$} & \multirow{2}{*}{$\begin{array}{l}\text { Atomic } \\
\text { Number }\end{array}$} & \multirow{2}{*}{$\begin{array}{c}\text { Atomic } \\
\text { Type }\end{array}$} & \multicolumn{3}{|c|}{ Coordinates (Angstroms) } \\
\hline & & & $\mathrm{X}$ & $\mathrm{Y}$ & Z \\
\hline 1 & 6 & 0 & 1.018193 & 1.069463 & -0.007216 \\
\hline 2 & 6 & 0 & 2.419381 & 1.419915 & 0.051869 \\
\hline 3 & 6 & 0 & 3.378091 & 0.441721 & 0.039995 \\
\hline 4 & 6 & 0 & 2.998288 & -0.920172 & -0.027616 \\
\hline 5 & 6 & 0 & 1.692242 & -1.315707 & -0.080904 \\
\hline 6 & 6 & 0 & 0.693531 & -0.325290 & -0.071462 \\
\hline
\end{tabular}




$\begin{array}{rrrrrr}7 & 6 & 0 & -0.689263 & -0.681126 & -0.126744 \\ 8 & 6 & 0 & -3.027542 & 0.034944 & -0.145086 \\ 9 & 6 & 0 & -3.521762 & -0.009008 & 1.320074 \\ 10 & 6 & 0 & -3.624536 & 1.237810 & -0.879780 \\ 11 & 6 & 0 & -3.421991 & -1.260028 & -0.870961 \\ 12 & 1 & 0 & 4.433183 & 0.671046 & 0.080960 \\ 13 & 1 & 0 & 1.440308 & -2.367989 & -0.129579 \\ 14 & 1 & 0 & -0.990172 & -1.728801 & -0.161454 \\ 15 & 1 & 0 & -2.848569 & -0.648506 & 1.892838 \\ 16 & 1 & 0 & -3.483492 & 0.998689 & 1.750114 \\ 17 & 1 & 0 & -5.417917 & -0.009133 & 0.947994 \\ 18 & 1 & 0 & -3.392763 & 1.171697 & -1.949775 \\ 19 & 1 & 0 & -3.178375 & 2.154957 & -0.479419 \\ 20 & 1 & 0 & -5.431434 & 1.979287 & -1.096038 \\ 21 & 1 & 0 & -2.818916 & -1.355863 & -1.778160 \\ 22 & 1 & 0 & -4.470770 & -1.181283 & -1.170223 \\ 23 & 1 & 0 & -3.884641 & -2.439896 & 0.582117 \\ 24 & 7 & 0 & -1.585140 & 0.258163 & -0.126640 \\ 25 & 8 & 0 & -4.808235 & -0.578653 & 1.431531 \\ 26 & 8 & 0 & -5.029190 & 1.224797 & -0.660352 \\ 27 & 8 & 0 & -3.199033 & -2.417849 & -0.096134 \\ 28 & 8 & 0 & 0.113071 & 1.966627 & -0.000954 \\ 29 & 1 & 0 & -0.998246 & 1.275963 & -0.058054 \\ 30 & 7 & 0 & 4.050971 & -1.933630 & -0.040164 \\ 31 & 8 & 0 & 5.203774 & -1.550367 & 0.011667 \\ 32 & 8 & 0 & 3.718319 & -3.100654 & -0.102591 \\ 33 & 8 & 0 & 2.655214 & 2.743589 & 0.117549 \\ 34 & 6 & 0 & 4.004618 & 3.154064 & 0.205220 \\ 35 & 1 & 0 & 4.480966 & 2.743974 & 1.101494 \\ 36 & 1 & 0 & 4.568152 & 2.845315 & -0.680968 \\ 37 & 1 & 0 & 3.985012 & 4.239389 & 0.265710 \\ ----------------------------1\end{array}$

\section{$\underline{\text { Structure of } 61}$}

Zero-point correction $=0.294337$ (Hartree/Particle) Thermal correction to Energy $=0.314676$

Thermal correction to Enthalpy $=0.315621$

Thermal correction to Gibbs Free Energy $=0.244287$ Sum of electronic and zero-point Energies= -1102.446908

Sum of electronic and thermal Energies= -1102.426569

Sum of electronic and thermal Enthalpies=-1102.425624

Sum of electronic and thermal Free Energies= -1102.496958

No imaginary frequencies

Standard orientation:

\begin{tabular}{|c|c|c|c|c|c|}
\hline \multirow{2}{*}{$\begin{array}{l}\text { Center } \\
\text { Number }\end{array}$} & \multirow{2}{*}{$\begin{array}{l}\text { Atomic } \\
\text { Number }\end{array}$} & \multirow{2}{*}{$\begin{array}{c}\text { Atomic } \\
\text { Type }\end{array}$} & \multicolumn{3}{|c|}{ Coordinates (Angstroms) } \\
\hline & & & $\mathrm{X}$ & $\mathrm{Y}$ & Z \\
\hline 1 & 6 & 0 & 1.082079 & 1.168465 & -0.004260 \\
\hline 2 & 6 & 0 & 2.529268 & 1.416645 & 0.048373 \\
\hline 3 & 6 & 0 & 3.420217 & 0.387499 & 0.041316 \\
\hline 4 & 6 & 0 & 2.953360 & -0.960022 & -0.017900 \\
\hline 5 & 6 & 0 & 1.634033 & -1.276877 & -0.068680 \\
\hline 6 & 6 & 0 & 0.678737 & -0.228627 & -0.064020 \\
\hline 7 & 6 & 0 & -0.685338 & -0.549771 & -0.117502 \\
\hline 8 & 6 & 0 & -3.072138 & 0.089327 & -0.131586 \\
\hline 9 & 6 & 0 & -3.542488 & -0.108219 & 1.327516 \\
\hline 10 & 6 & 0 & -3.726866 & 1.322964 & -0.757493 \\
\hline 11 & 6 & 0 & -3.421152 & -1.153420 & -0.967397 \\
\hline 12 & 1 & 0 & 4.487741 & 0.549009 & 0.078978 \\
\hline 13 & 1 & 0 & 1.323816 & -2.314057 & -0.111700 \\
\hline 14 & 1 & 0 & -0.999040 & -1.591738 & -0.153074 \\
\hline 15 & 1 & 0 & -2.835040 & -0.771407 & 1.828213 \\
\hline 16 & 1 & 0 & -3.534631 & 0.855781 & 1.850028 \\
\hline 17 & 1 & 0 & -5.444624 & -0.147953 & 0.985970 \\
\hline 18 & 1 & 0 & -3.515133 & 1.348800 & -1.833189 \\
\hline 19 & 1 & 0 & -3.308510 & 2.225125 & -0.295339 \\
\hline 20 & 1 & 0 & -5.565817 & 1.999949 & -0.892682 \\
\hline 21 & 1 & 0 & -2.819803 & -1.146763 & -1.880240 \\
\hline 22 & 1 & 0 & -4.474638 & -1.087386 & -1.252633 \\
\hline 23 & 1 & 0 & -3.837190 & -2.480293 & 0.370530 \\
\hline 24 & 7 & 0 & -1.635732 & 0.361086 & -0.125464 \\
\hline
\end{tabular}




\begin{tabular}{rrrrrr}
25 & 8 & 0 & -4.803936 & -0.735988 & 1.402654 \\
26 & 8 & 0 & -5.122699 & 1.238588 & -0.511875 \\
27 & 8 & 0 & -3.151160 & -2.365058 & -0.297938 \\
28 & 8 & 0 & 0.266347 & 2.105125 & 0.003030 \\
29 & 1 & 0 & -1.266984 & 1.326666 & -0.057738 \\
30 & 7 & 0 & 3.942562 & -2.033257 & -0.021726 \\
31 & 8 & 0 & 5.116299 & -1.717255 & 0.025526 \\
32 & 8 & 0 & 3.544062 & -3.180912 & -0.071456 \\
33 & 8 & 0 & 2.844260 & 2.722570 & 0.101501 \\
34 & 6 & 0 & 4.217325 & 3.050474 & 0.156400 \\
35 & 1 & 0 & 4.686013 & 2.624972 & 1.049648 \\
36 & 1 & 0 & 4.741765 & 2.691612 & -0.735127 \\
37 & 1 & 0 & 4.266672 & 4.135697 & 0.198541 \\
\hline
\end{tabular}

\section{$\underline{\text { Structure of } 84 \text { (open form) }}$}

Zero-point correction= 0.262506 (Hartree/Particle)
Thermal correction to Energy= 0.279341
Thermal correction to Enthalpy= 0.280285
Thermal correction to Gibbs Free Energy= 0.217768
Sum of electronic and zero-point Energies=-858.690661
Sum of electronic and thermal Energies=-858.673825
Sum of electronic and thermal Enthalpies= -858.672881
Sum of electronic and thermal Free Energies= -858.735398
No imaginary frequencies

Standard orientation:

\begin{tabular}{|c|c|c|c|c|c|}
\hline \multirow{2}{*}{$\begin{array}{l}\text { Center } \\
\text { Number }\end{array}$} & \multirow{2}{*}{$\begin{array}{l}\text { Atomic } \\
\text { Number }\end{array}$} & \multirow{2}{*}{$\begin{array}{c}\text { Atomic } \\
\text { Type }\end{array}$} & \multicolumn{3}{|c|}{ Coordinates (Angstroms) } \\
\hline & & & $\mathrm{X}$ & $\mathrm{Y}$ & Z \\
\hline & & & -- & - & ---------- \\
\hline 1 & 6 & 0 & -2.177262 & -0.979894 & 0.076662 \\
\hline 2 & 6 & 0 & -3.565761 & -1.033075 & 0.144938 \\
\hline 3 & 6 & 0 & -4.322305 & 0.128539 & 0.047368 \\
\hline 4 & 6 & 0 & -3.698980 & 1.362073 & -0.123898 \\
\hline 5 & 6 & 0 & -2.315584 & 1.399411 & -0.191399 \\
\hline 6 & 6 & 0 & -1.519470 & 0.255565 & -0.093269 \\
\hline 7 & 6 & 0 & -0.063116 & 0.440871 & -0.174009 \\
\hline 8 & 6 & 0 & 2.212994 & -0.155667 & -0.126335 \\
\hline 9 & 6 & 0 & 2.700414 & 0.031082 & 1.326907 \\
\hline 10 & 6 & 0 & 2.869816 & -1.392540 & -0.744801 \\
\hline 11 & 6 & 0 & 2.588873 & 1.080614 & -0.963296 \\
\hline 12 & 1 & 0 & -4.078799 & -1.979688 & 0.276749 \\
\hline 13 & 1 & 0 & -4.284368 & 2.271433 & -0.200479 \\
\hline 14 & 1 & 0 & -1.820144 & 2.355658 & -0.324082 \\
\hline 15 & 1 & 0 & 0.234011 & 1.484927 & -0.334685 \\
\hline 16 & 1 & 0 & 2.021661 & 0.719643 & 1.832524 \\
\hline 17 & 1 & 0 & 2.661926 & -0.931043 & 1.849705 \\
\hline 18 & 1 & 0 & 4.589957 & 0.026902 & 0.921353 \\
\hline 19 & 1 & 0 & 2.615051 & -1.449648 & -1.810441 \\
\hline 20 & 1 & 0 & 2.470178 & -2.275436 & -0.238916 \\
\hline 21 & 1 & 0 & 4.703842 & -2.081600 & -0.934601 \\
\hline 22 & 1 & 0 & 1.957085 & 1.109916 & -1.855351 \\
\hline 23 & 1 & 0 & 3.627308 & 0.975097 & -1.293177 \\
\hline 24 & 1 & 0 & 3.081818 & 2.343837 & 0.402152 \\
\hline 25 & 1 & 0 & -2.032634 & -2.859516 & 0.285706 \\
\hline 26 & 7 & 0 & 0.792045 & -0.489387 & -0.056961 \\
\hline 27 & 8 & 0 & 3.990569 & 0.613194 & 1.398543 \\
\hline 28 & 8 & 0 & 4.284622 & -1.299691 & -0.570763 \\
\hline 29 & 8 & 0 & 2.406235 & 2.309210 & -0.285633 \\
\hline 30 & 8 & 0 & -1.443062 & -2.108998 & 0.168177 \\
\hline 31 & 8 & 0 & -5.670753 & -0.008722 & 0.126160 \\
\hline 32 & 1 & 0 & -6.089797 & 0.853421 & 0.057190 \\
\hline
\end{tabular}

\section{$\underline{\text { Structure of } 84 \text { (closed form) }}$}

Zero-point correction $=0.263369$ (Hartree/Particle)

Thermal correction to Energy $=0.279593$

Thermal correction to Enthalpy $=0.280537$

Thermal correction to Gibbs Free Energy= 0.219857 
Sum of electronic and zero-point Energies $=-858.710719$

Sum of electronic and thermal Energies= -858.694496

Sum of electronic and thermal Enthalpies= -858.693552

Sum of electronic and thermal Free Energies= -858.754232

No imaginary frequencies

Standard orientation:

\begin{tabular}{|c|c|c|c|c|c|}
\hline \multirow{2}{*}{$\begin{array}{l}\text { Center } \\
\text { Number }\end{array}$} & \multirow{2}{*}{$\begin{array}{l}\text { Atomic } \\
\text { Number }\end{array}$} & \multirow{2}{*}{$\begin{array}{l}\text { Atomic } \\
\text { Type }\end{array}$} & \multicolumn{3}{|c|}{ Coordinates (Angstroms) } \\
\hline & & & $\mathrm{X}$ & $\mathrm{Y}$ & Z \\
\hline 1 & 6 & 0 & -2.096712 & -0.934488 & 0.078199 \\
\hline 2 & 6 & 0 & -3.478310 & -1.089403 & 0.148728 \\
\hline 3 & 6 & 0 & -4.301421 & 0.022493 & 0.054815 \\
\hline 4 & 6 & 0 & -3.769117 & 1.308021 & -0.110812 \\
\hline 5 & 6 & 0 & -2.397736 & 1.453447 & -0.179204 \\
\hline 6 & 6 & 0 & -1.535726 & 0.355373 & -0.088691 \\
\hline 7 & 6 & 0 & -0.097973 & 0.561531 & -0.162147 \\
\hline 8 & 6 & 0 & 2.170620 & -0.151525 & -0.132643 \\
\hline 9 & 6 & 0 & 2.654506 & 0.030257 & 1.323166 \\
\hline 10 & 6 & 0 & 2.785578 & -1.413637 & -0.742129 \\
\hline 11 & 6 & 0 & 2.589050 & 1.067250 & -0.975143 \\
\hline 12 & 1 & 0 & -3.904565 & -2.075625 & 0.277349 \\
\hline 13 & 1 & 0 & -1.967836 & 2.441940 & -0.305811 \\
\hline 14 & 1 & 0 & 0.238956 & 1.597329 & -0.276007 \\
\hline 15 & 1 & 0 & 1.992758 & 0.741378 & 1.820033 \\
\hline 16 & 1 & 0 & 2.586221 & -0.926860 & 1.853011 \\
\hline 17 & 1 & 0 & 4.548325 & -0.036123 & 0.941329 \\
\hline 18 & 1 & 0 & 2.542044 & -1.462747 & -1.810717 \\
\hline 19 & 1 & 0 & 2.351598 & -2.284710 & -0.241497 \\
\hline 20 & 1 & 0 & 4.599062 & -2.155680 & -0.922556 \\
\hline 21 & 1 & 0 & 1.965027 & 1.107453 & -1.872143 \\
\hline 22 & 1 & 0 & 3.626800 & 0.929057 & -1.293845 \\
\hline 23 & 1 & 0 & 3.114180 & 2.332728 & 0.377912 \\
\hline 24 & 1 & 0 & -0.387214 & -1.727901 & 0.109566 \\
\hline 25 & 7 & 0 & 0.732816 & -0.410315 & -0.087660 \\
\hline 26 & 8 & 0 & 3.958251 & 0.575927 & 1.397017 \\
\hline 27 & 8 & 0 & 4.196733 & -1.370364 & -0.546437 \\
\hline 28 & 8 & 0 & 2.433105 & 2.301903 & -0.304724 \\
\hline 29 & 8 & 0 & -1.329221 & -2.023367 & 0.172385 \\
\hline 30 & 1 & 0 & -4.425432 & 2.168182 & -0.181040 \\
\hline 31 & 8 & 0 & -5.638617 & -0.193358 & 0.131068 \\
\hline 32 & 1 & 0 & -6.108190 & 0.641943 & 0.058735 \\
\hline
\end{tabular}

\section{$\underline{\text { Structure of } \mathbf{T S}_{\mathbf{8} 4 / 38 \mathrm{~S}}}$}

Zero-point correction $=0.259078$ (Hartree/Particle)

Thermal correction to Energy $=0.275061$

Thermal correction to Enthalpy $=0.276005$

Thermal correction to Gibbs Free Energy= 0.215682

Sum of electronic and zero-point Energies $=-858.703687$

Sum of electronic and thermal Energies $=-858.687703$

Sum of electronic and thermal Enthalpies $=-858.686759$

Sum of electronic and thermal Free Energies= -858.747082

One imaginary frequency: 686i

Standard orientation:

\begin{tabular}{|c|c|c|c|c|c|}
\hline \multirow{2}{*}{$\begin{array}{l}\text { Center } \\
\text { Number }\end{array}$} & \multirow{2}{*}{$\begin{array}{l}\text { Atomic } \\
\text { Number }\end{array}$} & \multirow{2}{*}{$\begin{array}{c}\text { Atomic } \\
\text { Type }\end{array}$} & \multicolumn{3}{|c|}{ Coordinates (Angstroms) } \\
\hline & & & $\mathrm{X}$ & $\mathrm{Y}$ & Z \\
\hline 1 & 6 & 0 & -1.986781 & -0.904913 & 0.072605 \\
\hline 2 & 6 & 0 & -3.383855 & -1.134117 & 0.153001 \\
\hline 3 & 6 & 0 & -4.265919 & -0.084161 & 0.072323 \\
\hline 4 & 6 & 0 & -3.832654 & 1.258804 & -0.090509 \\
\hline 5 & 6 & 0 & -2.490809 & 1.511481 & -0.168752 \\
\hline 6 & 6 & 0 & -1.547531 & 0.464648 & -0.092377 \\
\hline 7 & 6 & 0 & -0.159815 & 0.722476 & -0.170395 \\
\hline 8 & 6 & 0 & 2.136380 & -0.138259 & -0.132267 \\
\hline 9 & 6 & 0 & 2.637146 & 0.013444 & 1.322129 \\
\hline 10 & 6 & 0 & 2.659409 & -1.437118 & -0.750048 \\
\hline 11 & 6 & 0 & 2.611722 & 1.055679 & -0.975871 \\
\hline 12 & 1 & 0 & -3.747860 & -2.145417 & 0.276523 \\
\hline
\end{tabular}




\begin{tabular}{|c|c|c|c|c|c|}
\hline 13 & 1 & 0 & -4.560178 & 2.060365 & -0.150585 \\
\hline 14 & 1 & 0 & -2.134101 & 2.529447 & -0.291650 \\
\hline 15 & 1 & 0 & 0.212755 & 1.741716 & -0.278542 \\
\hline 16 & 1 & 0 & 2.008603 & 0.747918 & 1.827607 \\
\hline 17 & 1 & 0 & 2.532183 & -0.943632 & 1.846245 \\
\hline 18 & 1 & 0 & 4.526665 & -0.144600 & 0.950086 \\
\hline 19 & 1 & 0 & 2.432488 & -1.454005 & -1.822778 \\
\hline 20 & 1 & 0 & 2.156039 & -2.283778 & -0.270594 \\
\hline 21 & 1 & 0 & 4.417887 & -2.303021 & -0.899541 \\
\hline 22 & 1 & 0 & 2.001832 & 1.113126 & -1.881344 \\
\hline 23 & 1 & 0 & 3.648123 & 0.875017 & -1.275846 \\
\hline 24 & 1 & 0 & 3.172081 & 2.313655 & 0.370765 \\
\hline 25 & 1 & 0 & 0.065054 & -1.225207 & 0.033044 \\
\hline 26 & 7 & 0 & 0.686116 & -0.271406 & -0.105715 \\
\hline 27 & 8 & 0 & 3.961627 & 0.503358 & 1.387071 \\
\hline 28 & 8 & 0 & 4.063335 & -1.494059 & -0.524750 \\
\hline 29 & 8 & 0 & 2.487568 & 2.293814 & -0.308823 \\
\hline 30 & 8 & 0 & -1.134640 & -1.857999 & 0.141299 \\
\hline 31 & 8 & 0 & -5.589911 & -0.368876 & 0.15383 \\
\hline 32 & 1 & 0 & -6.101975 & 0.440570 & 0.078521 \\
\hline
\end{tabular}

\section{$\underline{\text { Structure of } \mathbf{3 8 S}}$}

Zero-point correction= 0.262401 (Hartree/Particle)
Thermal correction to Energy= 0.278863
Thermal correction to Enthalpy= 0.279807
Thermal correction to Gibbs Free Energy= 0.218341
Sum of electronic and zero-point Energies=-858.701282
Sum of electronic and thermal Energies= -858.684819
Sum of electronic and thermal Enthalpies=-858.683875
Sum of electronic and thermal Free Energies= -858.745341
No imaginary frequencies

Standard orientation:

\begin{tabular}{|c|c|c|c|c|c|}
\hline \multirow{2}{*}{$\begin{array}{l}\text { Center } \\
\text { Number }\end{array}$} & \multirow{2}{*}{$\begin{array}{l}\text { Atomic } \\
\text { Number }\end{array}$} & \multirow{2}{*}{$\begin{array}{c}\text { Atomic } \\
\text { Type }\end{array}$} & \multicolumn{3}{|c|}{ Coordinates (Angstroms) } \\
\hline & & & $\mathrm{X}$ & $\mathrm{Y}$ & Z \\
\hline 1 & 6 & 0 & -2.039782 & -0.977183 & 0.064382 \\
\hline 2 & 6 & 0 & -3.463112 & -1.128044 & 0.151513 \\
\hline 3 & 6 & 0 & -4.290013 & -0.043977 & 0.076630 \\
\hline 4 & 6 & 0 & -3.794214 & 1.288701 & -0.088726 \\
\hline 5 & 6 & 0 & -2.451213 & 1.482101 & -0.173262 \\
\hline 6 & 6 & 0 & -1.542562 & 0.389830 & -0.102652 \\
\hline 7 & 6 & 0 & -0.172051 & 0.629080 & -0.181842 \\
\hline 8 & 6 & 0 & 2.170776 & -0.154135 & -0.132780 \\
\hline 9 & 6 & 0 & 2.643542 & 0.044978 & 1.324989 \\
\hline 10 & 6 & 0 & 2.758915 & -1.435829 & -0.726847 \\
\hline 11 & 6 & 0 & 2.604107 & 1.046629 & -0.990933 \\
\hline 12 & 1 & 0 & -3.872992 & -2.121670 & 0.276821 \\
\hline 13 & 1 & 0 & -4.488328 & 2.119863 & -0.143114 \\
\hline 14 & 1 & 0 & -2.051259 & 2.483993 & -0.296657 \\
\hline 15 & 1 & 0 & 0.202638 & 1.646382 & -0.282802 \\
\hline 16 & 1 & 0 & 1.973839 & 0.759034 & 1.806798 \\
\hline 17 & 1 & 0 & 2.573327 & -0.906037 & 1.866051 \\
\hline 18 & 1 & 0 & 4.545068 & -0.034804 & 0.988849 \\
\hline 19 & 1 & 0 & 2.554460 & -1.471372 & -1.803536 \\
\hline 20 & 1 & 0 & 2.284327 & -2.301308 & -0.250145 \\
\hline 21 & 1 & 0 & 4.556049 & -2.224242 & -0.834800 \\
\hline 22 & 1 & 0 & 1.999394 & 1.066296 & -1.901184 \\
\hline 23 & 1 & 0 & 3.649197 & 0.901486 & -1.279231 \\
\hline 24 & 1 & 0 & 3.104636 & 2.346314 & 0.340569 \\
\hline 25 & 7 & 0 & 0.725028 & -0.341357 & -0.134240 \\
\hline 26 & 8 & 0 & 3.943067 & 0.595686 & 1.401219 \\
\hline 27 & 8 & 0 & 4.157607 & -1.430456 & -0.471705 \\
\hline 28 & 8 & 0 & 2.427427 & 2.288729 & -0.344395 \\
\hline 29 & 8 & 0 & -1.255347 & -1.954990 & 0.128646 \\
\hline 30 & 1 & 0 & 0.280324 & -1.273563 & 0.004061 \\
\hline 31 & 8 & 0 & -5.627550 & -0.253679 & 0.164207 \\
\hline 32 & 1 & 0 & -6.093876 & 0.583903 & 0.101396 \\
\hline
\end{tabular}




\section{Cartesian coordinates for all optimized structures.}

\section{Computational calculations at the M06-2X/6-311++G(d,p) level including the solvent effect (SMD model, DMSO as solvent).}

\section{Structure of 4 (open form)}

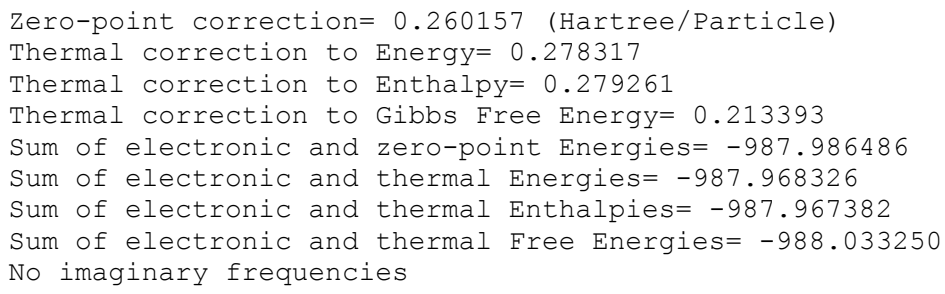

Standard orientation:

\begin{tabular}{|c|c|c|c|c|c|}
\hline \multirow{2}{*}{$\begin{array}{l}\text { Center } \\
\text { Number }\end{array}$} & \multirow{2}{*}{$\begin{array}{l}\text { Atomic } \\
\text { Number }\end{array}$} & \multirow{2}{*}{$\begin{array}{c}\text { Atomic } \\
\text { Type }\end{array}$} & \multicolumn{3}{|c|}{ Coordinates (Angstroms) } \\
\hline & & & $\mathrm{X}$ & $\mathrm{Y}$ & Z \\
\hline 1 & 6 & 0 & 1.434864 & 1.790652 & 0.064477 \\
\hline 2 & 6 & 0 & 2.786570 & 2.136093 & 0.186722 \\
\hline 3 & 6 & 0 & 3.770704 & 1.168675 & 0.164651 \\
\hline 4 & 6 & 0 & 3.390297 & -0.159054 & 0.007998 \\
\hline 5 & 6 & 0 & 2.061148 & -0.524442 & -0.124568 \\
\hline 6 & 6 & 0 & 1.056210 & 0.438885 & -0.085553 \\
\hline 7 & 6 & 0 & -0.329785 & -0.056271 & -0.224757 \\
\hline 8 & 6 & 0 & -2.686546 & 0.011987 & -0.096598 \\
\hline 9 & 6 & 0 & -3.171075 & -0.279167 & 1.340613 \\
\hline 10 & 6 & 0 & -3.564714 & 1.092888 & -0.734731 \\
\hline 11 & 6 & 0 & -2.771497 & -1.259572 & -0.952949 \\
\hline 12 & 1 & 0 & 3.058316 & 3.180660 & 0.298907 \\
\hline 13 & 1 & 0 & 4.814223 & 1.437315 & 0.261784 \\
\hline 14 & 1 & 0 & 1.796150 & -1.566918 & -0.255814 \\
\hline 15 & 1 & 0 & -0.379430 & -1.096955 & -0.560278 \\
\hline 16 & 1 & 0 & -2.385622 & -0.813891 & 1.877915 \\
\hline 17 & 1 & 0 & -3.353993 & 0.668272 & 1.859909 \\
\hline 18 & 1 & 0 & -4.994254 & -0.661601 & 0.841573 \\
\hline 19 & 1 & 0 & -3.301280 & 1.208743 & -1.793107 \\
\hline 20 & 1 & 0 & -3.383099 & 2.039869 & -0.218664 \\
\hline 21 & 1 & 0 & -5.490734 & 1.402658 & -0.959685 \\
\hline 22 & 1 & 0 & -2.148554 & -1.139010 & -1.842942 \\
\hline 23 & 1 & 0 & -3.803861 & -1.381278 & -1.293821 \\
\hline 24 & 1 & 0 & -2.984097 & -2.615171 & 0.401117 \\
\hline 25 & 1 & 0 & 0.905099 & 3.609379 & 0.146940 \\
\hline 26 & 7 & 0 & -1.363626 & 0.625437 & 0.043620 \\
\hline 27 & 8 & 0 & -4.319976 & -1.112605 & 1.366524 \\
\hline 28 & 8 & 0 & -4.932674 & 0.705531 & -0.600084 \\
\hline 29 & 8 & 0 & -2.331535 & -2.432915 & -0.286069 \\
\hline 30 & 8 & 0 & 0.488191 & 2.739308 & 0.070748 \\
\hline 31 & 7 & 0 & 4.418972 & -1.195980 & -0.024275 \\
\hline 32 & 8 & 0 & 4.066706 & -2.351513 & -0.169170 \\
\hline 33 & 8 & 0 & 5.580120 & -0.853095 & 0.096665 \\
\hline
\end{tabular}

\section{$\underline{\text { Structure of } 4 \text { (closed form) }}$}

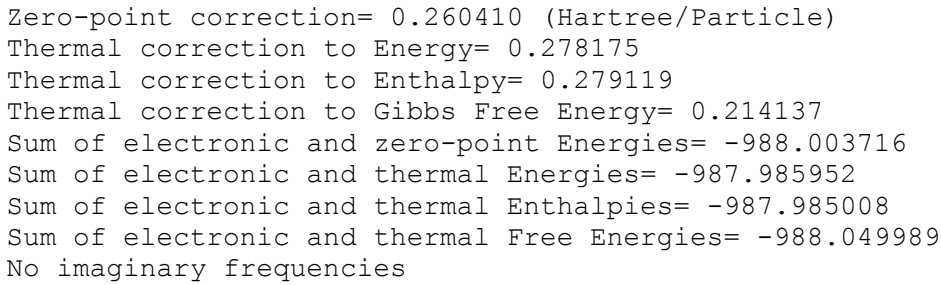




\begin{tabular}{|c|c|c|c|c|c|}
\hline \multirow{2}{*}{$\begin{array}{l}\text { Center } \\
\text { Number }\end{array}$} & \multirow{2}{*}{$\begin{array}{l}\text { Atomic } \\
\text { Number }\end{array}$} & \multirow{2}{*}{$\begin{array}{c}\text { Atomic } \\
\text { Type }\end{array}$} & \multicolumn{3}{|c|}{ Coordinates (Angstroms) } \\
\hline & & & $\mathrm{X}$ & Y & Z \\
\hline 1 & 6 & 0 & 1.283389 & 1.630461 & 0.166173 \\
\hline 2 & 6 & 0 & 2.590183 & 2.128520 & 0.274784 \\
\hline 3 & 6 & 0 & 3.668946 & 1.275245 & 0.185110 \\
\hline 4 & 6 & 0 & 3.445090 & -0.088312 & -0.013783 \\
\hline 5 & 6 & 0 & 2.167119 & -0.609508 & -0.121701 \\
\hline 6 & 6 & 0 & 1.072307 & 0.243216 & -0.032957 \\
\hline 7 & 6 & 0 & -0.280718 & -0.308157 & -0.149079 \\
\hline 8 & 6 & 0 & -2.663868 & -0.049852 & -0.170672 \\
\hline 9 & 6 & 0 & -3.260753 & 0.123420 & 1.244193 \\
\hline 10 & 6 & 0 & -3.387703 & 0.866831 & -1.161357 \\
\hline 11 & 6 & 0 & -2.799465 & -1.502243 & -0.638264 \\
\hline 12 & 1 & 0 & 2.734308 & 3.191075 & 0.427599 \\
\hline 13 & 1 & 0 & 4.679405 & 1.654056 & 0.265790 \\
\hline 14 & 1 & 0 & 2.012543 & -1.671212 & -0.274384 \\
\hline 15 & 1 & 0 & -0.353083 & -1.389803 & -0.281721 \\
\hline 16 & 1 & 0 & -2.555865 & -0.270943 & 1.978142 \\
\hline 17 & 1 & 0 & -3.400449 & 1.191462 & 1.446436 \\
\hline 18 & 1 & 0 & -5.078121 & -0.273798 & 0.735224 \\
\hline 19 & 1 & 0 & -3.054984 & 0.647681 & -2.182762 \\
\hline 20 & 1 & 0 & -3.141513 & 1.905999 & -0.922543 \\
\hline 21 & 1 & 0 & -5.260049 & 1.243498 & -1.615176 \\
\hline 22 & 1 & 0 & -2.144052 & -1.672329 & -1.495992 \\
\hline 23 & 1 & 0 & -3.826233 & -1.662435 & -0.978744 \\
\hline 24 & 1 & 0 & -3.142032 & -2.411813 & 1.027575 \\
\hline 25 & 1 & 0 & -0.574514 & 1.935752 & 0.148493 \\
\hline 26 & 7 & 0 & -1.296417 & 0.459435 & -0.087194 \\
\hline 27 & 8 & 0 & -4.469805 & -0.598147 & 1.412492 \\
\hline 28 & 8 & 0 & -4.789845 & 0.642260 & -1.028188 \\
\hline 29 & 8 & 0 & -2.449416 & -2.449963 & 0.357040 \\
\hline 30 & 8 & 0 & 0.265853 & 2.477013 & 0.252860 \\
\hline 31 & 7 & 0 & 4.587395 & -0.990334 & -0.109197 \\
\hline 32 & 8 & 0 & 4.372693 & -2.172931 & -0.303661 \\
\hline 33 & 8 & 0 & 5.703099 & -0.518060 & 0.009722 \\
\hline
\end{tabular}

\section{$\underline{\text { Structure of } \mathbf{T S}_{4 / 34}}$}

Zero-point correction=0.256251 (Hartree/Particle)

Thermal correction to Energy $=0.273708$

Thermal correction to Enthalpy $=0.274652$

Thermal correction to Gibbs Free Energy= 0.210419

Sum of electronic and zero-point Energies= -988.002559

Sum of electronic and thermal Energies= -987.985103

Sum of electronic and thermal Enthalpies= -987.984158

Sum of electronic and thermal Free Energies= -988.048391

one imaginary frequency: $1024 i$

Standard orientation:

\begin{tabular}{|c|c|c|c|c|c|}
\hline \multirow{2}{*}{$\begin{array}{l}\text { Center } \\
\text { Number }\end{array}$} & \multirow{2}{*}{$\begin{array}{l}\text { Atomic } \\
\text { Number }\end{array}$} & \multirow{2}{*}{$\begin{array}{c}\text { Atomic } \\
\text { Type }\end{array}$} & \multicolumn{3}{|c|}{ Coordinates (Angstroms) } \\
\hline & & & $\mathrm{X}$ & Y & Z \\
\hline 1 & 6 & 0 & 1.191393 & 1.570694 & 0.163847 \\
\hline 2 & 6 & 0 & 2.496268 & 2.118435 & 0.294890 \\
\hline 3 & 6 & 0 & 3.604702 & 1.316094 & 0.209749 \\
\hline 4 & 6 & 0 & 3.458232 & -0.065874 & -0.006475 \\
\hline 5 & 6 & 0 & 2.211806 & -0.648821 & -0.133023 \\
\hline 6 & 6 & 0 & 1.078981 & 0.154940 & -0.049680 \\
\hline 7 & 6 & 0 & -0.240725 & -0.419692 & -0.176264 \\
\hline 8 & 6 & 0 & -2.656688 & -0.054064 & -0.174282 \\
\hline 9 & 6 & 0 & -3.227299 & 0.126055 & 1.251395 \\
\hline 10 & 6 & 0 & -3.346438 & 0.901619 & -1.152284 \\
\hline 11 & 6 & 0 & -2.841427 & -1.494742 & -0.656019 \\
\hline 12 & 1 & 0 & 2.594263 & 3.184511 & 0.459940 \\
\hline 13 & 1 & 0 & 4.596866 & 1.738281 & 0.307159 \\
\hline 14 & 1 & 0 & 2.110791 & -1.715737 & -0.296395 \\
\hline 15 & 1 & 0 & -0.345975 & -1.494879 & -0.314640 \\
\hline 16 & 1 & 0 & -2.526176 & -0.299605 & 1.971035 \\
\hline 17 & 1 & 0 & -3.326116 & 1.196436 & 1.465878 \\
\hline 18 & 1 & 0 & -5.068147 & -0.191954 & 0.772328 \\
\hline
\end{tabular}




\begin{tabular}{|c|c|c|c|c|c|}
\hline 19 & 1 & 0 & -3.042534 & 0.666519 & -2.178633 \\
\hline 20 & 1 & 0 & -3.048623 & 1.929006 & -0.919050 \\
\hline 21 & 1 & 0 & -5.208680 & 1.346811 & -1.582350 \\
\hline 22 & 1 & 0 & -2.206722 & -1.671120 & -1.527906 \\
\hline 23 & 1 & 0 & -3.878580 & -1.618307 & -0.978470 \\
\hline 24 & 1 & 0 & -3.183465 & -2.422528 & 1.001316 \\
\hline 25 & 1 & 0 & -0.807856 & 1.486084 & 0.075318 \\
\hline 26 & 7 & 0 & -1.257783 & 0.365357 & -0.111238 \\
\hline 27 & 8 & 0 & -4.455848 & -0.555840 & 1.425261 \\
\hline 28 & 8 & 0 & -4.752179 & 0.741165 & -0.988853 \\
\hline 29 & 8 & 0 & -2.498595 & -2.459638 & 0.322632 \\
\hline 30 & 8 & 0 & 0.134669 & 2.305864 & 0.231499 \\
\hline 31 & 7 & 0 & 4.642381 & -0.898631 & -0.097575 \\
\hline 32 & 8 & 0 & 5.732507 & -0.367668 & 0.034302 \\
\hline 33 & 8 & 0 & 4.498937 & -2.092440 & -0.302963 \\
\hline
\end{tabular}

\section{$\underline{\text { Structure of } 34}$}

Zero-point correction $=0.260786$ (Hartree/Particle) Thermal correction to Energy $=0.278692$

Thermal correction to Enthalpy $=0.279636$

Thermal correction to Gibbs Free Energy= 0.213764

Sum of electronic and zero-point Energies= -988.002719

Sum of electronic and thermal Energies= -987.984814

Sum of electronic and thermal Enthalpies= -987.983870

Sum of electronic and thermal Free Energies= -988.049742

No imaginary frequencies

Standard orientation:

\begin{tabular}{|c|c|c|c|c|c|}
\hline \multirow{2}{*}{$\begin{array}{l}\text { Center } \\
\text { Number }\end{array}$} & \multirow{2}{*}{$\begin{array}{l}\text { Atomic } \\
\text { Number }\end{array}$} & \multirow{2}{*}{$\begin{array}{c}\text { Atomic } \\
\text { Type }\end{array}$} & \multicolumn{3}{|c|}{ Coordinates (Angstroms) } \\
\hline & & & $\mathrm{X}$ & Y & Z \\
\hline 1 & 6 & 0 & 1.298647 & 1.756328 & 0.104499 \\
\hline 2 & 6 & 0 & 2.684700 & 2.170638 & 0.178537 \\
\hline 3 & 6 & 0 & 3.705670 & 1.273413 & 0.131396 \\
\hline 4 & 6 & 0 & 3.433013 & -0.115005 & 0.003913 \\
\hline 5 & 6 & 0 & 2.143265 & -0.582111 & -0.075731 \\
\hline 6 & 6 & 0 & 1.072607 & 0.320954 & -0.028693 \\
\hline 7 & 6 & 0 & -0.239617 & -0.209609 & -0.117022 \\
\hline 8 & 6 & 0 & -2.693589 & 0.007887 & -0.138435 \\
\hline 9 & 6 & 0 & -3.159314 & -0.214872 & 1.317681 \\
\hline 10 & 6 & 0 & -3.528141 & 1.094280 & -0.821486 \\
\hline 11 & 6 & 0 & -2.798685 & -1.292117 & -0.948075 \\
\hline 12 & 1 & 0 & 2.883205 & 3.231706 & 0.275501 \\
\hline 13 & 1 & 0 & 4.734913 & 1.605199 & 0.188900 \\
\hline 14 & 1 & 0 & 1.947492 & -1.643933 & -0.174701 \\
\hline 15 & 1 & 0 & -0.363497 & -1.286746 & -0.208372 \\
\hline 16 & 1 & 0 & -2.372105 & -0.737922 & 1.863670 \\
\hline 17 & 1 & 0 & -3.321229 & 0.756479 & 1.798719 \\
\hline 18 & 1 & 0 & -5.006513 & -0.572029 & 0.890477 \\
\hline 19 & 1 & 0 & -3.296537 & 1.123466 & -1.892071 \\
\hline 20 & 1 & 0 & -3.288779 & 2.067587 & -0.378896 \\
\hline 21 & 1 & 0 & -5.445547 & 1.452261 & -1.013330 \\
\hline 22 & 1 & 0 & -2.175404 & -1.210611 & -1.841639 \\
\hline 23 & 1 & 0 & -3.835829 & -1.401738 & -1.275389 \\
\hline 24 & 1 & 0 & -3.036960 & -2.608055 & 0.443777 \\
\hline 25 & 7 & 0 & -1.317909 & 0.520080 & -0.092727 \\
\hline 26 & 8 & 0 & -4.314171 & -1.030504 & 1.384270 \\
\hline 27 & 8 & 0 & -4.896849 & 0.774883 & -0.603480 \\
\hline 28 & 8 & 0 & -2.373422 & -2.440036 & -0.237065 \\
\hline 29 & 8 & 0 & 0.355296 & 2.574083 & 0.151768 \\
\hline 30 & 1 & 0 & -1.156203 & 1.530015 & 0.023934 \\
\hline 31 & 7 & 0 & 4.529180 & -1.052488 & -0.041980 \\
\hline 32 & 8 & 0 & 5.667340 & -0.614937 & 0.038116 \\
\hline 33 & 8 & 0 & 4.279652 & -2.243181 & -0.158251 \\
\hline
\end{tabular}

\section{$\underline{\text { Structure of } 6 \text { (open form) }}$}

Zero-point correction $=0.248840$ (Hartree/Particle) 
Thermal correction to Energy $=0.265356$

Thermal correction to Enthalpy $=0.266300$

Thermal correction to Gibbs Free Energy $=0.204479$

Sum of electronic and zero-point Energies= -882.742173

Sum of electronic and thermal Energies= -882.725657

Sum of electronic and thermal Enthalpies $=-882.724713$

Sum of electronic and thermal Free Energies= -882.786534

No imaginary frequencies

\begin{tabular}{|c|c|c|c|c|c|}
\hline \multirow{2}{*}{$\begin{array}{l}\text { Center } \\
\text { Number }\end{array}$} & \multirow{2}{*}{$\begin{array}{l}\text { Atomic } \\
\text { Number }\end{array}$} & \multirow{2}{*}{$\begin{array}{c}\text { Atomic } \\
\text { Type }\end{array}$} & \multicolumn{3}{|c|}{ Coordinates (Angstroms) } \\
\hline & & & $\mathrm{X}$ & $\mathrm{Y}$ & Z \\
\hline & & & ---------- & ---------- & ---------- \\
\hline 1 & 6 & 0 & 2.154311 & 1.329367 & 0.154491 \\
\hline 2 & 6 & 0 & 3.537223 & 1.498643 & 0.245546 \\
\hline 3 & 6 & 0 & 4.402587 & 0.420984 & 0.122707 \\
\hline 4 & 6 & 0 & 3.860515 & -0.831433 & -0.093654 \\
\hline 5 & 6 & 0 & 2.502268 & -1.032564 & -0.187877 \\
\hline 6 & 6 & 0 & 1.617153 & 0.046491 & -0.064211 \\
\hline 7 & 6 & 0 & 0.182422 & -0.284702 & -0.175335 \\
\hline 8 & 6 & 0 & -2.152050 & 0.079253 & -0.152705 \\
\hline 9 & 6 & 0 & -2.700998 & 0.136842 & 1.289355 \\
\hline 10 & 6 & 0 & -2.871859 & 1.110149 & -1.027965 \\
\hline 11 & 6 & 0 & -2.380035 & -1.315271 & -0.751942 \\
\hline 12 & 1 & 0 & 3.939251 & 2.492726 & 0.414620 \\
\hline 13 & 1 & 0 & 5.475979 & 0.550473 & 0.192460 \\
\hline 14 & 1 & 0 & 2.117521 & -2.032804 & -0.355868 \\
\hline 15 & 1 & 0 & 0.007633 & -1.349601 & -0.359840 \\
\hline 16 & 1 & 0 & -2.001039 & -0.372341 & 1.954482 \\
\hline 17 & 1 & 0 & -2.770085 & 1.183242 & 1.607288 \\
\hline 18 & 1 & 0 & -4.549530 & -0.111918 & 0.796939 \\
\hline 19 & 1 & 0 & -2.567538 & 0.984722 & -2.074263 \\
\hline 20 & 1 & 0 & -2.587131 & 2.112066 & -0.694913 \\
\hline 21 & 1 & 0 & -4.739164 & 1.582100 & -1.415748 \\
\hline 22 & 1 & 0 & -1.736549 & -1.445488 & -1.625721 \\
\hline 23 & 1 & 0 & -3.415435 & -1.378979 & -1.099097 \\
\hline 24 & 1 & 0 & -2.777317 & -2.347997 & 0.826268 \\
\hline 25 & 1 & 0 & 1.850918 & 3.186629 & 0.414709 \\
\hline 26 & 7 & 0 & -0.767603 & 0.547804 & -0.060375 \\
\hline 27 & 8 & 0 & -3.947982 & -0.528398 & 1.427971 \\
\hline 28 & 8 & 0 & -4.280498 & 0.919308 & -0.889503 \\
\hline 29 & 8 & 0 & -2.095514 & -2.378912 & 0.144381 \\
\hline 30 & 8 & 0 & 1.323136 & 2.389950 & 0.272016 \\
\hline 31 & 9 & 0 & 4.691486 & -1.894399 & -0.216453 \\
\hline
\end{tabular}

\section{$\underline{\text { Structure of } 6 \text { (closed form) }}$}

Zero-point correction= 0.249266 (Hartree/Particle)

Thermal correction to Energy $=0.265370$

Thermal correction to Enthalpy $=0.266314$

Thermal correction to Gibbs Free Energy= 0.205035

Sum of electronic and zero-point Energies $=\mathbf{- 8 8 2 . 7 5 8 2 8 7}$

Sum of electronic and thermal Energies= -882.742183

Sum of electronic and thermal Enthalpies= -882.741239

Sum of electronic and thermal Free Energies= -882.802518

No imaginary frequencies

Standard orientation:

\begin{tabular}{|c|c|c|c|c|c|}
\hline \multirow{2}{*}{$\begin{array}{l}\text { Center } \\
\text { Number }\end{array}$} & \multirow{2}{*}{$\begin{array}{l}\text { Atomic } \\
\text { Number }\end{array}$} & \multirow{2}{*}{$\begin{array}{c}\text { Atomic } \\
\text { Type }\end{array}$} & \multicolumn{3}{|c|}{ Coordinates (Angstroms) } \\
\hline & & & $\mathrm{X}$ & Y & Z \\
\hline 1 & 6 & 0 & 2.014454 & 1.267415 & 0.178903 \\
\hline 2 & 6 & 0 & 3.369823 & 1.588577 & 0.293230 \\
\hline 3 & 6 & 0 & 4.339214 & 0.607412 & 0.156775 \\
\hline 4 & 6 & 0 & 3.943244 & -0.697458 & -0.095442 \\
\hline 5 & 6 & 0 & 2.620711 & -1.052324 & -0.215201 \\
\hline 6 & 6 & 0 & 1.634734 & -0.066683 & -0.078499 \\
\hline 7 & 6 & 0 & 0.226327 & -0.452096 & -0.199999 \\
\hline 8 & 6 & 0 & -2.114463 & 0.041118 & -0.167979 \\
\hline 9 & 6 & 0 & -2.664430 & 0.191110 & 1.268266 \\
\hline 10 & 6 & 0 & -2.768473 & 1.072546 & -1.092371 \\
\hline
\end{tabular}




$\begin{array}{rrrrrr}11 & 6 & 0 & -2.402895 & -1.364902 & -0.707324 \\ 12 & 1 & 0 & 3.649024 & 2.616431 & 0.492229 \\ 13 & 1 & 0 & 5.393217 & 0.844107 & 0.243971 \\ 14 & 1 & 0 & 2.344069 & -2.082646 & -0.412038 \\ 15 & 1 & 0 & 0.031626 & -1.512569 & -0.376372 \\ 16 & 1 & 0 & -1.986683 & -0.309985 & 1.961567 \\ 17 & 1 & 0 & -2.695487 & 1.254234 & 1.532697 \\ 18 & 1 & 0 & -4.521075 & -0.001521 & 0.785107 \\ 19 & 1 & 0 & -2.478416 & 0.876246 & -2.131404 \\ 20 & 1 & 0 & -2.419997 & 2.070009 & -0.808473 \\ 21 & 1 & 0 & -4.605017 & 1.643469 & -1.487574 \\ 22 & 1 & 0 & -1.779214 & -1.554780 & -1.584321 \\ 23 & 1 & 0 & -3.445292 & -1.402179 & -1.036318 \\ 24 & 1 & 0 & -2.817685 & -2.323846 & 0.913455 \\ 25 & 1 & 0 & 0.211228 & 1.825890 & 0.210138 \\ 26 & 7 & 0 & -0.703062 & 0.414580 & -0.091630 \\ 27 & 8 & 0 & -3.934271 & -0.419858 & 1.428397 \\ 28 & 8 & 0 & -4.183403 & 0.974851 & -0.937930 \\ 29 & 8 & 0 & -2.140280 & -2.396366 & 0.230266 \\ 30 & 8 & 0 & 1.106108 & 2.244778 & 0.320989 \\ 31 & 9 & 0 & 4.896269 & -1.653383 & -0.226353 \\ ---------------------------------------------------------\end{array}$

\section{$\underline{\text { Structure of } \mathbf{T S}_{6 / 36}}$}

Zero-point correction $=0.245100$ (Hartree/Particle) Thermal correction to Energy $=0.260907$

Thermal correction to Enthalpy $=0.261851$

Thermal correction to Gibbs Free Energy= 0.201582

Sum of electronic and zero-point Energies $=-882.753648$

Sum of electronic and thermal Energies $=-882.737841$

Sum of electronic and thermal Enthalpies= -882.736897

Sum of electronic and thermal Free Energies= -882.797166

one imaginary frequency: 951 i

Standard orientation:

\begin{tabular}{|c|c|c|c|c|c|}
\hline \multirow{2}{*}{$\begin{array}{l}\text { Center } \\
\text { Number }\end{array}$} & \multirow{2}{*}{$\begin{array}{l}\text { Atomic } \\
\text { Number }\end{array}$} & \multirow{2}{*}{$\begin{array}{c}\text { Atomic } \\
\text { Type }\end{array}$} & \multicolumn{3}{|c|}{ Coordinates (Angstroms) } \\
\hline & & & $\mathrm{X}$ & Y & Z \\
\hline 1 & 6 & 0 & 1.906685 & 1.247323 & 0.160179 \\
\hline 2 & 6 & 0 & 3.267373 & 1.636987 & 0.283554 \\
\hline 3 & 6 & 0 & 4.281629 & 0.712094 & 0.173816 \\
\hline 4 & 6 & 0 & 3.977212 & -0.632459 & -0.062346 \\
\hline 5 & 6 & 0 & 2.691642 & -1.075014 & -0.190006 \\
\hline 6 & 6 & 0 & 1.643750 & -0.139361 & -0.079883 \\
\hline 7 & 6 & 0 & 0.277763 & -0.562971 & -0.203159 \\
\hline 8 & 6 & 0 & -2.095283 & 0.040414 & -0.159841 \\
\hline 9 & 6 & 0 & -2.615482 & 0.089566 & 1.294332 \\
\hline 10 & 6 & 0 & -2.715687 & 1.165434 & -0.993106 \\
\hline 11 & 6 & 0 & -2.426251 & -1.308721 & -0.807105 \\
\hline 12 & 1 & 0 & 3.490000 & 2.681684 & 0.467181 \\
\hline 13 & 1 & 0 & 5.320740 & 1.008686 & 0.267111 \\
\hline 14 & 1 & 0 & 2.478178 & -2.123027 & -0.372995 \\
\hline 15 & 1 & 0 & 0.049635 & -1.614555 & -0.370602 \\
\hline 16 & 1 & 0 & -1.948059 & -0.496373 & 1.928367 \\
\hline 17 & 1 & 0 & -2.600282 & 1.126559 & 1.648723 \\
\hline 18 & 1 & 0 & -4.487533 & 0.015795 & 0.833642 \\
\hline 19 & 1 & 0 & -2.456922 & 1.029742 & -2.049661 \\
\hline 20 & 1 & 0 & -2.318546 & 2.126097 & -0.649773 \\
\hline 21 & 1 & 0 & -4.533191 & 1.827714 & -1.314441 \\
\hline 22 & 1 & 0 & -1.807165 & -1.443158 & -1.697648 \\
\hline 23 & 1 & 0 & -3.469359 & -1.284926 & -1.133603 \\
\hline 24 & 1 & 0 & -2.869786 & -2.385351 & 0.731589 \\
\hline 25 & 1 & 0 & -0.134234 & 1.354478 & 0.095255 \\
\hline 26 & 7 & 0 & -0.661537 & 0.319638 & -0.104457 \\
\hline 27 & 8 & 0 & -3.904674 & -0.484712 & 1. 419531 \\
\hline 28 & 8 & 0 & -4.127148 & 1.117361 & -0.806464 \\
\hline 29 & 8 & 0 & -2.192473 & -2.416212 & 0.044887 \\
\hline 30 & 8 & 0 & 0.936660 & 2.100271 & 0.258932 \\
\hline 31 & 9 & 0 & 5.006473 & -1.514916 & -0.165072 \\
\hline
\end{tabular}




\section{Structure of $\mathbf{3 6}$}

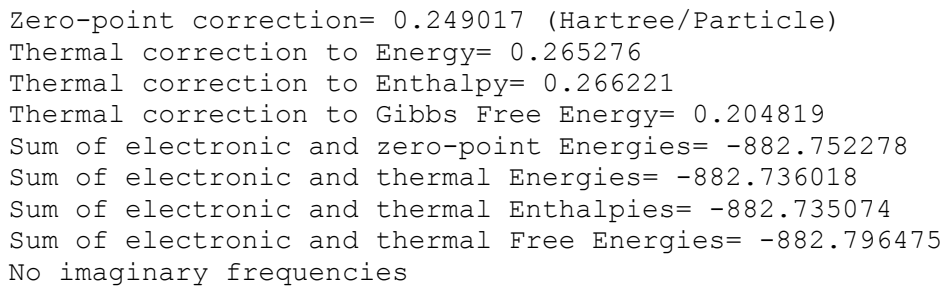

Standard orientation:

\begin{tabular}{|c|c|c|c|c|c|}
\hline \multirow{2}{*}{$\begin{array}{l}\text { Center } \\
\text { Number }\end{array}$} & \multirow{2}{*}{$\begin{array}{l}\text { Atomic } \\
\text { Number }\end{array}$} & \multirow{2}{*}{$\begin{array}{c}\text { Atomic } \\
\text { Type }\end{array}$} & \multicolumn{3}{|c|}{ Coordinates (Angstroms) } \\
\hline & & & $\mathrm{X}$ & Y & Z \\
\hline 1 & 6 & 0 & 2.005461 & 1.345833 & 0.095600 \\
\hline 2 & 6 & 0 & 3.424300 & 1.613095 & 0.150070 \\
\hline 3 & 6 & 0 & 4.347722 & 0.608179 & 0.100366 \\
\hline 4 & 6 & 0 & 3.926998 & -0.734685 & -0.009438 \\
\hline 5 & 6 & 0 & 2.617641 & -1.077416 & -0.072454 \\
\hline 6 & 6 & 0 & 1.631692 & -0.050747 & -0.022710 \\
\hline 7 & 6 & 0 & 0.275061 & -0.421905 & -0.102428 \\
\hline 8 & 6 & 0 & -2.135531 & 0.097046 & -0.125848 \\
\hline 9 & 6 & 0 & -2.606606 & -0.188502 & 1.315979 \\
\hline 10 & 6 & 0 & -2.839877 & 1.327995 & -0.700846 \\
\hline 11 & 6 & 0 & -2.410598 & -1.111182 & -1.034248 \\
\hline 12 & 1 & 0 & 3.737267 & 2.647497 & 0.233659 \\
\hline 13 & 1 & 0 & 5.411012 & 0.820758 & 0.143128 \\
\hline 14 & 1 & 0 & 2.318930 & -2.117062 & -0.159366 \\
\hline 15 & 1 & 0 & 0.014184 & -1.474522 & -0.193178 \\
\hline 16 & 1 & 0 & -1.889122 & -0.858745 & 1.793271 \\
\hline 17 & 1 & 0 & -2.627379 & 0.748930 & 1.883568 \\
\hline 18 & 1 & 0 & -4.488263 & -0.264767 & 0.895463 \\
\hline 19 & 1 & 0 & -2.611363 & 1.422387 & -1.768584 \\
\hline 20 & 1 & 0 & -2.480682 & 2.222302 & -0.179933 \\
\hline 21 & 1 & 0 & -4.699252 & 1.932996 & -0.836159 \\
\hline 22 & 1 & 0 & -1.781406 & -1.038747 & -1.924278 \\
\hline 23 & 1 & 0 & -3.454269 & -1.062973 & -1.356733 \\
\hline 24 & 1 & 0 & -2.813999 & -2.492693 & 0.251647 \\
\hline 25 & 7 & 0 & -0.711659 & 0.439758 & -0.077294 \\
\hline 26 & 8 & 0 & -3.861566 & -0.845669 & 1.346277 \\
\hline 27 & 8 & 0 & -4.237262 & 1.161411 & -0.491611 \\
\hline 28 & 8 & 0 & -2.135819 & -2.358743 & -0.422167 \\
\hline 29 & 8 & 0 & 1.152164 & 2.272026 & 0.147451 \\
\hline 30 & 1 & 0 & -0.412736 & 1.420949 & 0.039114 \\
\hline 31 & 9 & 0 & 4.888998 & -1.695898 & -0.053825 \\
\hline
\end{tabular}

\section{$\underline{\text { Structure of } 7 \text { (open form) }}$}

Zero-point correction $=0.247605$ (Hartree/Particle)

Thermal correction to Energy $=0.264532$

Thermal correction to Enthalpy $=0.265476$

Thermal correction to Gibbs Free Energy= 0.202229

Sum of electronic and zero-point Energies= -1243.104530

Sum of electronic and thermal Energies= -1243.087604

Sum of electronic and thermal Enthalpies= -1243.086659

Sum of electronic and thermal Free Energies= -1243.149907

No imaginary frequency

Standard orientation:

\begin{tabular}{|c|c|c|c|c|c|}
\hline \multirow{2}{*}{$\begin{array}{l}\text { Center } \\
\text { Number }\end{array}$} & \multirow{2}{*}{$\begin{array}{l}\text { Atomic } \\
\text { Number }\end{array}$} & \multirow{2}{*}{$\begin{array}{c}\text { Atomic } \\
\text { Type }\end{array}$} & \multicolumn{3}{|c|}{ Coordinates (Angstroms) } \\
\hline & & & $\mathrm{X}$ & Y & Z \\
\hline 1 & 6 & 0 & 1.708321 & 1.655281 & 0.089039 \\
\hline 2 & 6 & 0 & 3.069219 & 1.941929 & 0.210516 \\
\hline 3 & 6 & 0 & 4.020755 & 0.934557 & 0.171261 \\
\hline 4 & 6 & 0 & 3.602280 & -0.377541 & -0.002154 \\
\hline
\end{tabular}




$\begin{array}{rrrrrr}5 & 6 & 0 & 2.260058 & -0.681608 & -0.133645 \\ 6 & 6 & 0 & 1.286853 & 0.323235 & -0.078410 \\ 7 & 6 & 0 & -0.114053 & -0.123244 & -0.220956 \\ 8 & 6 & 0 & -2.469724 & 0.028114 & -0.101441 \\ 9 & 6 & 0 & -2.982836 & -0.222903 & 1.333497 \\ 10 & 6 & 0 & -3.297298 & 1.133773 & -0.764813 \\ 11 & 6 & 0 & -2.596646 & -1.250496 & -0.941287 \\ 12 & 1 & 0 & 3.385135 & 2.973011 & 0.336694 \\ 13 & 1 & 0 & 5.072822 & 1.172526 & 0.270445 \\ 14 & 1 & 0 & 1.944905 & -1.709641 & -0.276928 \\ 15 & 1 & 0 & -0.200917 & -1.160211 & -0.560727 \\ 16 & 1 & 0 & -2.223768 & -0.775355 & 1.890327 \\ 17 & 1 & 0 & -3.139676 & 0.738850 & 1.834908 \\ 18 & 1 & 0 & -4.812931 & -0.546591 & 0.820476 \\ 19 & 1 & 0 & -3.016961 & 1.223534 & -1.821389 \\ 20 & 1 & 0 & -3.083047 & 2.079919 & -0.259845 \\ 21 & 1 & 0 & -5.206755 & 1.516715 & -1.018297 \\ 22 & 1 & 0 & -1.968137 & -1.164755 & -1.831346 \\ 23 & 1 & 0 & -3.631791 & -1.341040 & -1.283597 \\ 24 & 1 & 0 & -2.862095 & -2.581690 & 0.427359 \\ 25 & 1 & 0 & 1.249830 & 3.495371 & 0.188616 \\ 26 & 7 & 0 & -1.126183 & 0.591834 & 0.046885 \\ 27 & 8 & 0 & -4.161037 & -1.015035 & 1.358086 \\ 28 & 8 & 0 & -4.681334 & 0.803728 & -0.641137 \\ 29 & 8 & 0 & -2.199605 & -2.429521 & -0.257435 \\ 30 & 8 & 0 & 0.792394 & 2.647322 & 0.113137 \\ 31 & 17 & 0 & 4.791279 & -1.663647 & -0.058573 \\ ---------------------------19\end{array}$

\section{$\underline{\text { Structure of } 7 \text { (closed form) }}$}

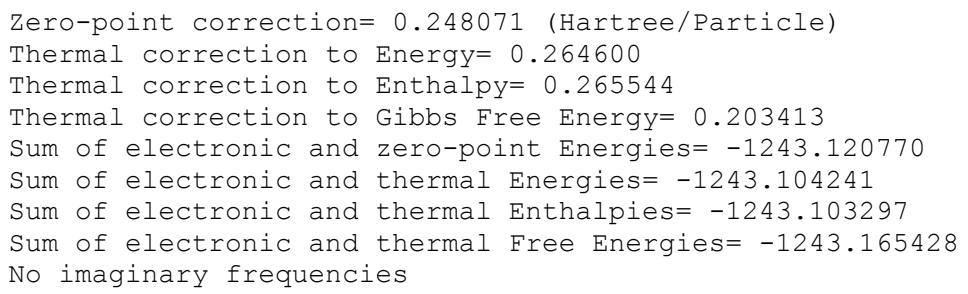

Standard orientation

\begin{tabular}{|c|c|c|c|c|c|}
\hline \multirow{2}{*}{$\begin{array}{l}\text { Center } \\
\text { Number }\end{array}$} & \multirow{2}{*}{$\begin{array}{l}\text { Atomic } \\
\text { Number }\end{array}$} & \multirow{2}{*}{$\begin{array}{l}\text { Atomic } \\
\text { Type }\end{array}$} & \multicolumn{3}{|c|}{ Coordinates (Angstroms) } \\
\hline & & & $\mathrm{X}$ & $Y$ & Z \\
\hline 1 & 6 & 0 & 1.567461 & 1.534959 & 0.182080 \\
\hline 2 & 6 & 0 & 2.891545 & 1.967862 & 0.296591 \\
\hline 3 & 6 & 0 & 3.937579 & 1.066816 & 0.187677 \\
\hline 4 & 6 & 0 & 3.665445 & -0.281045 & -0.036703 \\
\hline 5 & 6 & 0 & 2.366534 & -0.734195 & -0.151807 \\
\hline 6 & 6 & 0 & 1.302307 & 0.167753 & -0.043998 \\
\hline 7 & 6 & 0 & -0.068176 & -0.336252 & -0.166897 \\
\hline 8 & 6 & 0 & -2.442508 & -0.028470 & -0.172890 \\
\hline 9 & 6 & 0 & -3.025942 & 0.124970 & 1.249746 \\
\hline 10 & 6 & 0 & -3.158211 & 0.921457 & -1.137910 \\
\hline 11 & 6 & 0 & -2.612450 & -1.467048 & -0.671746 \\
\hline 12 & 1 & 0 & 3.086738 & 3.019164 & 0.472363 \\
\hline 13 & 1 & 0 & 4.961957 & 1.408626 & 0.276276 \\
\hline 14 & 1 & 0 & 2.156496 & -1.784146 & -0.325494 \\
\hline 15 & 1 & 0 & -0.171146 & -1.413537 & -0.318242 \\
\hline 16 & 1 & 0 & -2.323437 & -0.297771 & 1.970040 \\
\hline 17 & 1 & 0 & -3.143369 & 1.190921 & 1.475873 \\
\hline 18 & 1 & 0 & -4.855020 & -0.225308 & 0.748708 \\
\hline 19 & 1 & 0 & -2.837943 & 0.717395 & -2.166483 \\
\hline 20 & 1 & 0 & -2.891644 & 1.950983 & -0.880386 \\
\hline 21 & 1 & 0 & -5.027350 & 1.338246 & -1.571967 \\
\hline 22 & 1 & 0 & -1.963218 & -1.632379 & -1.535115 \\
\hline 23 & 1 & 0 & -3.643525 & -1.596668 & -1.012373 \\
\hline 24 & 1 & 0 & -2.970978 & -2.404536 & 0.974527 \\
\hline 25 & 1 & 0 & -0.275134 & 1.938941 & 0.181371 \\
\hline 26 & 7 & 0 & -1.065455 & 0.454734 & -0.088893 \\
\hline 27 & 8 & 0 & -4.247955 & -0.576321 & 1.413542 \\
\hline 28 & 8 & 0 & -4.563609 & 0.719564 & -0.998306 \\
\hline
\end{tabular}




\begin{tabular}{|c|c|c|c|c|c|}
\hline 29 & 8 & 0 & -2.282347 & -2.445978 & 0.300123 \\
\hline 30 & 8 & 0 & 0.582023 & 2.434127 & 0.291682 \\
\hline 31 & 17 & 0 & 4.994668 & -1.415591 & -0.172237 \\
\hline
\end{tabular}

\section{$\underline{\text { Structure of }} \mathbf{T S}_{7 / 37}$}

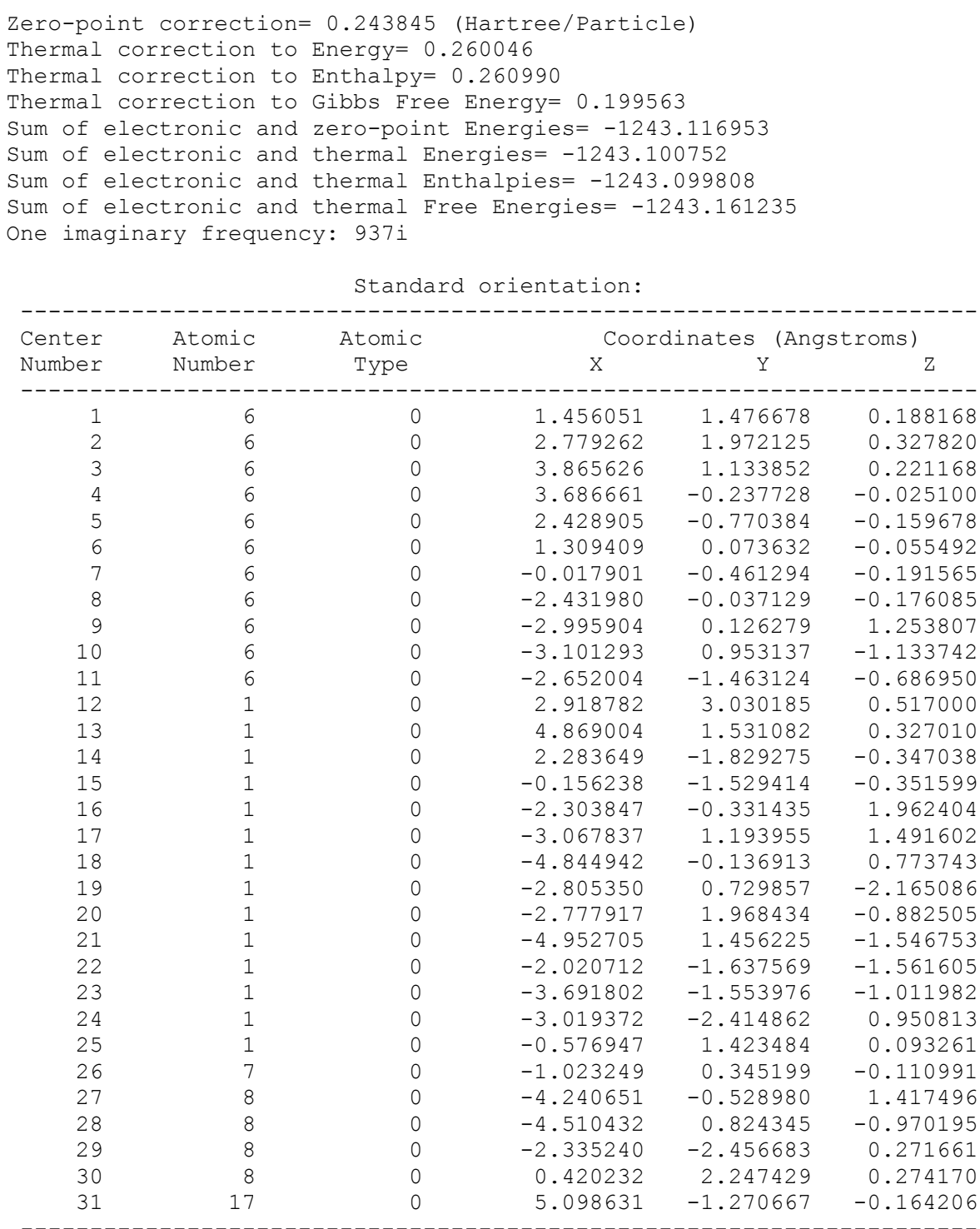

\section{$\underline{\text { Structure of } \mathbf{3 7}}$}

Zero-point correction $=0.247758$ (Hartree/Particle)

Thermal correction to Energy $=0.264465$

Thermal correction to Enthalpy $=0.265409$

Thermal correction to Gibbs Free Energy $=0.202527$

Sum of electronic and zero-point Energies= -1243.115941

Sum of electronic and thermal Energies= -1243.099234

Sum of electronic and thermal Enthalpies= -1243.098290

Sum of electronic and thermal Free Energies= -1243.161172

No imaginary frequencies

Standard orientation:

$\begin{array}{llcl}\text { Center } & \text { Atomic } & \text { Atomic } & \text { Coordinates (Angstroms) } \\ \text { Number } & \text { Number } & \text { Type } & \text { X }\end{array}$




$\begin{array}{rrrrrr}1 & 6 & 0 & 1.564285 & 1.634778 & 0.100926 \\ 2 & 6 & 0 & 2.960426 & 2.000996 & 0.167824 \\ 3 & 6 & 0 & 3.954461 & 1.066420 & 0.121471 \\ 4 & 6 & 0 & 3.646196 & -0.313192 & 0.001046 \\ 5 & 6 & 0 & 2.353888 & -0.734539 & -0.073897 \\ 6 & 6 & 0 & 1.297356 & 0.214125 & -0.027764 \\ 7 & 6 & 0 & -0.024922 & -0.266415 & -0.113833 \\ 8 & 6 & 0 & -2.470540 & 0.033585 & -0.132216 \\ 9 & 6 & 0 & -2.920732 & -0.256124 & 1.316081 \\ 10 & 6 & 0 & -3.284856 & 1.178120 & -0.740372 \\ 11 & 6 & 0 & -2.629511 & -1.218187 & -1.009025 \\ 12 & 1 & 0 & 3.199017 & 3.054594 & 0.260571 \\ 13 & 1 & 0 & 4.993832 & 1.372778 & 0.176141 \\ 14 & 1 & 0 & 2.112324 & -1.788312 & -0.168449 \\ 15 & 1 & 0 & -0.193110 & -1.337601 & -0.206952 \\ 16 & 1 & 0 & -2.141280 & -0.831167 & 1.819221 \\ 17 & 1 & 0 & -3.047342 & 0.690209 & 1.854109 \\ 18 & 1 & 0 & -4.784206 & -0.537371 & 0.900603 \\ 19 & 1 & 0 & -3.067127 & 1.261109 & -1.811250 \\ 20 & 1 & 0 & -3.010945 & 2.117603 & -0.247109 \\ 21 & 1 & 0 & -5.194250 & 1.596049 & -0.892576 \\ 22 & 1 & 0 & -2.005510 & -1.111227 & -1.899312 \\ 23 & 1 & 0 & -3.671390 & -1.272398 & -1.335490 \\ 24 & 1 & 0 & -2.911142 & -2.593171 & 0.314488 \\ 25 & 7 & 0 & -1.082777 & 0.504243 & -0.089724 \\ 26 & 8 & 0 & -4.099319 & -1.041202 & 1.359209 \\ 27 & 8 & 0 & -4.659926 & 0.886190 & -0.520986 \\ 28 & 8 & 0 & -2.246789 & -2.419837 & -0.364293 \\ 29 & 8 & 0 & 0.644864 & 2.491219 & 0.151216 \\ 30 & 1 & 0 & -0.879769 & 1.507129 & 0.028242 \\ 31 & 17 & 0 & 4.964317 & -1.472135 & -0.048634 \\ -----------------------------1\end{array}$

\section{$\underline{\text { Structure of } \mathbf{8} \text { (open form) }}$}

Zero-point correction= 0.247031 (Hartree/Particle)

Thermal correction to Energy $=0.264290$

Thermal correction to Enthalpy $=0.265234$

Thermal correction to Gibbs Free Energy $=0.200001$

Sum of electronic and zero-point Energies= -3357.077420

Sum of electronic and thermal Energies= -3357.060161

Sum of electronic and thermal Enthalpies= -3357.059217

Sum of electronic and thermal Free Energies= -3357.124450

No imaginary frequencies

Standard orientation:

\begin{tabular}{|c|c|c|c|c|c|}
\hline \multirow{2}{*}{$\begin{array}{l}\text { Center } \\
\text { Number }\end{array}$} & \multirow{2}{*}{$\begin{array}{l}\text { Atomic } \\
\text { Number }\end{array}$} & \multirow{2}{*}{$\begin{array}{c}\text { Atomic } \\
\text { Type }\end{array}$} & \multicolumn{3}{|c|}{ Coordinates (Angstroms) } \\
\hline & & & $\mathrm{X}$ & $\mathrm{Y}$ & Z \\
\hline 1 & 6 & 0 & 0.920754 & 1.945262 & 0.183866 \\
\hline 2 & 6 & 0 & 2.243376 & 2.386229 & 0.261317 \\
\hline 3 & 6 & 0 & 3.303504 & 1.497004 & 0.173075 \\
\hline 4 & 6 & 0 & 3.034691 & 0.144705 & 0.005895 \\
\hline 5 & 6 & 0 & 1.731121 & -0.311321 & -0.071385 \\
\hline 6 & 6 & 0 & 0.649478 & 0.574490 & 0.013885 \\
\hline 7 & 6 & 0 & -0.691908 & -0.035636 & -0.088596 \\
\hline 8 & 6 & 0 & -3.056883 & -0.103957 & -0.175669 \\
\hline 9 & 6 & 0 & -3.734732 & 0.087720 & 1.199164 \\
\hline 10 & 6 & 0 & -3.856522 & 0.632671 & -1.256163 \\
\hline 11 & 6 & 0 & -3.002971 & -1.593735 & -0.535273 \\
\hline 12 & 1 & 0 & 2.442785 & 3.445662 & 0.392549 \\
\hline 13 & 1 & 0 & 4.321164 & 1.862396 & 0.234313 \\
\hline 14 & 1 & 0 & 1.526688 & -1.368349 & -0.202445 \\
\hline 15 & 1 & 0 & -0.655098 & -1.126326 & -0.173011 \\
\hline 16 & 1 & 0 & -3.023129 & -0.171099 & 1.985501 \\
\hline 17 & 1 & 0 & -4.005538 & 1.142515 & 1.322128 \\
\hline 18 & 1 & 0 & -5.462031 & -0.564682 & 0.644601 \\
\hline 19 & 1 & 0 & -3.454297 & 0.389054 & -2.246847 \\
\hline 20 & 1 & 0 & -3.760500 & 1.708996 & -1.088971 \\
\hline 21 & 1 & 0 & -5.740321 & 0.721536 & -1.807875 \\
\hline 22 & 1 & 0 & -2.296834 & -1.750752 & -1.354281 \\
\hline 23 & 1 & 0 & -3.988803 & -1.899687 & -0.897355 \\
\hline 24 & 1 & 0 & -3.311738 & -2.408086 & 1.184098 \\
\hline
\end{tabular}




\begin{tabular}{rrrrrr}
25 & 1 & 0 & 0.258465 & 3.711246 & 0.394473 \\
26 & 7 & 0 & -1.782749 & 0.610297 & -0.080119 \\
27 & 8 & 0 & -4.860819 & -0.758918 & 1.376060 \\
28 & 8 & 0 & -5.224015 & 0.232462 & -1.159094 \\
29 & 8 & 0 & -2.593163 & -2.423770 & 0.540769 \\
30 & 8 & 0 & -0.100687 & 2.821970 & 0.272423 \\
31 & 35 & 0 & 4.474813 & -1.093687 & -0.123657 \\
\hline
\end{tabular}

\section{$\underline{\text { Structure of } 8 \text { (closed form) }}$}

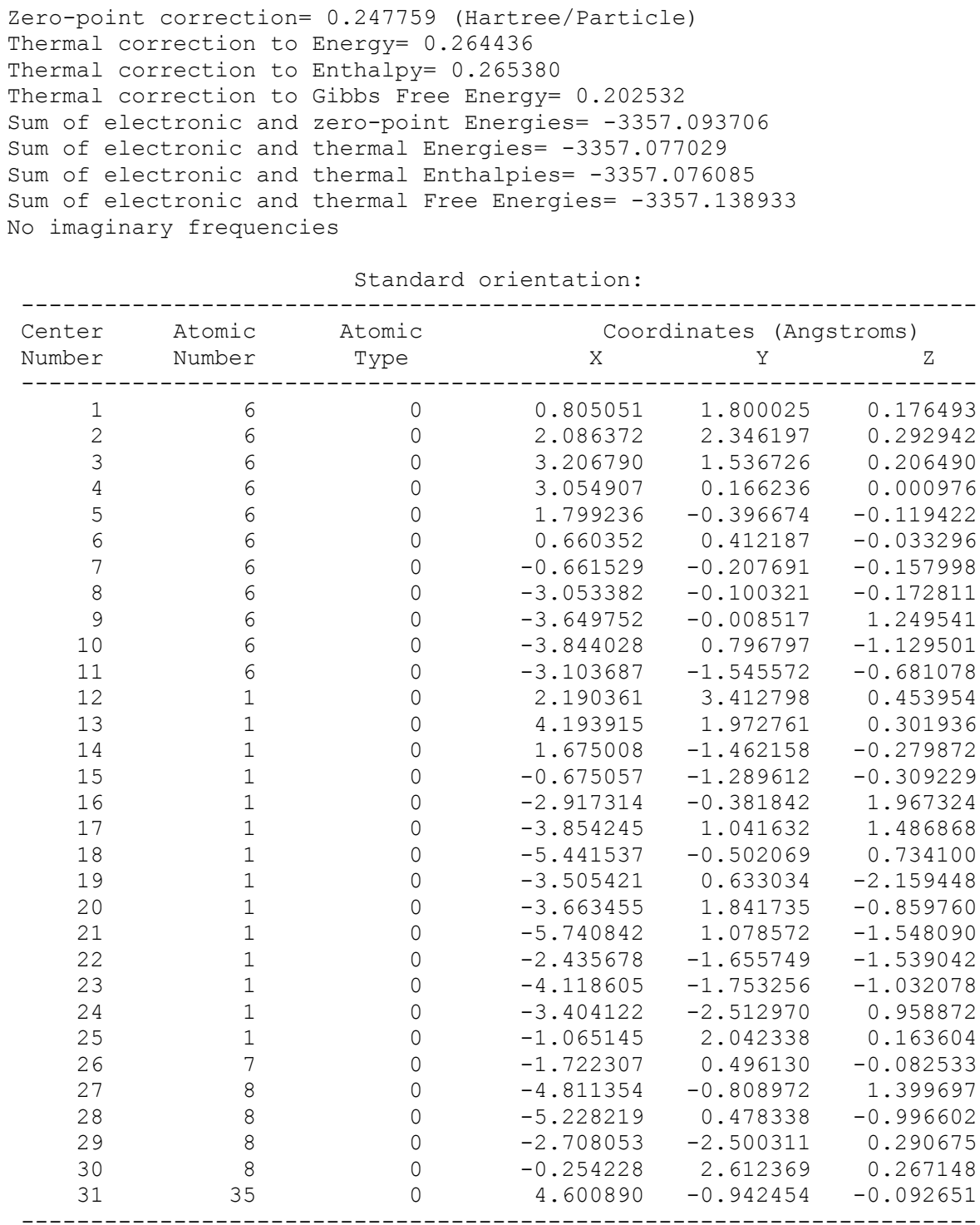

\section{$\underline{\text { Structure of } \mathbf{T S}} \mathbf{S}_{\mathbf{8} / 8}$}

Zero-point correction= 0.243426 (Hartree/Particle)

Thermal correction to Energy $=0.259841$

Thermal correction to Enthalpy $=0.260785$

Thermal correction to Gibbs Free Energy= 0.198061

Sum of electronic and zero-point Energies $=-3357.089997$

Sum of electronic and thermal Energies= -3357.073582

Sum of electronic and thermal Enthalpies= -3357.072637

Sum of electronic and thermal Free Energies= -3357.135362

One imaginary frequency: $943 i$ 


\begin{tabular}{|c|c|c|c|c|c|}
\hline \multirow{2}{*}{$\begin{array}{l}\text { Center } \\
\text { Number }\end{array}$} & \multirow{2}{*}{$\begin{array}{l}\text { Atomic } \\
\text { Number }\end{array}$} & \multirow{2}{*}{$\begin{array}{c}\text { Atomic } \\
\text { Type }\end{array}$} & \multicolumn{3}{|c|}{ Coordinates (Angstroms) } \\
\hline & & & $\mathrm{X}$ & Y & Z \\
\hline 1 & 6 & 0 & 0.705358 & 1.715703 & 0.175999 \\
\hline 2 & 6 & 0 & 1.986857 & 2.309861 & 0.317154 \\
\hline 3 & 6 & 0 & 3.133569 & 1.552453 & 0.234507 \\
\hline 4 & 6 & 0 & 3.060306 & 0.166776 & 0.009364 \\
\hline 5 & 6 & 0 & 1.845162 & -0.457671 & -0.131210 \\
\hline 6 & 6 & 0 & 0.665560 & 0.303010 & -0.051558 \\
\hline 7 & 6 & 0 & -0.616759 & -0.332331 & -0.187775 \\
\hline 8 & 6 & 0 & -3.055191 & -0.098290 & -0.177573 \\
\hline 9 & 6 & 0 & -3.621035 & -0.003718 & 1.257793 \\
\hline 10 & 6 & 0 & -3.809394 & 0.850846 & -1.113105 \\
\hline 11 & 6 & 0 & -3.166901 & -1.529622 & -0.709179 \\
\hline 12 & 1 & 0 & 2.044893 & 3.377818 & 0.492837 \\
\hline 13 & 1 & 0 & 4.100433 & 2.030150 & 0.346582 \\
\hline 14 & 1 & 0 & 1.773344 & -1.526658 & -0.302641 \\
\hline 15 & 1 & 0 & -0.672274 & -1.409149 & -0.340334 \\
\hline 16 & 1 & 0 & -2.889307 & -0.412672 & 1.956314 \\
\hline 17 & 1 & 0 & -3.783406 & 1.049806 & 1.512627 \\
\hline 18 & 1 & 0 & -5.446175 & -0.411139 & 0.783797 \\
\hline 19 & 1 & 0 & -3.506055 & 0.669181 & -2.150621 \\
\hline 20 & 1 & 0 & -3.565287 & 1.884450 & -0.847298 \\
\hline 21 & 1 & 0 & -5.696979 & 1.221127 & -1.495381 \\
\hline 22 & 1 & 0 & -2.525882 & -1.643308 & -1.586908 \\
\hline 23 & 1 & 0 & -4.197538 & -1.693566 & -1.034730 \\
\hline 24 & 1 & 0 & -3.459566 & -2.525141 & 0.916809 \\
\hline 25 & 1 & 0 & -1.317702 & 1.507849 & 0.079364 \\
\hline 26 & 7 & 0 & -1.681111 & 0.394976 & -0.114953 \\
\hline 27 & 8 & 0 & -4.805751 & -0.764334 & 1.415566 \\
\hline 28 & 8 & 0 & -5.202435 & 0.608314 & -0.941003 \\
\hline 29 & 8 & 0 & -2.776333 & -2.510549 & 0.235409 \\
\hline 30 & 8 & 0 & -0.386250 & 2.406433 & 0.248083 \\
\hline 31 & 35 & 0 & 4. 671721 & -0.847560 & -0.083905 \\
\hline
\end{tabular}

\section{Structure of $\mathbf{3 8}$}

Zero-point correction= 0.247781 (Hartree/Particle)
Thermal correction to Energy= 0.264540
Thermal correction to Enthalpy= 0.265484
Thermal correction to Gibbs Free Energy= 0.202290
Sum of electronic and zero-point Energies=-3357.088755
Sum of electronic and thermal Energies=-3357.071996
Sum of electronic and thermal Enthalpies=-3357.071052
Sum of electronic and thermal Free Energies=-3357.134246
No imaginary frequencies

Standard orientation:

\begin{tabular}{|c|c|c|c|c|c|}
\hline \multirow{2}{*}{$\begin{array}{l}\text { Center } \\
\text { Number }\end{array}$} & \multirow{2}{*}{$\begin{array}{l}\text { Atomic } \\
\text { Number }\end{array}$} & \multirow{2}{*}{$\begin{array}{c}\text { Atomic } \\
\text { Type }\end{array}$} & \multicolumn{3}{|c|}{ Coordinates (Angstroms) } \\
\hline & & & $\mathrm{x}$ & $\mathrm{Y}$ & $\mathrm{Z}$ \\
\hline 1 & 6 & 0 & 0.781548 & 1.888664 & 0.102443 \\
\hline 2 & 6 & 0 & 2.133334 & 2.385775 & 0.201204 \\
\hline 3 & 6 & 0 & 3.214005 & 1.552525 & 0.157791 \\
\hline 4 & 6 & 0 & 3.044391 & 0.151238 & 0.009931 \\
\hline 5 & 6 & 0 & 1.797877 & -0.390641 & -0.090122 \\
\hline 6 & 6 & 0 & 0.654377 & 0.451057 & -0.045933 \\
\hline 7 & 6 & 0 & -0.618092 & -0.151605 & -0.137939 \\
\hline 8 & 6 & 0 & -3.083958 & -0.054202 & -0.141198 \\
\hline 9 & 6 & 0 & -3.520814 & -0.322700 & 1.315388 \\
\hline 10 & 6 & 0 & -3.979471 & 0.999750 & -0.796944 \\
\hline 11 & 6 & 0 & -3.138611 & -1.345774 & -0.969920 \\
\hline 12 & 1 & 0 & 2.268723 & 3.455656 & 0.314433 \\
\hline 13 & 1 & 0 & 4.214801 & 1.962637 & 0.238451 \\
\hline 14 & 1 & 0 & 1.653317 & -1.460386 & -0.201637 \\
\hline 15 & 1 & 0 & -0.688934 & -1.232889 & -0.235855 \\
\hline 16 & 1 & 0 & -2.705381 & -0.823430 & 1.840669 \\
\hline 17 & 1 & 0 & -3.714089 & 0.632013 & 1.817935 \\
\hline 18 & 1 & 0 & -5.356717 & -0.750770 & 0.901766 \\
\hline 19 & 1 & 0 & -3.764388 & 1.053654 & -1.870149 \\
\hline 20 & 1 & 0 & -3.780035 & 1.977887 & -0.345203 \\
\hline
\end{tabular}




$\begin{array}{rrrrrr}21 & 1 & 0 & -5.913726 & 1.271498 & -0.962321 \\ 22 & 1 & 0 & -2.520475 & -1.223877 & -1.862485 \\ 23 & 1 & 0 & -4.171133 & -1.494956 & -1.296991 \\ 24 & 1 & 0 & -3.314006 & -2.685065 & 0.408190 \\ 25 & 7 & 0 & -1.737907 & 0.524264 & -0.106311 \\ 26 & 8 & 0 & -4.641624 & -1.185795 & 1.384470 \\ 27 & 8 & 0 & -5.329928 & 0.615933 & -0.565851 \\ 28 & 8 & 0 & -2.664013 & -2.486436 & -0.277557 \\ 29 & 8 & 0 & -0.216451 & 2.653770 & 0.143851 \\ 30 & 1 & 0 & -1.613223 & 1.540991 & 0.018019 \\ 31 & 35 & 0 & 4.590835 & -0.961631 & -0.032233 \\ -\end{array}$

\section{$\underline{\text { Structure of } 10 \text { (open form) }}$}

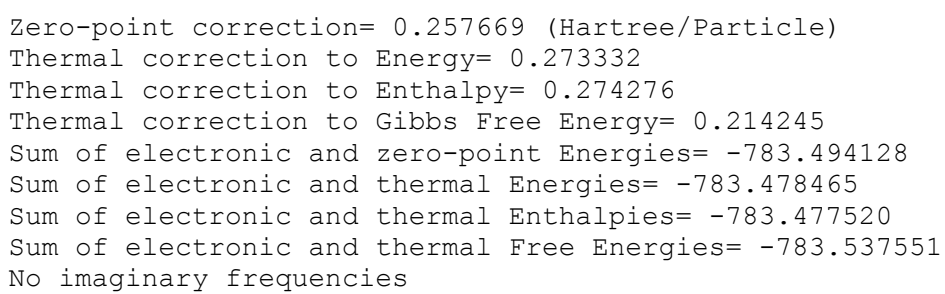

Standard orientation:

\begin{tabular}{|c|c|c|c|c|c|}
\hline \multirow{2}{*}{$\begin{array}{l}\text { Center } \\
\text { Number }\end{array}$} & \multirow{2}{*}{$\begin{array}{l}\text { Atomic } \\
\text { Number }\end{array}$} & \multirow{2}{*}{$\begin{array}{c}\text { Atomic } \\
\text { Type }\end{array}$} & \multicolumn{3}{|c|}{ Coordinates (Angstroms) } \\
\hline & & & $\mathrm{X}$ & Y & Z \\
\hline & & & & 0 & --------1 \\
\hline 1 & 6 & 0 & -2.576694 & -0.959245 & 0.147061 \\
\hline 2 & 6 & 0 & -3.971290 & -0.993929 & 0.211449 \\
\hline 3 & 6 & 0 & -4.719129 & 0.163846 & 0.049459 \\
\hline 4 & 6 & 0 & -4.083830 & 1.381226 & -0.181802 \\
\hline 5 & 6 & 0 & -2.698986 & 1.412583 & -0.247178 \\
\hline 6 & 6 & 0 & -1.914681 & 0.261653 & -0.086124 \\
\hline 7 & 6 & 0 & -0.454480 & 0.446896 & -0.185249 \\
\hline 8 & 6 & 0 & 1.836478 & -0.135059 & -0.135748 \\
\hline 9 & 6 & 0 & 2.378698 & -0.153075 & 1.309562 \\
\hline 10 & 6 & 0 & 2.456394 & -1.284424 & -0.937114 \\
\hline 11 & 6 & 0 & 2.195115 & 1.191176 & -0.821344 \\
\hline 12 & 1 & 0 & -4.465820 & -1.944640 & 0.389305 \\
\hline 13 & 1 & 0 & -5.800662 & 0.110396 & 0.103333 \\
\hline 14 & 1 & 0 & -4.658472 & 2.290203 & -0.310109 \\
\hline 15 & 1 & 0 & -2.191055 & 2.354592 & -0.428782 \\
\hline 16 & 1 & 0 & -0.173964 & 1.482742 & -0.403135 \\
\hline 17 & 1 & 0 & 1.735285 & 0.464899 & 1.938571 \\
\hline 18 & 1 & 0 & 2.345967 & -1.178489 & 1.694756 \\
\hline 19 & 1 & 0 & 4.238497 & -0.116330 & 0.798843 \\
\hline 20 & 1 & 0 & 2.158382 & -1.206541 & -1.989854 \\
\hline 21 & 1 & 0 & 2.082499 & -2.229473 & -0.533564 \\
\hline 22 & 1 & 0 & 4.264960 & -1.977325 & -1.259459 \\
\hline 23 & 1 & 0 & 1.559611 & 1.329227 & -1.699841 \\
\hline 24 & 1 & 0 & 3.228550 & 1.131694 & -1.175408 \\
\hline 25 & 1 & 0 & 2.701813 & 2.275193 & 0.689914 \\
\hline 26 & 1 & 0 & -2.463885 & -2.830557 & 0.445959 \\
\hline 27 & 7 & 0 & 0.413492 & -0.466504 & -0.031890 \\
\hline 28 & 8 & 0 & 3.686914 & 0.392465 & 1.407964 \\
\hline 29 & 8 & 0 & 3.877942 & -1.217203 & -0.813142 \\
\hline 30 & 8 & 0 & 2.023863 & 2.332504 & 0.005808 \\
\hline 31 & 8 & 0 & -1.856435 & -2.092760 & 0.302480 \\
\hline
\end{tabular}

\section{$\underline{\text { Structure of } \mathbf{1 0} \text { (closed form) }}$}

\footnotetext{
Zero-point correction $=0.257494$ (Hartree/Particle)

Thermal correction to Energy $=0.272795$

Thermal correction to Enthalpy $=0.273739$

Thermal correction to Gibbs Free Energy= 0.214729

Sum of electronic and zero-point Energies= -783.510899

Sum of electronic and thermal Energies= -783.495598

Sum of electronic and thermal Enthalpies= -783.494653
} 
Sum of electronic and thermal Free Energies $=-783.553663$ No imaginary frequencies

Standard orientation:

\begin{tabular}{|c|c|c|c|c|c|}
\hline \multirow{2}{*}{$\begin{array}{l}\text { Center } \\
\text { Number }\end{array}$} & \multirow{2}{*}{$\begin{array}{l}\text { Atomic } \\
\text { Number }\end{array}$} & \multirow{2}{*}{$\begin{array}{c}\text { Atomic } \\
\text { Type }\end{array}$} & \multicolumn{3}{|c|}{ Coordinates (Angstroms) } \\
\hline & & & $\mathrm{X}$ & $\mathrm{Y}$ & Z \\
\hline 1 & 6 & 0 & -2.447785 & -0.943067 & 0.161521 \\
\hline 2 & 6 & 0 & -3.829470 & -1.133964 & 0.247025 \\
\hline 3 & 6 & 0 & -4.691199 & -0.060279 & 0.080186 \\
\hline 4 & 6 & 0 & -4.200283 & 1.223431 & -0.173765 \\
\hline 5 & 6 & 0 & -2.831229 & 1.415219 & -0.259251 \\
\hline 6 & 6 & 0 & -1.938389 & 0.348499 & -0.095500 \\
\hline 7 & 6 & 0 & -0.499676 & 0.595331 & -0.200728 \\
\hline 8 & 6 & 0 & 1.786946 & -0.105032 & -0.146390 \\
\hline 9 & 6 & 0 & 2.320873 & -0.166188 & 1.301532 \\
\hline 10 & 6 & 0 & 2.350684 & -1.271156 & -0.963903 \\
\hline 11 & 6 & 0 & 2.197853 & 1.214267 & -0.812864 \\
\hline 12 & 1 & 0 & -4.206371 & -2.131326 & 0.442944 \\
\hline 13 & 1 & 0 & -5.760890 & -0.225380 & 0.148030 \\
\hline 14 & 1 & 0 & -4.881621 & 2.055280 & -0.302040 \\
\hline 15 & 1 & 0 & -2.424975 & 2.402879 & -0.455517 \\
\hline 16 & 1 & 0 & -0.204619 & 1.628759 & -0.400266 \\
\hline 17 & 1 & 0 & 1.696964 & 0.463805 & 1.937891 \\
\hline 18 & 1 & 0 & 2.250847 & -1.196238 & 1.669459 \\
\hline 19 & 1 & 0 & 4.183783 & -0.195111 & 0.803227 \\
\hline 20 & 1 & 0 & 2.072452 & -1.154355 & -2.018185 \\
\hline 21 & 1 & 0 & 1.921726 & -2.203844 & -0.585483 \\
\hline 22 & 1 & 0 & 4.130055 & -2.032056 & -1.295977 \\
\hline 23 & 1 & 0 & 1.577288 & 1.382766 & -1.696589 \\
\hline 24 & 1 & 0 & 3.232621 & 1.123031 & -1.155592 \\
\hline 25 & 1 & 0 & 2.726501 & 2.268370 & 0.713159 \\
\hline 26 & 1 & 0 & -0.704672 & -1.664446 & 0.230881 \\
\hline 27 & 7 & 0 & 0.347811 & -0.349364 & -0.061500 \\
\hline 28 & 8 & 0 & 3.645479 & 0.331861 & 1.408716 \\
\hline 29 & 8 & 0 & 3.769875 & -1.279140 & -0.816071 \\
\hline 30 & 8 & 0 & 2.052228 & 2.348174 & 0.027496 \\
\hline 31 & 8 & 0 & -1.636795 & -1.998264 & 0.325783 \\
\hline
\end{tabular}

\section{$\underline{\text { Structure of } \mathbf{T S}_{\mathbf{1 0 / 4 0}}}$}

Zero-point correction $=0.253404$ (Hartree/Particle)

Thermal correction to Energy $=0.268360$

Thermal correction to Enthalpy $=0.269304$

Thermal correction to Gibbs Free Energy= 0.210979

Sum of electronic and zero-point Energies $=-783.506514$

Sum of electronic and thermal Energies= -783.491559

Sum of electronic and thermal Enthalpies= -783.490614

Sum of electronic and thermal Free Energies= -783.548940

One imaginary frequency: 965i

Standard orientation:

\begin{tabular}{|c|c|c|c|c|c|}
\hline \multirow{2}{*}{$\begin{array}{l}\text { Center } \\
\text { Number }\end{array}$} & \multirow{2}{*}{$\begin{array}{l}\text { Atomic } \\
\text { Number }\end{array}$} & \multirow{2}{*}{$\begin{array}{c}\text { Atomic } \\
\text { Type }\end{array}$} & \multicolumn{3}{|c|}{ Coordinates (Angstroms) } \\
\hline & & & $\mathrm{X}$ & $\mathrm{Y}$ & Z \\
\hline 1 & 6 & 0 & -2.332350 & -0.948288 & 0.163985 \\
\hline 2 & 6 & 0 & -3.721520 & -1.217523 & 0.275711 \\
\hline 3 & 6 & 0 & -4.648032 & -0.208788 & 0.121118 \\
\hline 4 & 6 & 0 & -4.263057 & 1.119865 & -0.150385 \\
\hline 5 & 6 & 0 & -2.922112 & 1.413788 & -0.263244 \\
\hline 6 & 6 & 0 & -1.951249 & 0.405930 & -0.111662 \\
\hline 7 & 6 & 0 & -0.554125 & 0.709544 & -0.230492 \\
\hline 8 & 6 & 0 & 1.760871 & -0.090569 & -0.153639 \\
\hline 9 & 6 & 0 & 2.278636 & -0.174010 & 1.300059 \\
\hline 10 & 6 & 0 & 2.282509 & -1.271675 & -0.977359 \\
\hline 11 & 6 & 0 & 2.207592 & 1.219236 & -0.811146 \\
\hline 12 & 1 & 0 & -4.031054 & -2.235466 & 0.484939 \\
\hline 13 & 1 & 0 & -5.703699 & -0.444860 & 0.210606 \\
\hline 14 & 1 & 0 & -5.012442 & 1.892735 & -0.267688 \\
\hline 15 & 1 & 0 & -2.590614 & 2.427013 & -0.471502 \\
\hline 16 & 1 & 0 & -0.238671 & 1.734028 & -0.425108 \\
\hline
\end{tabular}




$\begin{array}{rrrrrr}17 & 1 & 0 & 1.661452 & 0.464789 & 1.933751 \\ 18 & 1 & 0 & 2.181818 & -1.205155 & 1.658990 \\ 19 & 1 & 0 & 4.147702 & -0.254374 & 0.832838 \\ 20 & 1 & 0 & 2.034829 & -1.124511 & -2.034925 \\ 21 & 1 & 0 & 1.806859 & -2.192884 & -0.625573 \\ 22 & 1 & 0 & 4.039134 & -2.091153 & -1.284522 \\ 23 & 1 & 0 & 1.607753 & 1.398748 & -1.706741 \\ 24 & 1 & 0 & 3.246866 & 1.105868 & -1.131574 \\ 25 & 1 & 0 & 2.731268 & 2.268137 & 0.721461 \\ 26 & 1 & 0 & -0.308973 & -1.226845 & 0.127575 \\ 27 & 7 & 0 & 0.308989 & -0.244748 & -0.097765 \\ 28 & 8 & 0 & 3.610617 & 0.294544 & 1.419466 \\ 29 & 8 & 0 & 3.693431 & -1.339493 & -0.791775 \\ 30 & 8 & 0 & 2.062490 & 2.349970 & 0.030439 \\ 31 & 8 & 0 & -1.436304 & -1.873508 & 0.302389 \\ --------1.304\end{array}$

\section{$\underline{\text { Structure of } 40}$}

Zero-point correction $=0.257667$ (Hartree/Particle) Thermal correction to Energy $=0.272990$

Thermal correction to Enthalpy $=0.273934$

Thermal correction to Gibbs Free Energy= 0.215058

Sum of electronic and zero-point Energies= -783.505038

Sum of electronic and thermal Energies= -783.489714

Sum of electronic and thermal Enthalpies= -783.488770

Sum of electronic and thermal Free Energies $=-783.547646$

No imaginary frequencies

Standard orientation:

\begin{tabular}{|c|c|c|c|c|c|}
\hline \multirow{2}{*}{$\begin{array}{l}\text { Center } \\
\text { Number }\end{array}$} & \multirow{2}{*}{$\begin{array}{l}\text { Atomic } \\
\text { Number }\end{array}$} & \multirow{2}{*}{$\begin{array}{l}\text { Atomic } \\
\text { Type }\end{array}$} & \multicolumn{3}{|c|}{ Coordinates (Angstroms) } \\
\hline & & & $\mathrm{X}$ & Y & $\mathrm{Z}$ \\
\hline 1 & 6 & 0 & -2.422739 & -1.012808 & 0.153328 \\
\hline 2 & 6 & 0 & -3.854469 & -1.157077 & 0.245984 \\
\hline 3 & 6 & 0 & -4.692580 & -0.085021 & 0.104228 \\
\hline 4 & 6 & 0 & -4.204646 & 1.228514 & -0.138073 \\
\hline 5 & 6 & 0 & -2.853870 & 1.420689 & -0.232398 \\
\hline 6 & 6 & 0 & -1.945427 & 0.336491 & -0.094005 \\
\hline 7 & 6 & 0 & -0.565003 & 0.599745 & -0.201693 \\
\hline 8 & 6 & 0 & 1.805019 & -0.118595 & -0.146590 \\
\hline 9 & 6 & 0 & 2.314916 & -0.107442 & 1.311394 \\
\hline 10 & 6 & 0 & 2.380509 & -1.312019 & -0.914413 \\
\hline 11 & 6 & 0 & 2.192352 & 1.178003 & -0.865875 \\
\hline 12 & 1 & 0 & -4.244602 & -2.152555 & 0.429213 \\
\hline 13 & 1 & 0 & -5.764848 & -0.239766 & 0.178371 \\
\hline 14 & 1 & 0 & -4.896234 & 2.054607 & -0.244463 \\
\hline 15 & 1 & 0 & -2.445470 & 2.410888 & -0.415288 \\
\hline 16 & 1 & 0 & -0.235227 & 1.622094 & -0.370244 \\
\hline 17 & 1 & 0 & 1.668392 & 0.538310 & 1.908112 \\
\hline 18 & 1 & 0 & 2.255623 & -1.121596 & 1.723220 \\
\hline 19 & 1 & 0 & 4.192126 & -0.140806 & 0.866482 \\
\hline 20 & 1 & 0 & 2.139521 & -1.219933 & -1.979666 \\
\hline 21 & 1 & 0 & 1.935727 & -2.235602 & -0.528510 \\
\hline 22 & 1 & 0 & 4.168946 & -2.072928 & -1.169668 \\
\hline 23 & 1 & 0 & 1.576917 & 1.291684 & -1.761496 \\
\hline 24 & 1 & 0 & 3.232200 & 1.086777 & -1.190520 \\
\hline 25 & 1 & 0 & 2.691093 & 2. 313227 & 0.612384 \\
\hline 26 & 7 & 0 & 0.355736 & -0.326075 & -0.105507 \\
\hline 27 & 8 & 0 & 3.626144 & 0.419754 & 1.413628 \\
\hline 28 & 8 & 0 & 3.788980 & -1.317317 & -0.708912 \\
\hline 29 & 8 & 0 & 2.014458 & 2.339254 & -0.075399 \\
\hline 30 & 8 & 0 & -1.638214 & -1.992441 & 0.278344 \\
\hline 31 & 1 & 0 & -0.019638 & -1.268762 & 0.088016 \\
\hline
\end{tabular}

\section{$\underline{\text { Structure of } 12 \text { (open form) }}$}

Zero-point correction $=0.290168$ (Hartree/Particle)

Thermal correction to Energy $=0.308343$

Thermal correction to Enthalpy $=0.309287$ 
Thermal correction to Gibbs Free Energy= 0.244019

Sum of electronic and zero-point Energies= -897.973113

Sum of electronic and thermal Energies= -897.954938

Sum of electronic and thermal Enthalpies $=-897.953994$

Sum of electronic and thermal Free Energies= -898.019262

No imaginary frequencies

Standard orientation

\begin{tabular}{|c|c|c|c|c|c|}
\hline \multirow{2}{*}{$\begin{array}{l}\text { Center } \\
\text { Number }\end{array}$} & \multirow{2}{*}{$\begin{array}{l}\text { Atomic } \\
\text { Number }\end{array}$} & \multirow{2}{*}{$\begin{array}{c}\text { Atomic } \\
\text { Type }\end{array}$} & \multicolumn{3}{|c|}{ Coordinates (Angstroms) } \\
\hline & & & $\mathrm{X}$ & $\mathrm{Y}$ & $\mathrm{Z}$ \\
\hline--1 & & & & & --------- \\
\hline 1 & 6 & 0 & 1.678591 & 1.598511 & 0.093695 \\
\hline 2 & 6 & 0 & 3.041981 & 1.843455 & 0.207503 \\
\hline 3 & 6 & 0 & 3.979137 & 0.814340 & 0.149115 \\
\hline 4 & 6 & 0 & 3.547011 & -0.496948 & -0.035682 \\
\hline 5 & 6 & 0 & 2.182699 & -0.744702 & -0.160061 \\
\hline 6 & 6 & 0 & 1.231993 & 0.275026 & -0.088418 \\
\hline 7 & 6 & 0 & -0.176526 & -0.147004 & -0.226554 \\
\hline 8 & 6 & 0 & -2.529436 & 0.026428 & -0.101954 \\
\hline 9 & 6 & 0 & -3.039821 & -0.219454 & 1.334745 \\
\hline 10 & 6 & 0 & -3.352020 & 1.136560 & -0.763792 \\
\hline 11 & 6 & 0 & -2.670329 & -1.252279 & -0.940048 \\
\hline 12 & 1 & 0 & 3.385338 & 2.864882 & 0.343041 \\
\hline 13 & 1 & 0 & 5.029637 & 1.056286 & 0.244274 \\
\hline 14 & 1 & 0 & 1.851355 & -1.767115 & -0.311580 \\
\hline 15 & 1 & 0 & -0.279919 & -1.183352 & -0.563781 \\
\hline 16 & 1 & 0 & -2.282687 & -0.776083 & 1.890044 \\
\hline 17 & 1 & 0 & -3.188839 & 0.743930 & 1.835486 \\
\hline 18 & 1 & 0 & -4.873823 & -0.531965 & 0.828451 \\
\hline 19 & 1 & 0 & -3.074828 & 1.223199 & -1.821483 \\
\hline 20 & 1 & 0 & -3.129866 & 2.081899 & -0.260777 \\
\hline 21 & 1 & 0 & -5.259971 & 1.532127 & -1.011319 \\
\hline 22 & 1 & 0 & -2.044046 & -1.173479 & -1.832282 \\
\hline 23 & 1 & 0 & -3.707390 & -1.333716 & -1.278946 \\
\hline 24 & 1 & 0 & -2.943051 & -2.578976 & 0.431442 \\
\hline 25 & 1 & 0 & 1.259171 & 3.449118 & 0.212713 \\
\hline 26 & 7 & 0 & -1.180780 & 0.580391 & 0.040962 \\
\hline 27 & 8 & 0 & -4.223560 & -1.003694 & 1.365149 \\
\hline 28 & 8 & 0 & -4.738060 & 0.816072 & -0.635190 \\
\hline 29 & 8 & 0 & -2.282515 & -2.434955 & -0.256934 \\
\hline 30 & 8 & 0 & 0.778623 & 2.614931 & 0.137078 \\
\hline 31 & 8 & 0 & 4.367757 & -1.578585 & -0.110511 \\
\hline 32 & 6 & 0 & 5.762343 & -1.344683 & 0.012508 \\
\hline 33 & 1 & 0 & 6.004777 & -0.903701 & 0.984110 \\
\hline 34 & 1 & 0 & 6.241225 & -2.318505 & -0.071921 \\
\hline 35 & 1 & 0 & 6.126684 & -0.692946 & -0.787287 \\
\hline
\end{tabular}

\section{$\underline{\text { Structure of } 12 \text { (closed form) }}$}

Zero-point correction= 0.290457 (Hartree/Particle)

Thermal correction to Energy $=0.308259$

Thermal correction to Enthalpy $=0.309203$

Thermal correction to Gibbs Free Energy= 0.244244

Sum of electronic and zero-point Energies $=-897.988853$

Sum of electronic and thermal Energies= -897.971051

Sum of electronic and thermal Enthalpies= -897.970107

Sum of electronic and thermal Free Energies= -898.035066

No imaginary frequencies

Standard orientation:

\begin{tabular}{|c|c|c|c|c|c|}
\hline \multirow{2}{*}{$\begin{array}{l}\text { Center } \\
\text { Number }\end{array}$} & \multirow{2}{*}{$\begin{array}{l}\text { Atomic } \\
\text { Number }\end{array}$} & \multirow{2}{*}{$\begin{array}{c}\text { Atomic } \\
\text { Type }\end{array}$} & \multicolumn{3}{|c|}{ Coordinates (Angstroms) } \\
\hline & & & $\mathrm{X}$ & $\mathrm{Y}$ & Z \\
\hline 1 & 6 & 0 & 1.553877 & 1.465578 & 0.173623 \\
\hline 2 & 6 & 0 & 2.887150 & 1.846524 & 0.284001 \\
\hline 3 & 6 & 0 & 3.913196 & 0.914176 & 0.163171 \\
\hline 4 & 6 & 0 & 3.616995 & -0.431255 & -0.072044 \\
\hline 5 & 6 & 0 & 2.287350 & -0.819835 & -0.185764 \\
\hline 6 & 6 & 0 & 1.250842 & 0.108029 & -0.066664 \\
\hline 7 & 6 & 0 & -0.132828 & -0.360666 & -0.188878 \\
\hline 8 & 6 & 0 & -2.499284 & -0.014241 & -0.168682 \\
\hline
\end{tabular}




$\begin{array}{rrrrrr}9 & 6 & 0 & -3.048692 & 0.074226 & 1.272246 \\ 10 & 6 & 0 & -3.221381 & 0.991103 & -1.070687 \\ 11 & 6 & 0 & -2.703067 & -1.424624 & -0.734301 \\ 12 & 1 & 0 & 3.118583 & 2.889243 & 0.469438 \\ 13 & 1 & 0 & 4.937133 & 1.252483 & 0.255483 \\ 14 & 1 & 0 & 2.053563 & -1.863777 & -0.369342 \\ 15 & 1 & 0 & -0.261777 & -1.433442 & -0.355810 \\ 16 & 1 & 0 & -2.341484 & -0.405935 & 1.950842 \\ 17 & 1 & 0 & -3.132855 & 1.128112 & 1.561133 \\ 18 & 1 & 0 & -4.895554 & -0.206727 & 0.792360 \\ 19 & 1 & 0 & -2.923898 & 0.833106 & -2.114281 \\ 20 & 1 & 0 & -2.934824 & 2.003317 & -0.769873 \\ 21 & 1 & 0 & -5.091850 & 1.445523 & -1.459285 \\ 22 & 1 & 0 & -2.061108 & -1.561425 & -1.608105 \\ 23 & 1 & 0 & -3.738751 & -1.515157 & -1.074108 \\ 24 & 1 & 0 & -3.073461 & -2.430756 & 0.868063 \\ 25 & 1 & 0 & -0.276386 & 1.934807 & 0.193823 \\ 26 & 7 & 0 & -1.114695 & 0.447806 & -0.092123 \\ 27 & 8 & 0 & -4.284457 & -0.605553 & 1.425706 \\ 28 & 8 & 0 & -4.626992 & 0.802512 & -0.914124 \\ 29 & 8 & 0 & -2.391139 & -2.457736 & 0.186463 \\ 30 & 8 & 0 & 0.591451 & 2.400292 & 0.300498 \\ 31 & 8 & 0 & 4.550879 & -1.413486 & -0.201121 \\ 32 & 6 & 0 & 5.912870 & -1.037366 & -0.067342 \\ 33 & 1 & 0 & 6.110329 & -0.612331 & 0.921545 \\ 34 & 1 & 0 & 6.491825 & -1.950943 & -0.188845 \\ 35 & 1 & 0 & 6.203286 & -0.318866 & -0.839631 \\ -----------------------------------------------1\end{array}$

\section{$\underline{\text { Structure of } \mathbf{T S}_{\underline{12 / 42}}}$}

Zero-point correction $=0.286689$ (Hartree/Particle)

Thermal correction to Energy $=0.303997$

Thermal correction to Enthalpy $=0.304941$

Thermal correction to Gibbs Free Energy $=0.241883$

Sum of electronic and zero-point Energies $=-897.982065$

Sum of electronic and thermal Energies= -897.964758

Sum of electronic and thermal Enthalpies $=-897.963814$

Sum of electronic and thermal Free Energies= -898.026871

One imaginary frequency: 809i

Standard orientation:

\begin{tabular}{|c|c|c|c|c|c|}
\hline \multirow{2}{*}{$\begin{array}{l}\text { Center } \\
\text { Number }\end{array}$} & \multirow{2}{*}{$\begin{array}{l}\text { Atomic } \\
\text { Number }\end{array}$} & \multirow{2}{*}{$\begin{array}{l}\text { Atomic } \\
\text { Type }\end{array}$} & \multicolumn{3}{|c|}{ Coordinates (Angstroms) } \\
\hline & & & $\mathrm{X}$ & Y & Z \\
\hline 1 & 6 & 0 & 1.444878 & 1.403370 & 0.161246 \\
\hline 2 & 6 & 0 & 2.784171 & 1.841153 & 0.301329 \\
\hline 3 & 6 & 0 & 3.847630 & 0.965277 & 0.195853 \\
\hline 4 & 6 & 0 & 3.643115 & -0.407255 & -0.055131 \\
\hline 5 & 6 & 0 & 2.353123 & -0.873773 & -0.192672 \\
\hline 6 & 6 & 0 & 1.259130 & 0.004733 & -0.088494 \\
\hline 7 & 6 & 0 & -0.079866 & -0.491114 & -0.218536 \\
\hline 8 & 6 & 0 & -2.486654 & -0.011691 & -0.170001 \\
\hline 9 & 6 & 0 & -2.997339 & 0.040530 & 1.287430 \\
\hline 10 & 6 & 0 & -3.167632 & 1.064268 & -1.020559 \\
\hline 11 & 6 & 0 & -2.752823 & -1.389151 & -0.786419 \\
\hline 12 & 1 & 0 & 2.964114 & 2.892860 & 0.494919 \\
\hline 13 & 1 & 0 & 4.851688 & 1.355254 & 0.311475 \\
\hline 14 & 1 & 0 & 2.178644 & -1.928461 & -0.382845 \\
\hline 15 & 1 & 0 & -0.253712 & -1.553922 & -0.380371 \\
\hline 16 & 1 & 0 & -2.295516 & -0.499283 & 1.925357 \\
\hline 17 & 1 & 0 & -3.028334 & 1.083860 & 1.621944 \\
\hline 18 & 1 & 0 & -4.868508 & -0.132090 & 0.850750 \\
\hline 19 & 1 & 0 & -2.912051 & 0.918758 & -2.076502 \\
\hline 20 & 1 & 0 & -2.814186 & 2.050095 & -0.701178 \\
\hline 21 & 1 & 0 & -5.021018 & 1.616666 & -1.350800 \\
\hline 22 & 1 & 0 & -2.132694 & -1.512001 & -1.677770 \\
\hline 23 & 1 & 0 & -3.798091 & -1.424358 & -1.104998 \\
\hline 24 & 1 & 0 & -3.131562 & -2.451074 & 0.779566 \\
\hline 25 & 1 & 0 & -0.620043 & 1.386659 & 0.071681 \\
\hline 26 & 7 & 0 & -1.069145 & 0.339232 & -0.128684 \\
\hline 27 & 8 & 0 & -4.255362 & -0.592934 & 1.437912 \\
\hline 28 & 8 & 0 & -4.573172 & 0.951141 & -0.818099 \\
\hline
\end{tabular}




\begin{tabular}{rrrrrr}
29 & 8 & 0 & -2.458908 & -2.464542 & 0.087711 \\
30 & 8 & 0 & 0.433230 & 2.209955 & 0.252946 \\
31 & 8 & 0 & 4.652612 & -1.321970 & -0.170246 \\
32 & 6 & 0 & 5.981922 & -0.846387 & -0.041749 \\
33 & 1 & 0 & 6.157330 & -0.415316 & 0.948941 \\
34 & 1 & 0 & 6.627665 & -1.712704 & -0.174894 \\
35 & 1 & 0 & 6.215169 & -0.102519 & -0.810067 \\
\hline
\end{tabular}

\section{$\underline{\text { Structure of } 42}$}

Zero-point correction $=0.289840$ (Hartree/Particle)
Thermal correction to Energy $=0.307816$
Thermal correction to Enthalpy= 0.308760
Thermal correction to Gibos Free Energy= 0.243719
Sum of electronic and zero-point Energies=-897.980430
Sum of electronic and thermal Energies=-897.962454
Sum of electronic and thermal Enthalpies= -897.961510
Sum of electronic and thermal Free Energies= -898.026551
No imaginary frequencies

Standard orientation:

\begin{tabular}{|c|c|c|c|c|c|}
\hline \multirow{2}{*}{$\begin{array}{l}\text { Center } \\
\text { Number }\end{array}$} & \multirow{2}{*}{$\begin{array}{l}\text { Atomic } \\
\text { Number }\end{array}$} & \multirow{2}{*}{$\begin{array}{c}\text { Atomic } \\
\text { Type }\end{array}$} & \multicolumn{3}{|c|}{ Coordinates (Angstroms) } \\
\hline & & & $\mathrm{x}$ & $\mathrm{Y}$ & Z \\
\hline & & & $5000-7$ & ---------1 & ---------- \\
\hline 1 & 6 & 0 & 1.530847 & 1.540910 & 0.094677 \\
\hline 2 & 6 & 0 & 2.927656 & 1.877984 & 0.146427 \\
\hline 3 & 6 & 0 & 3.913102 & 0.926698 & 0.090021 \\
\hline 4 & 6 & 0 & 3.604311 & -0.456936 & -0.026033 \\
\hline 5 & 6 & 0 & 2.291819 & -0.836247 & -0.088386 \\
\hline 6 & 6 & 0 & 1.246988 & 0.126652 & -0.032731 \\
\hline 7 & 6 & 0 & -0.084982 & -0.325711 & -0.112266 \\
\hline 8 & 6 & 0 & -2.521896 & 0.040711 & -0.124639 \\
\hline 9 & 6 & 0 & -2.973985 & -0.274080 & 1.317394 \\
\hline 10 & 6 & 0 & -3.308086 & 1.221428 & -0.699072 \\
\hline 11 & 6 & 0 & -2.719163 & -1.183631 & -1.032582 \\
\hline 12 & 1 & 0 & 3.187666 & 2.927145 & 0.235484 \\
\hline 13 & 1 & 0 & 4.947148 & 1.248676 & 0.134866 \\
\hline 14 & 1 & 0 & 2.040234 & -1.888443 & -0.181711 \\
\hline 15 & 1 & 0 & -0.280711 & -1.391828 & -0.210813 \\
\hline 16 & 1 & 0 & -2.210034 & -0.886562 & 1.799762 \\
\hline 17 & 1 & 0 & -3.068823 & 0.660778 & 1.881581 \\
\hline 18 & 1 & 0 & -4.846343 & -0.488816 & 0.901998 \\
\hline 19 & 1 & 0 & -3.091937 & 1.327689 & -1.768243 \\
\hline 20 & 1 & 0 & -3.006721 & 2.139117 & -0.182073 \\
\hline 21 & 1 & 0 & -5.205839 & 1.697317 & -0.829315 \\
\hline 22 & 1 & 0 & -2.090285 & -1.073844 & -1.919087 \\
\hline 23 & 1 & 0 & -3.761956 & -1.197759 & -1.360962 \\
\hline 24 & 1 & 0 & -3.044000 & -2.579788 & 0.257672 \\
\hline 25 & 7 & 0 & -1.123432 & 0.473978 & -0.078386 \\
\hline 26 & 8 & 0 & -4.176481 & -1.023903 & 1. 347242 \\
\hline 27 & 8 & 0 & -4.690328 & 0.961695 & -0.482248 \\
\hline 28 & 8 & 0 & -2.374924 & -2.412406 & -0.417924 \\
\hline 29 & 8 & 0 & 0.619031 & 2.415820 & 0.155397 \\
\hline 30 & 1 & 0 & -0.882121 & 1.471480 & 0.044078 \\
\hline 31 & 8 & 0 & 4.554663 & -1.441333 & -0.086500 \\
\hline 32 & 6 & 0 & 5.911821 & -1.051432 & 0.036602 \\
\hline 33 & 1 & 0 & 6.099994 & -0.553934 & 0.993282 \\
\hline 34 & 1 & 0 & 6.494243 & -1.970446 & -0.007401 \\
\hline 35 & 1 & 0 & 6.217232 & -0.395702 & -0.784623 \\
\hline
\end{tabular}

\section{$\underline{\text { Structure of } 13 \text { (open form) }}$}

Zero-point correction $=0.261310$ (Hartree/Particle)

Thermal correction to Energy $=0.278288$

Thermal correction to Enthalpy $=0.279232$

Thermal correction to Gibbs Free Energy $=0.216770$

Sum of electronic and zero-point Energies $=\mathbf{- 8 5 8 . 7 1 6 1 1 3}$

Sum of electronic and thermal Energies= -858.699135

Sum of electronic and thermal Enthalpies= -858.698191 
Sum of electronic and thermal Free Energies $=\mathbf{- 8 5 8 . 7 6 0 6 5 3}$ No imaginary frequencies

Standard orientation:

\begin{tabular}{|c|c|c|c|c|c|}
\hline \multirow{2}{*}{$\begin{array}{l}\text { Center } \\
\text { Number }\end{array}$} & \multirow{2}{*}{$\begin{array}{l}\text { Atomic } \\
\text { Number }\end{array}$} & \multirow{2}{*}{$\begin{array}{c}\text { Atomic } \\
\text { Type }\end{array}$} & \multicolumn{3}{|c|}{ Coordinates (Angstroms) } \\
\hline & & & $\mathrm{X}$ & Y & Z \\
\hline 1 & 6 & 0 & 2.141089 & 1.342999 & 0.175678 \\
\hline 2 & 6 & 0 & 3.522114 & 1.509905 & 0.256159 \\
\hline 3 & 6 & 0 & 4.388830 & 0.434335 & 0.112100 \\
\hline 4 & 6 & 0 & 3.876717 & -0.838803 & -0.117380 \\
\hline 5 & 6 & 0 & 2.503361 & -1.013557 & -0.193844 \\
\hline 6 & 6 & 0 & 1.613940 & 0.058521 & -0.050863 \\
\hline 7 & 6 & 0 & 0.180956 & -0.282814 & -0.152188 \\
\hline 8 & 6 & 0 & -2.157476 & 0.065011 & -0.161240 \\
\hline 9 & 6 & 0 & -2.741306 & 0.221972 & 1.259866 \\
\hline 10 & 6 & 0 & -2.862196 & 1.028749 & -1.121367 \\
\hline 11 & 6 & 0 & -2.368405 & -1.367856 & -0.667206 \\
\hline 12 & 1 & 0 & 3.926530 & 2.501979 & 0.433883 \\
\hline 13 & 1 & 0 & 5.461086 & 0.590797 & 0.177900 \\
\hline 14 & 1 & 0 & 2.108296 & -2.009142 & -0.368571 \\
\hline 15 & 1 & 0 & 0.013368 & -1.354290 & -0.300881 \\
\hline 16 & 1 & 0 & -2.051067 & -0.223043 & 1.978828 \\
\hline 17 & 1 & 0 & -2.833141 & 1.288474 & 1.494454 \\
\hline 18 & 1 & 0 & -4.574210 & -0.088617 & 0.747434 \\
\hline 19 & 1 & 0 & -2.540095 & 0.829261 & -2.150594 \\
\hline 20 & 1 & 0 & -2.584783 & 2.052521 & -0.855232 \\
\hline 21 & 1 & 0 & -4.723799 & 1.480748 & -1.558543 \\
\hline 22 & 1 & 0 & -1.717564 & -1.552236 & -1.525460 \\
\hline 23 & 1 & 0 & -3.400124 & -1.464817 & -1.017739 \\
\hline 24 & 1 & 0 & -2.769817 & -2.294822 & 0.974057 \\
\hline 25 & 1 & 0 & 1.832772 & 3.195757 & 0.468565 \\
\hline 26 & 7 & 0 & -0.776756 & 0.545630 & -0.071088 \\
\hline 27 & 8 & 0 & -3.982467 & -0.449505 & 1.420540 \\
\hline 28 & 8 & 0 & -4.272973 & 0.844685 & -0.994025 \\
\hline 29 & 8 & 0 & -2.081857 & -2.365386 & 0.301431 \\
\hline 30 & 8 & 0 & 1.304378 & 2.402391 & 0.313324 \\
\hline 31 & 8 & 0 & 4.671248 & -1.937623 & -0.272211 \\
\hline 32 & 1 & 0 & 5.597466 & -1.679238 & -0.191240 \\
\hline
\end{tabular}

\section{Structure of $\mathbf{1 3}$ (closed form)}

Zero-point correction $=0.261432$ (Hartree/Particle)

Thermal correction to Energy $=0.278083$

Thermal correction to Enthalpy $=0.279027$

Thermal correction to Gibbs Free Energy= 0.216615

Sum of electronic and zero-point Energies $=-858.732212$

Sum of electronic and thermal Energies= -858.715561

Sum of electronic and thermal Enthalpies $=-858.714617$

Sum of electronic and thermal Free Energies $=-858.777028$

No imaginary frequencies

Standard orientation:

\begin{tabular}{|c|c|c|c|c|c|}
\hline \multirow{2}{*}{$\begin{array}{l}\text { Center } \\
\text { Number }\end{array}$} & \multirow{2}{*}{$\begin{array}{l}\text { Atomic } \\
\text { Number }\end{array}$} & \multirow{2}{*}{$\begin{array}{c}\text { Atomic } \\
\text { Type }\end{array}$} & \multicolumn{3}{|c|}{ Coordinates (Angstroms) } \\
\hline & & & $\mathrm{X}$ & Y & Z \\
\hline 1 & 6 & 0 & -2.004750 & -1.282552 & 0.187314 \\
\hline 2 & 6 & 0 & -3.359074 & -1.595550 & 0.299420 \\
\hline 3 & 6 & 0 & -4.326731 & -0.611684 & 0.155128 \\
\hline 4 & 6 & 0 & -3.958967 & 0.710145 & -0.104480 \\
\hline 5 & 6 & 0 & -2.617468 & 1.034346 & -0.215729 \\
\hline 6 & 6 & 0 & -1.630787 & 0.051082 & -0.073312 \\
\hline 7 & 6 & 0 & -0.223638 & 0.441975 & -0.195173 \\
\hline 8 & 6 & 0 & 2.120904 & -0.034065 & -0.170762 \\
\hline 9 & 6 & 0 & 2.682811 & -0.217272 & 1.257046 \\
\hline 10 & 6 & 0 & 2.774926 & -1.037853 & -1.125155 \\
\hline 11 & 6 & 0 & 2.399317 & 1.385997 & -0.676051 \\
\hline 12 & 1 & 0 & -3.644701 & -2.621271 & 0.502284 \\
\hline 13 & 1 & 0 & -5.377163 & -0.872833 & 0.244658 \\
\hline 14 & 1 & 0 & -2.326744 & 2.060563 & -0.416134 \\
\hline 15 & 1 & 0 & -0.034953 & 1.504333 & -0.367910 \\
\hline
\end{tabular}




\begin{tabular}{|c|c|c|c|c|c|}
\hline 16 & 1 & 0 & 2.006199 & 0.259463 & 1.968414 \\
\hline 17 & 1 & 0 & 2.724168 & -1.286851 & 1.492693 \\
\hline 18 & 1 & 0 & 4.534355 & 0.003017 & 0.766759 \\
\hline 19 & 1 & 0 & 2.476600 & -0.818356 & -2.157142 \\
\hline 20 & 1 & 0 & 2.435186 & -2.044331 & -0.863501 \\
\hline 21 & 1 & 0 & 4.612399 & -1.593068 & -1.539694 \\
\hline 22 & 1 & 0 & 1.773762 & 1.594202 & -1.547469 \\
\hline 23 & 1 & 0 & 3.440889 & 1.438161 & -1.005751 \\
\hline 24 & 1 & 0 & 2.810804 & 2.305753 & 0.967809 \\
\hline 25 & 1 & 0 & -0.202216 & -1.846432 & 0.219931 \\
\hline 26 & 7 & 0 & 0.712700 & -0.418342 & -0.092247 \\
\hline 27 & 8 & 0 & 3.949072 & 0.399376 & 1.425205 \\
\hline 28 & 8 & 0 & 4.190452 & -0.934652 & -0.978149 \\
\hline 29 & 8 & 0 & 2.132041 & 2.392038 & 0.287495 \\
\hline 30 & 8 & 0 & -1.094103 & -2.263869 & 0.334423 \\
\hline 31 & 8 & 0 & -4.876331 & 1.710671 & -0.25563 \\
\hline 32 & 1 & 0 & -5.765801 & 1.350116 & -0.155416 \\
\hline
\end{tabular}

\section{$\underline{\text { Structure of } \mathbf{T S}_{13 / 43}}$}

Zero-point correction= 0.257350 (Hartree/Particle)
Thermal correction to Energy= 0.273685
Thermal correction to Enthalpy= 0.274629
Thermal correction to Gibbs Free Energy= 0.213529
Sum of electronic and zero-point Energies=-858.726284
Sum of electronic and thermal Energies= -858.709949
Sum of electronic and thermal Enthalpies=-858.709005
Sum of electronic and thermal Free Energies=-858.770104
One imaginary frequency: 890i

Standard orientation:

\begin{tabular}{|c|c|c|c|c|c|}
\hline \multirow{2}{*}{$\begin{array}{l}\text { Center } \\
\text { Number }\end{array}$} & \multirow{2}{*}{$\begin{array}{l}\text { Atomic } \\
\text { Number }\end{array}$} & \multirow{2}{*}{$\begin{array}{c}\text { Atomic } \\
\text { Type }\end{array}$} & \multicolumn{3}{|c|}{ Coordinates (Angstroms) } \\
\hline & & & $\mathrm{X}$ & Y & Z \\
\hline 1 & 6 & 0 & 1.893865 & 1.263066 & 0.155803 \\
\hline 2 & 6 & 0 & 3.254811 & 1.644330 & 0.282322 \\
\hline 3 & 6 & 0 & 4.266805 & 0.715365 & 0.175001 \\
\hline 4 & 6 & 0 & 3.993520 & -0.646059 & -0.063053 \\
\hline 5 & 6 & 0 & 2.689671 & -1.059022 & -0.190901 \\
\hline 6 & 6 & 0 & 1.638989 & -0.123727 & -0.084572 \\
\hline 7 & 6 & 0 & 0.276591 & -0.551335 & -0.207607 \\
\hline 8 & 6 & 0 & -2.102202 & 0.038444 & -0.160364 \\
\hline 9 & 6 & 0 & -2.617153 & 0.077765 & 1.295861 \\
\hline 10 & 6 & 0 & -2.733970 & 1.162402 & -0.986341 \\
\hline 11 & 6 & 0 & -2.427276 & -1.310358 & -0.811710 \\
\hline 12 & 1 & 0 & 3.483426 & 2.687965 & 0.466957 \\
\hline 13 & 1 & 0 & 5.301112 & 1.035006 & 0.275177 \\
\hline 14 & 1 & 0 & 2.462700 & -2.104804 & -0.373540 \\
\hline 15 & 1 & 0 & 0.050670 & -1.603823 & -0.372525 \\
\hline 16 & 1 & 0 & -1.943419 & -0.506791 & 1.924516 \\
\hline 17 & 1 & 0 & -2.606856 & 1.113214 & 1.654977 \\
\hline 18 & 1 & 0 & -4.490909 & -0.005949 & 0.843136 \\
\hline 19 & 1 & 0 & -2.478282 & 1.033512 & -2.044503 \\
\hline 20 & 1 & 0 & -2.342359 & 2.124395 & -0.640246 \\
\hline 21 & 1 & 0 & -4.557618 & 1.811801 & -1.300052 \\
\hline 22 & 1 & 0 & -1.808409 & -1.438627 & -1.703285 \\
\hline 23 & 1 & 0 & -3.470979 & -1.290693 & -1.136664 \\
\hline 24 & 1 & 0 & -2.862935 & -2.393363 & 0.724599 \\
\hline 25 & 1 & 0 & -0.159156 & 1.357056 & 0.086401 \\
\hline 26 & 7 & 0 & -0.670346 & 0.326362 & -0.110877 \\
\hline 27 & 8 & 0 & -3.902410 & -0.505045 & 1.424496 \\
\hline 28 & 8 & 0 & -4.144459 & 1.103734 & -0.794635 \\
\hline 29 & 8 & 0 & -2.187318 & -2.419900 & 0.036038 \\
\hline 30 & 8 & 0 & 0.922237 & 2.117710 & 0.252463 \\
\hline 31 & 8 & 0 & 4.995228 & -1.574537 & -0.171141 \\
\hline 32 & 1 & 0 & 5.847311 & -1.144348 & -0.033211 \\
\hline
\end{tabular}




\section{$\underline{\text { Structure of } 43}$}

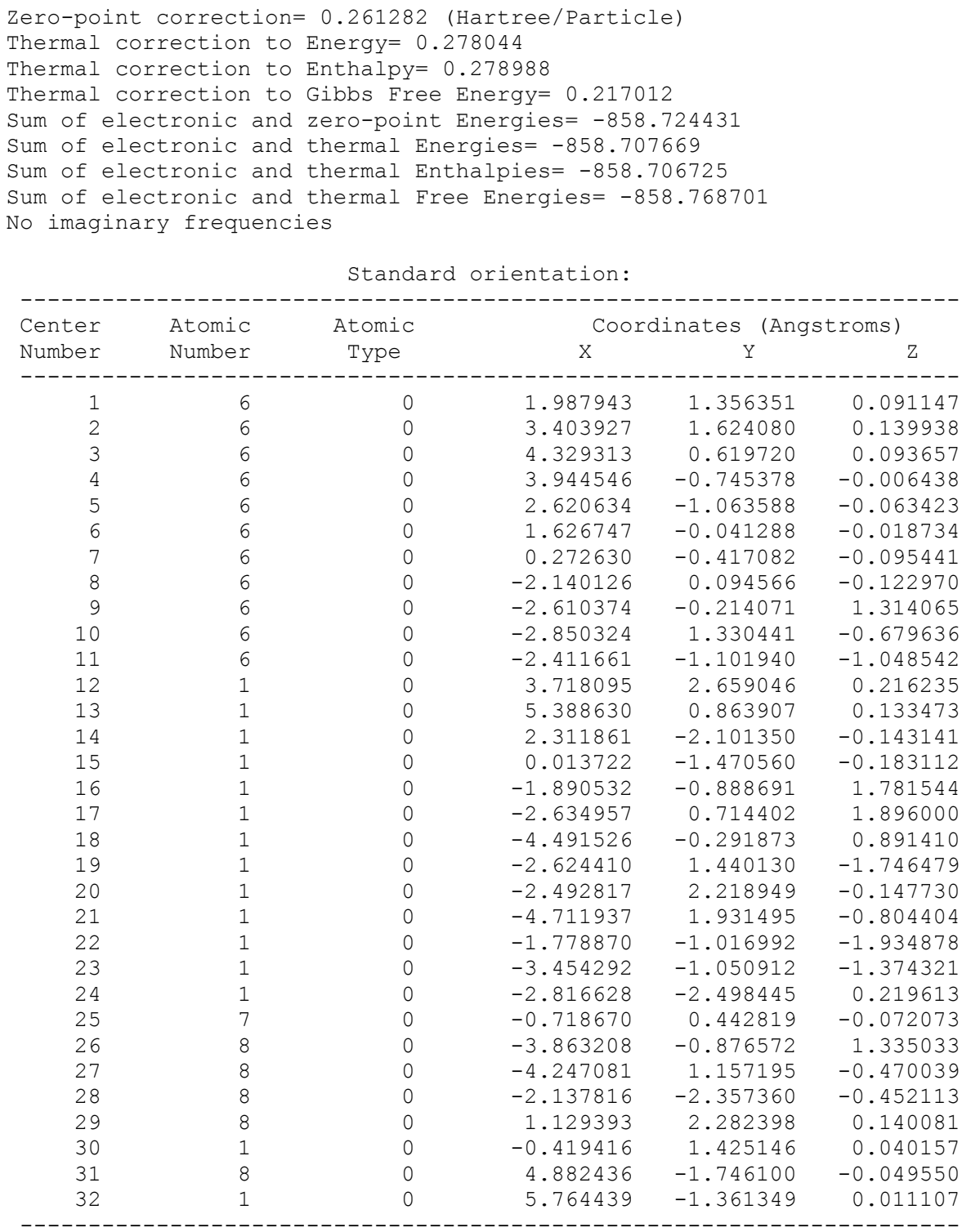

\section{$\underline{\text { Structure of } 14 \text { (open form) }}$}

Zero-point correction $=0.260314$ (Hartree/Particle)

Thermal correction to Energy $=0.278537$

Thermal correction to Enthalpy $=0.279481$

Thermal correction to Gibbs Free Energy= 0.213034

Sum of electronic and zero-point Energies $=-987.982717$

Sum of electronic and thermal Energies= -987.964495

Sum of electronic and thermal Enthalpies= -987.963551

Sum of electronic and thermal Free Energies= -988.029997

No imaginary frequencies

Standard orientation:

\begin{tabular}{|c|c|c|c|c|c|}
\hline \multirow{2}{*}{$\begin{array}{l}\text { Center } \\
\text { Number }\end{array}$} & \multirow{2}{*}{$\begin{array}{l}\text { Atomic } \\
\text { Number }\end{array}$} & \multirow{2}{*}{$\begin{array}{c}\text { Atomic } \\
\text { Type }\end{array}$} & \multicolumn{3}{|c|}{ Coordinates (Angstroms) } \\
\hline & & & $\mathrm{X}$ & $\mathrm{Y}$ & Z \\
\hline 1 & 6 & 0 & -1.542612 & -0.986564 & 0.053203 \\
\hline 2 & 6 & 0 & -2.933185 & -1.042806 & 0.125262 \\
\hline 3 & 6 & 0 & -3.659375 & 0.128595 & -0.000478 \\
\hline 4 & 6 & 0 & -3.070603 & 1.366090 & -0.199468 \\
\hline 5 & 6 & 0 & -1.687250 & 1.400955 & -0.267979 \\
\hline 6 & 6 & 0 & -0.898296 & 0.252729 & -0.140650 \\
\hline
\end{tabular}




\begin{tabular}{|c|c|c|c|c|c|}
\hline 7 & 6 & 0 & 0.563407 & 0.451740 & -0.234309 \\
\hline 8 & 6 & 0 & 2.851764 & -0.126858 & -0.115913 \\
\hline 9 & 6 & 0 & 3.373362 & -0.117936 & 1.338218 \\
\hline 10 & 6 & 0 & 3.478306 & -1.295462 & -0.884134 \\
\hline 11 & 6 & 0 & 3.221604 & 1.184180 & -0.822559 \\
\hline 12 & 1 & 0 & -3.436828 & -1.990774 & 0.275413 \\
\hline 13 & 1 & 0 & -3.666575 & 2.262284 & -0.296408 \\
\hline 14 & 1 & 0 & -1.190873 & 2.352173 & -0.425400 \\
\hline 15 & 1 & 0 & 0.837520 & 1.478865 & -0.493256 \\
\hline 16 & 1 & 0 & 2.719114 & 0.507200 & 1.948735 \\
\hline 17 & 1 & 0 & 3.340293 & -1.137000 & 1.739422 \\
\hline 18 & 1 & 0 & 5.240526 & -0.084638 & 0.855181 \\
\hline 19 & 1 & 0 & 3.196350 & -1.238650 & -1.942501 \\
\hline 20 & 1 & 0 & 3.096166 & -2.231045 & -0.466514 \\
\hline 21 & 1 & 0 & 5.290552 & -1.995584 & -1.165002 \\
\hline 22 & 1 & 0 & 2.609242 & 1.299420 & -1.720683 \\
\hline 23 & 1 & 0 & 4.263904 & 1.121604 & -1.148564 \\
\hline 24 & 1 & 0 & 3.687060 & 2.305819 & 0.674873 \\
\hline 25 & 1 & 0 & -1.397927 & -2.869318 & 0.267299 \\
\hline 26 & 7 & 0 & 1.427061 & -0.450984 & -0.021082 \\
\hline 27 & 8 & 0 & 4.676538 & 0.436015 & 1.442631 \\
\hline 28 & 8 & 0 & 4.897228 & -1.226753 & -0.739431 \\
\hline 29 & 8 & 0 & 3.023138 & 2.341046 & -0.024325 \\
\hline 30 & 8 & 0 & -0.808661 & -2.110517 & 0.157271 \\
\hline 31 & 7 & 0 & -5.130089 & 0.046183 & 0.077449 \\
\hline 32 & 8 & 0 & -5.762518 & 1.074577 & -0.050087 \\
\hline 33 & 8 & 0 & -5.631983 & -1.043708 & 0.263400 \\
\hline
\end{tabular}

\title{
$\underline{\text { Structure of } 14 \text { (closed form) }}$
}

\begin{abstract}
Zero-point correction $=0.260071$ (Hartree/Particle)
Thermal correction to Energy $=0.278008$

Thermal correction to Enthalpy $=0.278952$

Thermal correction to Gibbs Free Energy= 0.212634

Sum of electronic and zero-point Energies $=-987.999963$

Sum of electronic and thermal Energies= -987.982027

Sum of electronic and thermal Enthalpies= -987.981083

Sum of electronic and thermal Free Energies= -988.047401

No imaginary frequencies
\end{abstract}

Standard orientation:

\begin{tabular}{|c|c|c|c|c|c|}
\hline \multirow{2}{*}{$\begin{array}{l}\text { Center } \\
\text { Number }\end{array}$} & \multirow{2}{*}{$\begin{array}{l}\text { Atomic } \\
\text { Number }\end{array}$} & \multirow{2}{*}{$\begin{array}{l}\text { Atomic } \\
\text { Type }\end{array}$} & \multicolumn{3}{|c|}{ Coordinates (Angstroms) } \\
\hline & & & $\mathrm{X}$ & Y & Z \\
\hline 1 & 6 & 0 & -1.448350 & -0.853628 & 0.084282 \\
\hline 2 & 6 & 0 & -2.831839 & -1.024273 & 0.149129 \\
\hline 3 & 6 & 0 & -3.638347 & 0.088144 & 0.000024 \\
\hline 4 & 6 & 0 & -3.152877 & 1.372843 & -0.213552 \\
\hline 5 & 6 & 0 & -1.778789 & 1.526156 & -0.279144 \\
\hline 6 & 6 & 0 & -0.915509 & 0.436761 & -0.133900 \\
\hline 7 & 6 & 0 & 0.533110 & 0.655084 & -0.217399 \\
\hline 8 & 6 & 0 & 2.793258 & -0.120894 & -0.141141 \\
\hline 9 & 6 & 0 & 3.296417 & -0.204469 & 1.317943 \\
\hline 10 & 6 & 0 & 3.335092 & -1.301684 & -0.952512 \\
\hline 11 & 6 & 0 & 3.254912 & 1.188369 & -0.793438 \\
\hline 12 & 1 & 0 & -3.249595 & -2.008669 & 0.312851 \\
\hline 13 & 1 & 0 & -3.825823 & 2.210753 & -0.324856 \\
\hline 14 & 1 & 0 & -1.352750 & 2.509680 & -0.446327 \\
\hline 15 & 1 & 0 & 0.856659 & 1.683735 & -0.392886 \\
\hline 16 & 1 & 0 & 2.676522 & 0.437408 & 1.946237 \\
\hline 17 & 1 & 0 & 3.192937 & -1.234678 & 1.676848 \\
\hline 18 & 1 & 0 & 5.167328 & -0.283384 & 0.858217 \\
\hline 19 & 1 & 0 & 3.086142 & -1.169753 & -2.012183 \\
\hline 20 & 1 & 0 & 2.867735 & -2.222056 & -0.589548 \\
\hline 21 & 1 & 0 & 5.099453 & -2.108607 & -1.255096 \\
\hline 22 & 1 & 0 & 2.656519 & 1.379278 & -1.687824 \\
\hline 23 & 1 & 0 & 4.292782 & 1.067011 & -1.116929 \\
\hline 24 & 1 & 0 & 3.786155 & 2.220091 & 0.747202 \\
\hline 25 & 1 & 0 & 0.277382 & -1.606121 & 0.157189 \\
\hline 26 & 7 & 0 & 1.345923 & -0.317749 & -0.083666 \\
\hline 27 & 8 & 0 & 4.630026 & 0.259233 & 1.450705 \\
\hline 28 & 8 & 0 & 4.748724 & -1.354136 & -0.770569 \\
\hline
\end{tabular}




$\begin{array}{rrrrrr}29 & 8 & 0 & 3.126338 & 2.321365 & 0.050243 \\ 30 & 8 & 0 & -0.665611 & -1.925069 & 0.230349 \\ 31 & 7 & 0 & -5.101450 & -0.106643 & 0.072383 \\ 32 & 8 & 0 & -5.808456 & 0.877942 & 0.006017 \\ 33 & 8 & 0 & -5.521673 & -1.239136 & 0.193799 \\ -\end{array}$

\section{Structure of $\mathbf{T S}_{\mathbf{1} 1 / 44}$}

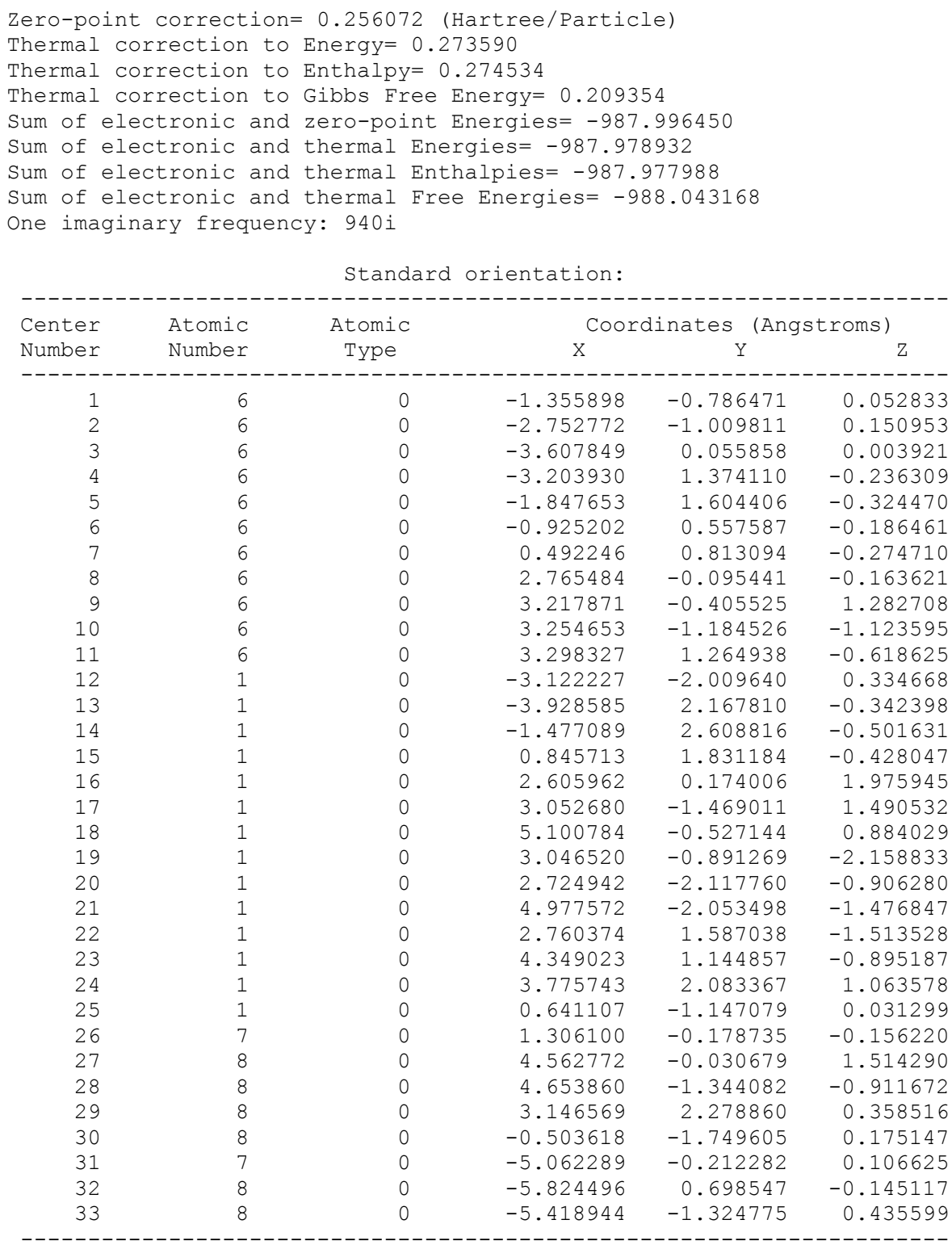

\section{$\underline{\text { Structure of } 44}$}

Zero-point correction $=0.260121$ (Hartree/Particle)

Thermal correction to Energy $=0.278134$

Thermal correction to Enthalpy $=0.279078$

Thermal correction to Gibbs Free Energy= 0.212604

Sum of electronic and zero-point Energies= -987.995493

Sum of electronic and thermal Energies= -987.977480

Sum of electronic and thermal Enthalpies= -987.976536

Sum of electronic and thermal Free Energies= -988.043010

No imaginary frequencies 


\begin{tabular}{|c|c|c|c|c|c|}
\hline \multirow{2}{*}{$\begin{array}{l}\text { Center } \\
\text { Number }\end{array}$} & \multirow{2}{*}{$\begin{array}{l}\text { Atomic } \\
\text { Number }\end{array}$} & \multirow{2}{*}{$\begin{array}{c}\text { Atomic } \\
\text { Type }\end{array}$} & \multicolumn{3}{|c|}{ Coordinates (Angstroms) } \\
\hline & & & $\mathrm{X}$ & Y & Z \\
\hline 1 & 6 & 0 & -1.430256 & -0.925784 & 0.089588 \\
\hline 2 & 6 & 0 & -2.867958 & -1.041045 & 0.150040 \\
\hline 3 & 6 & 0 & -3.642905 & 0.070407 & 0.001442 \\
\hline 4 & 6 & 0 & -3.152392 & 1.376070 & -0.214208 \\
\hline 5 & 6 & 0 & -1.792061 & 1.517315 & -0.281701 \\
\hline 6 & 6 & 0 & -0.921423 & 0.411252 & -0.136306 \\
\hline 7 & 6 & 0 & 0.474533 & 0.641300 & -0.219144 \\
\hline 8 & 6 & 0 & 2.819025 & -0.137810 & -0.134645 \\
\hline 9 & 6 & 0 & 3.310481 & -0.133349 & 1.330734 \\
\hline 10 & 6 & 0 & 3.370826 & -1.349527 & -0.891120 \\
\hline 11 & 6 & 0 & 3.245273 & 1.146118 & -0.854390 \\
\hline 12 & 1 & 0 & -3.305288 & -2.017126 & 0.313919 \\
\hline 13 & 1 & 0 & -3.827220 & 2.211587 & -0.323592 \\
\hline 14 & 1 & 0 & -1.357360 & 2.497607 & -0.450821 \\
\hline 15 & 1 & 0 & 0.831240 & 1.655714 & -0.378875 \\
\hline 16 & 1 & 0 & 2.670215 & 0.527462 & 1.917550 \\
\hline 17 & 1 & 0 & 3.226434 & -1.144656 & 1.744795 \\
\hline 18 & 1 & 0 & 5.192068 & -0.211829 & 0.912943 \\
\hline 19 & 1 & 0 & 3.150095 & -1.252632 & -1.960250 \\
\hline 20 & 1 & 0 & 2.894029 & -2.259346 & -0.510667 \\
\hline 21 & 1 & 0 & 5.144761 & -2.150267 & -1.123644 \\
\hline 22 & 1 & 0 & 2.645473 & 1.269234 & -1.759441 \\
\hline 23 & 1 & 0 & 4.287065 & 1.028231 & -1.163667 \\
\hline 24 & 1 & 0 & 3.751405 & 2.279333 & 0.623440 \\
\hline 25 & 7 & 0 & 1.362492 & -0.306546 & -0.108299 \\
\hline 26 & 8 & 0 & 4.629828 & 0.368037 & 1.443558 \\
\hline 27 & 8 & 0 & 4.774179 & -1.391176 & -0.660886 \\
\hline 28 & 8 & 0 & 3.082200 & 2.313878 & -0.071228 \\
\hline 29 & 8 & 0 & -0.682643 & -1.927268 & 0.224456 \\
\hline 30 & 1 & 0 & 0.963429 & -1.242005 & 0.073563 \\
\hline 31 & 7 & 0 & -5.116215 & -0.104070 & 0.079410 \\
\hline 32 & 8 & 0 & -5.562280 & -1.232329 & 0.097611 \\
\hline 33 & 8 & 0 & -5.799928 & 0.898024 & 0.122143 \\
\hline
\end{tabular}

\section{$\underline{\text { Structure of } 15 \text { (open form) }}$}

Zero-point correction= 0.249298 (Hartree/Particle) Thermal correction to Energy $=0.265844$

Thermal correction to Enthalpy $=0.266788$

Thermal correction to Gibbs Free Energy $=0.204006$

Sum of electronic and zero-point Energies $=\mathbf{- 8 8 2 . 7 4 4 0 9 6}$

Sum of electronic and thermal Energies $=-882.727550$

Sum of electronic and thermal Enthalpies $=-882.726606$

Sum of electronic and thermal Free Energies $=-882.789388$

No imaginary frequencies

Standard orientation:

\begin{tabular}{|c|c|c|c|c|c|}
\hline \multirow{2}{*}{$\begin{array}{l}\text { Center } \\
\text { Number }\end{array}$} & \multirow{2}{*}{$\begin{array}{l}\text { Atomic } \\
\text { Number }\end{array}$} & \multirow{2}{*}{$\begin{array}{c}\text { Atomic } \\
\text { Type }\end{array}$} & \multicolumn{3}{|c|}{ Coordinates (Angstroms) } \\
\hline & & & $\mathrm{X}$ & Y & $\mathrm{Z}$ \\
\hline 1 & 6 & 0 & -2.193945 & -0.976611 & 0.132114 \\
\hline 2 & 6 & 0 & -3.587160 & -1.025170 & 0.191326 \\
\hline 3 & 6 & 0 & -4.304330 & 0.141199 & 0.020514 \\
\hline 4 & 6 & 0 & -3.712105 & 1.368546 & -0.209656 \\
\hline 5 & 6 & 0 & -2.326914 & 1.394734 & -0.266045 \\
\hline 6 & 6 & 0 & -1.537667 & 0.249721 & -0.100013 \\
\hline 7 & 6 & 0 & -0.078640 & 0.441929 & -0.191946 \\
\hline 8 & 6 & 0 & 2.213895 & -0.131759 & -0.134312 \\
\hline 9 & 6 & 0 & 2.749333 & -0.151493 & 1.313504 \\
\hline 10 & 6 & 0 & 2.842302 & -1.276262 & -0.935955 \\
\hline 11 & 6 & 0 & 2.570082 & 1.197989 & -0.814425 \\
\hline 12 & 1 & 0 & -4.107227 & -1.960797 & 0.368071 \\
\hline 13 & 1 & 0 & -4.313937 & 2.258765 & -0.338353 \\
\hline 14 & 1 & 0 & -1.826197 & 2.340204 & -0.446086 \\
\hline 15 & 1 & 0 & 0.197670 & 1.479939 & -0.404196 \\
\hline 16 & 1 & 0 & 2.100665 & 0.462162 & 1.941386 \\
\hline 17 & 1 & 0 & 2.719128 & -1.178099 & 1.695707 \\
\hline 18 & 1 & 0 & 4.611448 & -0.105749 & 0.811740 \\
\hline
\end{tabular}




\begin{tabular}{rrrrrr}
19 & 1 & 0 & 2.548115 & -1.197093 & -1.989684 \\
20 & 1 & 0 & 2.471016 & -2.223950 & -0.536196 \\
21 & 1 & 0 & 4.654944 & -1.961520 & -1.250919 \\
22 & 1 & 0 & 1.938437 & 1.335596 & -1.695798 \\
23 & 1 & 0 & 3.605543 & 1.144111 & -1.163389 \\
24 & 1 & 0 & 3.063872 & 2.280018 & 0.702606 \\
25 & 1 & 0 & -2.068637 & -2.847932 & 0.434236 \\
26 & 7 & 0 & 0.791694 & -0.468940 & -0.038857 \\
27 & 8 & 0 & 4.054731 & 0.399306 & 1.419258 \\
28 & 8 & 0 & 4.262992 & -1.202961 & -0.806200 \\
29 & 8 & 0 & 2.389295 & 2.336120 & 0.015061 \\
30 & 8 & 0 & -1.470016 & -2.102282 & 0.292579 \\
31 & 9 & 0 & -5.649089 & 0.068726 & 0.082963 \\
\hline
\end{tabular}

\section{$\underline{\text { Structure of } 15 \text { (closed form) }}$}

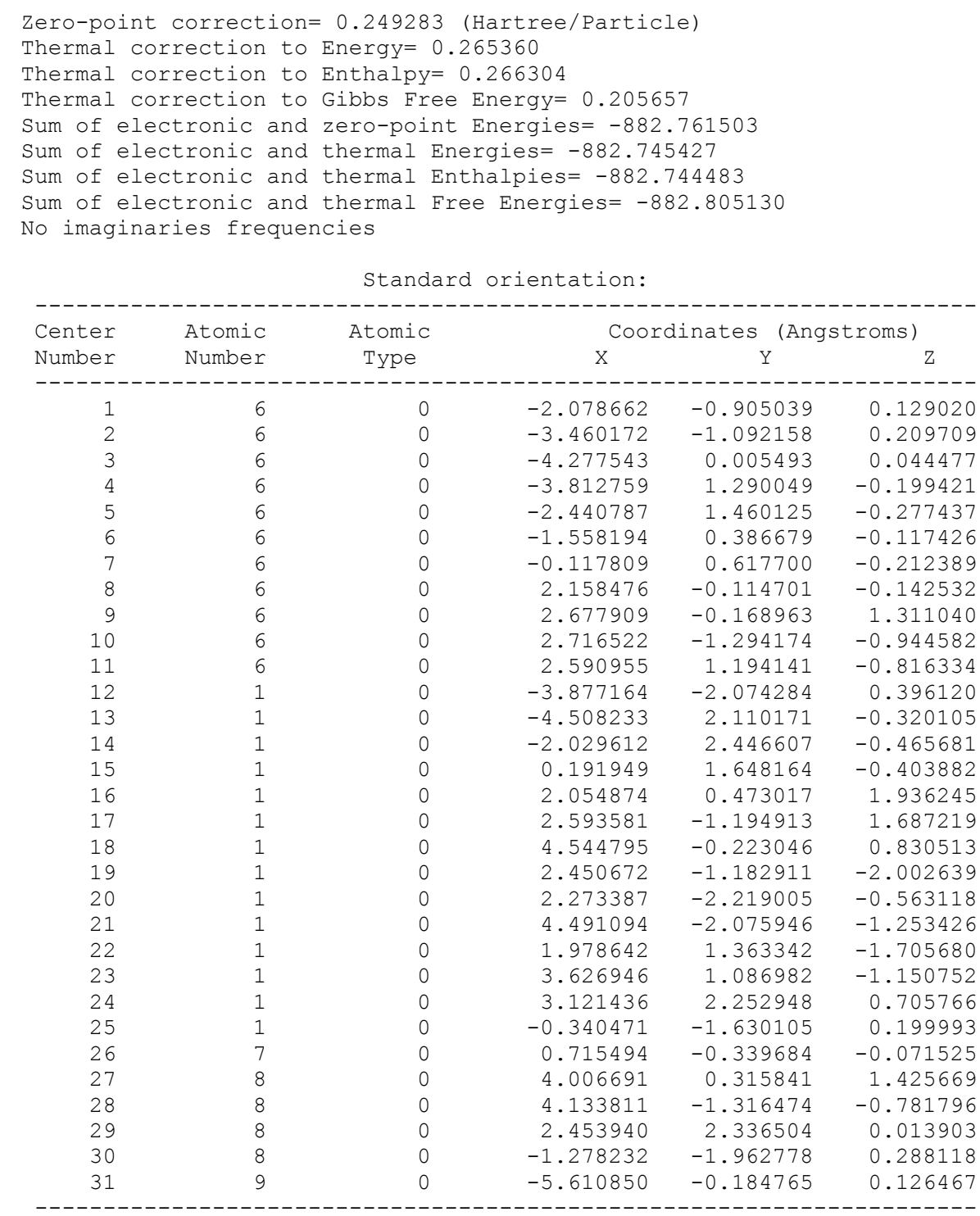

\section{$\underline{\text { Structure of } \mathbf{T S}_{15 / 45}}$}

Zero-point correction= 0.245102 (Hartree/Particle) Thermal correction to Energy $=0.260864$

Thermal correction to Enthalpy $=0.261808$

Thermal correction to Gibbs Free Energy= 0.201919

Sum of electronic and zero-point Energies $=\mathbf{- 8 8 2 . 7 5 8 7 6 8}$ 
Sum of electronic and thermal Energies= -882.743006

Sum of electronic and thermal Enthalpies= -882.742062

Sum of electronic and thermal Free Energies= -882.801951

One imaginary frequency: $987 i$

Standard orientation:

\begin{tabular}{|c|c|c|c|c|c|}
\hline \multirow{2}{*}{$\begin{array}{l}\text { Center } \\
\text { Number }\end{array}$} & \multirow{2}{*}{$\begin{array}{l}\text { Atomic } \\
\text { Number }\end{array}$} & \multirow{2}{*}{$\begin{array}{c}\text { Atomic } \\
\text { Type }\end{array}$} & \multicolumn{3}{|c|}{ Coordinates (Angstroms) } \\
\hline & & & $\mathrm{X}$ & $\mathrm{Y}$ & Z \\
\hline 1 & 6 & 0 & -1 981271 & -0.877443 & 0.123699 \\
\hline 2 & 6 & 0 & -3.371181 & -1.127274 & 0.226180 \\
\hline 3 & 6 & 0 & -4.242223 & -0.080446 & 0.067323 \\
\hline 4 & 6 & 0 & -3.865490 & 1.240460 & -0.192915 \\
\hline 5 & 6 & 0 & -2.515022 & 1.496595 & -0.293869 \\
\hline 6 & 6 & 0 & -1.568462 & 0.470763 & -0.141731 \\
\hline 7 & 6 & 0 & -0.162415 & 0.743617 & -0.246407 \\
\hline 8 & 6 & 0 & 2.130034 & -0.104613 & -0.150749 \\
\hline 9 & 6 & 0 & 2.629706 & -0.204215 & 1.308534 \\
\hline 10 & 6 & 0 & 2.637519 & -1.292813 & -0.972965 \\
\hline 11 & 6 & 0 & 2.611143 & 1.198586 & -0.797082 \\
\hline 12 & 1 & 0 & -3.735045 & -2.127600 & 0.426207 \\
\hline 13 & 1 & 0 & -4.617603 & 2.010003 & -0.305988 \\
\hline 14 & 1 & 0 & -2.165624 & 2.504704 & -0.493472 \\
\hline 15 & 1 & 0 & 0.175711 & 1.762646 & -0.431723 \\
\hline 16 & 1 & 0 & 2.017463 & 0.443133 & 1.938377 \\
\hline 17 & 1 & 0 & 2.508988 & -1.234945 & 1.661177 \\
\hline 18 & 1 & 0 & 4.502319 & -0.319038 & 0.863538 \\
\hline 19 & 1 & 0 & 2.405509 & -1.136815 & -2.032785 \\
\hline 20 & 1 & 0 & 2.139057 & -2.205399 & -0.630386 \\
\hline 21 & 1 & 0 & 4.381227 & -2.145319 & -1.264246 \\
\hline 22 & 1 & 0 & 2.025690 & 1.395593 & -1.698509 \\
\hline 23 & 1 & 0 & 3.651440 & 1.064643 & -1.106118 \\
\hline 24 & 1 & 0 & 3.138873 & 2.228209 & 0.747134 \\
\hline 25 & 1 & 0 & 0.013960 & -1.210780 & 0.105497 \\
\hline 26 & 7 & 0 & 0.674945 & -0.230970 & -0.110419 \\
\hline 27 & 8 & 0 & 3.968788 & 0.238416 & 1.445296 \\
\hline 28 & 8 & 0 & 4.044500 & -1.389924 & -0.770993 \\
\hline 29 & 8 & 0 & 2.480314 & 2.328100 & 0.048654 \\
\hline 30 & 8 & 0 & -1.105559 & -1.820601 & 0.263179 \\
\hline 31 & 9 & 0 & -5.564994 & -0.337720 & 0.169448 \\
\hline
\end{tabular}

\section{Structure of 45}

Zero-point correction $=0.249568$ (Hartree/Particle)

Thermal correction to Energy $=0.265727$

Thermal correction to Enthalpy $=0.266671$

Thermal correction to Gibbs Free Energy= 0.205815

Sum of electronic and zero-point Energies= -882.758154

Sum of electronic and thermal Energies= -882.741996

Sum of electronic and thermal Enthalpies= -882.741051

Sum of electronic and thermal Free Energies= -882.801908

No imaginary frequencies

Standard orientation:

\begin{tabular}{|c|c|c|c|c|c|}
\hline \multirow{2}{*}{$\begin{array}{l}\text { Center } \\
\text { Number }\end{array}$} & \multirow{2}{*}{$\begin{array}{l}\text { Atomic } \\
\text { Number }\end{array}$} & \multirow{2}{*}{$\begin{array}{c}\text { Atomic } \\
\text { Type }\end{array}$} & \multicolumn{3}{|c|}{ Coordinates (Angstroms) } \\
\hline & & & $\mathrm{X}$ & $\mathrm{Y}$ & Z \\
\hline 1 & 6 & 0 & -2.067053 & -0.986143 & 0.121670 \\
\hline 2 & 6 & 0 & -3.499247 & -1.112435 & 0.204040 \\
\hline 3 & 6 & 0 & -4.281783 & -0.009601 & 0.057952 \\
\hline 4 & 6 & 0 & -3.807603 & 1.299048 & -0.173720 \\
\hline 5 & 6 & 0 & -2.451467 & 1.457481 & -0.255424 \\
\hline 6 & 6 & 0 & -1.564806 & 0.359295 & -0.115719 \\
\hline 7 & 6 & 0 & -0.180701 & 0.603992 & -0.211265 \\
\hline 8 & 6 & 0 & 2.182685 & -0.129216 & -0.142165 \\
\hline 9 & 6 & 0 & 2.680282 & -0.113202 & 1.320123 \\
\hline 10 & 6 & 0 & 2.759006 & -1.328476 & -0.900030 \\
\hline 11 & 6 & 0 & 2.581590 & 1.162392 & -0.864476 \\
\hline 12 & 1 & 0 & -3.940897 & -2.086299 & 0.378116 \\
\hline 13 & 1 & 0 & -4.505405 & 2.119052 & -0.277573 \\
\hline 14 & 1 & 0 & -2.028652 & 2.442154 & -0.430318 \\
\hline
\end{tabular}




$\begin{array}{rrrrrr}15 & 1 & 0 & 0.160713 & 1.623530 & -0.372160 \\ 16 & 1 & 0 & 2.030833 & 0.537455 & 1.908322 \\ 17 & 1 & 0 & 2.614424 & -1.125169 & 1.736262 \\ 18 & 1 & 0 & 4.561350 & -0.156269 & 0.893133 \\ 19 & 1 & 0 & 2.528547 & -1.239313 & -1.967853 \\ 20 & 1 & 0 & 2.306385 & -2.248459 & -0.514705 \\ 21 & 1 & 0 & 4.546259 & -2.098992 & -1.133434 \\ 22 & 1 & 0 & 1.972891 & 1.275302 & -1.764822 \\ 23 & 1 & 0 & 3.623104 & 1.064018 & -1.181573 \\ 24 & 1 & 0 & 3.076641 & 2.301608 & 0.612049 \\ 25 & 7 & 0 & 0.731389 & -0.329427 & -0.112629 \\ 26 & 8 & 0 & 3.992109 & 0.410438 & 1.430419 \\ 27 & 8 & 0 & 4.165232 & -1.338997 & -0.680845 \\ 28 & 8 & 0 & 2.404605 & 2.327963 & -0.080238 \\ 29 & 8 & 0 & -1.302015 & -1.976067 & 0.247249 \\ 30 & 1 & 0 & 0.354916 & -1.270629 & 0.073821 \\ 31 & 9 & 0 & -5.622854 & -0.159723 & 0.138949 \\ -----------------------------------------------------\end{array}$

\section{$\underline{\text { Structure of } 16 \text { (open form) }}$}

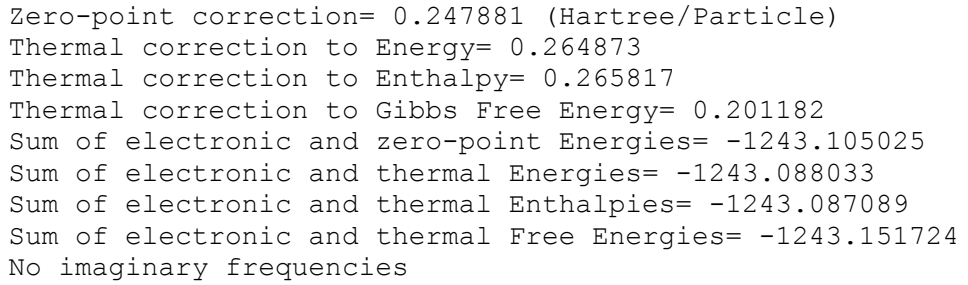

Standard orientation:

\begin{tabular}{|c|c|c|c|c|c|}
\hline \multirow{2}{*}{$\begin{array}{l}\text { Center } \\
\text { Number }\end{array}$} & \multirow{2}{*}{$\begin{array}{l}\text { Atomic } \\
\text { Number }\end{array}$} & \multirow{2}{*}{$\begin{array}{c}\text { Atomic } \\
\text { Type }\end{array}$} & \multicolumn{3}{|c|}{ Coordinates (Angstroms) } \\
\hline & & & $\mathrm{X}$ & $\mathrm{Y}$ & Z \\
\hline & & & 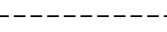 & -ー-ー---ー-ー & ---------- \\
\hline 1 & 6 & 0 & -1.808418 & -0.979011 & 0.121153 \\
\hline 2 & 6 & 0 & -3.202745 & -1.029207 & 0.172943 \\
\hline 3 & 6 & 0 & -3.935434 & 0.133017 & 0.000146 \\
\hline 4 & 6 & 0 & -3.327709 & 1.359760 & -0.225186 \\
\hline 5 & 6 & 0 & -1.942616 & 1.389546 & -0.275088 \\
\hline 6 & 6 & 0 & -1.152423 & 0.246706 & -0.106796 \\
\hline 7 & 6 & 0 & 0.307239 & 0.441420 & -0.195085 \\
\hline 8 & 6 & 0 & 2.599203 & -0.131016 & -0.131334 \\
\hline 9 & 6 & 0 & 3.131318 & -0.147126 & 1.317952 \\
\hline 10 & 6 & 0 & 3.230164 & -1.277062 & -0.928804 \\
\hline 11 & 6 & 0 & 2.955267 & 1.197604 & -0.813670 \\
\hline 12 & 1 & 0 & -3.702915 & -1.976224 & 0.346693 \\
\hline 13 & 1 & 0 & -3.914424 & 2.259080 & -0.358497 \\
\hline 14 & 1 & 0 & -1.444454 & 2.337092 & -0.452292 \\
\hline 15 & 1 & 0 & 0.582551 & 1.479349 & -0.408085 \\
\hline 16 & 1 & 0 & 2.480354 & 0.466563 & 1.943417 \\
\hline 17 & 1 & 0 & 3.102211 & -1.173072 & 1.701900 \\
\hline 18 & 1 & 0 & 4.994509 & -0.099306 & 0.820128 \\
\hline 19 & 1 & 0 & 2.937930 & -1.201020 & -1.983277 \\
\hline 20 & 1 & 0 & 2.859269 & -2.224121 & -0.527191 \\
\hline 21 & 1 & 0 & 5.044494 & -1.959648 & -1.239393 \\
\hline 22 & 1 & 0 & 2.325857 & 1.332099 & -1.697142 \\
\hline 23 & 1 & 0 & 3.991722 & 1.144287 & -1.159631 \\
\hline 24 & 1 & 0 & 3. 443559 & 2.284356 & 0.701856 \\
\hline 25 & 1 & 0 & -1.682521 & -2.851040 & 0.423294 \\
\hline 26 & 7 & 0 & 1.176969 & -0.468890 & -0.038071 \\
\hline 27 & 8 & 0 & 4.435328 & 0.406335 & 1.424904 \\
\hline 28 & 8 & 0 & 4.650345 & -1.201272 & -0.796266 \\
\hline 29 & 8 & 0 & 2.770400 & 2.337284 & 0.012670 \\
\hline 30 & 8 & 0 & -1.084372 & -2.104382 & 0.284222 \\
\hline 31 & 17 & 0 & -5.679624 & 0.043079 & 0.066652 \\
\hline
\end{tabular}

\section{$\underline{\text { Structure of } 16 \text { (closed form) }}$}

Zero-point correction= 0.247859 (Hartree/Particle) 
Thermal correction to Energy $=0.264344$

Thermal correction to Enthalpy $=0.265288$

Thermal correction to Gibbs Free Energy $=0.203226$

Sum of electronic and zero-point Energies $=-1243.122234$

Sum of electronic and thermal Energies= -1243.105749

Sum of electronic and thermal Enthalpies= -1243.104804

Sum of electronic and thermal Free Energies= -1243.166867

No imaginary frequencies

\begin{tabular}{|c|c|c|c|c|c|}
\hline \multirow{2}{*}{$\begin{array}{l}\text { Center } \\
\text { Number }\end{array}$} & \multirow{2}{*}{$\begin{array}{l}\text { Atomic } \\
\text { Number }\end{array}$} & \multirow{2}{*}{$\begin{array}{c}\text { Atomic } \\
\text { Type }\end{array}$} & \multicolumn{3}{|c|}{ Coordinates (Angstroms) } \\
\hline & & & $\mathrm{X}$ & $Y$ & Z \\
\hline & & & & & \\
\hline 1 & 6 & 0 & -1.706285 & -0.864679 & 0.112831 \\
\hline 2 & 6 & 0 & -3.091098 & -1.037979 & 0.180311 \\
\hline 3 & 6 & 0 & -3.910501 & 0.063860 & 0.014683 \\
\hline 4 & 6 & 0 & -3.415868 & 1.345357 & -0.215849 \\
\hline 5 & 6 & 0 & -2.041680 & 1.502913 & -0.281985 \\
\hline 6 & 6 & 0 & -1.170721 & 0.420928 & -0.122627 \\
\hline 7 & 6 & 0 & 0.273561 & 0.637252 & -0.211429 \\
\hline 8 & 6 & 0 & 2.539577 & -0.124581 & -0.138648 \\
\hline 9 & 6 & 0 & 3.054469 & -0.177839 & 1.316744 \\
\hline 10 & 6 & 0 & 3.084387 & -1.315472 & -0.933113 \\
\hline 11 & 6 & 0 & 2.989908 & 1.175373 & -0.817918 \\
\hline 12 & 1 & 0 & -3.498512 & -2.025758 & 0.357187 \\
\hline 13 & 1 & 0 & -1.621076 & 2.487259 & -0.461496 \\
\hline 14 & 1 & 0 & 0.595551 & 1.664499 & -0.399791 \\
\hline 15 & 1 & 0 & 2.437643 & 0.474671 & 1.937194 \\
\hline 16 & 1 & 0 & 2.957012 & -1.200774 & 1.697760 \\
\hline 17 & 1 & 0 & 4.921696 & -0.257460 & 0.841540 \\
\hline 18 & 1 & 0 & 2.822608 & -1.206604 & -1.992418 \\
\hline 19 & 1 & 0 & 2.628946 & -2.232731 & -0.547857 \\
\hline 20 & 1 & 0 & 4.850561 & -2.119372 & -1.234093 \\
\hline 21 & 1 & 0 & 2.381366 & 1.348133 & -1.709144 \\
\hline 22 & 1 & 0 & 4.025099 & 1.053224 & -1.149647 \\
\hline 23 & 1 & 0 & 3.531839 & 2.234694 & 0.699759 \\
\hline 24 & 1 & 0 & 0.021319 & -1.613871 & 0.191766 \\
\hline 25 & 7 & 0 & 1.093703 & -0.330778 & -0.070418 \\
\hline 26 & 8 & 0 & 4.388524 & 0.291752 & 1.431711 \\
\hline 27 & 8 & 0 & 4.500799 & -1.354008 & -0.766393 \\
\hline 28 & 8 & 0 & 2.865963 & 2.323087 & 0.006896 \\
\hline 29 & 8 & 0 & -0.920713 & -1.933939 & 0.272716 \\
\hline 30 & 1 & 0 & -4.087237 & 2.184561 & -0.339862 \\
\hline 31 & 17 & 0 & -5.643166 & -0.167115 & 0.095565 \\
\hline
\end{tabular}

\section{$\underline{\text { Structure of } \mathbf{T S}_{16 / 46}}$}

Zero-point correction $=0.243856$ (Hartree/Particle)

Thermal correction to Energy $=0.259966$

Thermal correction to Enthalpy $=0.260910$

Thermal correction to Gibbs Free Energy= 0.199864

Sum of electronic and zero-point Energies $=-1243.119110$

Sum of electronic and thermal Energies= -1243.103000

Sum of electronic and thermal Enthalpies= -1243.102056

Sum of electronic and thermal Free Energies= -1243.163102

One imaginary frequency: $976 i$

Standard orientation:

\begin{tabular}{|c|c|c|c|c|c|}
\hline \multirow{2}{*}{$\begin{array}{l}\text { Center } \\
\text { Number }\end{array}$} & \multirow{2}{*}{$\begin{array}{l}\text { Atomic } \\
\text { Number }\end{array}$} & \multirow{2}{*}{$\begin{array}{c}\text { Atomic } \\
\text { Type }\end{array}$} & \multicolumn{3}{|c|}{ Coordinates (Angstroms) } \\
\hline & & & $\mathrm{X}$ & $\mathrm{Y}$ & Z \\
\hline 1 & 6 & 0 & -1.617732 & -0.814557 & 0.100680 \\
\hline 2 & 6 & 0 & -3.014931 & -1.040702 & 0.184763 \\
\hline 3 & 6 & 0 & -3.878862 & 0.017416 & 0.027050 \\
\hline 4 & 6 & 0 & -3.461703 & 1.335562 & -0.213569 \\
\hline 5 & 6 & 0 & -2.105797 & 1.568723 & -0.297318 \\
\hline 6 & 6 & 0 & -1.178838 & 0.526124 & -0.147037 \\
\hline 7 & 6 & 0 & 0.233984 & 0.773642 & -0.241602 \\
\hline 8 & 6 & 0 & 2.507730 & -0.121458 & -0.144980 \\
\hline 9 & 6 & 0 & 3.000918 & -0.218634 & 1.316793 \\
\hline 10 & 6 & 0 & 2.993045 & -1.326725 & -0.955807 \\
\hline
\end{tabular}




$\begin{array}{rrrrrr}11 & 6 & 0 & 3.015625 & 1.167191 & -0.800368 \\ 12 & 1 & 0 & -3.377302 & -2.044521 & 0.368583 \\ 13 & 1 & 0 & -4.184968 & 2.131409 & -0.329466 \\ 14 & 1 & 0 & -1.738271 & 2.573361 & -0.482498 \\ 15 & 1 & 0 & 0.594059 & 1.786536 & -0.418203 \\ 16 & 1 & 0 & 2.399745 & 0.445945 & 1.939401 \\ 17 & 1 & 0 & 2.859087 & -1.243793 & 1.677503 \\ 18 & 1 & 0 & 4.872326 & -0.374407 & 0.878993 \\ 19 & 1 & 0 & 2.764301 & -1.176686 & -2.017184 \\ 20 & 1 & 0 & 2.476983 & -2.226197 & -0.604423 \\ 21 & 1 & 0 & 4.717912 & -2.220801 & -1.230184 \\ 22 & 1 & 0 & 2.434830 & 1.369624 & -1.703591 \\ 23 & 1 & 0 & 4.053200 & 1.009340 & -1.107023 \\ 24 & 1 & 0 & 3.563258 & 2.196989 & 0.736854 \\ 25 & 1 & 0 & 0.372047 & -1.185967 & 0.095092 \\ 26 & 7 & 0 & 1.050075 & -0.218487 & -0.108585 \\ 27 & 8 & 0 & 4.347821 & 0.199152 & 1.453213 \\ 28 & 8 & 0 & 4.397683 & -1.448638 & -0.752067 \\ 29 & 8 & 0 & 2.907457 & 2.305192 & 0.036986 \\ 30 & 8 & 0 & -0.763524 & -1.776923 & 0.239533 \\ 31 & 17 & 0 & -5.601992 & -0.290657 & 0.127252 \\ -----------------------------------------------------\end{array}$

\section{Structure of 46}

Zero-point correction= 0.247964 (Hartree/Particle) Thermal correction to Energy $=0.264591$

Thermal correction to Enthalpy $=0.265536$

Thermal correction to Gibbs Free Energy= 0.202829

Sum of electronic and zero-point Energies $=-1243.118671$

Sum of electronic and thermal Energies= -1243.102044

Sum of electronic and thermal Enthalpies= -1243.101099

Sum of electronic and thermal Free Energies= -1243.163806

No imaginary frequencies

Standard orientation:

\begin{tabular}{|c|c|c|c|c|c|}
\hline \multirow{2}{*}{$\begin{array}{l}\text { Center } \\
\text { Number }\end{array}$} & \multirow{2}{*}{$\begin{array}{l}\text { Atomic } \\
\text { Number }\end{array}$} & \multirow{2}{*}{$\begin{array}{c}\text { Atomic } \\
\text { Type }\end{array}$} & \multicolumn{3}{|c|}{ Coordinates (Angstroms) } \\
\hline & & & $\mathrm{X}$ & Y & Z \\
\hline 1 & 6 & 0 & -1.692106 & -0.948808 & 0.103331 \\
\hline 2 & 6 & 0 & -3.128963 & -1.064334 & 0.170078 \\
\hline 3 & 6 & 0 & -3.914251 & 0.041072 & 0.024963 \\
\hline 4 & 6 & 0 & -3.412176 & 1.348054 & -0.190921 \\
\hline 5 & 6 & 0 & -2.053728 & 1.494686 & -0.259447 \\
\hline 6 & 6 & 0 & -1.176802 & 0.389926 & -0.121030 \\
\hline 7 & 6 & 0 & 0.211736 & 0.621190 & -0.208588 \\
\hline 8 & 6 & 0 & 2.565029 & -0.138992 & -0.136934 \\
\hline 9 & 6 & 0 & 3.060550 & -0.117304 & 1.326145 \\
\hline 10 & 6 & 0 & 3.129218 & -1.349911 & -0.885263 \\
\hline 11 & 6 & 0 & 2.978482 & 1.143122 & -0.868307 \\
\hline 12 & 1 & 0 & -3.557287 & -2.046034 & 0.332355 \\
\hline 13 & 1 & 0 & -4.087155 & 2.186236 & -0.296312 \\
\hline 14 & 1 & 0 & -1.623101 & 2.478093 & -0.423782 \\
\hline 15 & 1 & 0 & 0.565089 & 1.637419 & -0.364571 \\
\hline 16 & 1 & 0 & 2.416718 & 0.544259 & 1.908292 \\
\hline 17 & 1 & 0 & 2.984180 & -1.125358 & 1.749874 \\
\hline 18 & 1 & 0 & 4.941711 & -0.183326 & 0.903118 \\
\hline 19 & 1 & 0 & 2.903607 & -1.264705 & -1.954426 \\
\hline 20 & 1 & 0 & 2.664370 & -2.262032 & -0.495753 \\
\hline 21 & 1 & 0 & 4.909680 & -2.137825 & -1.113248 \\
\hline 22 & 1 & 0 & 2.371201 & 1.256086 & -1.769618 \\
\hline 23 & 1 & 0 & 4.018993 & 1.030759 & -1.184126 \\
\hline 24 & 1 & 0 & 3.485817 & 2.287687 & 0.599794 \\
\hline 25 & 7 & 0 & 1.111309 & -0.322949 & -0.108677 \\
\hline 26 & 8 & 0 & 4.377236 & 0.394209 & 1.433877 \\
\hline 27 & 8 & 0 & 4.534246 & -1.375086 & -0.660633 \\
\hline 28 & 8 & 0 & 2.813968 & 2.316105 & -0.092589 \\
\hline 29 & 8 & 0 & -0.941600 & -1.949479 & 0.230499 \\
\hline 30 & 1 & 0 & 0.723271 & -1.260780 & 0.072322 \\
\hline 31 & 17 & 0 & -5.656999 & -0.145766 & 0.104907 \\
\hline
\end{tabular}




\section{$\underline{\text { Structure of } 17 \text { (open form) }}$}

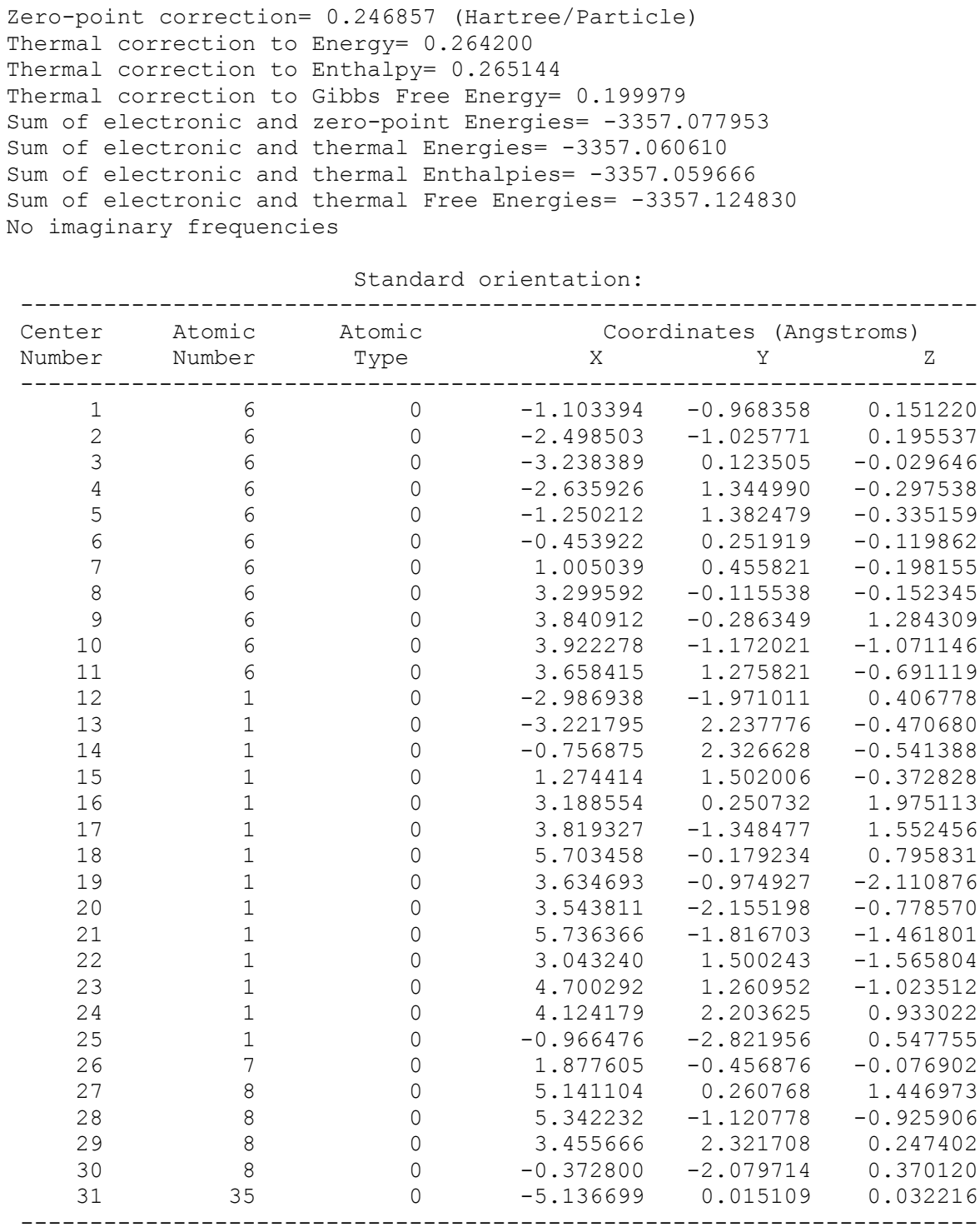

\section{$\underline{\text { Structure of } 17 \text { (closed form) }}$}

Zero-point correction= 0.247349 (Hartree/Particle)

Thermal correction to Energy $=0.264058$

Thermal correction to Enthalpy $=0.265002$

Thermal correction to Gibbs Free Energy $=0.201840$

Sum of electronic and zero-point Energies= -3357.094947

Sum of electronic and thermal Energies= -3357.078239

Sum of electronic and thermal Enthalpies= -3357.077294

Sum of electronic and thermal Free Energies= -3357.140457

No imaginary frequencies

Standard orientation:

\begin{tabular}{|c|c|c|c|c|c|}
\hline \multirow{2}{*}{$\begin{array}{l}\text { Center } \\
\text { Number }\end{array}$} & \multirow{2}{*}{$\begin{array}{l}\text { Atomic } \\
\text { Number }\end{array}$} & \multirow{2}{*}{$\begin{array}{c}\text { Atomic } \\
\text { Type }\end{array}$} & \multicolumn{3}{|c|}{ Coordinates (Angstroms) } \\
\hline & & & $\mathrm{X}$ & $\mathrm{Y}$ & $\mathrm{Z}$ \\
\hline 1 & 6 & 0 & -1.020541 & -0.829789 & 0.099419 \\
\hline 2 & 6 & 0 & -2.408111 & -0.989524 & 0.157946 \\
\hline 3 & 6 & 0 & -3.216679 & 0.120771 & -0.009180 \\
\hline 4 & 6 & 0 & -2.706223 & 1.398279 & -0.231209 \\
\hline 5 & 6 & 0 & -1.329614 & 1.541690 & -0.288586 \\
\hline 6 & 6 & 0 & -0.470569 & 0.450641 & -0.129035 \\
\hline
\end{tabular}




\begin{tabular}{|c|c|c|c|c|c|}
\hline 7 & 6 & 0 & 0.976341 & 0.652051 & -0.212721 \\
\hline 8 & 6 & 0 & 3.233440 & -0.133856 & -0.136044 \\
\hline 9 & 6 & 0 & 3.743534 & -0.189133 & 1.321049 \\
\hline 10 & 6 & 0 & 3.768860 & -1.331679 & -0.926574 \\
\hline 11 & 6 & 0 & 3.698701 & 1.160266 & -0.816590 \\
\hline 12 & 1 & 0 & -2.818638 & -1.977097 & 0.328592 \\
\hline 13 & 1 & 0 & -3.361512 & 2.249644 & -0.357409 \\
\hline 14 & 1 & 0 & -0.898550 & 2.522651 & -0.462373 \\
\hline 15 & 1 & 0 & 1.309921 & 1.676184 & -0.397861 \\
\hline 16 & 1 & 0 & 3.131025 & 0.470456 & 1.938300 \\
\hline 17 & 1 & 0 & 3.634962 & -1.210317 & 1.703673 \\
\hline 18 & 1 & 0 & 5.611350 & -0.287936 & 0.852003 \\
\hline 19 & 1 & 0 & 3.512158 & -1.221864 & -1.987006 \\
\hline 20 & 1 & 0 & 3.302816 & -2.243657 & -0.541450 \\
\hline 21 & 1 & 0 & 5.528949 & -2.150737 & -1.223565 \\
\hline 22 & 1 & 0 & 3.093950 & 1.337862 & -1.709435 \\
\hline 23 & 1 & 0 & 4.733309 & 1.026493 & -1.145742 \\
\hline 24 & 1 & 0 & 4.248159 & 2.216324 & 0.700641 \\
\hline 25 & 1 & 0 & 0.698613 & -1.598224 & 0.183427 \\
\hline 26 & 7 & 0 & 1.785392 & -0.325194 & -0.071770 \\
\hline 27 & 8 & 0 & 5.081574 & 0.267925 & 1.438972 \\
\hline 28 & 8 & 0 & 5.184149 & -1.383973 & -0.754478 \\
\hline 29 & 8 & 0 & 3.585113 & 2.310701 & 0.005830 \\
\hline 30 & 8 & 0 & -0.247326 & -1.907913 & 0.259878 \\
\hline 31 & 35 & 0 & -5.104776 & -0.112161 & 0.058518 \\
\hline
\end{tabular}

\section{$\underline{\text { Structure of } \mathbf{T S}_{17 / 47}}$}

Zero-point correction= 0.243378 (Hartree/Particle)
Thermal correction to Energy= 0.259706
Thermal correction to Enthalpy= 0.260650
Thermal correction to Gibbs Free Energy= 0.198514
Sum of electronic and zero-point Energies= -3357.091787
Sum of electronic and thermal Energies=-3357.075459
Sum of electronic and thermal Enthalpies=-3357.074515
Sum of electronic and thermal Free Energies=-3357.136651
One imaginary frequency: $974 i$

\begin{tabular}{|c|c|c|c|c|c|}
\hline \multirow{2}{*}{$\begin{array}{l}\text { Center } \\
\text { Number }\end{array}$} & \multirow{2}{*}{$\begin{array}{l}\text { Atomic } \\
\text { Number }\end{array}$} & \multirow{2}{*}{$\begin{array}{c}\text { Atomic } \\
\text { Type }\end{array}$} & \multicolumn{3}{|c|}{ Coordinates (Angstroms) } \\
\hline & & & $\mathrm{X}$ & Y & Z \\
\hline 1 & 6 & 0 & -0.941878 & -0.756763 & 0.082949 \\
\hline 2 & 6 & 0 & -2.344203 & -0.959778 & 0.154402 \\
\hline 3 & 6 & 0 & -3.189645 & 0.113480 & -0.004394 \\
\hline 4 & 6 & 0 & -2.746876 & 1.426359 & -0.231654 \\
\hline 5 & 6 & 0 & -1.385884 & 1.636137 & -0.302748 \\
\hline 6 & 6 & 0 & -0.478110 & 0.577061 & -0.153178 \\
\hline 7 & 6 & 0 & 0.939636 & 0.799590 & -0.239978 \\
\hline 8 & 6 & 0 & 3.195921 & -0.136645 & -0.141201 \\
\hline 9 & 6 & 0 & 3.682253 & -0.236798 & 1.322620 \\
\hline 10 & 6 & 0 & 3.662178 & -1.353360 & -0.946090 \\
\hline 11 & 6 & 0 & 3.728510 & 1.140666 & -0.799565 \\
\hline 12 & 1 & 0 & -2.716668 & -1.961653 & 0.327854 \\
\hline 13 & 1 & 0 & -3.449603 & 2.239913 & -0.349025 \\
\hline 14 & 1 & 0 & -0.999922 & 2.635701 & -0.478262 \\
\hline 15 & 1 & 0 & 1.319744 & 1.806314 & -0.409419 \\
\hline 16 & 1 & 0 & 3.091192 & 0.441458 & 1.940224 \\
\hline 17 & 1 & 0 & 3.520330 & -1.257738 & 1.686824 \\
\hline 18 & 1 & 0 & 5.552226 & -0.428644 & 0.892693 \\
\hline 19 & 1 & 0 & 3.436980 & -1.204367 & -2.008378 \\
\hline 20 & 1 & 0 & 3.130484 & -2.242240 & -0.590977 \\
\hline 21 & 1 & 0 & 5.369725 & -2.282695 & -1.208130 \\
\hline 22 & 1 & 0 & 3.152939 & 1.351247 & -1.704251 \\
\hline 23 & 1 & 0 & 4.763520 & 0.962585 & -1.103648 \\
\hline 24 & 1 & 0 & 4.292142 & 2.164197 & 0.736036 \\
\hline 25 & 1 & 0 & 1.040587 & -1.164312 & 0.085907 \\
\hline 26 & 7 & 0 & 1.736661 & -0.208067 & -0.109385 \\
\hline 27 & 8 & 0 & 5.036009 & 0.156893 & 1.462218 \\
\hline 28 & 8 & 0 & 5.064314 & -1.498188 & -0.740450 \\
\hline 29 & 8 & 0 & 3.640430 & 2.283117 & 0.034073 \\
\hline 30 & 8 & 0 & -0.106262 & -1.735292 & 0.221291 \\
\hline
\end{tabular}




\section{$\underline{\text { Structure of } 47}$} Zero-point correction $=0.247381$ (Hartree/Particle)
Thermal correction to Energy= 0.264259
Thermal correction to Enthalpy= 0.265203
Thermal correction to Gibbs Free Energy= 0.201042
Sum of electronic and zero-point Energies=-3357.091502
Sum of electronic and thermal Energies=-3357.074624
Sum of electronic and thermal Enthalpies=-3357.073679
Sum of electronic and thermal Free Energies=-3357.137841
No imaginary frequencies

\begin{tabular}{|c|c|c|c|c|c|}
\hline \multirow{2}{*}{$\begin{array}{l}\text { Center } \\
\text { Number }\end{array}$} & \multirow{2}{*}{$\begin{array}{l}\text { Atomic } \\
\text { Number }\end{array}$} & \multirow{2}{*}{$\begin{array}{c}\text { Atomic } \\
\text { Type }\end{array}$} & \multicolumn{3}{|c|}{ Coordinates (Angstroms) } \\
\hline & & & $\mathrm{X}$ & Y & Z \\
\hline 1 & 6 & 0 & -1.004889 & -0.919423 & 0.094939 \\
\hline 2 & 6 & 0 & -2.444803 & -1.022565 & 0.148902 \\
\hline 3 & 6 & 0 & -3.219262 & 0.090025 & 0.000167 \\
\hline 4 & 6 & 0 & -2.701993 & 1.393707 & -0.205075 \\
\hline 5 & 6 & 0 & -1.341348 & 1.527387 & -0.261512 \\
\hline 6 & 6 & 0 & -0.475469 & 0.414314 & -0.121413 \\
\hline 7 & 6 & 0 & 0.915660 & 0.632178 & -0.203180 \\
\hline 8 & 6 & 0 & 3.261576 & -0.147959 & -0.132878 \\
\hline 9 & 6 & 0 & 3.758926 & -0.124360 & 1.329452 \\
\hline 10 & 6 & 0 & 3.815663 & -1.365706 & -0.877526 \\
\hline 11 & 6 & 0 & 3.684047 & 1.128351 & -0.869666 \\
\hline 12 & 1 & 0 & -2.875245 & -2.004437 & 0.303578 \\
\hline 13 & 1 & 0 & -3.362187 & 2.242873 & -0.313626 \\
\hline 14 & 1 & 0 & -0.900838 & 2.507688 & -0.418859 \\
\hline 15 & 1 & 0 & 1.279153 & 1.645323 & -0.355945 \\
\hline 16 & 1 & 0 & 3.120514 & 0.543992 & 1.909786 \\
\hline 17 & 1 & 0 & 3.675999 & -1.130171 & 1.757261 \\
\hline 18 & 1 & 0 & 5.639079 & -0.205688 & 0.904767 \\
\hline 19 & 1 & 0 & 3.590187 & -1.282026 & -1.946846 \\
\hline 20 & 1 & 0 & 3.343829 & -2.272966 & -0.484951 \\
\hline 21 & 1 & 0 & 5.590715 & -2.165336 & -1.107562 \\
\hline 22 & 1 & 0 & 3.076279 & 1.242491 & -1.770500 \\
\hline 23 & 1 & 0 & 4.723255 & 1.006757 & -1.186344 \\
\hline 24 & 1 & 0 & 4.202567 & 2.274759 & 0.593051 \\
\hline 25 & 7 & 0 & 1.806351 & -0.320380 & -0.103349 \\
\hline 26 & 8 & 0 & 5.079357 & 0.378253 & 1.433541 \\
\hline 27 & 8 & 0 & 5.220505 & -1.400966 & -0.653424 \\
\hline 28 & 8 & 0 & 3.529591 & 2. 305441 & -0.098148 \\
\hline 29 & 8 & 0 & -0.265903 & -1.927889 & 0.224646 \\
\hline 30 & 1 & 0 & 1.410585 & -1.255283 & 0.074361 \\
\hline 31 & 35 & 0 & -5.118726 & -0.096563 & 0.059974 \\
\hline
\end{tabular}

\section{$\underline{\text { Structure of } 18 \text { (open form) }}$}

Zero-point correction=0.290616 (Hartree/Particle)

Thermal correction to Energy $=0.308827$

Thermal correction to Enthalpy=0.309771

Thermal correction to Gibbs Free Energy= 0.244090

Sum of electronic and zero-point Energies= -897.977681

Sum of electronic and thermal Energies= -897.959471

Sum of electronic and thermal Enthalpies= -897.958526

Sum of electronic and thermal Free Energies= -898.024208

No imaginary frequencies

Standard orientation:

\begin{tabular}{|c|c|c|c|c|c|}
\hline \multirow{2}{*}{$\begin{array}{l}\text { Center } \\
\text { Number }\end{array}$} & \multirow{2}{*}{$\begin{array}{l}\text { Atomic } \\
\text { Number }\end{array}$} & \multirow{2}{*}{$\begin{array}{c}\text { Atomic } \\
\text { Type }\end{array}$} & \multicolumn{3}{|c|}{ Coordinates (Angstroms) } \\
\hline & & & $\mathrm{X}$ & Y & Z \\
\hline 1 & 6 & 0 & -1.758222 & -1.135355 & 0.130564 \\
\hline 2 & 6 & 0 & -3.140828 & -1.233740 & 0.203355 \\
\hline
\end{tabular}




$\begin{array}{rrrrrr}3 & 6 & 0 & -3.949501 & -0.106208 & 0.045208 \\ 4 & 6 & 0 & -3.369016 & 1.142698 & -0.189719 \\ 5 & 6 & 0 & -1.985085 & 1.220356 & -0.257587 \\ 6 & 6 & 0 & -1.143074 & 0.115074 & -0.102500 \\ 7 & 6 & 0 & 0.302092 & 0.368777 & -0.198881 \\ 8 & 6 & 0 & 2.620678 & -0.091010 & -0.138109 \\ 9 & 6 & 0 & 3.150822 & -0.086969 & 1.311297 \\ 10 & 6 & 0 & 3.308781 & -1.200303 & -0.939992 \\ 11 & 6 & 0 & 2.914605 & 1.256438 & -0.813215 \\ 12 & 1 & 0 & -3.610656 & -2.196004 & 0.382252 \\ 13 & 1 & 0 & -3.966825 & 2.034319 & -0.316687 \\ 14 & 1 & 0 & -1.526577 & 2.187748 & -0.438194 \\ 15 & 1 & 0 & 0.531160 & 1.418537 & -0.411702 \\ 16 & 1 & 0 & 2.470547 & 0.495033 & 1.935952 \\ 17 & 1 & 0 & 3.166167 & -1.114430 & 1.692031 \\ 18 & 1 & 0 & 5.012699 & 0.047204 & 0.822628 \\ 19 & 1 & 0 & 3.013989 & -1.134145 & -1.994461 \\ 20 & 1 & 0 & 2.984573 & -2.166367 & -0.543150 \\ 21 & 1 & 0 & 5.155650 & -1.786997 & -1.260168 \\ 22 & 1 & 0 & 2.277959 & 1.368223 & -1.694618 \\ 23 & 1 & 0 & 3.951874 & 1.251685 & -1.161100 \\ 24 & 1 & 0 & 3.356955 & 2.352230 & 0.709341 \\ 25 & 1 & 0 & -1.561423 & -3.001761 & 0.421543 \\ 26 & 7 & 0 & 1.217377 & -0.499404 & -0.046112 \\ 27 & 8 & 0 & 4.428524 & 0.524249 & 1.426584 \\ 28 & 8 & 0 & 4.723922 & -1.056036 & -0.806002 \\ 29 & 8 & 0 & 2.681696 & 2.381912 & 0.020840 \\ 30 & 8 & 0 & -0.988999 & -2.236808 & 0.276897 \\ 31 & 8 & 0 & -5.277407 & -0.322816 & 0.135095 \\ 32 & 6 & 0 & -6.146002 & 0.790141 & -0.046700 \\ 33 & 1 & 0 & -7.156326 & 0.397407 & 0.047448 \\ 34 & 1 & 0 & -5.978007 & 1.549786 & 0.721175 \\ 35 & 1 & 0 & -6.016775 & 1.229957 & -1.039139 \\ ---------------------------------------------------\end{array}$

\section{$\underline{\text { Structure of } 18 \text { (closed form) }}$}

Zero-point correction= 0.290703 (Hartree/Particle)
Thermal correction to Energy= 0.308454
Thermal correction to Enthalpy= 0.309398
Thermal correction to Gibbs Free Energy= 0.245137
Sum of electronic and zero-point Energies= -897.994407
Sum of electronic and thermal Energies= -897.976656
Sum of electronic and thermal Enthalpies= -897.975712
Sum of electronic and thermal Free Energies= -898.039973
No imaginary frequencies

Standard orientation:

\begin{tabular}{|c|c|c|c|c|c|}
\hline \multirow{2}{*}{$\begin{array}{l}\text { Center } \\
\text { Number }\end{array}$} & \multirow{2}{*}{$\begin{array}{l}\text { Atomic } \\
\text { Number }\end{array}$} & \multirow{2}{*}{$\begin{array}{c}\text { Atomic } \\
\text { Type }\end{array}$} & \multicolumn{3}{|c|}{ Coordinates (Angstroms) } \\
\hline & & & $\mathrm{X}$ & $\mathrm{Y}$ & Z \\
\hline--- & & & --------- & 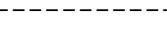 & ---------- \\
\hline 1 & 6 & 0 & -1.653627 & -1.008586 & 0.150350 \\
\hline 2 & 6 & 0 & -3.021665 & -1.233592 & 0.227318 \\
\hline 3 & 6 & 0 & -3.917926 & -0.177385 & 0.058072 \\
\hline 4 & 6 & 0 & -3.454411 & 1.123180 & -0.188387 \\
\hline 5 & 6 & 0 & -2.086631 & 1.333469 & -0.262665 \\
\hline 6 & 6 & 0 & -1.164018 & 0.297602 & -0.100013 \\
\hline 7 & 6 & 0 & 0.264188 & 0.573222 & -0.199469 \\
\hline 8 & 6 & 0 & 2.566207 & -0.081366 & -0.145932 \\
\hline 9 & 6 & 0 & 3.101719 & -0.146933 & 1.300667 \\
\hline 10 & 6 & 0 & 3.153521 & -1.226176 & -0.976704 \\
\hline 11 & 6 & 0 & 2.951257 & 1.253033 & -0.797885 \\
\hline 12 & 1 & 0 & -3.395574 & -2.233033 & 0.415105 \\
\hline 13 & 1 & 0 & -4.137814 & 1.950018 & -0.319621 \\
\hline 14 & 1 & 0 & -1.711642 & 2.334398 & -0.453353 \\
\hline 15 & 1 & 0 & 0.539655 & 1.613601 & -0.391466 \\
\hline 16 & 1 & 0 & 2.467527 & 0.467331 & 1.942247 \\
\hline 17 & 1 & 0 & 3.048191 & -1.181325 & 1.659298 \\
\hline 18 & 1 & 0 & 4.964892 & -0.139444 & 0.802147 \\
\hline 19 & 1 & 0 & 2.872779 & -1.101970 & -2.029547 \\
\hline 20 & 1 & 0 & 2.742274 & -2.171145 & -0.609295 \\
\hline 21 & 1 & 0 & 4.945120 & -1.954372 & -1.314303 \\
\hline 22 & 1 & 0 & 2.327606 & 1.419224 & -1.679897 \\
\hline
\end{tabular}




$\begin{array}{rrrrrr}23 & 1 & 0 & 3.987675 & 1.186161 & -1.141261 \\ 24 & 1 & 0 & 3.458866 & 2.300522 & 0.739765 \\ 25 & 1 & 0 & 0.106794 & -1.684019 & 0.221564 \\ 26 & 7 & 0 & 1.132567 & -0.355623 & -0.065122 \\ 27 & 8 & 0 & 4.418402 & 0.371471 & 1.413901 \\ 28 & 8 & 0 & 4.573038 & -1.210063 & -0.830037 \\ 29 & 8 & 0 & 2.783426 & 2.374875 & 0.054720 \\ 30 & 8 & 0 & -0.817786 & -2.043281 & 0.314464 \\ 31 & 8 & 0 & -5.224462 & -0.501932 & 0.148255 \\ 32 & 6 & 0 & -6.184121 & 0.530349 & -0.049636 \\ 33 & 1 & 0 & -7.158142 & 0.056619 & 0.054417 \\ 34 & 1 & 0 & -6.080389 & 1.314344 & 0.704873 \\ 35 & 1 & 0 & -6.093690 & 0.962982 & -1.049543 \\ -\end{array}$

\section{$\underline{\text { Structure of } \mathbf{T S}_{\mathbf{1 8 / 4 8}}}$}

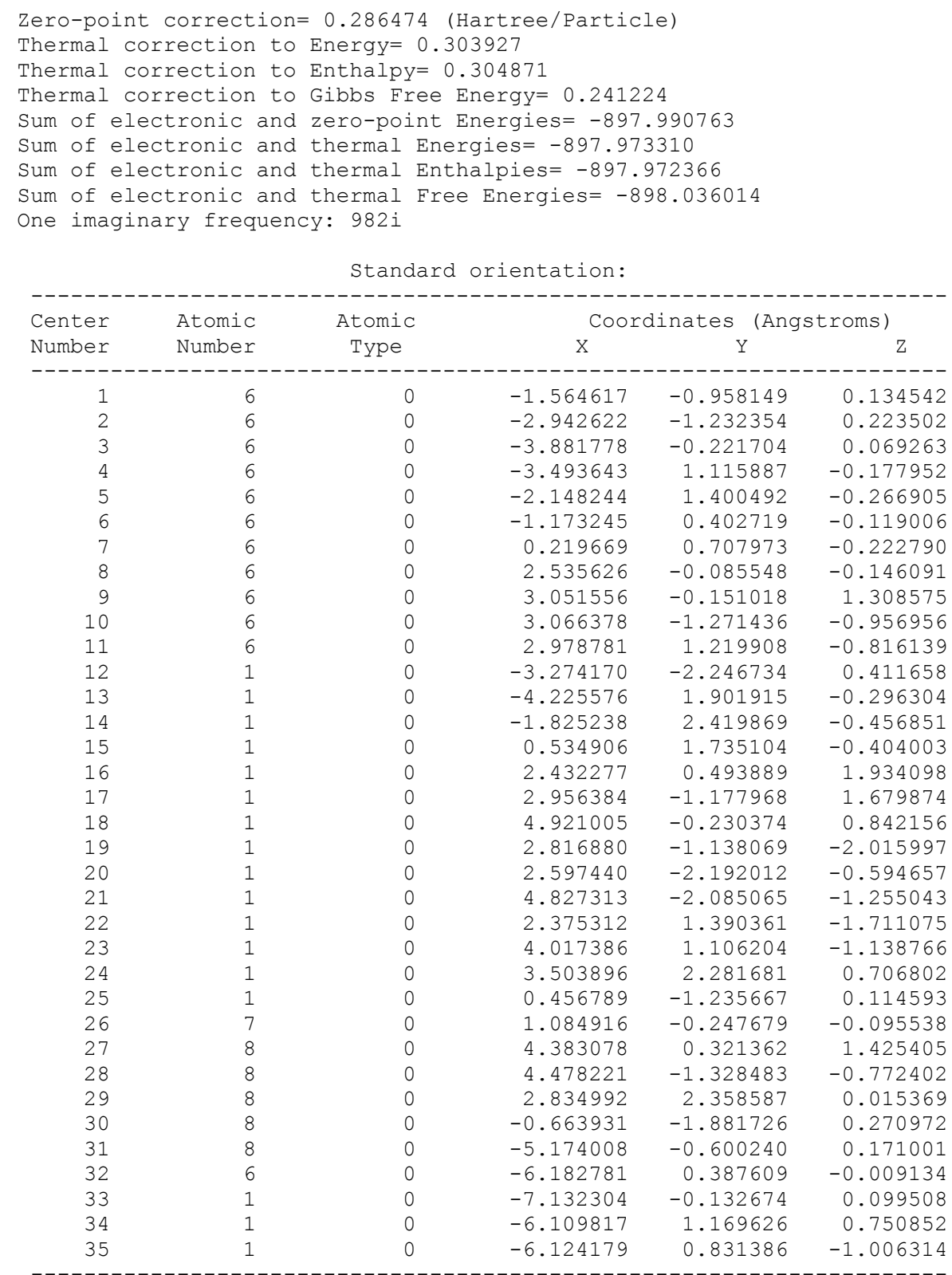

\section{Structure of 48}

Zero-point correction $=0.290806$ (Hartree/Particle) 
Thermal correction to Energy $=0.308657$

Thermal correction to Enthalpy $=0.309601$

Thermal correction to Gibbs Free Energy= 0.245157

Sum of electronic and zero-point Energies $=-897.989516$

Sum of electronic and thermal Energies= -897.971665

Sum of electronic and thermal Enthalpies= -897.970721

Sum of electronic and thermal Free Energies= -898.035165

No imaginary frequencies

\begin{tabular}{|c|c|c|c|c|c|}
\hline \multirow{2}{*}{$\begin{array}{l}\text { Center } \\
\text { Number }\end{array}$} & \multirow{2}{*}{$\begin{array}{l}\text { Atomic } \\
\text { Number }\end{array}$} & \multirow{2}{*}{$\begin{array}{c}\text { Atomic } \\
\text { Type }\end{array}$} & \multicolumn{3}{|c|}{ Coordinates (Angstroms) } \\
\hline & & & $\mathrm{X}$ & $\mathrm{Y}$ & Z \\
\hline & & & (1) & (1) & ---------- \\
\hline 1 & 6 & 0 & -1.629780 & -1.083030 & 0.140350 \\
\hline 2 & 6 & 0 & -3.045235 & -1.258467 & 0.219634 \\
\hline 3 & 6 & 0 & -3.919423 & -0.203548 & 0.069416 \\
\hline 4 & 6 & 0 & -3.456252 & 1.124822 & -0.167959 \\
\hline 5 & 6 & 0 & -2.106504 & 1.333710 & -0.246997 \\
\hline 6 & 6 & 0 & -1.171336 & 0.279460 & -0.102910 \\
\hline 7 & 6 & 0 & 0.199054 & 0.575164 & -0.204024 \\
\hline 8 & 6 & 0 & 2.586232 & -0.093332 & -0.144925 \\
\hline 9 & 6 & 0 & 3.096732 & -0.093650 & 1.312613 \\
\hline 10 & 6 & 0 & 3.186021 & -1.263183 & -0.930368 \\
\hline 11 & 6 & 0 & 2.947527 & 1.221085 & -0.844661 \\
\hline 12 & 1 & 0 & -3.436406 & -2.253977 & 0.395654 \\
\hline 13 & 1 & 0 & -4.146834 & 1.947635 & -0.282664 \\
\hline 14 & 1 & 0 & -1.727759 & 2.336062 & -0.425627 \\
\hline 15 & 1 & 0 & 0.505866 & 1.605117 & -0.369352 \\
\hline 16 & 1 & 0 & 2.438160 & 0.530513 & 1.918979 \\
\hline 17 & 1 & 0 & 3.057322 & -1.114796 & 1.709253 \\
\hline 18 & 1 & 0 & 4.974131 & -0.082088 & 0.866414 \\
\hline 19 & 1 & 0 & 2.940010 & -1.161670 & -1.993657 \\
\hline 20 & 1 & 0 & 2.762207 & -2.201242 & -0.555903 \\
\hline 21 & 1 & 0 & 4. 987511 & -1.988957 & -1.194177 \\
\hline 22 & 1 & 0 & 2.332687 & 1.334263 & -1.740817 \\
\hline 23 & 1 & 0 & 3.990097 & 1.157639 & -1.167026 \\
\hline 24 & 1 & 0 & 3.416713 & 2.345684 & 0.651535 \\
\hline 25 & 7 & 0 & 1.142233 & -0.330695 & -0.107760 \\
\hline 26 & 8 & 0 & 4.397646 & 0.457508 & 1.423427 \\
\hline 27 & 8 & 0 & 4.595247 & -1.242184 & -0.729255 \\
\hline 28 & 8 & 0 & 2.741751 & 2.366904 & -0.038062 \\
\hline 29 & 8 & 0 & -0.818738 & -2.042597 & 0.270364 \\
\hline 30 & 1 & 0 & 0.785575 & -1.280969 & 0.083227 \\
\hline 31 & 8 & 0 & -5.233181 & -0.500599 & 0.161927 \\
\hline 32 & 6 & 0 & -6.186385 & 0.538866 & -0.034429 \\
\hline 33 & 1 & 0 & -7.162512 & 0.068866 & 0.068613 \\
\hline 34 & 1 & 0 & -6.082000 & 1.320662 & 0.721811 \\
\hline 35 & 1 & 0 & -6.096114 & 0.972483 & -1.033593 \\
\hline
\end{tabular}

\section{$\underline{\text { Structure of } 21 \text { (open form) }}$}

Zero-point correction $=0.291005$ (Hartree/Particle)

Thermal correction to Energy $=0.309183$

Thermal correction to Enthalpy $=0.310127$

Thermal correction to Gibbs Free Energy= 0.244549

Sum of electronic and zero-point Energies= -897.975906

Sum of electronic and thermal Energies= -897.957727

Sum of electronic and thermal Enthalpies= -897.956783

Sum of electronic and thermal Free Energies= -898.022361

No imaginary frequencies

Standard orientation:

\begin{tabular}{|c|c|c|c|c|c|}
\hline \multirow{2}{*}{$\begin{array}{l}\text { Center } \\
\text { Number }\end{array}$} & \multirow{2}{*}{$\begin{array}{l}\text { Atomic } \\
\text { Number }\end{array}$} & \multirow{2}{*}{$\begin{array}{c}\text { Atomic } \\
\text { Type }\end{array}$} & \multicolumn{3}{|c|}{ Coordinates (Angstroms) } \\
\hline & & & $\mathrm{X}$ & $\mathrm{Y}$ & Z \\
\hline 1 & 6 & 0 & -2.007618 & -0.371134 & 0.007196 \\
\hline 2 & 6 & 0 & -3.408500 & -0.211326 & 0.034984 \\
\hline 3 & 6 & 0 & -3.986979 & 1.040954 & -0.084668 \\
\hline 4 & 6 & 0 & -3.165131 & 2.161948 & -0.238404 \\
\hline 5 & 6 & 0 & -1.792663 & 2.012700 & -0.268250 \\
\hline 6 & 6 & 0 & -1.185612 & 0.748342 & -0.147014 \\
\hline
\end{tabular}




\begin{tabular}{|c|c|c|c|c|c|}
\hline 7 & 6 & 0 & 0.287920 & 0.718291 & -0.196531 \\
\hline 8 & 6 & 0 & 2.459686 & -0.213654 & -0.152219 \\
\hline 9 & 6 & 0 & 2.932684 & -0.489527 & 1.292077 \\
\hline 10 & 6 & 0 & 2.933419 & -1.339971 & -1.076511 \\
\hline 11 & 6 & 0 & 3.043562 & 1.113671 & -0.656683 \\
\hline 12 & 1 & 0 & -5.063054 & 1.155700 & -0.060901 \\
\hline 13 & 1 & 0 & -3.612628 & 3.143687 & -0.334270 \\
\hline 14 & 1 & 0 & -1.153002 & 2.880722 & -0.387570 \\
\hline 15 & 1 & 0 & 0.728425 & 1.714314 & -0.307347 \\
\hline 16 & 1 & 0 & 2.354476 & 0.132249 & 1.978041 \\
\hline 17 & 1 & 0 & 2.738981 & -1.539128 & 1.540684 \\
\hline 18 & 1 & 0 & 4.801079 & -0.667229 & 0.846687 \\
\hline 19 & 1 & 0 & 2.703559 & -1.088574 & -2.118982 \\
\hline 20 & 1 & 0 & 2.401783 & -2.257272 & -0.808646 \\
\hline 21 & 1 & 0 & 4.634487 & -2.256147 & -1.434465 \\
\hline 22 & 1 & 0 & 2.479754 & 1.452101 & -1.529655 \\
\hline 23 & 1 & 0 & 4.072617 & 0.938325 & -0.984059 \\
\hline 24 & 1 & 0 & 3.631849 & 1.915515 & 0.993814 \\
\hline 25 & 1 & 0 & -2.253674 & -2.222735 & 0.225041 \\
\hline 26 & 7 & 0 & 1.000080 & -0.329383 & -0.115956 \\
\hline 27 & 8 & 0 & 4.298369 & -0.155798 & 1.494351 \\
\hline 28 & 8 & 0 & 4.341048 & -1.509444 & -0.902305 \\
\hline 29 & 8 & 0 & 3.004179 & 2.159449 & 0.303008 \\
\hline 30 & 8 & 0 & -1.504831 & -1.616018 & 0.132751 \\
\hline 31 & 8 & 0 & -4.081129 & -1.383577 & 0.186053 \\
\hline 32 & 6 & 0 & -5.501796 & -1.328631 & 0.259636 \\
\hline 33 & 1 & 0 & -5.837147 & -2.355419 & 0.388903 \\
\hline 34 & 1 & 0 & -5.820944 & -0.727224 & 1.114735 \\
\hline 35 & 1 & 0 & -5.922040 & -0.920076 & -0.662848 \\
\hline
\end{tabular}

\section{$\underline{\text { Structure of } \mathbf{2 1} \text { (closed form) }}$}

Zero-point correction $=0.290886$ (Hartree/Particle) Thermal correction to Energy $=0.308667$

Thermal correction to Enthalpy= 0.309611

Thermal correction to Gibbs Free Energy= 0.245183

Sum of electronic and zero-point Energies= -897.987478

Sum of electronic and thermal Energies= -897.969696

Sum of electronic and thermal Enthalpies= -897.968752

Sum of electronic and thermal Free Energies= -898.033180

No imaginary frequencies

Standard orientation:

\begin{tabular}{|c|c|c|c|c|c|}
\hline \multirow{2}{*}{$\begin{array}{l}\text { Center } \\
\text { Number }\end{array}$} & \multirow{2}{*}{$\begin{array}{l}\text { Atomic } \\
\text { Number }\end{array}$} & \multirow{2}{*}{$\begin{array}{c}\text { Atomic } \\
\text { Type }\end{array}$} & \multicolumn{3}{|c|}{ Coordinates (Angstroms) } \\
\hline & & & $\mathrm{X}$ & $\mathrm{Y}$ & Z \\
\hline 1 & 6 & 0 & -1.920919 & -0.320346 & -0.008379 \\
\hline 2 & 6 & 0 & -3.333067 & -0.287413 & 0.044359 \\
\hline 3 & 6 & 0 & -3.995254 & 0.928927 & -0.043351 \\
\hline 4 & 6 & 0 & -3.277926 & 2.125433 & -0.184392 \\
\hline 5 & 6 & 0 & -1.900485 & 2.102065 & -0.238415 \\
\hline 6 & 6 & 0 & -1.209875 & 0.881023 & -0.151447 \\
\hline 7 & 6 & 0 & 0.252972 & 0.884002 & -0.210130 \\
\hline 8 & 6 & 0 & 2.386987 & -0.194455 & -0.153500 \\
\hline 9 & 6 & 0 & 2.839188 & -0.443081 & 1.302623 \\
\hline 10 & 6 & 0 & 2.787388 & -1.379746 & -1.036933 \\
\hline 11 & 6 & 0 & 3.038280 & 1.080497 & -0.704895 \\
\hline 12 & 1 & 0 & -5.076450 & 0.961862 & -0.003834 \\
\hline 13 & 1 & 0 & -3.816650 & 3.062813 & -0.250394 \\
\hline 14 & 1 & 0 & -1.330594 & 3.018810 & -0.347161 \\
\hline 15 & 1 & 0 & 0.724766 & 1.863884 & -0.319564 \\
\hline 16 & 1 & 0 & 2.297344 & 0.236346 & 1.963031 \\
\hline 17 & 1 & 0 & 2.586137 & -1.470309 & 1.589115 \\
\hline 18 & 1 & 0 & 4.694106 & -0.752624 & 0.874132 \\
\hline 19 & 1 & 0 & 2.584073 & -1.145282 & -2.088589 \\
\hline 20 & 1 & 0 & 2.191507 & -2.250334 & -0.747244 \\
\hline 21 & 1 & 0 & 4.426546 & -2.416858 & -1.345591 \\
\hline 22 & 1 & 0 & 2.489039 & 1.414238 & -1.588870 \\
\hline 23 & 1 & 0 & 4.056180 & 0.838874 & -1.024457 \\
\hline 24 & 1 & 0 & 3.669180 & 1.912904 & 0.915406 \\
\hline 25 & 1 & 0 & -0.331216 & -1.324578 & 0.031832 \\
\hline 26 & 7 & 0 & 0.925276 & -0.198462 & -0.128950 \\
\hline
\end{tabular}




\begin{tabular}{rrrrrr}
27 & 8 & 0 & 4.220763 & -0.179540 & 1.491664 \\
28 & 8 & 0 & 4.176726 & -1.637030 & -0.838911 \\
29 & 8 & 0 & 3.051279 & 2.159014 & 0.216441 \\
30 & 8 & 0 & -1.309266 & -1.507904 & 0.081451 \\
31 & 8 & 0 & -3.936325 & -1.490831 & 0.181452 \\
32 & 6 & 0 & -5.353702 & -1.500179 & 0.269028 \\
33 & 1 & 0 & -5.640923 & -2.543534 & 0.384229 \\
34 & 1 & 0 & -5.698081 & -0.929880 & 1.136842 \\
35 & 1 & 0 & -5.808836 & -1.097129 & -0.640411 \\
\hline
\end{tabular}

\section{$\underline{\text { Structure of } \mathbf{T S}_{21 / 51}}$} Zero-point correction= 0.286766 (Hartree/Particle)
Thermal correction to Energy $=0.304084$
Thermal correction to Enthalpy= 0.305028
Thermal correction to Gibbs Free Energy= 0.241953
Sum of electronic and zero-point Energies=-897.983784
Sum of electronic and thermal Energies=-897.966466
Sum of electronic and thermal Enthalpies= -897.965521
Sum of electronic and thermal Free Energies= -898.028597
One imaginary frequency: $990 i$

\begin{tabular}{|c|c|c|c|c|c|}
\hline \multirow{2}{*}{$\begin{array}{l}\text { Center } \\
\text { Number }\end{array}$} & \multirow{2}{*}{$\begin{array}{l}\text { Atomic } \\
\text { Number }\end{array}$} & \multirow{2}{*}{$\begin{array}{c}\text { Atomic } \\
\text { Type }\end{array}$} & \multicolumn{3}{|c|}{ Coordinates (Angstroms) } \\
\hline & & & $\mathrm{x}$ & Y & Z \\
\hline 1 & 6 & 0 & -1.837741 & -0.296084 & -0.024419 \\
\hline 2 & 6 & 0 & -3.271202 & -0.321013 & 0.046444 \\
\hline 3 & 6 & 0 & -3.989818 & 0.854412 & -0.008339 \\
\hline 4 & 6 & 0 & -3.345361 & 2.108128 & -0.135215 \\
\hline 5 & 6 & 0 & -1.978119 & 2.169619 & -0.206567 \\
\hline 6 & 6 & 0 & -1.217602 & 0.979042 & -0.153042 \\
\hline 7 & 6 & 0 & 0.215406 & 1.020821 & -0.215188 \\
\hline 8 & 6 & 0 & 2.340541 & -0.189491 & -0.157464 \\
\hline 9 & 6 & 0 & 2.768808 & -0.409387 & 1.310819 \\
\hline 10 & 6 & 0 & 2.681649 & -1.417463 & -1.005887 \\
\hline 11 & 6 & 0 & 3.041380 & 1.041313 & -0.744280 \\
\hline 12 & 1 & 0 & -5.071314 & 0.830778 & 0.045482 \\
\hline 13 & 1 & 0 & -3.945333 & 3.009091 & -0.172686 \\
\hline 14 & 1 & 0 & -1.460742 & 3.118722 & -0.303483 \\
\hline 15 & 1 & 0 & 0.722842 & 1.981743 & -0.296084 \\
\hline 16 & 1 & 0 & 2.250836 & 0.315050 & 1.941611 \\
\hline 17 & 1 & 0 & 2.468713 & -1.414620 & 1.628792 \\
\hline 18 & 1 & 0 & 4.612910 & -0.818018 & 0.917342 \\
\hline 19 & 1 & 0 & 2.506256 & -1.198103 & -2.065351 \\
\hline 20 & 1 & 0 & 2.037330 & -2.249679 & -0.704625 \\
\hline 21 & 1 & 0 & 4.268172 & -2.545223 & -1.255001 \\
\hline 22 & 1 & 0 & 2.508014 & 1.365238 & -1.641420 \\
\hline 23 & 1 & 0 & 4.050885 & 0.750188 & -1.047452 \\
\hline 24 & 1 & 0 & 3.698726 & 1.906334 & 0.849442 \\
\hline 25 & 1 & 0 & 0.076133 & -0.955578 & -0.053638 \\
\hline 26 & 7 & 0 & 0.883469 & -0.086133 & -0.163618 \\
\hline 27 & 8 & 0 & 4.157384 & -0.196932 & 1.500698 \\
\hline 28 & 8 & 0 & 4.049902 & -1.739718 & -0.774435 \\
\hline 29 & 8 & 0 & 3.085414 & 2.144640 & 0.143559 \\
\hline 30 & 8 & 0 & -1.141710 & -1.385186 & 0.027698 \\
\hline 31 & 8 & 0 & -3.810046 & -1.557290 & 0.167759 \\
\hline 32 & 6 & 0 & -5.221958 & -1.637242 & 0.272506 \\
\hline 33 & 1 & 0 & -5.458902 & -2.694824 & 0.373393 \\
\hline 34 & 1 & 0 & -5.583148 & -1.098230 & 1.153908 \\
\hline 35 & 1 & 0 & -5.709102 & -1.239978 & -0.623345 \\
\hline
\end{tabular}

\section{$\underline{\text { Structure of } 51}$}

Zero-point correction $=0.290889$ (Hartree/Particle)

Thermal correction to Energy $=0.308670$

Thermal correction to Enthalpy=0.309614

Thermal correction to Gibbs Free Energy= 0.245268

Sum of electronic and zero-point Energies $=-897.983248$ 
Sum of electronic and thermal Energies= -897.965467

Sum of electronic and thermal Enthalpies= -897.964523

Sum of electronic and thermal Free Energies= -898.028869

No imaginary frequencies

Standard orientation:

\begin{tabular}{|c|c|c|c|c|c|}
\hline \multirow{2}{*}{$\begin{array}{l}\text { Center } \\
\text { Number }\end{array}$} & \multirow{2}{*}{$\begin{array}{l}\text { Atomic } \\
\text { Number }\end{array}$} & \multirow{2}{*}{$\begin{array}{c}\text { Atomic } \\
\text { Type }\end{array}$} & \multicolumn{3}{|c|}{ Coordinates (Angstroms) } \\
\hline & & & $\mathrm{X}$ & $Y$ & Z \\
\hline 1 & 6 & 0 & -1.916966 & -0.398123 & -0.022726 \\
\hline 2 & 6 & 0 & -3.374614 & -0.292915 & 0.042071 \\
\hline 3 & 6 & 0 & -3.998611 & 0.923846 & -0.004585 \\
\hline 4 & 6 & 0 & -3.258383 & 2.140984 & -0.118112 \\
\hline 5 & 6 & 0 & -1.900015 & 2.105482 & -0.180449 \\
\hline 6 & 6 & 0 & -1.207109 & 0.856784 & -0.133718 \\
\hline 7 & 6 & 0 & 0.197691 & 0.868269 & -0.189450 \\
\hline 8 & 6 & 0 & 2.413107 & -0.230432 & -0.143493 \\
\hline 9 & 6 & 0 & 2.866493 & -0.292842 & 1.331360 \\
\hline 10 & 6 & 0 & 2.820464 & -1.499775 & -0.896542 \\
\hline 11 & 6 & 0 & 3.025975 & 0.989670 & -0.844002 \\
\hline 12 & 1 & 0 & -5.079151 & 0.982983 & 0.044308 \\
\hline 13 & 1 & 0 & -3.795617 & 3.080706 & -0.151962 \\
\hline 14 & 1 & 0 & -1.317178 & 3.017724 & -0.263614 \\
\hline 15 & 1 & 0 & 0.709453 & 1.825458 & -0.259578 \\
\hline 16 & 1 & 0 & 2.312110 & 0.455512 & 1.900683 \\
\hline 17 & 1 & 0 & 2.625949 & -1.280397 & 1.741555 \\
\hline 18 & 1 & 0 & 4.728013 & -0.632748 & 0.956109 \\
\hline 19 & 1 & 0 & 2.632834 & -1.370537 & -1.968642 \\
\hline 20 & 1 & 0 & 2.222748 & -2.341915 & -0.530319 \\
\hline 21 & 1 & 0 & 4.472851 & -2.536552 & -1.091738 \\
\hline 22 & 1 & 0 & 2.455771 & 1.212372 & -1.748786 \\
\hline 23 & 1 & 0 & 4.043185 & 0.728624 & -1.147966 \\
\hline 24 & 1 & 0 & 3.672327 & 2.012384 & 0.659210 \\
\hline 25 & 7 & 0 & 0.948786 & -0.207828 & -0.158036 \\
\hline 26 & 8 & 0 & 4.241294 & 0.014619 & 1.482554 \\
\hline 27 & 8 & 0 & 4.202580 & -1.727310 & -0.644896 \\
\hline 28 & 8 & 0 & 3.030280 & 2.160377 & -0.046379 \\
\hline 29 & 8 & 0 & -1.338127 & -1.509803 & 0.018926 \\
\hline 30 & 1 & 0 & 0.427234 & -1.088253 & -0.055382 \\
\hline 31 & 8 & 0 & -4.001717 & -1.487069 & 0.151295 \\
\hline 32 & 6 & 0 & -5.415604 & -1.466651 & 0.245724 \\
\hline 33 & 1 & 0 & -5.730057 & -2.504983 & 0.335576 \\
\hline 34 & 1 & 0 & -5.744246 & -0.909134 & 1.128655 \\
\hline 35 & 1 & 0 & -5.866785 & -1.028741 & -0.650316 \\
\hline
\end{tabular}

\section{Structure of $\mathbf{2 3}$ (open form)}

Zero-point correction= 0.290097 (Hartree/Particle)

Thermal correction to Energy $=0.308394$

Thermal correction to Enthalpy= 0.309339

Thermal correction to Gibbs Free Energy $=0.243364$

Sum of electronic and zero-point Energies= -897.974922

Sum of electronic and thermal Energies= -897.956624

Sum of electronic and thermal Enthalpies= -897.955680

Sum of electronic and thermal Free Energies= -898.021654

No imaginary frequencies

Standard orientation:

\begin{tabular}{|c|c|c|c|c|c|}
\hline \multirow{2}{*}{$\begin{array}{l}\text { Center } \\
\text { Number }\end{array}$} & \multirow{2}{*}{$\begin{array}{l}\text { Atomic } \\
\text { Number }\end{array}$} & \multirow{2}{*}{$\begin{array}{c}\text { Atomic } \\
\text { Type }\end{array}$} & \multicolumn{3}{|c|}{ Coordinates (Angstroms) } \\
\hline & & & $\mathrm{X}$ & Y & Z \\
\hline 1 & 6 & 0 & -2.013953 & -1.692851 & 0.029102 \\
\hline 2 & 6 & 0 & -3.370275 & -2.004084 & 0.158889 \\
\hline 3 & 6 & 0 & -4.305874 & -0.984888 & 0.206203 \\
\hline 4 & 6 & 0 & -3.924098 & 0.351668 & 0.125250 \\
\hline 5 & 6 & 0 & -2.570381 & 0.655460 & -0.004149 \\
\hline 6 & 6 & 0 & -1.587669 & -0.358914 & -0.049545 \\
\hline 7 & 6 & 0 & -0.180882 & 0.044821 & -0.246806 \\
\hline 8 & 6 & 0 & 2.171174 & -0.177331 & -0.041131 \\
\hline 9 & 6 & 0 & 2.737724 & 0.193475 & 1.346620 \\
\hline 10 & 6 & 0 & 2.901745 & -1.408985 & -0.586978 \\
\hline
\end{tabular}




\begin{tabular}{|c|c|c|c|c|c|}
\hline 11 & 6 & 0 & 2.369967 & 0.983962 & -1.023136 \\
\hline 12 & 1 & 0 & -3.677155 & -3.043677 & 0.214033 \\
\hline 13 & 1 & 0 & -4.672451 & 1.130233 & 0.167590 \\
\hline 14 & 1 & 0 & -0.052216 & 0.976812 & -0.800697 \\
\hline 15 & 1 & 0 & 2.030758 & 0.852301 & 1.854568 \\
\hline 16 & 1 & 0 & 2.844314 & -0.717019 & 1.947124 \\
\hline 17 & 1 & 0 & 4.570817 & 0.337019 & 0.766582 \\
\hline 18 & 1 & 0 & 2.592619 & -1.595069 & -1.622794 \\
\hline 19 & 1 & 0 & 2.631335 & -2.275022 & 0.023464 \\
\hline 20 & 1 & 0 & 4.772490 & -1.960559 & -0.816782 \\
\hline 21 & 1 & 0 & 1.736849 & 0.836508 & -1.901400 \\
\hline 22 & 1 & 0 & 3.407688 & 0.971398 & -1.369641 \\
\hline 23 & 1 & 0 & 2.720440 & 2.440209 & 0.188473 \\
\hline 24 & 1 & 0 & -1.550627 & -3.530353 & -0.071684 \\
\hline 25 & 7 & 0 & 0.799401 & -0.622355 & 0.205849 \\
\hline 26 & 8 & 0 & 3.965430 & 0.902466 & 1.263995 \\
\hline 27 & 8 & 0 & 4.308764 & -1.170930 & -0.519846 \\
\hline 28 & 8 & 0 & 2.047618 & 2.253898 & -0.477698 \\
\hline 29 & 8 & 0 & -1.092369 & -2.680304 & -0.056506 \\
\hline 30 & 8 & 0 & -2.097815 & 1.920368 & -0.080924 \\
\hline 31 & 6 & 0 & -3.038327 & 2.987919 & -0.092645 \\
\hline 32 & 1 & 0 & -3.718660 & 2.902083 & -0.943991 \\
\hline 33 & 1 & 0 & -3.610506 & 3.015963 & 0.838287 \\
\hline 34 & 1 & 0 & -2.452489 & 3.900252 & -0.184928 \\
\hline 35 & 1 & 0 & -5.356948 & -1.230088 & 0.308631 \\
\hline
\end{tabular}

\title{
$\underline{\text { Structure of } \mathbf{2 3} \text { (closed form) }}$
}

\begin{abstract}
Zero-point correction= 0.291142 (Hartree/Particle) Thermal correction to Energy $=0.308783$

Thermal correction to Enthalpy $=0.309727$

Thermal correction to Gibbs Free Energy $=0.245946$

Sum of electronic and zero-point Energies= -897.991501

Sum of electronic and thermal Energies= -897.973861

Sum of electronic and thermal Enthalpies= -897.972917

Sum of electronic and thermal Free Energies= -898.036698

No imaginary frequencies
\end{abstract}

Standard orientation:

\begin{tabular}{|c|c|c|c|c|c|}
\hline \multirow{2}{*}{$\begin{array}{l}\text { Center } \\
\text { Number }\end{array}$} & \multirow{2}{*}{$\begin{array}{l}\text { Atomic } \\
\text { Number }\end{array}$} & \multirow{2}{*}{$\begin{array}{c}\text { Atomic } \\
\text { Type }\end{array}$} & \multicolumn{3}{|c|}{ Coordinates (Angstroms) } \\
\hline & & & $\mathrm{X}$ & $\mathrm{Y}$ & Z \\
\hline 1 & 6 & 0 & -1.824906 & -1.694219 & 0.125683 \\
\hline 2 & 6 & 0 & -3.138086 & -2.166601 & 0.222735 \\
\hline 3 & 6 & 0 & -4.185096 & -1.264619 & 0.174559 \\
\hline 4 & 6 & 0 & -3.973370 & 0.109301 & 0.031472 \\
\hline 5 & 6 & 0 & -2.668441 & 0.580410 & -0.063227 \\
\hline 6 & 6 & 0 & -1.572135 & -0.313249 & -0.017130 \\
\hline 7 & 6 & 0 & -0.208612 & 0.199304 & -0.115656 \\
\hline 8 & 6 & 0 & 2.167246 & -0.090791 & -0.156745 \\
\hline 9 & 6 & 0 & 2.752214 & -0.269964 & 1.261629 \\
\hline 10 & 6 & 0 & 2.895924 & -1.007659 & -1.143627 \\
\hline 11 & 6 & 0 & 2. 322272 & 1.362536 & -0.619730 \\
\hline 12 & 1 & 0 & -3.308523 & -3.230497 & 0.333410 \\
\hline 13 & 1 & 0 & -5.203423 & -1.630143 & 0.248905 \\
\hline 14 & 1 & 0 & -4.817429 & 0.783243 & -0.003159 \\
\hline 15 & 1 & 0 & -0.096602 & 1.279565 & -0.199364 \\
\hline 16 & 1 & 0 & 2.047467 & 0.134858 & 1.990051 \\
\hline 17 & 1 & 0 & 2.875991 & -1.339555 & 1.466751 \\
\hline 18 & 1 & 0 & 4.576980 & 0.106650 & 0.762202 \\
\hline 19 & 1 & 0 & 2.571539 & -0.785057 & -2.167103 \\
\hline 20 & 1 & 0 & 2.642943 & -2.046044 & -0.908927 \\
\hline 21 & 1 & 0 & 4.768978 & -1.396550 & -1.587085 \\
\hline 22 & 1 & 0 & 1.662347 & 1.545471 & -1.471330 \\
\hline 23 & 1 & 0 & 3.348987 & 1.508636 & -0.967125 \\
\hline 24 & 1 & 0 & 2.690103 & 2.259469 & 1.047272 \\
\hline 25 & 1 & 0 & 0.029636 & -2.057593 & 0.086148 \\
\hline 26 & 7 & 0 & 0.795939 & -0.592741 & -0.093120 \\
\hline 27 & 8 & 0 & 3.971187 & 0.434531 & 1.440007 \\
\hline 28 & 8 & 0 & 4.299591 & -0.792593 & -1.002421 \\
\hline 29 & 8 & 0 & 1.996160 & 2.315751 & 0.379443 \\
\hline 30 & 8 & 0 & -0.821184 & -2.577571 & 0.171931 \\
\hline
\end{tabular}




$\begin{array}{rrrrrr}31 & 8 & 0 & -2.352045 & 1.886215 & -0.202913 \\ 32 & 6 & 0 & -3.416444 & 2.828519 & -0.267805 \\ 33 & 1 & 0 & -4.067338 & 2.623736 & -1.121629 \\ 34 & 1 & 0 & -4.000930 & 2.823340 & 0.655837 \\ 35 & 1 & 0 & -2.945379 & 3.801183 & -0.394342 \\ -\end{array}$

\section{$\underline{\text { Structure of } \mathbf{T S}_{23 / 53}}$}

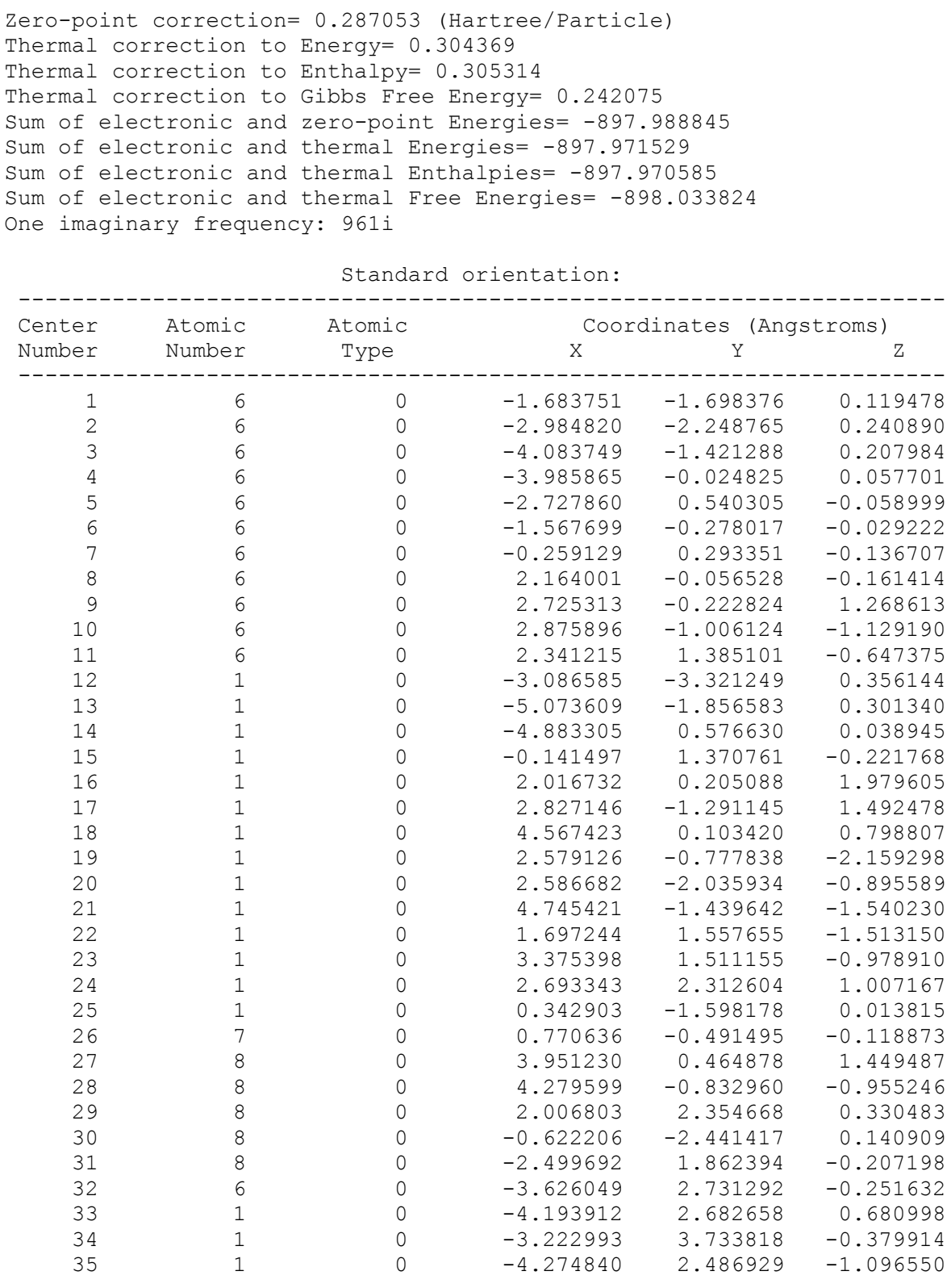

\section{$\underline{\text { Structure of } \mathbf{5 3}}$}

Zero-point correction $=0.290698$ (Hartree/Particle)

Thermal correction to Energy $=0.308549$

Thermal correction to Enthalpy= 0.309493

Thermal correction to Gibbs Free Energy= 0.244971

Sum of electronic and zero-point Energies= -897.988570

Sum of electronic and thermal Energies= -897.970719

Sum of electronic and thermal Enthalpies= -897.969775

Sum of electronic and thermal Free Energies= -898.034297

No imaginary frequencies 
Standard orientation:

\begin{tabular}{|c|c|c|c|c|c|}
\hline \multirow{2}{*}{$\begin{array}{l}\text { Center } \\
\text { Number }\end{array}$} & \multirow{2}{*}{$\begin{array}{l}\text { Atomic } \\
\text { Number }\end{array}$} & \multirow{2}{*}{$\begin{array}{c}\text { Atomic } \\
\text { Type }\end{array}$} & \multicolumn{3}{|c|}{ Coordinates (Angstroms) } \\
\hline & & & $\mathrm{X}$ & Y & Z \\
\hline-- & & & & & \\
\hline 1 & 6 & 0 & -1.847233 & -1.775398 & 0.071975 \\
\hline 2 & 6 & 0 & -3.235268 & -2.154768 & 0.139579 \\
\hline 3 & 6 & 0 & -4.218815 & -1.207043 & 0.132428 \\
\hline 4 & 6 & 0 & -3.956721 & 0.186840 & 0.059080 \\
\hline 5 & 6 & 0 & -2.649555 & 0.604067 & -0.011636 \\
\hline 6 & 6 & 0 & -1.573426 & -0.347587 & -0.007212 \\
\hline 7 & 6 & 0 & -0.254556 & 0.123577 & -0.082826 \\
\hline 8 & 6 & 0 & 2.188990 & -0.170800 & -0.127364 \\
\hline 9 & 6 & 0 & 2.631619 & 0.118036 & 1.322642 \\
\hline 10 & 6 & 0 & 3.014714 & -1.306642 & -0.735609 \\
\hline 11 & 6 & 0 & 2.345817 & 1.084890 & -1.000348 \\
\hline 12 & 1 & 0 & -3.467873 & -3.211910 & 0.196028 \\
\hline 13 & 1 & 0 & -5.256317 & -1.523318 & 0.184523 \\
\hline 14 & 1 & 0 & -4.779300 & 0.887000 & 0.057869 \\
\hline 15 & 1 & 0 & -0.074500 & 1.193124 & -0.135130 \\
\hline 16 & 1 & 0 & 1.846884 & 0.688052 & 1.823437 \\
\hline 17 & 1 & 0 & 2.759734 & -0.828930 & 1.859409 \\
\hline 18 & 1 & 0 & 4.495223 & 0.410401 & 0.916011 \\
\hline 19 & 1 & 0 & 2.803287 & -1.386259 & -1.808101 \\
\hline 20 & 1 & 0 & 2.743916 & -2.250291 & -0.248654 \\
\hline 21 & 1 & 0 & 4.926720 & -1.717482 & -0.874751 \\
\hline 22 & 1 & 0 & 1.722555 & 0.977720 & -1.891016 \\
\hline 23 & 1 & 0 & 3.387975 & 1.145135 & -1.325331 \\
\hline 24 & 1 & 0 & 2.618324 & 2.455778 & 0.329060 \\
\hline 25 & 7 & 0 & 0.805767 & -0.651696 & -0.095900 \\
\hline 26 & 8 & 0 & 3.806852 & 0.908737 & 1.375384 \\
\hline 27 & 8 & 0 & 4.387576 & -1.008755 & -0.508153 \\
\hline 28 & 8 & 0 & 1.957813 & 2.284585 & -0.353994 \\
\hline 29 & 8 & 0 & -0.918887 & -2.624865 & 0.079538 \\
\hline 30 & 1 & 0 & 0.605002 & -1.657344 & -0.015977 \\
\hline 31 & 8 & 0 & -2.270832 & 1.896761 & -0.090148 \\
\hline 32 & 6 & 0 & -3.290588 & 2.889177 & -0.106997 \\
\hline 33 & 1 & 0 & -3.954230 & 2.750048 & -0.964177 \\
\hline 34 & 1 & 0 & -3.870639 & 2.867880 & 0.819233 \\
\hline 35 & 1 & 0 & -2.777169 & 3.844463 & -0.194423 \\
\hline
\end{tabular}

\section{$\underline{\text { Structure of } \mathbf{2 4} \text { (open form) }}$}

Zero-point correction $=0.289616$ (Hartree/Particle) Thermal correction to Energy $=0.307966$ Thermal correction to Enthalpy $=0.308910$

Thermal correction to Gibbs Free Energy= 0.243698

Sum of electronic and zero-point Energies= -897.998085

Sum of electronic and thermal Energies= -897.979735

Sum of electronic and thermal Enthalpies= -897.978791

Sum of electronic and thermal Free Energies= -898.044003

No imaginary frequencies

Standard orientation:

\begin{tabular}{|c|c|c|c|c|c|}
\hline \multirow{2}{*}{$\begin{array}{l}\text { Center } \\
\text { Number }\end{array}$} & \multirow{2}{*}{$\begin{array}{l}\text { Atomic } \\
\text { Number }\end{array}$} & \multirow{2}{*}{$\begin{array}{c}\text { Atomic } \\
\text { Type }\end{array}$} & \multicolumn{3}{|c|}{ Coordinates (Angstroms) } \\
\hline & & & $\mathrm{x}$ & Y & $\mathrm{Z}$ \\
\hline 1 & 6 & 0 & -2.049063 & -1.213889 & -0.066774 \\
\hline 2 & 6 & 0 & -3.427992 & -1.342874 & 0.075912 \\
\hline 3 & 6 & 0 & -4.216556 & -0.203205 & 0.155873 \\
\hline 4 & 6 & 0 & -3.640714 & 1.064244 & 0.084138 \\
\hline 5 & 6 & 0 & -2.264933 & 1.195748 & -0.069248 \\
\hline 6 & 6 & 0 & -1.441495 & 0.053504 & -0.132932 \\
\hline 7 & 6 & 0 & 0.011515 & 0.242177 & -0.313255 \\
\hline 8 & 6 & 0 & 2.310911 & -0.240745 & -0.019939 \\
\hline 9 & 6 & 0 & 2.865656 & 0.175173 & 1.360320 \\
\hline 10 & 6 & 0 & 2.929234 & -1.576125 & -0.445247 \\
\hline 11 & 6 & 0 & 2.662983 & 0.819186 & -1.071000 \\
\hline 12 & 1 & 0 & -3.888598 & -2.324346 & 0.121316 \\
\hline 13 & 1 & 0 & -4.268033 & 1.947902 & 0.147408 \\
\hline 14 & 1 & 0 & 0.284158 & 1.117768 & -0.906972 \\
\hline
\end{tabular}




$\begin{array}{rrrrrr}15 & 1 & 0 & 2.209996 & 0.933376 & 1.792750 \\ 16 & 1 & 0 & 2.863740 & -0.694190 & 2.027523 \\ 17 & 1 & 0 & 4.723001 & 0.098699 & 0.850955 \\ 18 & 1 & 0 & 2.643608 & -1.806651 & -1.478850 \\ 19 & 1 & 0 & 2.547174 & -2.362291 & 0.212058 \\ 20 & 1 & 0 & 4.741481 & -2.326726 & -0.551815 \\ 21 & 1 & 0 & 2.052787 & 0.667705 & -1.964894 \\ 22 & 1 & 0 & 3.706956 & 0.682936 & -1.368172 \\ 23 & 1 & 0 & 3.108185 & 2.323956 & 0.048421 \\ 24 & 1 & 0 & -1.843346 & -3.100016 & -0.199730 \\ 25 & 7 & 0 & 0.889960 & -0.519068 & 0.197593 \\ 26 & 8 & 0 & 4.158534 & 0.756912 & 1.277075 \\ 27 & 8 & 0 & 4.349719 & -1.476097 & -0.329454 \\ 28 & 8 & 0 & 2.445502 & 2.151670 & -0.631658 \\ 29 & 8 & 0 & -1.274773 & -2.319467 & -0.178373 \\ 30 & 6 & 0 & -1.669701 & 2.579824 & -0.139270 \\ 31 & 1 & 0 & -0.893363 & 2.719353 & 0.617101 \\ 32 & 1 & 0 & -1.211316 & 2.765293 & -1.115166 \\ 33 & 1 & 0 & -2.442091 & 3.332857 & 0.018576 \\ 34 & 8 & 0 & -5.552947 & -0.380463 & 0.301914 \\ 35 & 1 & 0 & -5.991406 & 0.479317 & 0.338833 \\ -----------------------------------------------------\end{array}$

\section{$\underline{\text { Structure of } \mathbf{2 4} \text { (closed form) }}$}

Zero-point correction $=0.289453$ (Hartree/Particle) Thermal correction to Energy $=0.307465$

Thermal correction to Enthalpy $=0.308410$

Thermal correction to Gibbs Free Energy= 0.243912

Sum of electronic and zero-point Energies $=\mathbf{- 8 9 8 . 0 1 6 1 7 3}$

Sum of electronic and thermal Energies= -897.998162

Sum of electronic and thermal Enthalpies= -897.997217

Sum of electronic and thermal Free Energies= -898.061715

No imaginary frequencies

Standard orientation:

\begin{tabular}{|c|c|c|c|c|c|}
\hline \multirow{2}{*}{$\begin{array}{l}\text { Center } \\
\text { Number }\end{array}$} & \multirow{2}{*}{$\begin{array}{l}\text { Atomic } \\
\text { Number }\end{array}$} & \multirow{2}{*}{$\begin{array}{c}\text { Atomic } \\
\text { Type }\end{array}$} & \multicolumn{3}{|c|}{ Coordinates (Angstroms) } \\
\hline & & & $\mathrm{X}$ & Y & Z \\
\hline 1 & 6 & 0 & -1.893305 & -1.154462 & 0.109534 \\
\hline 2 & 6 & 0 & -3.252561 & -1.446952 & 0.200404 \\
\hline 3 & 6 & 0 & -4.172410 & -0.415463 & 0.099010 \\
\hline 4 & 6 & 0 & -3.758360 & 0.908453 & -0.092650 \\
\hline 5 & 6 & 0 & -2.408313 & 1.211443 & -0.183943 \\
\hline 6 & 6 & 0 & -1.449771 & 0.178424 & -0.081523 \\
\hline 7 & 6 & 0 & -0.024000 & 0.473523 & -0.171872 \\
\hline 8 & 6 & 0 & 2.287849 & -0.162468 & -0.152848 \\
\hline 9 & 6 & 0 & 2.826666 & -0.470094 & 1.262272 \\
\hline 10 & 6 & 0 & 2.878853 & -1.148151 & -1.165444 \\
\hline 11 & 6 & 0 & 2.667642 & 1.262605 & -0.567873 \\
\hline 12 & 1 & 0 & -3.579971 & -2.468798 & 0.346892 \\
\hline 13 & 1 & 0 & -4.500042 & 1.697351 & -0.172142 \\
\hline 14 & 1 & 0 & 0.258241 & 1.519801 & -0.290269 \\
\hline 15 & 1 & 0 & 2.181865 & 0.012281 & 1.999124 \\
\hline 16 & 1 & 0 & 2.788284 & -1.552010 & 1.433848 \\
\hline 17 & 1 & 0 & 4.692736 & -0.358086 & 0.791450 \\
\hline 18 & 1 & 0 & 2.600697 & -0.848493 & -2.182950 \\
\hline 19 & 1 & 0 & 2.469347 & -2.142868 & -0.965586 \\
\hline 20 & 1 & 0 & 4.671403 & -1.827679 & -1.588786 \\
\hline 21 & 1 & 0 & 2.068197 & 1.566994 & -1.429471 \\
\hline 22 & 1 & 0 & 3.713915 & 1.263403 & -0.886408 \\
\hline 23 & 1 & 0 & 3.119275 & 2.045884 & 1.135736 \\
\hline 24 & 1 & 0 & -0.108364 & -1.772764 & 0.117948 \\
\hline 25 & 7 & 0 & 0.857225 & -0.453195 & -0.102986 \\
\hline 26 & 8 & 0 & 4.133733 & 0.040891 & 1.470842 \\
\hline 27 & 8 & 0 & 4.297818 & -1.152365 & -1.013112 \\
\hline 28 & 8 & 0 & 2.459415 & 2.223988 & 0.454545 \\
\hline 29 & 8 & 0 & -1.025390 & -2.167354 & 0.208880 \\
\hline 30 & 6 & 0 & -1.993004 & 2.645512 & -0.395301 \\
\hline 31 & 1 & 0 & -1.355108 & 3.000142 & 0.418279 \\
\hline 32 & 1 & 0 & -1.434028 & 2.762935 & -1.327106 \\
\hline 33 & 1 & 0 & -2.871829 & 3.288652 & -0.442182 \\
\hline 34 & 8 & 0 & -5.484031 & -0.740677 & 0.191756 \\
\hline
\end{tabular}




\section{$\underline{\text { Structure of } \mathbf{T S}_{2}} \underline{\underline{2454}}$}

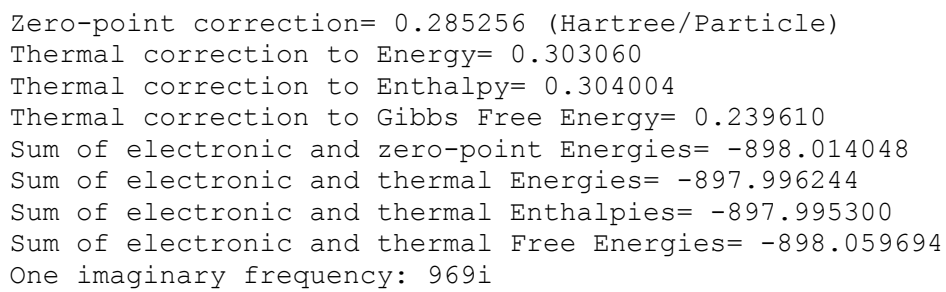

Standard orientation:

\begin{tabular}{|c|c|c|c|c|c|}
\hline \multirow{2}{*}{$\begin{array}{l}\text { Center } \\
\text { Number }\end{array}$} & \multirow{2}{*}{$\begin{array}{l}\text { Atomic } \\
\text { Number }\end{array}$} & \multirow{2}{*}{$\begin{array}{c}\text { Atomic } \\
\text { Type }\end{array}$} & \multicolumn{3}{|c|}{ Coordinates (Angstroms) } \\
\hline & & & $\mathrm{X}$ & Y & Z \\
\hline 1 & 6 & 0 & -1.813806 & -1.152581 & 0.093228 \\
\hline 2 & 6 & 0 & -3.183099 & -1.487142 & 0.161289 \\
\hline 3 & 6 & 0 & -4.134003 & -0.490666 & 0.092089 \\
\hline 4 & 6 & 0 & -3.782790 & 0.869660 & -0.047527 \\
\hline 5 & 6 & 0 & -2.455453 & 1.235315 & -0.120807 \\
\hline 6 & 6 & 0 & -1.454431 & 0.233135 & -0.050572 \\
\hline 7 & 6 & 0 & -0.064175 & 0.557766 & -0.136984 \\
\hline 8 & 6 & 0 & 2.264600 & -0.185809 & -0.134270 \\
\hline 9 & 6 & 0 & 2.790236 & -0.249502 & 1.316364 \\
\hline 10 & 6 & 0 & 2.823281 & -1.348927 & -0.958786 \\
\hline 11 & 6 & 0 & 2.670929 & 1.137380 & -0.796041 \\
\hline 12 & 1 & 0 & -3.478104 & -2.523946 & 0.267407 \\
\hline 13 & 1 & 0 & -4.564062 & 1.621660 & -0.099964 \\
\hline 14 & 1 & 0 & 0.251926 & 1.593329 & -0.245755 \\
\hline 15 & 1 & 0 & 2.161645 & 0.379364 & 1.949009 \\
\hline 16 & 1 & 0 & 2.718653 & -1.280306 & 1.682358 \\
\hline 17 & 1 & 0 & 4.657999 & -0.283533 & 0.835546 \\
\hline 18 & 1 & 0 & 2.561983 & -1.213605 & -2.014842 \\
\hline 19 & 1 & 0 & 2.382239 & -2.284792 & -0.600777 \\
\hline 20 & 1 & 0 & 4.601318 & -2.115662 & -1.276182 \\
\hline 21 & 1 & 0 & 2.045944 & 1.305904 & -1.676427 \\
\hline 22 & 1 & 0 & 3.705147 & 1.047976 & -1.139862 \\
\hline 23 & 1 & 0 & 3.202814 & 2.190778 & 0.730404 \\
\hline 24 & 1 & 0 & 0.200522 & -1.406722 & 0.053883 \\
\hline 25 & 7 & 0 & 0.819313 & -0.390321 & -0.082830 \\
\hline 26 & 8 & 0 & 4.112820 & 0.249879 & 1.429056 \\
\hline 27 & 8 & 0 & 4.237733 & -1.370659 & -0.786231 \\
\hline 28 & 8 & 0 & 2.522684 & 2.265484 & 0.049727 \\
\hline 29 & 8 & 0 & -0.894742 & -2.065593 & 0.156216 \\
\hline 30 & 6 & 0 & -2.086869 & 2.687861 & -0.273982 \\
\hline 31 & 1 & 0 & -1.453408 & 3.025258 & 0.550520 \\
\hline 32 & 1 & 0 & -1.536074 & 2.859841 & -1.202499 \\
\hline 33 & 1 & 0 & -2.983839 & 3.307034 & -0.290089 \\
\hline 34 & 8 & 0 & -5.435580 & -0.860393 & 0.163061 \\
\hline 35 & 1 & 0 & -5.999737 & -0.079011 & 0.098461 \\
\hline
\end{tabular}

\section{Structure of 54}

Zero-point correction $=0.289349$ (Hartree/Particle)

Thermal correction to Energy $=0.307592$

Thermal correction to Enthalpy $=0.308536$

Thermal correction to Gibbs Free Energy $=0.243410$

Sum of electronic and zero-point Energies= -898.013410

Sum of electronic and thermal Energies= -897.995168

Sum of electronic and thermal Enthalpies= -897.994223

Sum of electronic and thermal Free Energies= $\mathbf{- 8 9 8 . 0 5 9 3 5 0}$

No imaginary frequencies

Standard orientation:

Center Atomic Atomic Coordinates (Angstroms)




\begin{tabular}{|c|c|c|c|c|c|}
\hline Number & Number & Type & $\mathrm{X}$ & Y & Z \\
\hline 1 & 6 & 0 & -1.908608 & -1.253477 & 0.019630 \\
\hline 2 & 6 & 0 & -3.324728 & -1.456198 & 0.100575 \\
\hline 3 & 6 & 0 & -4.182562 & -0.390216 & 0.094748 \\
\hline 4 & 6 & 0 & -3.721715 & 0.955800 & 0.011364 \\
\hline 5 & 6 & 0 & -2.381000 & 1.218780 & -0.065938 \\
\hline 6 & 6 & 0 & -1.445537 & 0.130570 & -0.065330 \\
\hline 7 & 6 & 0 & -0.071740 & 0.403563 & -0.131428 \\
\hline 8 & 6 & 0 & 2.306748 & -0.240306 & -0.130004 \\
\hline 9 & 6 & 0 & 2.731267 & 0.015646 & 1.331246 \\
\hline 10 & 6 & 0 & 2.977815 & -1.499244 & -0.683761 \\
\hline 11 & 6 & 0 & 2.677490 & 0.957871 & -1.018951 \\
\hline 12 & 1 & 0 & -3.704848 & -2.468656 & 0.166522 \\
\hline 13 & 1 & 0 & -4.444247 & 1.766128 & 0.013530 \\
\hline 14 & 1 & 0 & 0.274702 & 1.432285 & -0.176418 \\
\hline 15 & 1 & 0 & 2.029736 & 0.720738 & 1.781663 \\
\hline 16 & 1 & 0 & 2.674628 & -0.924348 & 1.892442 \\
\hline 17 & 1 & 0 & 4.629862 & -0.003385 & 0.990810 \\
\hline 18 & 1 & 0 & 2.793185 & -1.575457 & -1.761444 \\
\hline 19 & 1 & 0 & 2.553081 & -2.378864 & -0.187546 \\
\hline 20 & 1 & 0 & 4.808646 & -2.198555 & -0.734542 \\
\hline 21 & 1 & 0 & 2.077004 & 0.923046 & -1.930728 \\
\hline 22 & 1 & 0 & 3.728585 & 0.859681 & -1.304621 \\
\hline 23 & 1 & 0 & 3.098905 & 2.307864 & 0.295312 \\
\hline 24 & 7 & 0 & 0.869118 & -0.516222 & -0.140183 \\
\hline 25 & 8 & 0 & 4.016913 & 0.604714 & 1.424264 \\
\hline 26 & 8 & 0 & 4.371344 & -1.403787 & -0.411147 \\
\hline 27 & 8 & 0 & 2.445369 & 2.216306 & -0.409165 \\
\hline 28 & 8 & 0 & -1.103302 & -2.222711 & 0.024172 \\
\hline 29 & 1 & 0 & 0.518739 & -1.481257 & -0.066391 \\
\hline 30 & 8 & 0 & -5.511452 & -0.638463 & 0.173133 \\
\hline 31 & 1 & 0 & -5.999705 & 0.194717 & 0.159279 \\
\hline 32 & 6 & 0 & -1.909039 & 2.646917 & -0.142477 \\
\hline 33 & 1 & 0 & -1.271157 & 2.899679 & 0.708810 \\
\hline 34 & 1 & 0 & -1.330345 & 2.827663 & -1.052182 \\
\hline 35 & 1 & 0 & -2.761929 & 3.325563 & -0.141309 \\
\hline
\end{tabular}

\section{$\underline{\text { Structure of } 27 \text { (open form) }}$}

Zero-point correction $=0.241095$ (Hartree/Particle)

Thermal correction to Energy $=0.258528$

Thermal correction to Enthalpy $=0.259472$

Thermal correction to Gibbs Free Energy= 0.195757

Sum of electronic and zero-point Energies= -981.986441

Sum of electronic and thermal Energies= -981.969008

Sum of electronic and thermal Enthalpies= -981.968064

Sum of electronic and thermal Free Energies= -982.031779

No imaginary frequencies

Standard orientation:

\begin{tabular}{|c|c|c|c|c|c|}
\hline \multirow{2}{*}{$\begin{array}{l}\text { Center } \\
\text { Number }\end{array}$} & \multirow{2}{*}{$\begin{array}{l}\text { Atomic } \\
\text { Number }\end{array}$} & \multirow{2}{*}{$\begin{array}{c}\text { Atomic } \\
\text { Type }\end{array}$} & \multicolumn{3}{|c|}{ Coordinates (Angstroms) } \\
\hline & & & $\mathrm{X}$ & $\mathrm{Y}$ & Z \\
\hline & & & & (1) & --ー-ー-ー-ー- \\
\hline 1 & 6 & 0 & -1.981931 & -1.008879 & 0.061226 \\
\hline 2 & 6 & 0 & -3.374465 & -1.045156 & 0.123374 \\
\hline 3 & 6 & 0 & -4.181673 & 0.067667 & 0.055977 \\
\hline 4 & 6 & 0 & -3.539458 & 1.284781 & -0.085496 \\
\hline 5 & 6 & 0 & -2.169431 & 1.390510 & -0.158303 \\
\hline 6 & 6 & 0 & -1.366029 & 0.243733 & -0.083660 \\
\hline 7 & 6 & 0 & 0.091838 & 0.462843 & -0.177494 \\
\hline 8 & 6 & 0 & 2.383272 & -0.110759 & -0.135220 \\
\hline 9 & 6 & 0 & 2.918441 & -0.152108 & 1.312712 \\
\hline 10 & 6 & 0 & 3.011291 & -1.242510 & -0.954946 \\
\hline 11 & 6 & 0 & 2.734404 & 1.230326 & -0.794965 \\
\hline 12 & 1 & 0 & -1.710957 & 2.366140 & -0.275349 \\
\hline 13 & 1 & 0 & 0.356437 & 1.508013 & -0.364688 \\
\hline 14 & 1 & 0 & 2.268149 & 0.450086 & 1.949867 \\
\hline 15 & 1 & 0 & 2.892014 & -1.184674 & 1.678447 \\
\hline 16 & 1 & 0 & 4.780619 & -0.095360 & 0.812851 \\
\hline 17 & 1 & 0 & 2.718441 & -1.145186 & -2.007493 \\
\hline 18 & 1 & 0 & 2.638980 & -2.196356 & -0.571122 \\
\hline
\end{tabular}




$\begin{array}{rrrrrr}19 & 1 & 0 & 4.824791 & -1.917019 & -1.285476 \\ 20 & 1 & 0 & 2.101424 & 1.378881 & -1.673604 \\ 21 & 1 & 0 & 3.769673 & 1.184855 & -1.145394 \\ 22 & 1 & 0 & 3.226615 & 2.292920 & 0.736673 \\ 23 & 1 & 0 & -1.879235 & -2.900213 & 0.213177 \\ 24 & 7 & 0 & 0.961331 & -0.449470 & -0.044582 \\ 25 & 8 & 0 & 4.221413 & 0.401591 & 1.424652 \\ 26 & 8 & 0 & 4.431301 & -1.170799 & -0.821498 \\ 27 & 8 & 0 & 2.549367 & 2.354357 & 0.052194 \\ 28 & 8 & 0 & -1.271458 & -2.152141 & 0.130271 \\ 29 & 9 & 0 & -4.291737 & 2.403611 & -0.157877 \\ 30 & 9 & 0 & -3.937005 & -2.266471 & 0.254664 \\ 31 & 1 & 0 & -5.260128 & -0.016816 & 0.108914 \\ -\end{array}$

\section{$\underline{\text { Structure of } \mathbf{2 7} \text { (closed form) }}$}

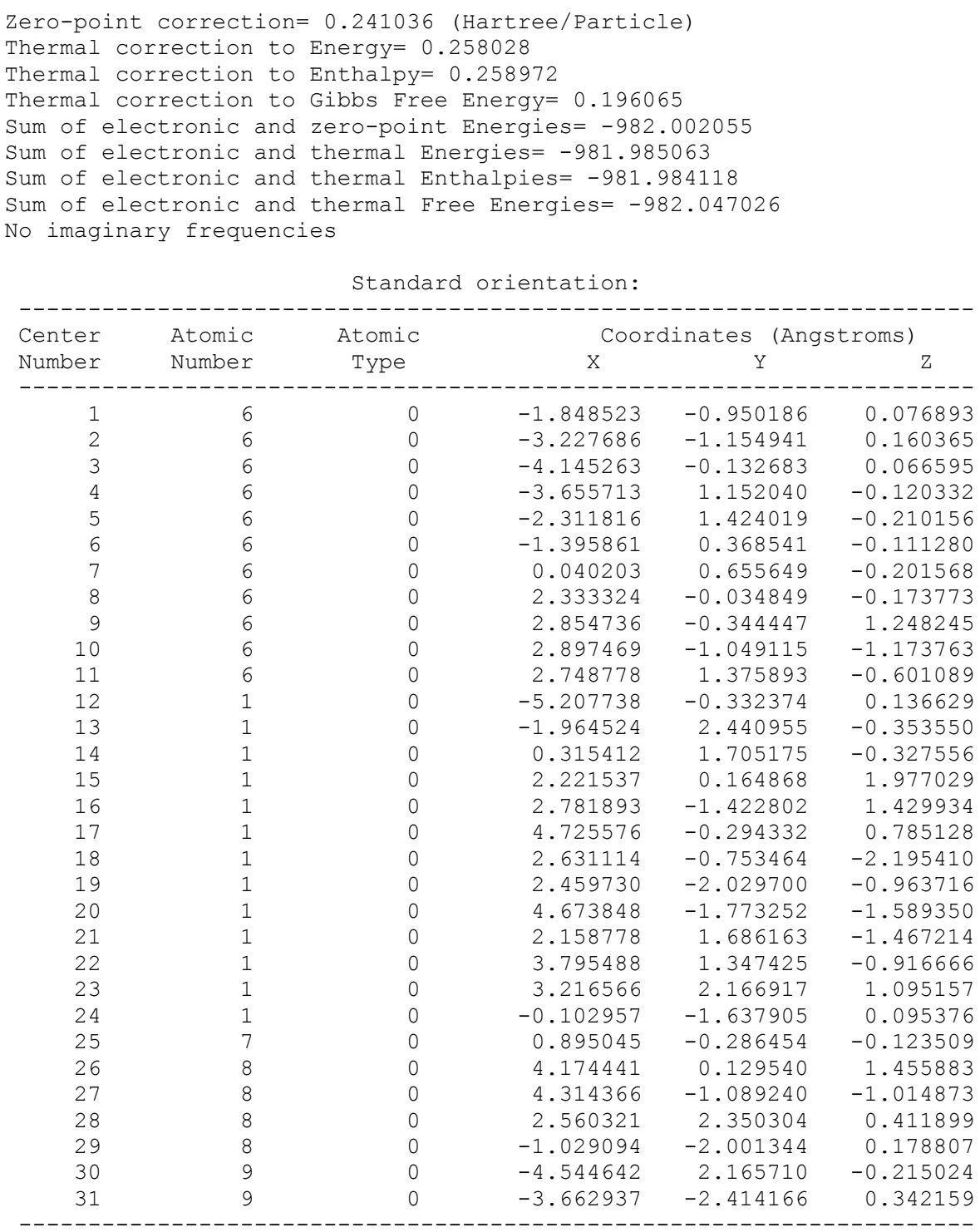

\section{$\underline{\text { Structure of } \mathbf{T S}} \underline{27157}_{27}$}

Zero-point correction= 0.237142 (Hartree/Particle)

Thermal correction to Energy $=0.253741$

Thermal correction to Enthalpy $=0.254685$

Thermal correction to Gibbs Free Energy= 0.192827

Sum of electronic and zero-point Energies= -981.998939 
Sum of electronic and thermal Energies=-981.982341

Sum of electronic and thermal Enthalpies= -981.981397

Sum of electronic and thermal Free Energies= -982.043255

one imaginary frequency: 968i

Standard orientation:

\begin{tabular}{|c|c|c|c|c|c|}
\hline \multirow{2}{*}{$\begin{array}{l}\text { Center } \\
\text { Number }\end{array}$} & \multirow{2}{*}{$\begin{array}{l}\text { Atomic } \\
\text { Number }\end{array}$} & \multirow{2}{*}{$\begin{array}{c}\text { Atomic } \\
\text { Type }\end{array}$} & \multicolumn{3}{|c|}{ Coordinates (Angstroms) } \\
\hline & & & $\mathrm{X}$ & $\mathrm{Y}$ & Z \\
\hline 1 & 6 & 0 & -1.757926 & -0.933076 & 0.074326 \\
\hline 2 & 6 & 0 & -3.146490 & -1.200889 & 0.155258 \\
\hline 3 & 6 & 0 & -4.108405 & -0.229717 & 0.077862 \\
\hline 4 & 6 & 0 & -3.699487 & 1.096056 & -0.092315 \\
\hline 5 & 6 & 0 & -2.383289 & 1.449601 & -0.184411 \\
\hline 6 & 6 & 0 & -1.408554 & 0.436060 & -0.101343 \\
\hline 7 & 6 & 0 & -0.007011 & 0.757902 & -0.196224 \\
\hline 8 & 6 & 0 & 2.306510 & -0.035056 & -0.167138 \\
\hline 9 & 6 & 0 & 2.816453 & -0.236380 & 1.278798 \\
\hline 10 & 6 & 0 & 2.833408 & -1.143451 & -1.083865 \\
\hline 11 & 6 & 0 & 2.749739 & 1.327341 & -0.708703 \\
\hline 12 & 1 & 0 & -5.159439 & -0.486035 & 0.146558 \\
\hline 13 & 1 & 0 & -2.092513 & 2.485710 & -0.317565 \\
\hline 14 & 1 & 0 & 0.299280 & 1.795656 & -0.320771 \\
\hline 15 & 1 & 0 & 2.193005 & 0.348386 & 1.957065 \\
\hline 16 & 1 & 0 & 2.720277 & -1.293348 & 1.552098 \\
\hline 17 & 1 & 0 & 4.690489 & -0.275649 & 0.824233 \\
\hline 18 & 1 & 0 & 2.589886 & -0.910122 & -2.126677 \\
\hline 19 & 1 & 0 & 2.357128 & -2.090817 & -0.811362 \\
\hline 20 & 1 & 0 & 4.592214 & -1.933714 & -1.446087 \\
\hline 21 & 1 & 0 & 2.158442 & 1.578569 & -1.592562 \\
\hline 22 & 1 & 0 & 3.793069 & 1.248090 & -1.025632 \\
\hline 23 & 1 & 0 & 3.253257 & 2.248697 & 0.910426 \\
\hline 24 & 1 & 0 & 0.231119 & -1.212847 & 0.033200 \\
\hline 25 & 7 & 0 & 0.854532 & -0.199389 & -0.124374 \\
\hline 26 & 8 & 0 & 4.144820 & 0.225885 & 1.443890 \\
\hline 27 & 8 & 0 & 4.243104 & -1.223394 & -0.897591 \\
\hline 28 & 8 & 0 & 2.586510 & 2.380108 & 0.225057 \\
\hline 29 & 8 & 0 & -0.867613 & -1.870718 & 0.153802 \\
\hline 30 & 9 & 0 & -4.661316 & 2.047756 & -0.166564 \\
\hline 31 & 9 & 0 & -3.520038 & -2.484674 & 0.319116 \\
\hline
\end{tabular}

\section{Structure of $\mathbf{5 7}$}

Zero-point correction $=0.241223$ (Hartree/Particle)

Thermal correction to Energy $=0.258369$

Thermal correction to Enthalpy $=0.259313$

Thermal correction to Gibbs Free Energy= 0.196203

Sum of electronic and zero-point Energies= -981.998779

Sum of electronic and thermal Energies= -981.981634

Sum of electronic and thermal Enthalpies= -981.980690

Sum of electronic and thermal Free Energies= -982.043799

No imaginary frequencies

Standard orientation:

\begin{tabular}{|c|c|c|c|c|c|}
\hline \multirow{2}{*}{$\begin{array}{l}\text { Center } \\
\text { Number }\end{array}$} & \multirow{2}{*}{$\begin{array}{l}\text { Atomic } \\
\text { Number }\end{array}$} & \multirow{2}{*}{$\begin{array}{c}\text { Atomic } \\
\text { Type }\end{array}$} & \multicolumn{3}{|c|}{ Coordinates (Angstroms) } \\
\hline & & & $\mathrm{X}$ & $\mathrm{Y}$ & Z \\
\hline 1 & 6 & 0 & -1.851018 & -1.026568 & 0.056807 \\
\hline 2 & 6 & 0 & -3.288574 & -1.153252 & 0.132055 \\
\hline 3 & 6 & 0 & -4.153867 & -0.109552 & 0.072933 \\
\hline 4 & 6 & 0 & -3.627742 & 1.193184 & -0.070589 \\
\hline 5 & 6 & 0 & -2.297656 & 1.433764 & -0.148880 \\
\hline 6 & 6 & 0 & -1.391875 & 0.334619 & -0.086832 \\
\hline 7 & 6 & 0 & -0.008868 & 0.605002 & -0.164878 \\
\hline 8 & 6 & 0 & 2.362756 & -0.091876 & -0.150164 \\
\hline 9 & 6 & 0 & 2.858506 & -0.106781 & 1.313017 \\
\hline 10 & 6 & 0 & 2.962321 & -1.258122 & -0.940910 \\
\hline 11 & 6 & 0 & 2.735406 & 1.227342 & -0.837112 \\
\hline 12 & 1 & 0 & -5.224473 & -0.270813 & 0.133378 \\
\hline 13 & 1 & 0 & -1.920952 & 2.444927 & -0.257215 \\
\hline 14 & 1 & 0 & 0.320136 & 1.637183 & -0.254321 \\
\hline
\end{tabular}




\begin{tabular}{|c|c|c|c|c|c|}
\hline 15 & 1 & 0 & 2.194939 & 0.512918 & 1.918666 \\
\hline 16 & 1 & 0 & 2.814956 & -1.131313 & 1.699831 \\
\hline 17 & 1 & 0 & 4.741190 & -0.097290 & 0.889133 \\
\hline 18 & 1 & 0 & 2.729785 & -1.143596 & -2.005845 \\
\hline 19 & 1 & 0 & 2.529374 & -2.197758 & -0.580969 \\
\hline 20 & 1 & 0 & 4.764560 & -1.983184 & -1.197413 \\
\hline 21 & 1 & 0 & 2.121864 & 1.352652 & -1.732526 \\
\hline 22 & 1 & 0 & 3.777845 & 1.157592 & -1.158404 \\
\hline 23 & 1 & 0 & 3.211700 & 2.337210 & 0.668404 \\
\hline 24 & 7 & 0 & 0.914393 & -0.320088 & -0.129652 \\
\hline 25 & 8 & 0 & 4.157866 & 0.442798 & 1.438391 \\
\hline 26 & 8 & 0 & 4.368170 & -1.245466 & -0.721456 \\
\hline 27 & 8 & 0 & 2.537464 & 2.367894 & -0.021663 \\
\hline 28 & 8 & 0 & -1.093811 & -2.026673 & 0.113616 \\
\hline 29 & 1 & 0 & 0.558239 & -1.279166 & -0.010495 \\
\hline 30 & 9 & 0 & -4.517477 & 2.216479 & -0.127506 \\
\hline 31 & 9 & 0 & -3.768497 & -2.404671 & 0.268789 \\
\hline
\end{tabular}

\title{
$\underline{\text { Structure of } \mathbf{3 0} \text { (open form) }}$
}

\begin{abstract}
Zero-point correction= 0.262694 (Hartree/Particle) Thermal correction to Energy $=0.283483$

Thermal correction to Enthalpy $=0.284428$

Thermal correction to Gibbs Free Energy $=0.211205$

Sum of electronic and zero-point Energies= -1192.469368

Sum of electronic and thermal Energies= -1192.448579

Sum of electronic and thermal Enthalpies= -1192.447635

Sum of electronic and thermal Free Energies= -1192.520858

No imaginary frequencies
\end{abstract}

Standard orientation:

\begin{tabular}{|c|c|c|c|c|c|}
\hline \multirow{2}{*}{$\begin{array}{l}\text { Center } \\
\text { Number }\end{array}$} & \multirow{2}{*}{$\begin{array}{l}\text { Atomic } \\
\text { Number }\end{array}$} & \multirow{2}{*}{$\begin{array}{c}\text { Atomic } \\
\text { Type }\end{array}$} & \multicolumn{3}{|c|}{ Coordinates (Angstroms) } \\
\hline & & & $\mathrm{X}$ & Y & Z \\
\hline 1 & 6 & 0 & 1.179701 & 1.058590 & -0.013397 \\
\hline 2 & 6 & 0 & 2.593227 & 1.121292 & 0.056159 \\
\hline 3 & 6 & 0 & 3.394807 & -0.011029 & 0.033523 \\
\hline 4 & 6 & 0 & 2.773965 & -1.233356 & -0.062076 \\
\hline 5 & 6 & 0 & 1.389532 & -1.348940 & -0.132218 \\
\hline 6 & 6 & 0 & 0.580687 & -0.225567 & -0.107010 \\
\hline 7 & 6 & 0 & -0.877784 & -0.472676 & -0.177803 \\
\hline 8 & 6 & 0 & -3.179291 & 0.054874 & -0.164024 \\
\hline 9 & 6 & 0 & -3.715695 & 0.352716 & 1.255700 \\
\hline 10 & 6 & 0 & -3.830782 & 1.005967 & -1.172817 \\
\hline 11 & 6 & 0 & -3.507378 & -1.388974 & -0.562597 \\
\hline 12 & 1 & 0 & 4. 471346 & 0.077303 & 0.088988 \\
\hline 13 & 1 & 0 & 0.935626 & -2.330302 & -0.207530 \\
\hline 14 & 1 & 0 & -1.116300 & -1.536653 & -0.262493 \\
\hline 15 & 1 & 0 & -3.037469 & -0.084582 & 1.990547 \\
\hline 16 & 1 & 0 & -3.735274 & 1.436769 & 1.413318 \\
\hline 17 & 1 & 0 & -5.577505 & 0.131248 & 0.806223 \\
\hline 18 & 1 & 0 & -3.552140 & 0.716890 & -2.193298 \\
\hline 19 & 1 & 0 & -3.470455 & 2.020357 & -0.980557 \\
\hline 20 & 1 & 0 & -5.665487 & 1.565550 & -1.597817 \\
\hline 21 & 1 & 0 & -2.899012 & -1.680436 & -1.422389 \\
\hline 22 & 1 & 0 & -4.553261 & -1.432273 & -0.879695 \\
\hline 23 & 1 & 0 & -3.925492 & -2.167536 & 1.150559 \\
\hline 24 & 1 & 0 & 0.971915 & 2.917108 & 0.073234 \\
\hline 25 & 7 & 0 & -1.764367 & 0.428929 & -0.127570 \\
\hline 26 & 8 & 0 & -4.990695 & -0.226057 & 1.485676 \\
\hline 27 & 8 & 0 & -5.246285 & 0.937072 & -1.001007 \\
\hline 28 & 8 & 0 & -3.262744 & -2.332796 & 0.468802 \\
\hline 29 & 8 & 0 & 0.405431 & 2.123373 & 0.000233 \\
\hline 30 & 7 & 0 & 3.598040 & -2.444434 & -0.094871 \\
\hline 31 & 8 & 0 & 4.803893 & -2.313246 & -0.040442 \\
\hline 32 & 8 & 0 & 3.027684 & -3.512935 & -0.177932 \\
\hline 33 & 7 & 0 & 3.280084 & 2.403253 & 0.147700 \\
\hline 34 & 8 & 0 & 4.485284 & 2.420229 & 0.183048 \\
\hline 35 & 8 & 0 & 2.596342 & 3.426225 & 0.184554 \\
\hline
\end{tabular}




\section{$\underline{\text { Structure of } \mathbf{3 0} \text { (closed form) }}$}

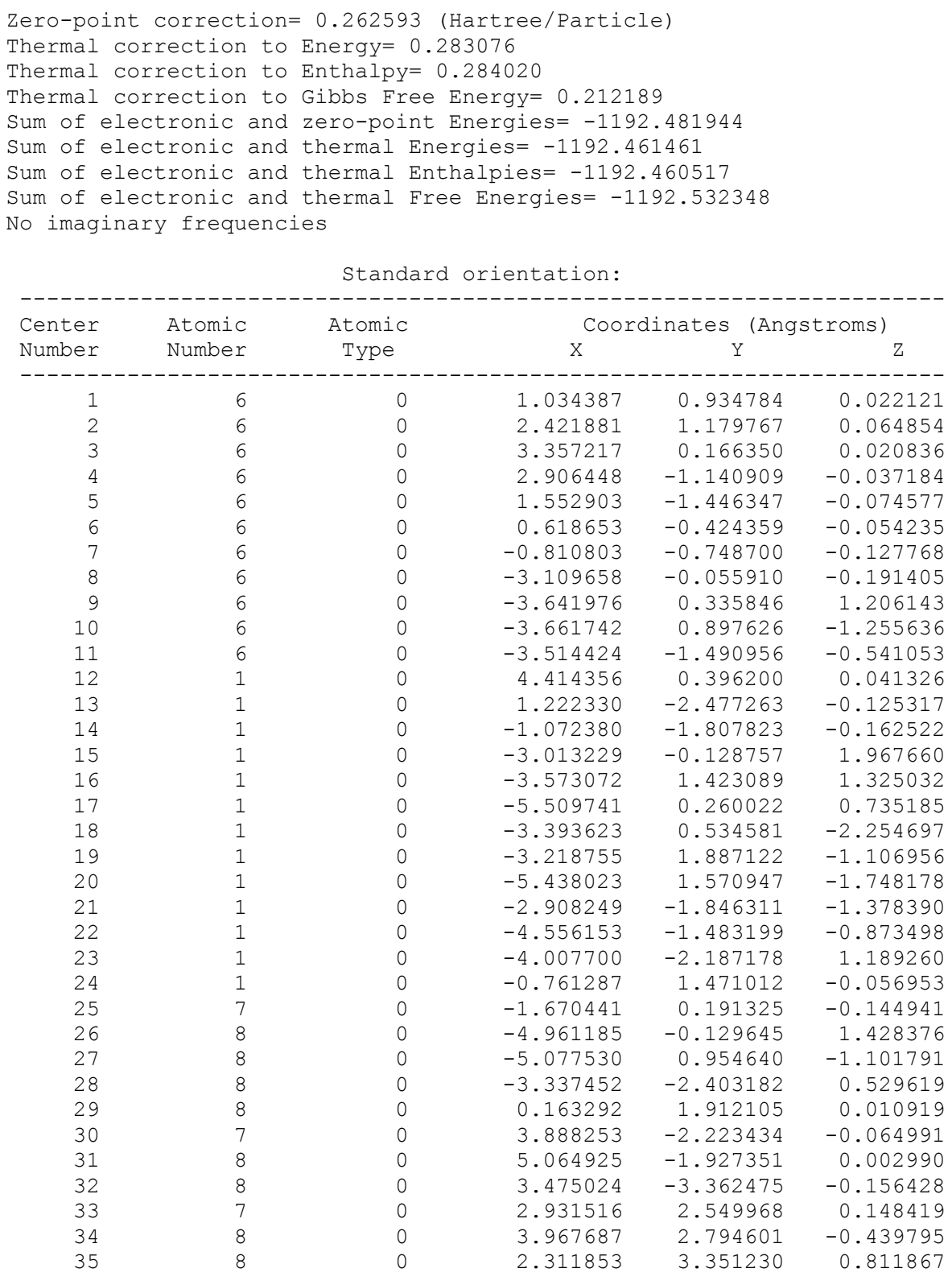

\section{$\underline{\text { Structure of } \mathbf{T S}_{30 / 60}}$}

Zero-point correction=0.258934 (Hartree/Particle)

Thermal correction to Energy $=0.279161$

Thermal correction to Enthalpy= 0.280105

Thermal correction to Gibbs Free Energy= 0.208632

Sum of electronic and zero-point Energies= -1192.483648

Sum of electronic and thermal Energies= -1192.463422

Sum of electronic and thermal Enthalpies= -1192.462477

Sum of electronic and thermal Free Energies= -1192.533951

one imaginary frequency: 1049i

Standard orientation:

\begin{tabular}{|c|c|c|c|c|c|}
\hline \multirow{2}{*}{$\begin{array}{l}\text { Center } \\
\text { Number }\end{array}$} & \multirow{2}{*}{$\begin{array}{l}\text { Atomic } \\
\text { Number }\end{array}$} & \multirow{2}{*}{$\begin{array}{c}\text { Atomic } \\
\text { Type }\end{array}$} & \multicolumn{3}{|c|}{ Coordinates (Angstroms) } \\
\hline & & & $\mathrm{X}$ & Y & Z \\
\hline 1 & 6 & 0 & 0.978894 & 0.912014 & 0.019575 \\
\hline 2 & 6 & 0 & 2.372218 & 1.197325 & 0.066659 \\
\hline
\end{tabular}




\begin{tabular}{|c|c|c|c|c|c|}
\hline 3 & 6 & 0 & 3.335516 & 0.214022 & 0.024775 \\
\hline 4 & 6 & 0 & 2.937475 & -1.114629 & -0.034631 \\
\hline 5 & 6 & 0 & 1.596390 & -1.470835 & -0.076396 \\
\hline 6 & 6 & 0 & 0.630983 & -0.480538 & -0.057670 \\
\hline 7 & 6 & 0 & -0.778586 & -0.830637 & -0.133929 \\
\hline 8 & 6 & 0 & -3.089927 & -0.073674 & -0.189584 \\
\hline 9 & 6 & 0 & -3.607440 & 0.281296 & 1.223852 \\
\hline 10 & 6 & 0 & -3.619491 & 0.925501 & -1.222857 \\
\hline 11 & 6 & 0 & -3.516214 & -1.489559 & -0.584416 \\
\hline 12 & 1 & 0 & 4.384892 & 0.478883 & 0.048095 \\
\hline 13 & 1 & 0 & 1.303176 & -2.513258 & -0.127647 \\
\hline 14 & 1 & 0 & -1.056586 & -1.883274 & -0.174518 \\
\hline 15 & 1 & 0 & -2.985160 & -0.222305 & 1.965569 \\
\hline 16 & 1 & 0 & -3.515648 & 1.362283 & 1.379489 \\
\hline 17 & 1 & 0 & -5.479288 & 0.262290 & 0.760919 \\
\hline 18 & 1 & 0 & -3.360456 & 0.588623 & -2.233106 \\
\hline 19 & 1 & 0 & -3.157685 & 1.901960 & -1.045055 \\
\hline 20 & 1 & 0 & -5.381879 & 1.658312 & -1.675183 \\
\hline 21 & 1 & 0 & -2.918693 & -1.824699 & -1.436186 \\
\hline 22 & 1 & 0 & -4.559008 & -1.454152 & -0.910564 \\
\hline 23 & 1 & 0 & -4.014596 & -2.241472 & 1.122317 \\
\hline 24 & 1 & 0 & -0.958791 & 1.189428 & -0.068599 \\
\hline 25 & 7 & 0 & -1.641019 & 0.116904 & -0.147769 \\
\hline 26 & 8 & 0 & -4.933550 & -0.166024 & 1.433218 \\
\hline 27 & 8 & 0 & -5.031960 & 1.004643 & -1.060259 \\
\hline 28 & 8 & 0 & -3.345597 & -2.436835 & 0.454731 \\
\hline 29 & 8 & 0 & 0.063780 & 1.811235 & 0.005144 \\
\hline 30 & 7 & 0 & 2.838466 & 2.579270 & 0.148562 \\
\hline 31 & 8 & 0 & 2.165140 & 3.375514 & 0.765536 \\
\hline 32 & 8 & 0 & 3.893878 & 2.848899 & -0.396638 \\
\hline 33 & 7 & 0 & 3.956383 & -2.154794 & -0.056114 \\
\hline 34 & 8 & 0 & 3.588794 & -3.310893 & -0.146920 \\
\hline 35 & 8 & 0 & 5.122514 & -1.815790 & 0.016370 \\
\hline
\end{tabular}

\section{$\underline{\text { Structure of } 60}$}

Zero-point correction $=0.263968$ (Hartree/Particle) Thermal correction to Energy $=0.284416$ Thermal correction to Enthalpy $=0.285360$

Thermal correction to Gibbs Free Energy $=0.213906$

Sum of electronic and zero-point Energies= -1192.486257 Sum of electronic and thermal Energies= -1192.465809

Sum of electronic and thermal Enthalpies= -1192.464865

Sum of electronic and thermal Free Energies= -1192.536319

No imaginary frequencies

Standard orientation:

\begin{tabular}{|c|c|c|c|c|c|}
\hline \multirow{2}{*}{$\begin{array}{l}\text { Center } \\
\text { Number }\end{array}$} & \multirow{2}{*}{$\begin{array}{l}\text { Atomic } \\
\text { Number }\end{array}$} & \multirow{2}{*}{$\begin{array}{c}\text { Atomic } \\
\text { Type }\end{array}$} & \multicolumn{3}{|c|}{ Coordinates (Angstroms) } \\
\hline & & & $\mathrm{X}$ & $\mathrm{Y}$ & Z \\
\hline 1 & 6 & 0 & 1.083349 & 1.060724 & -0.006216 \\
\hline 2 & 6 & 0 & 2.534710 & 1.164028 & 0.051412 \\
\hline 3 & 6 & 0 & 3.377298 & 0.091199 & 0.015030 \\
\hline 4 & 6 & 0 & 2.838319 & -1.202652 & -0.025410 \\
\hline 5 & 6 & 0 & 1.472073 & -1.407583 & -0.050454 \\
\hline 6 & 6 & 0 & 0.603856 & -0.319171 & -0.048778 \\
\hline 7 & 6 & 0 & -0.792646 & -0.601495 & -0.106583 \\
\hline 8 & 6 & 0 & -3.167900 & 0.049810 & -0.168534 \\
\hline 9 & 6 & 0 & -3.673762 & 0.141284 & 1.288953 \\
\hline 10 & 6 & 0 & -3.780840 & 1.153550 & -1.035707 \\
\hline 11 & 6 & 0 & -3.502577 & -1.317378 & -0.777795 \\
\hline 12 & 1 & 0 & 4.450398 & 0.237736 & 0.033504 \\
\hline 13 & 1 & 0 & 1.072557 & -2.415186 & -0.078197 \\
\hline 14 & 1 & 0 & -1.105902 & -1.642314 & -0.116912 \\
\hline 15 & 1 & 0 & -2.999103 & -0.425647 & 1.932579 \\
\hline 16 & 1 & 0 & -3.658945 & 1.187617 & 1.613788 \\
\hline 17 & 1 & 0 & -5.553530 & 0.062363 & 0.859619 \\
\hline 18 & 1 & 0 & -3.543449 & 0.971537 & -2.089929 \\
\hline 19 & 1 & 0 & -3.365817 & 2.122539 & -0.737817 \\
\hline 20 & 1 & 0 & -5.597455 & 1.814313 & -1.353153 \\
\hline 21 & 1 & 0 & -2.880853 & -1.480512 & -1.661412 \\
\hline 22 & 1 & 0 & -4.544244 & -1.290758 & -1.107076 \\
\hline
\end{tabular}




\begin{tabular}{rrrrrr}
23 & 1 & 0 & -3.965322 & -2.352401 & 0.785274 \\
24 & 7 & 0 & -1.719299 & 0.304972 & -0.145598 \\
25 & 8 & 0 & -4.957570 & -0.434394 & 1.435203 \\
26 & 8 & 0 & -5.185592 & 1.128546 & -0.816425 \\
27 & 8 & 0 & -3.281118 & -2.400255 & 0.106014 \\
28 & 8 & 0 & 0.317352 & 2.031774 & -0.053276 \\
29 & 1 & 0 & -1.391019 & 1.278419 & -0.108325 \\
30 & 7 & 0 & 3.148432 & 2.484217 & 0.136002 \\
31 & 8 & 0 & 2.605542 & 3.322372 & 0.823350 \\
32 & 8 & 0 & 4.188378 & 2.668869 & -0.473944 \\
33 & 7 & 0 & 3.734927 & -2.338004 & -0.034962 \\
34 & 8 & 0 & 4.935161 & -2.122958 & -0.016673 \\
35 & 8 & 0 & 3.250721 & -3.456440 & -0.063692 \\
\hline
\end{tabular}

\section{$\underline{\text { Structure of } \mathbf{3 1} \text { (open form) }}$}

Zero-point correction $=0.293156$ (Hartree/Particle)
Thermal correction to Energy $=0.314062$
Thermal correction to Enthalpy= 0.315006
Thermal correction to Gibbs Free Energy= 0.242639
Sum of electronic and zero-point Energies=-1102.468614
Sum of electronic and thermal Energies=-1102.447708
Sum of electronic and thermal Enthalpies=-1102.446764
Sum of electronic and thermal Free Energies=-1102.519130
No imaginary frequencies

Standard orientation:

\begin{tabular}{|c|c|c|c|c|c|}
\hline \multirow{2}{*}{$\begin{array}{l}\text { Center } \\
\text { Number }\end{array}$} & \multirow{2}{*}{$\begin{array}{l}\text { Atomic } \\
\text { Number }\end{array}$} & \multirow{2}{*}{$\begin{array}{c}\text { Atomic } \\
\text { Type }\end{array}$} & \multicolumn{3}{|c|}{ Coordinates (Angstroms) } \\
\hline & & & $\mathrm{X}$ & $\mathrm{Y}$ & Z \\
\hline & & & 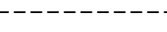 & ----- & ---------- \\
\hline 1 & 6 & 0 & 1.228668 & 1.194496 & 0.009226 \\
\hline 2 & 6 & 0 & 2.635237 & 1.350056 & 0.075407 \\
\hline 3 & 6 & 0 & 3.475320 & 0.258501 & 0.042466 \\
\hline 4 & 6 & 0 & 2.889427 & -1.004927 & -0.061099 \\
\hline 5 & 6 & 0 & 1.525711 & -1.189490 & -0.127095 \\
\hline 6 & 6 & 0 & 0.667479 & -0.084218 & -0.091985 \\
\hline 7 & 6 & 0 & -0.778145 & -0.378333 & -0.163223 \\
\hline 8 & 6 & 0 & -3.095594 & 0.078833 & -0.161851 \\
\hline 9 & 6 & 0 & -3.640490 & 0.330516 & 1.262638 \\
\hline 10 & 6 & 0 & -3.779364 & 1.026306 & -1.152534 \\
\hline 11 & 6 & 0 & -3.379540 & -1.367305 & -0.588824 \\
\hline 12 & 1 & 0 & 4.549968 & 0.362210 & 0.092177 \\
\hline 13 & 1 & 0 & 1.112040 & -2.186879 & -0.206921 \\
\hline 14 & 1 & 0 & -0.988896 & -1.449022 & -0.241365 \\
\hline 15 & 1 & 0 & -2.951557 & -0.105242 & 1.988458 \\
\hline 16 & 1 & 0 & -3.688183 & 1.409960 & 1.444876 \\
\hline 17 & 1 & 0 & -5.494077 & 0.065437 & 0.802025 \\
\hline 18 & 1 & 0 & -3.492750 & 0.764427 & -2.178138 \\
\hline 19 & 1 & 0 & -3.451302 & 2.048011 & -0.941977 \\
\hline 20 & 1 & 0 & -5.631678 & 1.528937 & -1.575776 \\
\hline 21 & 1 & 0 & -2.754328 & -1.626093 & -1.446919 \\
\hline 22 & 1 & 0 & -4.420895 & -1.433910 & -0.917258 \\
\hline 23 & 1 & 0 & -3.791644 & -2.187533 & 1.105941 \\
\hline 24 & 1 & 0 & 1.059692 & 3.062362 & 0.111445 \\
\hline 25 & 7 & 0 & -1.692926 & 0.497793 & -0.123724 \\
\hline 26 & 8 & 0 & -4.900922 & -0.285558 & 1.479286 \\
\hline 27 & 8 & 0 & -5.192538 & 0.910845 & -0.982652 \\
\hline 28 & 8 & 0 & -3.120241 & -2.324941 & 0.426498 \\
\hline 29 & 8 & 0 & 0.471313 & 2.294361 & 0.043660 \\
\hline 30 & 7 & 0 & 3.766074 & -2.174265 & -0.104060 \\
\hline 31 & 8 & 0 & 4.968370 & -1.988629 & -0.066985 \\
\hline 32 & 8 & 0 & 3.254687 & -3.275647 & -0.177097 \\
\hline 33 & 8 & 0 & 3.026789 & 2.640942 & 0.168415 \\
\hline 34 & 6 & 0 & 4.427308 & 2.907988 & 0.235503 \\
\hline 35 & 1 & 0 & 4.861204 & 2.452211 & 1.128717 \\
\hline 36 & 1 & 0 & 4.930267 & 2.535209 & -0.660222 \\
\hline 37 & 1 & 0 & 4.524337 & 3.989689 & 0.288660 \\
\hline
\end{tabular}




\section{$\underline{\text { Structure of } \mathbf{3 1} \text { (closed form) }}$}

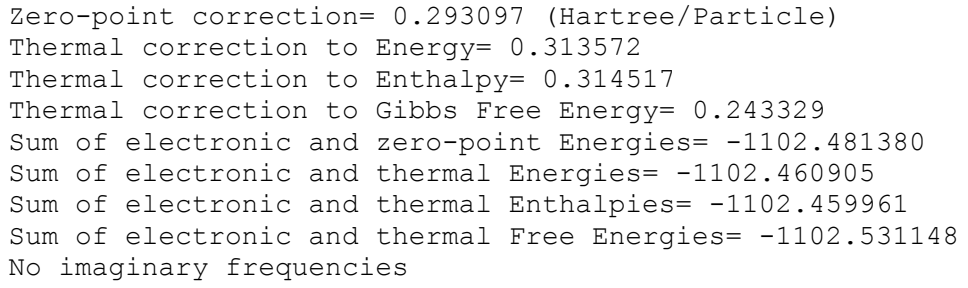

Standard orientation:

\begin{tabular}{|c|c|c|c|c|c|}
\hline \multirow{2}{*}{$\begin{array}{l}\text { Center } \\
\text { Number }\end{array}$} & \multirow{2}{*}{$\begin{array}{l}\text { Atomic } \\
\text { Number }\end{array}$} & \multirow{2}{*}{$\begin{array}{c}\text { Atomic } \\
\text { Type }\end{array}$} & \multicolumn{3}{|c|}{ Coordinates (Angstroms) } \\
\hline & & & $\mathrm{X}$ & $\mathrm{Y}$ & Z \\
\hline 1 & 6 & 0 & 1.104777 & 1.099485 & 0.014773 \\
\hline 2 & 6 & 0 & 2.490997 & 1.405373 & 0.064161 \\
\hline 3 & 6 & 0 & 3.418744 & 0.383587 & 0.041026 \\
\hline 4 & 6 & 0 & 2.969978 & -0.940578 & -0.039897 \\
\hline 5 & 6 & 0 & 1.634846 & -1.271269 & -0.096266 \\
\hline 6 & 6 & 0 & 0.689928 & -0.242958 & -0.066796 \\
\hline 7 & 6 & 0 & -0.735684 & -0.577036 & -0.133387 \\
\hline 8 & 6 & 0 & -3.047877 & 0.038526 & -0.144372 \\
\hline 9 & 6 & 0 & -3.564019 & 0.110379 & 1.309510 \\
\hline 10 & 6 & 0 & -3.669688 & 1.159007 & -0.983030 \\
\hline 11 & 6 & 0 & -3.402288 & -1.315578 & -0.774417 \\
\hline 12 & 1 & 0 & 4.479741 & 0.584145 & 0.080745 \\
\hline 13 & 1 & 0 & 1.314694 & -2.303650 & -0.159803 \\
\hline 14 & 1 & 0 & -0.984759 & -1.637735 & -0.215608 \\
\hline 15 & 1 & 0 & -2.908771 & -0.483290 & 1.949348 \\
\hline 16 & 1 & 0 & -3.529372 & 1.149659 & 1.655420 \\
\hline 17 & 1 & 0 & -5.433983 & 0.059862 & 0.838076 \\
\hline 18 & 1 & 0 & -3.401134 & 1.024696 & -2.037753 \\
\hline 19 & 1 & 0 & -3.272747 & 2.117285 & -0.634426 \\
\hline 20 & 1 & 0 & -5.481275 & 1.836299 & -1.313402 \\
\hline 21 & 1 & 0 & -2.769546 & -1.480764 & -1.650159 \\
\hline 22 & 1 & 0 & -4.438594 & -1.275245 & -1.121941 \\
\hline 23 & 1 & 0 & -3.893161 & -2.350939 & 0.777317 \\
\hline 24 & 1 & 0 & -0.676166 & 1.689751 & 0.006616 \\
\hline 25 & 7 & 0 & -1.618334 & 0.342938 & -0.093183 \\
\hline 26 & 8 & 0 & -4.866038 & -0.436732 & 1.441937 \\
\hline 27 & 8 & 0 & -5.085155 & 1.114128 & -0.814630 \\
\hline 28 & 8 & 0 & -3.212894 & -2.419127 & 0.096301 \\
\hline 29 & 8 & 0 & 0.241526 & 2.102782 & 0.044399 \\
\hline 30 & 7 & 0 & 3.965233 & -2.008366 & -0.065118 \\
\hline 31 & 8 & 0 & 5.140315 & -1.700195 & 0.018135 \\
\hline 32 & 8 & 0 & 3.575794 & -3.157358 & -0.167909 \\
\hline 33 & 8 & 0 & 2.790319 & 2.715611 & 0.133036 \\
\hline 34 & 6 & 0 & 4.169542 & 3.062173 & 0.192213 \\
\hline 35 & 1 & 0 & 4.640203 & 2.641431 & 1.085274 \\
\hline 36 & 1 & 0 & 4.696646 & 2.718607 & -0.702406 \\
\hline 37 & 1 & 0 & 4.203465 & 4.148342 & 0.239541 \\
\hline
\end{tabular}

\section{$\underline{\text { Structure of } \mathbf{T S}_{31 / 61}}$}

Zero-point correction= 0.289233 (Hartree/Particle)

Thermal correction to Energy $=0.309317$

Thermal correction to Enthalpy $=0.310261$

Thermal correction to Gibbs Free Energy= 0.239744

Sum of electronic and zero-point Energies= -1102.480652

Sum of electronic and thermal Energies= -1102.460568

Sum of electronic and thermal Enthalpies= -1102.459624

Sum of electronic and thermal Free Energies= -1102.530141

one imaginary frequency: 1073i

Standard orientation:

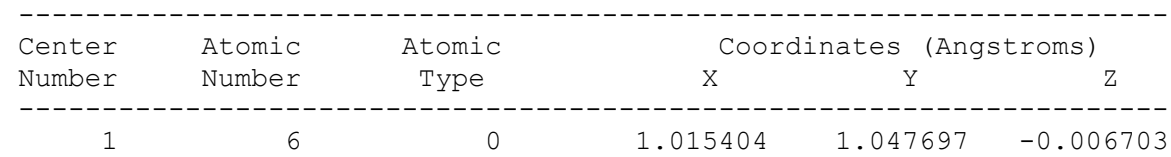




\begin{tabular}{|c|c|c|c|c|c|}
\hline 2 & 6 & 0 & 2.402752 & 1.420202 & 0.067375 \\
\hline 3 & 6 & 0 & 3.379045 & 0.454289 & 0.042486 \\
\hline 4 & 6 & 0 & 3.014115 & -0.905619 & -0.057255 \\
\hline 5 & 6 & 0 & 1.704603 & -1.314847 & -0.129396 \\
\hline 6 & 6 & 0 & 0.703617 & -0.339568 & -0.103661 \\
\hline 7 & 6 & 0 & -0.689669 & -0.716863 & -0.171837 \\
\hline 8 & 6 & 0 & -3.025827 & -0.015638 & -0.167628 \\
\hline 9 & 6 & 0 & -3.529505 & 0.266297 & 1.267040 \\
\hline 10 & 6 & 0 & -3.603371 & 1.008019 & -1.149712 \\
\hline 11 & 6 & 0 & -3.421289 & -1.427161 & -0.609605 \\
\hline 12 & 1 & 0 & 4.427853 & 0.710016 & 0.097262 \\
\hline 13 & 1 & 0 & 1.446241 & -2.364136 & -0.201889 \\
\hline 14 & 1 & 0 & -0.952997 & -1.772226 & -0.232234 \\
\hline 15 & 1 & 0 & -2.879011 & -0.246388 & 1.977783 \\
\hline 16 & 1 & 0 & -3.470476 & 1.342556 & 1.465334 \\
\hline 17 & 1 & 0 & -5.407762 & 0.205666 & 0.833855 \\
\hline 18 & 1 & 0 & -3.354071 & 0.720901 & -2.177711 \\
\hline 19 & 1 & 0 & -3.165517 & 1.989214 & -0.939652 \\
\hline 20 & 1 & 0 & -5.390368 & 1.721141 & -1.530445 \\
\hline 21 & 1 & 0 & -2.827038 & -1.716264 & -1.480099 \\
\hline 22 & 1 & 0 & -4.469116 & -1.408382 & -0.920775 \\
\hline 23 & 1 & 0 & -3.875070 & -2.250324 & 1.076200 \\
\hline 24 & 7 & 0 & -1.581860 & 0.210174 & -0.149863 \\
\hline 25 & 8 & 0 & -4.838091 & -0.231503 & 1.480043 \\
\hline 26 & 8 & 0 & -5.014659 & 1.041746 & -0.960558 \\
\hline 27 & 8 & 0 & -3.212498 & -2.406952 & 0.392026 \\
\hline 28 & 8 & 0 & 0.086677 & 1.939003 & 0.015956 \\
\hline 29 & 1 & 0 & -0.957729 & 1.270671 & -0.055432 \\
\hline 30 & 7 & 0 & 4.067352 & -1.904445 & -0.079846 \\
\hline 31 & 8 & 0 & 5.223784 & -1.526147 & 0.009990 \\
\hline 32 & 8 & 0 & 3.753801 & -3.078393 & -0.187374 \\
\hline 33 & 8 & 0 & 2.623951 & 2.745144 & 0.162604 \\
\hline 34 & 6 & 0 & 3.975746 & 3.172148 & 0.269150 \\
\hline 35 & 1 & 0 & 4.449360 & 2.753082 & 1.161821 \\
\hline 36 & 1 & 0 & 4.547825 & 2.890864 & -0.619782 \\
\hline 37 & 1 & 0 & 3.941976 & 4.256596 & 0.350712 \\
\hline
\end{tabular}

\section{$\underline{\text { Structure of } 61}$}

Zero-point correction $=0.293874$ (Hartree/Particle) Thermal correction to Energy $=0.314332$

Thermal correction to Enthalpy $=0.315276$

Thermal correction to Gibbs Free Energy= 0.244055

Sum of electronic and zero-point Energies= -1102.481818

Sum of electronic and thermal Energies $=-1102.461360$

Sum of electronic and thermal Enthalpies= -1102.460416

Sum of electronic and thermal Free Energies= -1102.531637

No imaginary frequencies

Standard orientation:

\begin{tabular}{|c|c|c|c|c|c|}
\hline \multirow{2}{*}{$\begin{array}{l}\text { Center } \\
\text { Number }\end{array}$} & \multirow{2}{*}{$\begin{array}{l}\text { Atomic } \\
\text { Number }\end{array}$} & \multirow{2}{*}{$\begin{array}{l}\text { Atomic } \\
\text { Type }\end{array}$} & \multicolumn{3}{|c|}{ Coordinates (Angstroms) } \\
\hline & & & $\mathrm{X}$ & Y & Z \\
\hline 1 & 6 & 0 & 1.117352 & 1.202650 & -0.023354 \\
\hline 2 & 6 & 0 & 2.570177 & 1.411033 & 0.039981 \\
\hline 3 & 6 & 0 & 3.437952 & 0.360442 & 0.045989 \\
\hline 4 & 6 & 0 & 2.936900 & -0.973052 & -0.016281 \\
\hline 5 & 6 & 0 & 1.599750 & -1.248000 & -0.079808 \\
\hline 6 & 6 & 0 & 0.680943 & -0.180228 & -0.083815 \\
\hline 7 & 6 & 0 & -0.698107 & -0.494997 & -0.136529 \\
\hline 8 & 6 & 0 & -3.088229 & 0.100171 & -0.135374 \\
\hline 9 & 6 & 0 & -3.508202 & -0.121169 & 1.333780 \\
\hline 10 & 6 & 0 & -3.773644 & 1.333702 & -0.727996 \\
\hline 11 & 6 & 0 & -3.431675 & -1.128837 & -0.990516 \\
\hline 12 & 1 & 0 & 4.507029 & 0.510558 & 0.094995 \\
\hline 13 & 1 & 0 & 1.243501 & -2.270112 & -0.124209 \\
\hline 14 & 1 & 0 & -0.997621 & -1.540614 & -0.166788 \\
\hline 15 & 1 & 0 & -2.795236 & -0.801879 & 1.803005 \\
\hline 16 & 1 & 0 & -3.468959 & 0.834390 & 1.869148 \\
\hline 17 & 1 & 0 & -5.407482 & -0.141409 & 0.994802 \\
\hline 18 & 1 & 0 & -3.590413 & 1.377465 & -1.807676 \\
\hline 19 & 1 & 0 & -3.360503 & 2.233923 & -0.259763 \\
\hline
\end{tabular}




\begin{tabular}{|c|c|c|c|c|c|}
\hline 20 & 1 & 0 & -5.614336 & 2.001154 & -0.800668 \\
\hline 21 & 1 & 0 & -2.836199 & -1.103615 & -1.905929 \\
\hline 22 & 1 & 0 & -4.485580 & -1.060563 & -1.273592 \\
\hline 23 & 1 & 0 & -3.823336 & -2.459068 & 0.353829 \\
\hline 24 & 7 & 0 & -1.650559 & 0.396568 & -0.146079 \\
\hline 25 & 8 & 0 & -4.781857 & -0.730101 & 1.436871 \\
\hline 26 & 8 & 0 & -5.163930 & 1.225434 & -0.449871 \\
\hline 27 & 8 & 0 & -3.166636 & -2.362077 & -0.347069 \\
\hline 28 & 8 & 0 & 0.325725 & 2.162234 & -0.022617 \\
\hline 29 & 1 & 0 & -1.333242 & 1.372783 & -0.090942 \\
\hline 30 & 7 & 0 & 3.881973 & -2.065286 & -0.008361 \\
\hline 31 & 8 & 0 & 5.072719 & -1.797501 & 0.05900 \\
\hline 32 & 8 & 0 & 3.460281 & -3.211089 & -0.06925 \\
\hline 33 & 8 & 0 & 2.926500 & 2.708007 & 0.089507 \\
\hline 34 & 6 & 0 & 4.316459 & 2.991928 & 0.157149 \\
\hline 35 & 1 & 0 & 4.759490 & 2.567513 & 1.06326 \\
\hline 36 & 1 & 0 & 4.838404 & 2.603225 & -0.722761 \\
\hline 37 & 1 & 0 & 4.404121 & 4.076169 & 0.18241 \\
\hline
\end{tabular}

\section{$\underline{\text { Structure of } 84 \text { (open form) }}$}

Zero-point correction= 0.261913 (Hartree/Particle) Thermal correction to Energy $=0.278754$

Thermal correction to Enthalpy $=0.279699$

Thermal correction to Gibbs Free Energy= 0.217335

Sum of electronic and zero-point Energies $=-858.720556$

Sum of electronic and thermal Energies= -858.703715

Sum of electronic and thermal Enthalpies $=-858.702770$

Sum of electronic and thermal Free Energies= -858.765134

No imaginary frequencies

Standard orientation:

\begin{tabular}{|c|c|c|c|c|c|}
\hline \multirow{2}{*}{$\begin{array}{l}\text { Center } \\
\text { Number }\end{array}$} & \multirow{2}{*}{$\begin{array}{l}\text { Atomic } \\
\text { Number }\end{array}$} & \multirow{2}{*}{$\begin{array}{c}\text { Atomic } \\
\text { Type }\end{array}$} & \multicolumn{3}{|c|}{ Coordinates (Angstroms) } \\
\hline & & & $\mathrm{X}$ & $\mathrm{Y}$ & Z \\
\hline 1 & 6 & 0 & -2.194591 & -0.982703 & 0.138051 \\
\hline 2 & 6 & 0 & -3.584961 & -1.028959 & 0.191093 \\
\hline 3 & 6 & 0 & -4.334510 & 0.129498 & 0.017561 \\
\hline 4 & 6 & 0 & -3.703861 & 1.352707 & -0.210912 \\
\hline 5 & 6 & 0 & -2.320910 & 1.381153 & -0.262461 \\
\hline 6 & 6 & 0 & -1.527705 & 0.238756 & -0.093793 \\
\hline 7 & 6 & 0 & -0.072150 & 0.429956 & -0.182586 \\
\hline 8 & 6 & 0 & 2.223868 & -0.131541 & -0.136721 \\
\hline 9 & 6 & 0 & 2.760575 & -0.155969 & 1.310130 \\
\hline 10 & 6 & 0 & 2.861020 & -1.265459 & -0.946319 \\
\hline 11 & 6 & 0 & 2.571651 & 1.204984 & -0.808700 \\
\hline 12 & 1 & 0 & -4.093685 & -1.971487 & 0.367900 \\
\hline 13 & 1 & 0 & -4.286886 & 2.257333 & -0.345076 \\
\hline 14 & 1 & 0 & -1.821954 & 2.328358 & -0.441015 \\
\hline 15 & 1 & 0 & 0.203441 & 1.471398 & -0.380338 \\
\hline 16 & 1 & 0 & 2.109285 & 0.451711 & 1.941132 \\
\hline 17 & 1 & 0 & 2.735103 & -1.184611 & 1.687338 \\
\hline 18 & 1 & 0 & 4.622196 & -0.098025 & 0.807430 \\
\hline 19 & 1 & 0 & 2.564935 & -1.181572 & -1.999215 \\
\hline 20 & 1 & 0 & 2.497361 & -2.218562 & -0.552460 \\
\hline 21 & 1 & 0 & 4.677892 & -1.937080 & -1.267129 \\
\hline 22 & 1 & 0 & 1.935214 & 1.346382 & -1.685964 \\
\hline 23 & 1 & 0 & 3.605771 & 1.157931 & -1.162723 \\
\hline 24 & 1 & 0 & 3.067172 & 2.277067 & 0.714578 \\
\hline 25 & 1 & 0 & -2.076333 & -2.852632 & 0.450277 \\
\hline 26 & 7 & 0 & 0.804035 & -0.479897 & -0.044612 \\
\hline 27 & 8 & 0 & 4.063954 & 0.400015 & 1.419225 \\
\hline 28 & 8 & 0 & 4.281666 & -1.183169 & -0.818331 \\
\hline 29 & 8 & 0 & 2.390691 & 2.337142 & 0.029246 \\
\hline 30 & 8 & 0 & -1.473088 & -2.111515 & 0.305900 \\
\hline 31 & 8 & 0 & -5.681487 & 0.008299 & 0.081390 \\
\hline 32 & 1 & 0 & -6.092183 & 0.872358 & -0.053950 \\
\hline
\end{tabular}




\section{$\underline{\text { Structure of } 84 \text { (closed form) }}$}

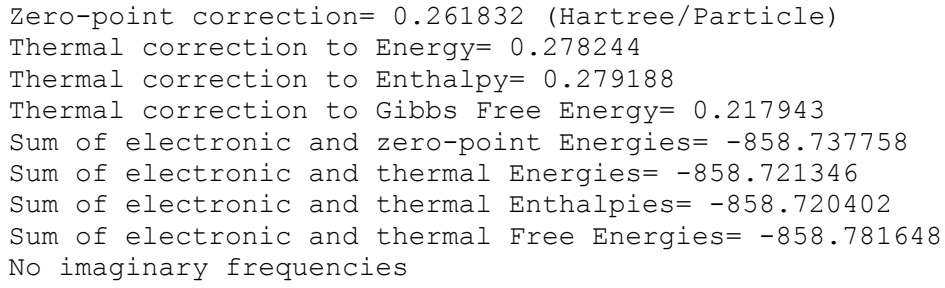

Standard orientation:

\begin{tabular}{|c|c|c|c|c|c|}
\hline \multirow{2}{*}{$\begin{array}{l}\text { Center } \\
\text { Number }\end{array}$} & \multirow{2}{*}{$\begin{array}{l}\text { Atomic } \\
\text { Number }\end{array}$} & \multirow{2}{*}{$\begin{array}{c}\text { Atomic } \\
\text { Type }\end{array}$} & \multicolumn{3}{|c|}{ Coordinates (Angstroms) } \\
\hline & & & $\mathrm{X}$ & Y & Z \\
\hline 1 & 6 & 0 & -2.078825 & -0.908558 & 0.132064 \\
\hline 2 & 6 & 0 & -3.456712 & -1.094024 & 0.208341 \\
\hline 3 & 6 & 0 & -4.307391 & -0.007435 & 0.044712 \\
\hline 4 & 6 & 0 & -3.805506 & 1.277951 & -0.196509 \\
\hline 5 & 6 & 0 & -2.436100 & 1.451250 & -0.271030 \\
\hline 6 & 6 & 0 & -1.548599 & 0.381103 & -0.111297 \\
\hline 7 & 6 & 0 & -0.112043 & 0.609903 & -0.204691 \\
\hline 8 & 6 & 0 & 2.167476 & -0.114859 & -0.140687 \\
\hline 9 & 6 & 0 & 2.691963 & -0.161479 & 1.310824 \\
\hline 10 & 6 & 0 & 2.728761 & -1.294458 & -0.940360 \\
\hline 11 & 6 & 0 & 2.594554 & 1.193030 & -0.820508 \\
\hline 12 & 1 & 0 & -3.860889 & -2.082522 & 0.391596 \\
\hline 13 & 1 & 0 & -2.028088 & 2.439919 & -0.457107 \\
\hline 14 & 1 & 0 & 0.197889 & 1.640924 & -0.394774 \\
\hline 15 & 1 & 0 & 2.070100 & 0.482758 & 1.934915 \\
\hline 16 & 1 & 0 & 2.609876 & -1.185652 & 1.692538 \\
\hline 17 & 1 & 0 & 4.557273 & -0.213655 & 0.823446 \\
\hline 18 & 1 & 0 & 2.457107 & -1.189174 & -1.997611 \\
\hline 19 & 1 & 0 & 2.291906 & -2.219846 & -0.553119 \\
\hline 20 & 1 & 0 & 4.504430 & -2.073548 & -1.250634 \\
\hline 21 & 1 & 0 & 1.977247 & 1.358588 & -1.707066 \\
\hline 22 & 1 & 0 & 3.629107 & 1.087226 & -1.159848 \\
\hline 23 & 1 & 0 & 3.130795 & 2.256636 & 0.695945 \\
\hline 24 & 1 & 0 & -0.340855 & -1.638588 & 0.204389 \\
\hline 25 & 7 & 0 & 0.725850 & -0.346360 & -0.066661 \\
\hline 26 & 8 & 0 & 4.021080 & 0.325114 & 1.420435 \\
\hline 27 & 8 & 0 & 4.147328 & -1.310330 & -0.785044 \\
\hline 28 & 8 & 0 & 2.460250 & 2.338147 & 0.006818 \\
\hline 29 & 8 & 0 & -1.277757 & -1.969231 & 0.291679 \\
\hline 30 & 1 & 0 & -4.484816 & 2.113987 & -0.321725 \\
\hline 31 & 8 & 0 & -5.637258 & -0.248740 & 0.129247 \\
\hline 32 & 1 & 0 & -6.126635 & 0.573253 & -0.004545 \\
\hline
\end{tabular}

\section{$\underline{\text { Structure of } \mathbf{T S}_{84 / 38 \mathrm{~S}}}$}

Zero-point correction $=0.257554$ (Hartree/Particle)

Thermal correction to Energy $=0.273650$

Thermal correction to Enthalpy $=0.274594$

Thermal correction to Gibbs Free Energy $=0.213466$

Sum of electronic and zero-point Energies $=-858.733712$

Sum of electronic and thermal Energies= -858.717617

Sum of electronic and thermal Enthalpies= -858.716673

Sum of electronic and thermal Free Energies= -858.777800

One imaginary frequency: $1008 i$

Standard orientation:

\begin{tabular}{|c|c|c|c|c|c|}
\hline \multirow{2}{*}{$\begin{array}{l}\text { Center } \\
\text { Number }\end{array}$} & \multirow{2}{*}{$\begin{array}{l}\text { Atomic } \\
\text { Number }\end{array}$} & \multirow{2}{*}{$\begin{array}{c}\text { Atomic } \\
\text { Type }\end{array}$} & \multicolumn{3}{|c|}{ Coordinates (Angstroms) } \\
\hline & & & $\mathrm{X}$ & $\mathrm{Y}$ & Z \\
\hline 1 & 6 & 0 & -1.962932 & -0.802042 & -0.254318 \\
\hline 2 & 6 & 0 & -3.342130 & -1.092402 & -0.324588 \\
\hline 3 & 6 & 0 & -4.275483 & -0.108552 & -0.058014 \\
\hline 4 & 6 & 0 & -3.898065 & 1.207669 & 0.290234 \\
\hline 5 & 6 & 0 & -2.558671 & 1.509173 & 0.363193 \\
\hline 6 & 6 & 0 & -1.578664 & 0.536712 & 0.099812 \\
\hline
\end{tabular}




$\begin{array}{rrrrrr}7 & 6 & 0 & -0.185530 & 0.848396 & 0.169911 \\ 8 & 6 & 0 & 2.132460 & 0.129205 & -0.067994 \\ 9 & 6 & 0 & 2.756871 & -1.237397 & -0.402996 \\ 10 & 6 & 0 & 2.482281 & 1.156671 & -1.150753 \\ 11 & 6 & 0 & 2.631540 & 0.625340 & 1.296882 \\ 12 & 1 & 0 & -3.665323 & -2.091859 & -0.590306 \\ 13 & 1 & 0 & -4.657290 & 1.955646 & 0.491055 \\ 14 & 1 & 0 & -2.240942 & 2.513073 & 0.628278 \\ 15 & 1 & 0 & 0.126657 & 1.860576 & 0.431694 \\ 16 & 1 & 0 & 2.248289 & -2.011190 & 0.175475 \\ 17 & 1 & 0 & 2.602971 & -1.454482 & -1.466728 \\ 18 & 1 & 0 & 4.569959 & -0.597811 & -0.539927 \\ 19 & 1 & 0 & 2.160495 & 2.157569 & -0.839507 \\ 20 & 1 & 0 & 1.960089 & 0.885744 & -2.074074 \\ 21 & 1 & 0 & 4.127024 & 1.721657 & -2.059028 \\ 22 & 1 & 0 & 1.991044 & 1.438471 & 1.644826 \\ 23 & 1 & 0 & 3.640610 & 1.029591 & 1.173608 \\ 24 & 1 & 0 & 3.315679 & -1.000116 & 2.071399 \\ 25 & 1 & 0 & 0.049585 & -1.061615 & -0.336736 \\ 26 & 7 & 0 & 0.683923 & -0.078567 & -0.079500 \\ 27 & 8 & 0 & 4.128072 & -1.305988 & -0.053334 \\ 28 & 8 & 0 & 3.893740 & 1.120945 & -1.343269 \\ 29 & 8 & 0 & 2.608512 & -0.381289 & 2.289552 \\ 30 & 8 & 0 & -1.058929 & -1.698994 & -0.497088 \\ 31 & 8 & 0 & -5.582772 & -0.452794 & -0.143569 \\ 32 & 1 & 0 & -6.135351 & 0.313025 & 0.059930 \\ ----------------------------------------1\end{array}$

\section{$\underline{\text { Structure of } \mathbf{3 8 S}}$}

Zero-point correction $=0.261972$ (Hartree/Particle) Thermal correction to Energy $=0.278523$

Thermal correction to Enthalpy= 0.279467

Thermal correction to Gibbs Free Energy= 0.217327

Sum of electronic and zero-point Energies $=\mathbf{- 8 5 8 . 7 3 4 0 3 9}$

Sum of electronic and thermal Energies $=-858.717488$

Sum of electronic and thermal Enthalpies $=-858.716543$

Sum of electronic and thermal Free Energies $=-858.778683$

No imaginary frequencies

Standard orientation:

\begin{tabular}{|c|c|c|c|c|c|}
\hline \multirow{2}{*}{$\begin{array}{l}\text { Center } \\
\text { Number }\end{array}$} & \multirow{2}{*}{$\begin{array}{l}\text { Atomic } \\
\text { Number }\end{array}$} & \multirow{2}{*}{$\begin{array}{c}\text { Atomic } \\
\text { Type }\end{array}$} & \multicolumn{3}{|c|}{ Coordinates (Angstroms) } \\
\hline & & & $\mathrm{X}$ & Y & Z \\
\hline 1 & 6 & 0 & -2.067231 & -0.994572 & 0.112575 \\
\hline 2 & 6 & 0 & -3.493106 & -1.118192 & 0.194092 \\
\hline 3 & 6 & 0 & -4.310678 & -0.024363 & 0.062835 \\
\hline 4 & 6 & 0 & -3.797952 & 1.287602 & -0.156558 \\
\hline 5 & 6 & 0 & -2.445568 & 1.450073 & -0.239051 \\
\hline 6 & 6 & 0 & -1.554620 & 0.351900 & -0.112668 \\
\hline 7 & 6 & 0 & -0.175628 & 0.595413 & -0.206720 \\
\hline 8 & 6 & 0 & 2.189672 & -0.132284 & -0.141957 \\
\hline 9 & 6 & 0 & 2.679524 & -0.083538 & 1.321713 \\
\hline 10 & 6 & 0 & 2.778672 & -1.342142 & -0.872412 \\
\hline 11 & 6 & 0 & 2.587269 & 1.147249 & -0.888390 \\
\hline 12 & 1 & 0 & -3.922293 & -2.099961 & 0.358466 \\
\hline 13 & 1 & 0 & -4.479561 & 2.125439 & -0.253284 \\
\hline 14 & 1 & 0 & -2.025795 & 2.438113 & -0.404370 \\
\hline 15 & 1 & 0 & 0.167924 & 1.616006 & -0.357384 \\
\hline 16 & 1 & 0 & 2.024259 & 0.577467 & 1.891767 \\
\hline 17 & 1 & 0 & 2.613653 & -1.086633 & 1.759138 \\
\hline 18 & 1 & 0 & 4.563554 & -0.128818 & 0.907708 \\
\hline 19 & 1 & 0 & 2.555801 & -1.275217 & -1.943482 \\
\hline 20 & 1 & 0 & 2.328176 & -2.257105 & -0.472938 \\
\hline 21 & 1 & 0 & 4.571226 & -2.109717 & -1.076052 \\
\hline 22 & 1 & 0 & 1.974448 & 1.244490 & -1.787714 \\
\hline 23 & 1 & 0 & 3.627763 & 1.043348 & -1.207557 \\
\hline 24 & 1 & 0 & 3.086064 & 2.309134 & 0.568284 \\
\hline 25 & 7 & 0 & 0.740591 & -0.340444 & -0.119347 \\
\hline 26 & 8 & 0 & 3.989511 & 0.446347 & 1.430619 \\
\hline 27 & 8 & 0 & 4.183569 & -1.341637 & -0.643288 \\
\hline 28 & 8 & 0 & 2.414802 & 2.327775 & -0.125055 \\
\hline 29 & 8 & 0 & -1.298127 & -1.986076 & 0.227936 \\
\hline
\end{tabular}


0.041468 


\section{References}

(1) a) Chumakov, Y. M.; Antosyak, B. Y.; Mazus, M. D.; Tsapkov, V. I.; Samus, N. M. Crystal Structures of N-(Salicylidene)Tris(hydroxymethyl)methylamine and $N$-(5-Chlorosalicylidene)-Tris(hydroxymethyl)methylamine. Crystal. Rep. 2000, 45, 945950; b) Asgedom, G.; Sreedhara, A.; Kivikoski, J.; Valkonen, J.; Kolehmainen, E.; Rao, C. P. Alkoxo Bound Monooxo- and Dioxovanadium(V) Complexes: Synthesis, Characterization, X-ray Crystal Structures, and Solution Reactivity Studies. Inorg. Chem. 1996, 35, 5674-5683; c) Cungen, Z.; Peizi, Z.; Dan, W.; Kaibei, Y. Evidence of Proton Transfer from the Hydroxy O Atom to the Imine N Atom, Crystal structure of N-Salicylideneamine-1-Tris(Hydroxymethyl)Methane. J. Chem. Res. 2000, 402-403; d) Odabasoglu, M.; Albayrak, M.; Büyükgüngör, O.; Lönnecke, P. 2-\{[Tris(hydroxymethyl)methyl]aminomethylene\}cyclohexa-3,5dien-1(2H)-one and its 6-hydroxy and 6-methoxy derivatives. Acta Cryst. 2003, C59, 616-619; e) Chumakov, Y. M.; Tsapkov, V. I.; Bocelli, G.; Antosyak, B. Y.; Gulya, A. P. Crystal Structures of 6-[(2-hydroxy-1,1-bis-hydroxymethyl-ethylamino)methylene]-4-nitro-cyclohexa-2,4-dienone hydrate and 6-[(2-hydroxy-1,1-bis-hydroxymethyl-ethylamino)-methylene]-4bromo-cyclohexa-2,4-dienone. J. Struct. Chem, 2006, 47, 346-351.

(2) Martínez, R. F.; Ávalos, M.; Babiano, R.; Cintas, P.; Jiménez, J. L.; Light, M. E.; Palacios, J. C. Schiff Bases from TRIS and ortho-Hydroxyarenecarbaldehydes: Structures and Tautomeric Equilibria in the Solid State and in Solution. Eur. J. Org. Chem. 2011, 3137-3145.

(3) Odababaşoğlu, M.; Albayrak, Ç.; Özkanza, R.; Aykan, F. Z.; Lonecke, P. Some Polyhydroxy Azo-Azomethine Derivatives of Salicylaldehyde: Synthesis, Characterization, Spectroscopic, Molecular Structure and Antimicrobial Activity Studies. J. Mol. Struct. 2007, 840, 71-89.

(4) Stepien, B. T.; Cyrański, M. K.; Krygowski, T. M. Aromaticity Strongly Affected by Substituents in Fulvene and Heptafulvene as a New Method of Estimating the Resonance Effect. Chem. Phys. Lett. 2001, 350, 537-542.

(5) a) Kamieński, B.; Schilf, W.; Dziembowska, T.; Rozwadoski, Z.; Szady-Chelmieniecka, A. The 15N and 13C Solid State NMR Study of Intramolecular Hydrogen Bond in Some Schiff's Bases. Solid State NMR 2000, 16, 285-289; b) Schilf, W.; Kamieński, B.; Dziembowska, T.; Rozwadoski, Z.; Szady-Chelmieniecka, A. ${ }^{15}$ N NMR Study of the Intramolecular Hydrogen Bond in $N$-Salicylidene-alkylamines. J. Mol. Struct. 2000, 552, 33-37.

(6) a) Martínez, R. F.; Ávalos, M.; Babiano, R.; Cintas, P.; Jiménez, J. L.; Light, M. E.; Palacios, J. C.; Pérez, E. M. S. An Anomeric Effect Drives the Regiospecific Ring-Opening of 1,3-Oxazolidines under Acetylating Conditions Eur. J. Org. Chem. 2010, 5263-5273. b) Ibid. Schiff Bases from TRIS and Formylpyridines: Structure and Mechanistic Rationale Aided by DFT Calculations Eur. J. Org. Chem. 2010, 6224-6232.

(7) Maiereanu, C.; Darabantu, M.; Plé, G.; Berghian, C.; Condamine, E.; Ramondenc, Y.; Silaghi-Dumitrescu, I.; Mager, S. Ringchain tautomerism and other versatile behaviour of 1,4-diimino- and 1,2-phenylene derivatives of some C-substituted serinols. Tetrahedron, 2002, 58, 2681-2693.

(8) a) Kruszewski, J.; Krygowski, T. M. Definition of Aromaticity Basing on the Harmonic Oscillator Model. Tetrahedron Lett. 1972, 13, 3839-3842; b) Krygowski, T. M. Crystallographic Studies of Inter- and Intramolecular Interactions Reflected in Aromatic Character of $\pi$-Electron Systems. J. Chem. Inf. Comput. Sci. 1993, 33, 70-78. 